\title{
THE EFFECTS OF SURROUNDING VEGETATION, BUILDING CONSTRUCTION AND HUMAN FACTORS ON THE THERMAL PERFORMANCE OF HOUSING IN A TROPICAL ENVIRONMENT
}

By

ALAMAH MISNI

A thesis

Submitted to the Victoria University of Wellington in fulfilment of the requirements for the degree of Doctor of Philosophy in Architecture

Victoria University of Wellington 


\begin{abstract}
Increasing energy consumption is having a detrimental effect on the environment. This issue combined with rising energy costs, is motivating people to reduce energy consumption. Moderating a building's surrounding microclimate naturally through strategic landscaping has the potential to benefit the environment, save energy, save money and provide comfortable living environments.
\end{abstract}

The urban heat island effect is a well documented phenomenon, which influences the climate of most of the major cities around the world. It occurs when the air temperature in densely built urban areas is higher by $2^{\circ} \mathrm{C}$ to $8^{\circ} \mathrm{C}$ compared to the temperature of the surrounding rural environment. This issue is of particular concern in tropical areas, which experience high temperatures and humidity all year round. In these areas, solar heat passes through a building's envelope via glazed windows and the walls and roofs resulting in uncomfortable interior spaces. The increased purchasing power of the population has resulted in greater use of air-conditioners to create and maintain a comfortable indoor temperature. This study found that the average household uses up to $37 \%$ of their electricity consumption for cooling.

Careful planning of exterior spaces can help reduce energy consumption for cooling by reducing the adverse impact of some climatic factors. Strategically placed vegetation around a building has long been recognised as a means of cooling. It can reduce temperatures and humidity through shading, evapotranspiration and wind channelling.

The aim of this study was to examine and quantify the relationship between surrounding vegetation, and the thermal performance of housing in a hot-humid tropical environment. The primary objective was to determine the energy saving potential of vegetation for the tropical residence. The secondary objective was to investigate the effect of vegetation on modifying the outdoor temperature around a single-family house in a hot-humid climate. 
Monitoring of household electricity use in the two Malaysian cities, Shah Alam and Putrajaya, has shown that at night time, when families are at home, is when airconditioning is used the most. Building surfaces on the east and west side are most affected by the sun, gaining and storing heat throughout the day until night time, when it is released into the house as the outdoor temperatures cool. Planting the right species, size and shape of trees, shrubs, vines, groundcover, and turf in strategic positions around a garden can greatly reduce the temperature around buildings. This in turn reduces the energy used for air conditioning. This study found that strategic landscaping, which resulted in shading and encouraged evapotranspiration and wind channelling, could reduce electricity use and costs by as much as $20 \%$.

The physical characteristics of buildings including their construction, size and age, combined with their landscape designs were looked at in 50 private houses in Malaysia. Measurements were taken from several outdoor and indoor locations around the houses. The findings showed that strategic design of landscaping could reduce heat build-up in a house, by shading, evapotranspiration and wind channelling by as much as $4^{\circ} \mathrm{C}$ for the exterior and $3^{\circ} \mathrm{C}$ for the interior spaces.

These results demonstrate how strategic landscaping can assist in creating a favourable microclimate in a house, which will help reduce energy consumption. Its effect can extend beyond the residential to have a positive influence on an area's macroclimate and at a regional scale. 


\section{ACKNOWLEDGEMENTS}

Many people deserve recognition for their support and guidance during my studies. I am especially indebted to my primary supervisor, Professor Dr. George Baird, who has been an outstanding advisor and mentor. His patience and understanding was extraordinary. Without his guidance my vision for this study would not have become a reality. I also wish to acknowledge his contribution in motivating and encouraging me. I would like to acknowledge my co-supervisor, Associate Professor Penny Allan, who provided invaluable feedback as the research progressed, and whose enthusiasm for my research was a great motivator. My heartfelt gratitude is also directed to my colleagues and fellow $\mathrm{PhD}$ candidates, especially to Associate Professor Dr. Ahmad Ezanee Hashim, Khadijah Khilmun Kamaruddin, Dr. Amar Dugar, Jestin Nordin, Faridah Muhamad Halil, Abbas Mahravan and others who have given their help and continuous support.

I would like to acknowledge to my sponsors, the Ministry of Higher Education, Malaysia and University of Technology Mara, for providing financial support. I also would like to acknowledge and give special thanks to Education New Zealand and Victoria University of Wellington for awards and grants that allowed me to participate in international conferences. Special thanks go to the Malaysian Meteorological Service Department, Putrajaya Corporation, Shah Alam City Council, Electric Utility Company (TNB) Shah Alam, Malaysian Remote Sensing Data Services, Technical Service Department, Faculty of Architecture, Planning and Surveying, University of Technology Mara and Faculty of Architecture and Design, Victoria University of Wellington, and others institutions and individuals in Malaysia and New Zealand for providing equipment, site study, and climate data.

My family deserves special recognition for their support and prayers during this long journey to complete my studies. Thanks to my parents, and brothers and sister for the support. Their support and inspiration gave me the stamina to be more diligent in my work, and follow my research schedule to complete my study on time. 


\section{TABLE OF CONTENTS}

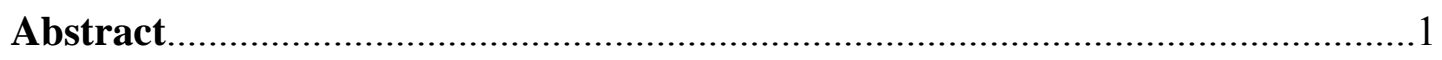

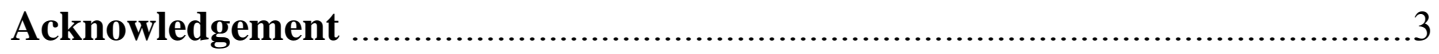

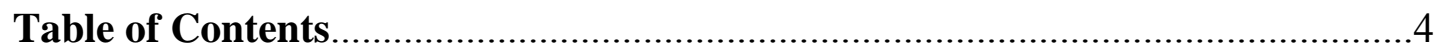

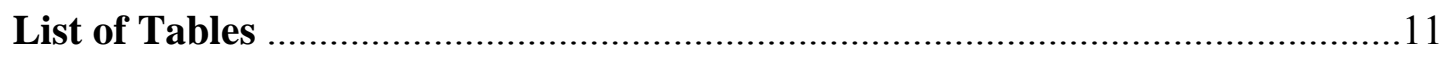

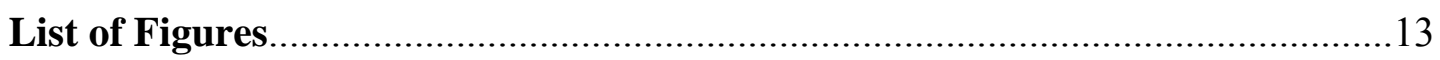

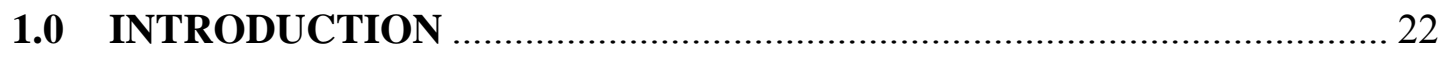

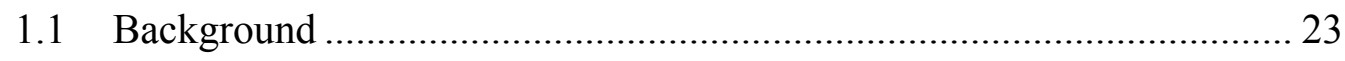

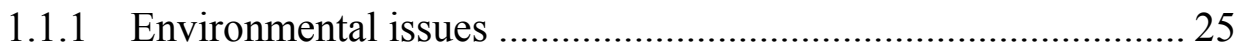

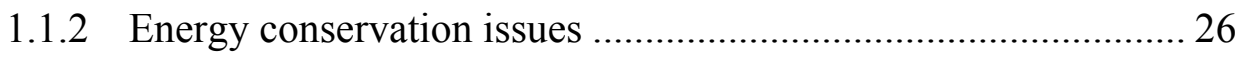

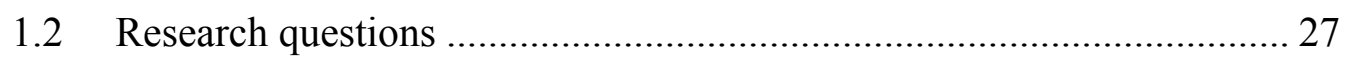

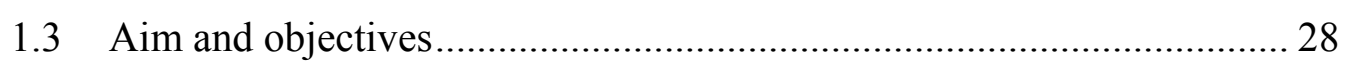

1.3.1 Examine landscaping patterns and structures...........................29

1.3.2 Quantify and validate the results ............................................. 29

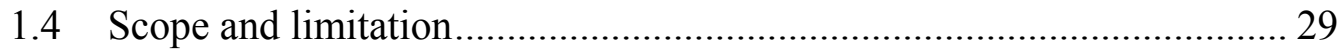

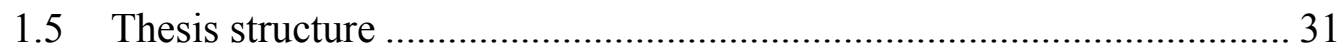

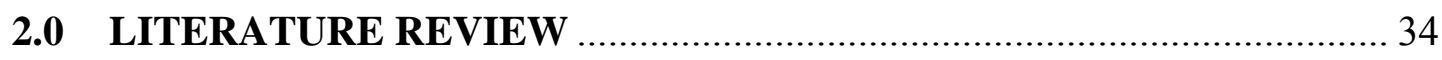

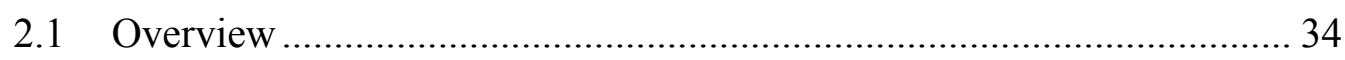

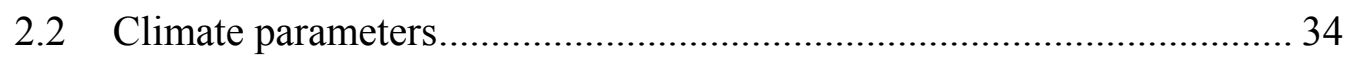

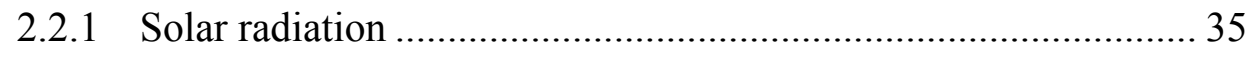

2.2.1.1 Sun path orientation.................................................... 38

2.2.1.2 Sensible heat flow .................................................... 40

2.2.2 Air temperature................................................................ 42

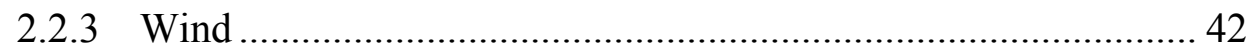

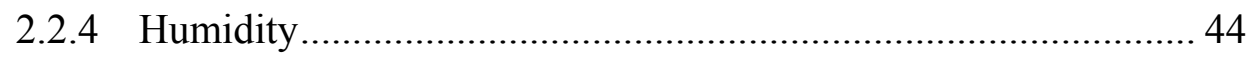

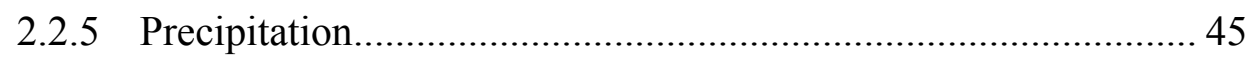

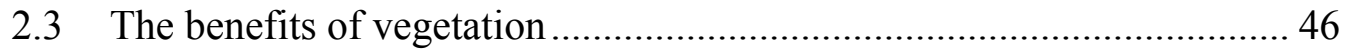

2.3.1 Direct effects of vegetation .................................................... 47

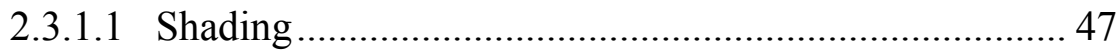


2.3.1.2 Shading and energy use .............................................. 49

2.3.1.3 Wind reduction and channelling............................... 50

2.3.1.4 Wind and energy use .............................................. 53

2.3.2 Indirect effects of vegetation.................................................. 54

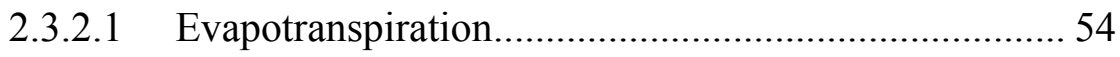

2.3.2.2 Evapotranspiration and energy use............................ 57

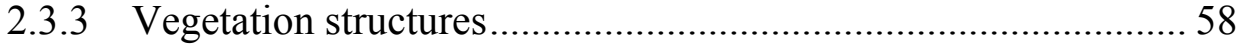

2.3.4 Landscape design typologies: the tropical garden..................... 59

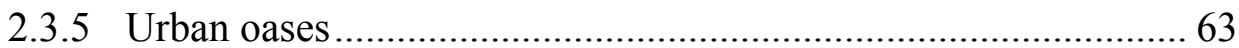

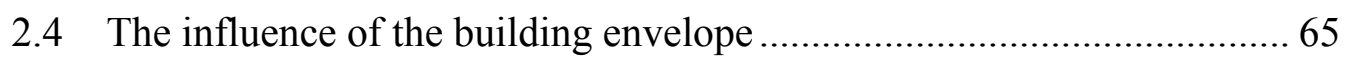

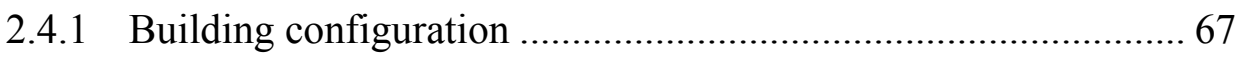

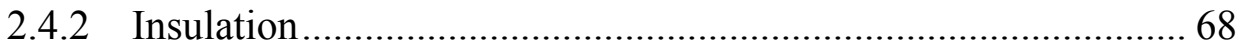

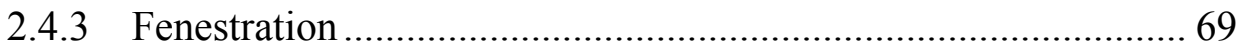

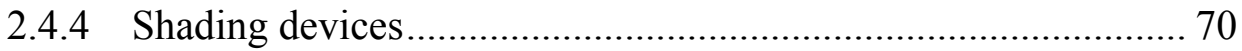

2.4.5 Surface colour ..................................................................... 72

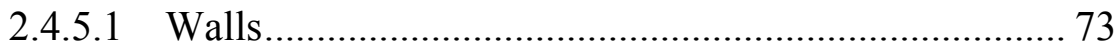

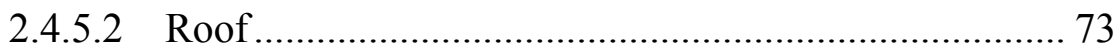

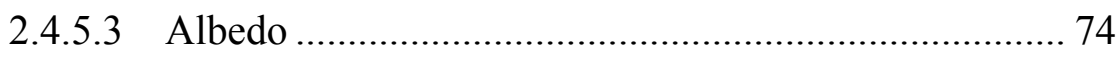

2.4.5.4 Light colour surfaces save energy ............................ 74

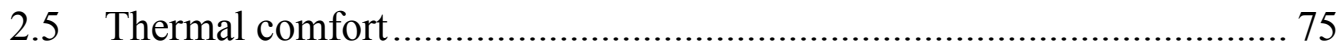

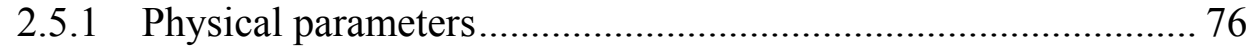

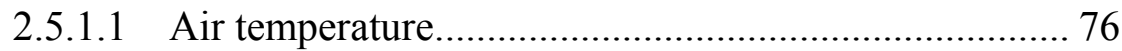

2.5.1.2 Mean radiant temperature .............................................. 77

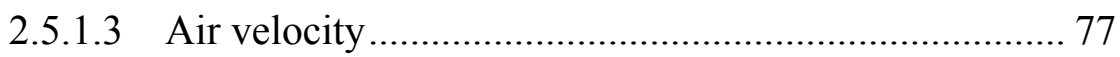

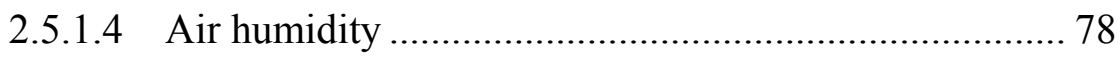

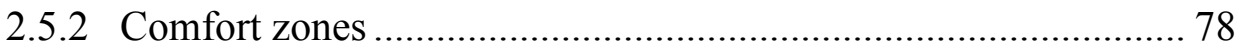

2.5.2.1 Bioclimate chart ........................................................ 79

2.5.2.2 Building Bioclimatic chart ......................................... 80

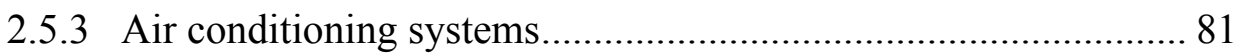

2.5.3.1 Energy efficiency use ............................................. 83

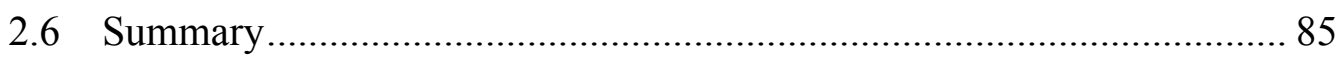




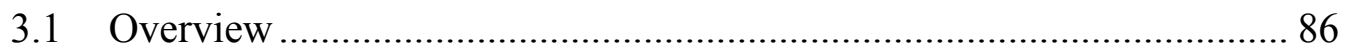

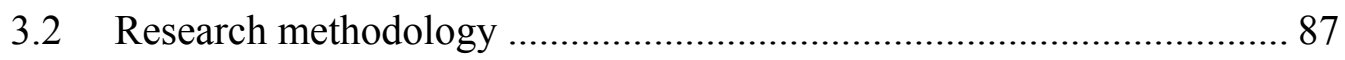

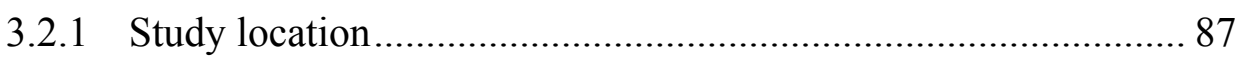

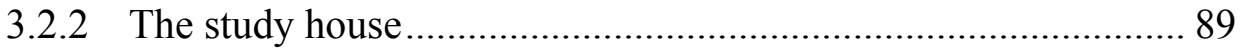

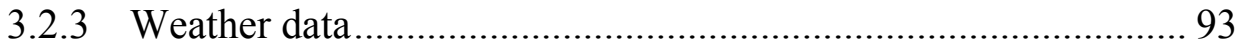

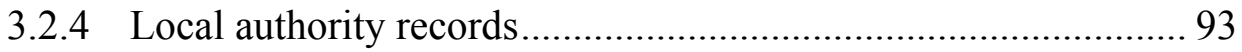

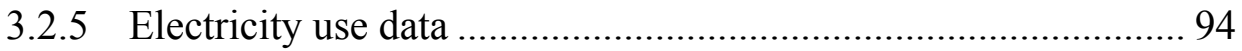

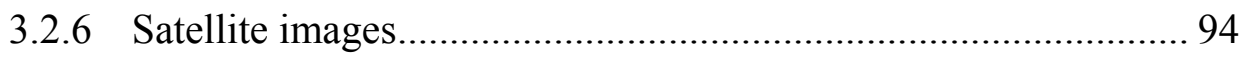

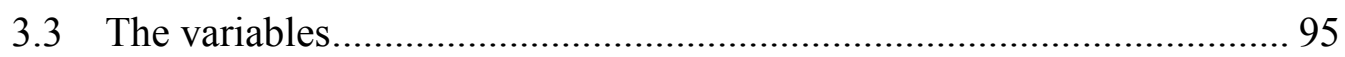

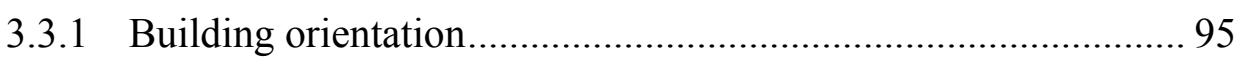

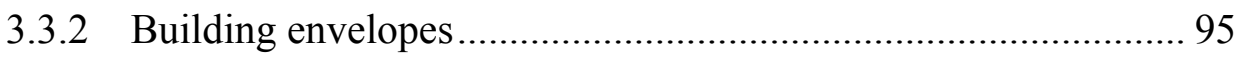

3.3.3 Surrounding vegetation - shading.......................................... 95

3.3.4 Surrounding vegetation - evapotranspiration ............................ 96

3.3.5 Surrounding vegetation - channelling wind .............................. 96

3.3.6 Human factors ..................................................................... 96

3.4 The effect of landscaping on the thermal performance of housing ......... 96

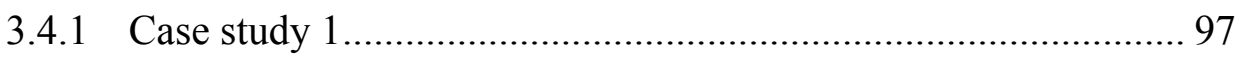

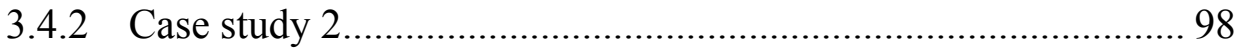

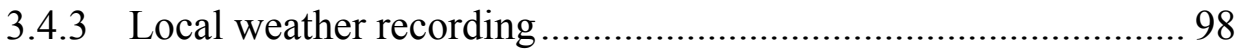

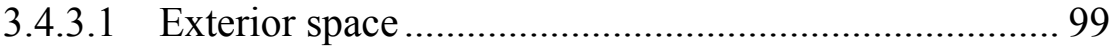

3.4.3.2 Interior space ........................................................ 100

3.4.4 Energy use measurements .................................................... 100

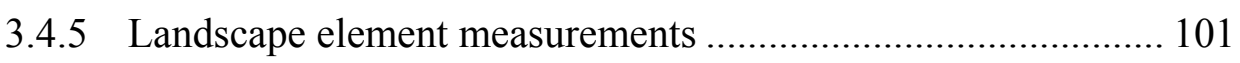

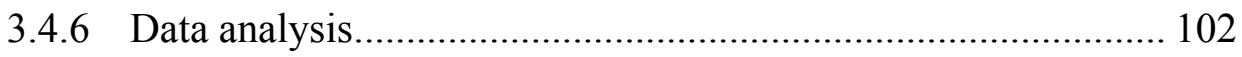

3.5 Landscaping to save air-conditioning energy ..................................... 103

3.5.1 Homeowner interviews.......................................................... 103

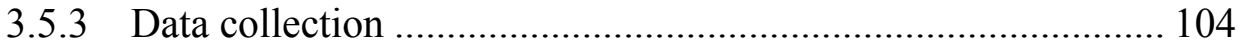

3.5.3.1 Building structure and construction .......................... 105

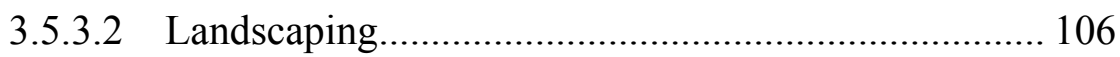

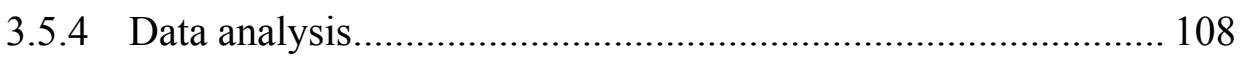

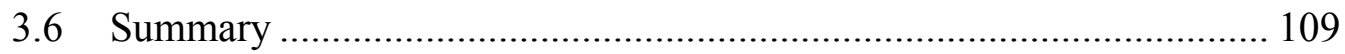




\subsection{THE EFFECTS OF LANDSCAPING ON THE THERMAL}

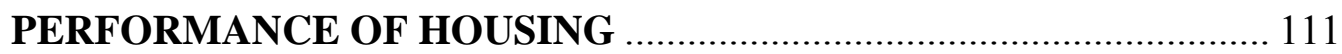

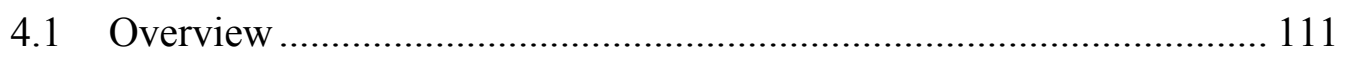

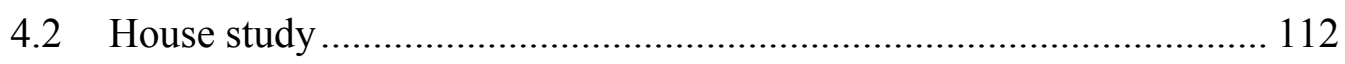

4.2.1 House selection................................................................. 112

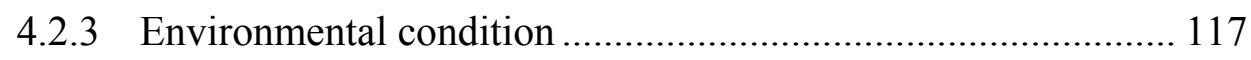

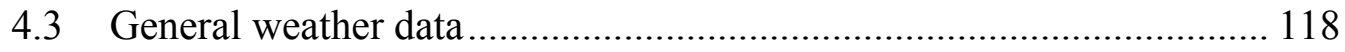

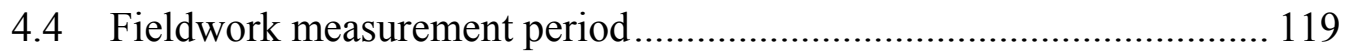

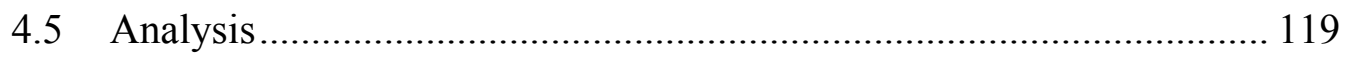

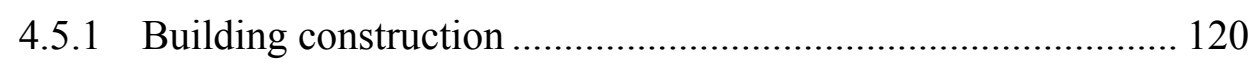

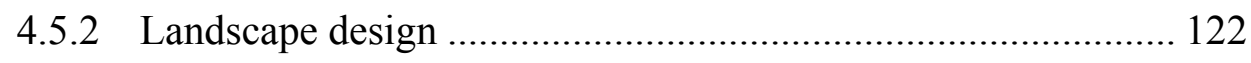

4.5.2.1 Trees ...................................................................... 122

4.5.2.2 Shrubs, vines, and groundcover ............................. 126

4.5.2.3 Other landscape elements ....................................... 132

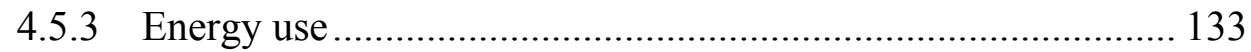

4.5.4 Weather data................................................................... 135

4.5.4.1 Solar radiation and cloud cover .............................. 137

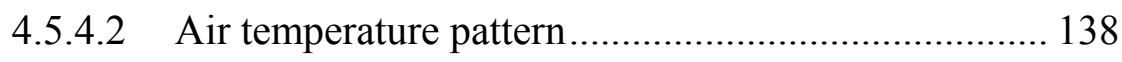

4.5.4.3 Wind speed ...................................................... 143

4.5.4.4 Humidity pattern .................................................... 149

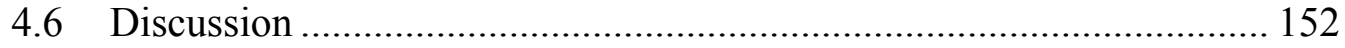

4.6.1 Building construction ............................................................ 153

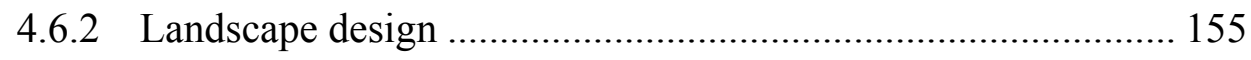

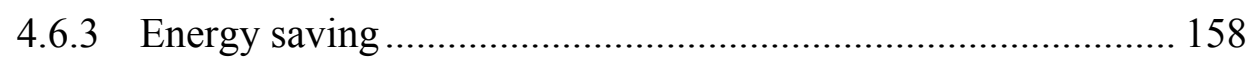

4.6.4 Modifying ambient conditions ............................................. 160

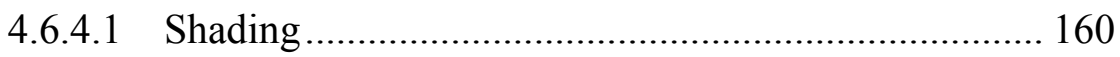

4.6.4.2 Channelling wind ................................................ 163

4.6.4.3 Evapotranspiration................................................... 166

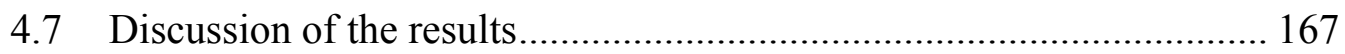

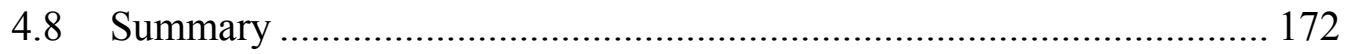




\subsection{THE EFFECTS OF LANDSCAPING ON THE OUTDOOR}

TEMPERATURE AROUND A SINGLE-FAMILY HOUSE ................. 174

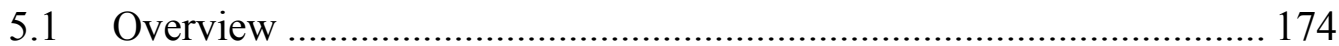

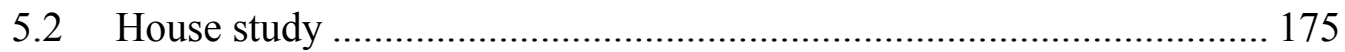

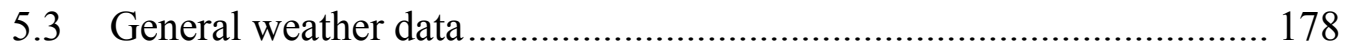

5.4 Fieldwork measurement period ........................................................ 179

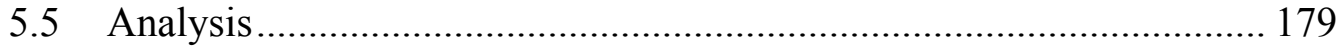

5.5.1 Building construction ........................................................ 180

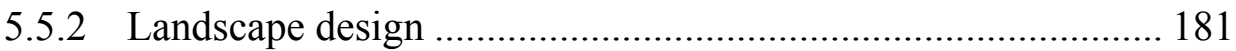

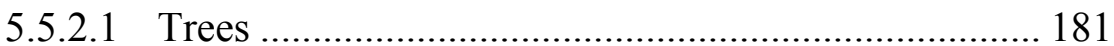

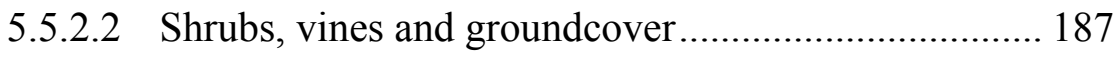

5.5.2.3 Other landscape elements ....................................... 192

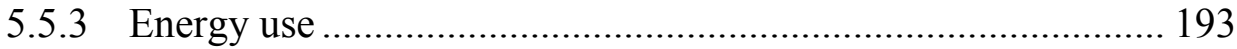

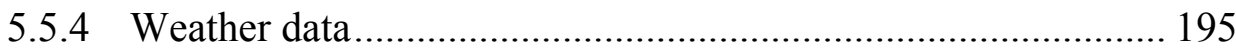

5.5.4.1 Solar radiation and cloud cover............................... 196

5.5.3.2 Air temperature ....................................................... 197

5.5.2.3 Wind speed ............................................................... 203

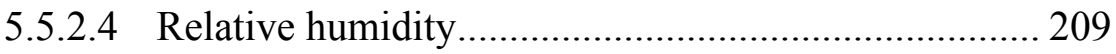

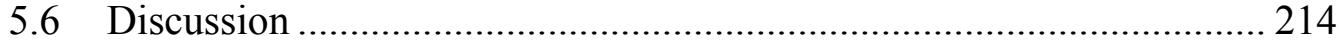

5.6.1 Building construction ........................................................... 214

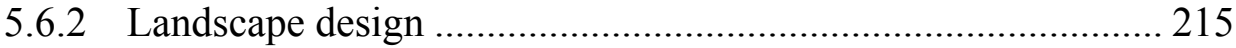

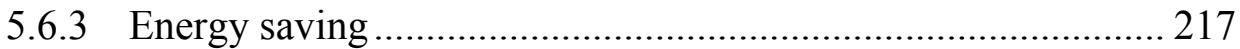

5.6.4 Modifying ambient conditions ............................................... 219

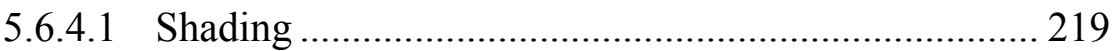

5.6.4.2 Channelling wind ................................................... 222

5.6.4.3 Evapotranspiration.............................................. 225

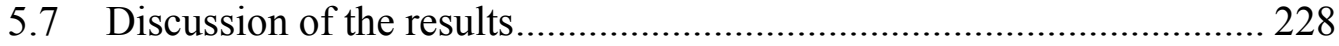

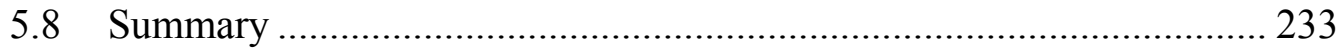

6.0 THE EFFECTS OF SURROUNDING LANDSCAPING ON THE

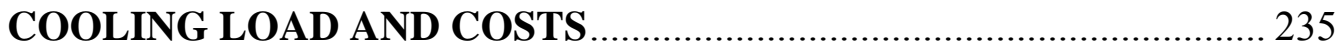

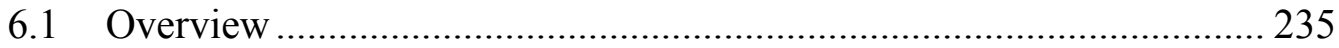




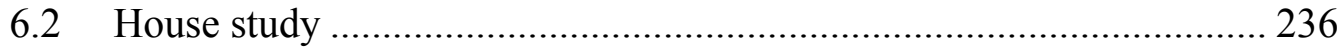

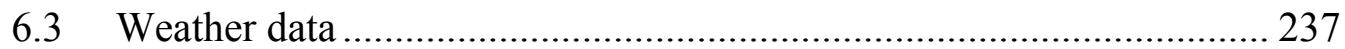

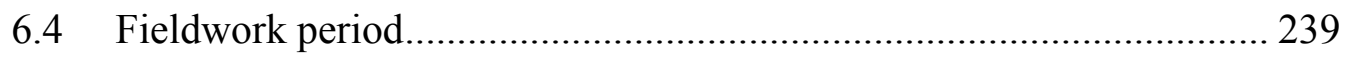

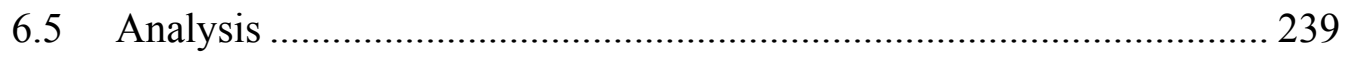

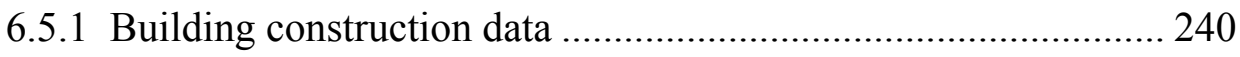

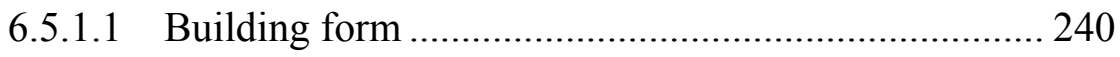

6.5.1.2 Construction types and materials ............................ 242

6.5.1.3 Interior and semi open spaces ............................... 243

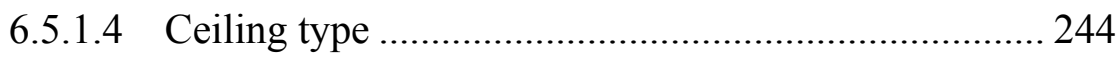

6.5.1.5 Building envelope............................................... 245

6.5.2 Landscape element (soft and hard) data.................................. 250

6.5.2.1 Garden size ........................................................ 250

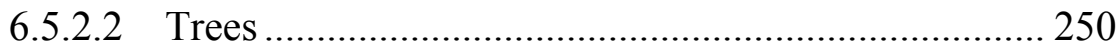

6.5.2.3 Shrubs, vines, groundcover and turf ....................... 255

6.5.2.4 Hard landscape elements ........................................ 261

6.5.3 Energy use data .................................................................. 262

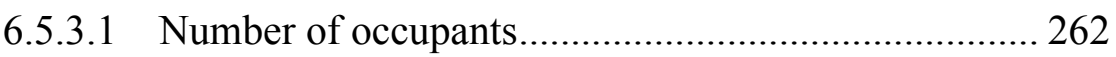

6.5.3.2 Air conditioning systems ....................................... 263

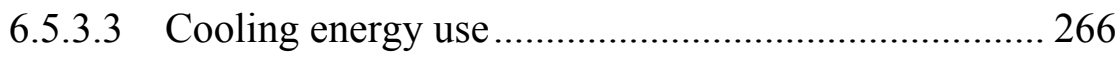

6.5.3.4 General domestic energy use.................................. 267

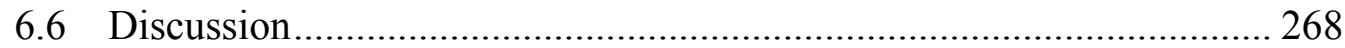

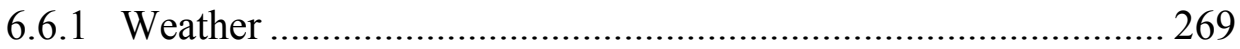

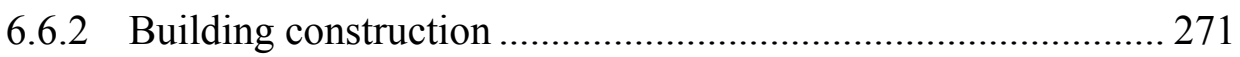

6.6.2.1 Construction type and materials ............................... 272

6.6.2.2 Building orientation............................................. 272

6.6.2.3 Interior and semi open spaces, and ceiling height...... 272

6.6.2.4 Building envelopes ................................................... 273

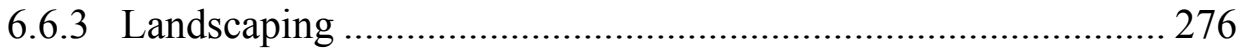

6.6.3.1 Landscaping in neighbourhood areas ....................... 276

4.6.3.2 Landscape design ................................................. 277

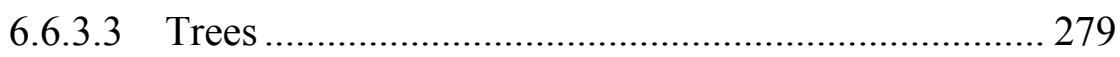

6.6.3.4 Shrubs, vines, groundcover and turf ....................... 282 
6.6.3.5 Hard landscape elements ........................................ 284

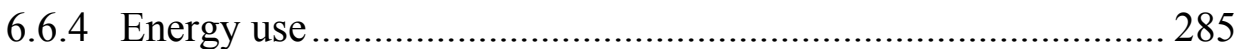

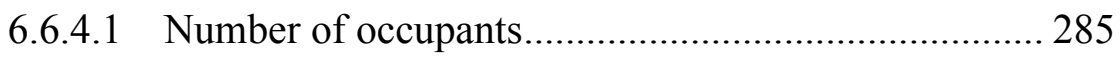

6.6.4.2 Air conditioning systems....................................... 286

6.6.4.3 Cooling energy use ................................................ 286

6.6.4.4 General domestic energy use .................................. 287

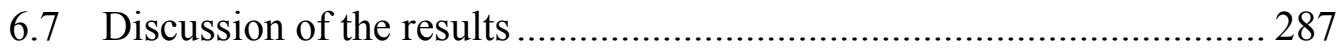

6.7.1 The effectiveness of using landscaping to reduce the cooling

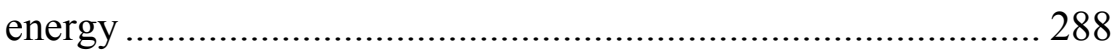

6.7.2 Strategic landscape designed around the house ........................ 291

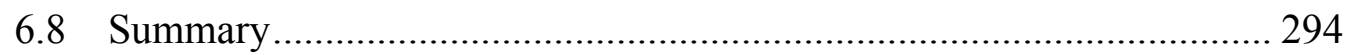

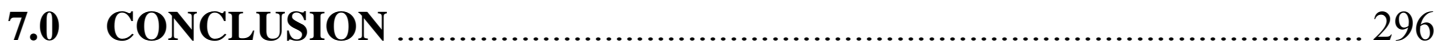

7.1 Examine and quantify the relationship between surrounding vegetation, and the thermal performance of housing ........................... 297

7.1.1 Determine the outside temperature reduction ........................... 298

7.1.1.1 Shading, channelling wind and evapotranspiration ... 298

7.1.2 Energy saving potential of vegetation ..................................... 300

7.1.2.1 Surrounding landscaping ........................................ 300

7.1.2.2 Building construction.............................................. 301

7.1.2.3 Human factors ................................................... 303

7.1.2.4 The effectiveness of using landscaping to reduce the

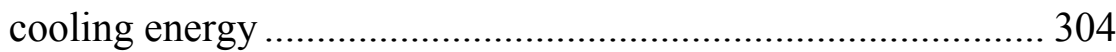

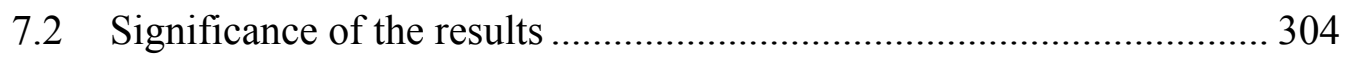

7.2.1 Modifying outdoor temperature and reduce urban heat island

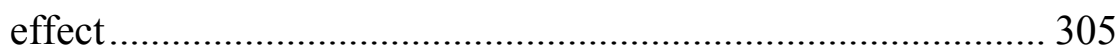

7.2.2 Save energy and costs …...................................................... 305

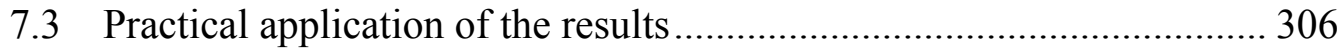

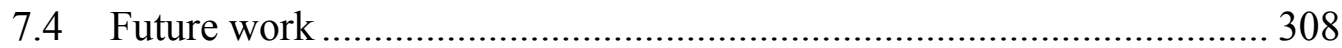

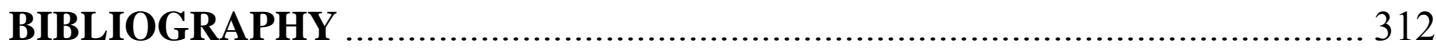


Appendix 1. List of papers

Appendix 2. Approvals to conduct research

Appendix 3. Interview and data collection sheets.

Appendix 4. Landscape elements around mature, ordinary and new

landscape house

Appendix 5. Landscape elements around heavily and sparsely landscaped house

Appendix 6. Soft landscape elements around all houses 361

\section{LIST OF TABLES}

Table 2.1. The U-value is based on an outdoor surface conductance of $22.7 \mathrm{~W} / \mathrm{m}^{2} .{ }^{\circ} \mathrm{C}$, and an outdoor surface conductance of $9.377 \mathrm{~W} / \mathrm{m}^{2} .{ }^{\circ} \mathrm{C}$ (adapted from Olgyay, 1963).

Table 2.2. Predicted electricity consumption, room air conditioners and the percentage fuel mix for electricity generation data (adapted from Mahlia, Masjuki, Choudhury, \& Ghazali, 2002) 84

Table 3.1. Canopy size, shape, and amount and size of leaves for trees. 107

Table 3.2. Size, shape, and amount and size of leaves for shrub, vines and groundcover

Table 4.1. Average daily maximum and minimum temperatures, relative humidity and wind for January, April, July, and October 2008-2009

Table 4.2. Building footprint, floor, roof, albedo and garden areas for the three landscape houses

Table 4.3. Area of brick walls and glazed surfaces for the three landscape houses 121

Table 4.4. Tree-growth indices at the mature landscape house 125

Table 4.5. Tree-growth indices at the ordinary landscape house 125

Table 4.6. Tree-growth indices at the new landscape house. 126

Table 4.7. Shrubs at the mature landscape house. 129

Table 4.8. Shrubs at the ordinary landscape house..... 129

Table 4.9. Shrubs at the new landscape house 130

Table 4.10. List of vines at the mature landscape house. 130 
Table 4.11. List of vines at the ordinary landscape house 130

Table 4.12. List of vines at the new landscape house

Table 4.13. List of groundcovers at the mature landscape house ....

Table 4.14. List of groundcovers at the ordinary landscape house.

Table 4.15. List of groundcovers at the new landscape house.............................. 131

Table 4.16. Air-conditioning use in the three houses............................................ 135

Table 4.17. Average indoor and outdoor air temperatures for all three houses ...... 143

Table 4.18. Averaged wind speeds affecting the three houses ............................. 148

Table 4.19. Averages of relative humidity for the three houses ........................... 152

Table 4.20. Averages of absolute humidity for the three house............................. 152

Table 4.21. Main façade orientation and glass surface areas, surrounding vegetation, weather data and human factors at the mature landscape house (M), ordinary landscape house $(\mathrm{O})$ and new landscape house $(\mathrm{N})$ 168

Table 5.1. Average daily maximum and minimum temperatures, relative humidity and wind for January, April, July and October in Putrajaya 2008-2009.

Table 5.2. Total area of brick walls and glazed surfaces for the heavily landscaped house (house A) and sparsely landscaped house (house B) ................................... 180

Table 5.3. Tree-growth indices at the heavily landscaped house............................ 185

Table 5.4. Tree-growth indices at the sparsely landscaped house .......................... 186

Table 5.5. Shrubs at the heavily landscaped house ............................................... 190

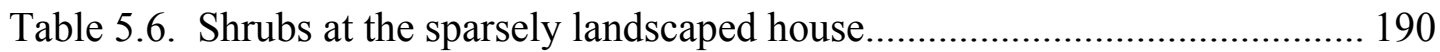

Table 5.7. List of the vines at the heavily landscaped house ................................. 192

Table 5.8. List of the groundcover plants at the heavily landscaped house............ 192

Table 5.9. Air-conditioning use of the heavily landscaped house (house A) and sparsely landscaped house (house B).....

Table 5.10. Façade orientation, surrounding vegetation, weather data and human factors at the heavily landscaped house (A) and the sparsely landscaped house (B)228 Table 6.1. An average floor and roof surfaces .................................................... 240

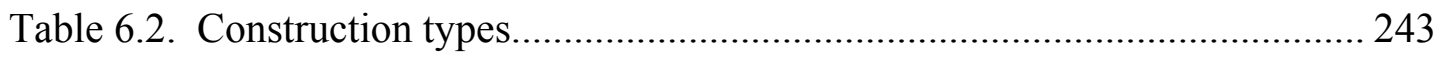

Table 6.3. Ceiling characteristics in the common rooms and porch ...................... 245

Table 6.4. Ceiling characteristic in the bedrooms.................................................. 245

Table 6.5. Average area for all walls for all house age groups ............................. 246 
Table 6.6. The average glazed area for all directions

Table 6.7. The average wall and roof colour, and albedo values.......................... 249

Table 6.8. Average garden size by age of construction ...................................... 250

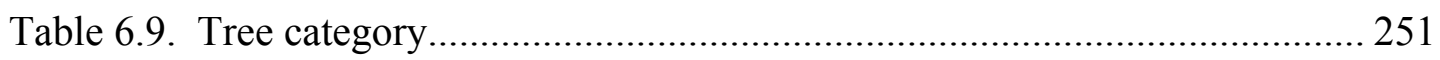

Table 6.10. Summary of tree distribution for all age groups of houses .................. 253

Table 6.11. Tree sizes and distances from the building for all azimuths for all age

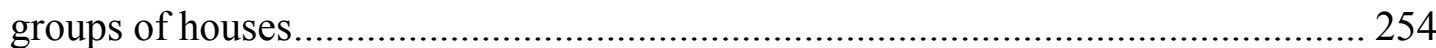

Table 6.12. Categories of shrubs, vines and groundcover .................................. 255

Table 6.13. Shrubs, vines and groundcover by sizes and distances from the building for all azimuths for all age groups of houses ........................................ 257

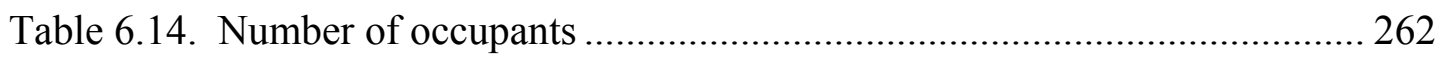

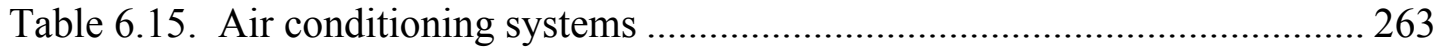

Table 6.16. Air conditioning systems in the common rooms .............................. 264

Table 6.17. Air conditioning systems in the bedrooms........................................ 264

Table 6.18. Electricity bills in the rainy and dry seasons..................................... 266

Table 6.19. Cooling cost in the rainy season and dry seasons .............................. 267

Table 6.20. Summary of two year's annual weather data for Putrajaya and Shah

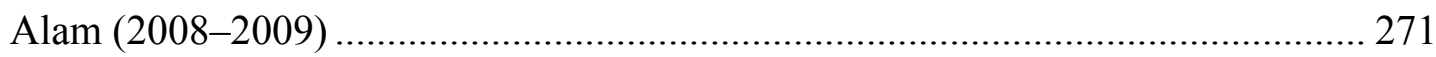

Table 6.21. Summary of the soft landscape elements in the different age groups of houses

\section{LIST OF FIGURES}

Figure 2.1. Passage of radiation through the atmosphere (Koenigsberger, Ingersoll, Mayhew, \& Szokolay, 1980, p. 7)

Figure 2.2. Heat release from the ground and the atmosphere (Koenigsberger, et al., 1980, p. 7)

Figure 2.3. Pattern of the sun's seasonal movement, altitude and azimuth angles at

Latitude $35^{\circ} \mathrm{S}$ (Nervegna, 2003, p. 6) 39

Figure 2.4. A stereographic sun-path diagram for Kuala Lumpur at latitude $3^{\circ} \mathrm{N}$ (GAISMA, 2009, p. 1) 
Figure 2.5. Global wind circulation, unequal heating of the Earth's surface and the Corialis deflection cause a zonal wind system to develop, arranged by the three circulation cells - Hadley, Ferrel and Polar cells (Pinet, 1998, p. 177)

Figure 2.6. In $40^{\circ} \mathrm{S}$ Latitude, deciduous trees on the northwest side of the house reduce indoor air temperature in summer through providing shade, and increase indoor air temperature in winter by allowing sun to enter a house (adapted from $\mathrm{Ha}$, 2009) 48

Figure 2.7. Wind speed reductions for different tree canopy densities as a ratio of wind speed at control site with no trees (McGinn, 1982, p. 65)

Figure 2.8. Use of evergreen trees in two to three rows in a staggered order, along with shrubs will deflect cold winds, while deciduous trees channel summer breezes (adapted from Ha, 2009)

Figure 2.9. Evergreens adjacent to the southwest sides of the house reduce wind speed and create insulation pocket air spaces (adapted from Ha, 2009). 52

Figure 2.10. The arrangement of trees and shrubs influences the movement of wind around and through a building (adapted from Ha, 2009).

Figure 2.11. Selected tropical trees and their "cooling factors" (Paul \& Dieter, 1993, p. 12) 56

Figure 2.12. Temperature reductions in Sacramento (left) and Phoenix (right) 57

Figure 2.13. A sketch-profile drawn of a fruit forest in West Sumatra, Indonesia showing the complex, forest-like structure of the vegetation (adapted from Michon, Foresta, Levang, \& Verdeaux, 2007)

Figure 2.14. The differing structures, types and shapes of shade trees (adapted from Hackett, 1979)

Figure 2.15. The oasis effects in a semi-arid environment. Average daily energy balance of an alfalfa crop in Jun 1964 located at Phoenix, Arizona (33N Latitude). The crop was irrigated by flooding in late May, which was followed by drought throughout June 1967. $\mathrm{Q}_{\mathrm{E}}=$ turbulent latent heat flux density $\left(\mathrm{Wm}^{-2}\right), \mathrm{Q}_{\mathrm{H}}=$ turbulent sensible heat flux density $\left(\mathrm{Wm}^{-2}\right), \mathrm{Q}^{*}=$ net all-wave radiation flux density $\left(\mathrm{Wm}^{-2}\right)$ (Oke, 1978, p. 144)

Figure 2.16. The result of analysis of the heat flow through various components of a typical single storey residential building envelope (adapted from Olgyay, 1963) .... 66 
Figure 2.17. The amount of shades are determine by the depth of overhang (EJCR, 2012)

Figure 2.18. Olgyay's bioclimatic chart (1963, p. 22).

Figure 2.19. Building Bioclimatic chart (Givoni 1969, cited in Lechner, 2000, p. 65).

This is the summary of design strategies as a function of ambient conditions

(climate)

Figure 2.20. Window air conditioner (McDowall, 2007, p. 81) ....

Figure 2.21. Split system unit (McDowall, 2007, p. 88)

Figure 3.1. Putrajaya and Shah Alam were the locations in Malaysia where fieldwork was conducted (Malaysia, 1993). These cities are located at approximately $101^{\circ} \mathrm{E}$ and $3^{\circ} \mathrm{N}$

Figure 3.2. Examples of modern tropical houses in the study areas ranging in age from 3 to 40 years with surrounding landscaping. 89

Figure 3.3. Example of a modern medium-size single-family house, 90

Figure 3.4. Front elevation (left) and left elevation (right) of the house 90

Figure 3.5. Right elevation (left) and rear elevation (right) of the house 91

Figure 3.6. Example the distribution of 5 year old immature vegetation around houses in Puterajaya neighbourhood area (Google Earth, 2011). 92

Figure 3.7. Example the distribution of 30 year old mature vegetation around houses in Shah Alam neighbourhood area (Google Earth, 2011). 92

Figure 3.8. Babuc A (left), mobile TSI VelociCalc Plus Meters (middle), and electronic Mini Thermohygrograph (right)

Figure 3.9. House configuration and the five metre intervals of vegetation measurements and other landscape elements around the house (modified from Simpson, 2002) 102

Figure 4.1. Location of the mature landscape house in Section 6, Shah Alam, Selangor (Google Earth, 2011)

Figure 4.2. Landscape plan of the mature landscape house

Figure 4.3. Front/west elevation of the mature landscape house (left) and view of the garden to the south (right) 
Figure 4.4. Looking towards south elevation of the mature landscape house (left) and view looking south from the car porch (right)

Figure 4.5. Landscape plan of the ordinary landscape house

Figure 4.6. Views of the front/east elevation of the ordinary landscape house looking at the entrance garden.

Figure 4.7. Views of the ordinary landscape house: swimming pool and gazebo at the rear/east side (left), and of the front garden view looking south (right) 116

Figure 4.8. Landscape of the new landscape house

Figure 4.9. Views of the new landscape house: front/south elevation of the house (left), garden view looking south (right).....

Figure 4.10. Views from the west towards the new landscape house

Figure 4.11. Tree distribution compared to tree size and tree azimuth, in relation to building for the three landscape houses: mature landscape house (M), new landscape house $(\mathrm{N})$ and ordinary landscape house $(\mathrm{O})$

Figure 4.12. Shrubs, vines and groundcover distributions in the three landscape houses

Figure 4.13. Window air-conditioning systems for the three houses by horsepower

Figure 4.14. Air-conditioning energy use for the three houses 134

Figure 4.15. Location plan (left) and landscape plan (right) of the mature landscape house

Figure 4.16. Location plan (left) and landscape plan (right) of the ordinary landscape house

Figure 4.17. Location plan (left) and landscape plan (right) of the new landscape house

Figure 4.18. The intensity of surface solar radiation from Subang. 138

Figure 4.19. Outdoor and indoor air temperatures adjacent to the north walls of the three houses

Figure 4.20. Outdoor and indoor air temperatures adjacent to the east walls of the three houses

Figure 4.21. Outdoor and indoor air temperatures adjacent to the south walls of the three houses. 
Figure 4.22. Outdoor and indoor air temperatures adjacent to the west walls of the three houses

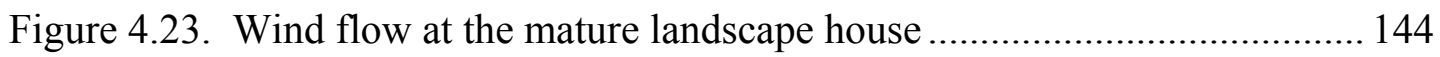

Figure 4.24. Wind flow at the ordinary landscape house..................................... 144

Figure 4.25. Wind flow at the new landscape house.......................................... 145

Figure 4.26. Wind speeds adjacent to the north walls of the three houses ............. 146

Figure 4.27. Wind speeds adjacent to the east walls of the three houses................ 147

Figure 4.28. Wind speeds adjacent to the south walls of the three houses ............. 147

Figure 4.29. Wind speeds adjacent to the west walls of the three houses................ 148

Figure 4.30. Relative humidity adjacent to the north walls of the three houses ..... 150

Figure 4.31. Relative humidity adjacent to the east walls of the three houses ....... 150

Figure 4.32. Relative humidity adjacent to the south walls of the three houses ..... 151

Figure 4.33. Relative humidity adjacent to the west walls of the three houses ...... 151

Figure 4.34. Example of French windows located on the west wall of the new

landscape house, to the living and dining spaces.....

Figure 4.35. East walls and windows exposed to direct sunlight. Low and small shrubs close to walls can only provide shade to small area of building underground, while trees and shrubs planted far away (15-20m distant) can only produce shade to the garden ground surfaces.

Figure 4.36 The arrangement of trees and shrubs around the new landscape house influences the movement of wind from south open space around the building

Figure 5.1. Location of two single-family residences in Precinct 14, Putrajaya (Google Earth, 2011)

Figure 5.2. General ground floor (left) and first floor plan (right) of the two houses

Figure 5.3. View of the front/south elevation of heavily landscaped house (left) and the front/east elevation of the sparsely landscaped house (right) 177

Figure 5.4. View of back/north elevation of the heavily landscaped house (left) and back/west elevation of the sparsely landscaped house (right)

Figure 5.5. Views of the west garden of the heavily landscaped house (left) and the south garden of the sparsely landscaped house (right) 178

Figure 5.6. Landscape plan for the heavily landscaped house. 182 
Figure 5.7. Landscape plan for the sparsely landscaped house.

Figure 5.8. Trees distribution compared by tree sizes and tree azimuth in relation to the building for the heavily landscaped house (A) and sparsely landscaped house (B)

Figure 5.9. Shrubs, vines and groundcover distributions at the heavily landscaped house (A) and the sparsely landscaped house (B)....

Figure 5.10. Similar capacity of the split system air-conditioning units in both houses

Figure 5.11. Air-conditioning use for the heavily landscaped house (house A) and sparsely landscaped house (house B)

Figure 5.12. Outdoor and indoor measurement points at the heavily landscaped house (house A) and sparsely landscaped house (house B

Figure 5.13. The intensity of the surface solar radiation from Sepang

Figure 5.14. Outdoor and indoor temperature adjacent to the north walls of the heavily landscaped house (house A) and the sparsely landscaped house (house B) 198 Figure 5.15. Outdoor and indoor temperature adjacent to the south walls of the heavily landscaped house (house A) and the sparsely landscaped house (house B) 199 Figure 5.16. Outdoor and indoor temperature adjacent to the east walls of the heavily landscaped house (house A) and the sparsely landscaped house (house B) 200 Figure 5.17. Outdoor and indoor temperature adjacent to the west walls of the heavily landscaped house (house A) and the sparsely landscaped house (house B) 201 Figure 5.18. Wind flow and average wind speed readings at the heavily landscaped house

Figure 5.19. Wind flow and average wind speed readings at the sparsely landscaped house 204

Figure 5.20. Wind speed adjacent to the north walls of the heavily landscaped house (house A) and the sparsely landscaped house (house B) 205 Figure 5.21. Wind speed adjacent to the south walls of the heavily landscaped house (house A) and the sparsely landscaped house (house B) 206 Figure 5.22. Wind speed adjacent to the east walls of the heavily landscaped house (house A) and the sparsely landscaped house (house B) 207 
Figure 5.23. Wind speed adjacent to the west walls of the heavily landscaped house (house A) and the sparsely landscaped house (house B) 208

Figure 5.24. Outdoor and indoor RH levels adjacent to the north walls of the heavily landscaped house (house A) and the sparsely landscaped house (house B) 210 Figure 5.25. Outdoor and indoor RH levels adjacent to the south walls of the heavily landscaped house (house A) and the sparsely landscaped house (house B) 211 Figure 5.26. Outdoor and indoor RH levels adjacent to the east walls of the heavily landscaped house (house A) and the sparsely landscaped house (house B) 212 Figure 5.27. Outdoor and indoor RH levels adjacent to the west walls of the heavily landscaped house (house A) and the sparsely landscaped house (house B) 213 Figure 5.28. A summary of air temperature adjacent to four side walls of the heavily landscaped house (house A) and the sparsely landscaped house (house B) 222 Figure 5.29. A summary of wind speed adjacent to the four side walls of the heavily landscaped house (house A) and the sparsely landscaped house (house B) 225 Figure 5.30. A summary of RH adjacent to the four side walls of the heavily landscaped house (house A) and the sparsely landscaped house (house B) Figure 5.31. Trees and shrubs of heavily landscaped house providing extensive shade to the windows and walls, and ground surfaces close to the house after 15.00 hours (west) in the afternoon and before 11.00 hours (east) in the morning.... 230

Figure 5.32. The arrangement of shrubs around heavily landscaped house influences the movement of wind from north-east through the building 231 Figure 5.33. The arrangement of trees and shrubs around heavily landscaped house influences the movement of wind from north-east around the building

Figure 5.34. The arrangement of trees with sufficient trunk heights and shrubs at heavily landscaped house influences the movement of wind from north-east around and through the building.... 232

Figure 6.1. Comparison of the monthly average temperature for 2008-2009 at Shah Alam and Putrajaya 237

Figure 6.2. Comparison of the monthly average relative humidity for 2008-2009 at Shah Alam and Putrajaya. 238

Figure 6.3. Comparison of an average 24 hours of the wind rose summary at Shah Alam (1975-2009) and Putrajaya (1999-2009). 
Figure 6.4. The average physical area by age of construction

Figure 6.5. House configuration

Figure 6.6. Generalized configuration of square/rectangular plan forms of the study houses

Figure 6.7. The average area for walls for all directions based on ages of construction

Figure 6.8. The average for glazed areas for all directions based on age of construction

Figure 6.9. The distribution of trees around the houses.

Figure 6.10. Tree sizes for the different age groupings of the houses . 253

Figure 6.11. Tree to building distance

Figure 6.12. Shrubs, vines and groundcover distribution around the houses in the study areas.....

Figure 6.13. Sizes of shrubs, vines and groundcover planted individually and in groups ( $\operatorname{spread~} \mathrm{m}^{2}$ ) 258

Figure 6.14. Shrubs, vines, and groundcover by distance from the buildings 259

Figure 6.15. Average shrub heights at each house. 260

Figure 6.16. Garden and turf cover areas around the houses 260

Figure 6.17. Hard landscape elements located around the gardens 261

Figure 6.18. Air temperature settings for the common room and bedrooms 265

Figure 6.19. Cooling energy use (per month) in the rainy and dry seasons 267

Figure 6.20. Summary of electricity use by tariff $(\mathrm{kWh})$ on a monthly basis for domestic buildings in the Shah Alam records of 24-hour mean temperatures (20082010) (TNB, 2010).

Figure 6.21. Example 1.5-2m shrub heights can provide shade to the windows before 11.00 hours (east) and after 15.00 hours (west) combined with hipped roof 275 Figure 6.22. Distances of trees providing extensive shade to the ground surfaces close to the house.

Figure 6.23. Example of pleasant tropical landscape; trees with diverse range of shrubs, groundcover and turf planted around the garden (modified from Kirsch, 2007; Ryan, 2012). 
Figure 6.24. The distribution of trees, shrubs and other vegetation compared with the cooling energy use 288

Figure 6.25. Tree numbers by size of group, compared with average cooling energy use

Figure 6.26. Landscape plan for strategically designed landscaping around the house that will maximise shade, wind flow towards the surrounding garden, and produce evapotranspiration cooling 292

Figure 6.27. Section/elevation of the house with landscaping to enable wind flow to get through to the house and surrounding garden, and to provide shade in the morning and afternoon to the building envelope (roof and walls) 


\subsection{INTRODUCTION}

Energy costs and environmental concerns have made it more important than ever to find ways to reduce energy consumption. As human consumption of energy continues to increase, it is important to improve the energy efficiency of our built environment. This will greatly benefit the environment, and the owners and users of buildings.

There are many ways to improve the energy efficiency of the built environment. Envelope technologies, such as wall, floor, and roof insulation, high-performance windows and doors, and air infiltration, have a priority role in producing a comfortable interior. However, buildings in hot-humid climates still frequently depend on energy use to power cooling and ventilation systems to control the environment.

The amount of energy used is dependent on a number of variables, such as climate, surrounding vegetation, a building's orientation, and the structure and materials used in the construction of the building envelope. Of these, climatic factors have the most significant effect on the balance of energy use in buildings. By controlling the microclimate, it is possible to control the amount of energy used for heating and cooling in buildings (McClenon, 1983). A microclimate space begins directly around an individual building, and spreads to its neighbours until its reach takes in a small community as a neighbourhood area.

In an individual building, architects can create a microclimate boundary by manipulating the building envelope and exterior environment (Bomberg \& Brown, 1993). The planning and development of exterior spaces can reduce the energy consumption of buildings by reducing the impact of some climate factors. If the microclimatic condition around the building is similar to the desired interior conditions, little extra energy is required. Conversely, if the microclimate is significantly different from the desired interior conditions, large amounts of energy may be required for heating or cooling. Awareness and knowledge of the potential 
of vegetation to modify microclimate could produce a new way of quantifying the energy saving potential of landscaping (Moffat \& Schiler, 1981). This in turn should also enable quantification of the moderating value of microclimate.

This introductory chapter will provide background on the significance and potential benefit of the effects of vegetation, building construction and human factors on the thermal performance of housing in a tropical environment by outlining the issues, hypothesis, scope and limitations, aim and objectives, and thesis structure of this research.

\subsection{Background}

The main concern of designers of low energy buildings has generally been focused on a building's thermal comfort and the protection it provides from the elements (ASHRAE, 2003; Nadel, 2006; Oral, Yener, \& Bayazit, 2003). Designers usually rely upon integration of the building envelope and the building's passive systems to improve thermal performance. This includes developing designs that focus on integrating building form and fabric with the passive systems of environmental control and the mechanical equipment for heating, ventilation and air-conditioning (HVAC).

A building's thermal performance and energy consumption is influenced by its layout, external shading devices, the surface colour, and insulation. Highly reflective finishes for walls, openings, and roofs, which provide a high amount of albedo, can also indirectly improve a building's thermal performance (Misni \& Allan, 2010). However, proper landscaping, water features, and other landscape structures can also be used to improve thermal performance. They reduce the amount of radiation falling on the building though the provision of shading, and by moderating temperatures, the evapotranspiration processes, and controlling wind direction to assist in keeping the building warm or cool. The design of external spaces needs to be prioritised because it will influence the building interior. 
The role of landscaping in moderating microclimate has been explored all over the world during recent years. Landscaping is an ecological measure used to combat the problems of heavy urban built environments (Wong \& Yu, 2005). Vegetation has the potential to increase environmental value by reducing energy consumption in individual buildings and increasing the energy efficiency of the community as a whole. Vegetation has a particularly effective influence on a building's microclimate and its thermal performance (McPherson, Herrington, \& Heisler, 1988). Therefore, landscaping has a significant role to play in providing a cool microclimate, and can directly influence the comfort level of an interior space.

Vegetation includes all the plant life in a particular region, such as trees, shrubs, grasses and lawns. Vegetation can influence solar radiation, air temperature, humidity and air flow. While there are a number of landscape strategies that can be implemented to modify the microclimate, the three main functions of vegetation used to do this are: shade, evapotranspiration, and wind control.

Using strategically placed shade trees of the right species and form around a building can potentially modify its microclimate and the building's energy use. The shading they provide can reduce the amount of radiant energy absorbed and stored by buildings and other built surfaces.

According to Simpson (2002), evapotranspiration is the process of transferring moisture from the earth to the atmosphere by evaporation of water and transpiration from plants. Evaporation and transpiration through vegetation contributes to lowering urban temperatures. In this process, the plant draws moisture from the ground, uses what it needs to grow, moderates its own temperature and transpires the excess. This process cools the surrounding air. Therefore, vegetation that is appropriately placed around a building can provide a cooling effect on the building and its microclimate. 
Vegetation can also be used as a barrier or windbreak to control wind, or by providing more effective ventilation and convective cooling of building surfaces by channelling or directing the flow of air.

However, there is a lack of data on the effects of vegetation on the thermal performance of buildings and their microclimates, and the methods for predicting the effect from shade, evapotranspiration, and channelling wind to reduce temperatures and energy use. According to McPherson et al. (1988), quantitative research is needed to better predict the impacts of vegetation and other landscape elements on buildings, microclimate and energy use. With quantitative evidence of energy savings, the likelihood of vegetation use in the design of landscaping could greatly increase.

In turn, policy makers would have a basis for implementing new landscaping requirements. Architects would have alternative methods and ideas for complying with energy saving guidelines and integrating the building with its site. Developers would be able to entice buyers with the future long-term benefits of energy-efficient houses, with only a potentially minimal increase in the early stage cost of construction of landscaping around the building.

This study will focus on two main issues: environmental and energy conservation.

\subsubsection{Environmental issues}

Global warming is defined as the increase in the average temperature on Earth (Diekmann, 2007). Lechner (2000) states that depending on the scenario (population, economic growth, energy requirements, etc.), the average global air temperature near the ground is predicted to increase by around 1.8 to $4.0^{\circ} \mathrm{C}$ by the year 2100 . An increase of $1^{\circ} \mathrm{C}$ will make the earth warmer now than it has been for at least a thousand years. Global warming and the resulting climate change are expected to have a serious effect on the planet. As the earth is getting hotter, disasters like hurricanes, droughts, and floods are becoming more frequent (Lindinger, 2007). 
Carbon dioxide is a major contributor to the greenhouse effect and global warming. Burning fossil fuels, such as natural gas, coal, oil, and gasoline, raises the level of carbon dioxide in the atmosphere. As more greenhouse gases are produced, they trap more of the sun's heat.

As humans alter the character of the natural landscape through the city-building process, the local energy exchanges that take place within the boundary layer are affected. Therefore, modification of the landscape influences the local (microscale), mesoscale, and macroscale climates.

At the urban level, the urban heat island effect is a phenomenon where air temperatures in densely built urban areas are higher than the temperatures of the surrounding rural environment. These heat islands influence most of the major cities around the world (Akbari, Davis, Dorsano, Huang, \& Winnet, 1992). In urban areas, buildings and paved surfaces have gradually replaced pre-existing natural landscapes. These structures and surfaces absorb solar energy, which is subsequently re-radiated to the surrounding area, increasing ambient temperatures at night causing the surface temperature of urban structure to become 5.5 to $10^{\circ} \mathrm{C}$ higher than surrounding areas (Akbari, et al., 1992). As the surface of an entire community becomes hotter, the overall ambient air temperature increases by 2 to $8^{\circ} \mathrm{C}$. In hothumid tropical climates, heat islands contribute significantly to the urban dweller's summer discomfort, and energy bills due to the increased cooling loads. Taha et al. (1988) state that houses are particularly sensitive to this effect since they are envelope-dominated structures. Urban heat islands are considered to be a mild asset in winter as they can contribute to a reduction in demand for heating. However, they can significantly increase demand for cooling energy during the summer.

\subsubsection{Energy conservation issues}

Improved living standards and the increased population in developing countries contribute to a dramatic increase in energy consumption worldwide. An increase in the urban population by $1 \%$ has been reported to increase energy consumption by 2.2\% (Santamouris, 2001a). According to Brown (2008), an annual energy 
consumption increase of $30 \%$ by the year 2020 was predicted by the International Energy Agency in 2008 A study of the tropical city of Singapore demonstrated that the anticipated increase in building energy consumption was mainly because of the use of air conditioning (Tso, 1994), where it has become a necessity.

Demand for electrical energy continues to grow, while the means of production remains limited. For example, use of fossil energies (coal, oil and gas) and uranium, are expected to decline in the coming decades (Bastide, Lauret, Garde, \& Boyer, 2006). As a result, energy efficiency is a critical factor in designing high-quality housing (Santamouris et al., 2005). Santamauris et al. stressed that by designing houses with energy efficiency in mind, the amount of energy needed to keep them comfortable can be reduced dramatically.

This issue is particularly important in tropical climates, which experience high temperatures and humidity all year round. In these areas the increase in the purchasing power of the population has resulted in greater use of air-conditioners. Air conditioning is often seen as the only means of achieving thermal comfort during the hot season, but unfortunately it consumes a high amount of energy.

In hot-humid tropical climates designers should provide a microclimate in any built environment to help people achieve an energy balance of "near zero", not overheated or under-heated (Bakar, 2002, p. 118). Having a cooler microclimate would reduce the temperature gradient between the exterior and interior of a building, thereby reducing the cooling energy balance to "near zero" for the building.

\subsection{Research questions}

The research will focus on two main research questions:

(1) How much can outdoor temperature be decreased by strategic landscaping around a tropical domestic building?

(2) How much energy can be saved through strategic landscaping around tropical domestic buildings and in their immediate neighbourhood? 
This research will evaluate how the planning of landscaping around domestic buildings can contribute to reducing the formation of hot air pockets, including heat islands, and encourage the formation of an acceptable outdoor temperature, and cooler and faster air flow into buildings. This will help to provide a comfortable environment for a building's inhabitants, and will contribute to cooler neighbourhood areas.

Vegetation can provide solar protection, which slows heat build-up and the city heat island effect through shading and evapotranspiration. Vegetation also moderates wind flow to individual houses, which provides natural ventilation and convective cooling to exterior building surfaces.

By using the best combinations of high-branching shade trees and low ground cover that ensure shade and moderate wind flow for buildings in hot-humid tropical areas, it is possible to reduce temperature and energy use. This can also create communities that are cooler, more aesthetically pleasing, and more energy efficient.

\subsection{Aim and objectives}

The aim of this study was to examine and quantify the relationship between surrounding vegetation, and the thermal performance of housing in a hot-humid tropical environment. The primary objectives were to determine, for the tropical residence, the outside temperature-reducing and the energy saving potential of vegetation. The literature (see for example Akbari, 1992; Parker, 1983; McPherson, 1988; Simpson, 2002; \& Taha, 1988) is fairly consistent in its recommendations for where trees should be placed for optimal energy benefits. However, in reality, houses vary considerably in orientation and homeowners generally make landscaping decisions based upon aesthetics.

To achieve the primary research objectives, two stages of research were undertaken: examining landscaping patterns and structures; and quantifying and validating the results. 


\subsubsection{Examine landscaping patterns and structures}

This stage involved examining landscaping patterns and structures in relation to the specific orientation of tropical domestic buildings to determine the benefits of vegetation. According to Simpson (2002) tree-shade alters building cooling loads by reducing solar radiation. A related question is whether the shading benefits of vegetation are of primary importance or if transpiration cooling plays a more important role in the building-energy balance equation. In other words, is there a measurable benefit from shrubs and groundcover or it is only trees that can effectively provide the cooling benefit? Another consideration is whether the wind flow and surrounding open spaces and landscaping of a neighbourhood area play a role. It is also important to determine the relationship between the quantity of vegetation and the energy consumption of the adjacent buildings.

\subsubsection{Quantify and validate the results}

This stage involved quantifying the relationship between vegetation, building construction and human factors on thermal performance, and validating those results. The key questions asked were:

- What is the impact of vegetation on a house?

- How much can the benefit of vegetation increase if the quantity, type or configuration of vegetation is increased?

The strategy for this study was to obtain a large enough sample of existing homes so that the building design and vagaries of the occupants' energy-use habits could be effectively averaged over the data set. By doing so, the statistical analysis should reveal the relative importance of each of the human and building construction variables, along with the vegetation variables to provide a comprehensive picture of residential cooling loads.

\subsection{Scope and limitation}

Malaysia is a tropical country close to the equator, and is also coastal. Generally, it has two distinct seasons. The dry season occurs during the southwest monsoon from 
May to September. The northeast monsoon brings the rainy season to the country from mid-November until March.

This study was undertaken in two cities in the hot-humid tropical climate region of Malaysia; Putrajaya and Shah Alam. Malaysia is a country with an abundance of solar radiation, an average of six hours of sunshine per day (Zakaria, Mokhtar, \& Mohamad, 2008), and high temperatures and humidity.

Putrajaya and Shah Alam were chosen for the fieldwork required in this study because their planning and landscape design around residential areas were appropriate for this study.

The case studies were taken in the actual site to assure the authenticity and accuracy of the research. The primary data on the physical characteristics of the houses, surrounding landscaping, and the lifestyle habits of the occupants were obtained by contacting the owners and observing each house directly. This method was used in preference to computer simulation whose accuracy is not yet sufficiently established in this field (Meier, 1990).

The fieldwork was done during the middle of the rainy season (January-February 2010). This period was chosen to ensure the maximum effects from the lush, green and fertile surrounding landscaping were observed. During the dry seasons the condition of surrounding vegetation is less fertile because rainfall is sparse and the air temperature is slightly higher. The housing estates used were chosen because they had similar climates and building construction, but buildings and landscaping of different ages. The distance between the two cities was around $24 \mathrm{~km}$.

In Malaysia $20 \%$ of the population from middle and upper classes generally live in single-family houses (Malaysia, 2007). The middle and upper class people are represent around $40 \%$ of the citizen of Malaysia (Malaysia, 2000). The findings of this study will be of benefit to them. Medium sized single-family houses (300 to 
$600 \mathrm{~m}^{2}$ ) were chosen for this study because they have individual lots and are surrounded by a garden.

The research looked at houses aged between 3 and 40 years old that had surrounding vegetation. Houses of different ages were chosen because they provided examples of different ages and maturity levels of surrounding landscaping, which would in turn have different influences on the energy used and thermal comfort for the houses' residents.

In Malaysia, almost all houses use air conditioning to deal with the extremes of an uncomfortably hot summer day. Air conditioners account for around 30\% of the total electricity consumption for residential buildings (Saidur, Hasanuzzaman, Hasan, \& Masjuki, 2009). This research evaluates the electricity consumption for every house in monthly and yearly figures. The total energy used for every house is calculated and analysed to determine the energy saved by the appropriate placement and use of vegetation. The rate of cost of energy used is in Malaysian Ringgit (NZ\$1 equal to MYR2.4).

\subsection{Thesis structure}

Chapter 1 provides a brief introduction and background to the effects of vegetation, building construction and human factors on the thermal performance of housing in a tropical environment. It also introduces the possibility of using landscaping around the building for passive cooling, and improving the energy efficiency of the built environment by modifying the microclimate. The research aim and objectives are also explained in this chapter.

Chapter 2 presents a literature review of the climatic criteria, the benefits of vegetation, and the influence of building envelopes and thermal comfort parameters on the thermal performance of a house. The theory of energy use is also elaborated in this chapter. 
Chapter 3 describes the research methodology. This was divided into two stages fieldwork, site measurements, and data collection at (a) 5 individual houses, and (b) 50 houses. The three main methods chosen for collecting data were by interview, direct observation, and physical artefacts evidence. Statistical analyses were used to measure and evaluate the thermal performance and energy consumption of the houses.

Chapter 4 examines the influence of landscaping on the thermal performance of single-family houses in a tropical environment. Three different ages of single-family houses were looked at in the tropical city of Shah Alam, Selangor, Malaysia ranging in age from five to 30 years old. These buildings had similar construction, but were relatively different in building size, design and landscaping because they were built in different eras of architecture and landscape styles.

Chapter 5 investigates the effect of vegetation in modifying outdoor temperature around two single-family houses in a hot-humid tropical climate. The two houses represented typical contemporary building and landscape design for medium-sized single-family houses in Malaysia. However, their surrounding landscape was significantly different and ranged from sparse to heavily landscaped. These areas were chosen to test the effects of landscape on modifying the outdoor and indoor temperature of houses.

Chapter 6 presents the results and discussion from interviews, observation, and fieldwork undertaken, using the data on the surrounding landscaping, building construction and human factors for 50 single-family houses. Each house was surrounded by varying amounts and sizes of landscaping. The results and discussion reveal that the amount of cooling energy used for each house was closely related to the amount of vegetation located around their house.

Chapter 7 provides the conclusion, and recommendations for better design for singlefamily housing and the surrounding landscaping in the context of a tropical city of Malaysia. The significance of the study to Malaysian communities, relevance of the 
results, and plans for the continuation of this research are also explained in this closing chapter. 


\subsection{LITERATURE REVIEW}

\subsection{Overview}

This chapter presents the literature review of the four factors that influence the thermal performance and energy saving of the house. These are: climate criteria, the benefits of vegetation, the influence of the building envelope, and thermal comfort parameters.

Solar radiation, air temperature, wind, humidity and precipitation are the main factors that can influence a building's thermal performance. The benefits of vegetation to the environment and its potential for energy saving are well known. It can directly influence urban microclimates by shading surfaces and altering wind speeds, and indirectly via the evapotranspiration of water. Therefore, landscaping is one of the simplest strategies that can be used to save energy and improve the quality of the environment in urban areas.

The building envelope is a critical component of any building since it both protects the occupants and plays a major role in regulating the indoor environment. It includes a building's roof, walls, windows, doors, construction details and ground surfaces. The envelope controls the flow of energy between the interior and exterior of the building. It can also be considered as the selective pathway for a building to work with the climate, responding to heating, cooling, ventilating and natural lighting needs.

Finally, the physiological parameters of thermal comfort are temperature, air speed, radiation, relative humidity, and people's clothing and the activities they may do in a house will be discussed. The positive effect of an urban design that accounts for human comfort is a reduction in the building's energy consumption.

\subsection{Climate parameters}

According to Szokolay (2004), climate can be defined as the integration in time of weather conditions, characteristic of a certain geographical location. The description 
of climate includes statistics and the averages, such as the day to day or year to year variations. Pidwirny (2008) classifies climate into five major climatic types: tropical, dry, temperate, continental and polar. These climate zone boundaries were selected according to vegetation distribution combined with average annual and monthly temperatures, and the seasonality of precipitation. For the purpose of this research, the topography, ground cover and vegetation, water and building densities will be examined because they also influence the climate of a specific site.

Building designers are usually interested in the aspects of climate that can increase human comfort and reduce energy needs for heating and cooling. The main factors that influence a building's thermal performance are solar radiation, air temperature, wind, humidity and precipitation. Appropriate planning based on these parameters can influence the ambient temperature in a neighbourhood and the energy requirement for each building.

This study will identify the climate parameters that have a significant effect on thermal performance of building.

\subsubsection{Solar radiation}

Solar radiation is radiant energy emitted by the sun, particularly electromagnetic energy. This is the dominant energy input to the Earth, and is intercepted by the atmosphere and absorbed by surfaces. It is also the single most important factor influencing climate and weather. Essentially, solar radiation is affected by the angle of the sun's rays to the ground, the distance from the sun, cloud coverage and the quality of the atmosphere through which the radiation passes (Koch-Nielsen, 2002).

There are two distinct types of wavelength radiation that will affect heat gain and loss in buildings: shortwave and longwave radiation. Shortwave radiation is an electromagnetic radiation with a wavelength of between 0.1 and 0.7 micrometers $(\mu \mathrm{m})$ that is used to describe the radiation emitted from the sun. It enters the Earth's surface-atmosphere system, and is ultimately returned to space as longwave 
radiation. Long wave radiation is the energy absorbed at the surface that is radiated by the Earth.

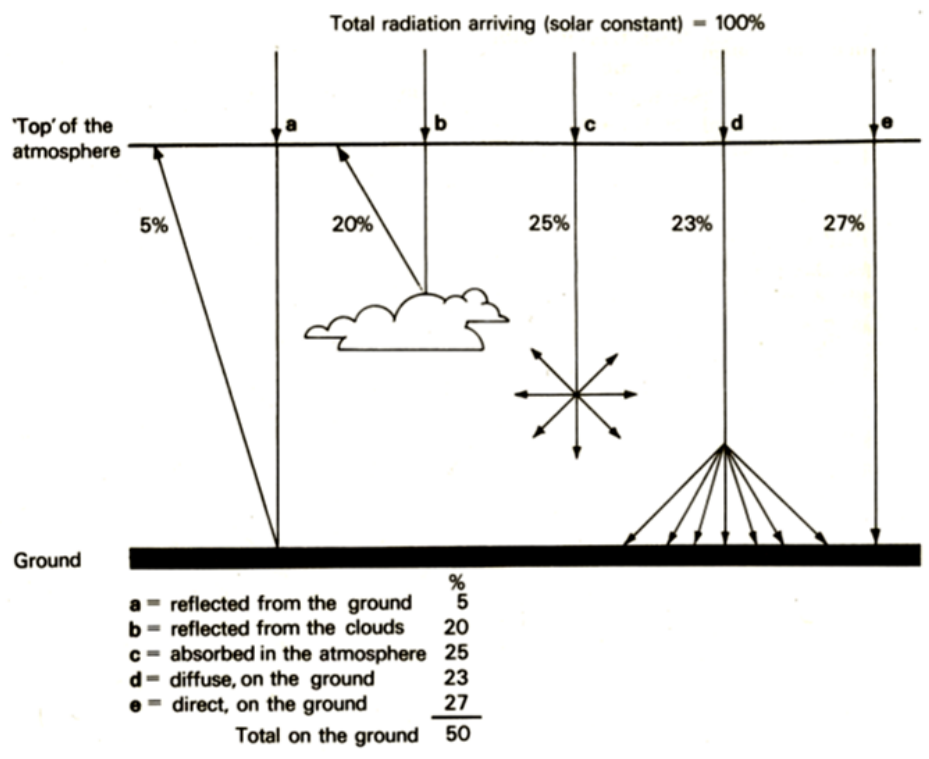

Figure 2.1. Passage of radiation through the atmosphere (Koenigsberger, Ingersoll, Mayhew, \& Szokolay, 1980, p. 7)

Figure 2.1 illustrates the average percentages of solar radiation received by a plane on the ground from diffused $(23 \%)$ and direct $(27 \%)$ radiation. At ground level the total intensity is $50 \%$.

Cloudiness is measured in terms of the proportion of the sky that is covered with cloud. On a scale of $0-8$, a cloudless sky is represented by 0 , while 8 means a completely overcast sky (Kukreja, 1978). Clouds also have an important effect on incoming radiation, as a considerable amount is reflected from their upper side, and the amount that passes through depends on the thickness and water content of the cloud. Clouds also greatly influence the strength of incoming and outgoing radiation. For example, in cloudy weather the total radiation received at ground level is reduced depending on cloud coverage and cloud thickness. The thicker and lower the clouds, the less incoming radiation that strikes the surface. Under extreme cloud conditions a significant proportion of radiation would be in the form of scattered or diffused light. Clouds also influence the temperature of the Earth's surface at night. 
Water droplets in clouds and invisible water vapour in the air absorb much of the outgoing thermal radiation, and some of this heat is reradiated back to the Earth. Thus, air ambient temperatures are normally higher on cloudy nights.

Most of the radiation from the Earth's surface is trapped within the atmosphere. According to Brown \& Gillespie (1995), the radiation is partly absorbed and partly reflected by natural and artificial surfaces. The absorption and reflection process is determined by the colour spectrum of the radiation, and the characteristics of the receiving surface (Kukreja, 1978). For example, plant matter appears to be green because it reflects the green part of the incident radiation and absorbs the other wavelengths of the visible spectrum. The proportion of reflected to incident light is called albedo.

The amount of energy emitted is dependent on the surface. The hotter the surface, the more radiant energy it will emit. Figure 2.2 shows how the Earth's surface releases heat through three processes: long wave radiation, evaporation, and convection.

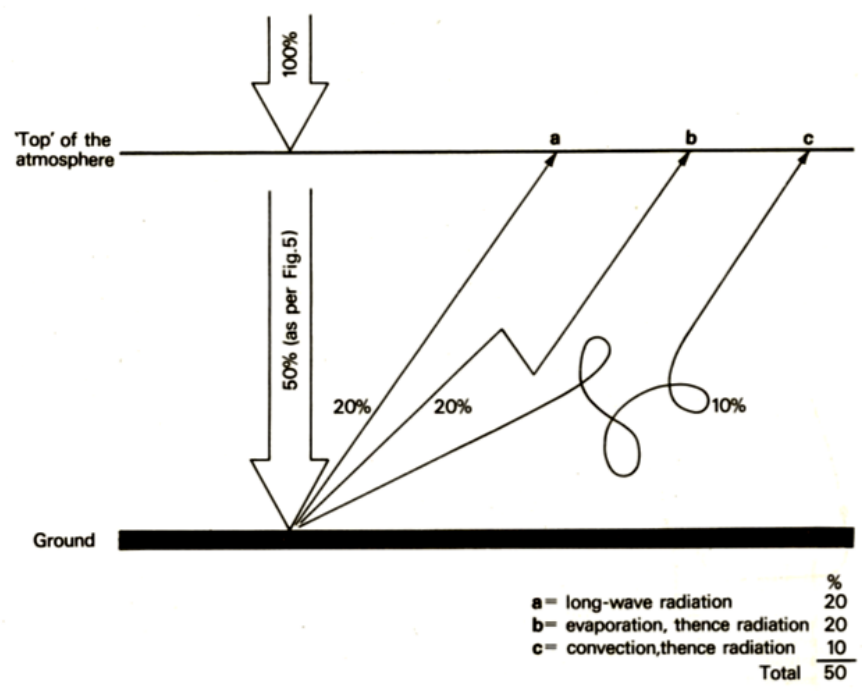

Figure 2.2. Heat release from the ground and the atmosphere (Koenigsberger, et al., 1980, p. 7) 
The $20 \%$ of longwave radiation goes to the cold outer space. Koenigsberger et al. (1980) state that $84 \%$ of this radiation is absorbed by the atmosphere, and only $16 \%$ escapes to space. The $20 \%$ of evaporation is when liquid water changes into vapour and mixes with air. The $10 \%$ convection is the process when air heated by contact with the warm Earth surface becomes lighter and rises to the upper atmosphere, where it dissipates its heat to space. Both air and water act as convecting agents for the transfer of energy. Such convection processes dominate the world's weather, including the production of rain and snow, thunderstorms, hurricanes and frontal systems. The energy from evaporation and sensible heat, together with longwave radiation, is absorbed by the atmosphere, which in turn radiates some of its energy back to space to complete the cycle.

According to Givoni (1969) the effect of solar radiation on internal temperatures can be divided for convenience into two parts: the effect on temperatures on the external surfaces and roof, and the resulting heat flow and indoor heating; and the heating caused by penetration of radiation through glazed or open areas. Givoni also stressed the architectural point of view that controlling solar radiation impact involves addressing three main problems: building orientation, external colour, and the shading of windows and other surfaces areas.

\subsubsection{Sun path orientation}

The sun's position in the sky changes throughout the day and seasonally. Sun path diagrams or solar charts are the simplest tools for depicting the sun's apparent movement. In Figure 2.3, the sky hemisphere is represented by a circle (the horizon). The sun's position in the sky can be specified by two angles (Koenigsberger, et al., 1980): solar altitude angle $(\gamma)$ and solar azimuth angle $(\alpha)$. The altitude angle is the vertical angle at the point of observation between the horizon plane and the line connecting the sun with the observer. The solar azimuth angle is the angle at the point of observation, which is measured on the horizontal plane between the northerly direction and a point on the horizon circle, where it is intersected by the arc of a vertical circle going through the zenith (the centre) and the sun's position. 


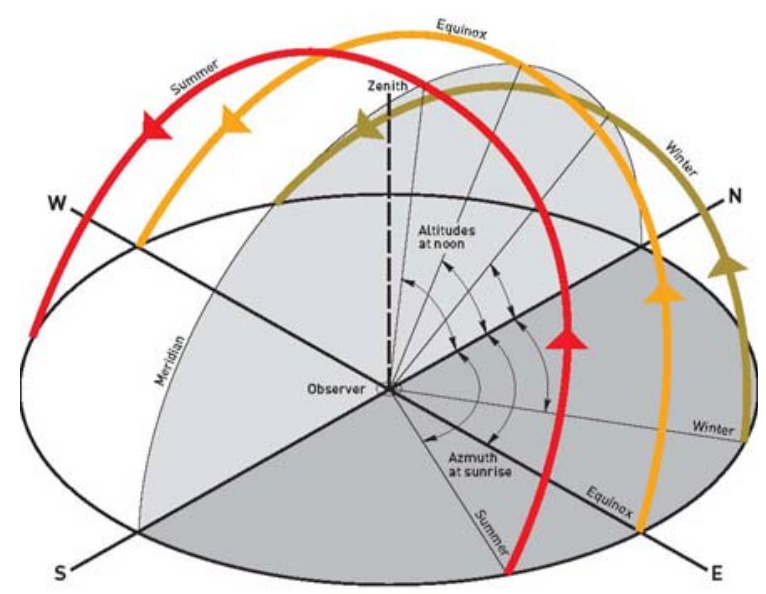

Figure 2.3. Pattern of the sun's seasonal movement, altitude and azimuth angles at Latitude $35^{\circ} \mathrm{S}$ (Nervegna, 2003, p. 6)

Conventionally, north $\alpha=0^{\circ}$ or $360^{\circ}$, east $\alpha=90^{\circ}$, south $\alpha=180^{\circ}$ and west $\alpha=270^{\circ}$ (Koenigsberger, et al., 1980). These two angles can be read directly for any date of the year or hour of the day from the solar charts or sun path diagram for latitude $3^{\circ} \mathrm{N}$ as shown in Figure 2.4.

The sun path lines in an equatorial location (Latitude $=0^{\circ}$ ) will be symmetrical around the equinox sun-path, which is a straight line. For higher latitudes the sun path lines will shift away from the equator. The date-lines (sun path lines) are intersected by hour lines. The vertical line at the centre is solar noon. On the equinox the sun rises at due east at 07:00 a.m. and sets at due west at 19:00 p.m.

Average daily amounts of solar radiation $\left(\mathrm{MJ} / \mathrm{m}^{2}\right.$.day) for each month of the year give an indication of climate conditions, including seasonal variations. Solar radiation can be measured in two ways: irradiance in $\mathrm{W} / \mathrm{m}^{2}$, which refers to intensity; and irradiance in $\mathrm{J} / \mathrm{m}^{2}$ or $\mathrm{Wh} / \mathrm{m}^{2}\left(\mathrm{~W} / \mathrm{m}^{2}=\mathrm{J} / \mathrm{m}^{2} \mathrm{~s}\right.$, as $\left.\mathrm{W}=\mathrm{J} / \mathrm{s}\right)$, an energy quantity integrated over a specified period of time (Szokolay, 2004). The sun's radiation is mainly concentrated within the wavelengths 0.3 and $2 \mu(1 \mu=1 / 10,000 \mathrm{~cm})$ (Kukreja, 1978). 


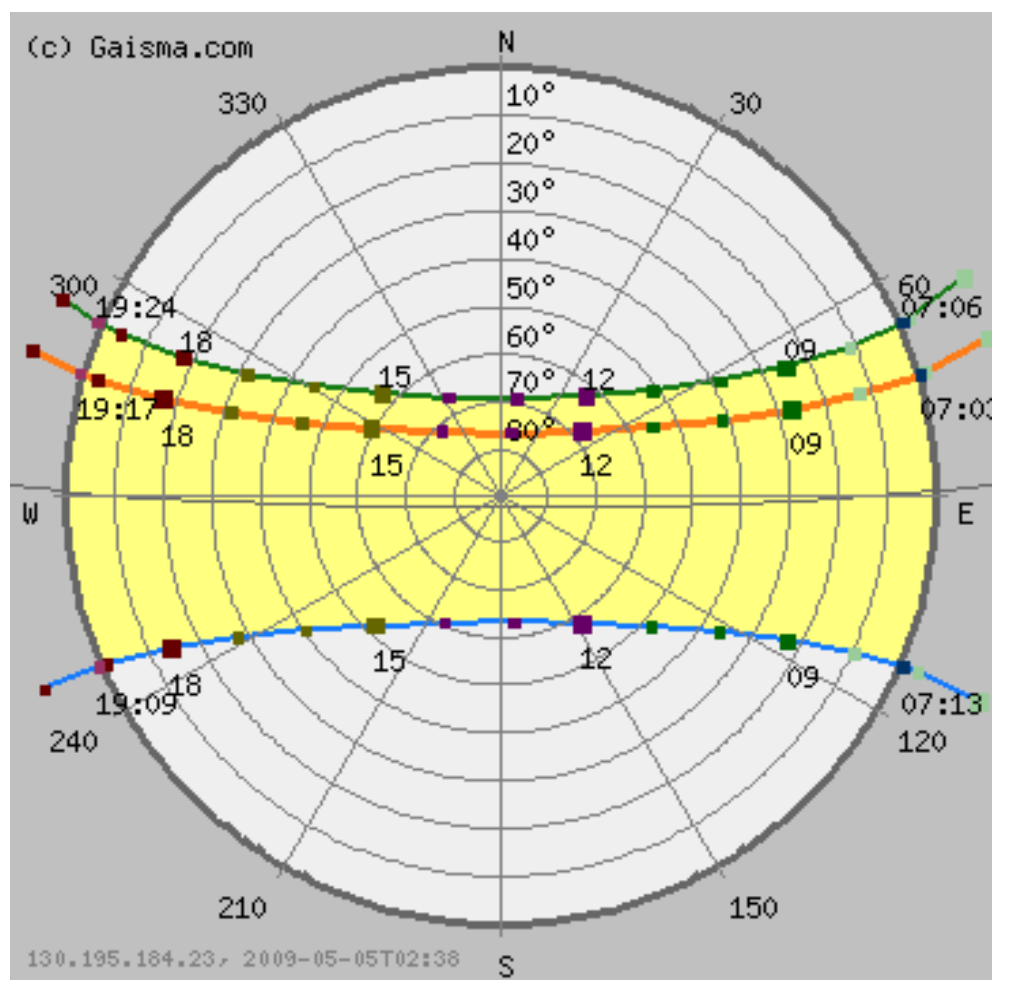

Sun path

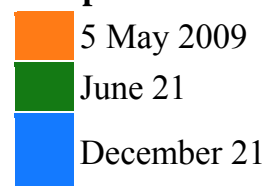

Annual variation

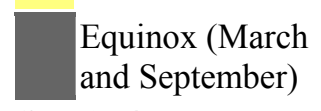

Sunrise/sunset

Sunrise

Time

\begin{tabular}{|l|l|}
\hline $00-02$ \\
$03-05$ \\
$06-08$ \\
\hline $09-11$ \\
$12-14$ \\
\hline $15-17$ \\
\hline $18-20$ \\
\hline $21-23$ \\
\hline
\end{tabular}

Figure 2.4. A stereographic sun-path diagram for Kuala Lumpur at latitude $3^{\circ} \mathrm{N}$ (GAISMA, 2009, p. 1)

The intensity of solar radiation is influenced by a combination of the angle of the sun's rays to the ground, distance from the sun, cloud coverage, and the quality of the atmosphere through which the radiation passes. The average monthly solar radiation in Malaysia is $7191.8 \mathrm{Wh} / \mathrm{m}^{2}$, with the monthly average daily sunshine duration ranges from 10-13.9 hours (ECOTECT 5.2v - weather file for Kuala Lumpur). High solar radiation occurs more frequently from October to November and January to February, while low solar radiation occurs from May to June.

\subsubsection{Sensible heat flow}

Local climate effects the rate of heat gain from buildings by contributing to heat flows throughout our environment. There are three ways that heat can be transmitted through any medium: conduction, convection and radiation. 


\section{i. Conduction}

Conduction involves the transformation of kinetic heat in a solid mass. This is the process of heat transfer from warmer to cooler molecules within a solid material. Heat flows through the materials or building fabric from which a house is built. The primary heat transfer paths between a building and the external environment are: conduction through walls, roofs, doors (opaque conduction) and windows (glazed conduction); solar radiation directly penetrating through windows; and sensible and latent heat gain from air exchange.

\section{ii. Convection}

Convection represents the transfer of heat by circulation or movement of hot particles to cooler areas. Heat transfer by convection can occur in liquids and gases when molecules move freely and independently (Moss, 2007). Convective heat exchange can occur when heat is transferred from a solid to the adjacent air, or between two surfaces at different temperatures by means of airflow. Convection is caused by temperature difference (natural) or forced air motion generated by wind or fans. Convective heat transfer takes place when the building is infiltrated by wall surfaces, when the outdoor air flows through the interior space. If the ambient temperature is different from the temperature of any element of the interior space, convective heat transfer takes place.

\section{iii. Radiation}

Radiation occurs without the involvement of a physical substance as the medium. Thermal radiation involves the interchange of electromagnetic waves between surfaces that are different temperatures (Moss, 2007). The sun transfers its heat to the surface of the Earth by radiation through space to the Earth's atmosphere. The ozone layer, atmospheric particles, condensing water vapour and dust, all act as a filter for solar radiation, reducing its intensity at the Earth's surface. In buildings, radiant heat transfer is the energy exchange between surfaces by electromagnetic waves across space. The radiation wave is transmitted through space until it strikes an opaque surface, where it is partly absorbed. The absorbed radiation increases the 
vibration of the surface molecules, and thus raises the temperature of the material where the absorption took place

\subsubsection{Air temperature}

Air temperature is the ambient temperature indicated by a thermometer exposed to the air, but sheltered from direct solar radiation, $1.5-2 \mathrm{~m}$ above ground (KochNielsen, 2002). The main factors that determine air temperature are season, latitude, the amount of cloud cover, and the time of year (Koenigsberger, et al., 1980). Generally the coldest air temperature during the course of the day is usually just before sunrise, while the hottest is two hours after noon. The air temperature then starts to decrease and continues to do so through the night. Krigger and Dorsi (2004) state that air temperature is the most noticeable characteristic of climate and the most important factor in determining heating and cooling energy use. Outdoor temperatures vary according to the season, weather and time of day.

Monthly mean temperatures are established by taking the average temperature between each day's maximum and minimum, and calculating the average of the month. The highest and lowest temperatures each month should be recorded to establish the extreme range of temperatures for each month. The values for each of the 12 months give a reasonably accurate picture of temperature conditions,

\subsubsection{Wind}

Wind is the natural movement of air. Winds are generally named according to their strength and the direction that they come from. Figure 2.5 shows how global wind patterns are driven by the sun (Pinet, 1998), and include three main circulation patterns or cells: Tropical cell (Hadley cell), Midlatitude cell (Ferrel cell), and the Polar cell. These are summarised below:

\section{i. Tropical cell (Hadley cell)}

In this cell, low latitude air moves towards the Equator and heats up. As it heats it rises vertically and moves towards the poles in the upper atmosphere, which forms a convection cell that dominates tropical and sub-tropical climates. 
ii. Midlatitude cell (Ferrel cell)

In the mid-latitude cell the air flows towards the poles and the east near the Earth's surface and the equator, but in a westerly direction at higher levels.

iii. Polar cell

In the polar cell air rises, spreads out and travels toward the poles where it sinks to form the polar highs. At the surface, the air spreads out from the polar highs. Surface winds in the polar cell are easterly (polar easterlies).

The atmospheric circulation of wind is very unstable as its characteristics fluctuate according to the prevailing weather conditions and depend on the origin of the wind. Koch-Nielsen (2002) states that direction, speed, gustiness and frequency are the most important characteristics of wind. Wind roses are normally used to show the characteristics of wind for a specific period. For example, for a day, month or year. Wind velocity is measured using an anemometer.

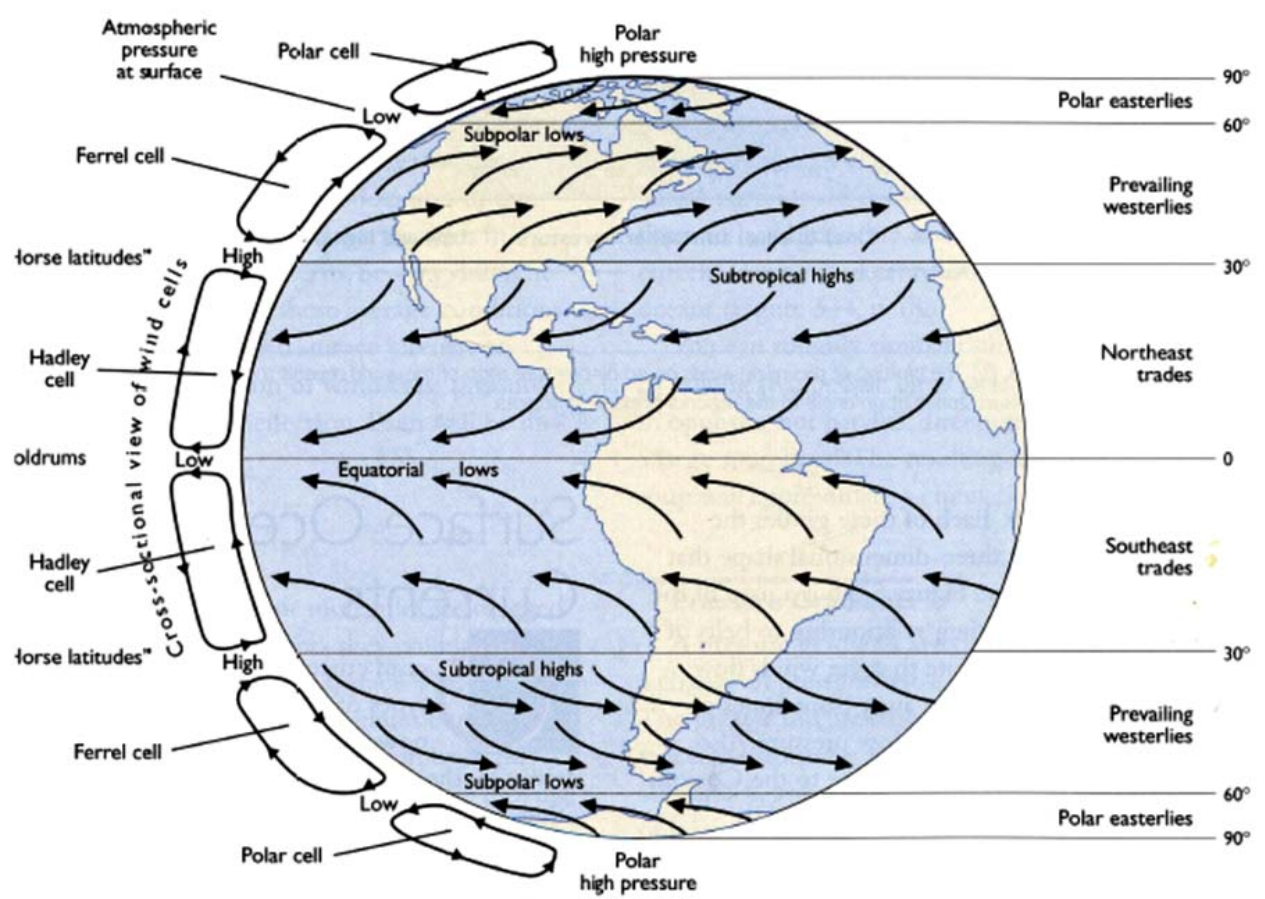

Figure 2.5. Global wind circulation, unequal heating of the Earth's surface and the Corialis deflection cause a zonal wind system to develop, arranged by the three circulation cells - Hadley, Ferrel and Polar cells (Pinet, 1998, p. 177) 
Olgyay (1963), stressed that the effects of wind must be considered with regard to the exterior of a house because of convection transfer and infiltration, and within the dwelling itself. However, Olgyay (1963) also suggests that positioning low buildings towards prevailing winds is not essential because using wind breaks and placing openings in high and low pressure areas helps to maximise air flow to the building. In a tropical environment wind is a crucial source of natural ventilation. It should be used for cooling in hot periods and as relief from vapour pressure during times of high absolute humidity. However, the effects of air movements on people's comfort levels have to be viewed as positive and negative.

To evaluate this and to determine the required architectural considerations, the variations in the prevailing annual and monthly wind direction and their temperatures must be analysed.

\subsubsection{Humidity}

Humidity refers to the amount of water vapour in the atmosphere, which is gained as a result of evaporation from exposed water surfaces, moist ground and plant transpiration (Koch-Nielsen, 2002). The higher the humidity at a given temperature, the more moisture the air holds. It can be described as absolute humidity (AH) or relative humidity $(\mathrm{RH})$. Absolute humidity is the density of water vapour (moisture), expressed as grams per cubic metre of air $\left(\mathrm{g} / \mathrm{m}^{3}\right)$. Relative humidity $(\mathrm{RH})$ measures the amount of water vapour in the air compared to the total amount of moisture that the air can hold at a specific temperature and pressure (Bobenhausen, 1994). It is usually expressed as a percentage. When the actual concentration of water vapour in air is equal to the water vapour concentration at saturation, the relative humidity is $100 \%$. Therefore, if air contains one-half of the total moisture it can hold, the relative humidity is said to be $50 \%$. Psychrometrics is the measurement of the heat and water vapour properties of air, which are determined by measuring two variables. For example, if wet-bulb and dry-bulb temperatures are measured then relative humidity can be determined with the aid of a psychrometric chart or tables. 
The relative humidity in a tropical city is high, averaging about $80 \%$ year round, and ranging between $58 \%$ and $97 \%$. Combined with high afternoon temperatures, this high humidity can create oppressive conditions when wind velocity is low. This is because evaporation of water from human skin is slow, causing a hot and muggy sensation. Maintaining an appropriate level of humidity is imperative. In general, the ideal comfortable relative humidity range is between 20 and $70 \%$ (Bobenhausen, 1994).

\subsubsection{Precipitation}

Szokolay (2004) describes precipitation as any product of the condensation of atmospheric water vapour that is deposited on the Earth's surface. This could be rain, hail, snow or dew, and is expressed in mm per unit of time. If the water vapour content in the atmosphere exceeds saturation point, some of it will be transformed into fluid or solid ice. This occurs when the atmosphere, a large gaseous solution, becomes saturated with water vapour and the water condenses and falls out of solution. The vapour condenses into droplets which form clouds. Two processes, possibly acting together, can lead to air becoming saturated: cooling the air or adding water vapour to the air and adding moisture. Rain gauges measure precipitation in inches or millimetres. Rainfall is usually described as light, moderate or heavy.

Koenigsberger et al. (1980) suggest that the total precipitation for each month of the year shows a pattern of dry and wet seasons. He added that the maximum rainfall over a 24-hour period can help to predict flooding, which is useful in the design of surface drainage from roofs and paved areas. Precipitation also naturally affects the amount and type of plant growth.

Precipitation in tropical latitudes usually comes in the form of rain, although big hail stones occasionally occur. Variations in precipitation are the most important factor for determining the microclimate. For example, the intensity of rainfall at lower latitudes is greater than at higher latitudes. This is in keeping with the high water vapour content of the tropical atmosphere (Kukreja, 1978). For example, Kuala Lumpur is located near a low latitude (Latitude $3^{\circ} \mathrm{N}$ ) and receives most of its rain 
each month from mid-November until March, when the northeast monsoon brings the rainy season. The dry season occurs from May to September during the southwest monsoon. Overall, rainfall is very heavy throughout the year, with an annual average of about $2000-3500 \mathrm{~mm}$.

\subsection{The benefits of vegetation}

Vegetation can influence urban microclimates directly by shading surfaces and altering wind speeds, and indirectly through evapotranspiration and by modifying the storage and exchange of heat between urban surfaces (Akbari, et al., 1992; DOE, 1993). Vegetation includes all plants or plant life, including trees, shrubs, and lawn.

Trees can have a canopy large enough to shade roofs and walls, which can reduce the amount of solar radiation reaching a house. Solar radiation is also absorbed by the tree canopy, generating evapotranspiration that cools the leaves and surrounding air. Air movement then disperses this cool air resulting in an overall cooling effect. Trees and shrubs can be planted to funnel or deflect wind away from or towards specific areas, as both vertical and horizontal concentrations of foliage can modify air movement patterns. How densely a group of trees or shrubs are planted, as well as their locations determine the amount of wind reduction. For example, dense clusters of trees can reduce wind speed compared to an area devoid of trees. By reducing wind velocity, a landscape aimed at assisting with energy conservation slows air infiltration into a building. In hot-humid tropical climates wind should be channelled for cooling and to provide relief from high vapour pressure. A few large trees with spreading branches can allow breezes to reach a building, and keep the area cool. Grasses and lawn also generate evapotranspiration and cool the surrounding air.

Careful landscape planning can reduce the amount of sunlight heating building surfaces, and prevent reflected solar radiation from entering the house. Trees, grass and shrubs will also reduce air temperatures near the house and provide evaporative cooling. 
This study will identify the direct and indirect effects of vegetation on buildings, and describe the vegetation structure, and the phenomenon of the urban oasis.

\subsubsection{Direct effects of vegetation}

Vegetation affects urban climates and building energy use directly by providing shade to help reduce temperatures, and by lowering and channelling wind speeds. It modifies the thermal interaction between a building and its surrounding environment.

\subsubsection{Shading}

Shading from trees is the cover or shelter provided when trees intercept solar radiation. How effectively this occurs depends on the species and density of the tree, its size and shape, and the location of the moving shadow that it casts. According to Hashem Akbari, et al. (1992), tree shade reduces the amount of cooling energy used inside a building in three ways. Firstly, window shading helps prevent direct solar radiation from entering the interior of the house. Secondly, trees can shade walls, windows and roofs keeping them from getting hot, thereby reducing the amount of heat reaching the interior. Finally, shade keeps the soil around the building cool, which can then act as a "heat sink" for the house.

To achieve efficient shading trees have to be placed strategically. For example, at $40^{\circ} \mathrm{N}$ Latitude the sun is at a low angle in the morning and late afternoon. To counter this evergreen trees should be placed facing southeast and southwest of the building. The best locations for deciduous trees are on the south and east sides of a house (VanDerZanden \& Rodie, 2008).

Conversely, at $40^{\circ} \mathrm{S}$ the most effective landscape planting strategy is to block the summer setting sun by planting dense deciduous trees and shrubs on the west and northwest sides of a house. The trees will cast long shadows that can effectively shade areas of houses, which are otherwise difficult to protect from the sun's heat at this time of the day. When these trees drop their leaves in the winter, sunlight can reach the house to assist with heating. Trees with high, spreading crowns can be planted to the south area to provide maximum summertime roof shading. 
Meanwhile, trees with crowns closer to the ground are beneficial in the west, where shade is needed from low afternoon sun. Evergreen trees on the north and west sides provide the best protection from the setting summer sun, as well as shelter from cold winter winds.

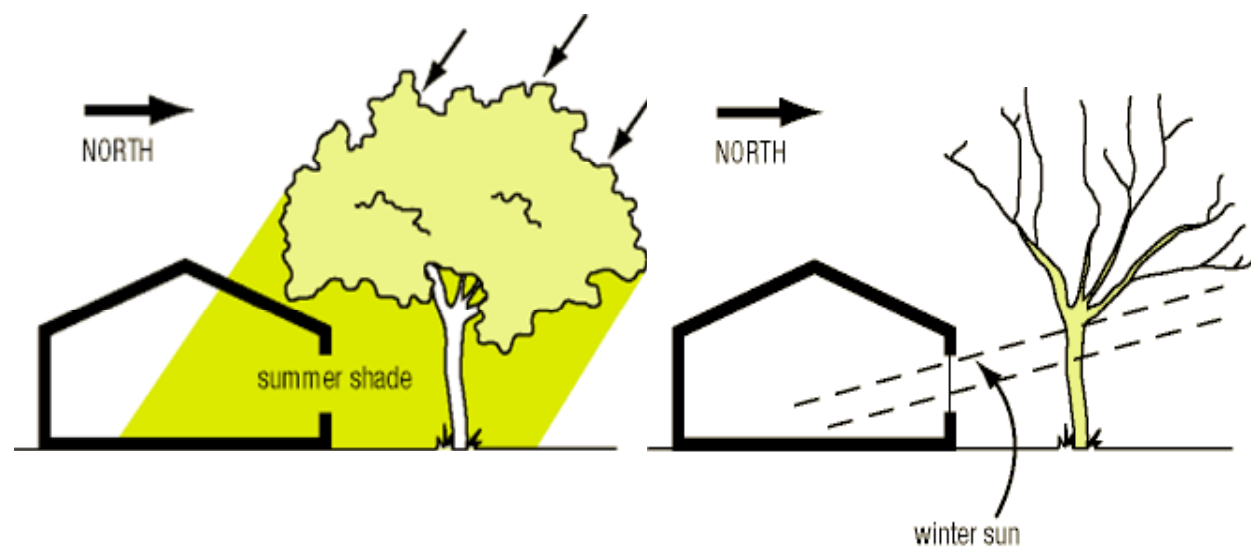

Figure 2.6. In $40^{\circ} \mathrm{S}$ Latitude, deciduous trees on the northwest side of the house reduce indoor air temperature in summer through providing shade, and increase indoor air temperature in winter by allowing sun to enter a house (adapted from Ha, 2009)

Similar principles may be applied in the tropics, which are located in low latitudes near to the equator line. To achieve efficient shading, trees also have to be placed strategically. In this area the sun is at low angles in the morning and late afternoon, and at high angle at noon. To counter this, evergreen trees should be planted facing the east and west sides of buildings. Where large trees are desired, those with a moderate growth rate should be selected because these varieties are sturdy in storms and generally more resistant to disease. A tree that will reach a medium to large size should be located $4.5-6 \mathrm{~m}$ from the side of a house and $3.7-4.6 \mathrm{~m}$ from the corner (LCC, 2011). Tree roots spread can harm the underground building structure if located too close to the building. Smaller trees can be planted closer to a house to shade walls and windows. The type and species of trees used is very important. They should be selected according to the amount of shade they could provide, as well as their aesthetic appearance. 
In addition to the obvious use of trees to provide shade and relief from the summer sun, when they are properly placed, trees can also channel summer breezes to desired locations.

In the tropics, shrubs and other low growing foliage provide shade during the morning and late afternoon when the sun is low in the sky. Shrubs planted close to the house will shade walls and windows. Vines are generally very fast growing and require little space. They are also a source of shade for a building.

\subsubsection{Shading and energy use}

Solar heat passing through windows and being absorbed through the roof are the main factors that result in increased temperatures inside a building, and are therefore a major reason for air-conditioner use and the associated energy costs. Shading is the most cost-effective way to reduce solar heat gain to these areas. Field measurements by Simpson \& McPherson (1996) have suggested that the shade provided by trees and shrubs planted immediately beside buildings can directly reduce cooling loads. Their study in Sacramento showed that trees shading a west exposure from afternoon sun had the greatest impact on cooling savings for all climate zones and insulation levels. Akbari et al. (1986), completed a study in Los Angeles that stated that planting trees can reduce as much as $34 \%$ of residential cooling demand on a hot summer day. They added that the net effect of shade in warm climates was beneficial. Shade from trees reduced annual energy costs by US\$60 compared to the no-tree condition for the conventional residence in Palm Springs, California. Buffington's study (1988) showed that the effect of uniform shading on the walls and roofs of a concrete block residence in central Florida reduced annual cooling costs by US\$108 (14\%).

Simulation studies using limited numbers of building and tree configurations for cities across the USA indicate that shade from a single well-placed, mature tree of about $8 \mathrm{~m}$ crown diameter, can reduce annual air-conditioning use by $2-8 \%$, and the peak cooling demand by $2-10 \%$ (Santamouris, 2001b). Proper, well designed landscaping around a house can save as much as $30 \%$ on heating and cooling costs, 
through energy saving. This can return the initial investment for the landscaping in less than eight years (Haque, Tai, \& Ham, 2000). Haque, Tai et al. (2000), also stated that according to computer models generated by the U.S. Department of Energy, three trees planted in strategic locations will save the average household between US\$100 and US\$250 annually in energy costs.

By blocking solar radiation, trees prevent structures and surfaces from heating up beyond the ambient temperature. Trees not only absorb carbon dioxide from the atmosphere, but also help prevent carbon dioxide emission in the first place through reducing the need for air conditioning. Akbari et al. (1992, p. xxii) suggest that "if enough trees are planted, we may be able to reduce our cooling energy enough to avoid both the costly construction of new power plants as well as their economic and environmental cost". Proper landscaping around the house could potentially save energy and protect our environment.

In addition, shrubs or small trees can be used to shade air conditioning condensers that are located outside a house. This will increase the equipment's efficiency by as much as $10 \%$ (Laumer, 2008).

\subsubsection{Wind reduction and channelling}

Windbreaks are barriers used to reduce the wind speed. They reduce air movement around a building, thereby reducing heat loss from the walls of the buildings. Windbreaks are taller than adjacent plants and are usually formed by only one or two rows of trees (Smith, Jarvis, \& Odongo, 1997). The reduction of wind speed by windbreaks can modify the microclimate in the sheltered zone, increasing the temperature and humidity levels. By reducing wind speed, an energy conserving landscape slows air infiltration. The best windbreaks block wind close to the ground using trees and shrubs that have low crowns. McGinn's (1982) study of urban microclimates in Davis, California, found that wind speed varies according to type of trees canopies and densities. He compared wind speeds at $5 \mathrm{~m}$ above ground in a variety of sites, including a control site of an open field. The results found that the 
density of the canopy reduces wind speeds from between $3 \%$ and $35 \%$ as shown in Figure 2.10.

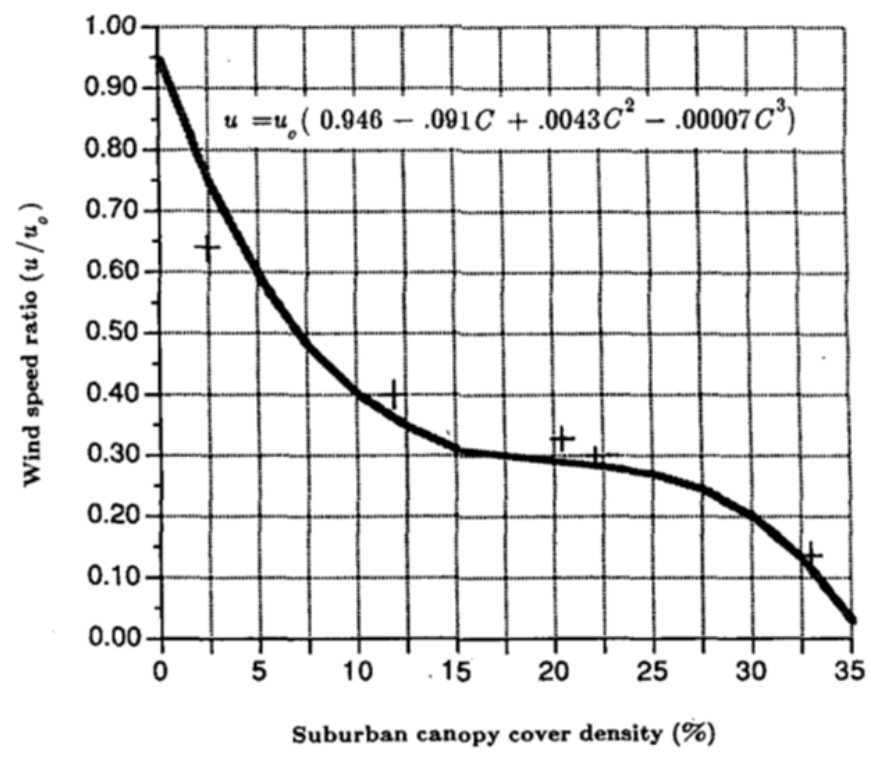

Figure 2.7. Wind speed reductions for different tree canopy densities as a ratio of wind speed at control site with no trees (McGinn, 1982, p. 65)

The effect of vegetation on wind conditions depends to a great extent on the type of vegetation and planting pattern. For example, at $40^{\circ} \mathrm{S}$ Latitude, evergreen trees and shrubs planted to the south and southeast of the house are the most common type of windbreak. Bushes, and shrubs are often planted together to block wind from ground level to the treetops. The most effective windbreaks can reduce wind velocity as much as $50 \%$, depending on the density of the planting (Starbuck, 2007). The predominant effect is a deflection of the airflow.

In addition to planting windbreaks away from houses, planting shrubs, bushes and vines next to a house creates dead air spaces along the walls that provide insulation in winter and summer. Foundation plantings of evergreens eliminates the movement of air close to the house and creates a layer of still air behind it. At least $1.5 \mathrm{~m}$ of space should be left between full-grown plants and a house's wall (Starbuck, 2007). 


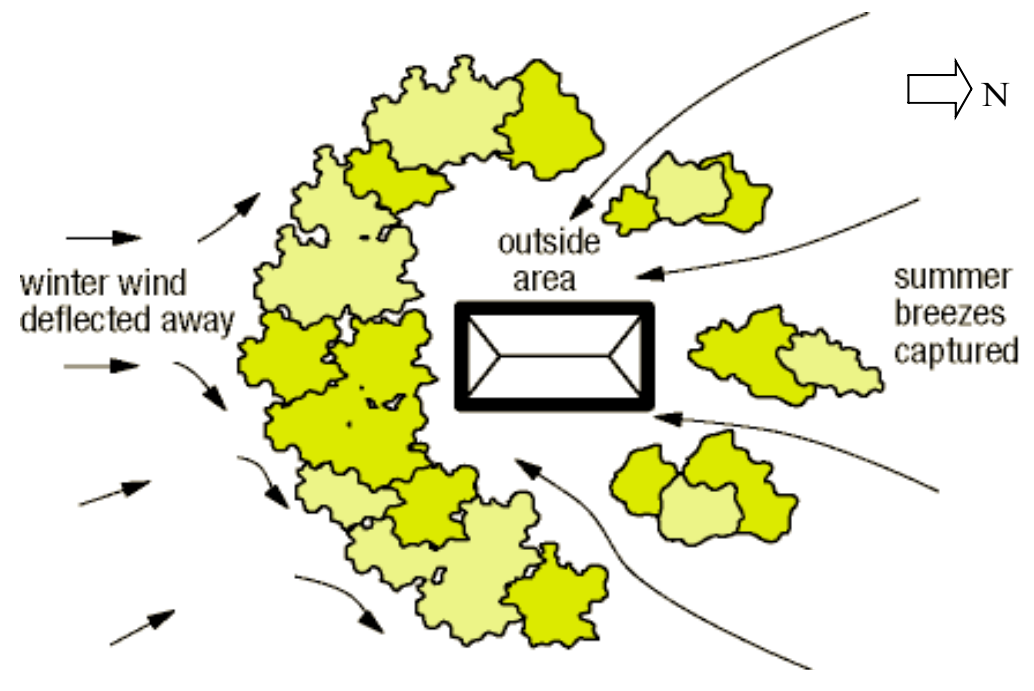

Figure 2.8. Use of evergreen trees in two to three rows in a staggered order, along with shrubs will deflect cold winds, while deciduous trees channel summer breezes (adapted from Ha, 2009)

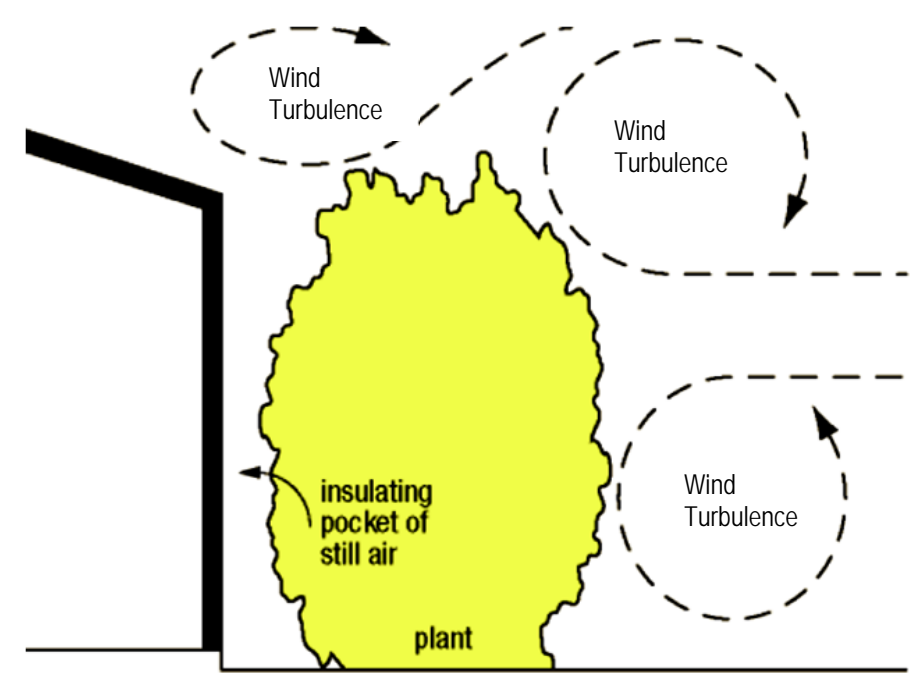

Figure 2.9. Evergreens adjacent to the southwest sides of the house reduce wind speed and create insulation pocket air spaces (adapted from Ha, 2009)

In hot-humid tropical climates, wind is an asset that can provide natural ventilation to buildings, and convective cooling of their hot exterior surfaces. Olgyay (1963) suggests that wind is particularly important for comfort when the temperature is above $29^{\circ} \mathrm{C}$ and relative humidity is above $50 \%$. In these cases, cooling needs are high, and landscaping around buildings can channel cool breezes and help to 
minimize humidity close to the house.

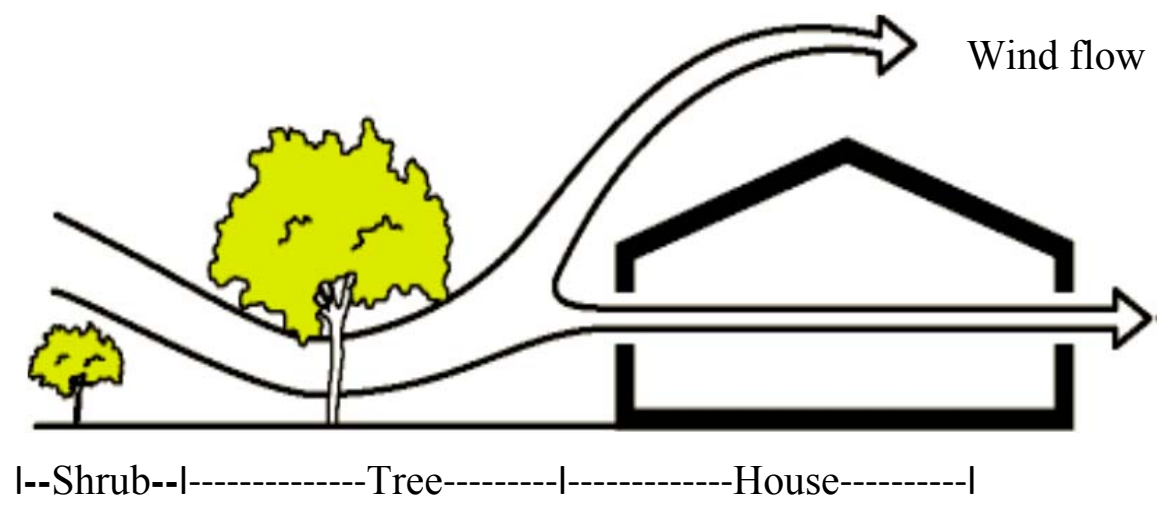

Figure 2.10. The arrangement of trees and shrubs influences the movement of wind around and through a building (adapted from Ha, 2009)

Heat removal from a building during the warmer months can be influenced by the placement of plants, and their shape. Airflow can be increased by the strategic placement of a combination of hedgerows and tree canopies. McPherson, Herrington and Heisler (1988) suggest that in hot-humid climates, high-branching and wide canopy trees and low ground covers should be used to promote shade and encourage wind. Trees with tall trunks and spreading canopies, in combination with shrubs and groundcover are the best plants for providing effective shade and influencing the movement of wind around and through a building. Directing wind through a building and against the walls and roof permits rapid removal of heat.

\subsubsection{Wind and energy use}

The reduction of wind speed that results from trees can increase or decrease cooling and heating energy use depending on local weather conditions (Akbari, et al., 1992). In studies in central Pennsylvania, a moderately dense windbreak that was a singlerow of white pine reduced heat energy use in a mobile house by about $12 \%$ (Heisler, 1986). A similar study of houses in Radisson, New York, by Reimann Buechner Partnership suggested that savings on heating of more than $20 \%$ were due to wind protection (cited in Heisler, 1986). 
A study by Huang et. al. using computer models to simulate wind-speed reduction found that the heating and cooling energy used in seven cities (Minneapolis, Pittsburgh, Chicago, Washington, Sacramento, Miami and Phoenix) could be lowered. Although the heating energy used was reduced, the cooling energy consumption was increased because reduced wind speed was more beneficial to heating energy use (Huang et. al (1990), cited in Akbari, et al., 1992). Akbari et al. revealed that the cooling and heating energy use will decrease if trees are used for wind protection during winter and shading during summer. Annual cooling and heating energy cost saving from three additional trees is estimated to be US\$75-175 per household in all seven cities in the study (Akbari, et al., 1992).

Properly selected and placed landscaping can provide excellent wind protection during winter and wind channelling during summer. This will reduce heating and cooling costs considerably. In a hot-humid tropical climate, planting trees in residential areas can act as a wind channel and potentially have a substantial impact on the energy performance of the houses.

\subsubsection{Indirect effects of vegetation}

Vegetation cools the environment indirectly through evapotranspiration. This is where plants release water vapour which helps to cool the ambient temperature. In general, this affects the whole microclimate.

\subsubsection{Evapotranspiration}

Evapotranspiration is the loss of water from vegetation to the atmosphere by evaporation and transpiration. This is a major part of how trees contribute to decreasing urban temperatures (Santamouris, 2001b). Evaporation accounts for the movement of water to the air from sources such as the soil, canopy interceptions, and waterbodies. Transpiration accounts for the movement of water within a plant and the subsequent loss of water as vapour through stomata of leaves. Transpiration puts moisture into the air and moderates changes in the air temperature. 
Having a large amount of vegetation can modify net radiation, sensible heat, latent heat and heat storage. According to Oke (1988) evapotranspiration increases the proportion of latent heat in comparison with the sensible heat in accordance with the Bowen ratio. The Bowen ratio relates to the ratio of sensible to latent heat fluxes of energy balance. To estimate the actual amount of evapotranspiration, the energy balance equation, $\lambda E=R_{n}+G-H$, where $\lambda E$ is the energy needed to change the phase of water from liquid to a gas in the atmosphere, $R_{n}$ is the net radiation, $G$ is the latent heat flux and heat storage, and $\mathrm{H}$ is the sensible heat flux was used. In a hothumid climate, latent heat may exceed net radiation by $10-50 \%$, with sensible heat derived from the air and converted to latent heat (ASCE, 1996).

The evapotranspiration process can reduce ambient air temperatures. Heisler has stated that trees are effective for cooling because they absorb $70-85 \%$ of the heat from solar radiation by transpiration (cited in Akbari, et al., 1992). Since leaves are generally dark and coarse, and thus reflect very little light, they make ideal solar radiation absorbers and controllers. Leaves absorb about $50 \%$ of the total solar energy (Taiz \& Zeiger, 2006). Therefore, planting three trees per house could reduce heat build-up, and the city heat island effect, by shading and evapotranspiration. This can reduce surrounding air temperatures by up to $5^{\circ} \mathrm{C}$ (DOE, 1995; Foster, 1994). Temperature measurements in Davis and Sacramento, showed that the air temperature in neighbourhoods with mature tree canopies are 1.7 to $3.3^{\circ} \mathrm{C}$ lower during the daytime than newer areas with no trees (Akbari, et al., 1992). Similarly, groundcover such as grasses or turf, also have a cooling effect from evapotranspiration. The temperature directly above groundcover plants can be 5.6 to $8.3^{\circ} \mathrm{C}$ cooler than above a heat absorbent material (Morgan et al., 2000).

However, the amount of water that plants transpire varies greatly geographically and over time. It is determined by a number of factors, such as temperature, relative humidity, wind, air movement, soil-moisture availability and type of plant. 

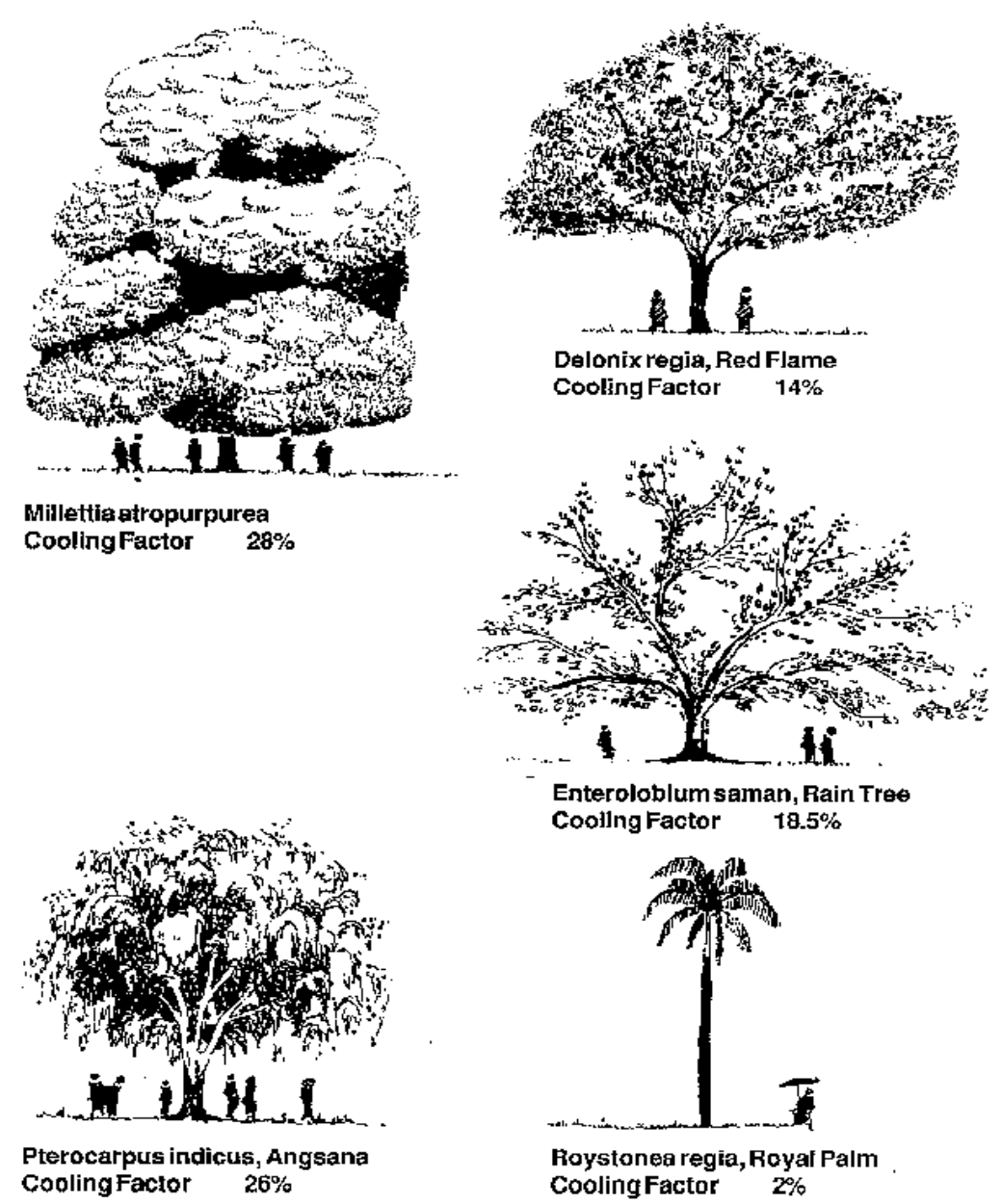

Figure 2.11. Selected tropical trees and their "cooling factors" (Paul \& Dieter, 1993, p. 12)

Temperature is an important for transpiration as it controls the opening of the stomata of leaves. Transpiration rates rise when the temperature is high and air is warmer from solar radiation. Plants transpire water at different rates depending on the type of plant and soil-moisture availability. Paul and Dieter (1993) define the "cooling factor" as the ratio of actual radiation intensity to un-shaded conditions for different species of plant, which are influenced by shade and evapotranspiration. This is illustrated in Figure 2.11. High temperatures cause plant cells to open, which allows water to be released to the atmosphere, whereas colder temperatures cause plant openings to close. As the relative humidity of the air surrounding a plant rises, the transpiration rate falls. When water transpires from a leaf, the water saturates the 
air surrounding the leaf. Wind movement increases the movement of air around a plant, which results in a higher transpiration rate.

\subsubsection{Evapotranspiration and energy use}

The evapotranspiration process from vegetation contributes significantly to cooling our cities and saving energy. Vegetation produces evapotranspiration around individual houses and their neighbourhoods, thus reducing urban temperatures at the microclimatic level. If ample soil moisture is present and environmental conditions are suitable, water in the leaves evaporates and the air is cooled.

The evapotranspirational cooling produced by a single tree is difficult to measure because the cool air is rapidly diffused into the larger volumes of air moving through the tree crown. However, computer simulations have shown that residential neighbourhoods that have a lot of vegetation can produce air temperature reductions of as much as $5.6^{\circ} \mathrm{C}$, compared to nearby areas with little vegetation (McPherson \& Simpson, 1995).
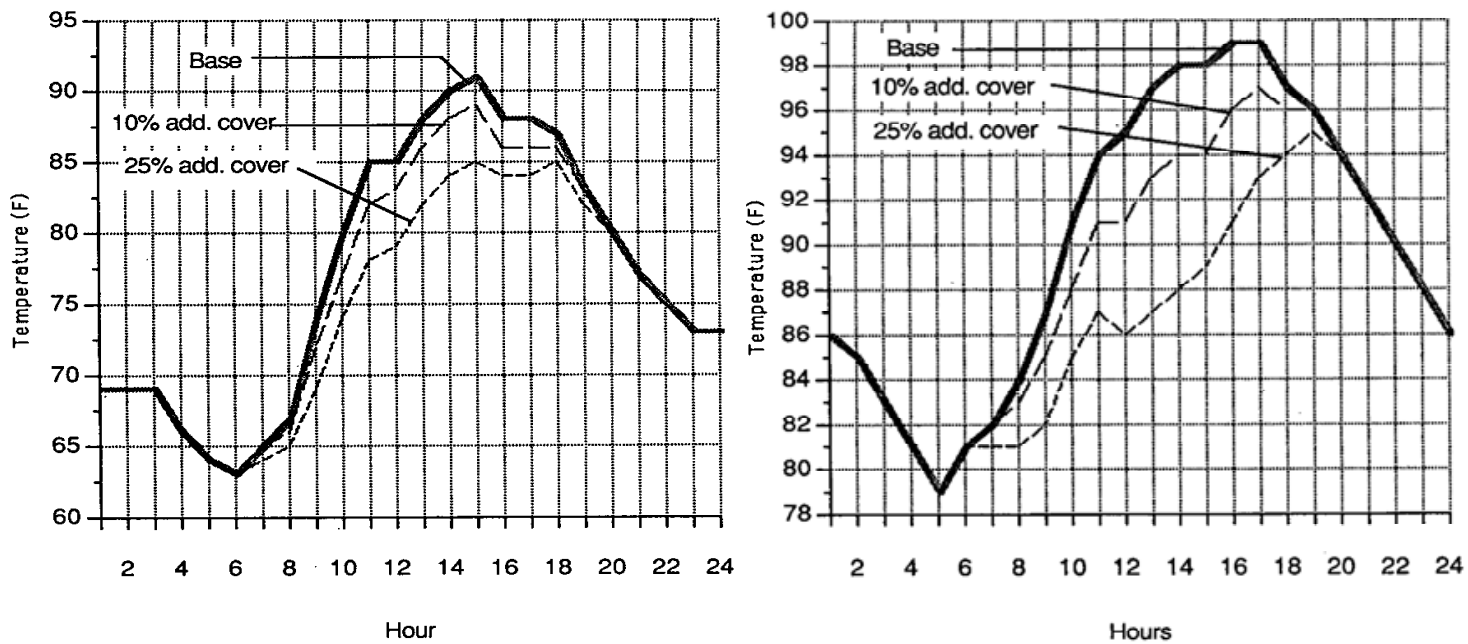

Figure 2.12. Temperature reductions in Sacramento (left) and Phoenix (right) due to added tree cover on a typical summer day in July, based on computer simulations: Increasing tree cover can significantly decrease city-wide temperatures (adapted from Huang, Akbari, Taha, \& Rosenfeld, 1987)

The amount and placement of trees helps to reduce the amount of cooling energy used in houses. Huang et al. (1987) found that a $10 \%$ increase in the tree cover per 
house would result in a cooling energy reduction of $24 \%$ in Sacramento, and $12 \%$ in both Phoenix and Lake Charles. This could result in a saving of between US\$40-90 a year. A $25 \%$ increase in tree cover, which equalled three trees per house on the southeast and west sides, was estimated to have an even more dramatic impact, with reductions in cooling energy use of 57\% in Sacramento, $17 \%$ in Phoenix and 23\% in Lake Charles. This corresponded to a saving of US\$100-250 a year per household (Huang, et al., 1987). According to Huang et al. (1987), shading accounted for only $10-35 \%$ of the total cooling energy saving. The remaining savings resulted from the lowered temperature due to evapotranspiration. This show that a minimum of three mature trees strategically placed around a residential building can reduce cooling energy use.

\subsubsection{Vegetation structures}

Vegetation is the general term for the plant life of a region. Plants do not exist by themselves in isolation; groups of plants living in the same area form a plant community. The vegetation structure relates to the number of vertical height layers of plants. An example of a complete vegetation structure is in the natural tropical rainforest ecosystem. Tropical rainforests, located around the equator and tropical areas of heavy rainfall, are the most productive and complex ecosystems on Earth. The canopy system, characteristic of tropical rainforests, increases diversity.

In a rainforest, there are four layers of vertical stratification of trees. According to Michael (2001), these layers are the emergent (A), upper canopy (B), under storey (C), and forest floor (D), as shown in Figure 2.13. The emergent category includes the tallest trees, which are usually over $45 \mathrm{~m}$ tall with umbrella-shaped canopies that extend above the general canopy of the forest. Layer B or the upper canopy is a closed canopy of trees that are around $24 \mathrm{~m}$ tall. Light is readily available at the top of this layer, but greatly reduced below it. Layer C or the understory, is a closed canopy of trees that are around $20 \mathrm{~m}$ tall. Layer D, the forest floor, comprises of dense growth of shrubs, ferns and other plants that need less light at ground level of the rainforest. The ground layer is sparse plant growth. 
In landscape design, the plant types configuration and species that are chosen can be similar to the natural plant structure of the rain forest. The use of trees, shrubs and groundcovers in the production of shade, evapotranspiration, and channelling wind depends on their size, species and location. Trees are used where the "habit" aspect of appearance is the most prominent, while with shrubs the "form" aspect is the most prominent (Hackett, 1979). Habit is a pattern of tree behaviour or appearance acquired through frequent repetition, such as a weeping willow. Form is more like a rigid framework with branches from each trunk. Hackett (1979) describes how the globular form created by the leaves at the extremities of the branch system is clearly observable, as is the weeping or drooping habit of the branches. Another example of tree form is the Douglas Fir, which has a pyramidical form, or the fountain form for Roystonea Regia.

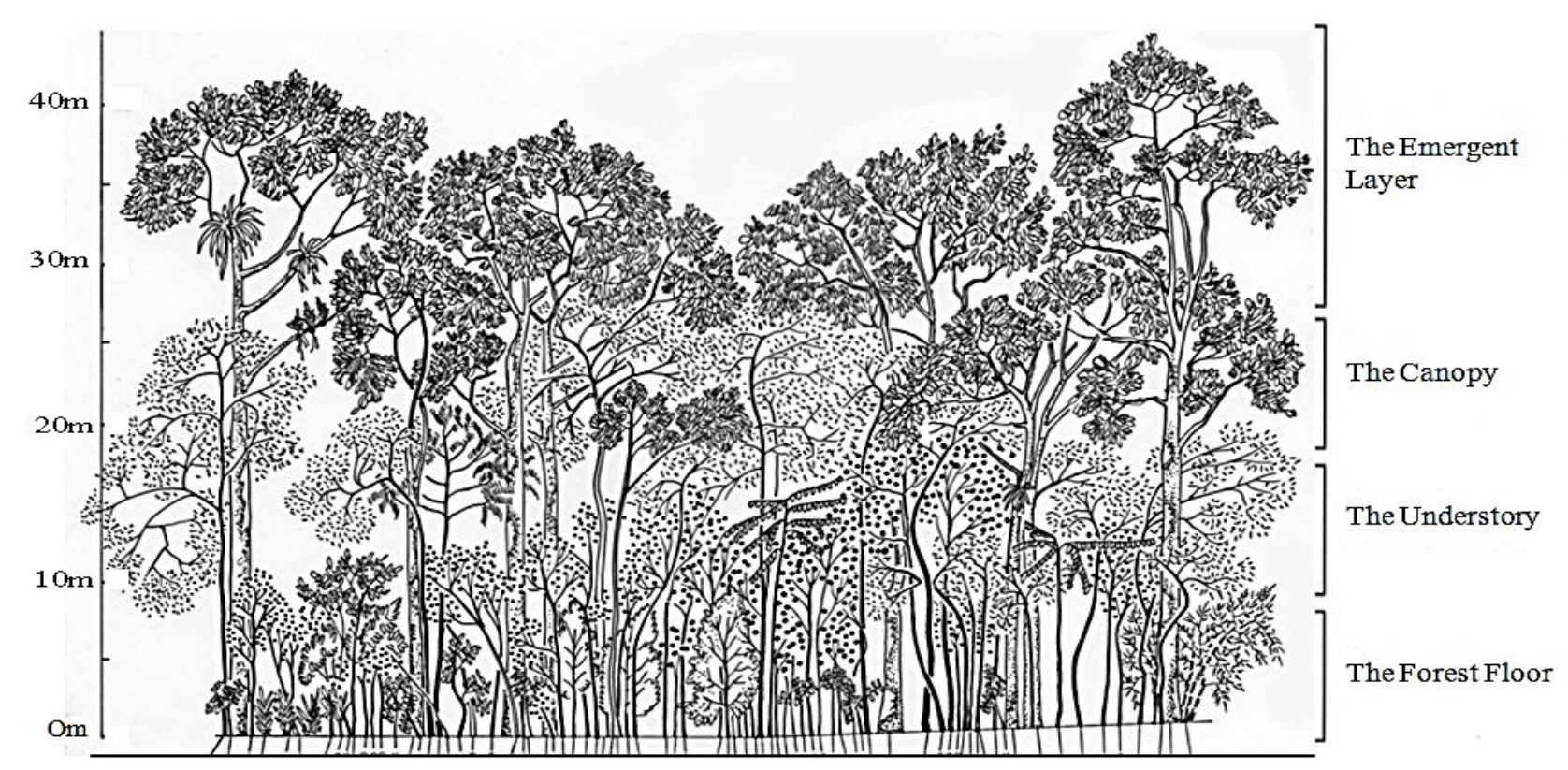

Figure 2.13. A sketch-profile drawn of a fruit forest in West Sumatra, Indonesia showing the complex, forest-like structure of the vegetation (adapted from Michon, Foresta, Levang, \& Verdeaux, 2007)

\subsubsection{Landscape design typologies: the tropical garden}

Generally every garden design was different and had its own identity and style. They were dictated by site, size, microclimate and the preferences of their creators (Sparrow \& Hanly, 2002). Tropical gardens have strong elements, including bold 
foliage, dramatic colours and flair, which create formal and informal style of design. The use of colourful vistas, linear pathways and focal points create the structure of the gardens, and help to provide a link between the indoor spaces and their surrounding outdoor spaces (Ross, 2011).

The tropical and subtropical zones contain the greatest variety of plants in the world, with an estimated minimum of 200,000 species (Sparrow \& Hanly, 2002). According to Casper (2007), besides having several canopies, the favourable conditions of the tropics encourages diverse plant life, which include trees, palms, shrubs, climbers, grasses, ground cover and lawn. These native tropical plants are typically lush and exotic, with thick and waxy leaves (Dawn, 2011). They grow rapidly, and frequently dominate tropical landscaping. Tropical plants also typically bloom over a long period because the average length of days and temperature changes little with the seasons near the equator (Brandies, 2004).

For the most part, tropical gardens rely on foliage rather than flowers to create interest throughout the year. Foliage should be flamboyant, lively and colourful, and plants must be chosen on the basis of the size, shape and texture of their leaves (Ross, 2011). According to Wijaya (2000), when planning the planting scheme in a tropical garden it is important to add a few "wet-season flowers" so that the garden is not just green throughout the year. In the wet months a tropical garden relies on shapes and silhouettes for dramatic effect.

Herbs and spices are synonymous with tropical gardens. As well as contributing their wonderful flavours and aromas to a wide variety of dishes, they provide fragrant garden areas.

Ross (2011) stressed that planting in groups of odd numbers is a common trick employed by tropical garden designers, as it gives a broad brushstroke of colour and texture, and makes a huge difference to the feel of the garden. Placing plants with contrasting foliage next to each other will create drama and interest. 


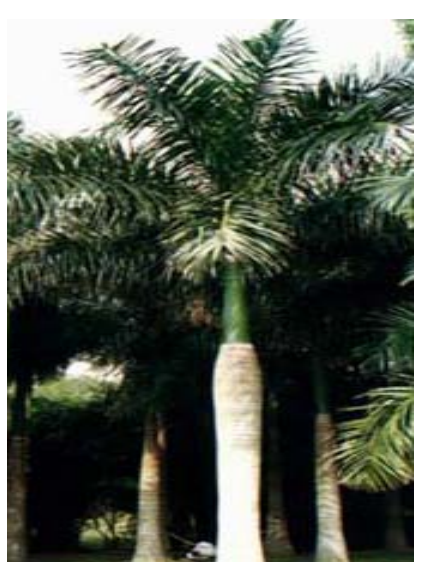

Fountain

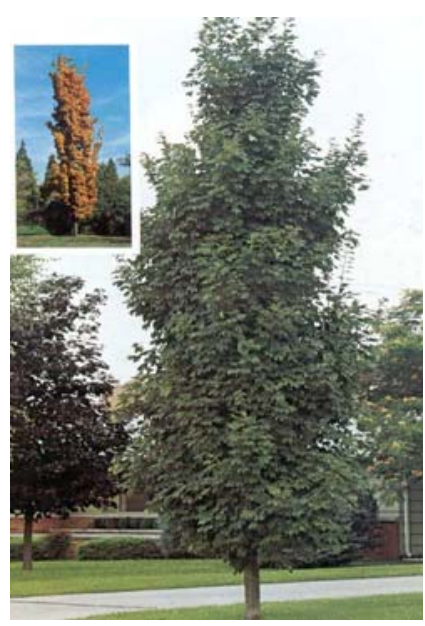

Columnar

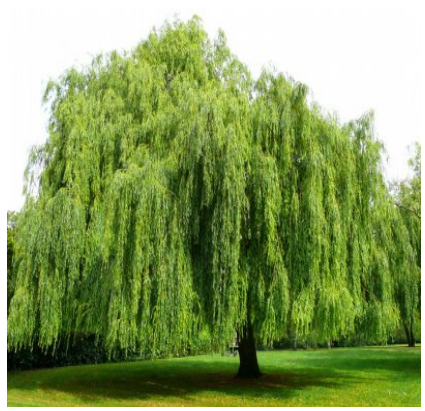

Weeping

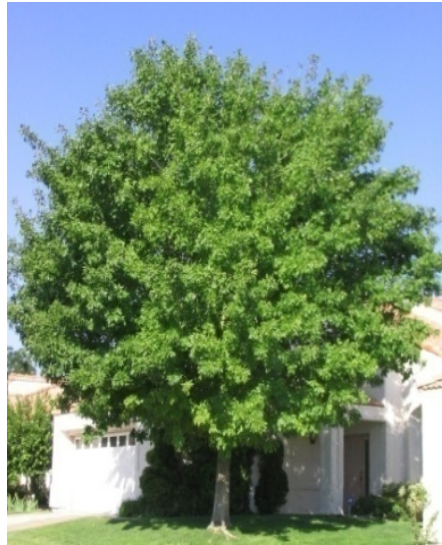

Round

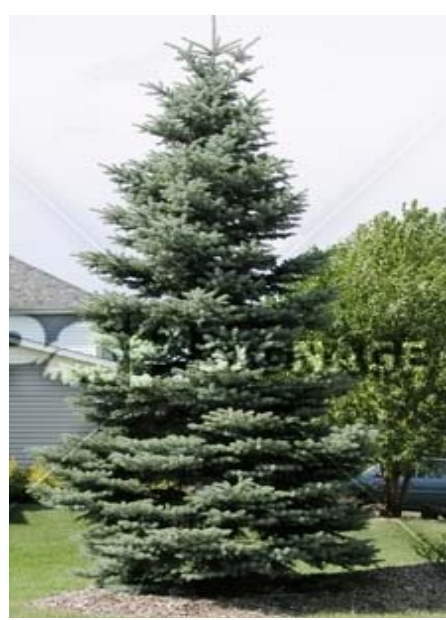

Pyramidal

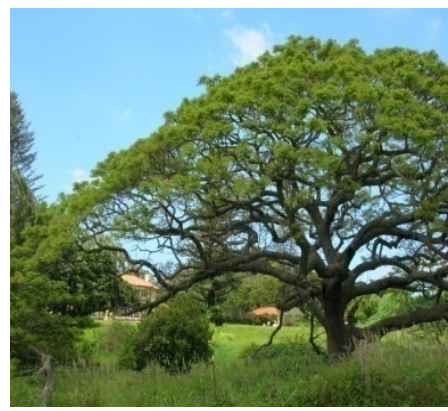

Spreading

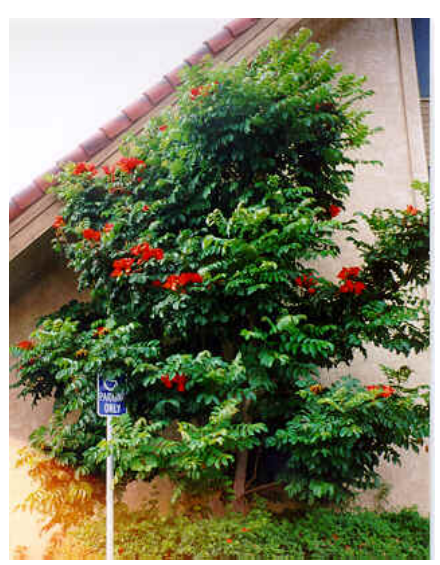

Layered

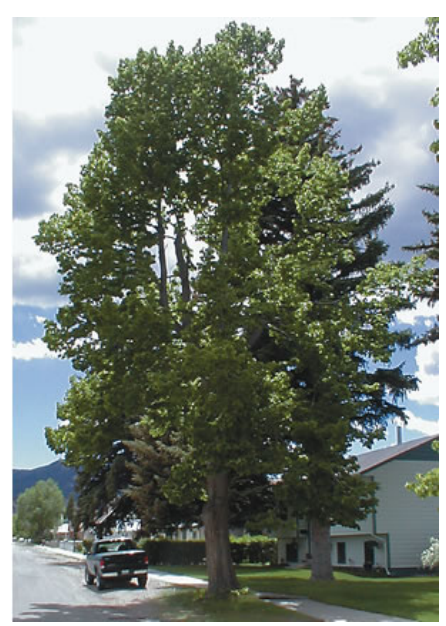

Oval

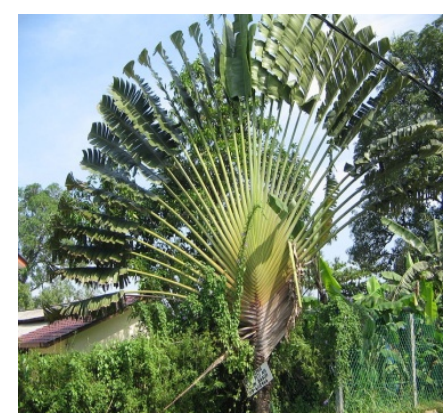

Vast or Fan-like

Figure 2.14. The differing structures, types and shapes of shade trees (adapted from Hackett, 1979)

In tropical regions, trees are the most important plant structure in a garden. They often grow particularly fast, and can be the most satisfying plants in the garden (Sparrow \& Hanly, 2002). However, while large trees with spreading branches help to keep houses cool, there should not be so many of them to exclude breezes. The 
length and patterns of shade provided by trees vary with the seasons. Foliage-canopy structures and vertical woody species distribution are also important factors, as they can influence shading, evaportanspiration and the channelling of wind.

Mature trees (those whose height is more than $15 \mathrm{~m}$ ) are classified into three major distinctive forms: round, oval or vertical. Round shaped treess are where the spread and the height is roughly the same. Oval shaped trees are where the spread is nearly half the height and the foliage takes an oval shape. Vertical shapes are where the trees do not spread very wide, and are known as columnar trees. Tall, full crowned trees are best for blocking the rays of the sun when it is shining directly overhead at mid-day. Oval shaped trees are best used on the eastern and western sides of buildings to provide the maximum protection to the roof and walls from solar heat gain, while allowing the access to cool breezes. Vertical or columnar trees can be used as wind barriers and dust screens.

In the domestic landscaping, palms convey instant opulence, having become fashionable symbols of luxury and pleasure (Sparrow \& Hanly, 2002). Their shapes are fountain-like. Sparrow \& Hanly also state that the range of palms is overwhelming, with over 200 genera and numerous species. Most palm species enjoy the humid subtropics and tropics.

Shrubs are woody plants that have numerous stem structures, with dense, bushy, open willow-like shapes (Sparrow \& Hanly, 2002). While their height varies, they are usually no higher than $3 \mathrm{~m}$. Shrubs can be trained to grow against walls, as hedge plants, and in containers. Some shrubs do well in shade, but many prefer sunlight.

Climbers, vines and creepers are flexible and versatile plants. They are ideal for many positions in the subtropical and tropical garden.

Ground cover plants provide a variety of textures and colours to gardens, and can provide a transition between landscape spaces. Lawns are created for aesthetic and 
recreational use, including for sports. Many different species of grass are used, often depending on the intended use of the lawn and partly on climate.

Integral to the tropical garden style are structures and accessories such as pools, fountains, pergolas and gazebos. In Malaysia, tropical garden design has been influenced by various styles of garden, depending of the era when the garden was built. Examples include formal English style gardens, which were popular during seventies and early eighties; and blooming tropical Balinese style gardens which were popular during the nineties. Currently most houses built in Malaysia apply a contemporary style of tropical landscaping that suits small sized gardens and is simple and practical in design. A new tropical garden style, neo-archipelago is being introduced; the design is influenced by Malay culture and heritage.

\subsubsection{Urban oases}

The "oasis effect" is defined as a change in the microclimatological conditions in a vegetated (green) area (Saaroni, Bitan, Dor, \& Feller, 2004). The change is manifested in lower temperatures and higher relative humidity (Oke, 1988). Oke states that green areas are more humid and therefore cooler than their surroundings due to evapotranspiration processes. Shade from vegetation also prevents direct radiation from reaching the ground surface and warming it, thereby resulting in lower air temperatures above these surfaces. As a result of the evapotranspiration process, the air near the ground in green areas is cooler.

According to Santamouris (2001b), evapotranspiration contributes to creating lower temperature spaces in the urban environment. This is known as "the oasis phenomenon". In this process, the plant draws moisture from the ground and uses what it needs for growth and moderates its own temperature as it transpires the excess water. This then cools the surrounding air. Evaporation of water from the leaves that are exposed to the sun consumes most of the absorbed solar radiation. The evaporation cools the leaves and the air in contact with them, and at the same time increases the humidity of the air (Givoni, 1991). Givoni stresses that the importance of this effect depends on the local humidity and temperature conditions. Similarly, 
Montgomery's (1987) study showed that energy transfer to latent heat from plants is very high: $2324 \mathrm{~kJ} / \mathrm{kg}$ of water evaporated compared to area without plants. This is supported by Moffat and Schiller (1981), who stated that $1460 \mathrm{~kg}$ of water is evaporated from an average tree during a sunny summer day.

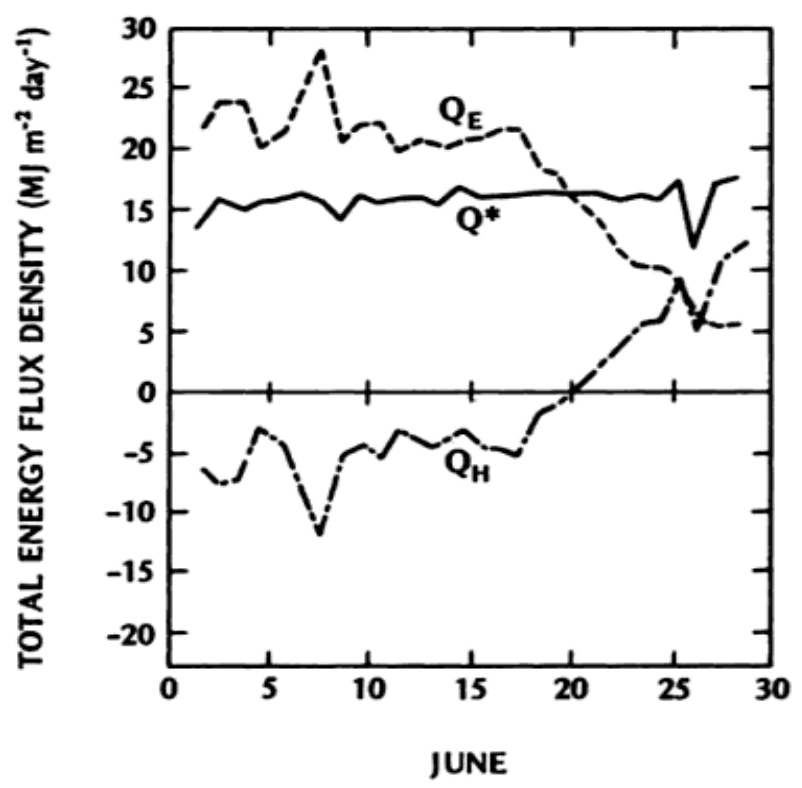

Figure 2.15. The oasis effects in a semi-arid environment. Average daily energy balance of an alfalfa crop in Jun 1964 located at Phoenix, Arizona (33 ${ }^{\circ} \mathrm{N}$ Latitude).

The crop was irrigated by flooding in late May, which was followed by drought throughout June 1967. $\mathrm{Q}_{\mathrm{E}}=$ turbulent latent heat flux density $\left(\mathrm{Wm}^{-2}\right), \mathrm{Q}_{\mathrm{H}}=$ turbulent sensible heat flux density $\left(\mathrm{Wm}^{-2}\right), \mathrm{Q}^{*}=$ net all-wave radiation flux density $\left(\mathrm{Wm}^{-2}\right)$

(Oke, 1978, p. 144)

Santamouris (2001b) states that the magnitude of the temperature reduction is related to the overall energy balance of the area, but in general the oasis phenomenon is characterised by the Bowen ratio. Any surface planted with vegetation has a different Bowen ratio from a mineral surface. This is because the incoming solar radiation is converted into energy for transpiration and photosynthesis by the plants, and the sensible heat flux is consequently lower (Taha, et al., 1988; Wong \& Yu, 2005). Taha (1997) reported that in average oasis conditions, the Bowen ratio in vegetative canopies is within $0.5-2$. A Bowen ratio of two was measured in a pine forest in England in July around noon, which corresponds to sensible and latent heat fluxes of 400 and $200 \mathrm{~W} / \mathrm{m}^{2}$ respectively. Taiz and Zeiger (2006) demonstrate that in 
well-watered crops, transpiration and hence water evaporation from the leaf is high, so the Bowen ratio is low. Conversely, when the evaporation rate is low because water supply is limited, the Bowen ratio tends to be high. Lower Bowen ratios signify a cooler environment.

Numerous studies simulating the effect of additional vegetation on urban temperatures have been performed by various researchers and provide very useful information. The computer simulation by Huang et al. (1987) predicted that increasing tree cover by $25 \%$ in Sacramento and Phoenix, USA, would decrease air temperature at 2.00 p.m. in July by 3.3 to $5.6^{\circ} \mathrm{C}$. Taha (1997) reported that the factors that affect temperature reduction are evaporative cooling and shading of the ground, whereas temperature increase during the night is the result of the reduced sky factor within the canopy. He concluded that the results of the simulations he carried out show that vegetation cover of $30 \%$ could produce a noontime oasis of up to $6^{\circ} \mathrm{C}$ cooler in favourable conditions, and night time heat island of $2^{\circ} \mathrm{C}$.

Evapotranspiration and shading play a role in creating the oasis effect (Saaroni, et al., 2004). Oke states that it can be modified by wind. He explains that it is well known that microclimatic conditions, such as radiation frost and urban heat islands, are well developed under calm conditions, while an increase in wind speed weakens and even prevents their development (cited in Saaroni, et al., 2004). The magnitude of the temperature modification and the size of the oasis boundary layer are dependent upon the size and physical properties of moisture sources, such as large irrigated fields and heavy urban vegetation. According to Bernatzky (1982) they reduce the local temperature by $2-4^{\circ} \mathrm{C}$. In hot-humid tropical climates, landscape design plays an important role in determining the extent of the oasis effects on a neighbourhood scale. The most important implications are the need for more shaded areas and to minimise the sun-exposed grass area.

\subsection{The influence of the building envelope}

The building envelope is the interface between the exterior of the building and the interior environment. It includes the walls, roof, glazing and floor foundation. The 
core purpose of the building envelope is to improve comfort level for occupants when compared to the conditions in the exterior environment by protecting them from moisture, temperature and weather elements (Alread \& Leslie, 2007). According to Qiu \& Haghighat (2005), the building envelope isolates a controlled indoor environment from the outdoors, and is designed to be airtight to minimize the costs of conditioning air. The building envelope's skin consists of structural materials and finishes that enclose a space, separating the outside environment from inside and includes walls, windows, doors, roofs and floor surfaces. The envelope should enable ventilation and daylight penetration, while providing thermal and moisture protection that is appropriate for the climate conditions (Hartweg, 2007). Envelope design is the integration of building form and materials to achieve optimum comfort and energy savings (Okba, 2005).

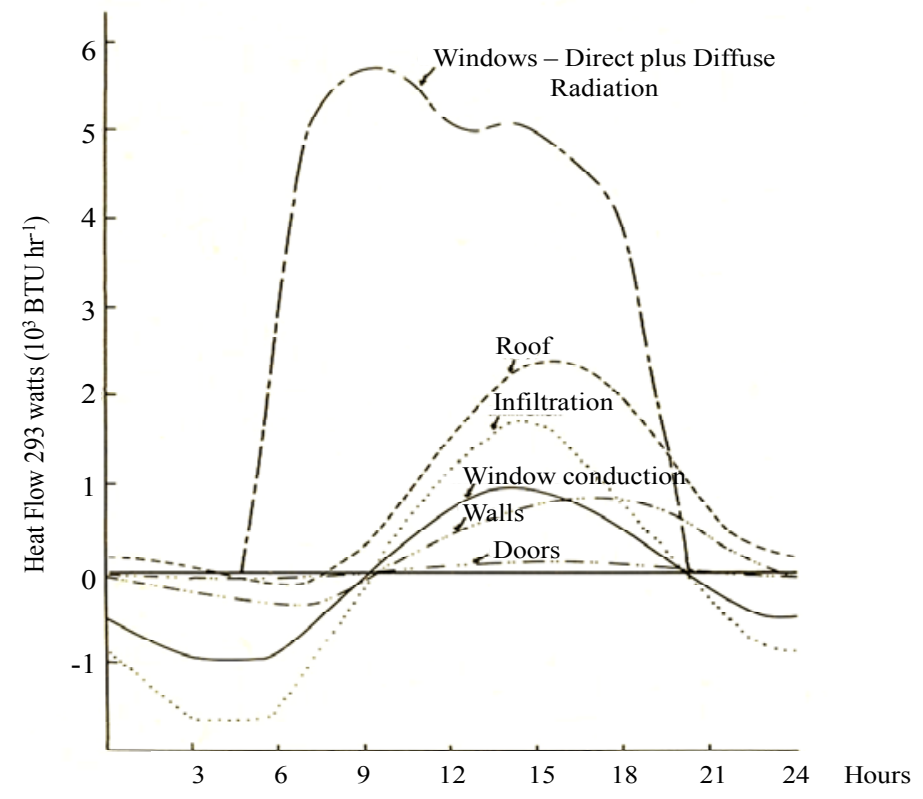

Figure 2.16. The result of analysis of the heat flow through various components of a typical single storey residential building envelope (adapted from Olgyay, 1963)

One of the most important factors affecting building envelope design is climate. The envelope can be considered as the selective pathway for a building to work with the climate, as it responds to heating, cooling, ventilating and natural lighting needs (DOE 2004, cited in Okba, 2005). Each type of climate will require different building envelope design strategies, and different designs and materials can 
potentially provide solutions for any given climatic conditions. An optimal design of the building envelope may provide significant reductions in cooling and heating loads.

The environment affects building envelope design by influencing building configuration, insulation, fenestration, the type of shading devices used, the choice of colour for the wall and roof, albedo and energy use.

\subsubsection{Building configuration}

A building's shape, solar orientation, interior layout and size are factors that affect its energy use and the requirements of the building envelope. The configuration of the house should be designed to suit the particular climate it is located in to ensure the comfort of its occupants and for energy efficiency (Nervegna, 2003). For example, in $40^{\circ} \mathrm{N}$ Latitude climates buildings should be compact to reduce heat loss caused by winter winds, and elongated on the east west axis to maximise solar gain. The heavy wall should be to the north or northwest.

In a hot-humid climate, proper house orientation in relation to the sun will enable energy savings and more comfortable conditions. Heat gain through the building envelope, particularly through windows, should be minimised and shading maximised. This can be done by blocking the sun, which is at a steeper angle in the summer, by using louvers and overhangs. Air movement should be maximised with cross ventilation.

The optimum level of solar access and the need to capture cool breezes varies with climate. Ideally heavy walls should face east and west. Positioning a long solid wall to the east-west position minimises the amount of daylight that enters the internal space, which reduces heating effects. A common recommendation for residential dwellings is to place living and kitchen areas to face solar noon, and bedrooms on the opposite side. Appropriately a building designed in tropical country should also aim to have well shaded north or south facing glazing to reduce diffused solar radiation. 
It is important that building designers understand the path of the sun at the building site. Other than near the poles, the sun will rise in the east and set in the west. In the tropics, passive solar radiation passing through windows will heat the interior of the building, affecting the occupants' comfort. This in turn increases the energy used by air conditioners. An ideal building configuration will minimise internal energy use by optimising the envelope design to suit the annual path of the sun and the local climate. Optimising building shape, orientation and shading will save energy and produce a more comfortable, and less expensive house to run.

\subsubsection{Insulation}

The material use for building envelopes conducts heat at different rates. The different components of the envelope, such as foundation, walls, sills, studs, joists, beams, connectors and roofing materials, can create paths for the transfer of thermal energy, which conduct heat in or out of the envelope (Hartweg, 2007). It is possible to choose and place insulation materials that will assure thermal efficiency.

Insulation is essential for an energy-efficient house. Heat flows naturally from a warmer to a cooler space; insulation provides resistance to heat flow, thereby reducing the amount of energy needed to keep a building warm in the winter and cool in the summer. Properly insulating your house will decrease heat flow by providing an effective resistance to the flow of heat.

Insulation is frequently discussed in terms of its ability to resist heat flow, its R-value (DOE, 2008). R-value is a measure of thermal resistance used in the building. The inverse of the R-value is the U-value. The U-value, or coefficient of heat transmission, is a measure of the rate of non-solar heat loss or gain through a material or assembly. U-values gauge how well a material allows heat to pass through it. Their ratings generally fall between 0.20 and 1.20 , where the lower the U-value, the greater a product's resistance to heat flow and the better its insulating value (DOE, 2009). A variety of insulation options exist, including blanket, concrete block, insulating concrete forms, spray foam, rigid foam and natural fibre insulation (DOE, 2008). 
Table 2.1. The U-value is based on an outdoor surface conductance of $22.7 \mathrm{~W} / \mathrm{m}^{2} .{ }^{\circ} \mathrm{C}$, and an outdoor surface conductance of $9.377 \mathrm{~W} / \mathrm{m}^{2} .{ }^{\circ} \mathrm{C}$ (adapted from Olgyay, 1963).

\section{OVERALL HEAT TRANSMISSION COEFFICIENT (U) AND TIME LAG CHARACTERISTIC DATA FOR HOMOGENEOUS WALLS}

\begin{tabular}{lccc}
\hline Material & $\begin{array}{c}\text { Thickness, } \\
\text { Metres }\end{array}$ & $\begin{array}{c}\mathrm{U} \text { value, } \\
\mathrm{W} / \mathrm{m}^{2} .{ }^{\circ} \mathrm{C}\end{array}$ & $\begin{array}{c}\text { Time Lag, } \\
\text { Hours }\end{array}$ \\
\hline Stone & 0.20 & 3.80 & 5.5 \\
& 0.30 & 3.12 & 8.0 \\
& 0.41 & 2.67 & 10.5 \\
Concrete & 0.61 & 2.04 & 15.5 \\
& 0.05 & 5.56 & 1.1 \\
& 0.10 & 4.77 & 2.5 \\
& 0.15 & 4.20 & 3.8 \\
Common brick & 0.20 & 3.75 & 5.1 \\
& 0.30 & 3.07 & 7.8 \\
& 0.41 & 2.61 & 10.2 \\
Face brick & 0.10 & 3.41 & 2.3 \\
Wood & 0.20 & 2.31 & 5.5 \\
& 0.30 & 1.76 & 8.5 \\
& 0.41 & 1.42 & 12.0 \\
Insulating board & 0.10 & 4.37 & 2.4 \\
& 0.01 & 3.86 & 0.17 \\
& 0.03 & 2.73 & 0.45 \\
& 0.05 & 1.70 & 1.3 \\
& 0.01 & 2.38 & 0.08 \\
& 0.03 & 1.48 & 0.23 \\
& 0.05 & 0.79 & 0.77 \\
& 0.10 & 0.45 & 2.7 \\
& 0.15 & 0.28 & 5.0 \\
\hline
\end{tabular}

\subsubsection{Fenestration}

Openings are located in the building envelope to provide access to a building, admit daylight and solar energy for heating, supply natural fresh air for ventilation, and allow views to the outside. Openings include windows, doors, and vents. Commonly, window openings must, by law, have a combined area of not less than one-tenth of the floor area of each room (UBBL, 2006). The form, size and location of the openings vary depending upon the role they play in the building envelope. Window glazing can be used to affect occupant comfort, and heating and cooling requirements 
by controlling the type and amount of light that passes through windows as seasons change. According to Lechner (2000), a skylight window collects a great amount of solar radiation in the summer and little in the winter. In the northern hemisphere roughly $40 \%$ of the unwanted heat that builds up in houses comes in through windows, particularly during summer (DOE, 1994).

Solar gain is often desirable in the winter and undesirable in the summer. The design must be location-specific and strongly influenced by the local annual path of the sun. In the tropics, windows are often the major source of solar heat gain. Krigger and Dorsi (2004) stated that window glass transmits between $20-84 \%$ of the sun's heat, depending on whether it is clear glass, low-e glass, reflective glass or heat-absorbing glass. The building's window surface area and R-values determine the level and comfort, and extent of energy problems associated with it. Usually windows have an $\mathrm{R}$-value of between R-0.18 and R-0.53 $\mathrm{m}^{2} .{ }^{\circ} \mathrm{C} / \mathrm{W}$

\subsubsection{Shading devices}

Shading is a key strategy of achieving thermal comfort in the summer by preventing unwanted passive solar heating. In warm, sunny climates excess solar gain often results in high cooling energy consumption; in cold and temperate climates winter sun entering windows can contribute to passive solar heating; and in nearly all climates, controlling and diffusing natural illumination will improve day light (Prowler \& Prowler, 2008).

Shading window glass affects the quantity of radiation that enters a room, and hence modifies the heat flow to the interior (Givoni, 1969). To get the best effect, windows must be shaded from direct solar radiation, as well as diffused solar radiation (Lechner, 2000). How effective a shading device is, depends on the proportion of the window surface it covers during the overheated period, without intercepting the sun's energy during cooler periods (Olgyay \& Olgyay, 1976). It also depends on the location of the shading, with respect to the glass, and if it is internal or external (Givoni, 1969). External shading is generally more effective at keeping a house cool than internal blinds or curtains because it does not allow sunlight to reach the 
windows. However, using both external and internal window coverings provides the maximum protection.

There are six types of exterior shading devices: roof overhangs, awnings, shutters, louvers, blinds and solar screens. Overhangs are very effective at mid-day for windows facing the sun at that time. If properly dimensioned, they can block sunlight during the time of maximum solar radiation. The depth of the overhang is an important design element, since it determines the amount of shade, as shown in Figure 2.17. Awnings are also very effective because they block direct sunlight. They are usually made of fabric or metal and are attached above the window, and extend down and out. A properly installed awning can reduce heat gain up to $65 \%$ on southern windows and $77 \%$ on eastern windows (DOE, 1994). A light-coloured awning does double duty by also reflecting sunlight.
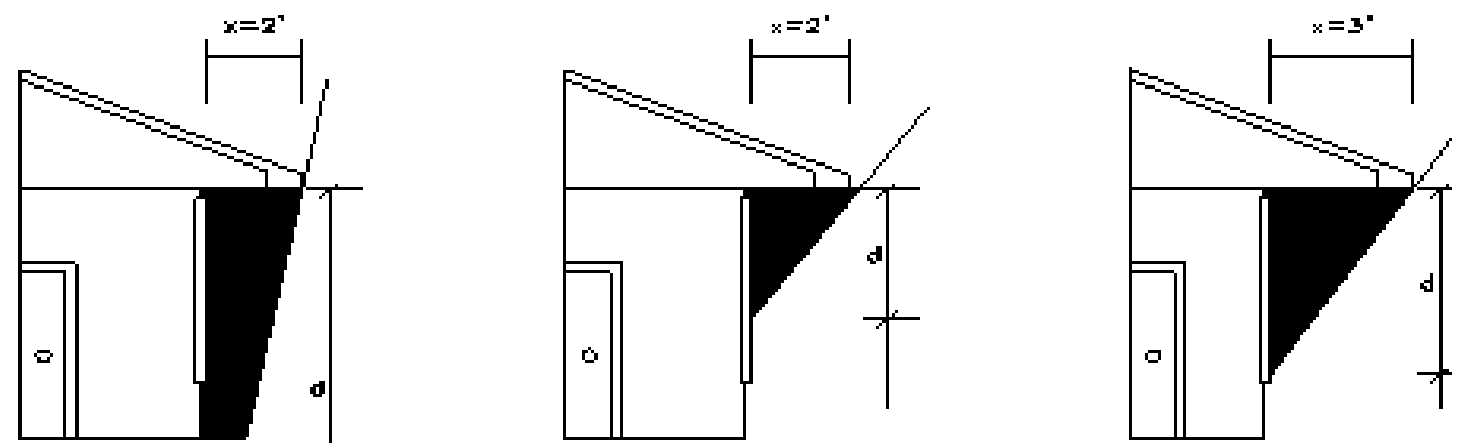

Figure 2.17. The amount of shades are determine by the depth of overhang (EJCR, 2012)

Shutters are moveable wooden or metal coverings which, when closed, cover a window and prevent sunlight from entering a house. Shutters are either solid or slatted with fixed or adjustable slats. Besides blocking out the summer heat, shutters provide some insulation for windows in winter. Louvers have slats like shutters, but instead of having to move an entire shutter, just the slats are closed or opened to prevent or allow sunlight to enter the room. The slats can be vertical or horizontal, and, depending on the design, are controlled from inside or outside the house. Exterior Rolled Blinds also have a series of slats that are lowered along a track when shading is desired. 
Similarly, in hot-humid climate areas, shading devices are needed during peak times of the day on the east and west sides. Well-designed sun control and shading devices can dramatically reduce heat gain to a building a peak times and therefore the amount of energy used for cooling. They can also improve the natural lighting quality of building interiors. Depending on the amount and location of fenestration, reductions in annual cooling energy consumption of $5-15 \%$ have been reported (Prowler \& Prowler, 2008). To properly design shading devices it is necessary to understand the position of the sun in the sky, which is expressed in terms of altitude and azimuth angles.

While interior shading is not as effective as exterior shading, it is still worthwhile for a number of practical reasons as these devices also provide numerous other benefits such as glare control and insulation. Interior shading that can block the sun's heat includes draperies, curtains, roller shades, venetian blinds and shutters. Using draperies and curtains made of tightly-woven, light-coloured and opaque fabrics can reflect more of the sun's rays than they let through. The tighter the curtain is against the wall around the window, the better its ability in preventing heat gain. However, this form of shading cannot block the sun while admitting the view.

Internal shading is not as effective as external shading, as solar radiation is transmitted through the glass before interception. Lechner (2000) suggests that the side of the shade facing the glass should be as light as possible to reflect solar radiation back out through the glass before it is converted to heat.

\subsubsection{Surface colour}

The use of light colour surfaces on buildings and outdoor urban areas is practical in areas which have a large amount of sunshine because they can reflect solar radiation and reduce the amount of heat absorbed by the building. Modifying the value of albedo for a building's envelope, particularly the walls and roof, can potentially reduce the ambient temperature in individual buildings and their surrounding environment, helping to keep entire neighbourhoods cool. 


\subsubsection{Walls}

Walls are major part of the building envelope. They are usually a solid structure of masonry, wood, plaster, or other building material, and generally have window and door openings. They have two main purposes: to support roofs and ceilings, and to divide spaces providing security against intrusion and weather. In addition, they also house various types of electrical wiring and outlets or plumbing. In conventional construction, a building's wall will usually have structural elements, insulation, and finish elements, or surface.

Wall thickness, materials, including insulation, and finish should be chosen based on the heating and cooling needs of the building, as appropriate thermal insulation and air cavities in a wall reduce heat transmission into the building. The thermal comfort conditions of buildings are dependent on the heat storage capacity and heat conduction properties of walls.

\subsubsection{Roof}

A roof is the top covering on the uppermost part of building envelopes. It protects the building from weather. Roofs can either be pitched, low slope or flat. Their shapes and pitch are often the product of design, material constraints and climatic considerations. Pitched roofs are the primary design element on residential building. Roofs are covered with weather proofing material, which could include asphalt shingles, thatch, wood shakes, steel, corrugated galvanised iron, slate and tile roofs. Drainage on roofs is typically installed on the exterior wall.

About a third of the unwanted heat that builds up in a house comes in through the roof (DOE, 1994). The need for greater thermal resistance will dictate the type of roof design, including colour. Light colour roofing material has been used widely in hot climates to reduce heating from solar energy. One good solution is to apply waterproof and reflective coating properties to a roof. Another way to reflect heat is to install a radiant barrier on the underside of a roof. This is simply a sheet of aluminium foil with a paper backing, that when installed correctly can reduce heat gain through the ceiling by about 25\% (DOE, 1994). 


\subsubsection{Albedo}

The measure of a surface's reflectivity is called albedo. Brown \& Gillespie (1995) explain that is the fraction of sunshine (shortwave radiation), reflected from any surface back into space in all directions. Albedo is measured as the fraction of incident light that the surface reflects back in all directions. In building surfaces and components, the importance of albedo varies depending on the location's latitude. For example in the near tropics, the roof plays the most critical role in sensible heat exchange (Taha, et al., 1988). An increase in the surface albedo level has a direct impact on the energy balance of the building. Santamouris (2005) states that in all cases, the temperature of reflective roof surfaces are significantly reduced, but the degree to which the cooling load decreases depends on the structure of the roof and the overall thermal balance of the building.

Using high albedo materials reduces the amount of solar radiation absorbed through a building envelope, which keeps the surface cooler. For example, white elastomeric coatings (with an albedo of 0.72 ) were $45^{\circ} \mathrm{C}$ cooler than black coatings (with an albedo 0.08 ) in the early afternoon of a clear day in summer (Taha, 1997). Taha also added that using a white surface with an albedo of 0.61 was only $5^{\circ} \mathrm{C}$ warmer than the ambient air, whereas conventional gravel with an albedo of 0.09 was $30^{\circ} \mathrm{C}$ warmer. The difference in temperature between dark coloured insulated residential building's surfaces in Washington $\mathrm{DC}$ is about $8^{\circ} \mathrm{C}$ higher than light coloured surfaces during peak time at 14.00 (Connor 1985, cited in Taha, et al., 1988).

\subsubsection{Light colour surfaces save energy}

In general, light-coloured surfaces have a high albedo and dark-colour surfaces have a low albedo. Modifying the albedo by using light-coloured surfaces on a building will lower the heat build-up from sunlight on the walls and roofs, and reduce the amount of electricity needed for air-conditioning. The reduction of surface temperature also reduces the intensity of long wave radiation. Local and downwind ambient air temperatures would be lower because of smaller convective heat fluxes from cooler surfaces. Taha (1997) suggested that reasonable increases in urban albedo can achieve a decrease of up to $2^{\circ} \mathrm{C}$ in air temperature. Reductions in 
temperatures and energy use from albedo modifications affect individual buildings and entire neighbourhoods (Akbari, et al., 1992).

According to Taha et al. (1988), the effect of albedo on building energy use can be categorised as either local or global. Local is a direct effect, in reference to particular modifications in the reflective building envelopes. Global is an indirect effect, which refers to micro-climatic changes where urban albedo is modified mainly in dry bulb temperature. Using a computer simulation of a typical individual house in Sacramento, Akbari (1992) found that the total air-conditioning bill could be reduced by up to $20 \%$ if the albedo of the roof and wall are increased from a typical 0.30 to a light-coloured 0.90. If similar albedo modifications are implemented at large scale in an urban environment, the in direct impact of lower air temperatures in the neighbourhood will occur. Akbari (1992) showed that the indirect energy saving for cooling in typical houses could be $3-5 \%$ for each 0.01 increase in overall city albedo. When the direct and indirect savings of albedo changes are combined, the simulated total energy savings approach 50\% during average hours and $31 \%$ during the peak cooling period (Akbari, et al., 1992). The overall ranges for simple changes in albedo levels were to reduce house energy use between 10 to 50\% (Akbari, et al., 1992). These studies show that using albedo carefully potentially can contribute to creating a comfortable environment in a building by reducing the air temperature, and save energy by reducing air-conditioning use.

\subsection{Thermal comfort}

Thermal comfort in occupied spaces is important for building occupants. ASHRAE (2005, p. 45) defines thermal comfort as "that condition of mind which expresses satisfaction with the thermal environment". Thermal comfort concerns the interior temperature of rooms, including maintaining and distributing it evenly, and air quality. The feeling of comfort varies depending on the person. According to Chan (2008), the thermal sensation is linked to the intensity of solar radiation, orientation of windows, and surface reflectance from the walls. Comfort is a mixture of culture, time of year, health, the amount of fat an individual carries, clothing, and most importantly, physical activity (Lechner, 2000). The aim in buildings, with regard to 
thermal comfort conditions, is to achieve internal thermal neutrality for the occupants and assure their comfort, using minimal power consumption for heating and cooling. Although considerations relating to thermal comfort in building design are mostly for indoor climate, the outdoor climate should be taken into consideration to achieve the occupants' comfort and reduce energy consumption.

\subsubsection{Physical parameters}

The four physical parameters are air temperature, mean radiant temperature, air velocity, and humidity (Alread \& Leslie, 2007; Fanger, 1973; Lechner, 2000; Moffat \& Schiler, 1981). While they may be independent from each other, together they contribute to a person's thermal comfort.

\subsubsection{Air temperature}

Air temperature is the temperature of the air surrounding the body, which can be measured by a dry bulb thermometer. If a sensor is placed in a room it will register a temperature between the air temperatures and mean radiant temperature. Commonly air temperature is used as an indicator of thermal comfort. Dry bulb temperatures between approximately 20 and $27^{\circ} \mathrm{C}$ are the boundaries of the human comfort zone (Givoni, 1969; Montgomery, 1987; Olgyay, 1963). Under still-air conditions temperature ranges for people's comfort when they were engaged in a range of household activities wearing ordinary clothing was between $24-33^{\circ} \mathrm{C}$ (Mallick, 1996).

The comfort zone varies depending on the person, their age, sex and climate conditioning. Generally women prefer air temperatures a few degrees warmer than men; young people prefer temperatures a few degrees cooler than older people; people who live in arctic climates prefer cooler temperatures than people from tropical climates (Furuta, 1978). Air temperature should always be considered in relation to other environmental and personal factors. 


\subsubsection{Mean radiant temperature}

Mean radiant temperature is the area weighted mean temperature of all the objects surrounding the body. It will be positive when the surrounding objects are warmer than the average skin temperature and negative when they are colder. Posture plays a certain role in this, as does a person's location and orientation in the room.

Radiant temperature has a greater influence on how people lose or gain heat to the environment than air temperature. Examples of radiant heat sources include the sun, fires, furnaces, ovens, cookers, dryers, hot surfaces and machinery. According to Fanger (1970), the absorptance of the outer surface of the clothed body will influence the mean radiant temperature. If people are clothed in black, the mean radiant temperature from solar radiation will be higher than if they are wearing light colours. For normal longwave radiation, the absorptance is equal to the emittance where the emittance of the person has no influence on the mean radiant temperature. Radiant temperature is measured with a globe thermometer.

\subsubsection{Air velocity}

Air velocity describes the speed of air moving across people. It may help to cool them if it is cooler than the environment. Air velocity is an important factor in thermal comfort because people are sensitive to it. For example, still or stagnant air in indoor environments that are artificially heated may cause people to feel stuffy. The instrument most commonly used to measure air velocity indoors is the thermal anemometer, of a type suitable for measuring the small velocities that normally occur in room $(0-0.5 \mathrm{~m} / \mathrm{s})$.

Moving air in warm or humid conditions can increase heat loss without any change in air temperature through convection. If the air temperature is less than the skin temperature it will significantly increase convective heat loss. Physical activity also increases air movement, so air velocity may be corrected to account for a person's level of physical activity. 


\subsubsection{Air humidity}

Microclimate comfort is directly influenced by humidity. A comfortable relative humidity range is generally between 20 and 80\% (Givoni, 1969; Olgyay, 1963; Salmon, 1999). According to Oughton \& Hodkinson (2002), levels of between 40 and $70 \%$ relative humidity are considered to be the limits of acceptability for humidity, with 45 to $65 \%$ humidity being the levels for optimum comfort.

Relative humidity is the ratio between the actual amount of water vapour in the air and the maximum amount of water vapour that the air can hold at that air temperature. This measurement is taken with a psychrometer.

Relative humidity of between 40 and $70 \%$ does not have a major impact on thermal comfort. In some spaces, such as office areas, humidity is usually kept between this range. However, in places that are not air conditioned or where the climatic conditions outdoors influence the indoor thermal environment, relative humidity may be higher than $70 \%$ on warm and hot-humid days. Humidity in indoor environments, such as houses, can vary greatly and may be dependent on whether there are drying processes where water vapour is given off.

Environments that have high levels of humidity have a lot of vapour in the air, which prevents the evaporation of sweat from the skin. In warm and hot environments, humidity is important because less sweat evaporates when humidity is high (above $80 \%$ ). The evaporation of sweat is one of the main methods of heat loss in humans.

\subsubsection{Comfort zones}

The human-comfort condition is dependent on various environmental and physiological factors, which also contribute substantially to making houses comfortable. This can only be achieved when the air temperature, humidity and air movement are within a specified range, often referred to as the 'comfort zone'. The human comfort zone is the climatic condition where a person is comfortable and can function efficiently. Physical strength and mental activity of all people are improved within a comfort zone (Furuta, 1978), whereas outside the comfort zone their 
efficiency decreases. This study will explain the Bioclimatic chart by Olgyay (1963) and Building Bioclimatic chart (Givoni, 1976) to evaluate the comfort condition and to formulate strategies to respond to it.

\subsubsection{Bioclimate chart}

Olgyay's (1963) bioclimatic chart is very effective at analysing the comfort condition. It is a graphical representation of the effect of climatic elements on human physiology. In Figure 2.18, the bioclimatic chart shows the zones of human comfort in relation to ambient temperature and humidity, mean radiant temperature, wind speed, solar radiation and evaporative cooling, where the human body with average clothing at rest in the shade will be comfortable.

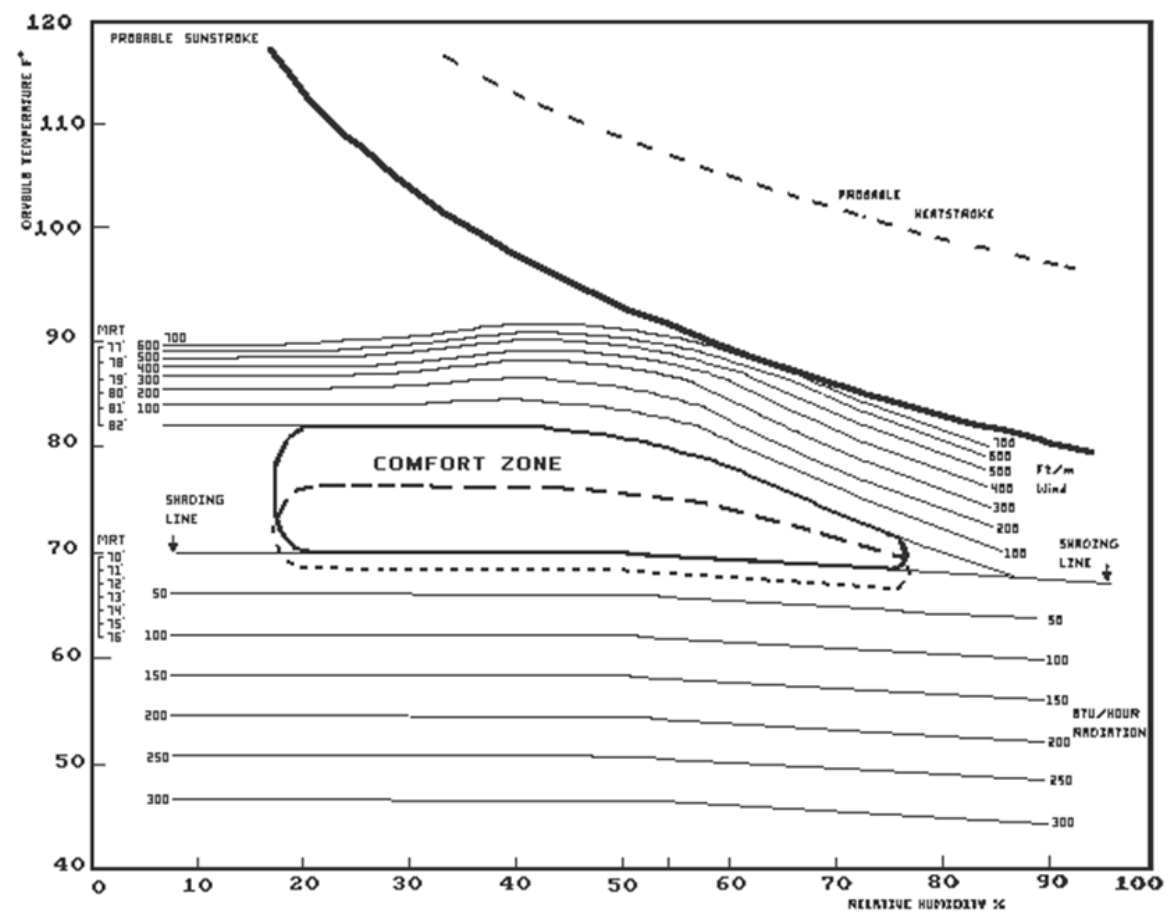

Figure 2.18. Olgyay's bioclimatic chart (1963, p. 22)

The bioclimatic chart was built up using dry-bulb temperature as ordinate and relative humidity as abscissa. The comfort zone is in an oval area that exists between $20^{\circ} \mathrm{C}$ and $28^{\circ} \mathrm{C}$, and $20-80 \%$ of relative humidity. At higher humidity levels the temperature lowers, due to the inability of the body to use evaporative cooling. The winter comfort zone lies a little lower. If the temperature and relative humidity fall 
below the lower limit, shading is required to maintain comfort. If they rise above the upper limit the cooling effects of the wind is usually the only element that can balance the comfort zone. Any climatic condition determined by its dry-bulb temperature and relative humidity can be plotted on the chart to determine their comfort zone.

The bioclimatic chart is important because it allows engineers and architects to figure out the right specifications for design factors, such as building orientation, location, size, shading, and form. In addition, the effectiveness of the methods suggested also depends on the design and structure of the building envelop and the surrounding area.

\subsubsection{Building Bioclimatic chart}

Givoni’s (1969) Building Bioclimatic chart, in Figure 2.19, provides suggestions for building design that take into consideration the prevailing outdoor local climate conditions to help predict indoor conditions. The study is based on the linear relationship between the temperature amplitude and vapour pressure of the outdoor air in various regions. In this psychrometric chart, different zones are plotted to indicate different strategies, depending upon the monthly temperature-humidity relationship.

The comfort zone is supposed to be between 20 and $27^{\circ} \mathrm{C}$ and relative humidity between 20 and $80 \%$. However, the comfort range could be extended by wearing more clothes in low temperatures at night time, increasing internal loads inside the room, or by changing the activity level. The periods where the temperature is down to $20^{\circ} \mathrm{C}$ or over $27^{\circ} \mathrm{C}$, and relative humidity is below $20 \%$ and over $80 \%$ are outside of the comfort zone. This chart shows the relationship between strategies and discomfort conditions. For example, the strategy of evaporative cooling corresponds to the hot and dry discomfort zone. Lechner (2000) mentions that when the climate conditions are to the right of the shade line, the sun should be prevented from entering windows because the sun's radiation may increase the temperature and modify the thermal comfort zone. Due to the differences between day and night 
temperatures, it is important to raise the comfort level at night. For domestic buildings, the most critical rooms are probably the bedrooms and living room.

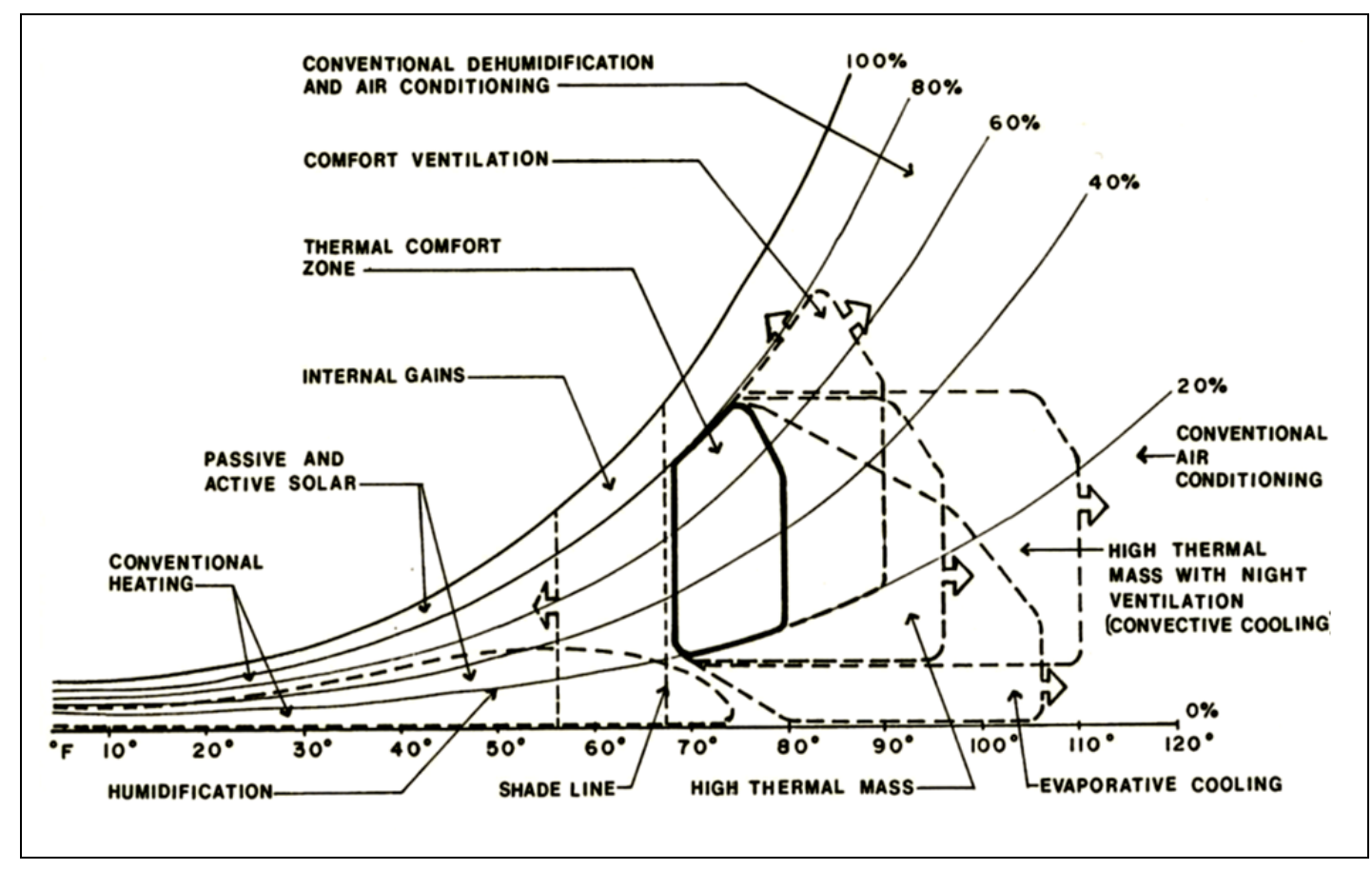

Figure 2.19. Building Bioclimatic chart (Givoni 1969, cited in Lechner, 2000, p. 65). This is the summary of design strategies as a function of ambient conditions (climate)

The Building Bioclimatic chart is correlated with specific boundaries of the passive cooling techniques overlaid on the chart. It combines different temperature amplitudes with the vapour pressure of the ambient air plotted on the psychrometric chart. The relationship between the average monthly vapour pressure and temperature amplitude of the outdoor air will define passive strategies according to the climatic conditions prevailing outside the building envelope.

\subsubsection{Air conditioning systems}

Air conditioning can be defined as providing and maintaining a desirable internal atmospheric environment irrespective of external conditions (Oughton \& Hodkinson, 2002). According to McDowall (2007), there are seven main processes required to achieve full air-conditioning: heating, cooling, humidifying, dehumidifying, 
cleaning, ventilating and air movement. The requirements and importance of these processes vary with climate. The desired atmospheric conditions for comfort involves a temperature of $18-22^{\circ} \mathrm{C}$ in winter and $21-24^{\circ} \mathrm{C}$ in summer, and a relative humidity of about $40-60 \%$ and a high degree of air purity (Oughton \& Hodkinson, 2002). There are four types of air conditioning systems: window air conditioning systems, split air conditioning systems, packaged air conditioners and central air conditional plants. What type of system to use in a particular environment depends upon a number of factors, such as area to be cooled and the total heat generated inside the enclosed area. The designer would consider all the related factors and suggest the most suitable system.

There are two systems of air-condition in usually used for residential building; window and split. Window air conditioners are one of the most popular and the cheapest of all air conditioning systems. They can be used for almost all types of small spaces, and are commonly used for single rooms. These types of air conditioners contain a compressor, condenser, expansion valve or coil, evaporator, fans and a cooling coil, which are all enclosed in a single box, as shown in Figure 2.20. This unit is fitted in a slot made in the wall of the room in a standard of window frame.

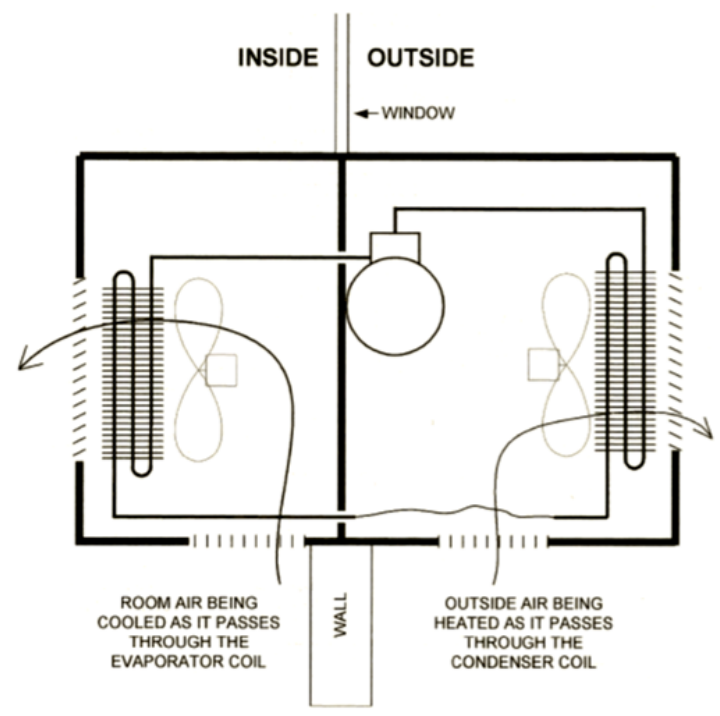

Figure 2.20. Window air conditioner (McDowall, 2007, p. 81) 


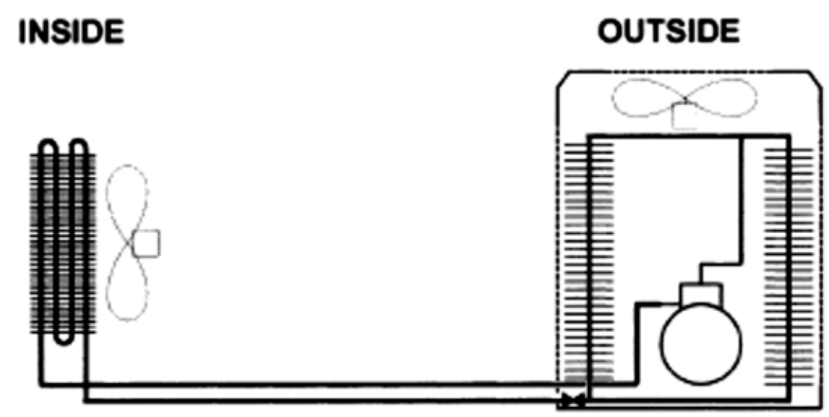

Figure 2.21. Split system unit (McDowall, 2007, p. 88)

When buildings need larger air-conditioning applications, split-system units are the most appropriate. A split-system air conditioner splits the hot side from the cold side of the system, as can be seen in Figure 2.21. The indoor unit part of contains an expansion valve, evaporator coil or cooling coil and the blower. The outdoor unit contains the compressor and the condenser, and is the hot side of the unit. The unit consists of a long, spiral coil shaped like a cylinder. Inside the coil is a fan to blow air through the coil, along with a weather-resistant compressor, and some control logic. This approach has evolved over the years because it is low-cost and normally results in reduced noise inside the house.

Mechanical ventilation systems are unavoidable in many buildings, but much can be done to lessen their impact and expense. Most buildings could be run with half of the energy they currently consume if they had been designed with efficiency and energy conversation in mind (Alread \& Leslie, 2007). This includes considering the activities and locations of people in a building, and providing comfort where it is most needed.

\subsubsection{Energy efficiency use}

Electrical energy is an essential energy source that has been drastically increasing in cost. In Malaysia, demand for electrical energy is increasing each year, at an average rate of $11.2 \%$ per annum; parallel to the development of technology in this country (Nasution \& Hassan, 2009). The consumption of electricity includes the application of lighting, electrical appliances, machinery, and air conditioning systems, which are 
now widely used in Malaysia. The role of air conditioning systems in the tropics is using the energy for cooling, as there is no need for heating in Malaysia due to its year round hot-humid climate.

Growth in the use of air conditioning has increased in residential buildings in Malaysia from 13251 units in 1970 to 253399 in 1991 . This figure is predicted to reach about 1511276 in the year 2020. Population growth, improved living standards and urban heat islands effects in cities are the main factors that have contributed to the dramatic increase in air conditioner use in residential buildings in Malaysia.

Maximising shade, channelling wind and evapotranspiration from vegetation potentially could significantly contribute to cooling our cities and therefore saving energy. If vegetation is planted in sufficient amounts and strategic locations around residential areas, it will reduce heat gain to a building, but also produce evapotranspiration around individual houses and their neighbourhood areas, thus reducing the urban temperature at the microclimatic scales. If this was implemented on a large scale throughout this country, it would be beneficial to the whole country as it could reduce future electricity demand in the residential sector, as well as the environmental impact caused by developing new power plants.

Table 2.2. Predicted electricity consumption, room air conditioners and the percentage fuel mix for electricity generation data (adapted from Mahlia, Masjuki, Choudhury, \& Ghazali, 2002)

\begin{tabular}{cccccccc}
\hline Year & $\begin{array}{c}\text { Total } \\
(\mathrm{GWh})\end{array}$ & $\begin{array}{c}\text { Residential } \\
(\mathrm{GWh})\end{array}$ & Room AC & $\begin{array}{c}\text { Coal } \\
(\%)\end{array}$ & $\begin{array}{c}\text { Petroleum } \\
(\%)\end{array}$ & $\begin{array}{c}\text { Gas } \\
(\%)\end{array}$ & $\begin{array}{c}\text { Hydro } \\
(\%)\end{array}$ \\
\hline 2002 & 66159 & 11986 & 604044 & 14.96 & 4.24 & 65.20 & 15.60 \\
2003 & 71368 & 12927 & 643586 & 15.06 & 3.89 & 62.95 & 18.10 \\
2004 & 76779 & 13905 & 684406 & 15.24 & 3.56 & 60.80 & 20.40 \\
2005 & 82390 & 14919 & 726504 & 15.50 & 3.25 & 58.75 & 22.50 \\
2006 & 88203 & 15969 & 769879 & 15.84 & 2.96 & 56.80 & 24.40 \\
2007 & 94217 & 17055 & 814532 & 16.26 & 2.69 & 54.95 & 26.10 \\
2008 & 100433 & 18178 & 860462 & 16.76 & 2.44 & 53.20 & 27.60 \\
2009 & 106850 & 19337 & 907670 & 17.34 & 2.21 & 51.55 & 28.90 \\
2010 & 113468 & 20532 & 956155 & 18.00 & 2.00 & 50.00 & 30.00 \\
2020 & 190721 & 34480 & 1511276 & 29.00 & 1.00 & 40.00 & 30.00 \\
\hline
\end{tabular}




\subsection{Summary}

Climate parameters, the benefits of vegetation, the influence of the building envelope and thermal comfort have been looked at in this literature review. These factors are closely related to the thermal performance of a house, and can combine to contribute to energy saving. One of the major factors that increases daytime temperatures in a residential area is limited amounts of vegetation planted around buildings and low albedo in the building envelopes. These factors also significantly influence thermal performance and energy use. Using high albedo materials reduces the amount of solar radiation absorbed through building envelopes and urban structures, and keeps their surface cooler. Vegetation reduces the cooling requirements, not only by providing shade, but also by reducing warm air infiltration and creating cool microclimates around buildings through evapotranspiration. In addition, during the summer season, vegetation in urban housing areas can act as a wind channel, which can potentially have a substantial impact on a residential building's energy performance. However, the thermal comfort level inside a house is mainly influenced by the outdoor environment. If the outdoor environment is extremely hot, and directly influences the indoor spaces, air-conditioning systems are used to modify the indoor environmental so that the occupants can live in comfortable temperatures. Maximising shade, channelling wind and evapotranspiration from vegetation could potentially significantly contribute to cooling our environment and therefore save energy. 


\subsection{METHODOLOGY}

\subsection{Overview}

This chapter describes the methodology used to measure the effects of surrounding vegetation, building construction and human factors on a small number of individual houses and a large number of single-family houses. The primary data was obtained from fieldwork and site measurements during a two-stage process. All supporting data for this study was sourced from various Malaysian government departments.

Fieldwork in the first stage focused on 10 individual single-family houses. Quantitative measurements were taken to record the construction of the buildings, their surrounding vegetation, human factors, and weather data during the day time. In this report only five houses of different ages and types of landscaping were chosen to compare the effect of landscaping on the thermal performance of the house. These five houses had used different styles and designs of tropical landscaping, which could produce different conditions of microclimates.

The air temperature, relative humidity and wind speed data were obtained from the exterior and interior of houses during similar weather conditions to ensure they could be accurately compared. This data was automatically recorded at half hour intervals by static and mobile sensors of the data loggers. The albedo data for every type of building were also measured during the day time.

In the second stage, the fieldwork measurements were divided into four categories: building construction, landscaping, human factors, and weather data. The annual energy consumption for every house was also evaluated by analysing electricity consumption for different single-family houses ranging in age from 3 to 40 years. The target number of respondents was 50 houses. Statistical calculation and analysis was used to measure and evaluate the energy saving. 


\subsection{Research methodology}

The research methodology was divided into the two fieldwork stages using a process called triangulation. This is where more than one research method or type of data is used to answer the research questions (Evans \& Gruba, 2002).

In this study, two stages of methodology were used to answer two research questions. According to Yin (2003), at least three principles for collecting data must be used from the six possible sources of documented evidence. The three main sources chosen for collecting data in this study were interview, direct observation, and physical artefacts evidence.

The first step was to record data about each building's construction and its surrounding landscaping. To measure the effect of surrounding landscaping on the thermal performance of a house, site measurements were made of the physical features that surrounded the building, and equipment was used to record weather data inside and outside of the houses. The outdoor weather data included temperature, relative humidity and wind speeds while the indoor data focussed on temperature and relative humidity.

The second step in assessing the influence of landscaping on reducing the amount of energy used for air-conditioning, was to calculate the average proportion of electricity used for cooling. Data on landscaping, building construction and energy use by air-conditioning systems, and the number of occupants in each house was obtained through observation and interviews with the homeowners.

\subsubsection{Study location}

The single-family houses sampled, were randomly chosen from two well planned Malaysian cities: Putrajaya and Shah Alam (Samad, Zain, Maarof, Hashim, \& Adnan, 2011). The city of Putrajaya is situated at latitude $2^{\circ} \mathrm{N}$ and longitude $101^{\circ} \mathrm{E}$, at an elevation of between 75 and $94 \mathrm{~m}, 25 \mathrm{~km}$ south of Kuala Lumpur. Shah Alam is located at latitude $3^{\circ} \mathrm{N}$ and longitude $101^{\circ} \mathrm{E}$, at an elevation of between 24 and $48 \mathrm{~m}$, 
about $25 \mathrm{~km}$ west of Kuala Lumpur. The distance between Putrajaya and Shah Alam is approximately $23.7 \mathrm{~km}$.

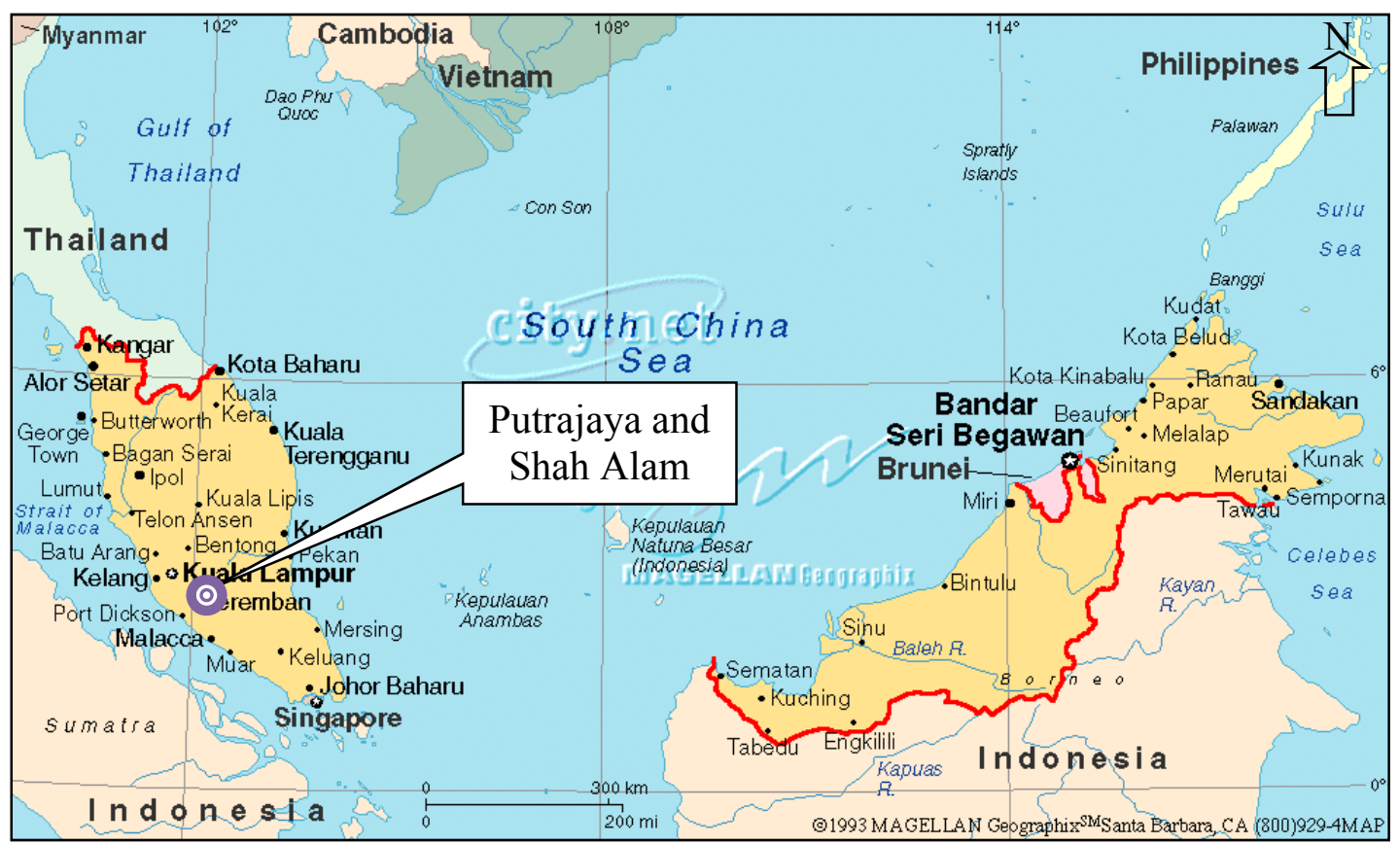

Figure 3.1. Putrajaya and Shah Alam were the locations in Malaysia where fieldwork was conducted (Malaysia, 1993). These cities are located at approximately $101^{\circ} \mathrm{E}$ and $3^{\circ} \mathrm{N}$.

Five locations of low-density housing development were selected to be the case study areas. The specific locations used were in Precinct 14, in the Federal Territory of Putrajaya, and Sections 3, 6, 9 and 11 in Shah Alam, Selangor.

Development of Putrajaya started in the 1990s. It is a well planned city, and is known as an "intelligent city" because of its extensive information and communication technologies (Malek, 2011). It was also planned as a garden city, with $38 \%$ of the city reserved for green space areas that emphasise and enhance the natural landscape. Shah Alam is the capital of Selangor State, and is also one of the most well planned cities in Malaysia (Aziz \& Hadi, 2007). It has around 10\% of its area dedicated to green space areas. 


\subsubsection{The study house}

In Putrajaya and Shah Alam the most common type of house is the two-storey singlefamily house that is surrounded by a garden. These houses are lived in by the middle and upper classes. Generally these types of house represent around 10 to $30 \%$ of all houses in a housing development. In this study the houses looked at were medium sized, single-family houses (around $300-600 \mathrm{~m}^{2}$ ). Figures 3.3 to 3.5 show an example of the detailed design of a single-family house used. The garden area includes the entire space around the building and is private to the owner and family.
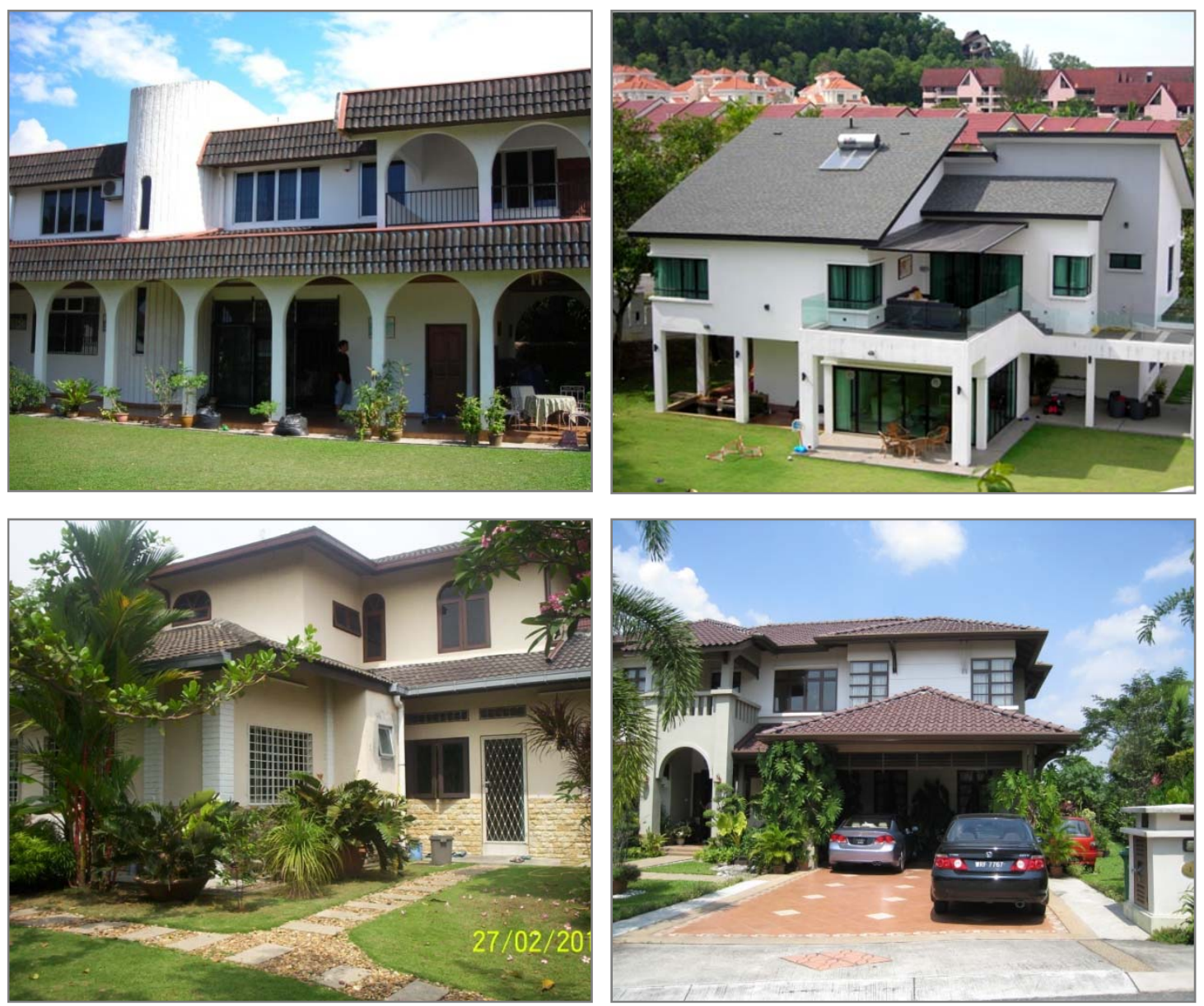

Figure 3.2. Examples of modern tropical houses in the study areas ranging in age from 3 to 40 years with surrounding landscaping

This research was undertaken looking at single-family houses ranging in age from 3 to 40 years. The different ages were chosen because they provided differences in the 
maturity of the surrounding landscaping, which affects the amount of energy used and thermal comfort and performance of the house.

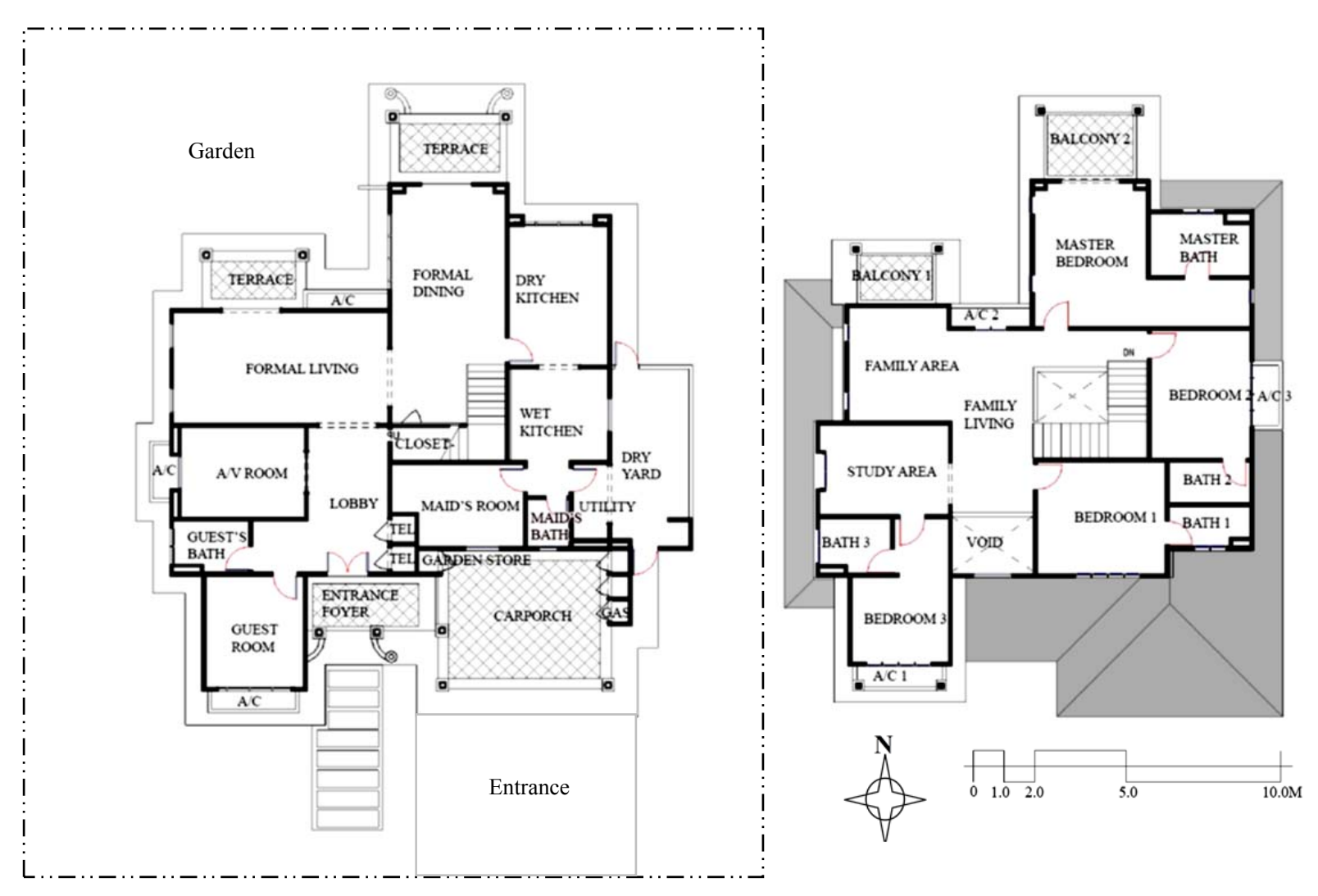

Figure 3.3. Example of a modern medium-size single-family house, ground floor plan (left) and upper floor plan (right)
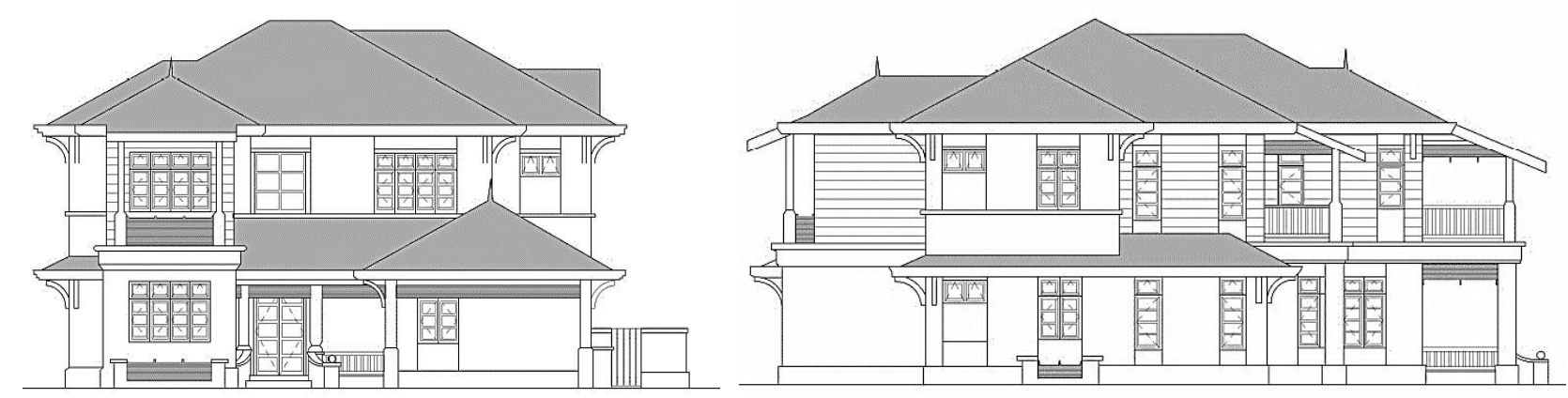

Figure 3.4. Front elevation (left) and left elevation (right) of the house 

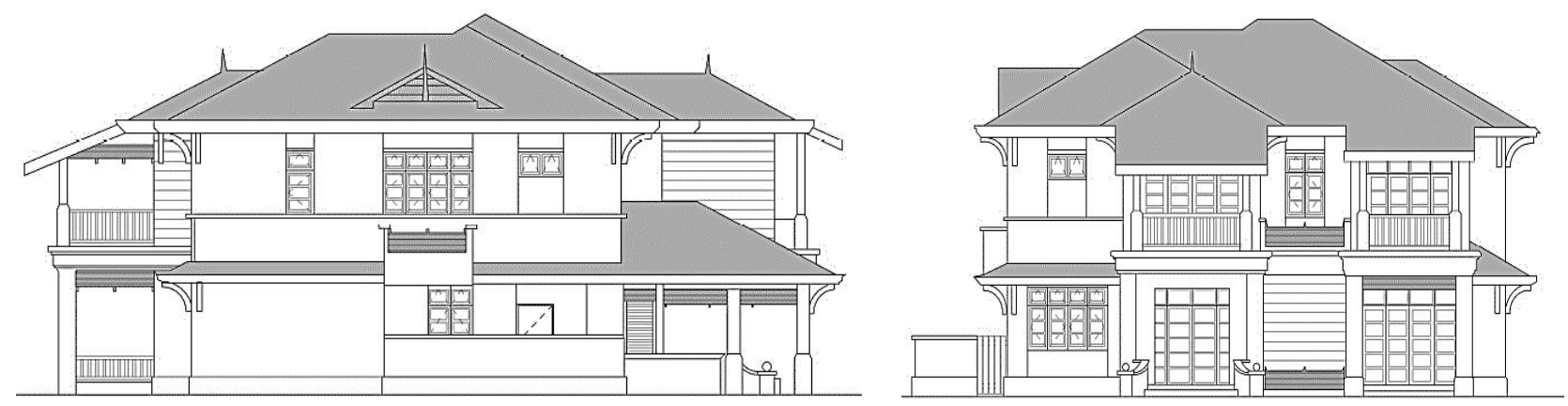

Figure 3.5. Right elevation (left) and rear elevation (right) of the house

In both cities air conditioning is an effective way of dealing with the extremes of an uncomfortably hot-humid day. It is used more during the dry season because of the increase in air temperature. Consumption of energy from the use of air conditioning will increase every year as more new residential developments are built and because of climate change issues facing the world today.

The current practice of domestic building construction and the materials typically used in Malaysia were considered in this study. Buildings are usually constructed using reinforced concrete as a main structure, with brick walls laid in cement mortar, and hardwood construction timber and roof coverings of clay or concrete tiles. Roof insulation is generally used as a barrier to reflect heat gain from radiating down into the house's habitable space. Shading includes roof eaves (overhangs) and window eaves (awnings) that may reduce direct solar radiation onto the walls. The colour of the building envelope was also considered because it influences heat gain to the buildings.

The impact of tropical and subtropical plants from different types of foliage, ranging from ground cover vegetation, through to different kinds of shrubs, grasses, to trees of various sizes and types will be looked at, along with the length and patterns of shade which varies with the time of day. In tropical regions in Malaysia trees are the most important plants. They often grow particularly fast and can be the most satisfying plants in the garden (Sparrow \& Hanly, 2002). Large trees with spreading branches assist in keeping the house cool, but there should not be so many of them as 
to exclude breezes. The length and patterns of shade vary with the time of day. The distribution and age of vegetation in the surrounding neighbourhood also may create cooling effects on the houses as can be seen in Figures 3.6 and 3.7.

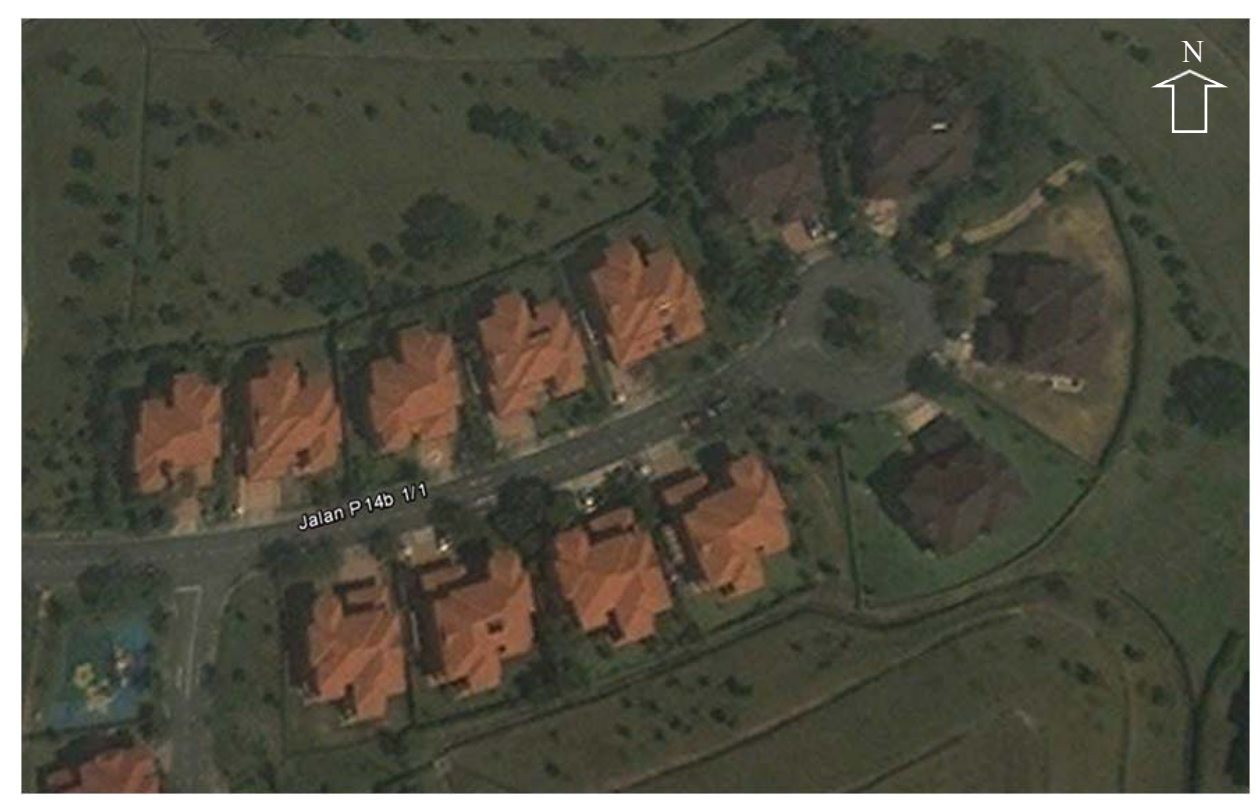

Figure 3.6. Example the distribution of 5 year old immature vegetation around houses in Puterajaya neighbourhood area (Google Earth, 2011)

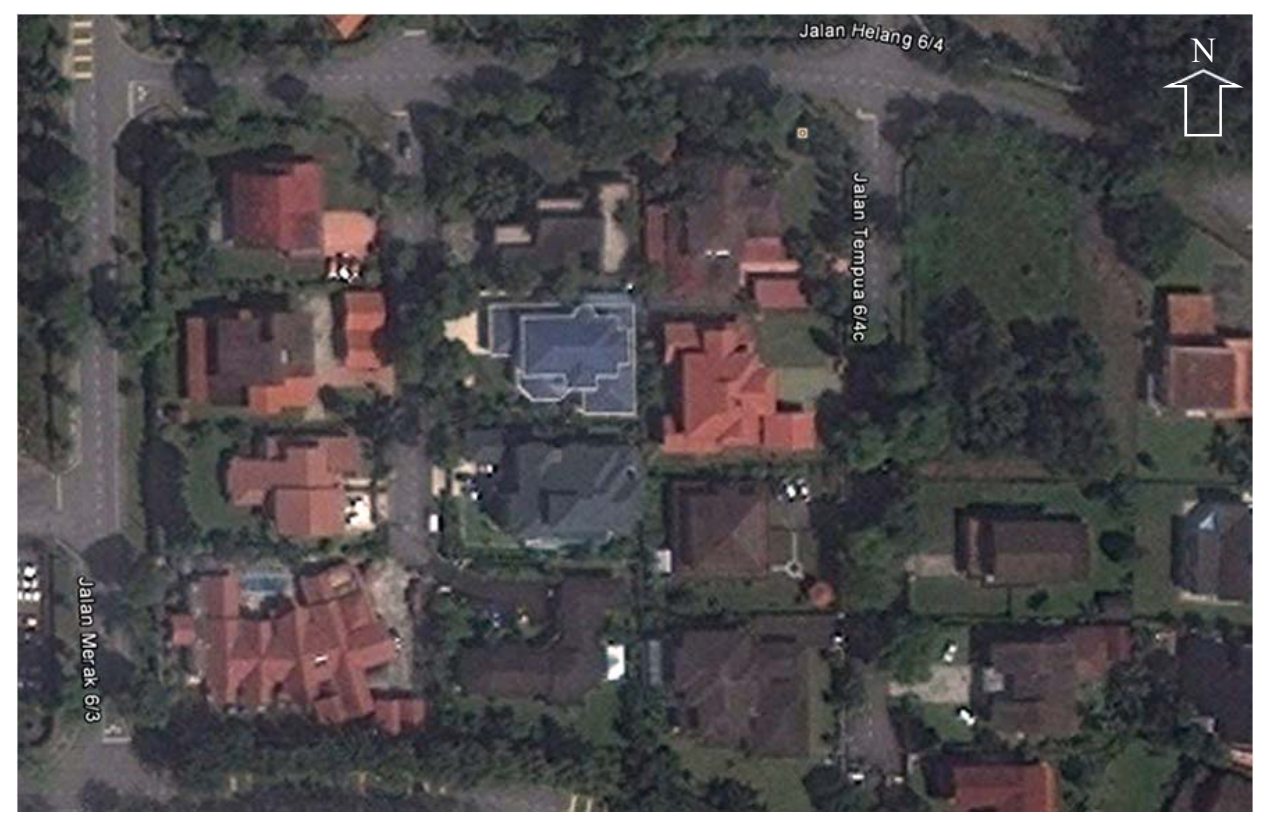

Figure 3.7. Example the distribution of 30 year old mature vegetation around houses in Shah Alam neighbourhood area (Google Earth, 2011) 


\subsubsection{Weather data}

In the first stage of fieldwork, which included site measurements for 10 individual houses, weather data from the Malaysian Meteorology Department was gained for the same days as when the climate data was recorded in the houses. This weather data was required to compare it with the study location. The dates this data was gathered were the $16^{\text {th }}$ and $17^{\text {th }}$ of January, 2010 in Putrajaya, and the $23^{\text {rd }}, 24^{\text {th }}$ and $28^{\text {th }}$ of February, 2010 for the Shah Alam study areas. The data included air temperature, relative humidity, rainfall, daily global radiation, cloud cover and wind.

In the second stage of fieldwork, which included site measurements of 50 houses, weather data was obtained from the Malaysian Meteorology Department for 2008 and 2009. Weather data was recorded at two airports: Sepang Weather Station for the Putrajaya study area, and Subang Weather Station for Shah Alam. Averages from this data were used to determine the exact tropical weather conditions in the study areas.

\subsubsection{Local authority records}

Putrajaya Cooperation and Shah Alam City Council were the two local authorities involved in this study. Their Planning, Architecture and Landscape Departments provided the master plans, constructions details and landscaping information for the sample houses.

In the Putrajaya study area, the whole housing development was designed and constructed by a developer. The sizes of the houses are slightly different, but are similar in design and are typical of tropical architecture.

In Shah Alam, every homeowner is free to build their own house. Developers only provide the infrastructure and land for sale. Detailed plans for houses in Shah Alam are done privately, and when owners plan to start building, they need to submit a design proposal and construction detail to the Shah Alam City Council for approval. 
In this study, the architects, landscape architects and planners who managed the areas of study were interviewed to gather information about the general construction, basic building design, landscaping, and the exact date and age of construction for every development. This primary data was validated with on-site interviews, data collection, and observation of each of the sample houses.

\subsubsection{Electricity use data}

Domestic electricity tariff information was obtained from the Malaysian Electric Utility Company (National Energy Limited-TNB). Data for the monthly amount of electricity used by the 50 residential houses was collected as the cost of electricity in Malaysian Ringgit (MYR). These costs were converted into energy used in kWh. Electricity used for air-conditioning was also calculated in $\mathrm{kWh}$ based on current local tariff. Annual domestic electricity use in the city of Shah Alam was analysed to validate the amount of energy used in relation to the increase in air temperature and humidity levels in the two main seasons: rainy and dry. The increase in cooling energy use was closely related to air temperature changes in the hot-humid tropical environment.

\subsubsection{Satellite images}

Satellite images for the study areas were obtained from Remote Sensing Data Services, Malaysian Remote Sensing Agency, Kuala Lumpur. These satellite images were formatted in high resolution, 'Quickbird' data series $(0.6 \mathrm{~m}$ resolution), and were taken in 2007 . The images show the natural condition of the housing estates and their surrounding landscaping.

Vegetation information was observed by the author during the fieldwork stages, and was compared with the digital satellite images. The satellites images came from geographic information system (Archview@GIS). This data was compared with the master plans from each house's local authority to confirm their location, orientation and surrounding landscaping; and to record the overall condition of the houses and landscaping in each location. 


\subsection{The variables}

Several variables were measured in order to assess their influence on the temperatures around the houses and the cooling energy use as follows:

- Building: orientation and envelope surfaces

- Surrounding vegetation: shading, evapotranspiration and wind channelling

- Human factors

\subsubsection{Building orientation}

The configuration of the house should be designed to ensure the comfort of its occupants and for energy efficiency. In a hot-humid tropical climate, proper house orientation in relation to the sun and it devices will enable energy savings and more comfortable conditions. In this study four main categories of wall orientation/direction are North, East, South and West and also the in between of the main directions include North-east, South-east, South-east and North-west.

\subsubsection{Building envelopes}

Building envelopes includes the walls, roof, glazing and floor foundation. Walls are the major part of the building envelope and in these houses faced in all directions, while roof is the top covering and is exposed to the direct solar radiation throughout the day. In the tropics, heat gain through glass windows and doors is often the major source of solar heat gain. Hence if such glazing located facing the direct solar radiation orientation, it should be minimised and shading maximised. Hence, not only were the materials of the building noted but the direction of the glazing was also assessed. The measurements taken in the the study areas were the areas of building envelopes include walls, roof, glazing and floors.

\subsubsection{Surrounding vegetation - shading}

Strategically placed shade trees around the building can potentially modify microclimate and building energy use through shading. This shading can minimize the amount of radiant heat absorbed and stored by buildings and other built surfaces. Shading in outdoor areas will be provided by all type of vegetation to the building 
envelopes and surrounding garden earth surfaces. The plants shading areas and their angles were recorded during morning, afternoon, and the peak time of the day.

\subsubsection{Surrounding vegetation - evapotranspiration}

The measurement of evaporation and transpiration in the study areas refers to the amount of water vapour in the atmosphere. The evapotranspiration is gained as a result of evaporation from exposed water surfaces, moist ground and plant transpiration. The measurements in the study areas were relative humidity or absolute humidity reading.

\subsubsection{Surrounding vegetation - channelling wind}

In a hot-humid tropical climate wind is generally of moderate speeds and is not steady all the time. Strategic outdoor designs to promote wind are required. Strategic location of vegetation can capture and directing the flow of air and provide effective ventilation and convective cooling of surrounding garden and building surfaces. The measurements taken in the the study areas was wind speeds.

\subsubsection{Human factors}

Human factors in this study are the number of occupants who need cooling and their daily living habits of using air-conditioning system. The cooling energy use will be calculated based on the horse power of the air-conditioning system, temperature setting and the duration of daily use.

\subsection{The effect of landscaping on the thermal performance of housing}

In this first stage, the measurements focused on individual houses. Research methods have been divided into three parts: selection of houses with suitable surrounding landscape; observation of building construction and landscape design; and field measurements of building construction, landscaping and day time weather data.

The selection of houses was done to ensure similar building construction and site locations, but which provided sufficiently different amounts of landscape structures and design. The aim of the field measurement aspect was to evaluate and compare 
the surrounding microclimate, which would directly influence the interior thermal performance of the house.

Quantitative measurements were gathered for 10 individual houses to document weather data during the day. However, only five houses were analysed in detail. In case study 1, houses were analysed to illustrate the three different ages of construction and surrounding landscaping, while in case study 2 analysis was done to compare heavily and sparsely landscaped areas. This was to assess how these factors, along with the different types of building constructions and surrounding landscaping could influence the ambient air and thermal performance of the houses.

Finally, the amount of energy used was also calculated, and the exact amount of cooling energy revealed.

\subsubsection{Case study 1}

Case study 1 involved looking at the construction and surrounding landscaping of three single-family houses aged 5, 10 and 30 years old in Shah Alam, Selangor, Malaysia. While their construction was similar, the design and size of each house varied because they were built in different eras of construction methods, and styles of architecture and landscaping. As mentioned before, the design and construction for single-family houses in Shah Alam are up to each homeowner to decide on.

The 30 year old house was located in Section 6, Shah Alam, and was surrounded with mature tropical landscaping, while the 10 year old house was located in Section 11, Shah Alam, and was surrounded by tropical landscaping of intermediate age. The 5 year old house was located in Section 9, Shah Alam, and was surrounded by immature tropical landscaping. The houses were labelled as mature, ordinary, and new landscaped houses. The three housing estates were close in location, as they were around $3 \mathrm{~km}$ apart. 
The three ages of construction were chosen because they provided different vegetation and landscaping styles, which would have different effects on the thermal performance of the houses by shading, evapotranspiration and channelling wind.

\subsubsection{Case study 2}

In case study two, two similar house constructions and locations were chosen, but the landscape of each house was totally different. This was to help demonstrate the effects of the surrounding landscape on a house's thermal performance. These houses were located in Precinct 14, Putrajaya, Malaysia, and were labelled as a heavily landscaped house and a sparsely landscaped house.

The heavily landscaped house had strategic landscaping, while the sparsely landscaped house had a minimum of landscaping in the garden. A heavily landscaped house will usually produce cooler ambient air than a sparsely landscaped house. This is because large quantities of vegetation located in strategic areas of a property could lower the ambient air temperature by providing shade, channelling wind and through evapotranspiration.

A well designed tropical house will also reflect heat from solar radiation from the building envelope, while providing shade for every opening and by being orientated to allow wind to blow through the building's interior spaces.

\subsubsection{Local weather recording}

In case study 1, which was conducted in Shah Alam, weather recordings were carried out during daytime on the $23^{\text {rd }}, 24^{\text {th }}$, and $28^{\text {th }}$ of February, 2010. These dates were chosen because they had similar weather conditions each day, with six to eight hours of sunshine during the day and drizzle at night.

In case study 2, which was conducted in Putrajaya, the weather recordings were carried out during daytime on the $16^{\text {th }}$ and $17^{\text {th }}$ of January, 2010. The weather on these two days was similar, with sunshine for the whole day, and soft rain starting at 
19.30 hours. Cloud is very typical in this tropical region, measuring approximately 6.9-7 okta.

The study measured climatic parameters and the physical characteristic of the four azimuths of the houses. Each measurement point represents an area of $90 \mathrm{~m}^{2}$, and an approximate radius of $3 \mathrm{~m}$ to $10 \mathrm{~m}$ around the fixed/mobile weather station. The measurements were taken at 30-minute intervals in all locations a metre above the ground and in the shade. The albedo data for every type of building envelope was also measured during the daytime.

\subsubsection{Exterior space}

The weather measurements taken for both studies were not influenced by shadows or reflected solar radiation. The gardens were covered by various plants, including trees, shrubs, vines, groundcover and turf.

The basic measuring equipment that was used to do the field measurements included:

i. Two sets of mobile TSI VelociCalc Plus Meters, model 8386, data loggers and sensors. This was to measure surface temperature, wetbulb temperature, drybulb temperature, relative humidity and wind velocity.

ii. Two sets of portable data acquisition devices: Model Babuc A code BSA014 multi-datalogger and sensors to measure air temperature, global temperature, relative humidity and wind velocity.

iii. Two sets of Lux meters PCE-172 to take the albedo measurement.

iv. Two sets of compasses and measuring tapes to ensure and confirm the measurement of the house configurations.

v. A set of digital cameras.

vi. A set of drawing equipment to draw and record the house configurations and landscape plans in scale and detail on site. 


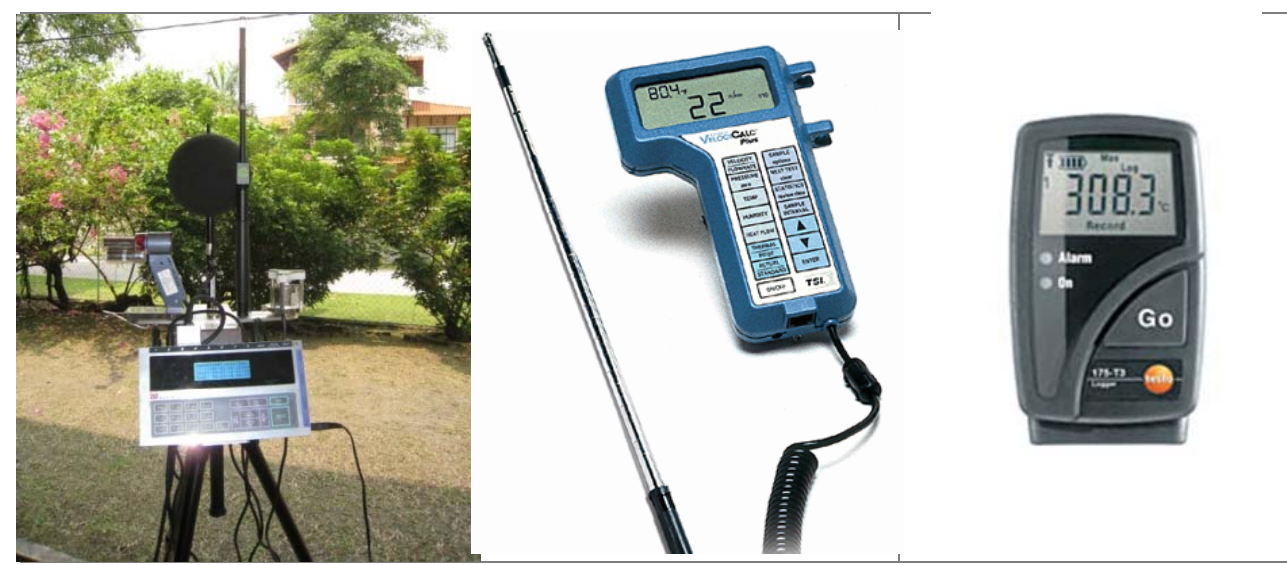

Figure 3.8. Babuc A (left), mobile TSI VelociCalc Plus Meters (middle), and electronic Mini Thermohygrograph (right)

Figure 3.2 shows the Babuc Automatic and sensor, the mobile TSI VelociCalc Plus Meters data loggers, and sensor that were used to measure current weather condition around houses in the shade. The same data was recorded from two types of equipment at the same time to validate the data and ensure accuracy.

\subsubsection{Interior space}

Four sets of Electronic Mini Thermohygrograph, model testo 175-T2 (Figure 3.2), were used to measure air temperature and air humidity data inside the buildings. This equipment was set automatically, and placed at a metre above the floor near the windows that faced the four azimuths on the ground floor of the houses. The purpose of these measurements was to compare with outside weather data. The data was recorded every 30 minutes during the day, at the same time as weather data that was gathered for the exterior of the house.

\subsubsection{Energy use measurements}

This experiment used quantitative measurements to document the energy used for air conditioning, while considering the influence of surrounding vegetation including trees, shrubs, vines, groundcover and lawn. Data about the internal temperature of each house was obtained during days with similar climate conditions. This was to ensure the data for houses with different designs and landscaping was comparable. 
Interviews with home owners and observations revealed that air conditioning units were used in every room of the houses looked at. The type of air-conditioning units used and their horse power were recorded, and energy consumption for these units was given by the owner of the house.

The cost for air conditioner use has been determined by National Electricity Limited (TNB). This, along with an analysis of the reduction of ambient temperature and air conditioning consumption during the day and on the hottest afternoons, would reveal the potential savings associated with the corresponding landscape.

\subsubsection{Landscape element measurements}

The study of all landscape elements was conducted through observation and interviews with the owner of each house. Landscape elements and house plans were drawn in detail and to scale. Landscape elements included soft landscape and hard landscape. These two main categories of landscaping are explained in detail in subtopic 3.5.3.2 - Data collection - landscaping.

The location of every type of vegetation was recorded at five metre intervals extending out from the building for each of four azimuths, as shown in Figure 3.3. Tree azimuth classes were defined with reference to building wall orientation. According to Simpson (2002), a wall is cardinally oriented if the normal distance to the wall is within $\pm 45^{\circ} \mathrm{E}$ of a cardinal direction (North, East, South or West), otherwise, it is inter-cardinal (NE, SE, SW or NW).

A detailed landscape plan was recorded for every type of vegetation because shading, evapotranspiration and wind flow are all affected by different types of landscaping. 


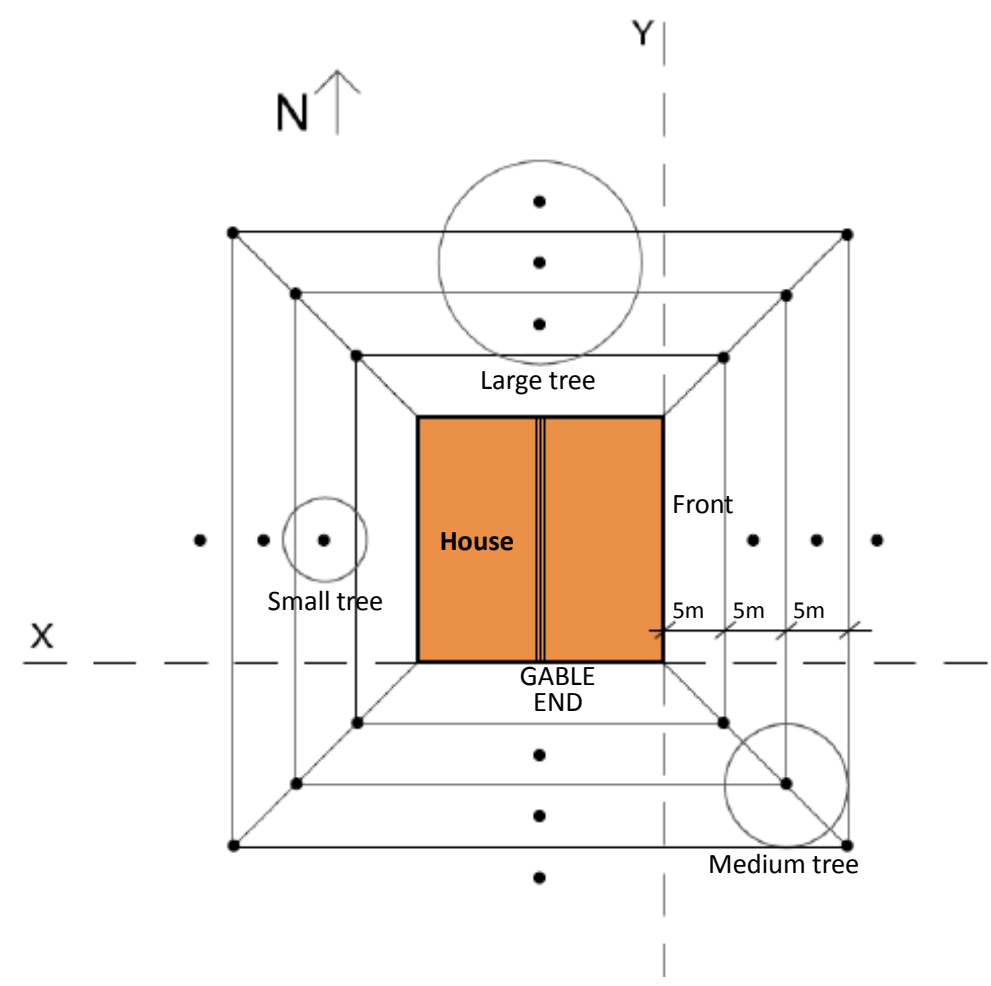

Figure 3.9. House configuration and the five metre intervals of vegetation measurements and other landscape elements around the house (modified from Simpson, 2002)

\subsubsection{Data analysis}

Once all of the data was collected, master spreadsheets were developed using Microsoft Excel 12.0 and Origin 8.0. All vegetation data gathered from the four azimuths of each house and was recorded numerically in this spreadsheet as percentages or figures. This data was analysed based on a few variables, including vegetation structure and typology or biomass, which was representative of the real conditions of vegetation on site. The actual amount and biomass of every type of vegetation could appear as an average or mean to represent the surrounding landscape and design for every sample house.

The types of equipment used for cooling, and resident's living habits were given different codes and numerical values in the spreadsheets to convert the actual values to energy consumption. Energy consumption for cooling was represented as a total value for the study period of $\mathrm{kWh}$ in Malaysian Ringgit (MYR). All drawings used as supporting documents were generated by Autodesk Revit Architecture 2011 and AutoCAD 2011. 
All exterior and interior weather data gathered using devices such as the Babuc A, mobile TSI VelociCalc data loggers, and the Mini Thermohygrograph were transferred and formatted to Microsoft Excel 12.0. The daily to yearly weather data obtained from Malaysian Meteorology Department and monthly electricity costs data from National Energy Limited (TNB) were also put into this format.

The best weather data from five individual houses was chosen for case studies 1 and 2 , and the results of these studies were presented in various types of tables and figures from Microsoft Excel 12.0 and Origin 8.0.

\subsection{Landscaping to save air-conditioning energy}

The second stage of fieldwork and site measurements was conducted at a neighbourhood scale. The methodology to evaluate air-conditioning energy saving was done using interviews and data collection in 50 single-family houses in Putrajaya and Shah Alam.

The site measurements included recording plant type and configuration (species, size, location, etc.), building characteristics (building orientation and construction, including surface and insulation properties, etc.), human factors (living habits and styles), and general weather conditions (temperature, humidity and wind speed). All data collected were used to estimate the effect of vegetation on thermal performance and cooling energy use in the houses.

Survey data was divided into two categories: data was collected by interviewing the owners of the houses, and from measurements and observation of the properties by the author.

\subsubsection{Homeowner interviews}

Homeowner interviews were conducted to find information on human factors such as the numbers of occupants, and lifestyle habits, including the time and numbers of airconditioning units used every day. This provided information on the exact amount of 
cooling energy used in the house on a daily and monthly basis. Appendix 3.3 shows the interview sheets that were used. These took approximately 10 to 15 minutes to complete, and asked three main questions.

The first question asked about the number of residents in the house, and included how many were children, adolescents or adults. The second question focused on a set number of electricity bills during the two main seasons in the tropics to evaluate the monthly and annual energy consumption for every house. Details about the type of air-conditioning systems used were also gathered, including each system's capacity, the total time it is used, the times it is turned on during the day and night, and the average temperature setting of the system. From this data the total amount of cooling energy used was calculated.

\subsubsection{Data collection}

To obtain accurate data about each house in the study, the fieldwork information was collected through observation and by taking measurements. The aim of the fieldwork was to collect all the possible data about the physical characteristics of the house, including the structure and construction method, and the surrounding landscaping. The data sheets used can be seen in Appendix 3.4. These were filled out after consent was gained from the participants.

Primary data from homeowners through interviews, along with direct observations of each house's environment were essential. The main goal was to observe the physical characteristics of the properties, including building construction and the surrounding landscape, and the lifestyle habits of the occupants that could not be obtained without going to each property and contacting them directly.

Approval from the Victoria University's Human Ethics Committee was gained prior to conducting interviews and surveys, as was approval from the Malaysian Economic Planning Unit, Department of the Prime Minister. The approval letters are in Appendices 2.1-2.2. Permission to interview homeowners, access the property, and collect, record and publish the data was obtained from each home owner. Consent 
forms and information letters for this study are in Appendices 3.1 and 3.2 respectively.

The need for voluntary participation in the study created some difficulty in ensuring a representative sample. Some of the people approached were not willing to be interviewed because they wanted to keep all of the information about their house private. Also, some housing areas did not allow researchers to enter their community to protect the residents' privacy. To achieve the target number of respondents, surveys were carried out in five housing estates with houses chosen at random. Approximately 50 data collection sheets were filled in, and interviews completed from throughout the metropolitan Shah Alam and Putrajaya areas from January through March of 2010. Overall, only one-fifth of the number of householders approached cooperated and answered the interviews.

The fieldwork was divided into two categories; building structure and construction, and landscaping.

\subsubsection{Building structure and construction}

The first data collected was general information about the building structure and construction of each house in the study. This data can be validated against construction records from local authorities, but the on-site data collected is the most up to date information about the houses, as some houses in the sample have had extensions built, such as enlarged car porches and additional kitchen spaces.

The year of construction and house sizes were the main data variables used to classify the houses to ensure there was a range of ages and that they were medium sized. Information about the main structure and materials used was gathered to ensure the houses had similar construction and materials.

Data on the building envelope of every house was also gathered. This included information on windows and doors, wall and roof area and colour, and insulation. 
This information is significant because the building envelope influences the amount of heat absorbed by a house, which indirectly influences the cooling system.

Data collected about windows, sliding doors and common external doors included actual size, materials and locations. For wall and roof areas, the biggest building envelope surfaces of every building, the areas were calculated in square metres. Their colour was also noted because colour provides different abilities to reflect sunlight, as well as an albedo value for every house sample. Every house in the study areas used a layer of aluminium foil as an insulation layer for roof. Ceiling type and the material used were also noted, as these provide another insulation layer for the roof. Data about the type of ceiling was collected for every room in each house, include heights, ceiling types, materials, and colours.

\subsubsection{Landscaping}

The second part of the data collection stage focused on the surrounding landscaping for each house. This included common space roadside landscaping at the entrance of each house, which consists of four azimuths of garden space around the house up to the boundary of the houses. The size of each garden was noted, as it was usually different for each house and influenced the amount of vegetation planted around the buildings.

Data about the surrounding landscaping was divided into two types: soft landscape and hard landscape. Soft landscape included any type of vegetation that was planted around the houses, and is divided into five categories of vegetation: trees, shrubs, vines, groundcover and turf. Trees were divided into six types: roadside tree, roadside palm, garden tree, edible fruits tree, garden palm and bamboo. The explanation for every species of tree includes location, tree to building distance, trunk height and overall height, canopy size, shape, and amount and size of leaves. Shrubs, vines and groundcover were also categorised, as well as trees with slight differences in size. Tables 3.1 and 3.2 show the categories of tree size, shrubs, vines and groundcover. Turf species were stated in areas. The botanical name and common name for vegetation types were updated later using photographs. 
Table 3.1. Canopy size, shape, and amount and size of leaves for trees

\begin{tabular}{|l|l|l|l|l|l|l|}
\hline \multicolumn{2}{|l|}{ Canopy size (diameter) } & Shape & \multicolumn{2}{l|}{$\begin{array}{l}\text { Amount of leaves } \\
\text { (density \%) }\end{array}$} & \multicolumn{2}{l|}{ Size of leaves $\left(\mathrm{mm}^{2}\right)$} \\
\hline Small & Below 3 & Spreading & Few & Below 40 & Small & Below 150 \\
\hline Small moderate & $3-7$ & Round & Medium & $40-70$ & Medium & $150-1000$ \\
\hline Medium & $8-11$ & Columnar & Dense & Above 70 & Large & Above 1000 \\
\hline Large moderate & $12-15$ & Pyramidal & & & & \\
\hline Large & Above 15 & Fountain & & & & \\
\hline
\end{tabular}

Table 3.2. Size, shape, and amount and size of leaves for shrub, vines and groundcover

\begin{tabular}{|l|l|l|l|l|l|}
\hline Spread $\left(\mathrm{m}^{2}\right)$ & Shape & \multicolumn{2}{|l|}{$\begin{array}{l}\text { Amount of leaves } \\
(\text { density \% }\end{array}$} & \multicolumn{2}{l|}{ Size of leaves $\left(\mathrm{mm}^{2}\right)$} \\
\hline Individual/potted & Spreading & Few & Below 40 & Small & Below 150 \\
\hline Group below 5 & Upright & Medium & $40-70$ & Medium & $150-1000$ \\
\hline Group 6-10 & Rounded & Dense & Above 70 & Large & Above 1000 \\
\hline Group 10-20 & Vase-shaped & & & & \\
\hline Group 21-30 & Sword leaf & & & & \\
\hline Above 30 & & & & & \\
\hline
\end{tabular}

Hard landscaping in the study areas included paved surfaces, swimming pools, fish ponds, fountains, and shelters such as pergolas or gazebos. These are located among soft landscaping, and are intended to complement and beautify garden design and provide spaces for outdoor activities. The data collected included recording the type of landscaping feature, and its location and distance from the house, and construction material, size and colour and total area.

The final portion of the data collection sheet contained a space to sketch the house plan and surrounding landscaping elements to provide a detailed reference about their exact location and sizes. The plan of the house was also based on satellite images, master plans and detail drawings found from government departments. A scale of 1:200 was used as can be seen in Figure 3.3. This data collection took approximately three to four hours to complete per house, depending on the size of the house and surrounding landscape. For example, if the house was large and had many 
types and species of vegetation, it usually took a long time to draw and name each type of vegetation in detail.

\subsubsection{Data analysis}

Quantitative methods were used to evaluate the result of the effect of vegetation, building construction and human factors on cooling energy used in a hot-humid environment. All of the data gathered from 50 houses and their surrounding landscapes, from five housing estates in two cities, was analysed using Statistical Package for the Social Sciences (SPSS 19.0). SPSS is one of the most frequently used research tools in statistics analyses used by social scientists. According to Mills (2003) the program offers a base feature that provides the most basic statistical procedures, as well as advanced features that allow the user to tackle more sophisticated statistical techniques. Mills (2003) also states that the users may choose to use the syntax methods to write code and variables for specific analyses as opposed to the point-and-click method of generating the output.

SPSS uses a wide variety of tests and analysis to break down the statistical trends of a dataset. Several methods of analysis were used in this study, such as descriptive statistics, and cross tabulation tests of association and regression analysis. The descriptive statistics of a dataset included the mean (average), median (middle value), range (minimum and maximum values), and standard deviation, among others. Cross tabulation was also used where a table of two (or more) categorical variables was produced to compare the incidence of one characteristic against another.

Microsoft Excel 12.0 and Origin 8.0 were used to generate figures and tables from SPSS data. The effects of surrounding landscape, together with building construction and human factors data, was calculated and evaluated by these statistical analyses systems. The cooling energy used in an individual single-family house was revealed in parallel with the neighbourhood trends of energy used for cooling. 


\subsection{Summary}

The direct and indirect thermal impact of the vegetation surrounding single-family houses and their neighbourhoods in tropical climates was investigated using three research methods to collect data: interview, direct observation, and physical artefacts evidence. The primary data on the effects of vegetation on the thermal performance of buildings and their microclimates on site were gathered to predict the effects of shade, evapotranspiration, and channelling wind on temperature and energy use. The secondary data was obtained from government agencies, and included information such as each neighbourhood's master plans, building plans, satellite images, climate data, and energy use. These were used to confirm that all of the data was accurate.

Five single-family housing developments ranging in age from 3 to 40 years old were chosen for this study. The maturity, number of species and location of the surrounding landscaping would affect the amount of energy used by each house, and its performance in relation to thermal comfort. Two stages of research were done on two different scales; neighbourhood areas or a microclimate scale was used to quantify the energy saving potential, and the temperature reductions were evaluated around individual houses.

In the first stage of fieldwork and measurements, the physical condition of five houses and their surrounding landscaping and weather data was recorded. The outdoor and indoor weather data were recorded for approximately 12 hours during the daytime in each of the four azimuths of the houses. Air temperature, relative humidity and wind flow around the houses was recorded automatically in 30 minute intervals using scientific equipment. The outdoor weather conditions can directly influence the thermal performance of a house.

In the second stage, 50 homeowners were interviewed to gain information about the human factors aspect of this study. On-site observations, data collection and information about building construction and the surrounding landscaping of each house were made manually and recorded by photographs. Statistical analysis was used to quantify and analyse the cooling-energy savings potential. This was based on 
the comparison between the surrounding landscaping, building construction and cooling energy use.

The next chapter will report in detail stage 1 of the fieldwork, how the surrounding landscaping modified the outdoor ambient air in three individual single-family houses that have similar building construction, but are different ages of construction and landscaping. 


\subsection{THE EFFECTS OF LANDSCAPING ON THE THERMAL PERFORMANCE OF HOUSING}

\subsection{Overview}

The previous chapter explained how the research was divided into two stages fieldwork, site measurements, and data collection at (a) 5 individual houses, and (b) 50 houses. This chapter examines the first stage of fieldwork measurement, the effectiveness of landscaping on the thermal performance of houses in a tropical environment as case study 1

This case study involved looking at the construction and landscaping of three singlefamily houses in three sections of Shah Alam, Selangor, Malaysia. The houses ranged in age from 5 to 30 years old, which provided different examples of construction and maturity levels of the surrounding landscaping. Landscaping affects the thermal performance of houses, in how it provides shade, channels wind, and by evapotranspiration. While the construction of the three houses was similar, they were different in size and design, including their landscape design. These houses were chosen because they are representative of single-family tropical houses and landscaping styles in Malaysia.

The focus in this study was on the effectiveness of landscaping on the thermal performance of the houses. Sets of instrument were placed in several exterior and interior locations around the houses to record the weather data during the day. The 30 year old house and its property was labelled as a mature landscape house; the 10 year old house, ordinary landscape house; and the 5 year old house, new landscape house.

In a tropical country, landscaping is used to reduce the effects of the hot, humid climate. Every type of vegetation, such as trees, grass, shrubs, groundcover, and turf, contributes to reducing air temperatures near the house, and providing evaporative cooling. This study will investigate the effects of different amounts of tropical 
landscaping around houses on their ability to provide a cool and comfortable environment.

\subsection{House study}

The three houses were located in Shah Alam $\left(3^{\circ} \mathrm{N}\right.$ Latitude and $101^{\circ} \mathrm{E}$ Longitude), at an elevation of 27 to $47 \mathrm{~m}$ in a hot-humid tropical climate. Shah Alam is the capital of Selangor State, and is known as one of the most well planned cities in Malaysia (Aziz \& Hadi, 2007). Landscaping has featured strongly in Shah Alam's development. Every development in the city must be balanced with green space.

\subsubsection{House selection}

The houses looked at as part of this case study are in Sections 6, 9 and 11 of Shah Alam, in the central city.

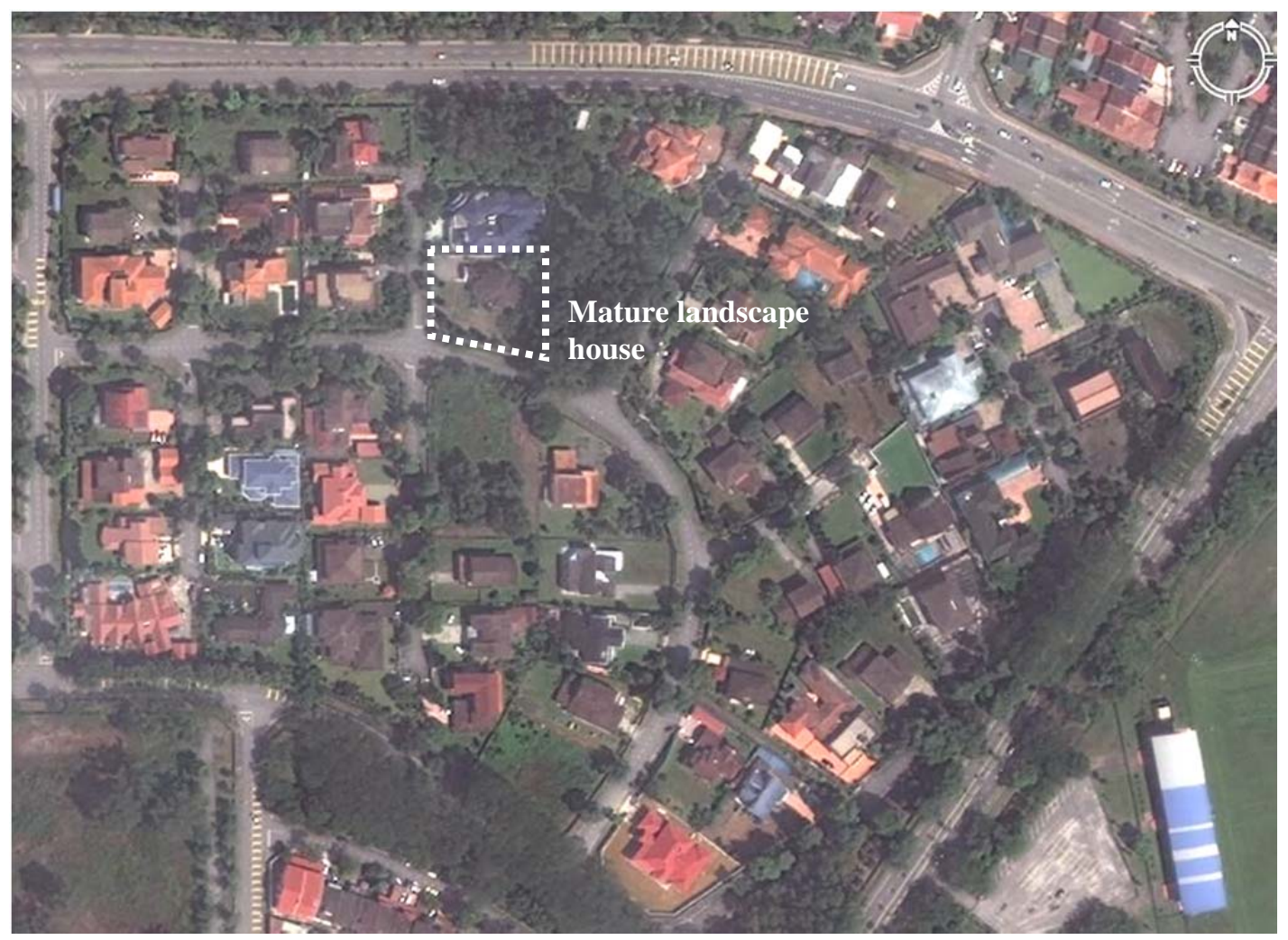

Figure 4.1. Location of the mature landscape house in Section 6, Shah Alam, Selangor (Google Earth, 2011) 
These sections each have around 50 low density single-family houses, and each take up an area of around $0.5 \mathrm{~km}^{2}$. They represent around $20-30 \%$ of the total residential development in each section. One house from each section was chosen for the study, each with a different age of construction and landscaping. As previously noted, these were labelled mature, ordinary and new. The house with mature landscape was 30 years old, and located in Section 6 (Figure 4.1); the house with the ordinary landscape was 10 years old and in Section 11 (Appendix 4.1); while the new landscape house was five years old and located in Section 9 (Appendix 4.2). Each house was surrounded by houses of similar age and landscaping.

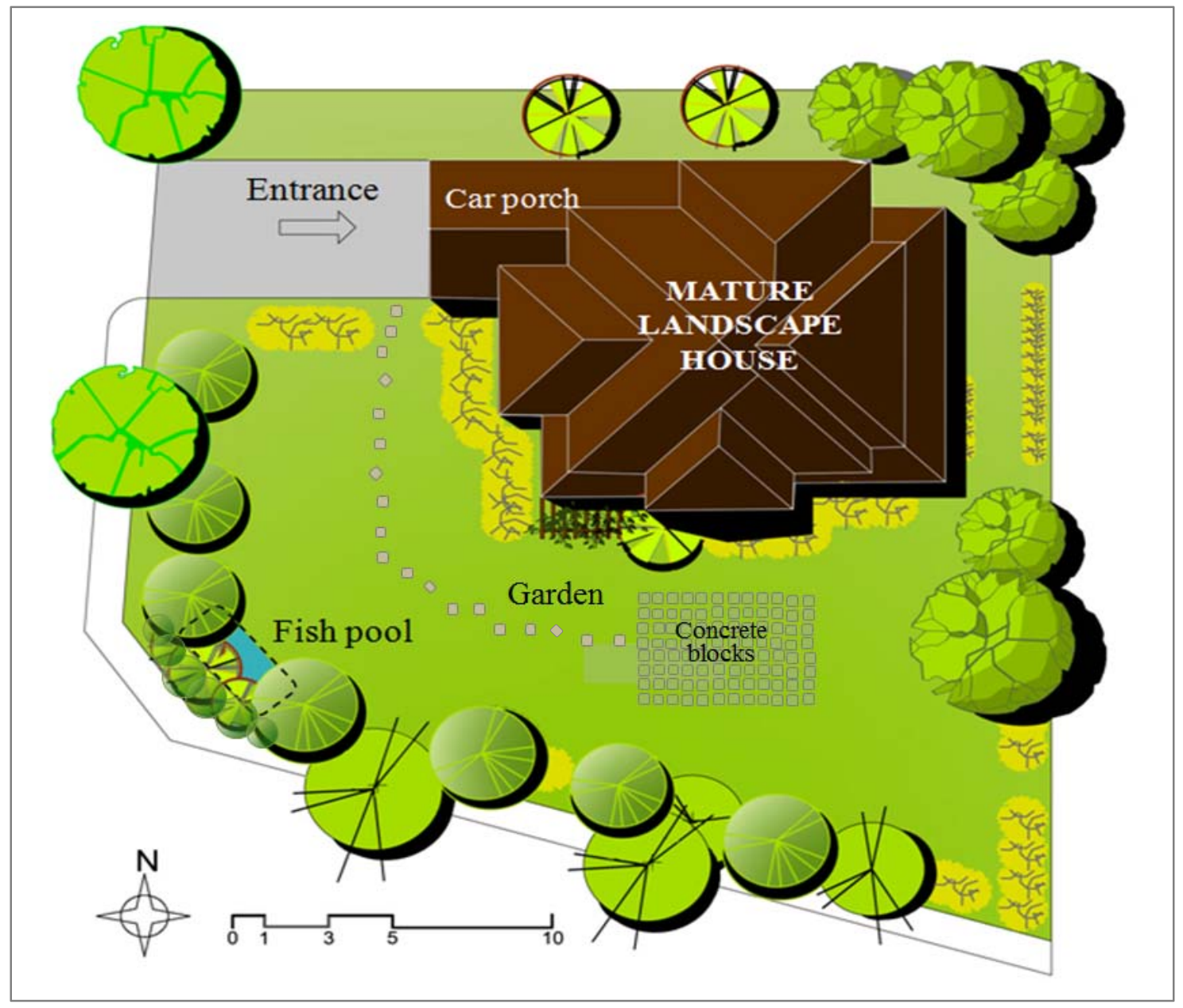

Figure 4.2. Landscape plan of the mature landscape house 

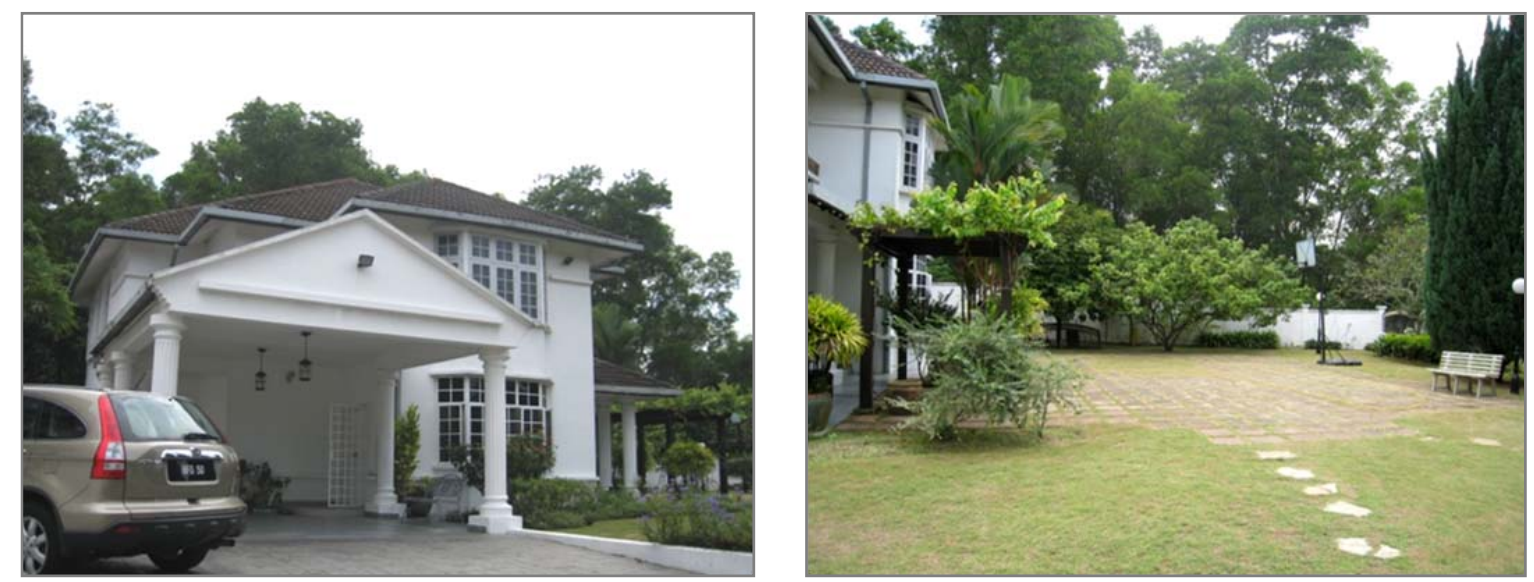

Figure 4.3. Front/west elevation of the mature landscape house (left) and view of the garden to the south (right)
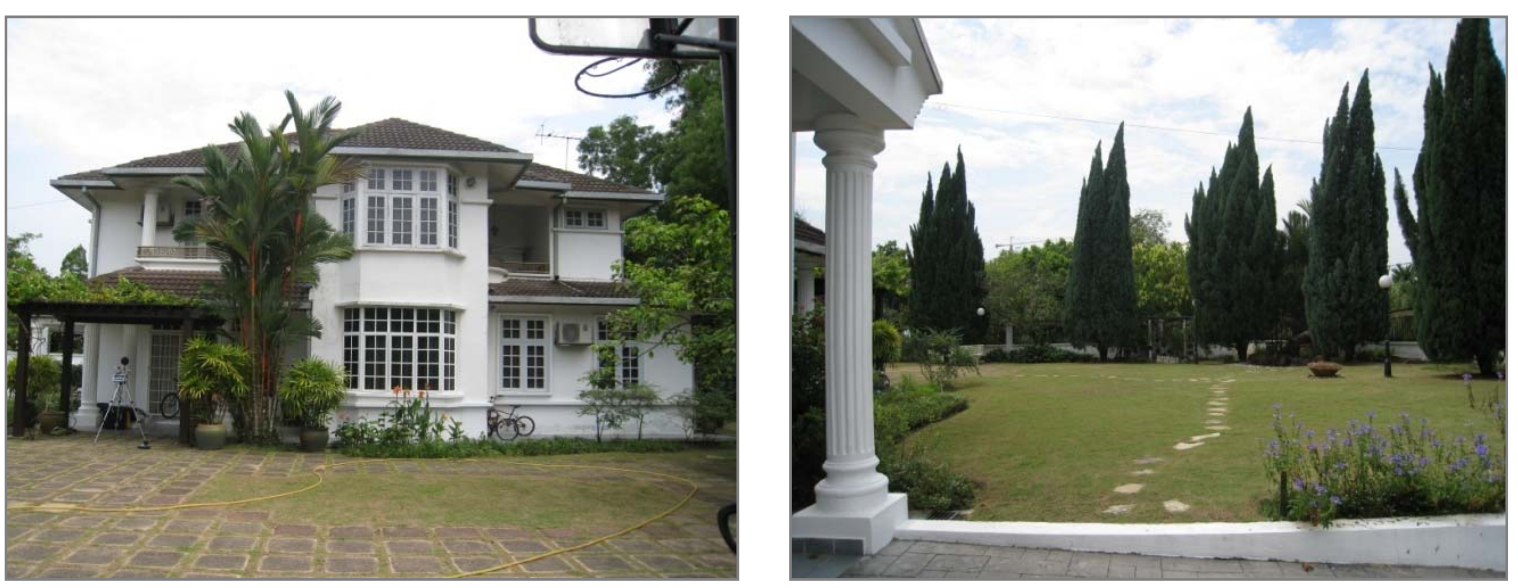

Figure 4.4. Looking towards south elevation of the mature landscape house (left) and view looking south from the car porch (right) 


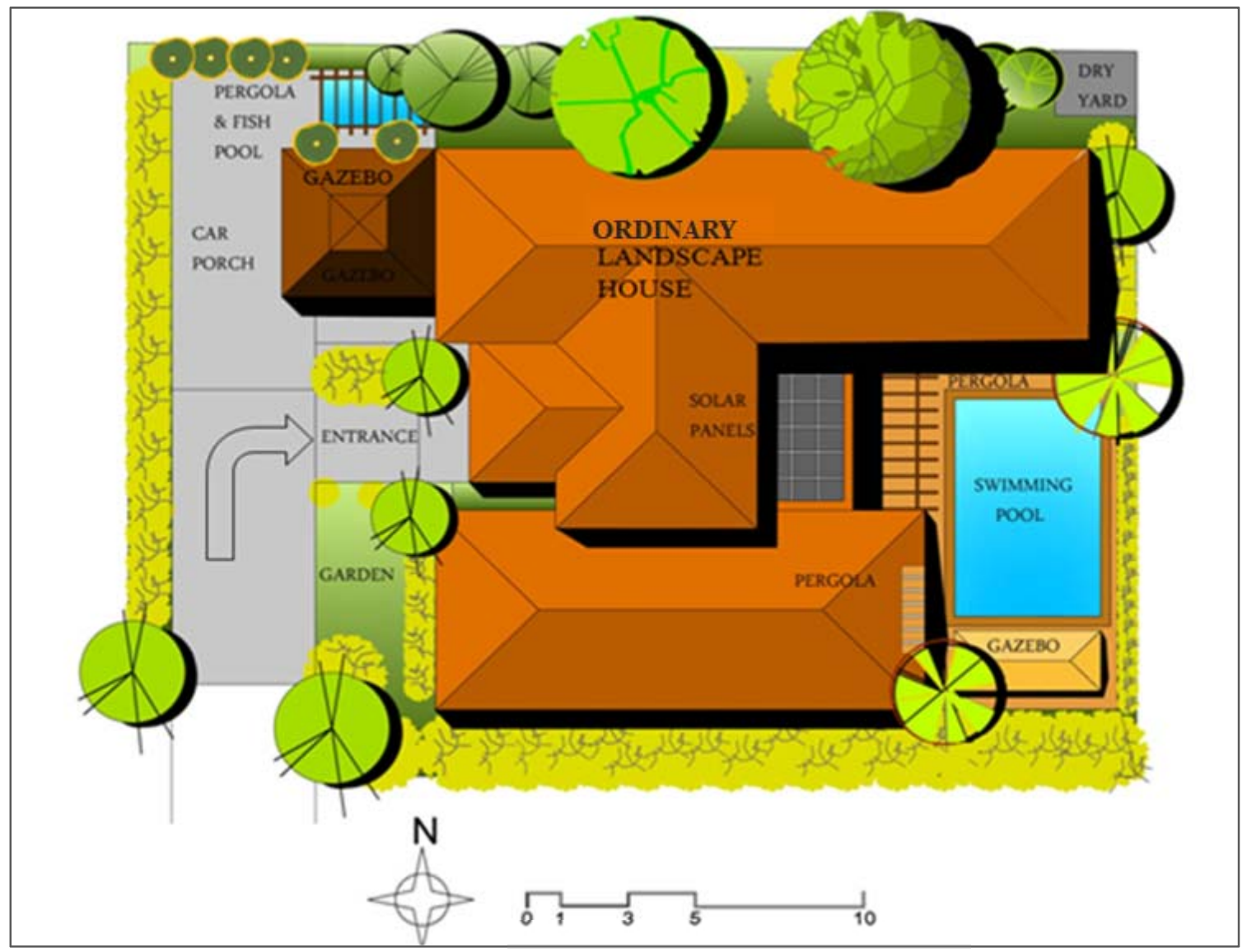

Figure 4.5. Landscape plan of the ordinary landscape house
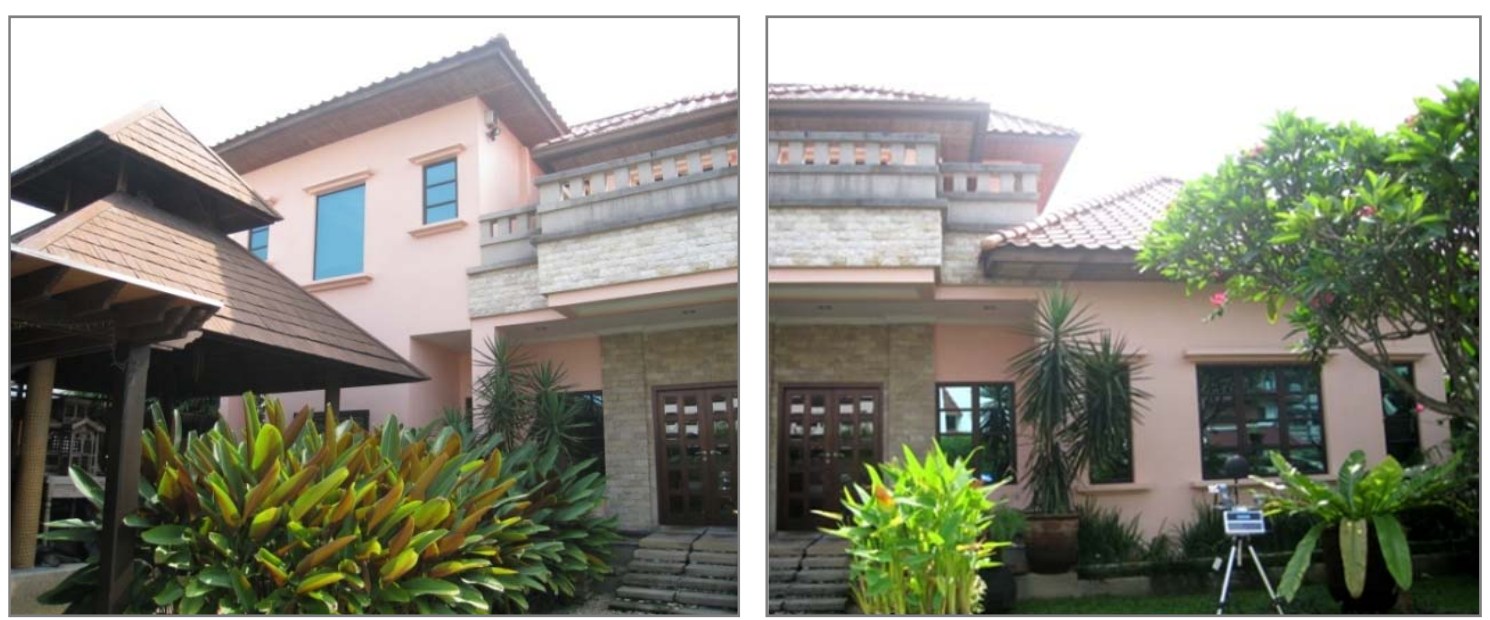

Figure 4.6. Views of the front/east elevation of the ordinary landscape house looking at the entrance garden 

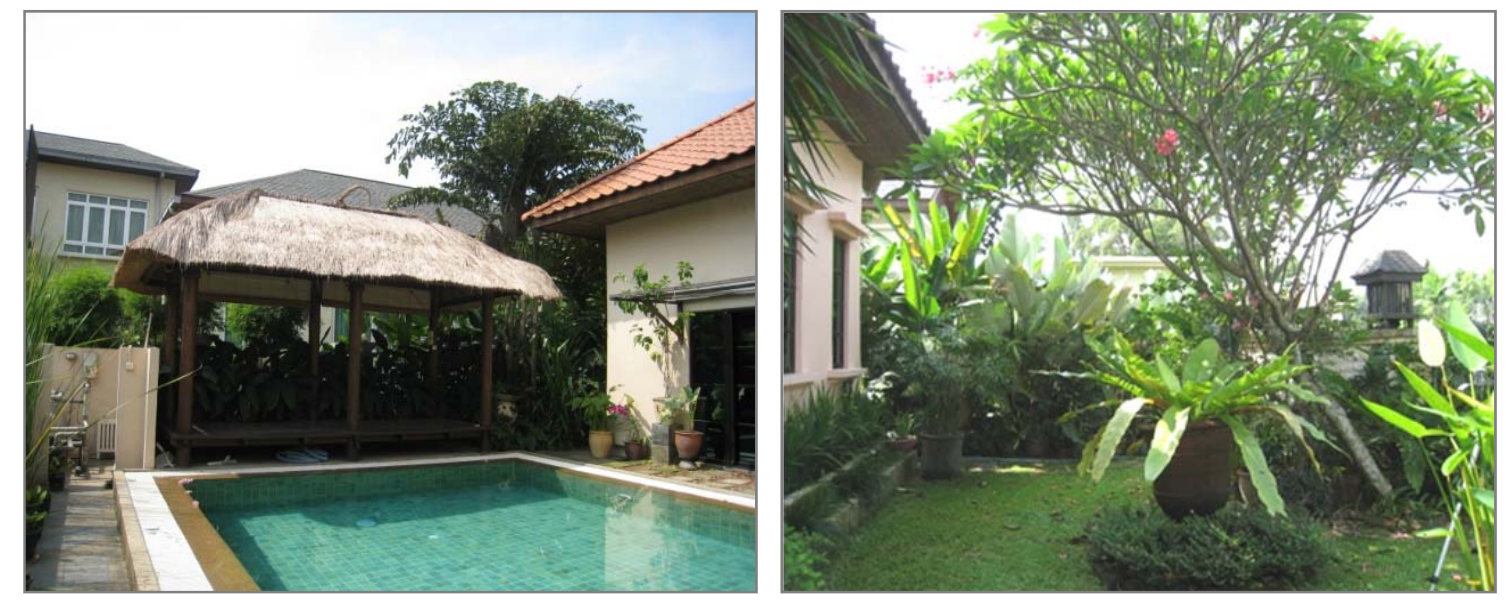

Figure 4.7. Views of the ordinary landscape house: swimming pool and gazebo at the rear/east side (left), and of the front garden view looking south (right)

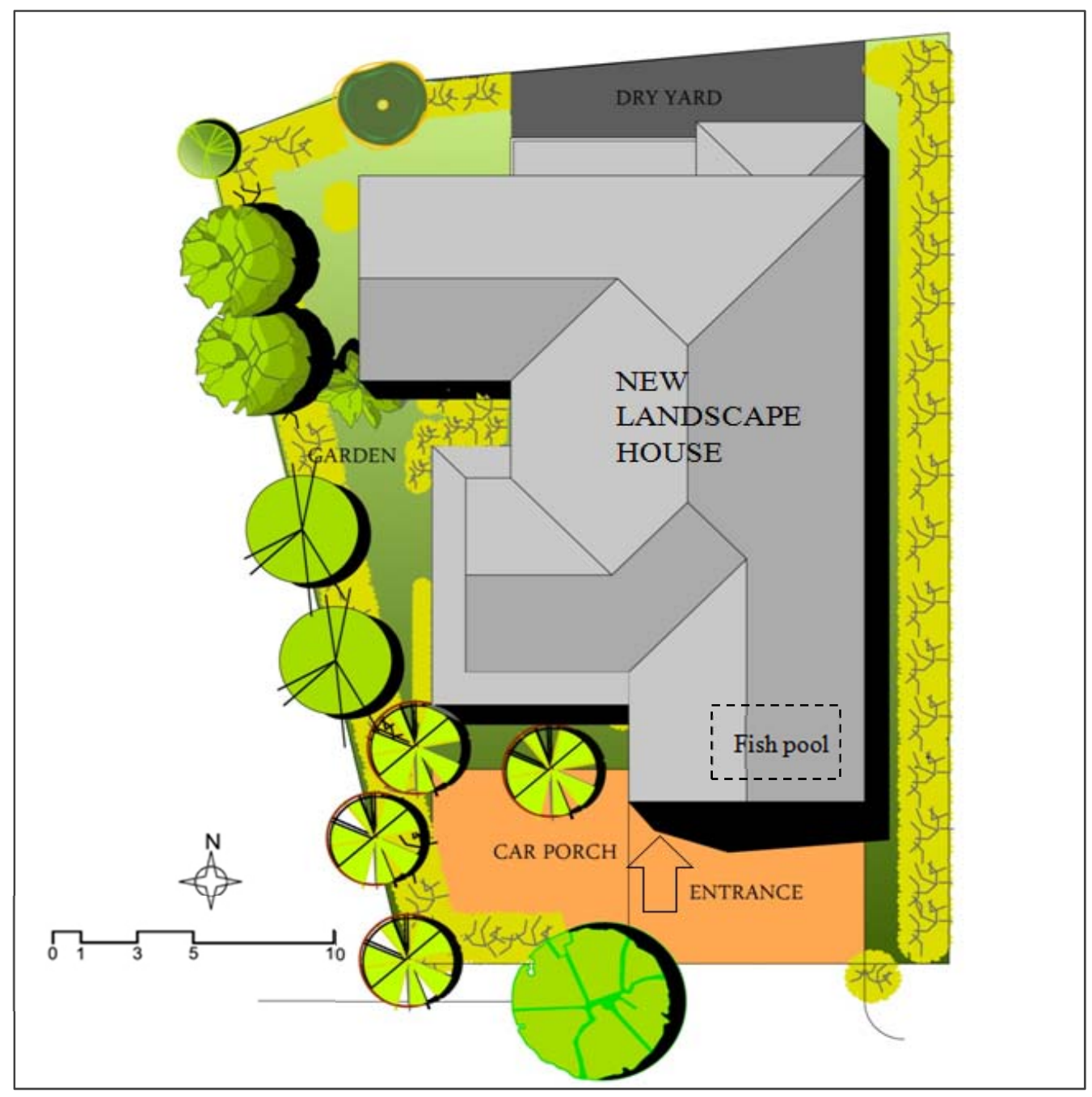

Figure 4.8. Landscape of the new landscape house 

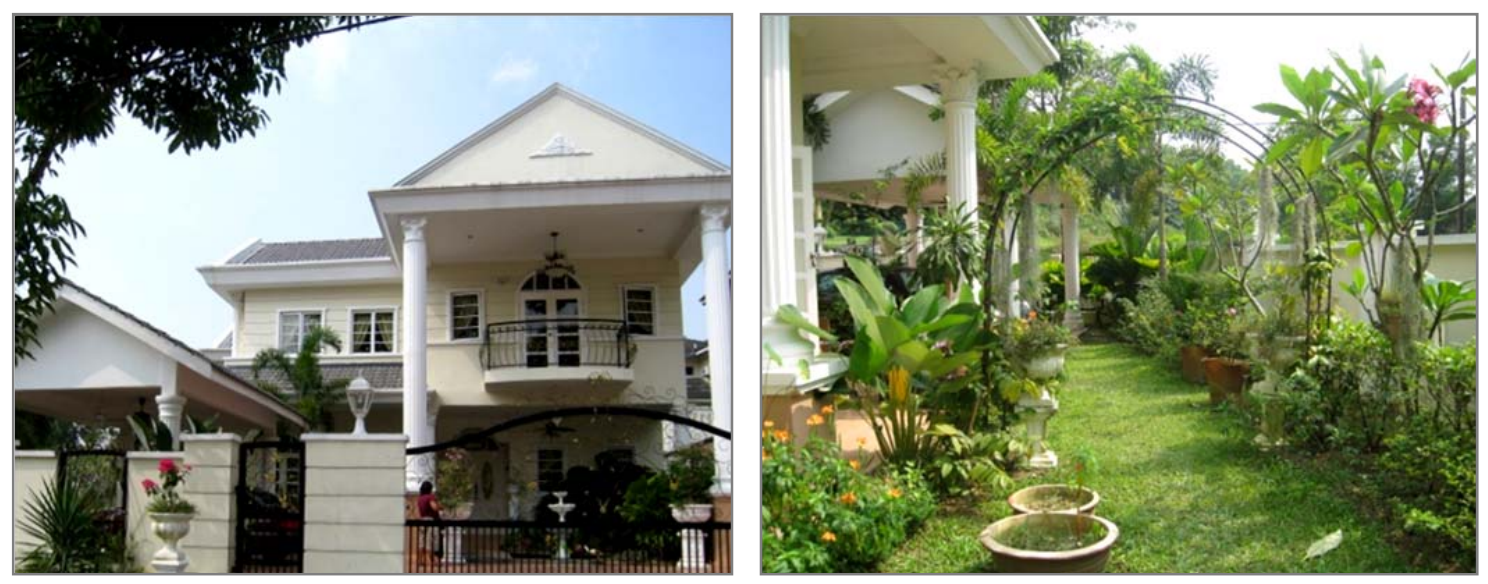

Figure 4.9. Views of the new landscape house: front/south elevation of the house (left), garden view looking south (right)

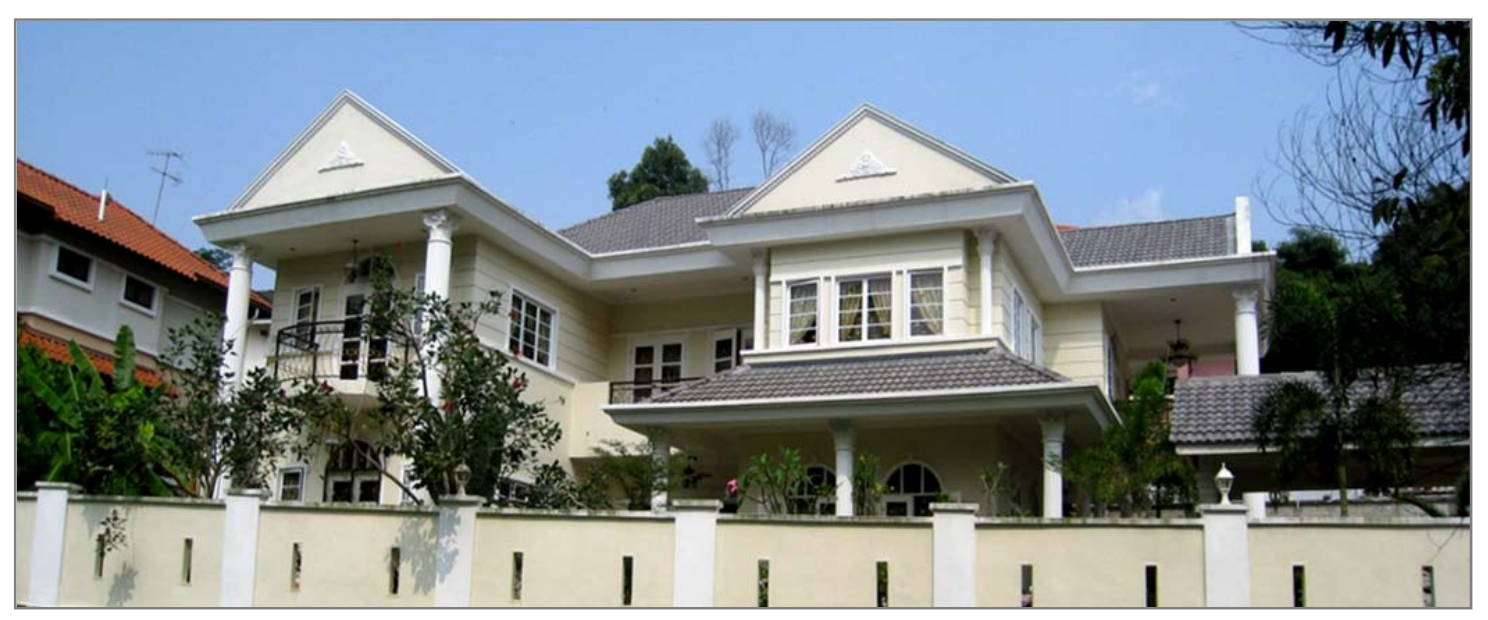

Figure 4.10. Views from the west towards the new landscape house

\subsubsection{Environmental condition}

The environment around each of the houses was different, reflecting the age and maturity of their landscaping. The mature landscape house was surrounded by neighbouring houses that had similar mature landscaping in their gardens, as well as along roadsides and open spaces. The ordinary and new landscaped houses were surrounded by moderate landscaping, and most of their shade trees were medium or small. Therefore, the environment of the mature landscape house appears to be greener. In addition, the mature landscape house had open space on its east and south sides, the new landscape house on its south and west sides, while the ordinary landscape house was completely surrounded by other single-family houses. The 
open space on the east side of the mature landscape house was planted with mature shade trees of up to $10 \mathrm{~m}$ high, and the open space on the south side had been planted with a few trees, shrubs and turf. The open space beside the new landscape house was moderately planted with mature trees, shrubs, grasses and turf. There were no water surfaces in these neighbourhoods.

\subsection{General weather data}

Two years' worth of climate data (2008-2009) was obtained from the nearby Subang Airport Weather Station. The average temperature (maximum and minimum), wind direction and speed, and humidity levels for the dry and rainy season are shown in Table 4.1. The climates in the study areas are typical of a hot-humid tropical climate, with temperatures ranging from 24 to $33^{\circ} \mathrm{C}$, and a relative humidity of between 72 and $78 \%$. Precipitation was very heavy throughout the year, with an annual average of about $3100 \mathrm{~mm}$, and 202 days of rain. The dry season occurs with the south-west monsoon, which starts in the latter half of May and ends in September. The prevailing wind flow is generally south and south-westerly and light, around $22 \%$ of the time. The north-east monsoon in the rainy season commences in early November and ends in March, with steady prevailing north and north-westerly winds $27 \%$ of the time. During the two shorter periods of inter-monsoon season in April and October, the winds are generally light and variable.

Table 4.1. Average daily maximum and minimum temperatures, relative humidity and wind for January, April, July, and October 2008-2009

\begin{tabular}{|c|c|c|c|c|c|}
\hline \multirow[t]{2}{*}{ Month } & \multicolumn{2}{|c|}{ Temperature $\left({ }^{\circ} \mathrm{C}\right)$} & \multicolumn{2}{|l|}{ Wind } & \multirow{2}{*}{$\begin{array}{l}\text { Relative } \\
\text { Humidity } \\
(\%)\end{array}$} \\
\hline & $\begin{array}{l}\text { Daily } \\
\text { Maximum }\end{array}$ & $\begin{array}{l}\text { Daily } \\
\text { Minimum }\end{array}$ & Direction & $\begin{array}{l}\text { Frequency } \\
\text { (\% of the time) }\end{array}$ & \\
\hline January & 32.05 & 23.55 & NW & 14.50 & 77.40 \\
\hline April & 32.95 & 24.35 & $\mathrm{~N}$ & 13.10 & 78.05 \\
\hline July & 32.30 & 23.40 & $\mathrm{~S}$ & 15.80 & 72.05 \\
\hline October & 32.50 & 23.40 & NW & 17.60 & 76.83 \\
\hline
\end{tabular}

The study areas also have an abundance of solar radiation, with about six to eight hours of sunshine per day, typically between 8.00 and 18.00 hours. The mean daily global radiation is around $18.22 \mathrm{MJ}^{-2} \mathrm{~m}^{-2}$, with 7.1 Okta of cloud cover. 
The uniformly high average temperatures and humidity levels, combined with low wind flow and high solar radiation create an uncomfortable microclimate. This contributes to radiant heat gain in buildings, which Barker (1987) noted is a significant and dominant factor with regards to human comfort. Therefore, one of the most important microclimate modifications will be to protect the building envelope and exterior environment from solar heat gain.

\subsection{Fieldwork measurement period}

Fieldwork measurements and data collection were conducted during the same day for each house on the $23^{\text {rd }}, 24^{\text {th }}$, and $28^{\text {th }}$ of February, 2010. The fieldwork site measurements were related to ambient air conditions, including solar radiation and cloud cover, air temperature, relative or absolute humidity, and wind speed while the data collection included building, landscaping and information about the airconditioning system.

\subsection{Analysis}

The three houses in this case study had differences in their architecture and construction. While their general building construction was similar, each house followed the construction trends of the time they were built.

Measurements taken for building construction included house orientation and configuration, size, general construction, and the materials used for floors, walls and the roof. The colour of the building envelopes and their areas were also recorded, including wall and roof area, and all windows and doors, and their shading devices. The information gathered on the interior settings included interior spaces, and ceiling heights and types. In addition, the weather data measurement taken for the ambient air included solar radiation and cloud cover, air temperature, relative humidity, and wind speed, all of which can influence the thermal performance of the house.

The landscape design of the garden for each house was developed by the individual owners, and was characteristic of the time each garden was planted and designed. Each had been planted with tropical plants that served multiple purposes: to enhance 
aesthetics, provide food, provide shade, and produce a pleasant, comfortable and healthy environment. The owners had followed a tropical landscape style, using every type of vegetation structure including trees, palms, bamboos, shrubs, vines, groundcover and turf.

The houses also had different external structures. The mature landscape house had a pergola, and the ordinary landscape house had two gazebos and a swimming pool, while each house had a small fish pool. The main entrances to all the houses were permanently paved with concrete and finished with cement screed and tiles. These were located in front of the houses, and attached to the car porches.

Each house's landscape design used different amounts, species and configurations of vegetation. Appropriate choice of vegetation and strategic location will potentially influence their shade, evapotranspiration, and wind channelling characteristics.

\subsubsection{Building construction}

The architecture of the three houses was typical of the Malaysian tropical style. However, the house configurations were different. The front façade of the mature landscape house and the ordinary landscape faced the east or west direction, while the new landscape house faced south. Also, while the houses were all two-storey, medium-sized houses, they had a slightly different sized building footprint and garden area.

The houses were built using similar building materials and construction methods. The main structures were built on reinforced concrete pad foundations (R$\left.1.7 \mathrm{~m}^{2} .{ }^{\circ} \mathrm{C} / \mathrm{W}\right)$; all had brick walls $\left(\mathrm{R}-1.3 \mathrm{~m}^{2} .{ }^{\circ} \mathrm{C} / \mathrm{W}\right)$; and a pitched timber-framed roof covered by concrete tiles with an insulation layer of aluminium foil paper underneath (R-1.7 $\left.\mathrm{m}^{2} .{ }^{\circ} \mathrm{C} / \mathrm{W}\right)$. Table 4.2 shows the different building footprints, floor, roof, and garden areas, and albedo values.

The three houses had different coloured building envelopes. The mature landscape house had white walls and a dark brown roof, the ordinary landscape had peach for 
the walls and a light orange roof, while the new landscape house had cream walls with light grey concrete roof tiles. The albedo values fell in the range of 0.12 to 0.42 , as can be seen in Table 4.2. Solid hardwood material was used for the doors (R$\left.0.34 \mathrm{~m}^{2} .{ }^{\circ} \mathrm{C} / \mathrm{W}\right)$.

Table 4.2. Building footprint, floor, roof, albedo and garden areas for the three landscape houses

\begin{tabular}{lllllllll}
\hline House type & $\begin{array}{l}\text { Building } \\
\text { footprint } \\
\left(\mathrm{m}^{2}\right)\end{array}$ & $\begin{array}{l}\text { Floor } \\
\left(\mathrm{m}^{2}\right)\end{array}$ & $\begin{array}{l}\text { Roof } \\
\left(\mathrm{m}^{2}\right)\end{array}$ & $\begin{array}{l}\text { Wall } \\
\text { colour }\end{array}$ & $\begin{array}{l}\text { Wall } \\
\text { albedo }\end{array}$ & $\begin{array}{l}\text { Roof } \\
\text { colour }\end{array}$ & $\begin{array}{l}\text { Roof } \\
\text { albedo }\end{array}$ & $\begin{array}{l}\text { Garden } \\
\left(\mathrm{m}^{2}\right)\end{array}$ \\
\hline Mature landscape house & 210 & 350 & 225 & white & 0.30 & dark brown & 0.12 & 500 \\
Ordinary landscape house & 355 & 550 & 375 & peach & 0.42 & light orange & 0.33 & 350 \\
New landscape house & 285 & 400 & 300 & crème & 0.33 & light grey & 0.30 & 250 \\
\hline
\end{tabular}

Table 4.3. Area of brick walls and glazed surfaces for the three landscape houses

\begin{tabular}{lllllll}
\hline \multirow{2}{*}{ Azimuth } & \multicolumn{2}{l}{ House type@Wall $\left(\mathrm{m}^{2}\right)$} & \multicolumn{4}{l}{ House type $@$ Glass $\left(\mathrm{m}^{2}\right)$} \\
\cline { 2 - 7 } & Mature & Ordinary & New & Mature & Ordinary & New \\
\hline North & 90 & 150 & 90 & 09.75 & 21.30 & 12.30 \\
East & 80 & 120 & 100 & 07.70 & 31.10 & 25.40 \\
South & 90 & 150 & 100 & 23.10 & 14.90 & 22.00 \\
West & 80 & 120 & 120 & 10.80 & 20.00 & 34.80 \\
\hline Total & 340 & 540 & 410 & 51.35 & 87.30 & 94.50 \\
\hline
\end{tabular}

Ceiling heights for the three houses were around $2.9 \mathrm{~m}$, and were finished with gypsum board, cement plaster, and asbestos free fibre cement ceiling sheets. Threemillimetre thick, single-layer glass, with metal window frames $\left(\mathrm{R}-0.13 \mathrm{~m}^{2} .{ }^{\circ} \mathrm{C} / \mathrm{W}\right)$ were used for all casement windows, the sliding doors, and a few of the external doors.

The houses had shading devices over almost all their windows and doors to provide shade during the peak times of the day. They also had at least $0.8 \mathrm{~m}$ of overhanging gable or hip roof, and a $2-3 \mathrm{~m}$ wide veranda built over the sliding doors, back windows and doors. Each house had a porch covering the main entrance, which provided shade to the windows and doors facing this space. Table 4.3 shows that the total areas for glass and plastered brick walls were different for each house. While 
the mature and the ordinary landscape house have $15 \%$ and $16 \%$ glass surfaces respectively, the new house has $24 \%$ glass surface.

\subsubsection{Landscape design}

Each house had different landscaping, which included various combinations of trees, shrubs, vines, groundcovers and turf. These were recorded for each house for the four facing directions of the house. The mature landscape house had a large garden, which was planted with a limited amount of vegetation. The majority of the garden space was lawn. A total of 22 trees were identified in the garden, 38 individual and groups of shrubs, a planting of vines and two groups of groundcover plants.

The ordinary landscape house had a moderate amount of landscaping, which comprised only seven trees, and 61 individual and groups of shrubs, five plantings of vines and six groups of groundcover plants.

The new landscape house also had a moderate amount of landscaping, which was still immature. It had 11 trees, and was surrounded by 63 individual and groups of shrubs, a planting of vines, and four groups of groundcover plants. The rest of the soil surfaces for the three houses were covered by Axonopus compressus.

\subsubsection{Trees}

The main function of the trees planted around the houses was to provide shade to the surrounding garden and house, and reduce heat gain to the building during the day. The garden of the mature landscape house had 22 trees that were planted between $3-$ $19 \mathrm{~m}$ from the house. They were planted individually and in groups, which were more concentrated on the north, south and west sides. Their canopies covered approximately $30 \%$ of the garden area. The sizes of the tree canopies varied. Fifty percent were small to moderate size, $41 \%$ were small, and $9 \%$ were medium (refer to Table 3.1). Their canopies were maintained by pruning the branches to keep the trees at an appropriate size and in a safe condition. Some of the garden trees were naturally small such as Juniperus chinensis. 
The most common type of trees were edible fruit trees ( $36 \%$ of trees in the garden), followed by garden trees $(28 \%)$, and a small number of palms and roadside trees (18\% each). The majority of trees were below $4 \mathrm{~m}$ in height (60\%), and the remaining trees were between $5-9 \mathrm{~m}$ in height, with an average trunk height of about $2 \mathrm{~m}$. The shape of the trees varied, with round shapes representing $36 \%$ of the trees, followed by columnar shapes at $23 \%$, and finally spreading and fountain shapes at $18 \%$ each.

The amount of leaves for every tree has been divided into three classes: $50 \%$ of trees had a dense canopy of leaves, $36 \%$ a medium canopy, and $14 \%$ trees had a sparse canopy, with few leaves (refer to Table 3.1). Fifty-nine percent of the trees had small sized leaves, and the balance had medium sized leaves (41\%). All trees were from evergreen tropical plant species that were aged approximately 30 years.

Table 4.5 shows the ordinary landscape house had a minimum amount of trees, with less than one-third of the amount of the mature landscape house. Most of the trees were situated within $4 \mathrm{~m}$ of the building, and covered around $10 \%$ of the garden. More trees were found on the north side of the house. Approximately $42 \%$ of the tree canopies were of medium size, and $29 \%$ of them were small to moderate and small sized respectively. $43 \%$ of the trees were more than $10 \mathrm{~m}$ tall, with $6 \mathrm{~m}$ trunk heights, while the rest (57\%) were $4 \mathrm{~m}$ in height, with $1-2 \mathrm{~m}$ trunk heights. Seventy percent of the trees were spreading in shape, and 15\% were round and fountain shaped respectively. There were generally medium amounts of leaves, and medium sized leaves in the tree canopies.

The amount of planted species at the new landscape house was moderate and the overall amount of trees was around half of that of the mature landscape. Most of the trees were situated within $5 \mathrm{~m}$ of the building. They covered around $20 \%$ of the garden's surface and were more focused on the elevations of the west and south sides of the house. All of the garden tree canopies were small in size with an overall height of between 2 and $4 \mathrm{~m}$ and a trunk height around 1-2m. There was only one round shaped tree of small to moderate size planted as a roadside tree with an overall height of $6 \mathrm{~m}$ and a $3.5 \mathrm{~m}$ trunk height on the south side. This tree produced a sparse 
amount of medium sized leaves. 50\% of the garden trees were of spreading shape and $50 \%$ of fountain shape. The amount of leaves in each canopy was generally sparse, and the leaf-size was generally medium. All the garden trees and palms were planted in small groups or individually placed and were still in the process of growing, needing a few years to mature and produce sufficient shade.

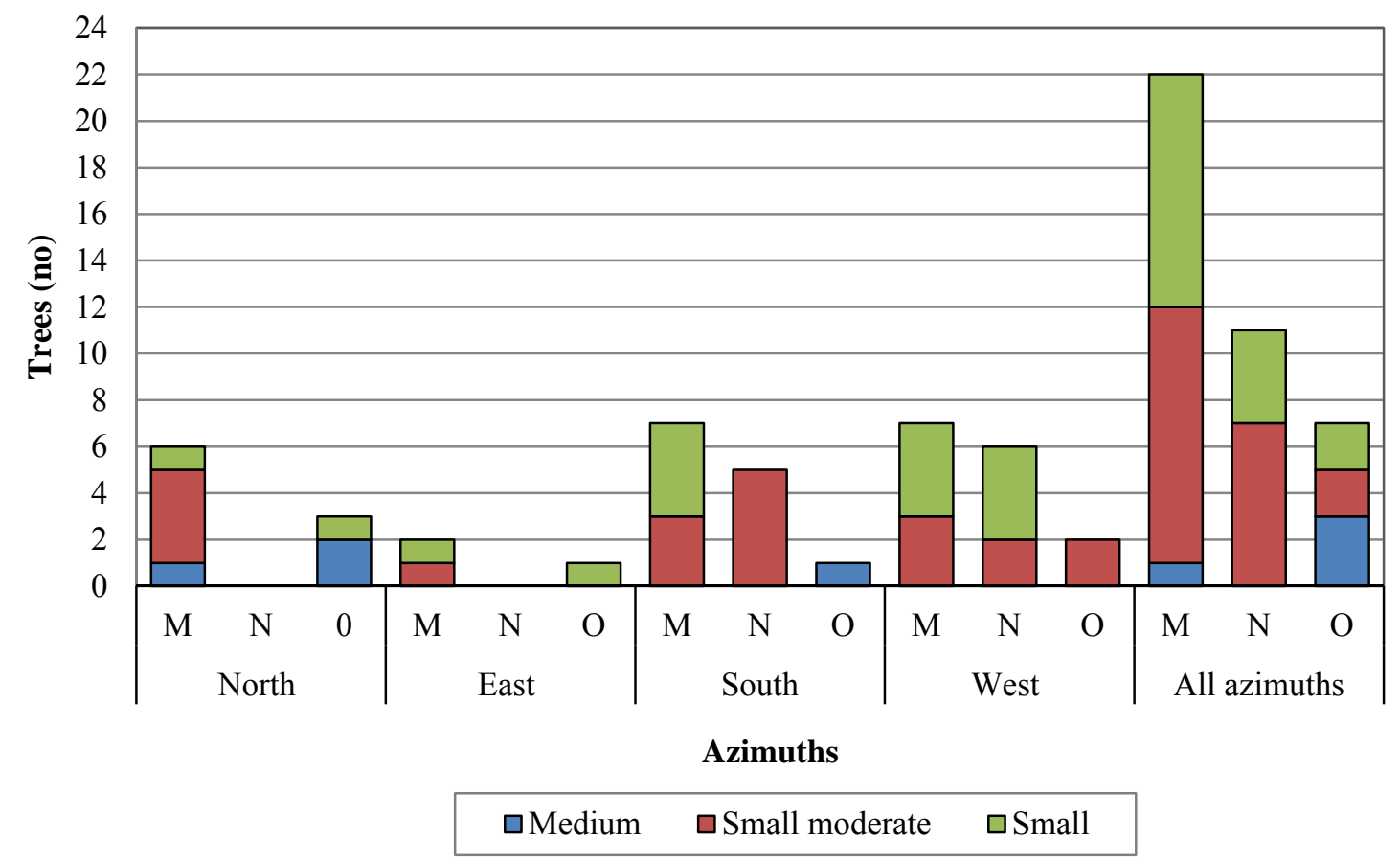

Figure 4.11. Tree distribution compared to tree size and tree azimuth, in relation to building for the three landscape houses: mature landscape house $(\mathrm{M})$, new landscape house $(\mathrm{N})$ and ordinary landscape house $(\mathrm{O})$ 
Table 4.4. Tree-growth indices at the mature landscape house

\begin{tabular}{|c|c|c|c|c|c|c|c|c|c|c|}
\hline \multirow[t]{2}{*}{ No. } & \multirow[t]{2}{*}{ Species } & \multirow[t]{2}{*}{ Type $^{1}$} & \multirow[t]{2}{*}{ Azimuth $^{2}$} & \multirow{2}{*}{$\begin{array}{l}\text { Tree to } \\
\text { building } \\
\text { Distance }^{3}\end{array}$} & \multicolumn{6}{|c|}{ Tree-growth index/typology/biomass structure } \\
\hline & & & & & $\begin{array}{l}\text { Trunk } \\
\text { height }^{4}\end{array}$ & $\begin{array}{l}\text { Overall } \\
\text { height }^{5}\end{array}$ & $\begin{array}{l}\text { Canopy } \\
\text { size }^{6}\end{array}$ & Shape $^{7}$ & $\begin{array}{l}\text { Amount } \\
\text { of leaves }\end{array}$ & $\begin{array}{l}\text { Size of } \\
\text { leaves }\end{array}$ \\
\hline 1 & Tabebuia rosea & A & 7 & 4.00 & 3.00 & 3.00 & 2.00 & 2.00 & 2.00 & 2.00 \\
\hline 2 & Tabebuia rosea & A & 7 & 4.00 & 3.00 & 3.00 & 2.00 & 2.00 & 2.00 & 2.00 \\
\hline 3 & Cinnamomum iners & $\mathrm{A}$ & 5 & 4.00 & 2.00 & 3.00 & 2.00 & 2.00 & 2.00 & 1.00 \\
\hline 4 & Cinnamomum iners & A & 5 & 4.00 & 2.00 & 3.00 & 2.00 & 2.00 & 2.00 & 1.00 \\
\hline 5 & Juniperus chinensis & B & 7 & 3.00 & - & 2.00 & 1.00 & 3.00 & 3.00 & 1.00 \\
\hline 6 & Juniperus chinensis & B & 7 & 3.00 & - & 2.00 & 1.00 & 3.00 & 3.00 & 1.00 \\
\hline 7 & Juniperus chinensis & B & 7 & 3.00 & - & 2.00 & 1.00 & 3.00 & 3.00 & 1.00 \\
\hline 8 & Juniperus chinensis & B & 7 & 3.00 & - & 2.00 & 1.00 & 3.00 & 3.00 & 1.00 \\
\hline 9 & Juniperus chinensis & B & 5 & 2.00 & - & 2.00 & 1.00 & 3.00 & 3.00 & 1.00 \\
\hline 10 & Juniperus chinensis & B & 5 & 3.00 & - & 2.00 & 1.00 & 3.00 & 3.00 & 1.00 \\
\hline 11 & Erythrina glauca & B & 5 & 3.00 & 1.00 & 3.00 & 1.00 & 1.00 & 1.00 & 2.00 \\
\hline 12 & Erythrina glauca & B & 5 & 3.00 & 1.00 & 3.00 & 1.00 & 1.00 & 1.00 & 2.00 \\
\hline 13 & Nephelium lappaceum & $\mathrm{C}$ & 3 & 1.00 & 1.00 & 2.00 & 3.00 & 1.00 & 3.00 & 2.00 \\
\hline 14 & Garcinia manqostana & $\mathrm{C}$ & 3 & 1.00 & 1.00 & 2.00 & 2.00 & 2.00 & 3.00 & 2.00 \\
\hline 15 & Mangifera indica & $\mathrm{C}$ & 1 & 1.00 & 3.00 & 3.00 & 2.00 & 2.00 & 3.00 & 2.00 \\
\hline 16 & Mangifera indica & $\mathrm{C}$ & 1 & 1.00 & 3.00 & 3.00 & 2.00 & 2.00 & 3.00 & 2.00 \\
\hline 17 & Mangifera indica & $\mathrm{C}$ & 1 & 1.00 & 3.00 & 3.00 & 3.00 & 2.00 & 3.00 & 2.00 \\
\hline 18 & Averrhoa bilimbi & $\mathrm{C}$ & 1 & 1.00 & 2.00 & 2.00 & 1.00 & 1.00 & 1.00 & 1.00 \\
\hline 19 & Veitchia merillii & $\mathrm{D}$ & 1 & 1.00 & 1.00 & 2.00 & 2.00 & 5.00 & 2.00 & 1.00 \\
\hline 20 & Veitchia merillii & $\mathrm{D}$ & 1 & 1.00 & 1.00 & 2.00 & 2.00 & 5.00 & 2.00 & 1.00 \\
\hline 21 & Cyrtostachys renda & $\mathrm{D}$ & 5 & 1.00 & 1.00 & 2.00 & 2.00 & 5.00 & 2.00 & 1.00 \\
\hline 22 & Cyrtostachys renda & $\mathrm{D}$ & 7 & 3.00 & 1.00 & 2.00 & 2.00 & 5.00 & 2.00 & 1.00 \\
\hline & Total & & & 51.00 & 29.00 & 53.00 & 37.00 & 58.00 & 52.00 & 31.00 \\
\hline & Average & & & 2.32 & 1.81 & 2.41 & 1.68 & 2.64 & 2.36 & 1.41 \\
\hline
\end{tabular}

Table 4.5. Tree-growth indices at the ordinary landscape house

\begin{tabular}{|c|c|c|c|c|c|c|c|c|c|c|}
\hline \multirow[t]{2}{*}{ No. } & \multirow[t]{2}{*}{ Species } & \multirow[t]{2}{*}{ Type $^{1}$} & \multirow[t]{2}{*}{ Azimuth $^{2}$} & \multirow{2}{*}{$\begin{array}{l}\text { Tree to } \\
\text { building } \\
\text { Distance }\end{array}$} & \multicolumn{6}{|c|}{ Tree-growth index/typology/biomass structure } \\
\hline & & & & & $\begin{array}{l}\text { Trunk } \\
\text { height }^{4}\end{array}$ & $\begin{array}{l}\text { Overall } \\
\text { height }^{5}\end{array}$ & $\begin{array}{l}\text { Canopy } \\
\text { size }^{6}\end{array}$ & Shape $^{7}$ & $\begin{array}{l}\text { Amount } \\
\text { of leaves } 8\end{array}$ & $\begin{array}{l}\text { Size of } \\
\text { leaves } 9\end{array}$ \\
\hline 1 & Plumeria rubra & B & 7 & 1.00 & 1.00 & 2.00 & 2.00 & 1.00 & 3.00 & 3.00 \\
\hline 2 & Plumeria rubra & B & 7 & 2.00 & 1.00 & 2.00 & 2.00 & 1.00 & 1.00 & 3.00 \\
\hline 3 & Plumeria rubra & B & 3 & 1.00 & 1.00 & 2.00 & 1.00 & 1.00 & 1.00 & 3.00 \\
\hline 4 & Persia americana & $\mathrm{C}$ & 1 & 1.00 & 8.00 & 4.00 & 3.00 & 2.00 & 3.00 & 2.00 \\
\hline 5 & Eugenia polyantha & $\mathrm{C}$ & 1 & 1.00 & 8.00 & 4.00 & 3.00 & 1.00 & 2.00 & 2.00 \\
\hline 6 & Manilkara zapota & $\mathrm{C}$ & 1 & 1.00 & 1.00 & 2.00 & 1.00 & 1.00 & 2.00 & 2.00 \\
\hline 7 & Caryota mitis & $\mathrm{D}$ & 5 & 1.00 & 8.00 & 3.00 & 3.00 & 5.00 & 3.00 & 2.00 \\
\hline & Total & & & 8.00 & 28.00 & 19.00 & 15.00 & 12.00 & 15.00 & 17.00 \\
\hline & Average & & & 1.14 & 4.00 & 2.71 & 2.14 & 1.71 & 2.14 & 2.43 \\
\hline
\end{tabular}


Table 4.6. Tree-growth indices at the new landscape house

\begin{tabular}{|c|c|c|c|c|c|c|c|c|c|c|}
\hline \multirow[t]{2}{*}{ No. } & \multirow[t]{2}{*}{ Species } & \multirow[t]{2}{*}{ Type $^{1}$} & \multirow[t]{2}{*}{ Azimuth $^{2}$} & \multirow{2}{*}{$\begin{array}{l}\text { Tree to } \\
\text { building } \\
\text { Distance }^{3}\end{array}$} & \multicolumn{6}{|c|}{ Tree-growth index/typology/biomass structure } \\
\hline & & & & & $\begin{array}{l}\text { Trunk } \\
\text { height }^{4}\end{array}$ & $\begin{array}{l}\text { Overall } \\
\text { height }^{5}\end{array}$ & $\begin{array}{l}\text { Canopy } \\
\text { size }^{6}\end{array}$ & Shape $^{7}$ & $\begin{array}{l}\text { Amount } \\
\text { of leaves }\end{array}$ & $\begin{array}{l}\text { Size of } \\
\text { leaves }\end{array}$ \\
\hline 1 & Tabebuia rosea & A & 5 & 2.00 & 6.00 & $3 . .00$ & 2.00 & 2.00 & 3.00 & 2.00 \\
\hline 2 & Erythrina fusca & $\mathrm{B}$ & 7 & 1.00 & 2.00 & 2.00 & 1.00 & 1.00 & 1.00 & 2.00 \\
\hline 3 & Erythrina fusca & B & 7 & 1.00 & 2.00 & 2.00 & 1.00 & 1.00 & 1.00 & 2.00 \\
\hline 4 & Bucida buceras & B & 7 & 1.00 & 1.00 & 1.00 & 1.00 & 1.00 & 1.00 & 1.00 \\
\hline 5 & Bucida buceras & B & 7 & 1.00 & 1.00 & 1.00 & 1.00 & 1.00 & 1.00 & 1.00 \\
\hline 6 & Plumeria rubra & $\mathrm{B}$ & 7 & 1.00 & 2.00 & 2.00 & 1.00 & 1.00 & 1.00 & 3.00 \\
\hline 7 & Wodyetia bifurcate & $\mathrm{D}$ & 5 & 2.00 & 3.00 & 2.00 & 1.00 & 5.00 & 1.00 & 1.00 \\
\hline 8 & Wodyetia bifurcate & $\mathrm{D}$ & 5 & 1.00 & 3.00 & 2.00 & 1.00 & 5.00 & 1.00 & 1.00 \\
\hline 9 & Wodyetia bifurcate & $\mathrm{D}$ & 5 & 1.00 & 3.00 & 2.00 & 1.00 & 5.00 & 1.00 & 1.00 \\
\hline 10 & Wodyetia bifurcate & $\mathrm{D}$ & 5 & 1.00 & 3.00 & 2.00 & 1.00 & 5.00 & 1.00 & 1.00 \\
\hline 11 & Ravenala madagascariensis & $\mathrm{D}$ & 7 & 1.00 & - & 2.00 & 1.00 & 5.00 & 2.00 & 3.00 \\
\hline & Total & & & 13.00 & 26.00 & 18.00 & 12.00 & 32.00 & 14.00 & 18.00 \\
\hline & Average & & & 1.18 & 2.36 & 1.64 & 1.09 & 2.91 & 1.27 & 1.64 \\
\hline
\end{tabular}

1 Type of trees; $\mathrm{A}=$ Roadside trees, $\mathrm{B}=\mathrm{Garden}$ trees, $\mathrm{C}=$ Edible fruit trees, $\mathrm{D}=\mathrm{Palm}, \mathrm{E}=\mathrm{Bamboo}$ $21=$ North, $3=$ East, $5=$ South, $7=$ West

$31.0=$ below $3 \mathrm{~m} .2 .0=3-5 \mathrm{~m}, 3.0=5-9 \mathrm{~m}, 4.0=10-14 \mathrm{~m}, 5.0=15-19 \mathrm{~m}$

$41.0=1 \mathrm{~m}, 2.0=1.5 \mathrm{~m}, 3.0=2 \mathrm{~m}, 4.0=2.5 \mathrm{~m}, 5.0=3.0 \mathrm{~m}, 6=3.5 \mathrm{~m}, 7=4.0 \mathrm{~m}, 8=>5 \mathrm{~m}$

$52.0=2-4 \mathrm{~m}, 3.0=5-9 \mathrm{~m}, 4.0=10-14,5.0=15-19 \mathrm{~m}$

$61.0=$ small, $2.0=$ small moderate, $3.0=$ medium

$71.0=$ spreading, $2.0=$ round, $3.0=$ columnar, $4.0=$ Pyramidal, $5.0=$ fountain

$81.0=$ rare, $2.0=$ medium, $3.0=$ dense

$91.0=$ small, $2.0=$ medium, $3.0=$ large

\subsubsection{Shrubs, vines, and groundcover}

The shrubs, vines and groundcover planted around the houses were a fundamental element of the landscape design of each. Shrubs and vines planted close to the walls of a house can produce shade and reduce heat gain in the building, especially during the morning and afternoon. The groundcover in the gardens was planted to create a transition between the shrubs and turf surfaces, as well as a balanced, harmonious and lively landscape design. 


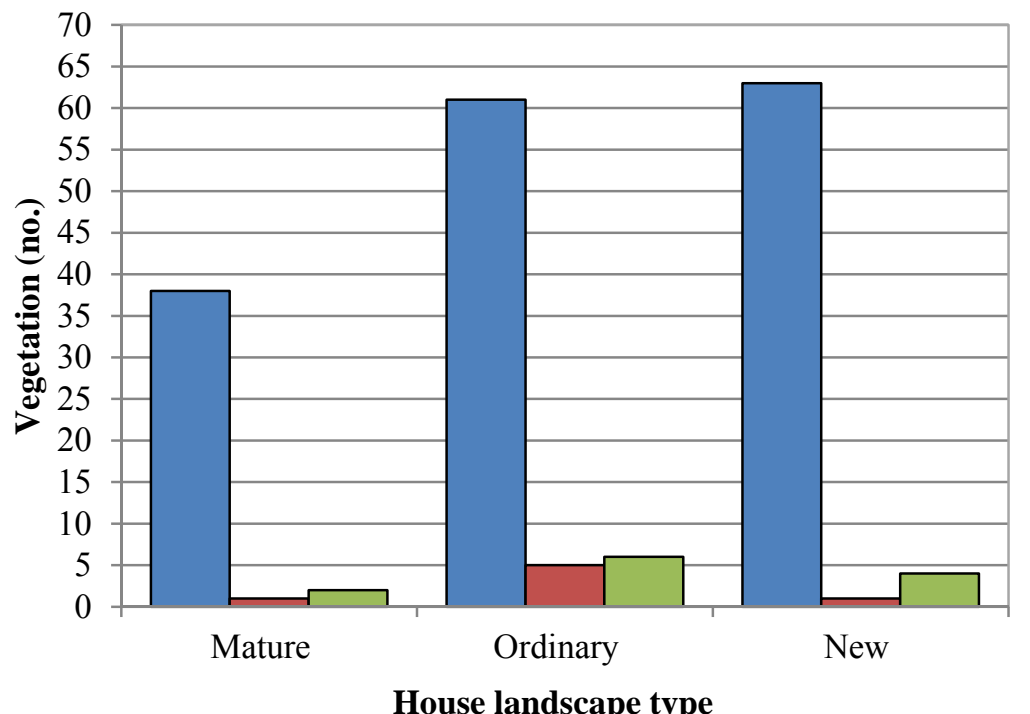

House landscape type

$\square$ Shrubs $\quad \square$ Vines $\quad \square$ Groundcovers

Figure 4.12. Shrubs, vines and groundcover distributions in the three landscape houses

A total of 38 individual and groups of shrubs, 1 group of vines, and 2 groups of groundcover were identified in the garden of the mature landscape house. The majority of shrub types was from the garden shrub category at $68 \%$; followed by $27 \%$ edible shrubs. Only $13 \%$ were palms shrubs. Shrubs were spread on four sides of the house, with $34 \%$ of the shrubs located to the south side, $29 \%$ to the east, $21 \%$ to the west side and $16 \%$ to the north side. $80 \%$ of the shrubs were grown at a distance of within $5 \mathrm{~m}$ from the building; $16 \%$ of the shrubs were positioned at $9 \mathrm{~m}$ and $5 \%$ were close to walls and at a distance of $14 \mathrm{~m}$ respectively.

Shrubs located within $9-14 \mathrm{~m}$ were located underneath shade trees. A planting of vines was located to the south side of the house. Quisqualis indica was climbing on a pergola structure close to the building walls covering $5 \mathrm{~m}^{2}$ with a medium amount and size of leaves. Only 2 groups of groundcover were scattered in the garden of the mature landscape house. Cuphea hyssopifolia and Zephyranthes candida were located to the south and west side respectively within a distance of $5 \mathrm{~m}$ from the building. The two groups of groundcover had been planted in groups of $5-9 \mathrm{~m}^{2}$. All of them have a medium amount of small sized leaves. On average all shrubs, vines 
and groundcover were of moderate size and condition, but the total amount of them was small compared to the total area of the garden.

The total amount of shrubs, vines and groundcover around the ordinary landscape house was almost double that of the mature landscape house. There were 61 individual and groups of shrubs, 4 groups of vines, and 6 groups of groundcover were identified. The majority of shrub types were from the garden shrubs category at $70 \%$, followed by $20 \%$ edible shrubs and finally only $7 \%$ and $3 \%$ were bamboo and palms shrubs respectively. $36 \%$ of the shrubs were located to the north side, $30 \%$ to the west side, $19 \%$ and $15 \%$ to the east and south sides respectively. $67 \%$ of the shrubs were grown within a distance of $5 \mathrm{~m}$ distance from the building. $25 \%$ of the shrubs were positioned close to walls and windows, and finally $8 \%$ of the shrubs was scattered at a distance between 5 and $9 \mathrm{~m}$ from the building. 5 groups of vines were climbing on the pergolas and in individual garden structures at the ordinary landscape house.

Vines situated to the north and east sides were placed within $5 \mathrm{~m}$ distance from the building. 3 groups of vines had been planted in groups below $5 \mathrm{~m}^{2}$, and another 2 groups of vines were in groups of $6-10 \mathrm{~m}^{2}$. All of them had a sparse leaves. The majority $(60 \%)$ had medium sized leaves and the rest had small sized leaves. 6 groups of groundcover were scattered in the garden of the ordinary landscape house. Groundcover was only planted to the west side at different distances from the building. 4 groups of groundcover were located within $5 \mathrm{~m}, 1$ group was close to the walls and 1 group within 5-9m from the building. All had a medium amount of small sized leaves.

In comparison, the shrub population in the new landscape house was similar to that of the ordinary landscape house, but different in maturity and size of plant. 63 individual and groups of shrub had been planted around the house. They were categorised into 4 groups: $57 \%$ were garden shrubs, $21 \%$ edible shrubs, $17 \%$ palm shrubs and $5 \%$ bamboo shrubs. $35 \%$ were located to the south of the garden at a distance of $5 \mathrm{~m}, 24 \%$ were close to walls and 19\% were between a 6 and $9 \mathrm{~m}$ distance 
from the building. A group of vines with a few small sized leaves was climbing on garden structures. Tristellateia australasiae was situated to the west side, placed within a $5 \mathrm{~m}$ distance from the building covering less than $5 \mathrm{~m}^{2} .2$ groups of groundcover species, Hemigraphis alternate, with a medium amount and size of leaves, was located to the south and west side. All groups were within $5 \mathrm{~m}$ from the building, each approximately $4 \mathrm{~m}^{2}$ in area.

Table 4.7. Shrubs at the mature landscape house

\begin{tabular}{|c|c|c|c|c|c|c|c|c|c|}
\hline \multirow[t]{2}{*}{ Azimuth } & \multirow[t]{2}{*}{ No. } & \multirow{2}{*}{$\begin{array}{l}\text { Distance } \\
\text { from } \\
\text { building }^{1}\end{array}$} & \multirow[t]{2}{*}{ No. } & \multirow[t]{2}{*}{ Type $^{2}$} & \multicolumn{5}{|c|}{ Shrub-growth index/typology/biomass structure } \\
\hline & & & & & Height $^{3}$ & Spread $^{4}$ & Shape ${ }^{5}$ & $\begin{array}{l}\text { Amount of } \\
\text { leaves }\end{array}$ & $\begin{array}{l}\text { Size of } \\
\text { leaves }\end{array}$ \\
\hline \multirow[t]{2}{*}{ North } & \multirow[t]{2}{*}{6} & \multirow[t]{2}{*}{2} & 5 & $\mathrm{~F}$ & 2.40 & 1.40 & 2.00 & 1.80 & 1.80 \\
\hline & & & 1 & G & 2.00 & 1.00 & 2.00 & 3.00 & 1.00 \\
\hline \multirow[t]{3}{*}{ East } & \multirow[t]{3}{*}{11} & \multirow[t]{3}{*}{2} & 3 & $\mathrm{~F}$ & 3.67 & 1.00 & 1.67 & 1.67 & 2.00 \\
\hline & & & 1 & G & 2.00 & 1.00 & 1.00 & 1.00 & 1.00 \\
\hline & & & 7 & $\mathrm{H}$ & 3.86 & 1.43 & 1.86 & 1.57 & 1.57 \\
\hline \multirow[t]{3}{*}{ South } & \multirow[t]{3}{*}{13} & \multirow[t]{2}{*}{2} & 5 & $\mathrm{~F}$ & 2.00 & 1.80 & 1.00 & 1.20 & 1.20 \\
\hline & & & 3 & $\mathrm{G}$ & 2.00 & 1.00 & 2.00 & 3.00 & 1.00 \\
\hline & & 3 & 5 & $\mathrm{~F}$ & 2.00 & 2.00 & 2.20 & 2.40 & 1.60 \\
\hline \multirow[t]{3}{*}{ West } & \multirow[t]{3}{*}{8} & 2 & 5 & $\mathrm{~F}$ & 2.00 & 1.80 & 1.20 & 2.00 & 1.60 \\
\hline & & 3 & 1 & $\mathrm{~F}$ & 2.00 & 2.00 & 2.00 & 1.00 & 1.00 \\
\hline & & 4 & 2 & $\mathrm{~F}$ & 2.00 & 2.00 & 3.00 & 2.00 & 1.50 \\
\hline Total & 38 & & 38 & & 96.00 & 59.00 & 69.00 & 71.00 & 57.00 \\
\hline Mean & & & & & 2.53 & 1.55 & 1.82 & 1.87 & 1.50 \\
\hline
\end{tabular}

Table 4.8. Shrubs at the ordinary landscape house

\begin{tabular}{|c|c|c|c|c|c|c|c|c|c|}
\hline \multirow[t]{2}{*}{ Azimuth } & \multirow[t]{2}{*}{ No. } & \multirow{2}{*}{$\begin{array}{l}\text { Distance } \\
\text { from } \\
\text { building } 1\end{array}$} & \multirow[t]{2}{*}{ No. } & \multirow[t]{2}{*}{ Type $^{2}$} & \multicolumn{5}{|c|}{ Shrub-growth index/typology/biomass structure } \\
\hline & & & & & Height $^{3}$ & Spread $^{4}$ & Shape ${ }^{5}$ & $\begin{array}{l}\text { Amount of } \\
\text { leaves }^{6}\end{array}$ & $\begin{array}{l}\text { Size of } \\
\text { leaves }\end{array}$ \\
\hline \multirow[t]{5}{*}{ North } & \multirow[t]{5}{*}{22} & \multirow[t]{2}{*}{1} & 2 & $\mathrm{H}$ & 2.00 & 1.00 & 1.00 & 2.50 & 2.50 \\
\hline & & & 1 & I & 5.00 & 2.00 & 2.00 & 2.00 & 1.00 \\
\hline & & \multirow[t]{3}{*}{2} & 9 & $\mathrm{~F}$ & 2.00 & 1.78 & 2.22 & 1.44 & 1.89 \\
\hline & & & 2 & G & 4.00 & 2.50 & 2.00 & 2.00 & 1.00 \\
\hline & & & 8 & $\mathrm{H}$ & 2.25 & 1.25 & 1.25 & 1.50 & 1.75 \\
\hline \multirow[t]{4}{*}{ East } & \multirow[t]{4}{*}{12} & 1 & 2 & $\mathrm{H}$ & 5.50 & 3.00 & 1.00 & 3.00 & 3.00 \\
\hline & & \multirow[t]{2}{*}{2} & 5 & $\mathrm{~F}$ & 1.80 & 1.20 & 2.00 & 1.00 & 2.00 \\
\hline & & & 1 & $\mathrm{H}$ & 3.00 & 1.00 & 1.00 & 1.00 & 2.00 \\
\hline & & 3 & 4 & $\mathrm{~F}$ & 2.50 & 1.50 & 2.00 & 1.00 & 1.75 \\
\hline \multirow[t]{3}{*}{ South } & \multirow[t]{3}{*}{9} & 1 & 7 & $\mathrm{~F}$ & 3.14 & 2.29 & 2.14 & 1.86 & 2.14 \\
\hline & & 2 & 1 & $\mathrm{~F}$ & 4.00 & 4.00 & 2.00 & 3.00 & 3.00 \\
\hline & & 3 & 1 & I & 5.00 & 3.00 & 2.00 & 3.00 & 1.00 \\
\hline \multirow[t]{2}{*}{ West } & \multirow[t]{2}{*}{18} & \multirow[t]{2}{*}{2} & 16 & $\mathrm{~F}$ & 2.81 & 1.44 & 3.38 & 2.13 & 1.75 \\
\hline & & & 2 & I & 5.00 & 2.50 & 2.00 & 2.50 & 1.00 \\
\hline Total & 61 & & 61 & & 170.00 & 100.00 & 136.00 & 110.00 & 113.00 \\
\hline Mean & & & & & 2.79 & 1.64 & 2.23 & 1.80 & 1.85 \\
\hline
\end{tabular}


Table 4.9. Shrubs at the new landscape house

\begin{tabular}{|c|c|c|c|c|c|c|c|c|c|}
\hline \multirow[t]{2}{*}{ Azimuth } & \multirow[t]{2}{*}{ No. } & \multirow{2}{*}{$\begin{array}{l}\text { Distance } \\
\text { from } \\
\text { building }{ }^{1}\end{array}$} & \multirow[t]{2}{*}{ No. } & \multirow[t]{2}{*}{ Type $^{2}$} & \multicolumn{5}{|c|}{ Shrub-growth index/typology/biomass structure } \\
\hline & & & & & Height $^{3}$ & Spread $^{4}$ & Shape ${ }^{5}$ & $\begin{array}{l}\text { Amount of } \\
\text { leaves }\end{array}$ & $\begin{array}{l}\text { Size of } \\
\text { leaves }\end{array}$ \\
\hline North & 11 & 2 & 11 & $\mathrm{H}$ & 2.18 & 1.18 & 2.45 & 1.45 & 1.91 \\
\hline \multirow[t]{3}{*}{ East } & \multirow[t]{3}{*}{10} & 1 & 2 & $\mathrm{~F}$ & 2.00 & 2.50 & 2.50 & 3.00 & 2.50 \\
\hline & & \multirow[t]{2}{*}{2} & 5 & $\mathrm{~F}$ & 1.20 & 2.00 & 2.20 & 1.20 & 1.40 \\
\hline & & & 3 & I & 1.67 & 1.00 & 1.80 & 2.00 & 1.00 \\
\hline \multirow[t]{5}{*}{ South } & \multirow[t]{5}{*}{22} & \multirow[t]{2}{*}{1} & 1 & $\mathrm{~F}$ & 3.00 & 1.00 & 1.00 & 3.00 & 3.00 \\
\hline & & & 2 & G & 4.00 & 3.00 & 2.50 & 3.00 & 2.50 \\
\hline & & \multirow[t]{2}{*}{2} & 9 & F & 2.22 & 1.44 & 2.00 & 1.89 & 1.89 \\
\hline & & & 2 & $\mathrm{G}$ & 4.00 & 3.00 & 2.50 & 3.00 & 2.50 \\
\hline & & 3 & 8 & $\mathrm{~F}$ & 2.38 & 1.50 & 3.38 & 2.13 & 2.13 \\
\hline \multirow[t]{5}{*}{ West } & \multirow[t]{5}{*}{20} & \multirow[t]{2}{*}{1} & 7 & $\mathrm{~F}$ & 2.00 & 2.00 & 2.57 & 1.86 & 2.29 \\
\hline & & & 3 & $\mathrm{G}$ & 2.67 & 3.00 & 2.33 & 2.33 & 2.33 \\
\hline & & \multirow[t]{2}{*}{2} & 4 & $\mathrm{~F}$ & 2.25 & 2.75 & 1.75 & 1.50 & 1.25 \\
\hline & & & 2 & $\mathrm{H}$ & 3.50 & 1.00 & 1.50 & 2.50 & 1.50 \\
\hline & & 3 & 4 & $\mathrm{G}$ & 4.00 & 2.00 & 2.25 & 2.25 & 2.25 \\
\hline Total & 63 & & 63 & & 151.00 & 113.00 & 149.00 & 123.00 & 123.00 \\
\hline Mean & & & & & 2.40 & 1.79 & 2.33 & 1.95 & 1.95 \\
\hline
\end{tabular}

$11=$ proximity to walls. $2=$ below $5 \mathrm{~m}, 3=5-9 \mathrm{~m}, 4=10-14 \mathrm{~m}$

${ }^{2} \mathrm{~F}=$ garden shrubs, $\mathrm{G}=$ palm shrubs, $\mathrm{H}=$ edible shrubs

${ }^{3}$ The mean of height scores of all shrubs for the given distance from building

${ }^{4}$ The mean of spread scores of all shrubs for the given distance from building

${ }^{5}$ The mean of shape scores of all shrubs for the given distance from building

${ }^{6}$ The mean of amount of leaves scores of all shrubs for the given distance from building

${ }^{7}$ The mean of size of leaves scores of all shrubs for the given distance from building

Table 4.10. List of vines at the mature landscape house

\begin{tabular}{|c|c|c|c|c|c|c|}
\hline \multirow[t]{2}{*}{ No. } & \multirow[t]{2}{*}{ Species } & \multirow[t]{2}{*}{ Azimuth $^{\mathrm{b}}$} & \multirow[t]{2}{*}{ Distance $^{c}$} & \multicolumn{3}{|c|}{ Vines-growth index/typology/biomass structure } \\
\hline & & & & Area/spread ${ }^{\mathrm{i}}$ & $\begin{array}{l}\text { Amount of } \\
\text { leaves }^{\mathrm{j}}\end{array}$ & Size of leaves ${ }^{k}$ \\
\hline \multirow[t]{3}{*}{1} & Quisqualis indica & 5 & 2.00 & 3.00 & 2.00 & 2.00 \\
\hline & Total & & 2.00 & 3.00 & 2.00 & 2.00 \\
\hline & Average & & 2.00 & 3.00 & 2.00 & 2.00 \\
\hline
\end{tabular}

Table 4.11. List of vines at the ordinary landscape house

\begin{tabular}{|c|c|c|c|c|c|c|}
\hline \multirow[t]{2}{*}{ No. } & \multirow[t]{2}{*}{ Species } & \multirow[t]{2}{*}{ Azimuth $^{b}$} & \multirow[t]{2}{*}{ Distance $^{c}$} & \multicolumn{3}{|c|}{ Vines-growth index/typology/biomass structure } \\
\hline & & & & Area/spread ${ }^{\mathrm{i}}$ & $\begin{array}{l}\text { Amount of } \\
\text { leaves }^{j}\end{array}$ & Size of leaves ${ }^{k}$ \\
\hline 1 & Vallaris glabra & 3 & 2.00 & 1.00 & 1.00 & 2.00 \\
\hline 2 & Quisqualis indica & 3 & 2.00 & 1.00 & 1.00 & 2.00 \\
\hline 3 & Scindapsus aureus & 1 & 2.00 & 1.00 & 1.00 & 2.00 \\
\hline 4 & Piper betle & 1 & 2.00 & 2.00 & 1.00 & 1.00 \\
\hline \multirow[t]{3}{*}{5} & Piper betle & 1 & 2.00 & 2.00 & 1.00 & 1.00 \\
\hline & Total & & 10.00 & 7.00 & 5.00 & 8.00 \\
\hline & Average & & 2.00 & 1.40 & 1.00 & 1.60 \\
\hline
\end{tabular}


Table 4.12. List of vines at the new landscape house

\begin{tabular}{|l|l|c|c|c|c|c|}
\hline No. & Species & Azimuth $^{\mathrm{b}}$ & Distance $^{\mathrm{c}}$ & & \multicolumn{3}{|c|}{ Vines-growth index/typology/biomass structure } & & $\begin{array}{l}\text { Area/ } \\
\text { spread }^{\mathrm{i}}\end{array}$ & $\begin{array}{l}\text { Amount of } \\
\text { leaves }^{\mathrm{j}}\end{array}$ & Size of leaves $^{\mathrm{k}}$ \\
\hline 1 & Tristellateia australasiae & 7 & 2.00 & 2.00 & 1.00 & 1.00 \\
\hline & Total & & 2.00 & 2.00 & 1.00 & 1.00 \\
\hline & Average & & 2.00 & 2.00 & 1.00 & 1.00 \\
\hline
\end{tabular}

Table 4.13. List of groundcovers at the mature landscape house

\begin{tabular}{|c|c|c|c|c|c|c|}
\hline \multirow[t]{2}{*}{ No. } & \multirow[t]{2}{*}{ Species } & \multirow[t]{2}{*}{ Azimuth $^{b}$} & \multirow[t]{2}{*}{ Distance $^{c}$} & \multicolumn{3}{|c|}{$\begin{array}{l}\text { Groundcovers-growth } \\
\text { index/typology/biomass structure }\end{array}$} \\
\hline & & & & Area $^{\mathrm{i}}$ & $\begin{array}{l}\text { Amount of } \\
\text { leaves }^{\mathrm{j}}\end{array}$ & Size of leaves \\
\hline 1 & Cuphea hyssopifolia & 7 & 2 & 3.00 & 2.00 & 1.00 \\
\hline \multirow[t]{3}{*}{2} & Zephyranthes candida & 7 & 2 & 3.00 & 2.00 & 1.00 \\
\hline & Total & & 4.00 & 6.00 & 4.00 & 2.00 \\
\hline & Average & & 2.00 & 3.00 & 2.00 & 1.00 \\
\hline
\end{tabular}

Table 4.14. List of groundcovers at the ordinary landscape house

\begin{tabular}{|c|c|c|c|c|c|c|}
\hline \multirow[t]{2}{*}{ No. } & \multirow[t]{2}{*}{ Species } & \multirow[t]{2}{*}{ Azimuth $^{\mathrm{b}}$} & \multirow[t]{2}{*}{ Distance $^{c}$} & \multicolumn{3}{|c|}{$\begin{array}{l}\text { Groundcovers-growth } \\
\text { index/typology/biomass structure }\end{array}$} \\
\hline & & & & Area $^{i}$ & $\begin{array}{l}\text { Amount of } \\
\text { leaves }^{j}\end{array}$ & Size of leaves ${ }^{k}$ \\
\hline 1 & Rheo discolor & 7 & 2 & 3.00 & 2.00 & 1.00 \\
\hline 2 & Rheo discolor & 7 & 2 & 3.00 & 2.00 & 1.00 \\
\hline 3 & Cuphea hyssopifolia & 7 & 2 & 4.00 & 2.00 & 1.00 \\
\hline 4 & Zephyranthes candida & 7 & 1 & 3.00 & 2.00 & 1.00 \\
\hline 5 & Pandanus pygmaeus & 7 & 3 & 4.00 & 2.00 & 1.00 \\
\hline 6 & Piper sarmentosum roxb & 7 & 2 & 4.00 & 2.00 & 1.00 \\
\hline & Total & & 38.00 & 21.00 & 12.00 & 6.00 \\
\hline & Average & & 6.33 & 3.50 & 2.00 & 1.00 \\
\hline
\end{tabular}

Table 4.15. List of groundcovers at the new landscape house

\begin{tabular}{|c|c|c|c|c|c|c|}
\hline \multirow[t]{2}{*}{ No. } & \multirow[t]{2}{*}{ Species } & \multirow[t]{2}{*}{ Azimuth $^{b}$} & \multirow[t]{2}{*}{ Distance $^{c}$} & \multicolumn{3}{|c|}{$\begin{array}{l}\text { Groundcovers-growth } \\
\text { index/typology/biomass structure }\end{array}$} \\
\hline & & & & Area $^{i}$ & $\begin{array}{l}\text { Amount of } \\
\text { leaves }^{j}\end{array}$ & Size of leaves ${ }^{k}$ \\
\hline \multirow[t]{3}{*}{1} & Hemigraphis alternate & 7 & 2 & 2.00 & 2.00 & 2.00 \\
\hline & Total & & 2 & 2.00 & 2.00 & 2.00 \\
\hline & Average & & 2 & 2.00 & 2.00 & 2.00 \\
\hline
\end{tabular}

\footnotetext{
${ }^{\mathrm{b}} 1$, North, 3, East, 5, South, 7, West.

c $1=$ proximity to walls, $2=$ below $5 \mathrm{~m}, 3=5-9 \mathrm{~m}$.

i $1=$ below $1 \mathrm{~m}^{2}, 2=1-4 \mathrm{~m}^{2}, 3=5-9 \mathrm{~m}^{2}, 4=10-14 \mathrm{~m}^{2}, 5=15-19 \mathrm{~m}^{2}$

${ }^{\mathrm{j}} 1=$ rare, $2=$ medium, $3=$ dense

${ }^{\mathrm{k}} 1=$ small, $2=$ medium, $3=$ large
} 


\subsubsection{Other landscape elements}

Every house had different, built, hard landscape structures to suit the soft landscape and the concept of landscape design. The mature landscape house had a pergola in the south side garden which was moderately covered by Bauhinia kockiana. The pergola was constructed of hardwood, and covered an area of $10 \mathrm{~m}^{2}$, and was $2.5 \mathrm{~m}$ high. It faced an open lawn paved partly with concrete blocks with a grass inlay that covered an area of approximately $50 \mathrm{~m}^{2}$. There was also a fish pool for Cyprinus carpio (Japanese Carp) situated on the west side of the garden, which had an approximate area of $5 \mathrm{~m}^{2}$, and depth of $0.6 \mathrm{~m}$. It was constructed out of concrete and was decorated with artificial stones, and surrounded by palms and garden shrubs.

The ordinary landscape house has six hard landscape elements around the house including two pergolas, a fish pool, two gazebos and a swimming pool. A pergola with fish pool for Cyprinus carpio (Japanese Carp) with a small waterfall underneath was located to the north-west and was partly covered by Scindapsus aureus and surrounded by Phyllostachys sulphurea. The pool had been built in concrete with an approximate area of $10 \mathrm{~m}^{2}$, and $0.6 \mathrm{~m}$ depth. Another pergola, partly covered by Vallaris glabra and Quisqualis indica, was located to the east side facing the swimming pool. Both of the pergolas were of similar size and constructed in hardwood with an area of $12 \mathrm{~m}^{2}$ and $2.5 \mathrm{~m}$ height. A $20 \mathrm{~m}^{2}$ area and $2.5 \mathrm{~m}$ high gazebo was situated at the west elevation, built in hardwood with a double layer pitched roof covered with slate tiles. The second gazebo with an approximate area of $16 \mathrm{~m}^{2}$ was located to the east side, also built of hardwood with a hip roof covered by sago leaf thatch. A small sized $\left(54 \mathrm{~m}^{2}\right.$ and $1.5 \mathrm{~m}$ deep) swimming pool was located near the gazebo, built in concrete and finished with light blue mosaic tiles.

Similarly, the new landscape house had a fish pool and artificial fountain decoration that were located to the south of the house, with an approximate area of $10 \mathrm{~m}^{2}$, and depth of $0.6 \mathrm{~m}$. 


\subsubsection{Energy use}

All three houses use window air-conditioning systems of between 1.5-3 horse power, which were located in their common and private rooms. Figure 4.13 shows that the mature landscape house had four air-conditioning units, the ordinary landscape house had eight, and the new landscape house had three. Window air conditioning units have components such as a compressor, condenser, expansion valve and evaporator, all housed in a single box. The air-conditioning units had different capacities (horse power), as shown in Figure 4.13.

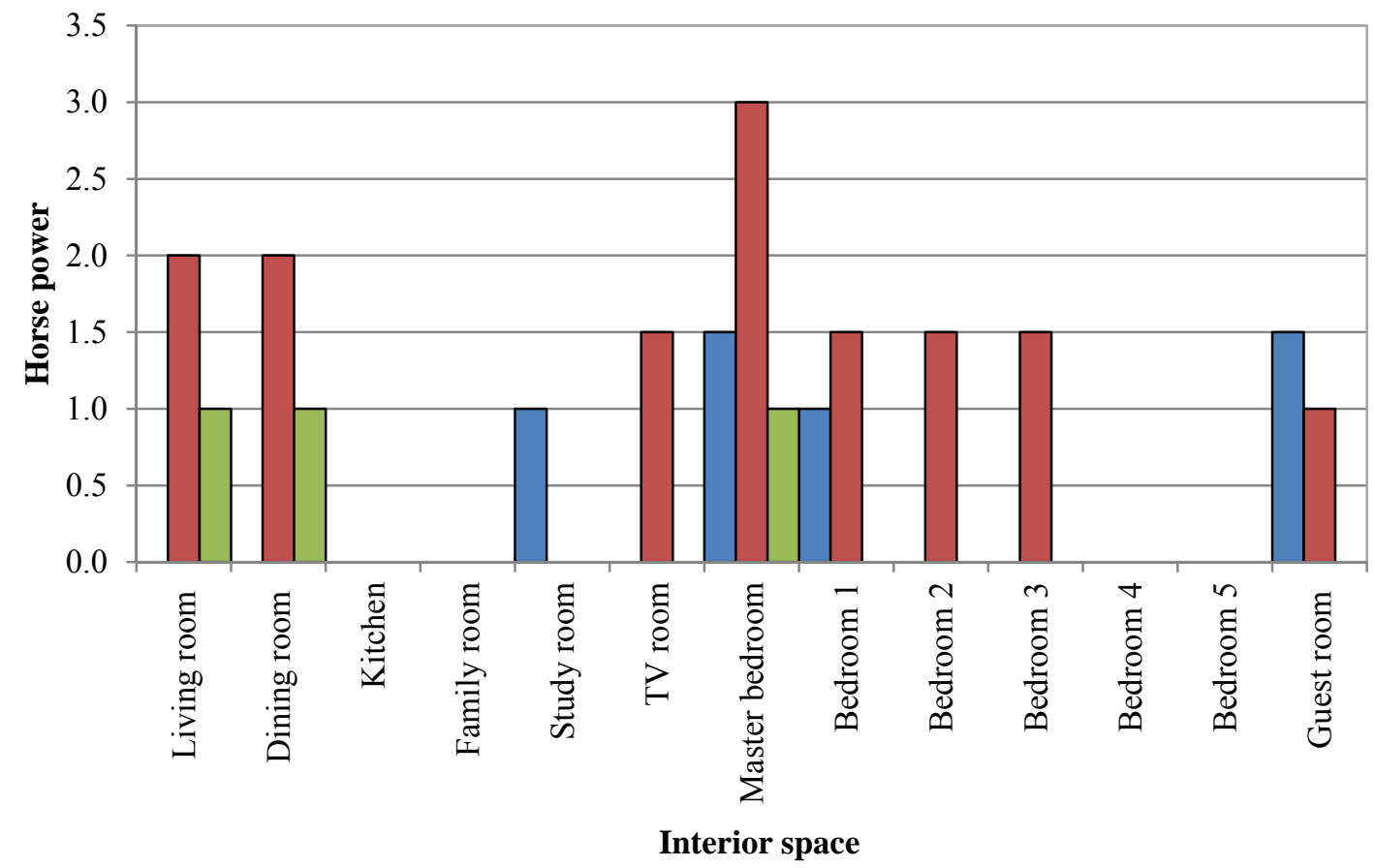

$\square$ Mature landscape house $\quad \square$ Ordinary landscape house $\quad \square$ New landscape house

Figure 4.13. Window air-conditioning systems for the three houses by horsepower

Figure 4.14 and Table 4.16 show the cooling energy for the three houses was around $15-46 \%$ of the total electricity used, although this was dependent on the number of occupants of each house and their living habits. All the houses used natural ventilation and ceiling fans for cooling during the day, with air-conditioning units only used during the night through until early morning (22.00-10.00 hours). 
Figure 4.14 shows the energy used for air-conditioning in $\mathrm{kWh}$ per month for all three houses. The ordinary landscape house used energy for cooling of $1221 \mathrm{kWh}$ per month. This house had five adults and a teenage occupant, as shown in Table 4.16. An example of the calculation for cooling energy use for the ordinary landscape house is listed in Appendix 4.6. The temperature settings of the system were between 21 and $24^{\circ} \mathrm{C}$. The air-conditioner use was mainly in the four bedrooms at night for up to six hours per day (24.00-6.00 hours), with a small amount of use in the audio room for around two hours per day during the night. A small amount of air-conditioning was also used in the living and dining rooms. The total energy used for air-conditioning was approximately $46 \%$ of the total of energy use per month for the ordinary landscape house.

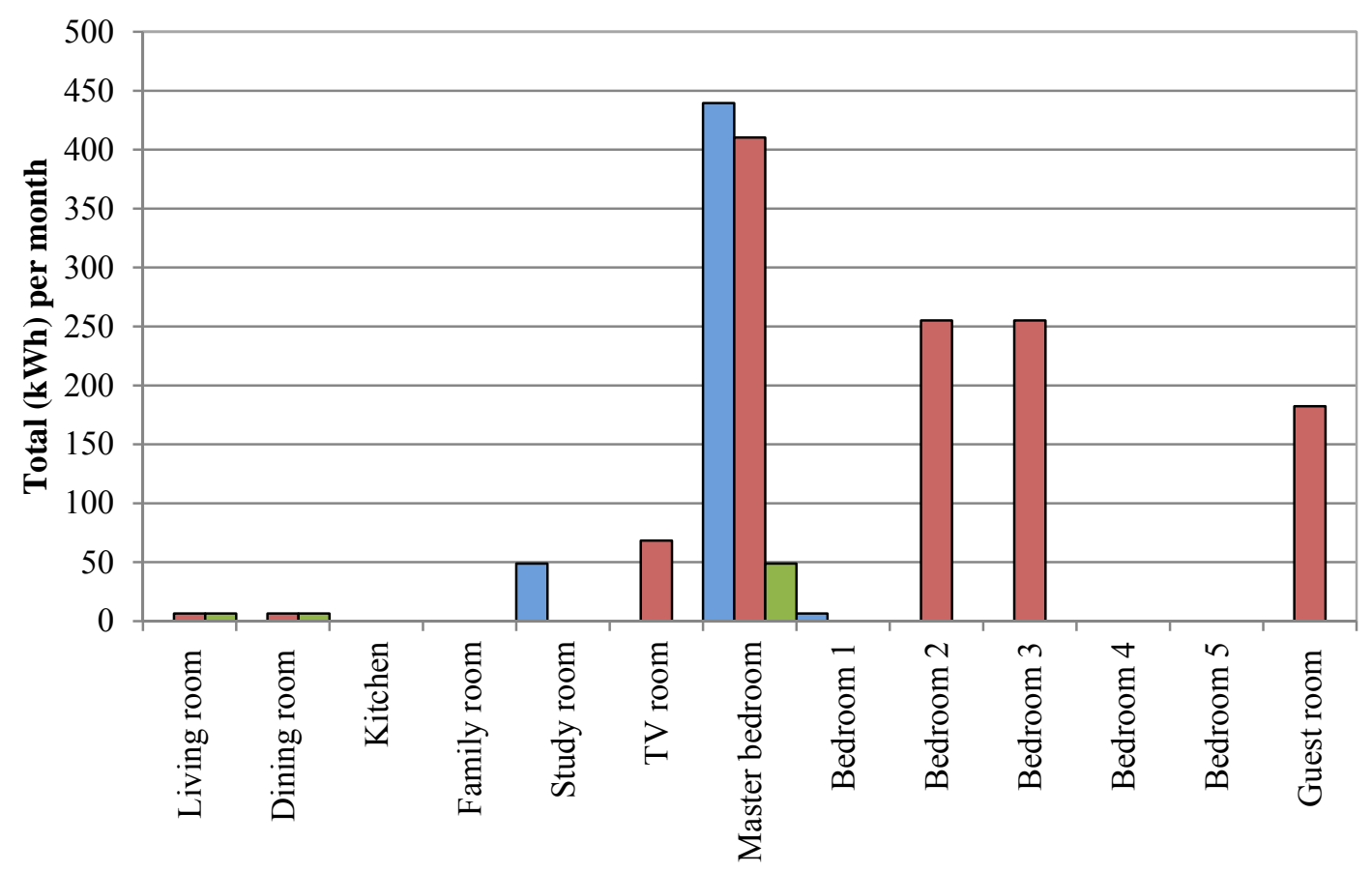

Interior space

$\square$ Mature landscape house $\quad \square$ Ordinary landscape house $\square$ New landscape house

Figure 4.14. Air-conditioning energy use for the three houses

The mature landscape house had six adult occupants, and used $495 \mathrm{kWh}$ per month. Air-conditioning was mainly used in the master bedroom for approximately 12 hours per day at night and in the morning (22.00-10.00 hours). It was also used in the study 
room for around two hours per day during the night time operating at a temperature of around $24^{\circ} \mathrm{C}$. A small amount of air-conditioning was also used in bedroom 1 . The total energy used for air-conditioning was around $35 \%$ of the total energy used per month.

The new landscape house used approximately $86 \mathrm{kWh}$ for air conditioning, which is equal to $15 \%$ of its total energy use per month. This house was occupied by three adults and three children. Air-conditioning was mainly used in the master bedroom, at a temperature of $18^{\circ} \mathrm{C}$ for three hours per day from 22.00 to 1.00 hours. The living and dining rooms also had minimal air conditioning use, as illustrated in Figure 4.14. Natural cross ventilation by opening windows was also used during the night.

Table 4.16. Air-conditioning use in the three houses

\begin{tabular}{|l|c|c|l|l|l|l|l|l|l|}
\hline \multirow{2}{*}{$\begin{array}{l}\text { House } \\
\text { type }\end{array}$} & \multicolumn{2}{|l|}{ Occupant } & $\begin{array}{l}\text { Cooling } \\
\text { energy }\end{array}$ & $\begin{array}{l}\text { House } \\
\text { energy }\end{array}$ & $\begin{array}{l}\text { HP } \\
\text { per } \\
\text { room }\end{array}$ & $\begin{array}{l}\text { Temp. } \\
\text { setting } \\
\left({ }^{\circ} \mathrm{C}\right)\end{array}$ & $\begin{array}{l}\text { Bed } \\
\text { room } \\
(\mathrm{hr})\end{array}$ & $\begin{array}{l}\text { Common } \\
\text { room (hr) }\end{array}$ & $\begin{array}{l}\text { Total } \\
\text { per day } \\
(\mathrm{hr})\end{array}$ \\
\hline Mature & 6 & 0 & 495 & 35 & 1.0 & 24 & 12 & 2 & 14 \\
\hline Ordinary & 5 & 1 & 1221 & 46 & 1.5 & 24 & 30 & 2 & 32 \\
\hline New & 3 & 3 & 86 & 15 & 1.0 & 18 & 3 & - & 3 \\
\hline
\end{tabular}

The different living habits of the occupants of the three houses directly influence the amount of energy consumption used for cooling. At this stage no clear effect from the vegetation around the houses on cooling energy use was apparent.

\subsubsection{Weather data}

To quantify and characterise the thermal effects of the vegetation in the gardens and adjacent to the walls of the houses, a few experimental measurements were made on various combinations of trees, shrubs, vines, groundcover and turf. The measurement points are indicated in Figures 4.15-4.17. At one point for the south elevation of the new landscape house the measuring points could not be close together for privacy reasons (Figure 4.17). The procedure involved continual recording of air temperature, wind speed, and humidity at all azimuths outside and inside the 
buildings. Solar radiation and cloud cover were obtained from the weather station nearby.
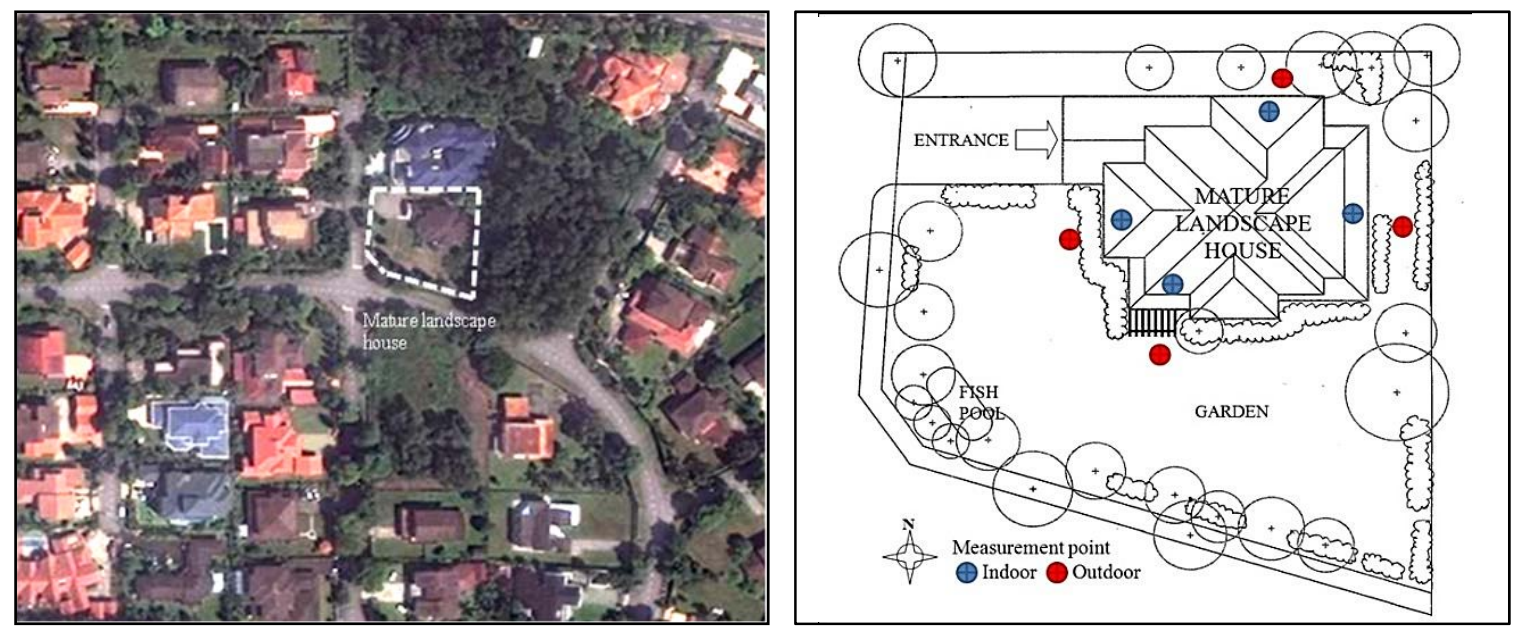

Figure 4.15. Location plan (left) and landscape plan (right) of the mature landscape house
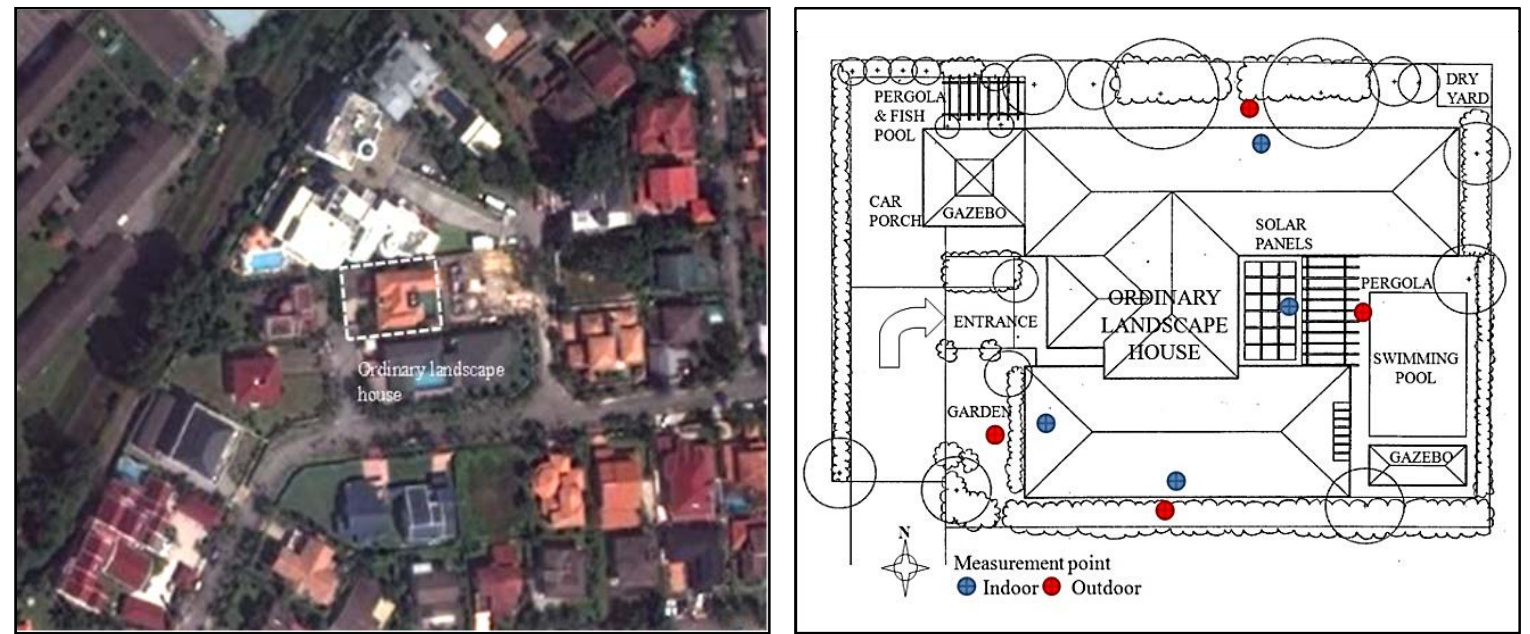

Figure 4.16. Location plan (left) and landscape plan (right) of the ordinary landscape house 

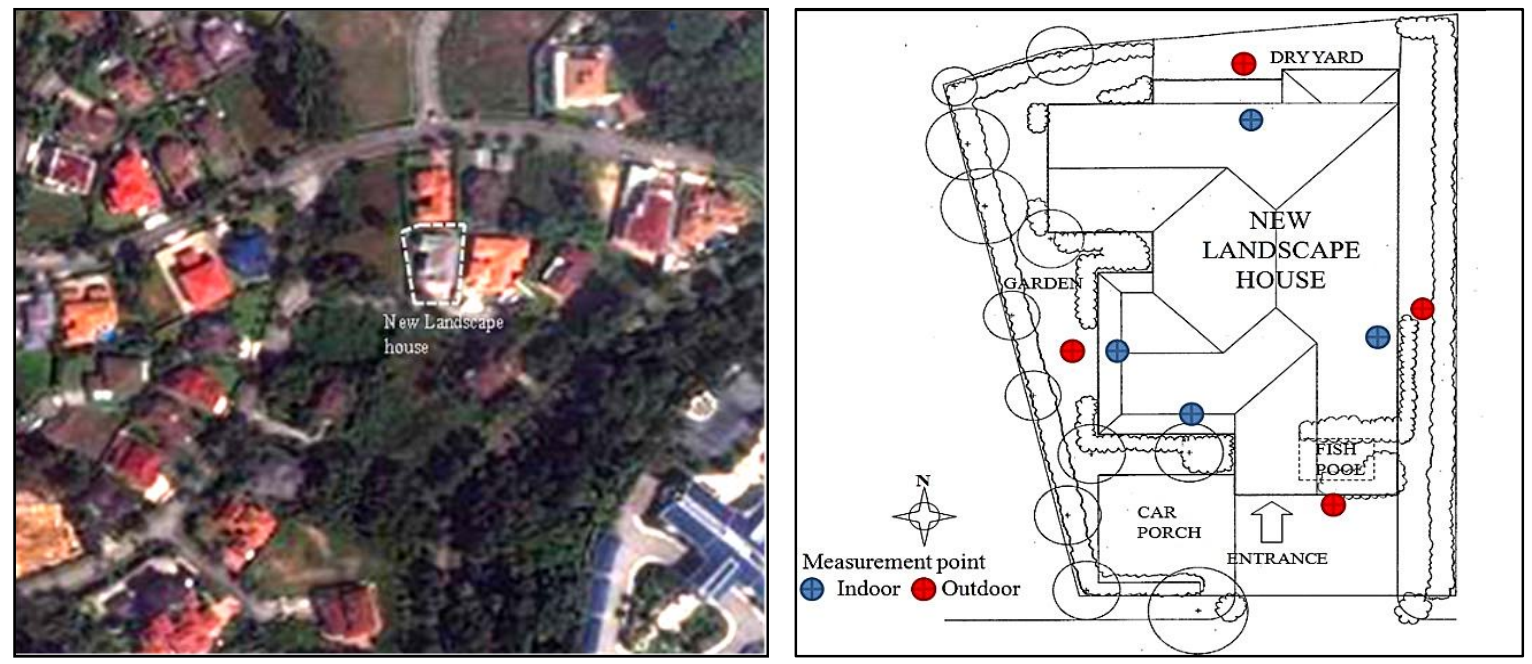

Figure 4.17. Location plan (left) and landscape plan (right) of the new landscape house

\subsubsection{Solar radiation and cloud cover}

The data on solar radiation and cloud cover was obtained from Subang Weather Station. In the case study areas, day length is the interval between sunrise and sunset from around 8.00 to 20.00 hours (12 hours). The solar radiation readings start at 8.00 hours, when the reading was as low as $0.04 \mathrm{MJ}_{\mathrm{m}} \mathrm{m}^{-2}$. It rises within two hours to $1.59 \mathrm{MJ} . \mathrm{m}^{-2}$, and continued to rise to its highest level of $3.69 \mathrm{MJ} . \mathrm{m}^{-2}$ at 13.00 hours. After 13.00 hours the levels of solar radiation uniformly decreased until they reached the lowest radiation of $0.04 \mathrm{MJ} \cdot \mathrm{m}^{-2}$ at 20.00 hours. Overall, solar radiation in the study area was most active between 10.00-18.00 hours, which is a total of approximately eight hours per day, with radiation ranges of $1.34-3.69 \mathrm{MJ} . \mathrm{m}^{-2}$. During the peak time of the day the solar radiation ranged from 1.75 to $3.69 \mathrm{MJ} . \mathrm{m}^{-2}$.

Cloud cover is relatively extensive in the study area, as is typical of a tropical region. The cloud fraction throughout the day for the study areas was 7 Okta. 


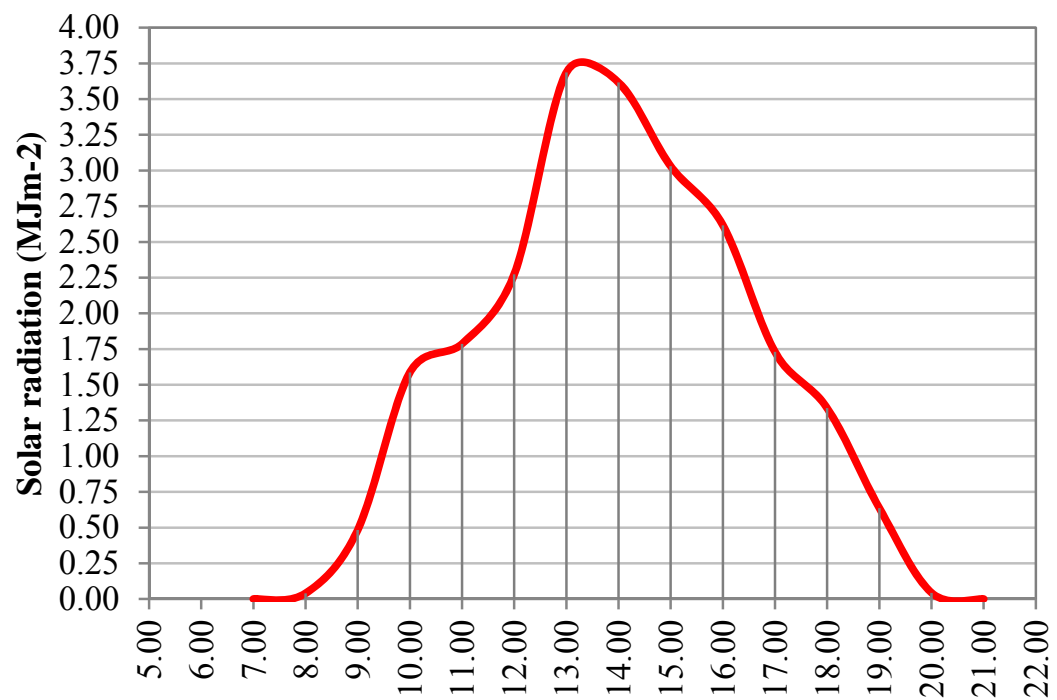

Time of the day (LST)

Solar radiation

Figure 4.18. The intensity of surface solar radiation from Subang

\subsubsection{Air temperature pattern}

To provide an analytical comparison of air temperatures between the three houses, the average external and internal temperatures for each house was recorded at various points and regular intervals during the day. According to Parker (1981), wall temperatures are usually obtained because they reflect the impact of direct and indirect solar radiation, as well as the ambient temperature. Therefore, the difference between the exterior and interior wall temperatures determines the rate of heat transfer through the walls and windows. The external temperature readings were taken a metre above the ground on four azimuths located next to the walls. These recordings were done between 7.30 and 19.30 hours, as can be seen in Figures 4.194.22. The maximum cooling effect of as much as $3.2^{\circ} \mathrm{C}$ at noon $(12.00$ hours $)$ was found in the exterior of the mature landscape house. This house had an air temperature of $30^{\circ} \mathrm{C}$, compared with $33.2^{\circ} \mathrm{C}$ at the new landscape house, as shown in Figure 4.21. Overall, the mature landscape house had the lowest outdoor temperature during the morning until noon. The three houses reached similar readings of air temperature at 14.00 hours, with the highest reading being between 36 and $37^{\circ} \mathrm{C}$. The air temperature readings declined during the afternoon and into the evening, when the readings were about the same for each house. However, the ordinary landscape 
house appeared to have slightly lower readings when compared to the two other houses, with a difference of up to $1.5^{\circ} \mathrm{C}$.

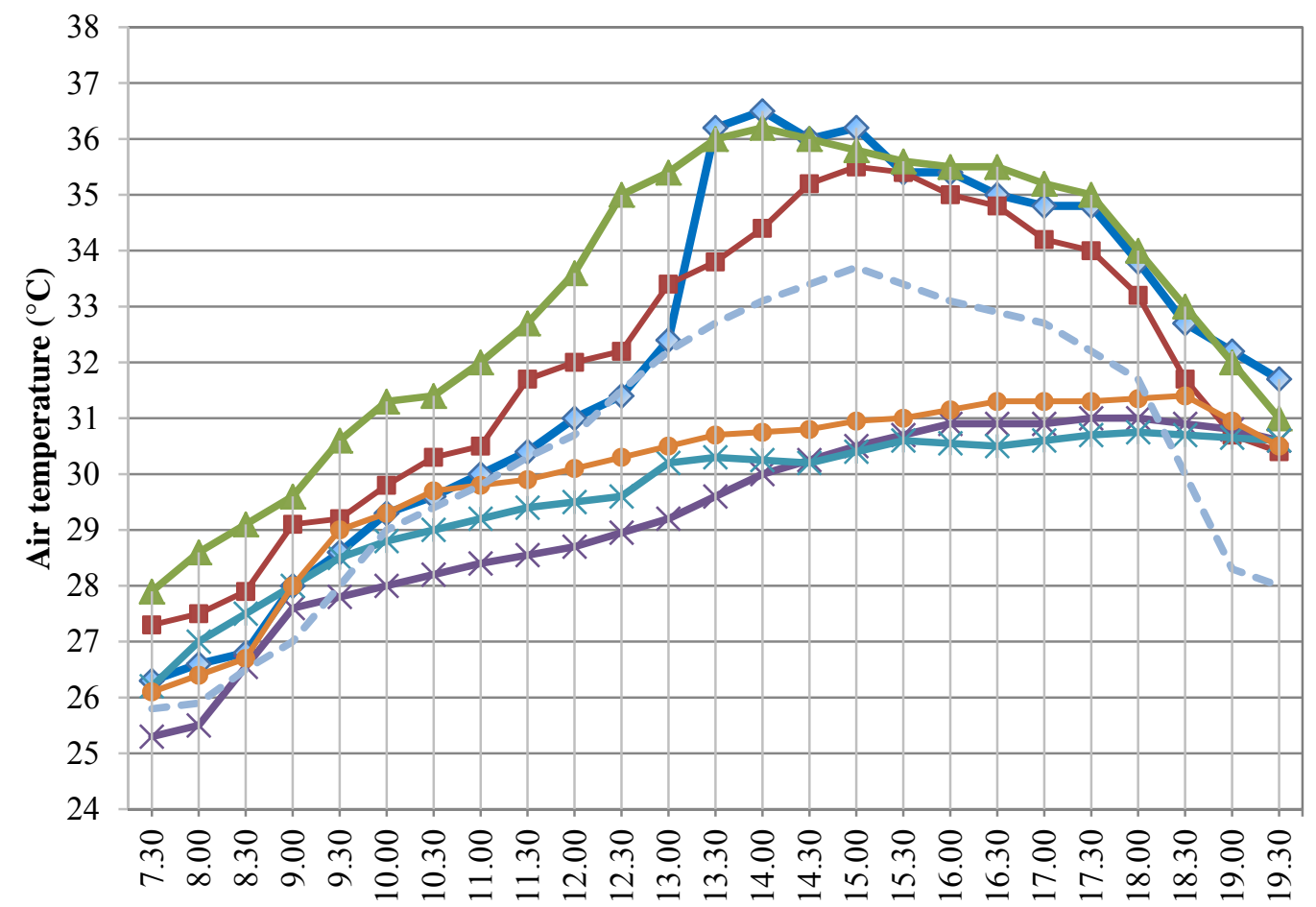

Time of the day (LST)

- Outdoor mature landscape house
- Outdoor new landscape house
- Indoor ordinary landscape house

Figure 4.19. Outdoor and indoor air temperatures adjacent to the north walls of the three houses 


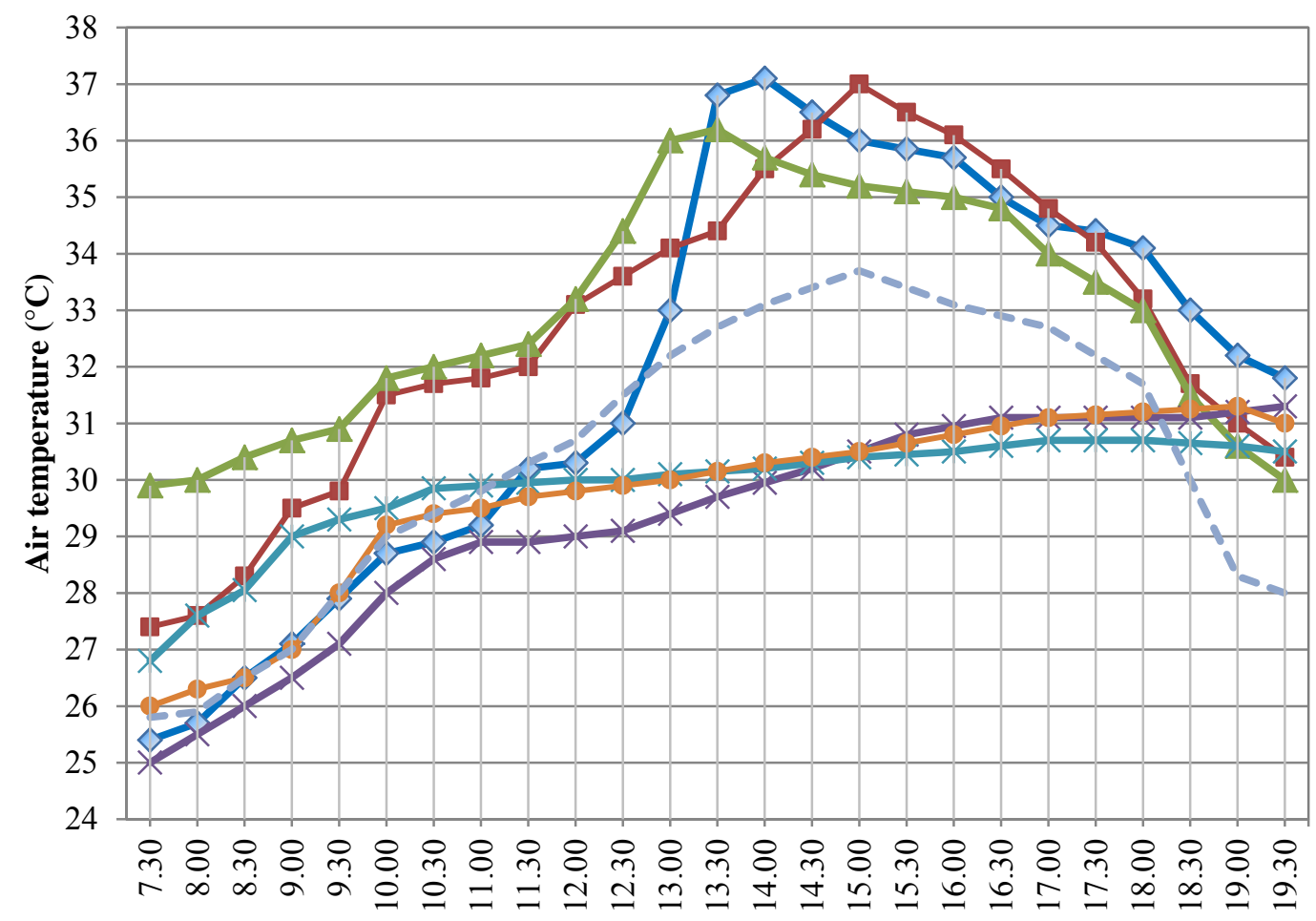

Time of the day (LST)

\begin{tabular}{|c|c|}
\hline $\begin{array}{l}- \text { Outdoor mature landscape house } \\
- \text { Outdoor new landscape house } \\
\approx-\text { Indoor ordinary landscape house } \\
-\infty \text { Airport }\end{array}$ & $\begin{array}{l}\longrightarrow \text { Outdoor ordinary landscape house } \\
\simeq \text { Indoor mature landscape house } \\
\longrightarrow \text { Indoor new landscape house }\end{array}$ \\
\hline
\end{tabular}

Figure 4.20. Outdoor and indoor air temperatures adjacent to the east walls of the three houses 


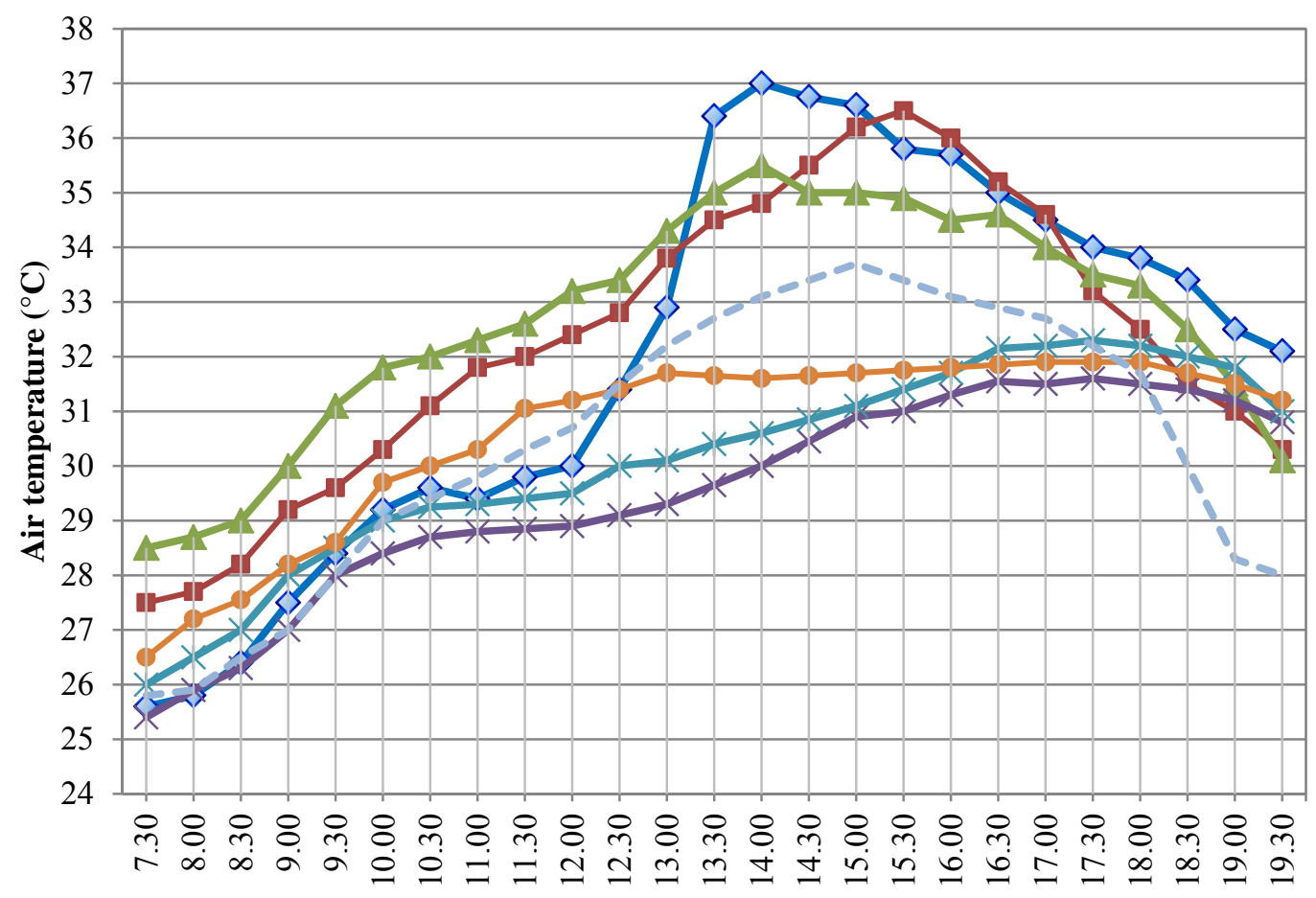

Time of the day (LST)

\begin{tabular}{|c|c|}
\hline $\begin{array}{l}\approx \text { Outdoor mature landscape house } \\
\approx \text { Outdoor new landscape house } \\
* \text { Indoor ordinary landscape house }\end{array}$ & $\begin{array}{l}\text { — Outdoor ordinary landscape house } \\
\leftarrow \text { Indoor mature landscape house } \\
\ldots \text { Indoor new landscape house }\end{array}$ \\
\hline & \\
\hline
\end{tabular}

Figure 4.21. Outdoor and indoor air temperatures adjacent to the south walls of the three houses 


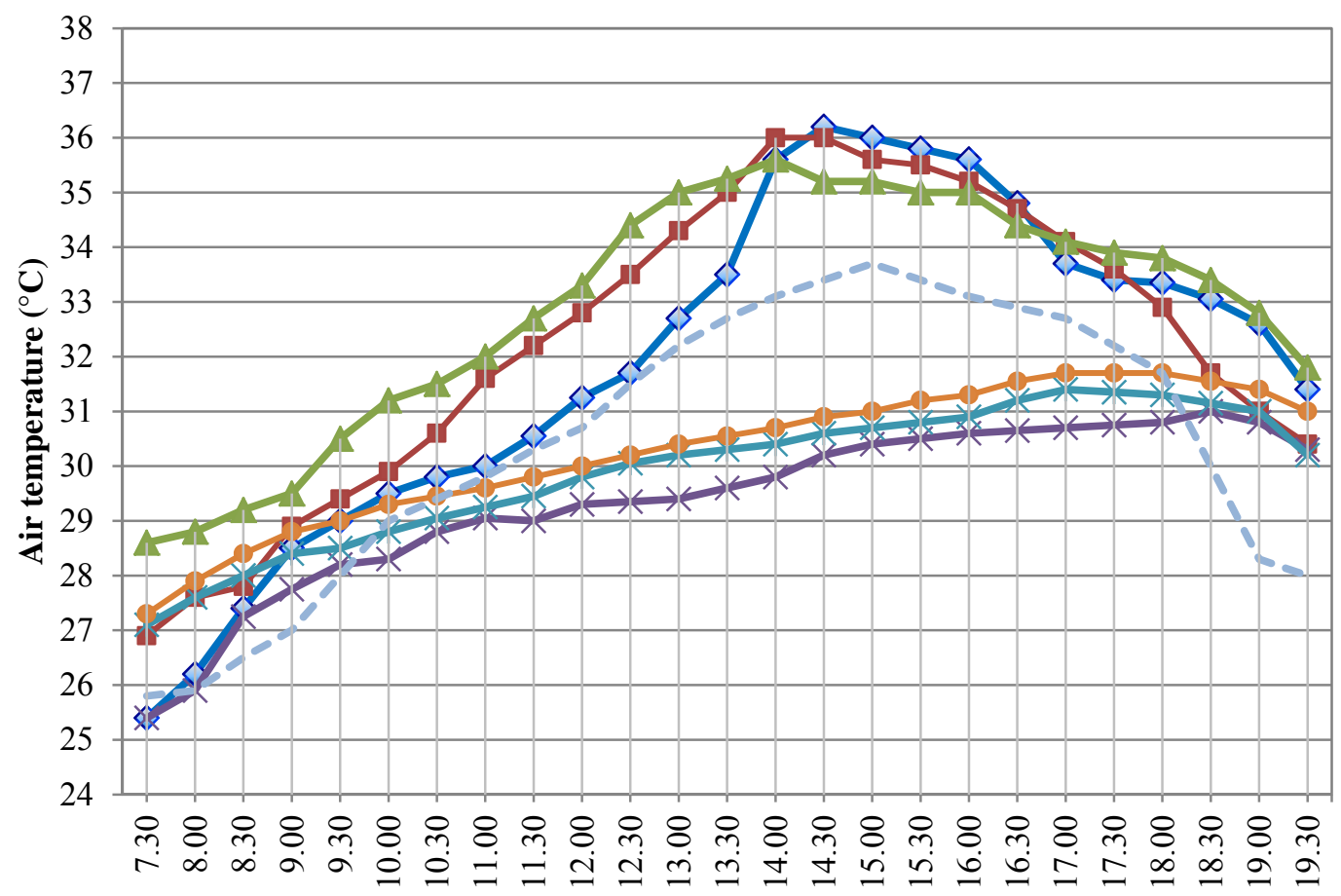

Time of the day (LST)

$\begin{array}{ll}-\checkmark \text { Outdoor mature landscape house } & - \text { Outdoor ordinary landscape house } \\ - \text { Outdoor new landscape house } & \text { Indoor mature landscape house } \\ * \text { Indoor ordinary landscape house } & - \text { Indoor new landscape house } \\ -\infty \text { Airport }\end{array}$

Figure 4.22. Outdoor and indoor air temperatures adjacent to the west walls of the three houses

Air temperature patterns in the interiors of the three houses were similar for all four azimuths. From the early morning air temperatures gradually increased at an average of $0.5^{\circ} \mathrm{C}$ per hour, until they reached a maximum in the evening (18.00 hours). This represents a delay of around 3.5 hours to reach maximum temperature when compared to outdoor temperature. The gap in air temperature between the three houses was up to $0.5^{\circ} \mathrm{C}$ during the peak time of the day. The mature landscape house had the lowest temperature, followed by the ordinary landscape house. The mature landscape house had the lowest temperature for the exterior and interior of the house, with minor differences of temperature (up to $1^{\circ} \mathrm{C}$ ) when compared to the other two houses. In comparison, the temperature at the airport was generally lower by approximately $1.5-2.0^{\circ} \mathrm{C}$, during the peak time of the day until the evening (13.30- 
19.30 hours). However, in the morning until noon (8.00-13.00 hours) the airport temperature was much closer to that of the mature landscape house.

Table 4.17. Average indoor and outdoor air temperatures for all three houses

\begin{tabular}{llccccc}
\hline Case Study & \multicolumn{6}{c}{ Time $@$ Air temperature $\left({ }^{\circ} \mathrm{C}\right)$} \\
\cline { 3 - 6 } Location & House & $08: 00$ & $12: 00$ & $14: 00$ & $18: 00$ & Mean \\
\hline Outdoor & Mature landscape house & 26.08 & 30.64 & 36.55 & 33.76 & 31.76 \\
& Ordinary landscape house & 27.60 & 32.58 & 35.18 & 32.95 & 32.08 \\
& New landscape house & 29.03 & 33.60 & 35.75 & 33.53 & 32.98 \\
\hline \multirow{2}{*}{ Indoor } & Mature landscape house & 25.70 & 28.98 & 29.94 & 31.10 & 28.93 \\
& Ordinary landscape house & 27.18 & 29.70 & 30.36 & 31.23 & 29.62 \\
& New landscape house & 26.95 & 30.28 & 30.84 & 31.54 & 29.90 \\
\hline
\end{tabular}

\subsubsection{Wind speed}

The three houses received wind from the same direction. In the early morning until noon most of the wind came from the south-east, and changed early afternoon to come from the south-west. From late afternoon through to the evening, wind flow was from the east. Because the mature and new landscape houses faced an open space and were located at the end of a lot site, they were more exposed to the prevailing winds than the ordinary landscape house. 


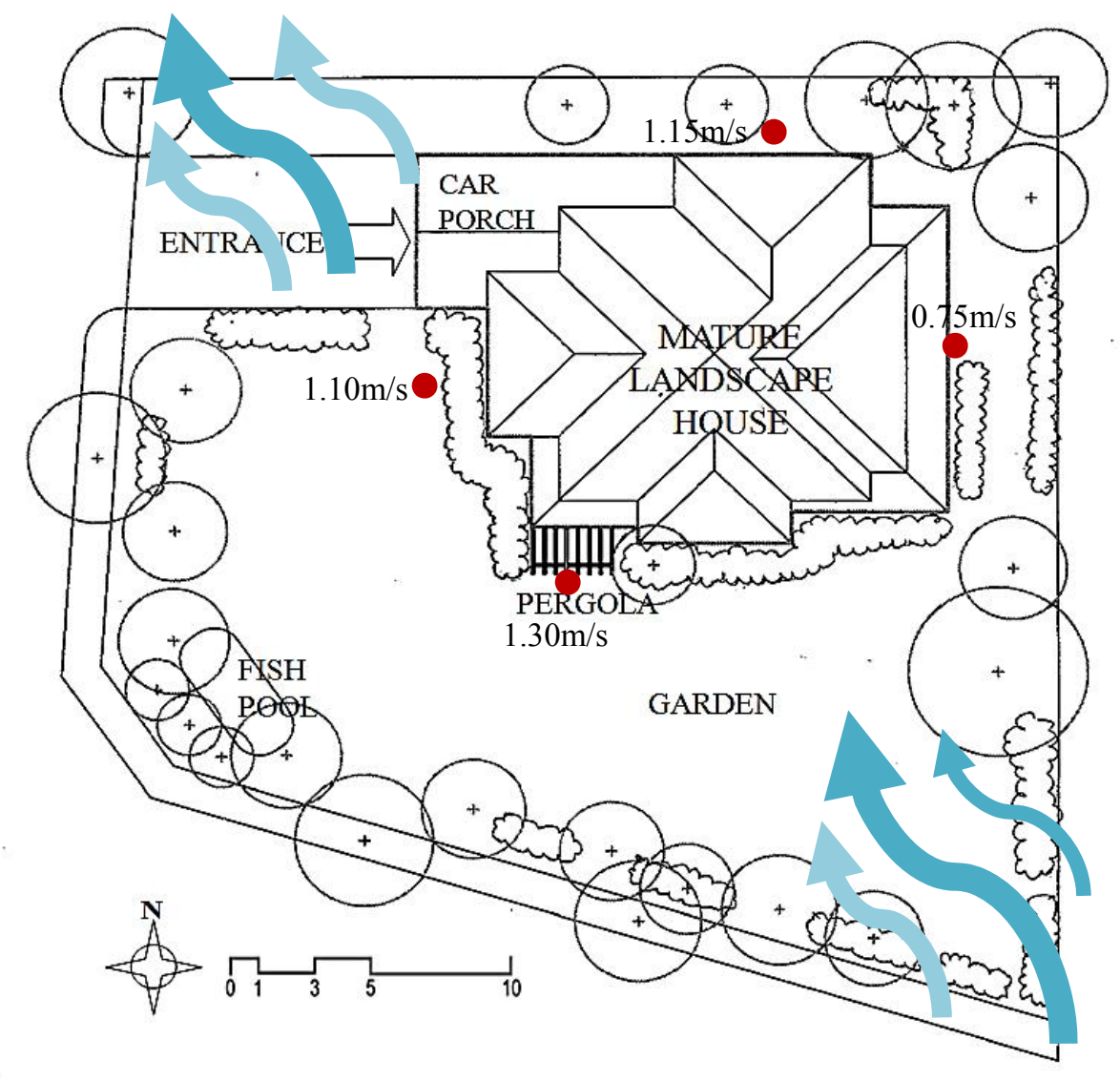

Figure 4.23. Wind flow at the mature landscape house

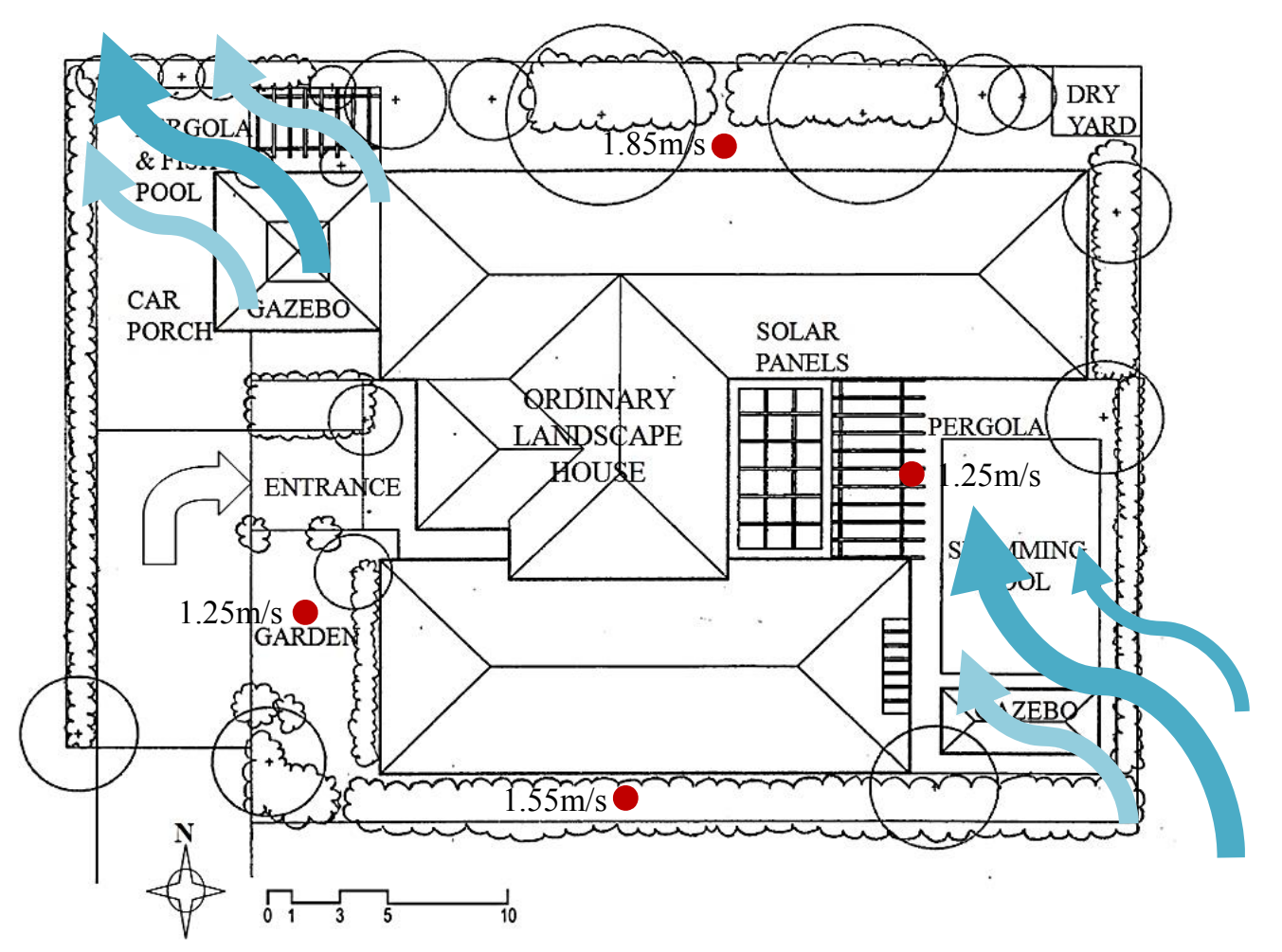

Figure 4.24. Wind flow at the ordinary landscape house 


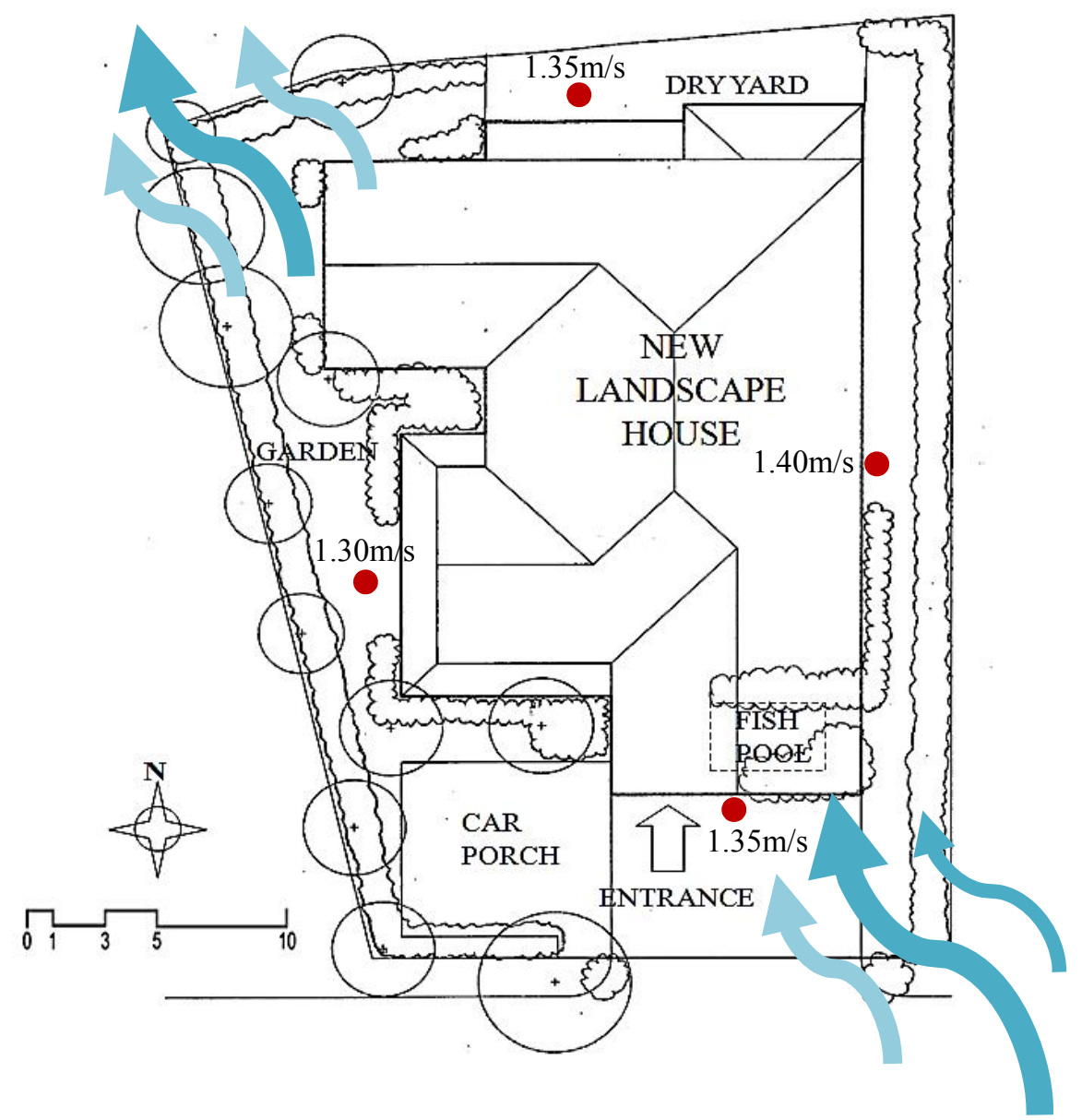

Figure 4.25. Wind flow at the new landscape house

Figures 4.26 to 4.29 show the wind speeds adjacent to each of the four walls of the three houses, and Table 4.18 summarizes the average wind speed around the houses at a height of $1 \mathrm{~m}$. Figures 4.23 to 4.25 show the houses' locations and landscape plans, and the wind flow and direction around houses.

The wind speeds around the three houses fluctuated throughout the day, measuring between 0.04 and $2.05 \mathrm{~m} / \mathrm{s}$. The mature landscape house recorded light to moderate wind speeds on each azimuth, with the speed fluctuating between 0.04 to $1.2 \mathrm{~m} / \mathrm{s}$. Wind speed adjacent to the north and east walls was lower, below $0.5 \mathrm{~m} / \mathrm{s}$, during the afternoon, while wind speed adjacent to the south walls was lighter during the morning until noon. During the rest of the day and in the other locations around the 
house, wind flow was moderate $(0.5-1.2 \mathrm{~m} / \mathrm{s})$. The highest wind speed was approximately $2.05 \mathrm{~m} / \mathrm{s}$, and occurred at 18.30 hours adjacent to the south wall.

The ordinary landscape house had different wind speed patterns, which also fluctuated throughout the day. The south and west sides of the house had low wind speeds during the morning until noon, while the east and north sides had light wind speeds recorded in the afternoon ranging from 0.04 to $1.75 \mathrm{~m} / \mathrm{s}$. The wind speed on the north side of the house fluctuated in the higher ranges until it reached the highest level recorded around the house of approximately $1.85 \mathrm{~m} / \mathrm{s}$ at 18.00 hours.

Wind speed around the new landscape house was consistent on all sides. Wind speeds were slow (below $1.4 \mathrm{~m} / \mathrm{s}$ ), and fluctuated constantly around the north and east sides of the house. However, on the south and west sides, a lighter wind blew (below $0.5 \mathrm{~m} / \mathrm{s}$ ) in the late morning and noon. The east side of the house had the highest wind speed, of $1.38 \mathrm{~m} / \mathrm{s}$ at 13.00 hours.

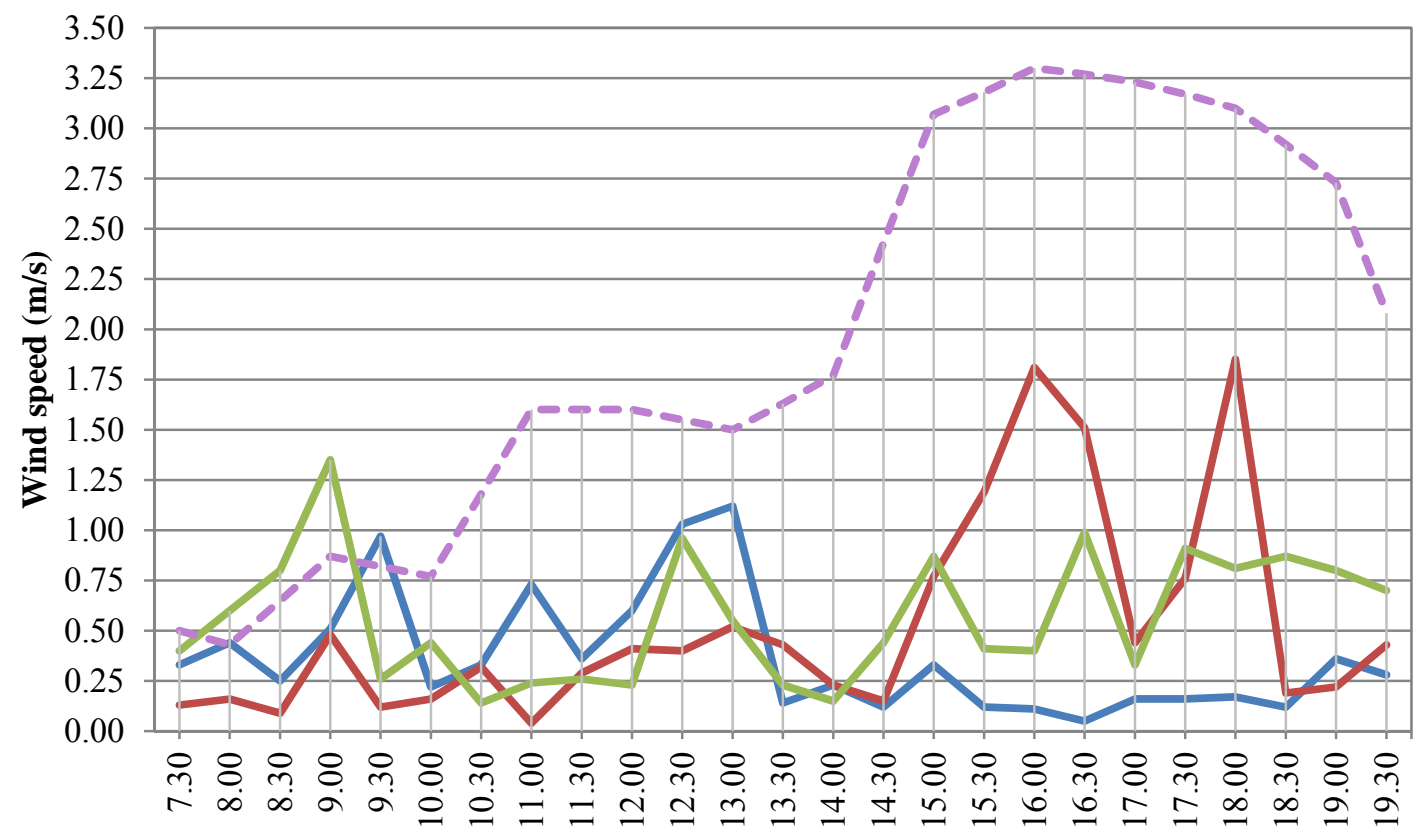

Time of the day (LST)

\begin{tabular}{ll|}
\hline Mature landscape house & Ordinary landscape house \\
New landscape house & -- Airport
\end{tabular}

Figure 4.26. Wind speeds adjacent to the north walls of the three houses 


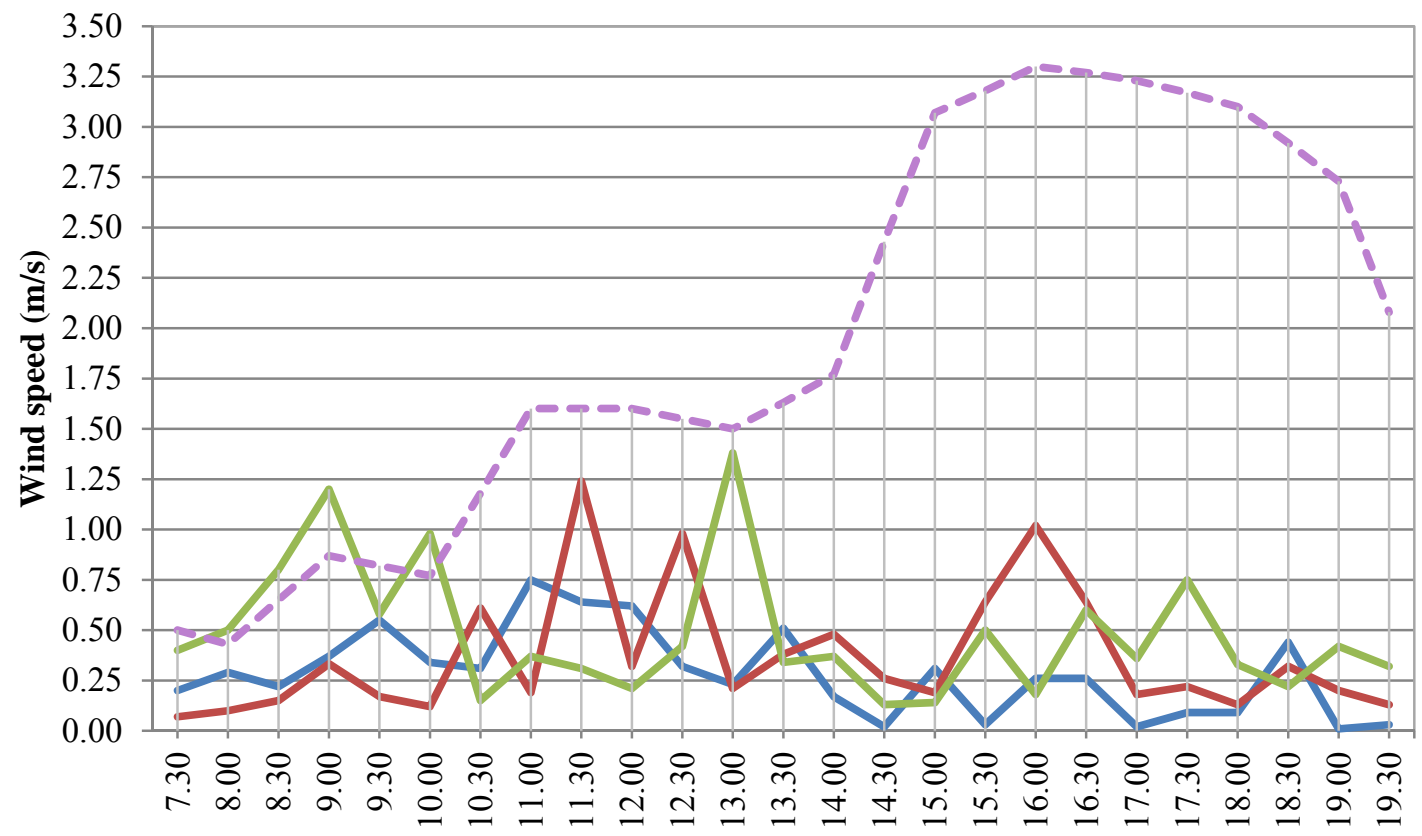

Time of the day (LST)

\begin{tabular}{|ll|}
\hline Mature landscape house & - Ordinary landscape house \\
New landscape house & $-\infty$ Airport
\end{tabular}

Figure 4.27. Wind speeds adjacent to the east walls of the three houses

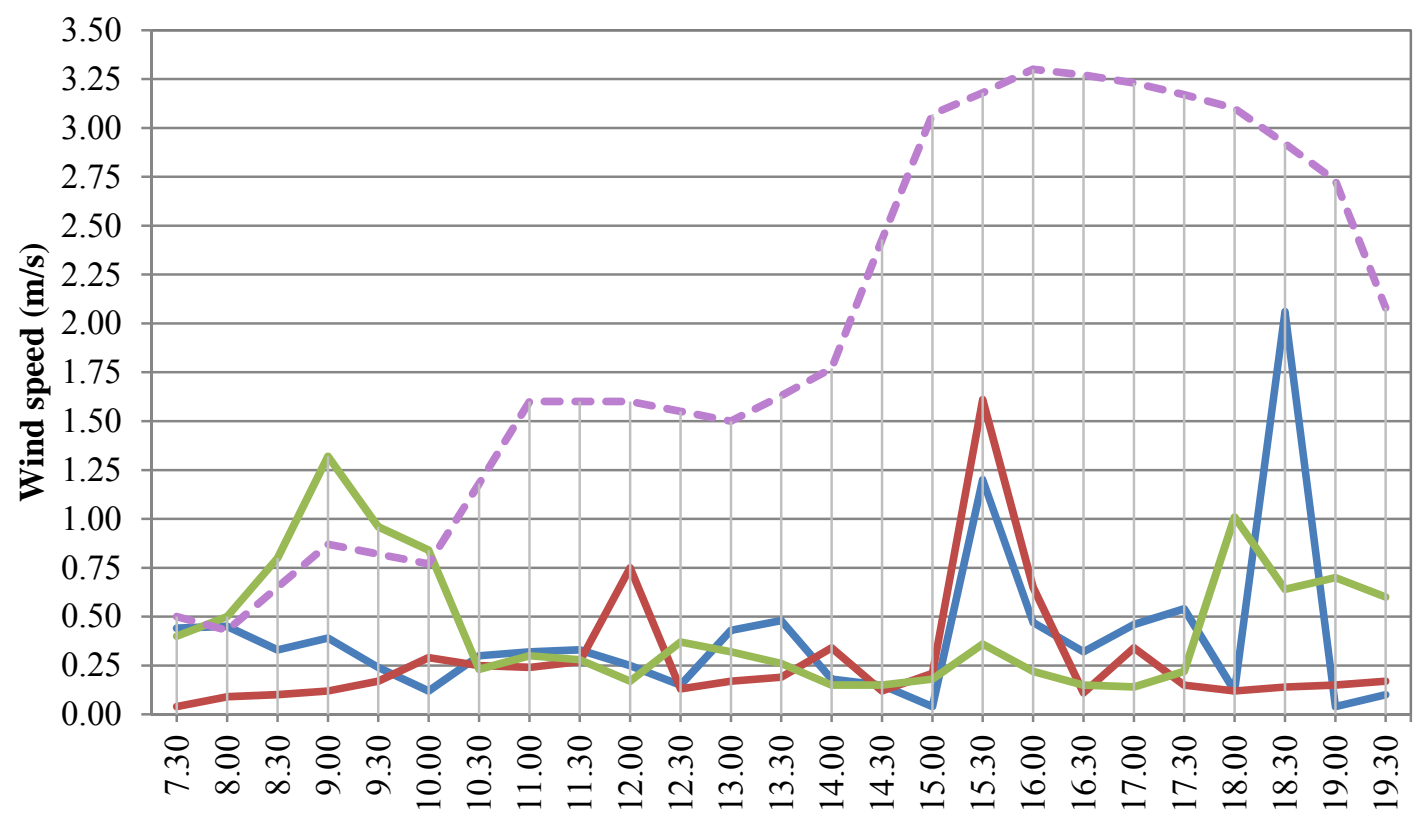

Time of the day (LST)

$\begin{array}{ll}\text { Mature landscape house } & \text { Ordinary landscape house } \\ \text { New landscape house } & \ldots \text { Airport }\end{array}$

Figure 4.28. Wind speeds adjacent to the south walls of the three houses 


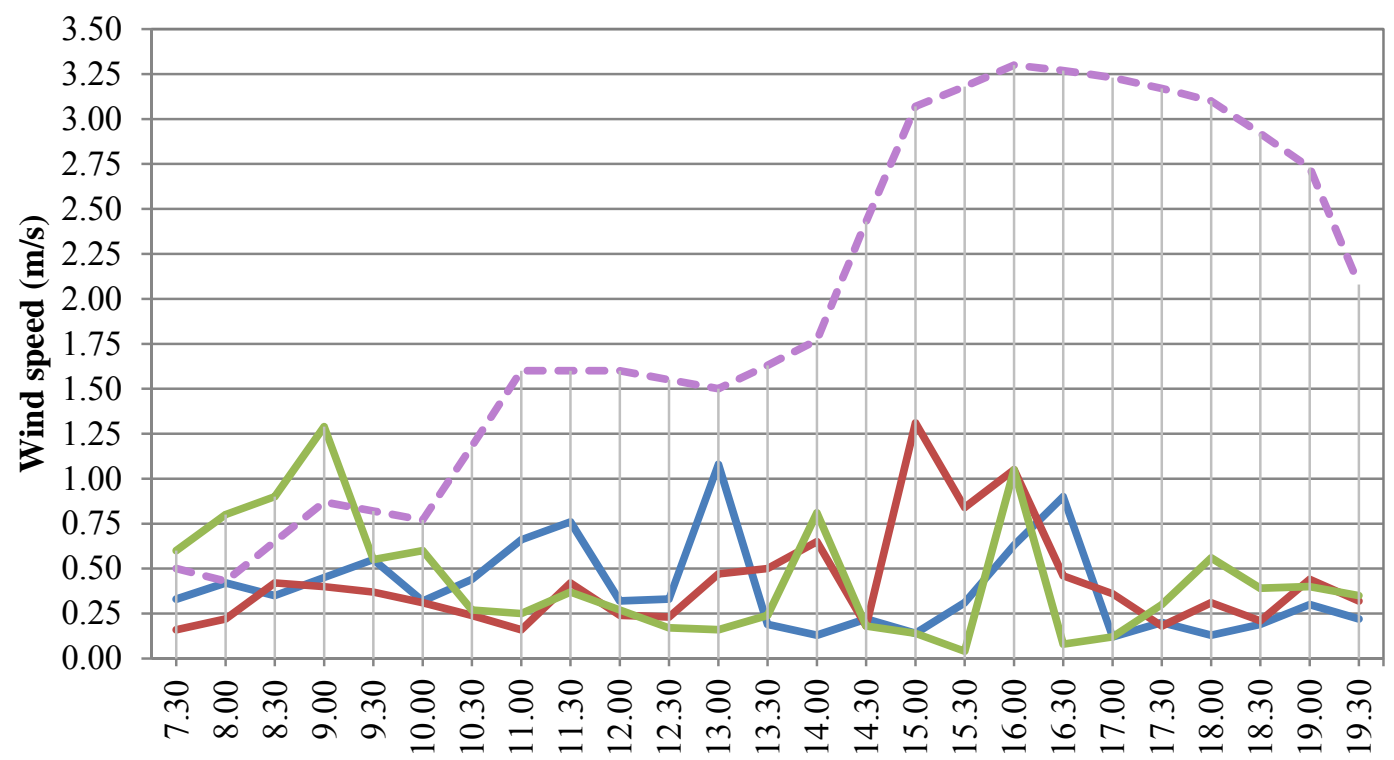

Time of the day (LST)

$\begin{array}{ll}\text { Mature landscape house } & \text { Ordinary landscape house } \\ \text { New landscape house } & -\infty \text { Airport }\end{array}$

Figure 4.29. Wind speeds adjacent to the west walls of the three houses

Table 4.18. Averaged wind speeds affecting the three houses

\begin{tabular}{llllll}
\hline Case study & \multicolumn{5}{c}{ Time@,Wind speed (m/s) } \\
\cline { 2 - 6 } House@Airport & $08: 00$ & $12: 00$ & $14: 00$ & $18: 00$ & Mean \\
\hline Mature landscape house & 0.40 & 0.56 & 0.18 & 0.13 & 0.32 \\
Ordinary landscape house & 0.14 & 0.43 & 0.43 & 0.60 & 0.40 \\
New landscape house & 0.60 & 0.40 & 0.37 & 0.68 & 0.51 \\
Airport & 0.43 & 1.60 & 1.77 & 3.10 & 1.73 \\
\hline
\end{tabular}

The average wind speeds for the three houses were similar, with the new landscape house recording the highest wind speed $(0.51 \mathrm{~m} / \mathrm{s})$, followed by the ordinary landscape house then the mature landscape house. The difference in wind speed between the three houses was up to $0.10 \mathrm{~m} / \mathrm{s}$, as described in Table 4.18 . The fluctuating patterns of wind speed in the study areas show that even where the flow was light and slow, the wind actively kept the ambient air moving and refreshed throughout the day.

In comparison, the wind speed at the airport station was higher than at the three houses. During the peak time of the day the wind speed was above $1.5 \mathrm{~m} / \mathrm{s}$, with the 
highest wind speed of $3.30 \mathrm{~m} / \mathrm{s}$ recorded at 16.00 hours. Overall the wind blew in a consistent pattern throughout the day in ranges of $0.43-3.30 \mathrm{~m} / \mathrm{s}$, as shown in Figures 4.26-4.29 and Table 4.18.

\subsubsection{Humidity pattern}

Relative humidity (RH) measures the partial pressure of water vapour in the air at the given temperature, compared to the partial pressure of saturation air at the same temperature. The ambient RH pattern at the study areas was typical for the tropical region. The RH levels adjacent to the four exterior walls of each house were similar. Each house had a stable RH reading of about $80-98 \%$ in the early morning. This gradually declined to $55-68 \%$ by noon and throughout the afternoon, and rose to 70 $82 \%$ in the evening. The three ages of landscaping had only minor differences in humidity levels at noon and during the afternoon, where the RH levels fluctuated between 55 and 70\%. However, there was a clear RH difference between the new landscape house and the other two houses, as the new landscape house's RH was approximately $18-20 \%$ higher. The mature landscape house recorded the lowest RH (70\%) towards the evening, and when compared to the other houses there were differences of up to $10 \%$. Table 4.19 lists the average $\mathrm{RH}$ for the three houses. Overall, the ordinary landscape house recorded the highest RH when compared to the other two houses. 


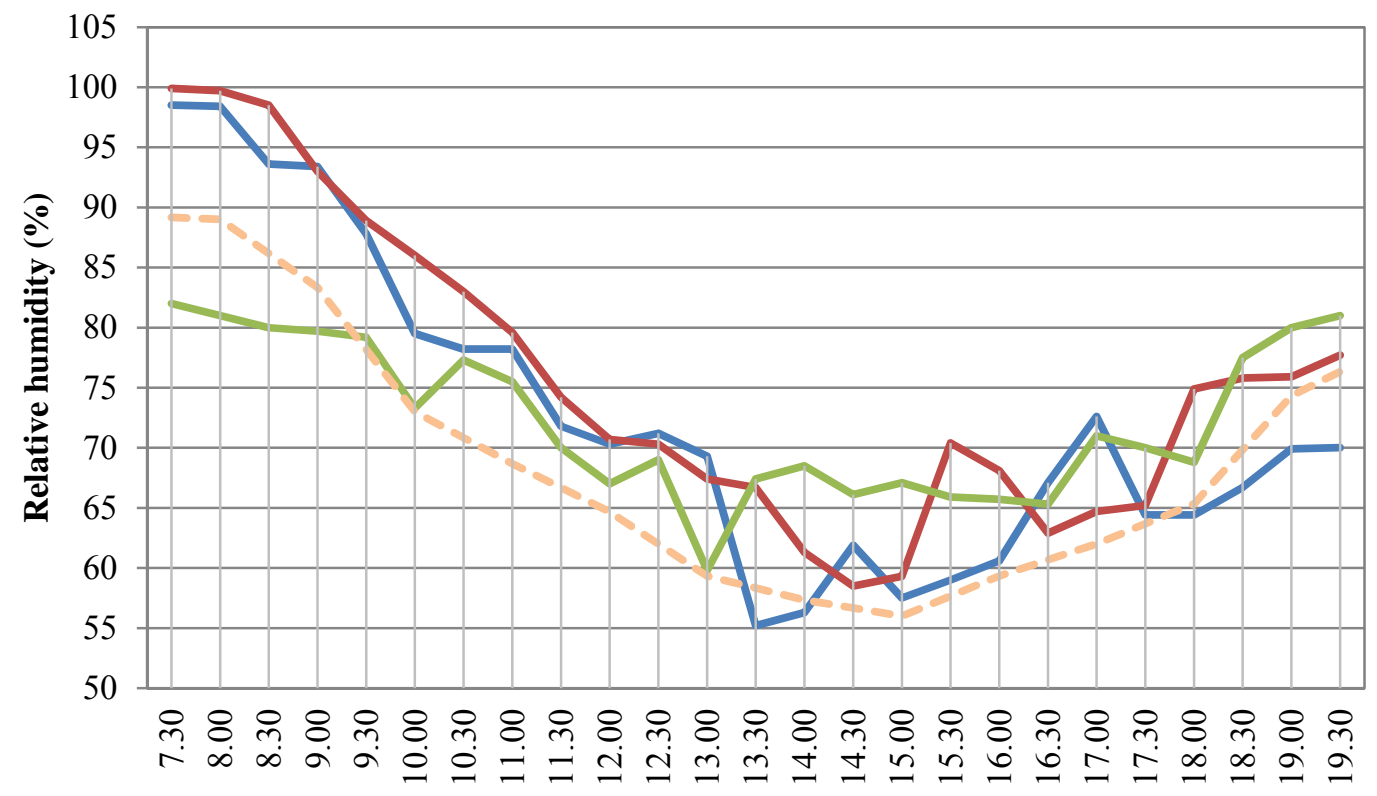

Time of the day (LST)

\begin{tabular}{|ll|}
\hline Mature landscape house & Ordinary landscape house \\
New landscape house & Airport \\
\hline
\end{tabular}

Figure 4.30. Relative humidity adjacent to the north walls of the three houses

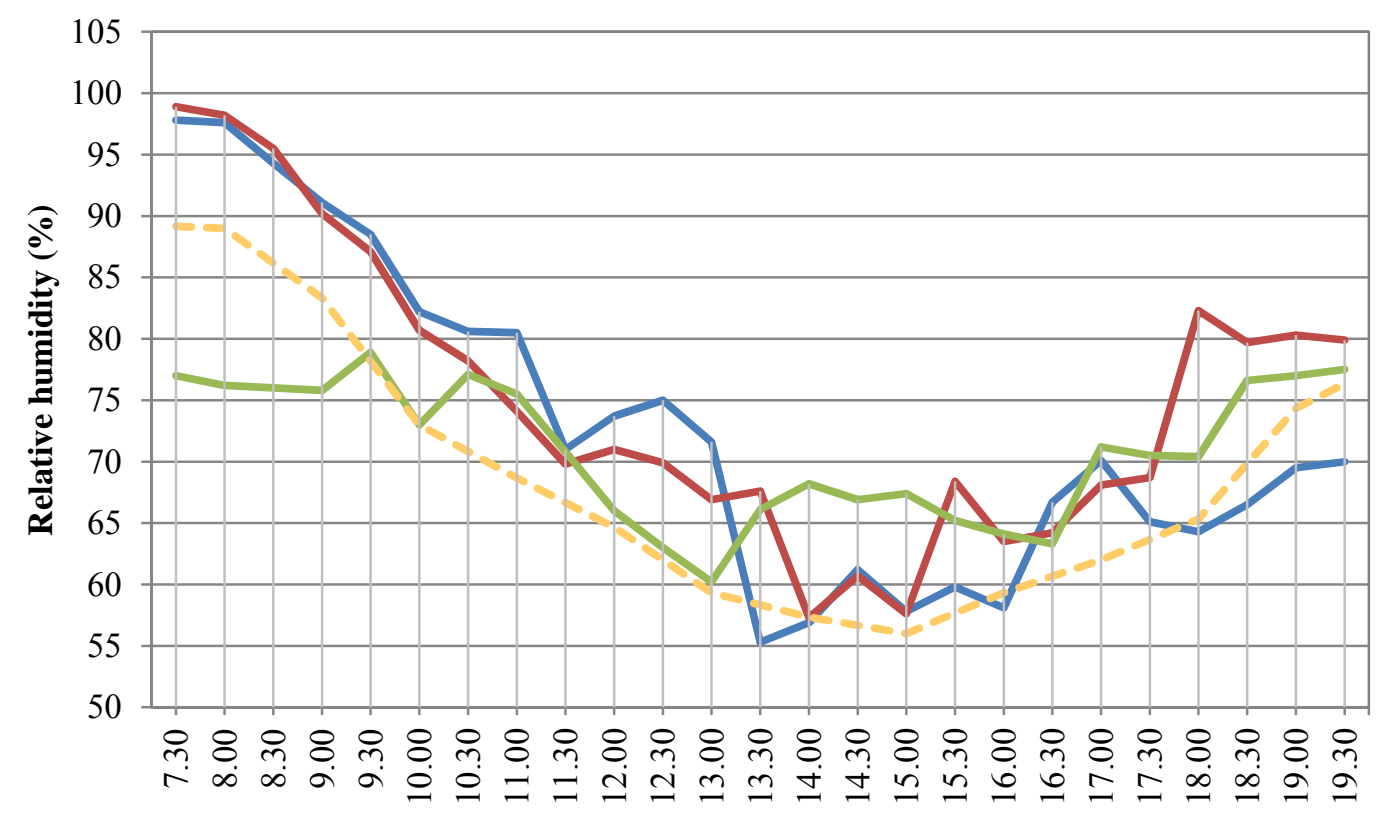

Time of the day (LST)

\begin{tabular}{|ll|}
\hline Mature landscape house & Ordinary landscape house \\
New landscape house & $-\infty$ Airport \\
\hline
\end{tabular}

Figure 4.31. Relative humidity adjacent to the east walls of the three houses 


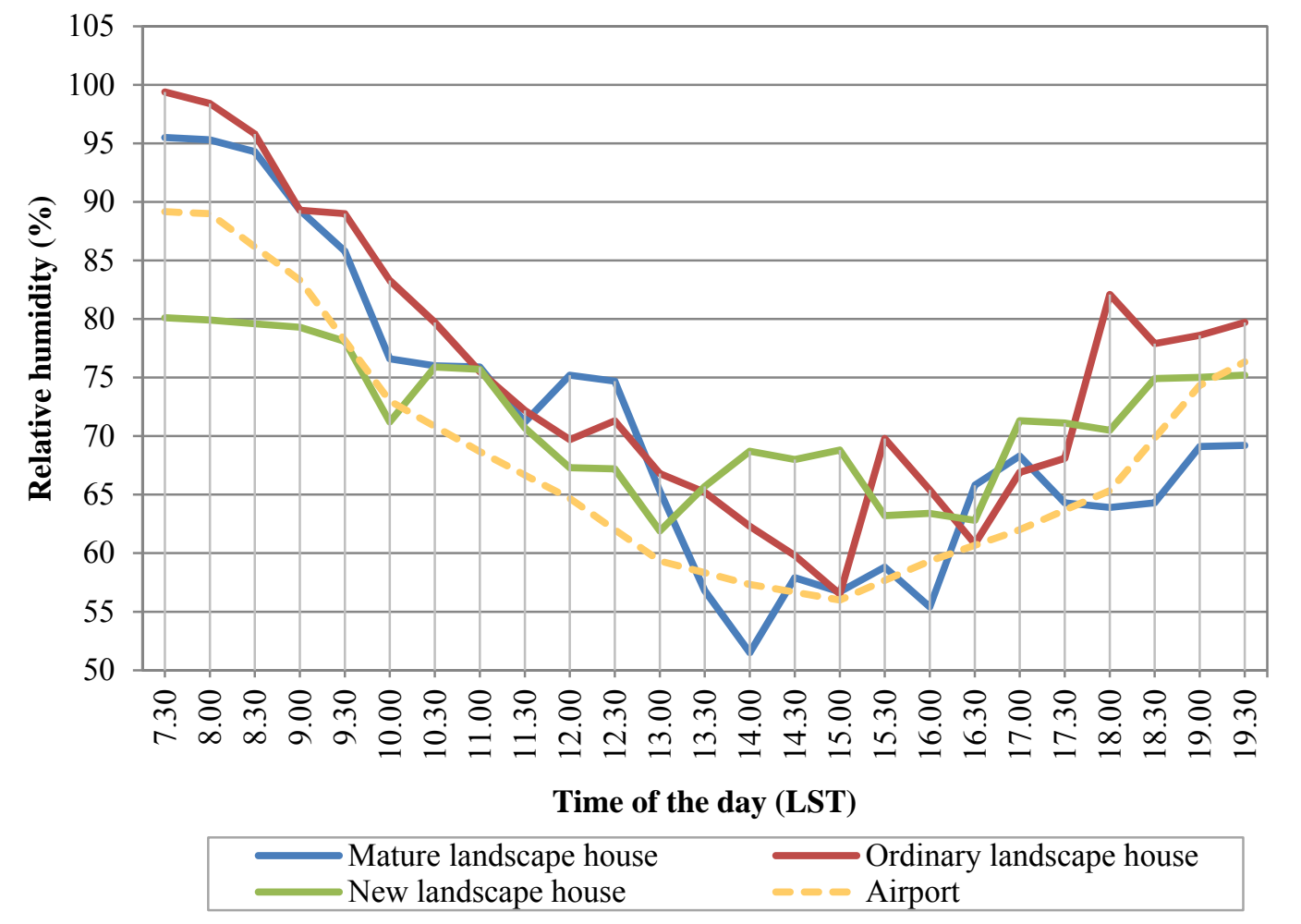

Figure 4.32. Relative humidity adjacent to the south walls of the three houses

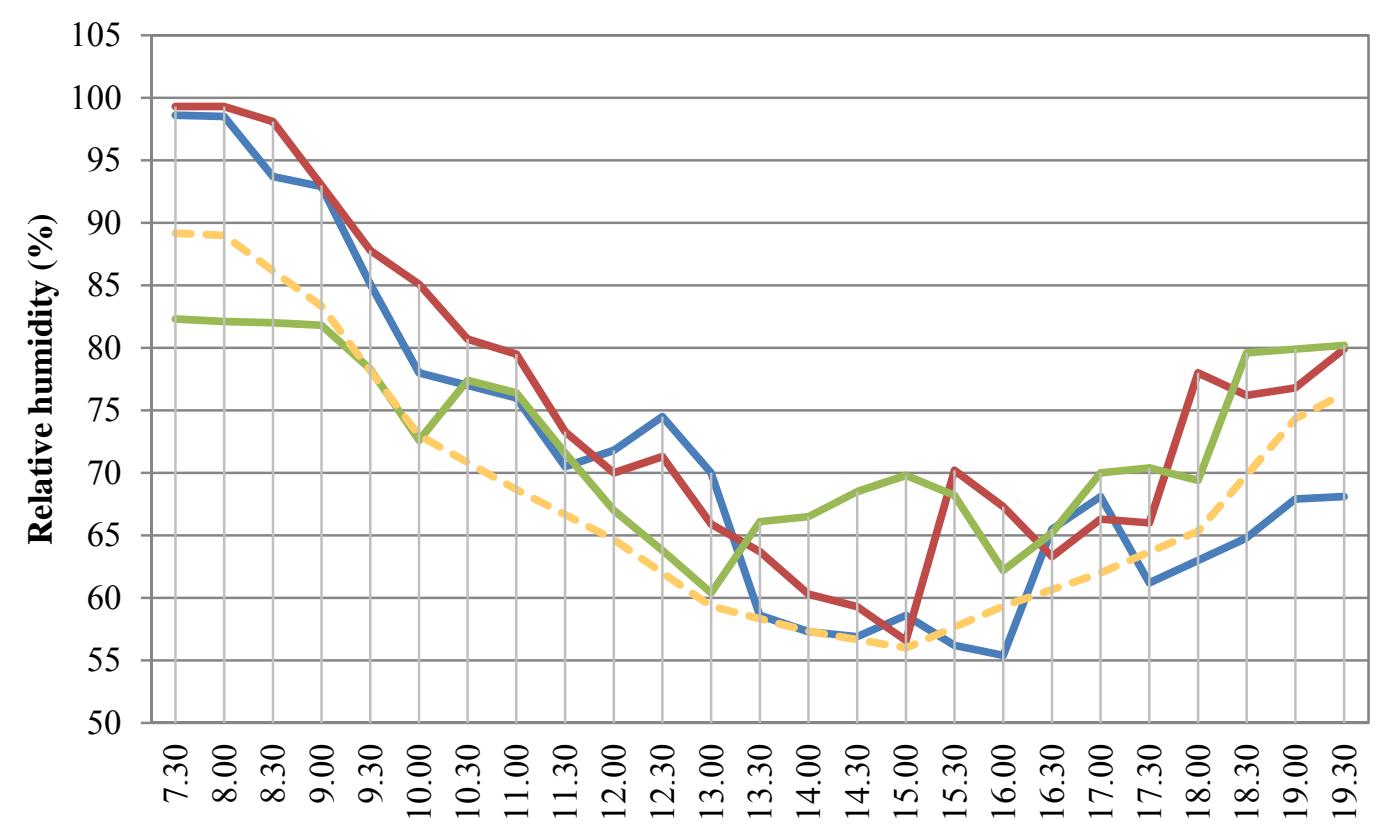

Time of the day (LST)

\begin{tabular}{|c|c|}
\hline $\begin{array}{l}\text { Mature landscape house } \\
\text { New landscape house }\end{array}$ & $\begin{array}{l}- \text { Ordinary landscape house } \\
- \text { Airport }\end{array}$ \\
\hline
\end{tabular}

Figure 4.33. Relative humidity adjacent to the west walls of the three houses 
$\mathrm{RH}$ levels in the interior of each house had uniform, stable reading patterns, averaging $70 \%$ on all four sides of the houses throughout the day. The minor differences in RH level at the exterior of the three houses did not affect the interior spaces.

Absolute humidity is the vapour concentration in the air as grams per kilogram $(\mathrm{g} / \mathrm{kg})$. Overall, for the three houses the results were similar to the RH results, where the ordinary landscape house had the highest area of water adjacent the building was expected had higher average absolute humidity, at $22.94 \mathrm{~g} / \mathrm{kg}$, when compared to the new landscape house and the mature landscape house, which had average readings of 22.02 and $21.03 \mathrm{~g} / \mathrm{kg}$ respectively.

Table 4.19. Averages of relative humidity for the three houses

\begin{tabular}{llllll}
\hline Case study & \multicolumn{6}{l}{ Time $@$ Relative humidity (\%) } \\
\cline { 2 - 6 } house & $08: 00$ & $12: 00$ & $14: 00$ & $18: 00$ & Mean \\
\hline Mature landscape house & 97.45 & 72.75 & 55.50 & 63.90 & 72.40 \\
Ordinary landscape house & 98.90 & 70.35 & 60.30 & 79.33 & 77.22 \\
New landscape house & 79.80 & 66.83 & 67.98 & 69.78 & 71.10 \\
\hline
\end{tabular}

Table 4.20. Averages of absolute humidity for the three house

\begin{tabular}{llllll}
\hline Case study & \multicolumn{7}{l}{ Time $@$ Absolute humidity $(\mathrm{g} / \mathrm{kg})$} \\
\cline { 2 - 6 } house & $08: 00$ & $12: 00$ & $14: 00$ & $18: 00$ & Mean \\
\hline Mature landscape house & 21.00 & 20.10 & 21.50 & 21.50 & 21.03 \\
Ordinary landscape house & 22.00 & 22.50 & 22.20 & 25.05 & 22.94 \\
New landscape house & 20.00 & 21.00 & 24.50 & 22.60 & 22.02 \\
\hline
\end{tabular}

The airport area's RH had a pattern similar to the three houses, but its form was smoother, and the RH was slightly lower by approximately $1-8 \%$. However, during the peak periods and towards the evening, the $\mathrm{RH}$ of the mature landscape house fluctuated and was slightly lower (5-10\%) than the RH at the airport.

\subsection{Discussion}

The results from the data gathered from the three single-family houses illustrates that different ages or eras of landscaping affect the surrounding ambient air and thermal 
performance of a house. These can also be affected by building construction and design, and the occupant's style of living.

The differences in air temperature, $\mathrm{RH}$ and wind speeds around and inside the three houses were minor. This was because each house had a moderate amount of plants that were different in location and number, and the size of shade trees were different, while the other plants such as shrubs, groundcover, and vines were of similar size. This section will discuss the effects of these variables, including landscaping from different eras and styles, on the ambient air and the thermal performance of houses.

\subsubsection{Building construction}

This study looked at three typical tropical style Malaysian houses that were built in different eras of design and construction. The basic style of each house was similar. They were all two storeys, of medium size, built in concrete, with a pitched timberframed roof, glass windows, and a square layout. The design theme for each was different, as can be seen in plans and the facades of the houses shown in Figures 4.24.4 and Figures 4.6-4.11.

The façade orientation of the mature and ordinary landscape houses faced east or west, while the new landscape house faced north and south. According to Givoni (1994), the main consideration affecting the orientation of the main facades and windows of a building arise from the relative importance of solar and ventilation issues. The strategic location of glass windows can minimise solar penetration, and allow natural cross-ventilation during the day and night, reducing air temperature and RH levels. Shading devices for windows provide shade and protect the glass surfaces from direct sunlight at the peak times of the day. For the three houses glass windows were located in all direction/facades. However, most of the windows or the sliding doors for each house faced their main garden areas. In the mature landscape house the windows were mainly on the south side, and only received indirect sunlight. In addition, this side faced the main garden area where soft landscape elements such as vines, shrubs and palms are located close to the walls and help to produce a cooler environment. In the ordinary landscape house windows were mainly on the east side, 
and in the new landscape house on the west side, with both houses receiving direct sunlight on these windows. This resulted in the indoor temperature in the mature landscape house being slightly lower by up to $1^{\circ} \mathrm{C}$ during the peak time of the day.

The roof and wall surfaces of the houses were the biggest areas exposed to solar radiation, receiving and leading to storing of heat gain throughout the day. This was followed by ground floor areas, which were not exposed to direct solar radiation which can absorb heat from the surrounding earth surfaces. In a hot-humid region, to minimise heat gain the building should be compact, with a minimum envelope surface area relative to the occupied space, which will reduce the resultant load on the cooling equipment (Givoni, 1994). Table 5.3 shows all wall areas in the four cardinal directions for the three landscape houses. The ordinary landscape house had the biggest envelope, with a total of $540 \mathrm{~m}^{2}$ of wall, $375 \mathrm{~m}^{2}$ roof, and $355 \mathrm{~m}^{2}$ ground floor areas. According to Talarosha (2002) the greater the building surfaces receiving direct solar radiation, the greater the heat gain to the building. At the peak time of day the temperatures adjacent to the east and west walls (directly receiving solar radiation), where there was less vegetation, were slightly higher $\left(0.5-1^{\circ} \mathrm{C}\right)$ when compared to the other two houses.

Glass surfaces generally allow solar radiation to penetrate directly into the interior spaces. Although every house was built with a gable or hip roof as a shading device at the windows and doors, which can provide shade on the east and west sides from late morning until the early afternoon, the solar radiation could still penetrate. This was because the walls did not have shrubs shading them to a sufficient height during the early morning and late afternoon. According to Olgyay (1963), the highest heat gains entering the building are through glass windows. Of the three houses the new landscape house had the highest proportion of glass surfaces (24\%) (Table 4.3), and the majority of glass surfaces were located on the west side (facing the main garden area) resulting in the interior temperature on this side being slightly higher by approximately $0.1-0.5^{\circ} \mathrm{C}$. 
In a tropical region, the solar reflectance ratio of the surface of the walls and roof is very important. This is expressed by the albedo value. The colour of the external envelope surfaces of a house has a tremendous effect on the impact of the sun on the building, and therefore on the indoor temperature. In this study the albedo values in the houses ranged from 0.12 to 0.42 as all the buildings used light-coloured paint for the walls, and slightly different colours of roof covering.

The three houses were also insulated in the roof, with all three houses having a layer of aluminium foil underneath the roof covering. Ceiling heights were also high at $2.9 \mathrm{~m}$, helping to maintain more comfortable temperatures at person height in the room. The insulation layer and high albedo value were intended to reflect and reduce heat gain from the walls and roof surfaces. The indoor temperatures of the three houses were similar and lower than the outdoor ambient air temperatures by approximately $3-6^{\circ} \mathrm{C}$ at the peak time of the day. However, the surrounding landscaping and their neighbourhood areas can also have a significant influence on the indoor spaces air temperatures.

\subsubsection{Landscape design}

The three houses looked at in this case study had different sized gardens, and had followed different trends of landscape design and style, that had been influenced by the different eras the houses were built in. The mature landscape house had the biggest garden, at approximately $500 \mathrm{~m}^{2}$, while the other houses had smaller gardens $\left(250-350 \mathrm{~m}^{2}\right)$. Each house had different species and types of vegetation, which had been scattered around the gardens to suit the size and theme of the gardens.

The mature landscape house followed an old fashioned, conventional, tropical style of landscaping, and was planned in square form. Most plants were positioned at the boundary area of the garden and the selection of species was influenced by the English garden style. This style was suited to the local context of landscape architecture during the era it was planted. This was a common style used during the early nineteen eighties in Malaysia, when housing lots for single-family houses were bigger than current lots. The mature landscape house had a huge lawn area to its 
south side, and was surrounded by mature Juniperus Chinensis trees, which were the main type of tree in the garden. A pergola structure by the veranda space near the living room acted as a transition space between the house, and the main garden area.

While some of the trees were approximately 30 years old, they were maintained at a medium size within 3-19m from building. Regular maintenance to prune branches was done to keep the trees in a good and safe condition.

Trees provided a small amount of shade to the house on the north side. However, in general the location of the trees, shrubs and ground cover was not strategic, and most of the walls and windows were exposed to the sunlight. Many of the shrubs were located close to the house walls, and were small in size and number so could only provide a small amount of shade to the walls of the ground floor of the building structure.

The ordinary landscape house followed a conventional style of landscaping that has been influenced by the tropical landscape of Bali. This concept was adapted to the local context of landscaping, and became quite a common style during late the 1990s (Idris, 2008). It tended to use lush species of plants along with hard landscape structures. In the ordinary landscape house only seven shade trees were planted. The trees in the area to the north of the house were close to the building (within $3 \mathrm{~m}$ ), and were tall enough to provide shade to the roof areas. They also had a dense canopy cover, with medium sized leaves, which contributed to their shading ability. There were also medium-sized trees that shaded a small part of the roof surfaces. Therefore, the ambient temperature at the north wall was lower than the other two houses by as much as $2^{\circ} \mathrm{C}$ during the early afternoon (13.30-14.30 hours) as shown in Figure 5.19.

The ordinary landscape house had 61 individual and groups of shrubs, five plantings of vines, and six groups of groundcover, which created a well-populated, luxuriant garden landscape. The overall condition of the shrubs was very good, but they were not situated strategically with regard to solar orientation. The shrubs were mainly 
located to the north and south of the house, rather than the east and west areas, which would have been more useful for shading the house from solar radiation. Gazebos were located to the east and west that provided shade, but they could not reduce the temperatures as much as the shade trees, which produce an evapotranspiration effect. The water element, the swimming pool located to the east of the house, was exposed to direct sunlight which resulted in an evaporation effect, with less transpiration and shade effect from plants.

The new landscape house had the smallest garden, and followed a contemporary style of tropical landscaping. Contemporary tropical landscaping involves planting fauna of various colours and textures to form patterns and create unique designs (Faulkner, 2012). The vegetation planted was concentrated on the south and west sides of the house. Only 11 shade trees, of small canopy sizes, had been individually planted around the garden. In addition, the average amount of leaves and their size was medium. This house had no roof shade because the majority of trees in the garden had an overall height of 2-4m. Figures 4.9-4.11 reveal that at noon, with the small number of shade trees around the new landscape house, the surface temperature at the north wall was as much as $3^{\circ} \mathrm{C}$ higher than the other two houses.

The new landscape house had 63 individual and groups of shrubs, an area with vines, and two groups of groundcover and turf cover. This helped to create a harmonious landscape design. In this garden some shrubs were growing well, and were strategically placed with regard to solar positions. However, some shrubs were still small, as can be seen in Figure 4.12. The majority of them were located around $5 \mathrm{~m}$ from the building, with the others closer to the walls. Shrubs and groundcover close to the walls also provided shade to the underground (floors and foundations) of the building. During the afternoon, the water element on the south wall and main entrance of the house produced evaporation the helped to reduce the surrounding temperature, which reached a maximum of $35.4^{\circ} \mathrm{C}$. As shown in Figure 4.14, the evaporation from the water element, combined with transpiration and shading from plants can produce a more stable reading of relative humidity. When the air is moderately moving, the air temperature will be lowered. The air temperature 
measured here was approximately $0.5^{\circ} \mathrm{C}$ lower than the temperature on the other sides of the house. Therefore, the small amount of vegetation surrounding the house could not provide sufficient shade and evapotranspiration cooling, contributing to as much as a $3.2^{\circ} \mathrm{C}$ increase in temperature when compared to the mature landscape house at noon.

\subsubsection{Energy saving}

One of the main factors that influences the energy used for cooling in a tropical city is the human factor. The living habits recorded for the three houses included the number of occupants and their ages, the occupants' preferred temperature, and the amount of time they used the active ventilation systems during the day and night.

When the external temperature around a house decreases during the night, the house will release the heat it stored in its building envelope throughout the day. This heat contributes to the discomfort of occupants in the house, so using an active ventilation system is the only way to ease this.

All of the occupants of the three houses in this study used air-conditioning at slightly different times, but mainly when most of the family members were at home. In all houses the occupants closed external openings at night for security reasons, to prevent noise, and to protect themselves and their house interiors from extreme climate phenomena such as heavy rain or thunderstorms. As a result, they depend entirely on active ventilation systems to achieve their preferred thermal comfort levels.

The three houses used similar window air-conditioning systems, but they had different models and capacities. The ordinary landscape house had eight air conditioning units, and five adults and a teenage occupant. The mature landscape house had four air-conditioning units for six adults. Finally, the new landscape had three air-conditioning units for three adults and three children. As shown in Figure 5.14, all three houses used their air conditioning systems extensively at night, especially in the bedrooms while the occupants were sleeping. The ordinary 
landscape house used the highest amount of energy for cooling, followed by the mature landscape house, then the new landscape house.

The houses used air-conditioning with different temperatures settings and for different amounts of time as explained in subtopic 4.5.3 and shown in Table 4.16. As a result, the ordinary landscape house spent $46 \%$ of the electricity bill on airconditioning; the mature landscape house used approximately 35\%; and the new landscape only used $15 \%$. This shows that the different temperatures and times airconditioners were used influenced the amount of cooling energy used. The longer air conditioning was use, and lower temperature settings will affect the amount of cooling energy used.

In a tropical city, air conditioning is a basic need for the people to be able to live comfortably and safely, especially during the night. However, in the new landscape house, natural ventilation is also used some nights when the occupants open the windows of bedrooms. This building is designed to use as much natural ventilation as possible to minimize the need for air-conditioning. The building configuration was perpendicular to the prevailing southerly wind during day time while the prevailing wind at night was from the east, and the house was strategically located with large, open green spaces to the south and west. The house was also surrounded by a private garden. This is illustrated in Appendix 4.2. These factors promoted convective cooling of the building.

The design of the window openings meant the interior was relatively safe from rain or strong wind at night while providing ventilation. For example, the master bedroom was located on the first floor, and the windows were protected by shading devices and a veranda roof, as illustrated in Figure 4.9 (left). All of these factors mean that the use of natural ventilation probably contributed to less cooling energy being used in the houses. 


\subsubsection{Modifying ambient conditions}

The results from the ambient conditions of the three study houses showed that air temperature, relative humidity, and wind speed were influenced by the surrounding vegetation. Vegetation was planted in each house's garden, and in the surrounding neighbourhood's green spaces. The intensity of the global solar radiation and cloud cover contributed to a microclimate effect in the case study areas. However, the surrounding vegetation exerted a direct influence by shading and channelling wind, and indirectly through evapotranspiration.

\subsubsection{Shading}

In typical hot and humid tropical climates, the east and west walls of houses are exposed to direct sunlight in the morning and afternoon, while the north and south walls receive indirect sunlight. However, as Malaysia is near the equator, the sunlight is angled such that during peak times of the day intense solar radiation can affect the north and south walls as they receive indirect/diffused solar radiation. Figure 4.18 showed that solar radiation is actively radiated for approximately eight hours per day, with a maximum radiation level of approximately $3.69 \mathrm{MJ} . \mathrm{m}^{-2}$ at 13.00 hours, with cloud cover of 7 Okta and with no rainfall. This means that to be most effective in reducing the temperatures around a house, shade trees and shrubs must be cultivated right around it to achieve the maximum shading for its walls and grounds to keep the outdoor and indoor environments as cool as possible.

One of the primary building envelope components of a house is the roof, which needs to be properly shaded to reduce heat gain throughout the day. For the three houses in this case study, all of their shade trees ranged from small to medium sized, and failed to provide extensive shading to the roofs. Only the ordinary landscape house had trees that could offer some shading during the middle of the day. These were Persia americana and Eugenia polyantha, which were located within $5 \mathrm{~m}$ of the north side of the house and were around $10 \mathrm{~m}$ high, with a medium sized spreading shape. 
Each house had small sized shade trees planted individually near their walls, which could provide shade to the building's walls. They also had shade trees that provided shade to the garden areas. However, the provision of quality shade depended on the canopy size, the height and the amount of leaves.

Most of the shade trees around the mature landscape house were located along the boundary of the house. The majority of them were small, and could only provide shade to the garden area. The four shade trees located to the north of the house were close to the house, of a medium size and height, and had a dense canopy. These trees provided shade to the garden and part of the house walls during the morning, but were not strategically placed to maximise shade to the house.

In the new landscape house, the majority of shade trees were small in canopy size, less than $4 \mathrm{~m}$ high, and had a sparse amount of leaves, so could only provide an insignificant amount of shade to the garden. Again, the trees were not strategically placed to provide shade to the house. The ordinary landscape house also got little shade from its surrounding trees.

The second largest building envelope surface on a house is the walls. If these surfaces are strategically protected by vegetation this will reduce building heat gain and lower surface temperatures of the internal walls. Shrubs, vines and groundcover are suitable types of vegetation to provide shade to the walls and foundation surrounds. These fast-growing plant species will not harm the foundations of a building because their roots are small and soft. Shrubs, vines and groundcover planted immediately adjacent to the exterior walls can act as a thermal barrier throughout the day. This type of vegetation can improve the insulation properties of walls, windows, and underneath the house if they are strategically planted. This will minimise the heating up of the house interior during the day. They can also be effective to help maintain cool temperatures, whether the house is being cooled via natural ventilation or mechanically air conditioned. 
The ordinary landscape house had lush shrubs, vines and groundcover planted immediately beside the north-south walls. These were on average $1-1.5 \mathrm{~m}$ high, and were of medium amounts and with medium sized leaves. Many species were planted together and created shade to the adjacent walls, windows and foundation surrounds. There were also shrubs and groundcover plants beside the east and west walls, but the majority of them were small and not well-located.

Generally, the most strategic sides of a house to plant lush shrubs are along the east and west facades, which receive direct solar radiation in the morning and late afternoon. By contrast, the north and south facades only receive indirect solar radiation. Shrubs must be planted continuously alongside the walls to create a natural insulation layer for the walls and foundation surrounds, and priority should be given to the east and west sides. If this is not done the high temperatures will transmit heat to the walls. Although the ordinary landscape house had a large amount of lush shrubs adjacent to its walls, this was focused on the north and south wall areas. This resulted in slightly different outdoor and indoor air temperatures compared to the other two houses as shown in Figures 4.15 and 4.17.

The new landscape house was oriented to the south-west, where the garden was located. It had shrubs of varying sizes planted to its east, south and west sides, with two layers of small shrubs planted on the east side. In the garden area shrubs were planted close to the house walls and along the fence underneath small shade trees. They ranged in size from small to mature, and were placed at intervals among the groundcover. Shrubs along the boundary and underneath the shade trees were of medium size. The north side of the house had a paved concrete area used for clothes drying and was planted with edible plants. Because of this area there was not continuous shade and insulation along the walls and floors of the house.

The mature landscape house had small amounts of sparsely planted, small shrubs and groundcover that were located close to its walls focused on its east, south and west sides. This situation would provide less quality shade to the walls and floors. Shrubs 
were also planted underneath the shade trees along the fence areas to the east, south, and west directions, but were located very far from the building.

Differences in the amount of shade-providing vegetation around the three houses resulted in slight variations in air temperature readings between the three houses. In the morning, the differences in air temperature were quite clear. In the afternoon and towards the evening the air temperature readings were similar because vegetation around the houses was not strategically placed.

The surrounding landscaping and the mass material of construction of the three houses delayed the effect of the outdoor temperature on the indoor temperature by 3.5 hours. The indoor temperatures reached their highest points at 18.00 hours, compared to 14.30 hours for the outdoor temperature. On average, the mature landscape house recorded the lowest outdoor and indoor air temperatures, at 31.8 and $28.9^{\circ} \mathrm{C}$ respectively. This was followed by the ordinary landscape house and the new landscape house, which had progressively higher temperatures of approximately $1^{\circ} \mathrm{C}$ as shown in Table 4.17.

It can be assumed that air-conditioners are used during the night time in each house because their temperatures remain high during the late evening, as can be seen in Figures 4.14-4.17, due to the release of heat stored in the walls during the day. In addition, all openings are closed for security reasons, which reduces natural air flow and increases the need for air-conditioning to ensure the indoor spaces are comfortable.

\subsubsection{Channelling wind}

In the hot temperatures and high humidity levels of a tropical climate, wind is needed to assist people's comfort levels. Appropriately sized and placed landscaping, together with an appropriate building orientation, will allow good wind flow into a building and through its surrounding garden. 
The mature landscape house was located in the centre of Section 6, Shah Alam, and was surrounded by green spaces to the east and south sides, while the north and west sides were surrounded by two-storey houses. The landscaping around this house had been arranged so that the majority of its trees were within $10-19 \mathrm{~m}$ of the building, to the south and west sides where the prevailing winds come from during the day. A small number of trees were also located to the north and east sides of the house. Their trunk heights were up to $1.5 \mathrm{~m}$, which channelled the prevailing wind through the garden area. Some shade trees were planted individually and others in small groups with shrubbery underneath. The huge lawn in the middle of the garden allowed wind to move freely into the building. Shrubs located along the building's walls were small, thus the wind could easily move straight to the building. However, on the east side, the prevailing breeze was diverted from the house by a small area of mature forest in a nearby area of open space. This caused the mature landscape house to record a fluctuating pattern of wind speed. Moreover, the wind speed moved to the south when it reached the highest speed of approximately $2.05 \mathrm{~m} / \mathrm{s}$ at 18.30 hours. On average, wind speed around the mature landscape house from all directions was $0.32 \mathrm{~m} / \mathrm{s}$. Therefore, with a minimum of landscaping adjacent to the house walls, natural ventilation was moderately effective.

The ordinary landscape house was located in the middle of a housing estate in Section 11. It was surrounded by two-storey houses, which caused changes of wind direction around the house. For example, the local prevailing wind direction should be from the east. However, the northern areas of the garden recorded the highest wind speed of approximately $1.85 \mathrm{~m} / \mathrm{s}$ at 18.00 hours. This area's landscape included shrubs of various sizes, ranging from $0.5 \mathrm{~m}$ to $2.5 \mathrm{~m}$ high, and trees with trunk heights up to $5 \mathrm{~m}$. The shade trees planted around this house averaged $2.5 \mathrm{~m}$ high, which allowed breezes to pass through to the building in most areas. However, lush shrubs close to the south walls blocked some breezes, diverting them to the north. Figure 4.28 reveals that wind speeds in the southern area of the ordinary landscape house were higher than other areas, reading approximately $1.62 \mathrm{~m} / \mathrm{s}$ at 15.30 hours. On average, wind speeds around the ordinary landscape house were approximately 
$0.40 \mathrm{~m} / \mathrm{s}$ for each direction, which shows that moderate landscaping adjacent to the house walls channelling air flow for natural ventilation was effective.

The new landscape house was situated in the middle of Section 9. It faced a huge green open space to the south and west sides, and had two-storey houses on the north and east sides. In some areas of the house natural ventilation was used during the day and night. Windows in the west side were open from morning until afternoon to allow natural ventilation. The design of the house included French windows, which were practical for allowing wind to pass through the building as can be seen in Figure 4.34. The house was also strategically located, facing an open space where in the morning the wind came from the south-east and then changed to the south-west in the afternoon. During the evening, wind flow was from the east.
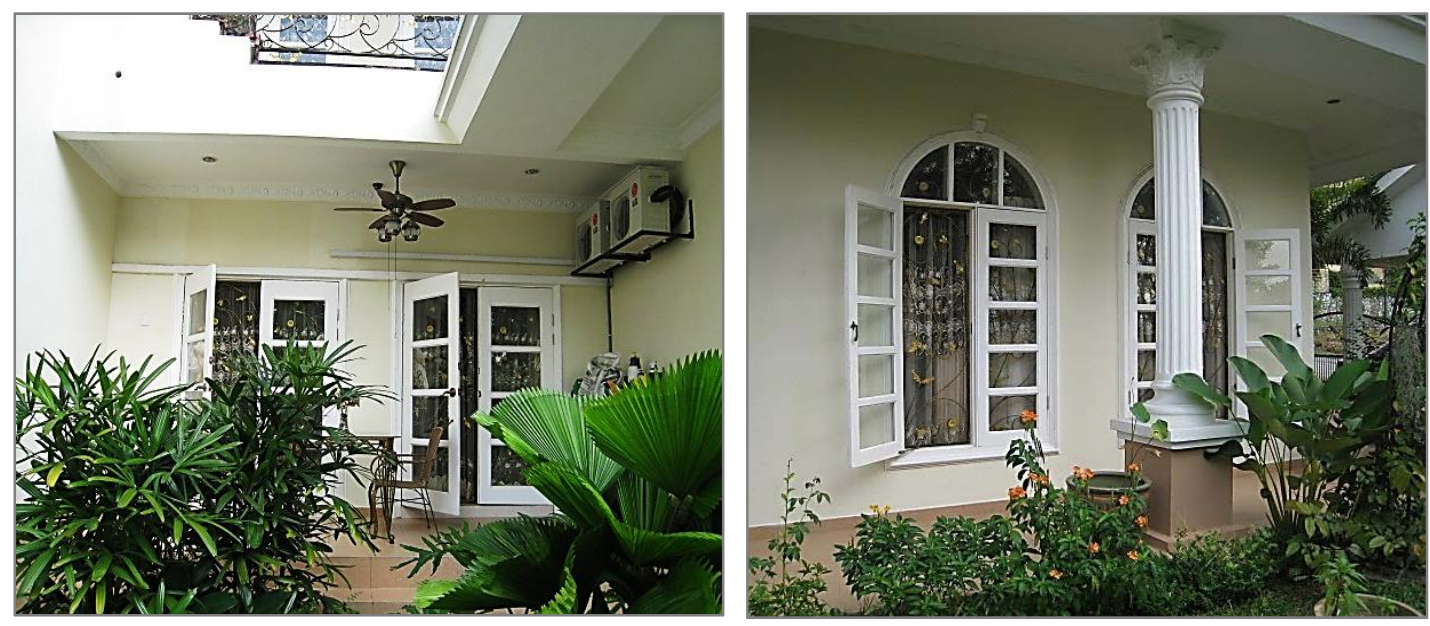

Figure 4.34. Example of French windows located on the west wall of the new landscape house, to the living and dining spaces

The size of all trees and shrubs around the house was small, so they could not prevent wind from passing through to the building. The trunk height of trees could channel wind effectively to the garden area and the buildings, to help release hot and humid ambient air especially during the time of day where the temperature was at its peak. Wind from the east and northerly directions also worked well to channel wind flow when the wind speed was actively blowing in fluctuating patterns throughout the day. The wind fluctuations were in the ranges of $0.04-1.38 \mathrm{~m} / \mathrm{s}$. The new 
landscape house received the highest wind speed compared to the mature and ordinary landscape houses. On average, wind speeds around the new landscape house were approximately $0.50 \mathrm{~m} / \mathrm{s}$. Hence a moderate amount of landscaping and large open spaces around the building should enable effective natural ventilation to the house as can be seen in Figure 4.25.

Overall there were slight differences in wind speed around the three houses, which was influenced by each of the house's landscaping. Trees with a trunk height of 1.5$2 \mathrm{~m}$, allow wind flow through to the house. The small to medium sizes of leaves allow wind flow to the garden and house.

\subsubsection{Evapotranspiration}

The measurements taken in and around the three case study houses revealed that there was a slight difference in the RH levels. The RH levels at the three houses were influenced by evaporation from water elements, and transpiration from the various amounts, placement and size of the landscaping. Figures 4.30-4.33 shows the results of the half and hourly interval relative humidity readings, which was measured in and around the three houses.

The general RH pattern reached a higher level during the morning, and gradually fell in fluctuating patterns from noon through the afternoon then increased towards the evening. The fluctuating patterns in the different garden configurations occurred at the peak time of the day. They were caused by the landscaping around the house not being strategically placed, which resulted in different impacts on the amount of water vapour in the ambient air around the four sides of each house. For example, the ordinary landscape house had lush and green shrubs and shade trees to its north-south side, but fewer shrubs on the east and west sides. This arrangement had also been used in the gardens around the other two houses. If the vegetation around the house was located more strategically and arranged in an organised way, the RH pattern would be smoother, with stable readings throughout the day, similar to the way in which the natural arrangement in a tropical rain forest creates a stable pattern of relative humidity (CCRR, 2012). Table 4.19 shows that the ordinary landscape house 
produced a higher average $\mathrm{RH}$ level of approximately $77 \%$, while the average $\mathrm{RH}$ levels in mature and new landscape house were 71 and $72 \%$ respectively. These levels were influenced by surrounding landscaping and nearby green open spaces.

Each house had a water element in the garden. However, the ordinary landscape house had the largest amount, with its swimming pool and fish pool, which contributed to evaporation. The transpiration process at this house was also influenced by the amount, placement and size of plants around the garden. This combination made evapotranspiration slightly higher than at the other two houses which only had small sized water elements.

Table 4.20 shows that the higher absolute humidity throughout the day at the ordinary landscape house was because of high levels of evapotranspiration. The vapour concentration in the air was increased to reduce air temperatures. The average absolute humidity was approximately $22.94 \mathrm{~g} / \mathrm{kg}$, which is slightly higher at $1 \mathrm{~g} / \mathrm{kg}$ than at the other two houses. This suggests that medium-sized shade trees, lush shrubs, vines, groundcover and turf located close to the house, together with water landscape elements, will produce a higher relative humidity or absolute humidity level.

\subsection{Discussion of the results}

The three houses were of similar construction, but had different main façade orientations, glass areas, and age (30,10 and 5 years old). The main façade orientations for the mature landscape house were facing north and south. The majority of the glass openings were located facing the main garden area. This orientation can minimize the amount of solar radiation penetrating the internal spaces. In contrast the main façades for the ordinary landscape house and the new landscape house were facing the east and west respectively, with the majority of their glass surfaces facing the main garden area as can be seen in Table 4.21. Immature vegetation around the main garden area (west) of the new landscape house was not sufficient to provide shade cover to walls and glass surfaces; while sparse medium maturity plants around the main garden area (east) of the ordinary landscape house 
had similar effects on the surrounding ambient air. Thus, in these case studies, the best orientation for the main garden area was facing north and south where the majority of glass should be located to receive diffuss solar radiation in a similar way to the mature landscape house. This can provide cooler temperatures - as low as $31.8^{\circ} \mathrm{C}\left(0.3-1.2^{\circ} \mathrm{C}\right.$ different $)$. Table 4.21 shows that the new landscape house had the highest glass surface areas. Most of the glass areas were locared on the east and west sides where in adequate strategic landscaping not only allowed solar penetration to the interior spaces, but also resulted in the highest outdoor temperature of $33^{\circ} \mathrm{C}$.

The colour of roof covering in the mature landscape house was dark brown and generated the lowest albedo value $(0.12)$. This may affects the heat gain to the building and may increase the outdoor and indoor air temperatures. A light colour of roof covering such as light grey (albedo value -0.30 ) and light orange (albedo value -0.33 ) which were used at the new landscape house and ordinary landscape house respectively were more effective at reflecting solar radiation.

Table 4.21. Main façade orientation and glass surface areas, surrounding vegetation, weather data and human factors at the mature landscape house (M), ordinary landscape house $(\mathrm{O})$ and new landscape house $(\mathrm{N})$

\begin{tabular}{|c|c|c|c|c|c|c|c|c|c|}
\hline House & \multicolumn{2}{|c|}{ Building } & Surrounding & \multicolumn{2}{|c|}{ Average daily microclimate data } & \multicolumn{2}{|c|}{ Human factors } \\
\cline { 2 - 8 } & $\begin{array}{c}\text { Main } \\
\text { façade } \\
\text { orientation }\end{array}$ & $\begin{array}{c}\text { Glass } \\
\text { surfaces } \\
\left(\mathrm{m}^{2}\right)\end{array}$ & Vegetation & $\begin{array}{c}\text { Temp. } \\
\text { outdoor } \\
\left({ }^{\circ} \mathrm{C}\right)\end{array}$ & $\begin{array}{c}\text { Temp. } \\
\text { indoor } \\
\left({ }^{\circ} \mathrm{C}\right)\end{array}$ & $\begin{array}{c}\text { Wind } \\
\text { speed } \\
(\mathrm{m} / \mathrm{s})\end{array}$ & $\begin{array}{c}\text { RH } \\
(\%)\end{array}$ & $\begin{array}{c}\text { Cooling } \\
\text { energy } \\
\text { use }(\%)\end{array}$ & $\begin{array}{c}\text { Occupant } \\
\text { number }\end{array}$ \\
\hline $\mathrm{M}$ & $\begin{array}{c}\text { North and } \\
\text { South }\end{array}$ & 51.35 & $\begin{array}{c}\text { Moderate/ } \\
\text { Mature }\end{array}$ & 31.76 & 28.93 & 0.32 & 72.40 & 35 & 6 \\
\hline $\mathrm{O}$ & $\begin{array}{c}\text { East and } \\
\text { West }\end{array}$ & 87.30 & $\begin{array}{c}\text { Moderate/ } \\
\text { Medium } \\
\text { maturity }\end{array}$ & 32.08 & 29.62 & 0.40 & 77.22 & 46 & 6 \\
\hline $\mathrm{N}$ & $\begin{array}{c}\text { East and } \\
\text { West }\end{array}$ & 94.50 & $\begin{array}{c}\text { Moderate/ } \\
\text { Immature }\end{array}$ & 32.98 & 29.90 & 0.51 & 71.10 & 15 & 6 \\
\hline
\end{tabular}

The three different ages of vegetation located around the three houses create moderating effects by providing shade, chanelling wind and evapotranspiration cooling. Table 4.2 shows that there are slight differences in the microclimate data for each house. 
Non-strategic placement of vegetation around the mature landscape house and the ordinary landscape house, and immature vegetation around the new landscape house generated the dramatic increase of temperatures during the peak time of the day as explained in subtopic 4.5.4.2. As listed in Table 4.21 the non-strategic placement of landscaping also results in similar temperature readings even though the age of vegetation is different.

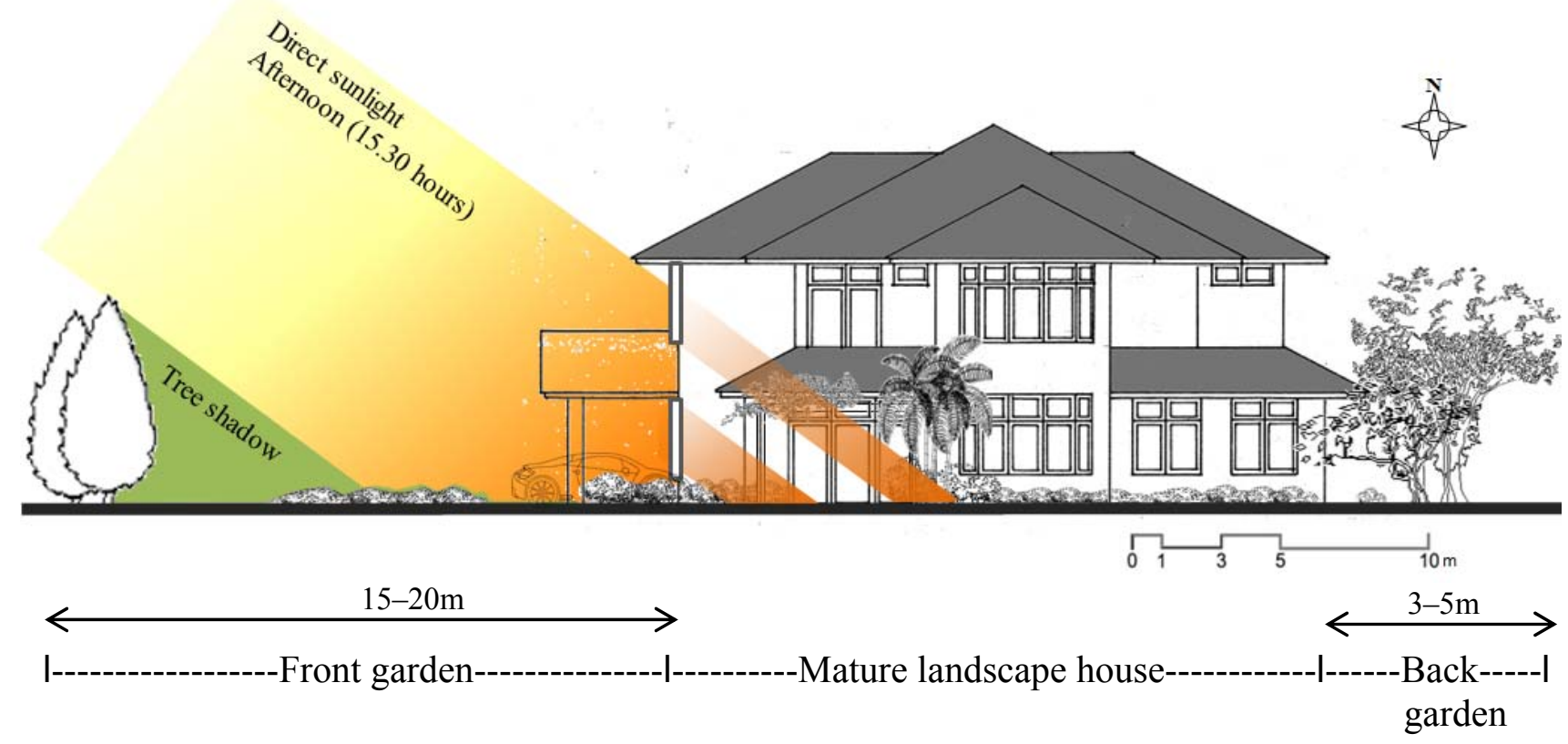

Figure 4.35. East walls and windows exposed to direct sunlight. Low and small shrubs close to walls can only provide shade to small area of building underground, while trees and shrubs planted far away (15-20m distant) can only produce shade to the garden ground surfaces

In this study, roofs, walls and glass surfaces are the most exposed parts of the building envelopes to direct solar radiation. This is due to non-strategic placement and design of landscaping. Medium sized trees and a round or spreading form of canopy can potentially provide shade to walls surfaces nearby. Most of the trees in the mature landscape house were located at $15-20 \mathrm{~m}$ from the house and therefore cannot provide shade to the building. This suggested the need for two or three trees of medium size with heights 6 to $9 \mathrm{~m}$ be located on the east and west sides 3-5m from the house. This amount of shade trees would protect part of the roofs and walls in early morning and shade most of the earth surfaces close to the house over a day, and 
furthermore can reduce heat gain to the building and surrounding garden, and decrease the surrounding temperature during the peak time of the day.

Figures 4.35 illustrate the non-strategic location of shade trees and shrubs for the mature landscape house. One result of this study clearly shows that small and low shrubs, and ground cover located close to walls, only provide shade to the nearest underground floors and foundations, while the walls and windows of the house are too exposed to the direct sunlight and can result in temperatures of $37^{\circ} \mathrm{C}$ (Figure 4.20 - east outdoors at the mature landscape house). They also affect the temperatures in interior spaces. The temperatures in the three houses gradually increased with the peak inside temperature delayed by approximately three hours. The warmer outdoor environment resulting from sparse and non-strategic landscaping can directly promote warmer indoor spaces as shown in Table 4.21. In all cases the differences in average temperature between outsides and insides was around $3{ }^{\circ} \mathrm{C}$ due mainly to the mass of the building construction, which was similar for all buildings.

A unique feature of the three houses was that each house had a water element in the garden. Table 4.19 and Table 4.21 show that the ordinary landscape house produced the highest average $\mathrm{RH}$ level of approximately $77 \%$. These levels were influenced by the surrounding landscaping including soft and hard landscape elements. The larger amounts of water at the ordinary landscape house included a swimming pool and a fish pool. These produce higher evaporation than at the other two houses which only had small sized water elements. However sparse and non-strategic placement of vegetation at the east side of the ordinary landscape house produced less plant shading resulting in a high temperature $\left(37^{\circ} \mathrm{C}-\right.$ similar to the mature landscape house and higher than the new landscape house during the peak time of the day). This suggested that high humidity must be combined with plant shading to produce lower temperatures. Finally a moderate wind is needed to circulate and refresh the surrounding air, and furthermore to produce a cool and comfortable environment. Overall the neighbourhood area around the three houses and the nearby green open spaces around the mature and new landscape houses also contributed to the evapotranspiration cooling. 


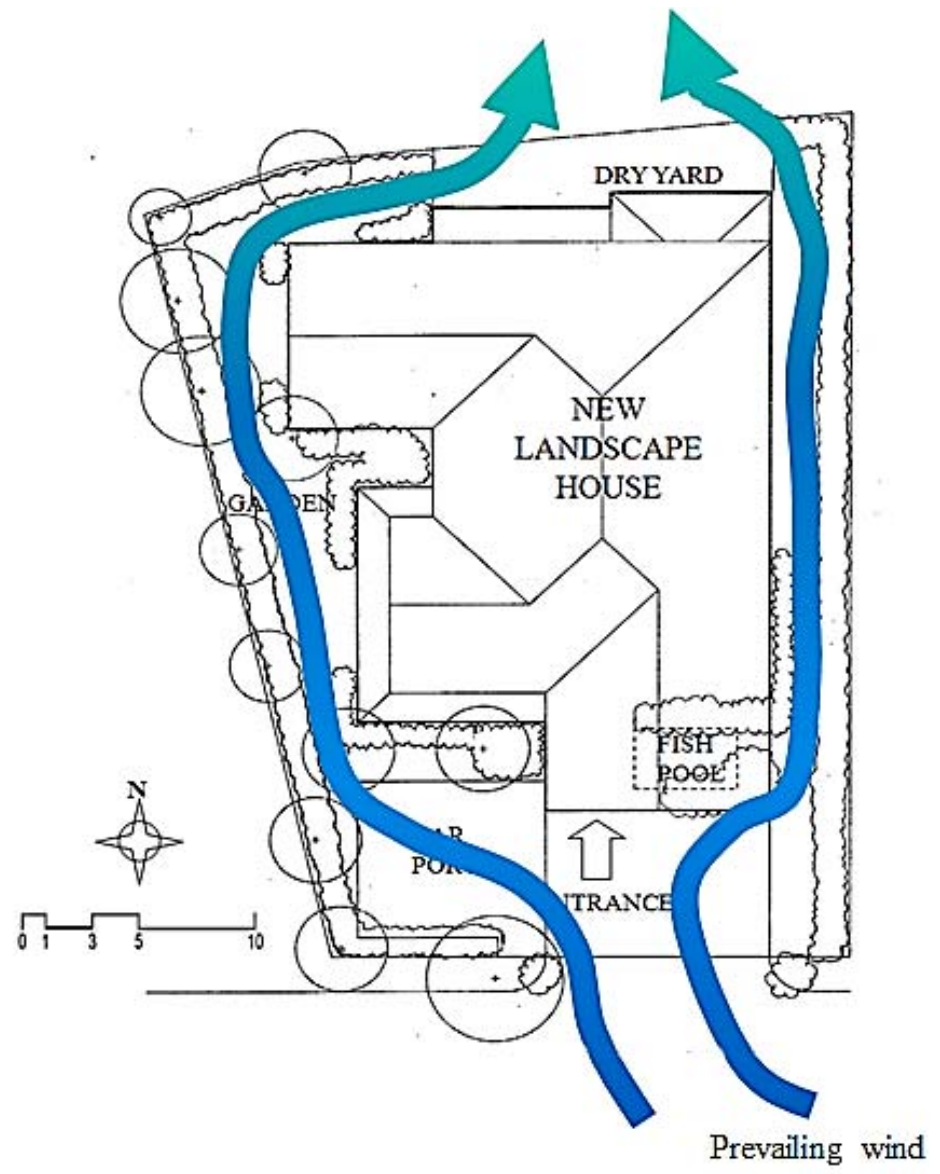

Figure 4.36 The arrangement of trees and shrubs around the new landscape house influences the movement of wind from south open space around the building

The human factors for the three houses were similar in terms of the number of occupants but they had different total cooling energy use, as can be seen in Table 4.21. The average indoor temperature for the three houses was up to $29^{\circ} \mathrm{C}$ showing that the occupants need air-conditioning system to achieve their comfort level in the evening. For example, the two houses with similar living habits, the mature landscape house and the ordinary landscape house used 100\% air-conditioning for their master bedrooms, while the new landscape house used the air-conditioning system for only 3 hours during night time, although the set temperature was low when it was used. At other times the new landscape house used ceiling fan and the passive ventilation system. This reduced cooling energy use as a percentage of total electricity used, although this will also be influence by other occupancy habits. The advantage of the new landscape house is the house is located facing a large open space where the prevailing winds can refresh the surrounding air all the time. The 
strategic position of immature vegetation around the new landscape house provides cool ambient air by channelling wind to the house and its surrounding garden as shown in Figure 4.36.

Overall a strategic placement of vegetation at the east and west sides close to the building is very important to provide shade to the building envelope especially walls and windows. Evapotranspiration cooling was produced by a combination of plants and water elements. The positioning and arrangement of plants around houses should be compatible with prevailing wind directions to promote and channel wind to the appropriate places through and around the house. The combination of shading by plants, evapotranspiration cooling, and compatible moderate wind flow can produce fresh, cool and comfortable environments.

\subsection{Summary}

This case study investigates the construction and surrounding landscaping of three single-family houses aged 30, 10 and 5 years old in Shah Alam, Selangor, Malaysia. The houses were labelled as mature, ordinary and new landscaped houses. The three ages of construction were chosen because they provided different vegetation and landscaping styles, which would have different effects on the thermal performance of the houses through shading, channelling wind and evapotranspiration.

The materials used and construction methods of these houses were similar, following typical styles of tropical architecture, while the design and size of each house varied because they were built in different eras. The façade orientation of the mature and ordinary landscape houses was east or west, while the new landscape house was orientated north to south. Every house had a medium amount and average size of glazed openings that covered an area of approximately $15-24 \%$ compared to the floor area, while roofs were insulated. The albedo values in the houses ranged from 0.12 to 0.42 , with all of the buildings using light-coloured paint for the walls, and slightly different colours for the roof coverings. 
A range of factors influences how effective landscaping can be on the thermal performance of a house. The results of this research show that the strategic location of landscaping to provide shade and reduce heat gain in buildings is of primary concern. The east and west sides of the houses were the main areas to protect from direct solar radiation during morning and afternoon, while the north and south areas needed protection from diffuse solar radiation. The size and amount of plants, as well as their lushness and condition, were also important factors, as these can affect the ability of the plants to provide shade and promote the evapotranspiration process. The different styles of landscaping in this case study affected the channelling of wind flow into each house. Neighbouring houses and the open green spaces around them also had an influence on the amount of prevailing wind and evapotranspiration cooling that affected each house. At the mature landscape house, greater evapotranspiration, combined with sufficient shade to the building envelope, and moderate wind flow helped to provide cool and comfortable ambient air as much as $3^{\circ} \mathrm{C}$ lower than at the other houses around mid-day. However, the temperature for the three houses appeared to be similar in the afternoon because the non-strategic location of vegetation around this house could not maintain the low temperature throughout the day.

The next chapter will compare two individual single-family houses that have similar styles of building construction, but different amounts and locations of surrounding landscaping. 


\subsection{THE EFFECTS OF LANDSCAPING ON THE OUTDOOR TEMPERATURE AROUND A SINGLE-FAMILY HOUSE}

\subsection{Overview}

In the previous chapter, case study 1 showed how the different surrounding landscaping of three single-family houses aged 30,10 and 5 years old only produced slight differences in the outdoor and indoor air temperatures of the houses. The landscaping at the three houses represented typical styles of landscaping used for single-family houses in Malaysia. In this chapter case study 2 will continue to investigate the effect of vegetation on modifying outdoor temperatures by looking at two single-family houses in a hot-humid climate that have significant differences in the amount of surrounding landscaping.

In this case study, two private houses in a low-density development of single-family homes in Precinct 14, Putrajaya, Malaysia were investigated. They were representative of contemporary and modern building and landscape design for medium-sized houses in Malaysia. Their climatic parameters and physical characteristics, including their building construction, size and configuration, were measured and surveyed to test the effects of landscaping on modifying their indoor and outdoor temperatures. While the two houses were similar, they had significant differences in their surrounding landscaping. They were labelled as a heavily landscaped house and a sparsely landscaped house. This case study differed from case study 1, where the three different houses had all used a moderate amount of landscaping.

This study focused on the impact of shade trees and different types of foliage on the outdoor temperature of the houses. In tropical climates vegetation can significantly affect the microclimate around a house. Trees, grass, shrubs, groundcover, and turf will reduce air temperatures near the house, and provide evaporative cooling. In addition to the obvious use of trees to provide shade, if they are properly placed they can channel breezes towards the house. Shrubs and other low growing foliage can provide shade to a house during the morning and late afternoon when the sun is low 
in the sky, and vines can provide shade to the building's walls. Through careful landscape planning these factors can be optimised. This study will show how this can be done, by demonstrating how different amounts and strategic locations of vegetation can help provide a cool and comfortable environment. Weather data was recorded using instruments that were placed in several exterior and interior locations around the houses.

\subsection{House study}

The area studied was in Putrajaya, in the Federal Territory Putrajaya, Malaysia. Its elevation is around $80 \mathrm{~m}$, at $2^{\circ} \mathrm{N}$ Latitude and $101^{\circ} \mathrm{E}$ Longitude. This is in the equatorial doldrums area, so Putrajaya has a hot-humid climate.

In this study area, the approach that had been taken was to conserve as best as possible the natural environment, and allow the buildings to blend with the existing site characteristics (PJH, 2001). Landscape architecture and design plays an important role in providing a cool and comfortable indoor and outdoor space.

The Chief Architect at the Architecture Department, Putrajaya Corporation was interviewed to determine potential study houses for this investigation. The houses chosen were two single-family residences in Precinct 14, as can be seen in Figure 5.1. This residential development is comprised of 27 single-family houses located in a well planned community area (Malek, 2011). These houses represent approximately $15 \%$ of the overall development together with some semi-detached bungalow houses.

This area is a low density development, which is surrounded by wide areas of green, open space, is located on gentle slopes and has been planted with grass lawn and immature tropical garden trees. Roadside trees, turf, and shrubs have been planted at the boundary of the properties by the developer. The houses are approximately three years old. 


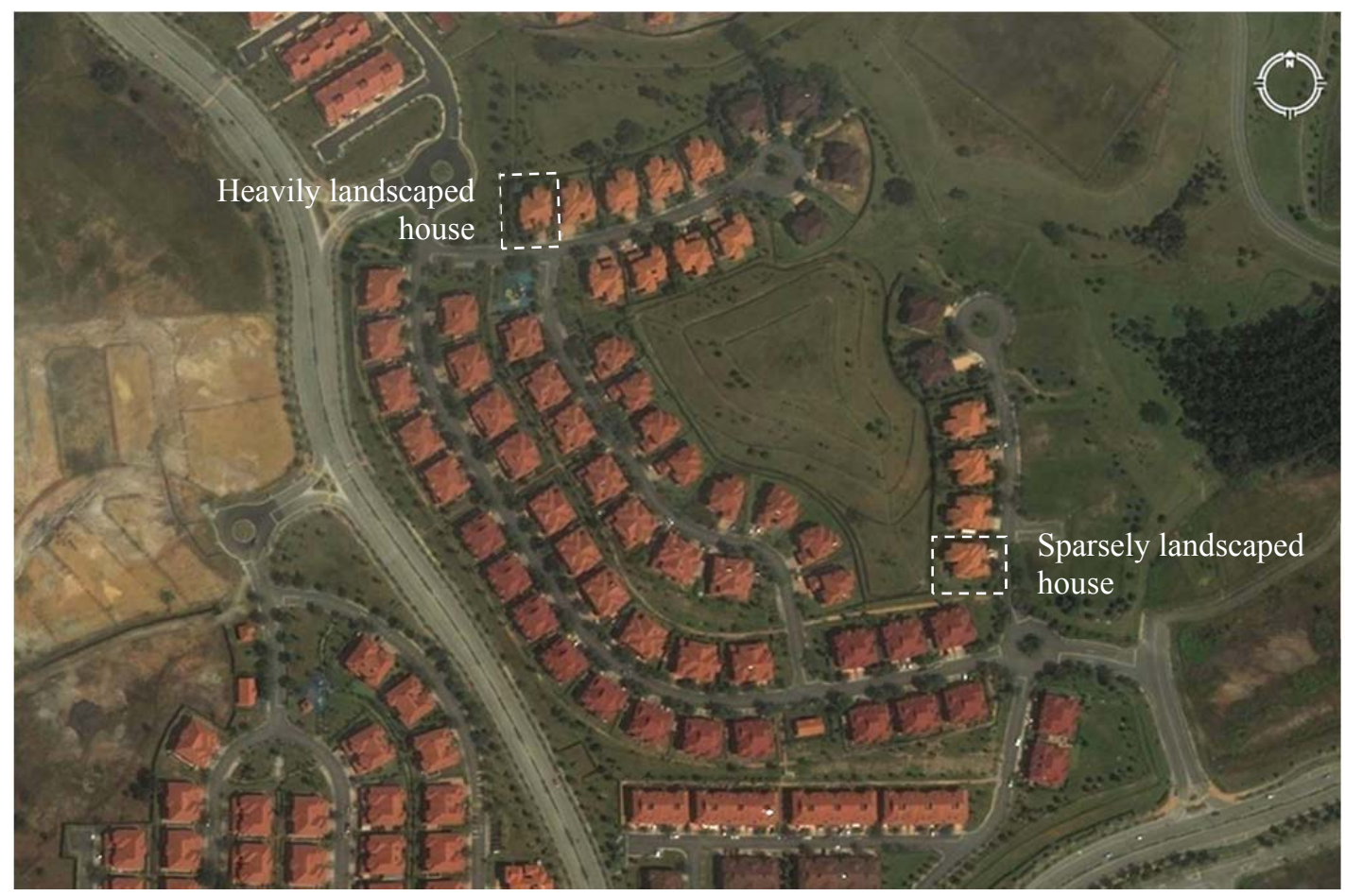

Figure 5.1. Location of two single-family residences in Precinct 14, Putrajaya (Google Earth, 2011)

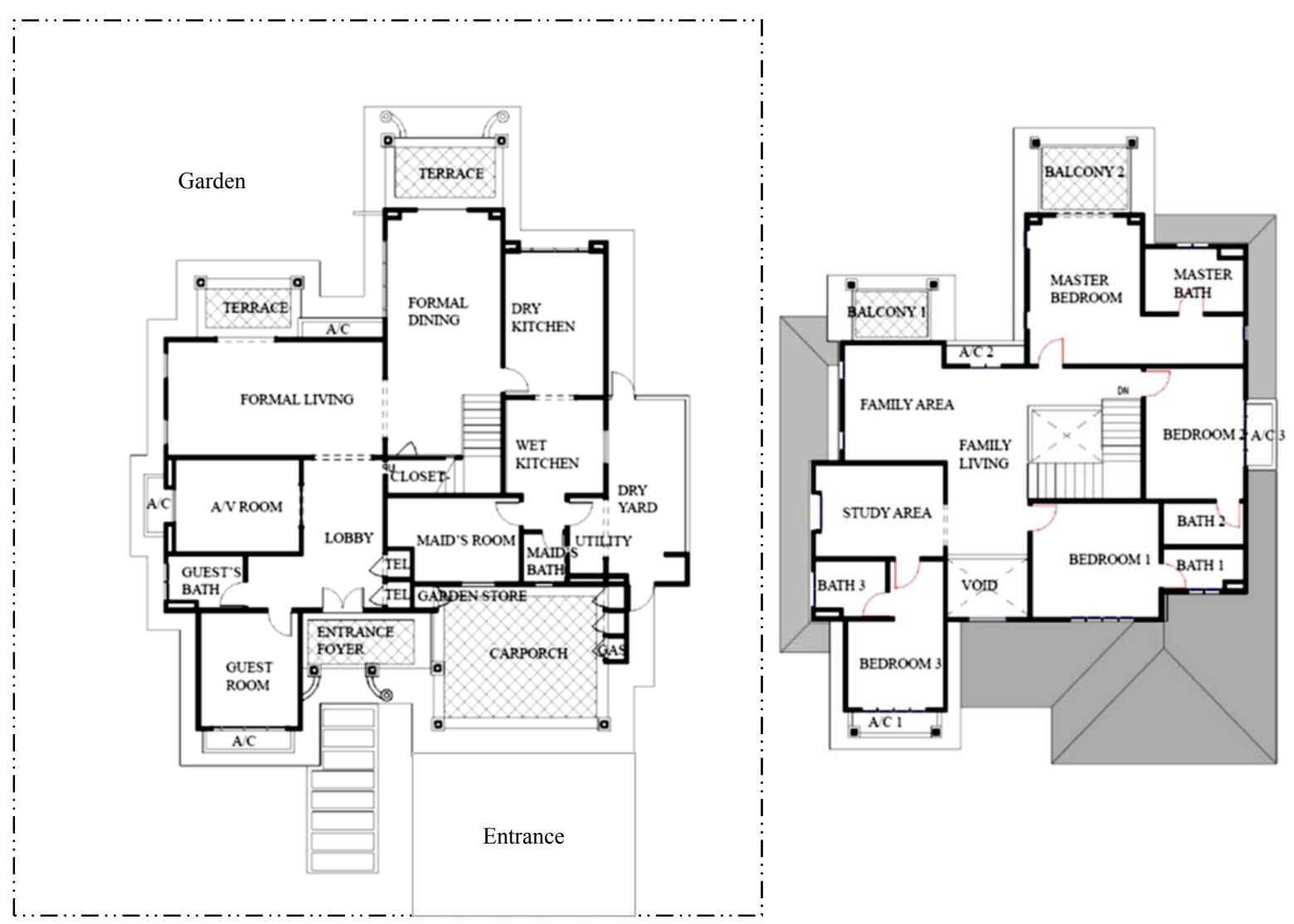

Figure 5.2. General ground floor (left) and first floor plan (right) of the two houses 
The two houses in this study were chosen because they were both medium sized, and built to a typical design for modern tropical architecture. They had similar standards of construction, light coloured walls and roof, and were located at the end of their streets, but they clearly had differences in their landscape treatments. The heavily landscaped house was surrounded by various types of plants strategically placed around the house and garden, while the sparsely landscaped house had fewer plants, with most located in non-strategic locations. The two types of landscape treatments were expected to produce significantly different effects on the ambient air around the houses, as well as their thermal performance and energy use.
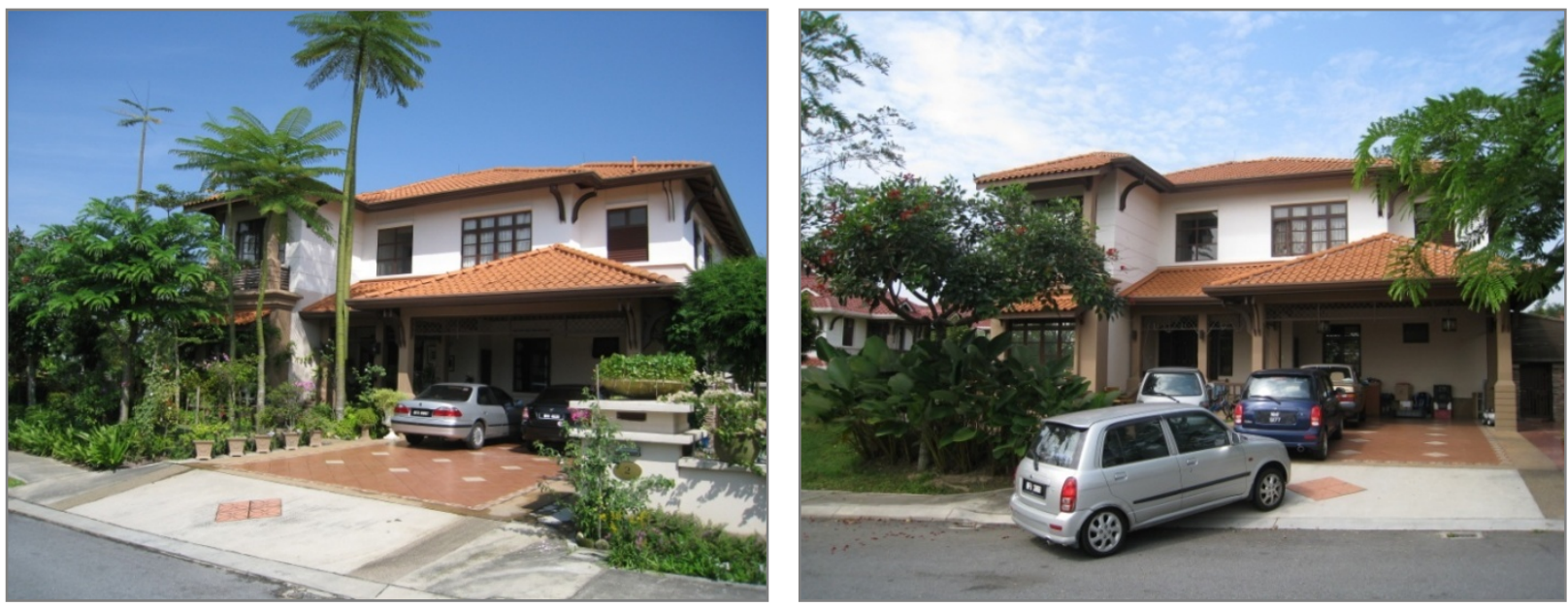

Figure 5.3. View of the front/south elevation of heavily landscaped house (left) and the front/east elevation of the sparsely landscaped house (right)
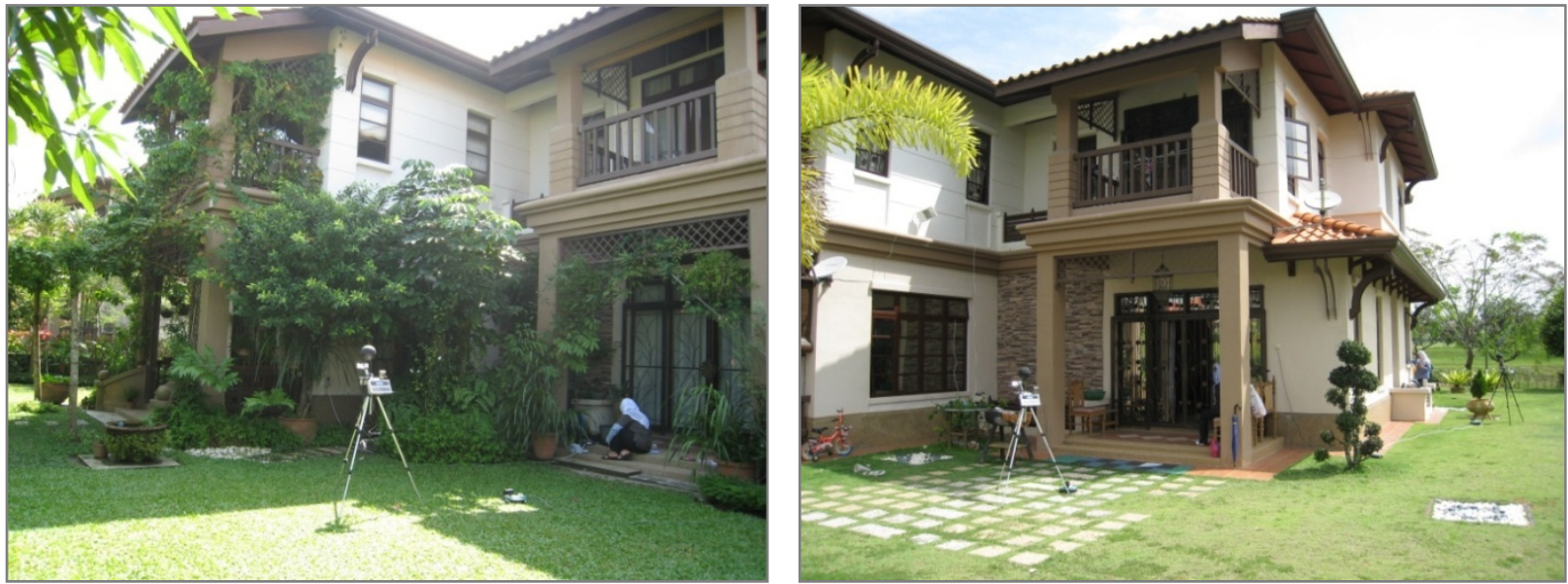

Figure 5.4. View of back/north elevation of the heavily landscaped house (left) and back/west elevation of the sparsely landscaped house (right) 

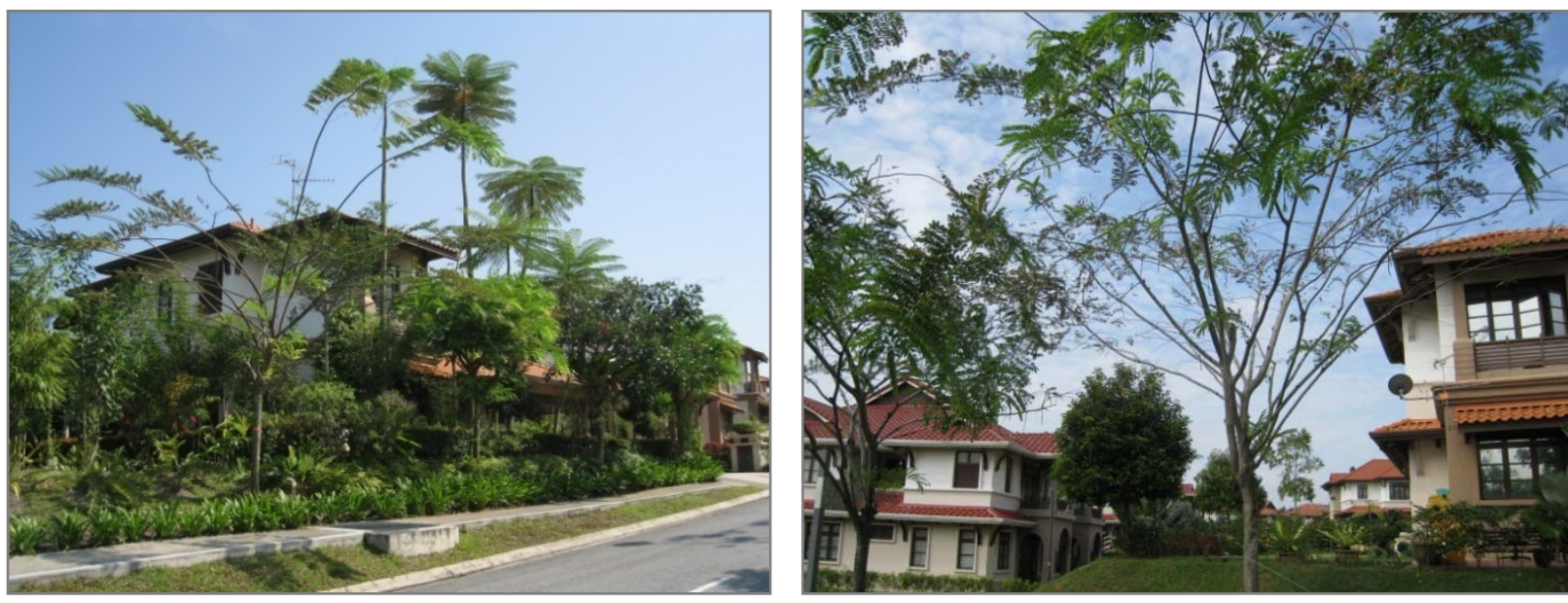

Figure 5.5. Views of the west garden of the heavily landscaped house (left) and the south garden of the sparsely landscaped house (right)

\subsection{General weather data}

Two years of climate data (2008-2009) was obtained from Sepang Airport Weather Station, which is near to Precinct 14. Malaysia is a hot-humid climate, so its weather characteristics include uniform temperature, high humidity, and copious amounts of rainfall in two main seasons (MMD, 2010). The dry season, with its southwest monsoon starts in the latter half of May or early June, and ends in September. Its prevailing wind is generally a moderate south and south-easterly, for $41 \%$ of the time. The northeast monsoon comes with the rainy season in early November, and ends in March. Steady east or north-easterly winds prevail for 38\% of the time. During the two shorter inter-monsoon seasons, the winds were generally light and variable.

In 2008-09 precipitation was very heavy throughout the year, with an annual average of about $2100 \mathrm{~mm}$. Solar radiation was abundant, with about six to eight hours of

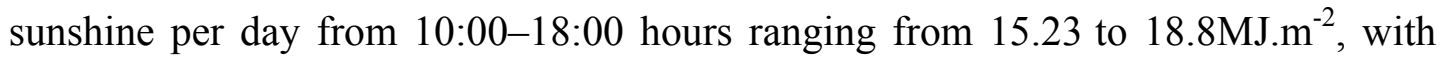
6.9 Okta of cloud cover. The averages in the dry and rainy seasons are shown in Table 5.1. Temperatures usually range from $23.8-32.2^{\circ} \mathrm{C}$, and relative humidity is between 75.1 and $84.4 \%$, which combine to produce extremely hot-humid weather.

In areas such as this, where there are high temperatures and relative humidity, and low wind speed, the best way to modify the microclimate is by reducing the amount 
of heat gained from solar radiation. Direct solar radiation should be kept out of the building and off its exterior surfaces to achieve low interior temperatures.

Table 5.1. Average daily maximum and minimum temperatures, relative humidity and wind for January, April, July and October in Putrajaya 2008-2009

\begin{tabular}{|c|c|c|c|c|c|}
\hline \multirow[t]{2}{*}{ Month } & \multicolumn{2}{|c|}{ Temperature $\left({ }^{\circ} \mathrm{C}\right)$} & \multicolumn{2}{|l|}{ Wind } & \multirow{2}{*}{$\begin{array}{l}\text { Relative } \\
\text { Humidity } \\
(\%)\end{array}$} \\
\hline & $\begin{array}{l}\text { Daily } \\
\text { Maximum }\end{array}$ & $\begin{array}{l}\text { Daily } \\
\text { Minimum }\end{array}$ & Direction & $\begin{array}{l}\text { Frequency } \\
\text { (\% of the time) }\end{array}$ & \\
\hline January & 31.90 & 23.80 & $\mathrm{NE}$ & 20.70 & 75.10 \\
\hline April & 32.20 & 24.10 & $\mathrm{~N}$ & 17.90 & 82.30 \\
\hline July & 31.50 & 24.00 & $\mathrm{SE}$ & 22.30 & 82.20 \\
\hline October & 31.70 & 24.20 & $\mathrm{~N}$ & 21.80 & 84.40 \\
\hline
\end{tabular}

\subsection{Fieldwork measurement period}

Data collection and experimental measurements were carried out on the $16^{\text {th }}$ and $17^{\text {th }}$ of January 2010. Data collection involved building, landscaping and air-conditioning system information while the experimental measurements were taken near wall areas where there were various combinations of trees, shrubs, vines, groundcovers, and turf. The procedure used to take these measurements involved continually recording the air temperature, humidity and wind velocity for all azimuths inside and outside the buildings. Information on solar radiation and cloud cover was obtained from the nearby weather station.

\subsection{Analysis}

The information gathered about the two houses' building construction included house configuration, size, general construction and materials for the floors, walls and roof. The building envelope areas were also recorded, and included wall and roof areas, and all windows and doors. Information gathered on the interior of the houses included interior spaces, ceiling heights and types.

For each of the houses their tropical landscape design was conceived and developed by the owners. They planted tropical plants that serve multiple functions: aesthetic, edible, to provide shade, and to produce a pleasant, comfortable and healthy environment. Their landscaping incorporated every type of vegetation structure 
including trees, palms, bamboos, shrubs, vines, groundcovers and turf. They also used different amounts, species, and configurations of vegetation. Aside from $30 \mathrm{~m}^{2}$ of tiles from the road straight to the porch of the house, the rest of the garden is a green area.

\subsubsection{Building construction}

The houses were medium-sized, single-family homes that are examples of typical Malaysian tropical architecture, as shown in Figure 5.2. They are both aged approximately three years old. Figure 5.1 shows the differences in house configuration. The heavily landscaped house faces south, with its main outdoor area at the rear of the house to the north. The sparsely landscaped house faces east, with its garden on the west side. Both houses are two storeys, medium sized, and were similar in size $\left(360 \mathrm{~m}^{2}\right)$ and garden area $\left(350 \mathrm{~m}^{2}\right)$. They are also built with similar building materials and construction techniques. The main structure was built on reinforced concrete pad foundation $\left(\mathrm{R}-1.7 \mathrm{~m}^{2} .{ }^{\circ} \mathrm{C} / \mathrm{W}\right)$, and had bricks walls (R$\left.1.3 \mathrm{~m}^{2} .{ }^{\circ} \mathrm{C} / \mathrm{W}\right)$, and a pitched timber-framed roof covered by concrete tiles $\left(225 \mathrm{~m}^{2}\right)$ with an insulation layer of aluminium foil paper underneath $\left(\mathrm{R}-1.7 \mathrm{~m}^{2} .{ }^{\circ} \mathrm{C} / \mathrm{W}\right)$.

Table 5.2. Total area of brick walls and glazed surfaces for the heavily landscaped house (house A) and sparsely landscaped house (house B)

\begin{tabular}{lllll}
\hline Azimuth & Wall $\left(\mathrm{m}^{2}\right)$ & \multicolumn{3}{l}{ Glass $\left(\mathrm{m}^{2}\right)$} \\
\cline { 2 - 5 } & House A & House B & House A & House B \\
\hline North & 57.83 & 83.76 & 29.77 & 07.05 \\
East & 83.76 & 72.75 & 07.05 & 15.24 \\
South & 72.75 & 99.95 & 15.24 & 14.85 \\
West & 99.95 & 57.83 & 14.85 & 29.77 \\
\hline Total & 314.29 & 314.29 & 66.91 & 66.91 \\
\hline
\end{tabular}

Both houses had the same coloured building envelope, with cream walls (albedo 0.25 ) and a light orange roof (albedo 0.33). All casement windows and sliding doors on each house were made from $3 \mathrm{~mm}$ thick single-layer glass, with metal frame (R$0.13 \mathrm{~m}^{2} .{ }^{\circ} \mathrm{C} / \mathrm{W}$ ), which makes up $21 \%$ of their surface areas, as shown in Figures $5.3-$ 5.5 . 
Shading devices are used over all windows and doors, there is a hip roof overhang of at least $1 \mathrm{~m}$, and there is a $2-3 \mathrm{~m}$ wide veranda over the sliding doors and entrance door. A porch covered the entrance of the house, which provides shading for the windows and doors facing this area. These features provided shade for windows, doors, and sliding doors during the morning and until afternoon. All external doors were made from solid hardwood $\left(\mathrm{R}-0.34 \mathrm{~m}^{2} .{ }^{\circ} \mathrm{C} / \mathrm{W}\right)$. Ceiling heights were $2.9-3 \mathrm{~m}$, and were finished by fibrous plaster, cement plaster, and asbestos free fibre cement ceiling sheets. Table 5.2 shows the areas of plastered brick walls, and glass for windows and sliding doors for the four azimuths of the two houses.

\subsubsection{Landscape design}

The landscape surrounding the two houses used a combination of trees, shrubs and vines, groundcover and turf. The heavily landscaped house had a lush, green garden, which had 47 types of trees, 104 individual and groups of shrubs, 10 areas with vines, and 10 areas of groundcover. This is illustrated in Figure 5.6. The sparsely landscaped house had only 11 trees and 36 individual and groups of shrubs, which can be seen in Figure 5.7. The lawns at both houses were covered by Axonopus compressus.

\subsubsection{Trees}

Trees are the most important plants in the study area for providing shade to the building and garden areas. Figures 5.3-5.5 show the heavily landscaped house has a diverse range of trees. All were planted 3-9m from the building in groups, so their canopies touched and overlapped, as shown in Figure 5.6. They covered approximately $60 \%$ of the garden area. 


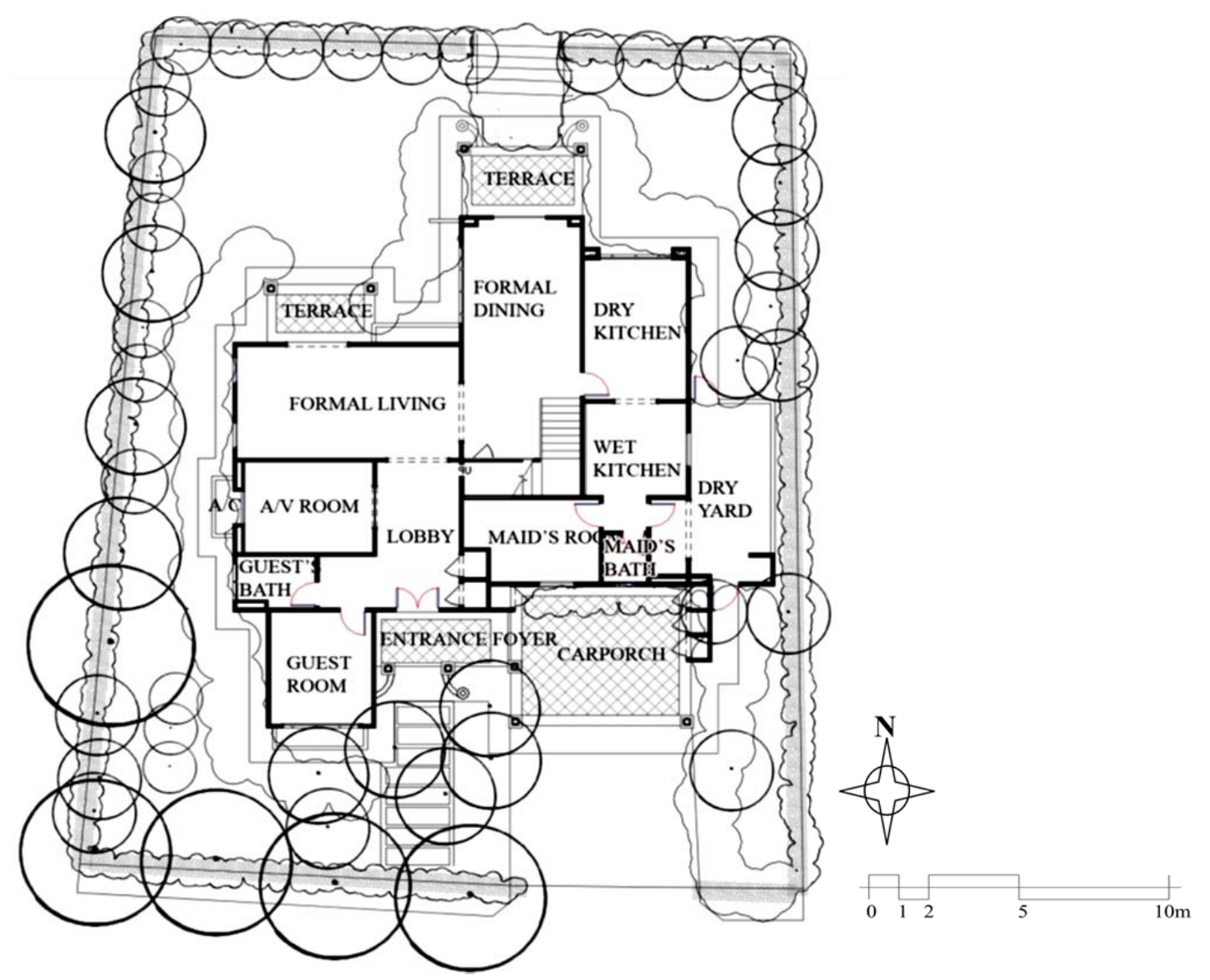

Figure 5.6. Landscape plan for the heavily landscaped house 


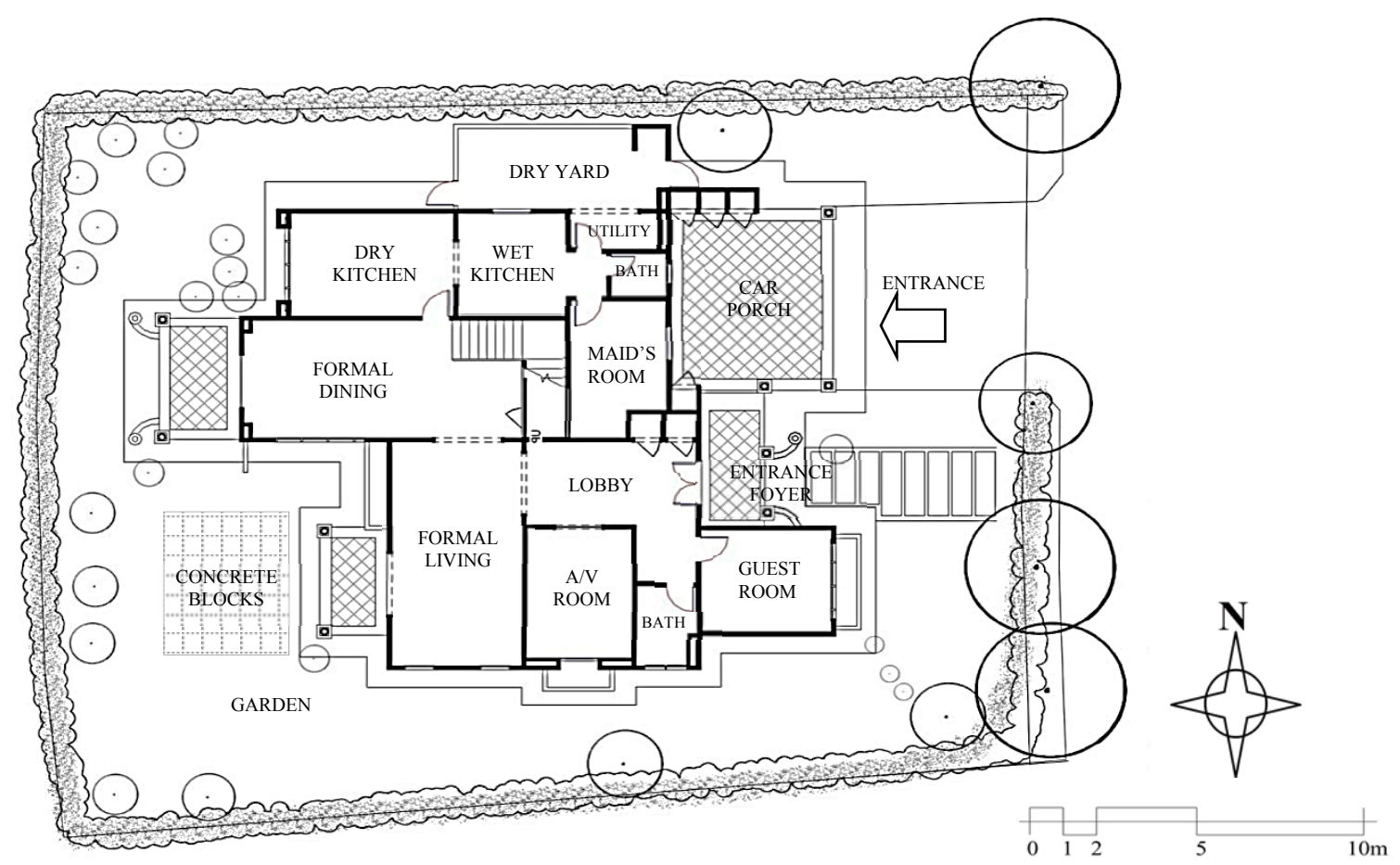

Figure 5.7. Landscape plan for the sparsely landscaped house

Most of the trees at the heavily landscaped house (89\%) were still young (three years), with their canopy sizes below $3 \mathrm{~m}$ (small), 9\% were small to moderately sized $(3-7 \mathrm{~m})$, and $2 \%$ were medium sized. This is shown in Figure 5.8 and Table 5.3. The garden did not have many different species of trees, but they were all native evergreen tropical species, which can survive in annual dry and rainy seasons.

The majority were in the edible fruit and garden tree categories $(36 \%$ and $34 \%$ respectively). There were also a small amount of palms (19\%), 9\% were roadside trees and $2 \%$ were bamboo trees. Fruit trees are commonly planted around traditional Malay houses. This was a reason the owner of the house decided to plant these types of trees, as well as to use the fruits produced. Most of the trees, around 18, were planted on the west side, while the south and north sides had 12 trees each. Only five trees were planted on the east side.

Trees with a sufficient trunk height will allow wind to flow to the garden and building surfaces. Trunk height combined with a good canopy size and shape can 
also provide shade to the garden and nearby buildings. The average trunk height of trees in this garden was about $1.5 \mathrm{~m}$ and their overall height was $2-4 \mathrm{~m}$. However, a few trees such as Dalbergia oliveri had an overall height of around 9m, and small groups of Schizolobium parahyba can reach more than $15 \mathrm{~m}$ high.

The most common shaped trees around the heavily landscaped house were spreading and fountain shapes (35\% of the trees, and 30\% respectively), followed by $26 \%$ round shaped, and finally $9 \%$ were columnar shaped. The amount and size of leaves are important to determine because they influence the ability of plants to produce shade and channel wind. The majority of trees in this garden have a medium amount of leaves (40\%), while $30 \%$ of the trees had few or a dense amount of leaves respectively. Trees with small sized leaves were dominant in this garden (64\%) and the balance was medium sized (36\%) leaves. The sparsely landscaped house had a small number of plant species, which are listed in Table 5.4. The trees were located $5-9 \mathrm{~m}$ from the house, and $73 \%$ of the tree canopy was small in size and $27 \%$ small to moderately-sized. The tree canopy only covered around 5\% of the garden's surfaces and was focused mostly on the front elevation on the east side of the house. The overall amount of trees was less than one-quarter of that around the heavily landscaped house.

Samanea saman and Erythrina fusca were planted along the roadside by the developer. These plants look lush when they are small to moderately sized, and have a spreading shape, as shown in Figures 5.3-5.5. The three garden trees planted on the south side of the house were round and columnar in shape, and the four Wodyetia bifurcate on the west side were small and had a fountain shape. The majority of the trees $(64 \%)$ had an overall height of around $2-4 \mathrm{~m}$, and the remaining $36 \%$ were between $5-9 \mathrm{~m}$ from the house, with trunk heights around $2-2.5 \mathrm{~m}$.

On average the trees had a sparse amount of leaves that were small. However, three of the trees had a dense amount of leaves, and one tree had a medium amount of leaves. The canopy size of the garden trees and palms in this garden (B) look smaller 
and less fertile compared to the heavily landscaped house's garden (A) because the trees have been planted a long distance away from each other.

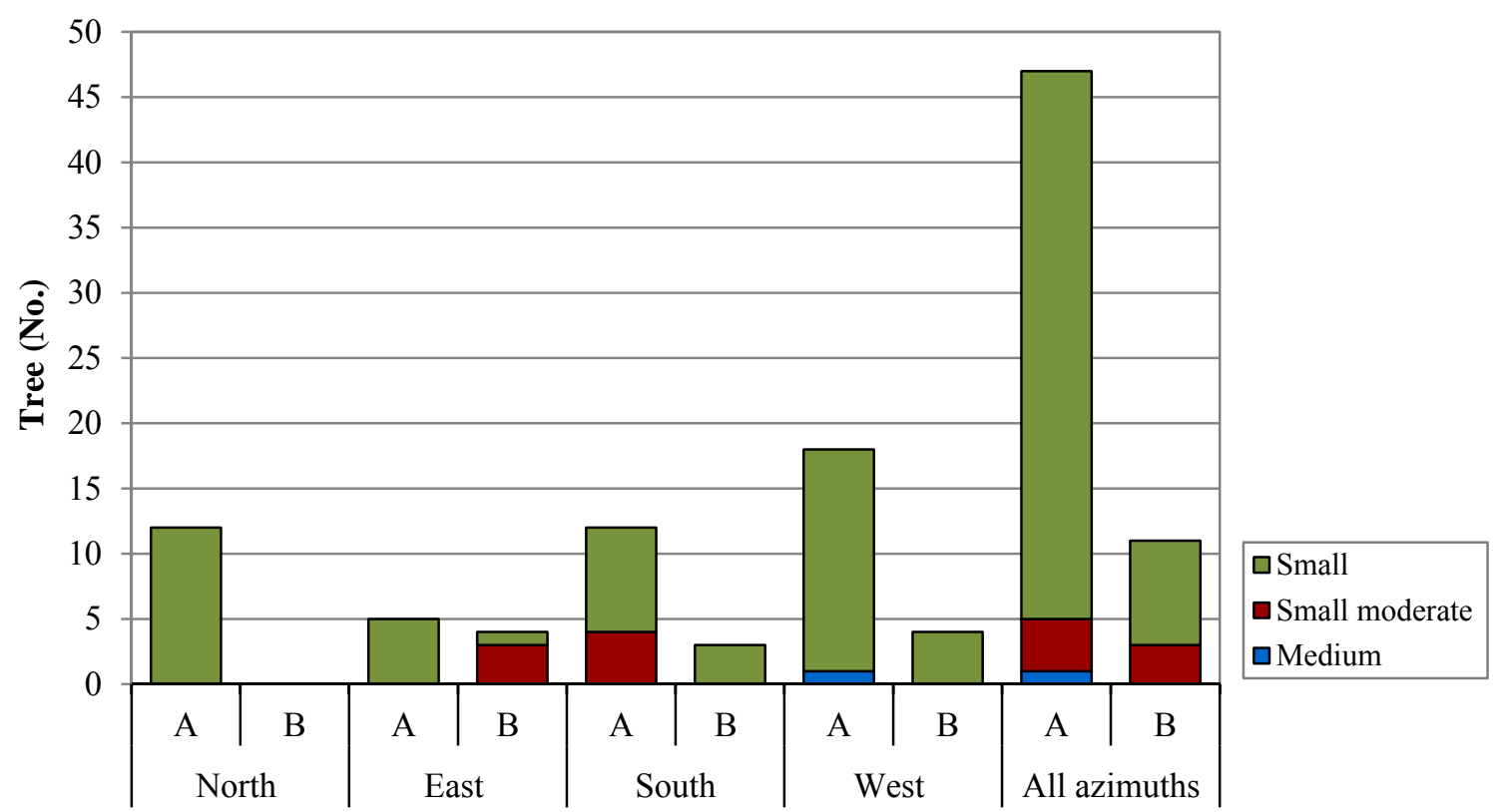

Azimuth

Figure 5.8. Trees distribution compared by tree sizes and tree azimuth in relation to the building for the heavily landscaped house (A) and sparsely landscaped house

(B)

Table 5.3. Tree-growth indices at the heavily landscaped house

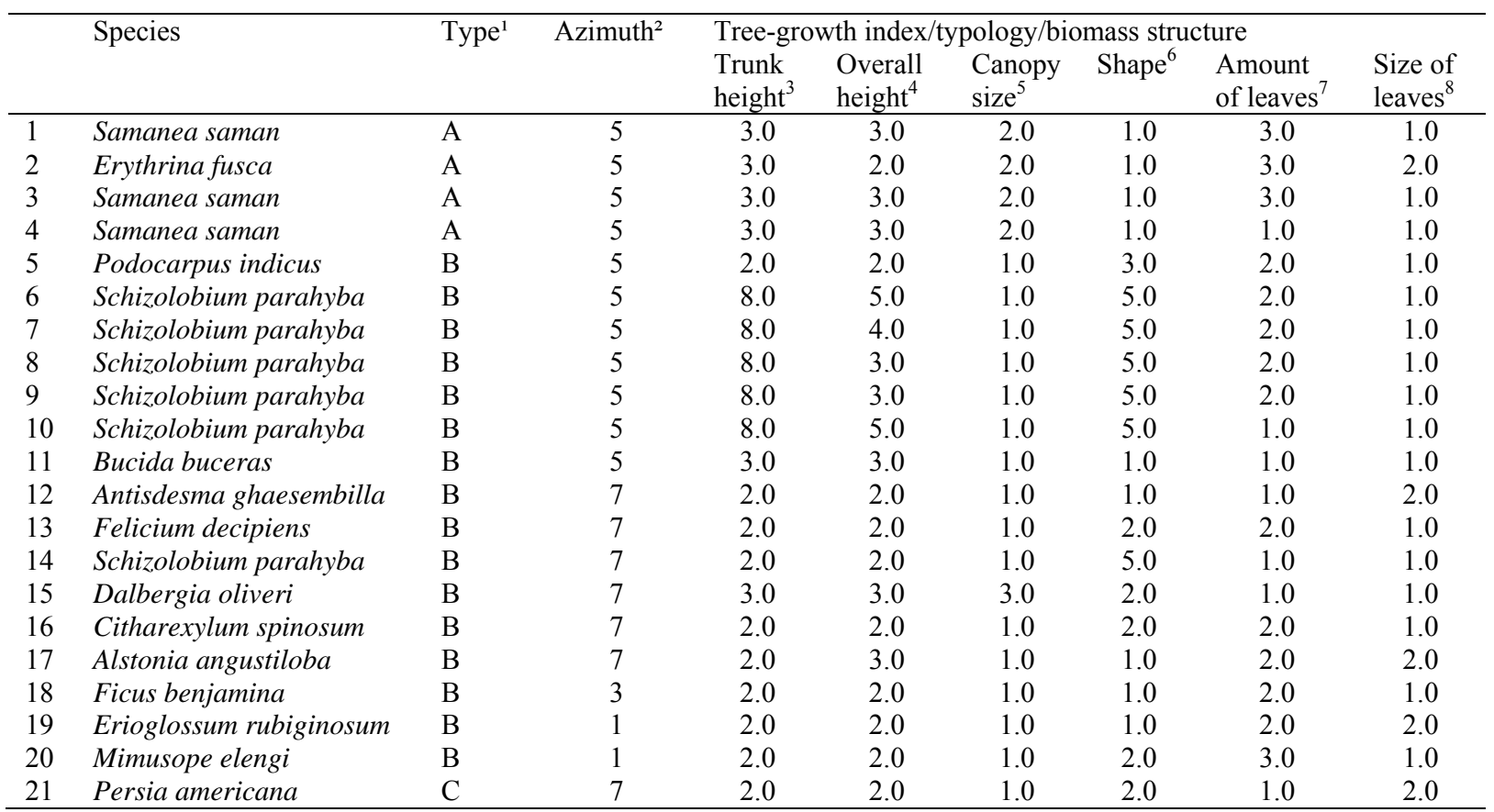




\begin{tabular}{|c|c|c|c|c|c|c|c|c|c|}
\hline 22 & Eugenia malaccensis & $\mathrm{C}$ & 7 & 2.0 & 2.0 & 1.0 & 2.0 & 1.0 & 2.0 \\
\hline 23 & Lansium domesticum & $\mathrm{C}$ & 7 & 2.0 & 2.0 & 1.0 & 3.0 & 2.0 & 1.0 \\
\hline 24 & Persia americana & $\mathrm{C}$ & 7 & 2.0 & 2.0 & 1.0 & 2.0 & 1.0 & 2.0 \\
\hline 25 & Nephelium lappaceum & $\mathrm{C}$ & 7 & 2.0 & 2.0 & 1.0 & 1.0 & 1.0 & 2.0 \\
\hline 26 & Castanea sativa & $\mathrm{C}$ & 7 & 2.0 & 2.0 & 1.0 & 1.0 & 1.0 & 2.0 \\
\hline 27 & Syzygium cumini & $\mathrm{C}$ & 7 & 2.0 & 2.0 & 1.0 & 1.0 & 1.0 & 2.0 \\
\hline 28 & Flacourtia Jagomas & $\mathrm{C}$ & 7 & 2.0 & 2.0 & 1.0 & 1.0 & 1.0 & 1.0 \\
\hline 29 & Artocarpus integer & $\mathrm{C}$ & 3 & 2.0 & 2.0 & 1.0 & 3.0 & 2.0 & 2.0 \\
\hline 30 & Morinda citrifolia & $\mathrm{C}$ & 3 & 2.0 & 2.0 & 1.0 & 3.0 & 3.0 & 2.0 \\
\hline 31 & Tamarindus indica & $\mathrm{C}$ & 3 & 3.0 & 2.0 & 1.0 & 1.0 & 1.0 & 1.0 \\
\hline 32 & Phyllanthus acidus & $\mathrm{C}$ & 3 & 3.0 & 2.0 & 1.0 & 1.0 & 2.0 & 1.0 \\
\hline 33 & Mangifera indica & $\mathrm{C}$ & 1 & 2.0 & 2.0 & 1.0 & 2.0 & 3.0 & 2.0 \\
\hline 34 & Mangifera indica & $\mathrm{C}$ & 1 & 2.0 & 2.0 & 1.0 & 2.0 & 3.0 & 2.0 \\
\hline 35 & Garcinia mangostana & $\mathrm{C}$ & 1 & 2.0 & 2.0 & 1.0 & 2.0 & 3.0 & 2.0 \\
\hline 36 & Mangifera indica & $\mathrm{C}$ & 1 & 2.0 & 2.0 & 1.0 & 2.0 & 3.0 & 2.0 \\
\hline 37 & Anacardium occidentale & $\mathrm{C}$ & 1 & 2.0 & 2.0 & 1.0 & 1.0 & 2.0 & 2.0 \\
\hline 38 & Wodyetia bifurcata & $\mathrm{D}$ & 7 & 2.0 & 2.0 & 1.0 & 5.0 & 2.0 & 1.0 \\
\hline 39 & Wodyetia bifurcata & $\mathrm{D}$ & 7 & 2.0 & 2.0 & 1.0 & 5.0 & 2.0 & 1.0 \\
\hline 40 & Wodyetia bifurcata & $\mathrm{D}$ & 7 & 2.0 & 2.0 & 1.0 & 5.0 & 2.0 & 1.0 \\
\hline 41 & Wodyetia bifurcata & $\mathrm{D}$ & 7 & 2.0 & 2.0 & 1.0 & 5.0 & 2.0 & 1.0 \\
\hline 42 & Wodyetia bifurcata & $\mathrm{D}$ & 1 & 2.0 & 2.0 & 1.0 & 5.0 & 3.0 & 1.0 \\
\hline 43 & Wodyetia bifurcata & $\mathrm{D}$ & 1 & 2.0 & 2.0 & 1.0 & 5.0 & 3.0 & 1.0 \\
\hline 44 & Wodyetia bifurcata & $\mathrm{D}$ & 1 & 2.0 & 2.0 & 1.0 & 5.0 & 3.0 & 1.0 \\
\hline 45 & Veitchia merillii & $\mathrm{D}$ & 1 & 2.0 & 2.0 & 1.0 & 5.0 & 3.0 & 1.0 \\
\hline 46 & Cocos nucifera & $\mathrm{D}$ & 1 & 1.0 & 2.0 & 1.0 & 5.0 & 2.0 & 1.0 \\
\hline \multirow[t]{3}{*}{47} & Bambusa vulgaris & $\mathrm{E}$ & 5 & 2.0 & 2.0 & 1.0 & 2.0 & 3.0 & 1.0 \\
\hline & Total & & & 131.0 & 110.0 & 53.0 & 127.0 & 94.0 & 64.0 \\
\hline & Average & & & 2.80 & 2.30 & 1.10 & 2.70 & 2.00 & 1.40 \\
\hline
\end{tabular}

Table 5.4. Tree-growth indices at the sparsely landscaped house

\begin{tabular}{|c|c|c|c|c|c|c|c|c|c|}
\hline & Species & Type $^{1}$ & Azimuth $^{2}$ & Tree-gr & th index/t & ology/bic & lass struc & & \\
\hline & & & & $\begin{array}{l}\text { Trunk } \\
\text { height }^{3}\end{array}$ & $\begin{array}{l}\text { Overall } \\
\text { height }^{4}\end{array}$ & $\begin{array}{l}\text { Canopy } \\
\text { size }^{5}\end{array}$ & Shape ${ }^{6}$ & $\begin{array}{l}\text { Amount } \\
\text { of leaves }\end{array}$ & $\begin{array}{l}\text { Size of } \\
\text { leaves }\end{array}$ \\
\hline 1 & Samanea saman & $\mathrm{A}$ & 3 & 3.0 & 3.0 & 2.0 & 1.0 & 3.0 & 1.0 \\
\hline 2 & Samanea saman & A & 3 & 3.0 & 3.0 & 2.0 & 1.0 & 1.0 & 1.0 \\
\hline 3 & Samanea saman & A & 3 & 3.0 & 3.0 & 2.0 & 1.0 & 1.0 & 1.0 \\
\hline 4 & Erythrina fusca & A & 3 & 3.0 & 2.0 & 1.0 & 1.0 & 1.0 & 2.0 \\
\hline 5 & Mimusope elengi & $\mathrm{B}$ & 5 & 3.0 & 2.0 & 1.0 & 2.0 & 3.0 & 1.0 \\
\hline 6 & Mimusope elengi & $\mathrm{B}$ & 5 & 3.0 & 2.0 & 1.0 & 2.0 & 3.0 & 1.0 \\
\hline 7 & Fagraea fragrans & $\mathrm{B}$ & 5 & 4.0 & 3.0 & 1.0 & 3.0 & 1.0 & 1.0 \\
\hline 8 & Wodyetia bifurcata & $\mathrm{D}$ & 7 & 3.0 & 2.0 & 1.0 & 5.0 & 1.0 & 1.0 \\
\hline 9 & Wodyetia bifurcata & $\mathrm{D}$ & 7 & 3.0 & 2.0 & 1.0 & 5.0 & 1.0 & 1.0 \\
\hline 10 & Wodyetia bifurcata & $\mathrm{D}$ & 7 & 3.0 & 2.0 & 1.0 & 5.0 & 1.0 & 1.0 \\
\hline 11 & Wodyetia bifurcata & $\mathrm{D}$ & 7 & 3.0 & 2.0 & 1.0 & 5.0 & 1.0 & 1.0 \\
\hline & Total & & & 34.0 & 26.0 & 14.0 & 31.0 & 17.0 & 12.0 \\
\hline & Average & & & 3.10 & 2.40 & 1.30 & 2.80 & 1.50 & 1.10 \\
\hline
\end{tabular}

1 Type of trees; $\mathrm{A}=$ Roadside trees, $\mathrm{B}=$ Garden trees, $\mathrm{C}=$ Edible fruit trees, $\mathrm{D}=\mathrm{Palm}, \mathrm{E}=\mathrm{Bamboo}$ $21=$ North, $3=$ East, $5=$ South, $7=$ West

$341.0=1 \mathrm{~m}, 2.0=1.5 \mathrm{~m}, 3.0=2 \mathrm{~m}, 4.0=2.5 \mathrm{~m}, 5.0=3.0 \mathrm{~m}, 6=3.5 \mathrm{~m}, 7=4.0 \mathrm{~m}, 8=>5 \mathrm{~m}$

$42.0=2-4 \mathrm{~m}, 3.0=5-9 \mathrm{~m}, 4.0=10-14,5.0=15-19 \mathrm{~m}$

$51.0=$ small, $2.0=$ small moderate, $3.0=$ medium

$61.0=$ spreading, $2.0=$ round, $3.0=$ columnar, $5.0=$ fountain

$71.0=$ rare, $2.0=$ medium, $3.0=$ dense

$81.0=$ small, $2.0=$ medium 


\subsubsection{Shrubs, vines and groundcover}

The function of the shrubs, vines and groundcover that were planted around the houses are to blend with the trees, palms and the building to produce a balanced and harmonious landscape design.

The heavily landscaped house had 104 individual and group of shrubs, 10 plantings of vines, and 10 groups of groundcover which can be seen in Figure 5.9. A layer of shrubs surrounding the house was planted as a natural fence or boundary for the property. A group of Hymenocallis littoralis was located on the roadside areas, and a few groups of Acalypha wilkensiana 'cultivar', Baphia nitida, and Eugenia oleina were placed to the left and right sides, and backyard boundaries of the house.

By comparison the shrub population in the sparsely landscaped house was very low, as shown in Figure 5.9, Table 5.6 and Appendix 5.2. Only 34 individual and groups of shrubs were planted around the house.

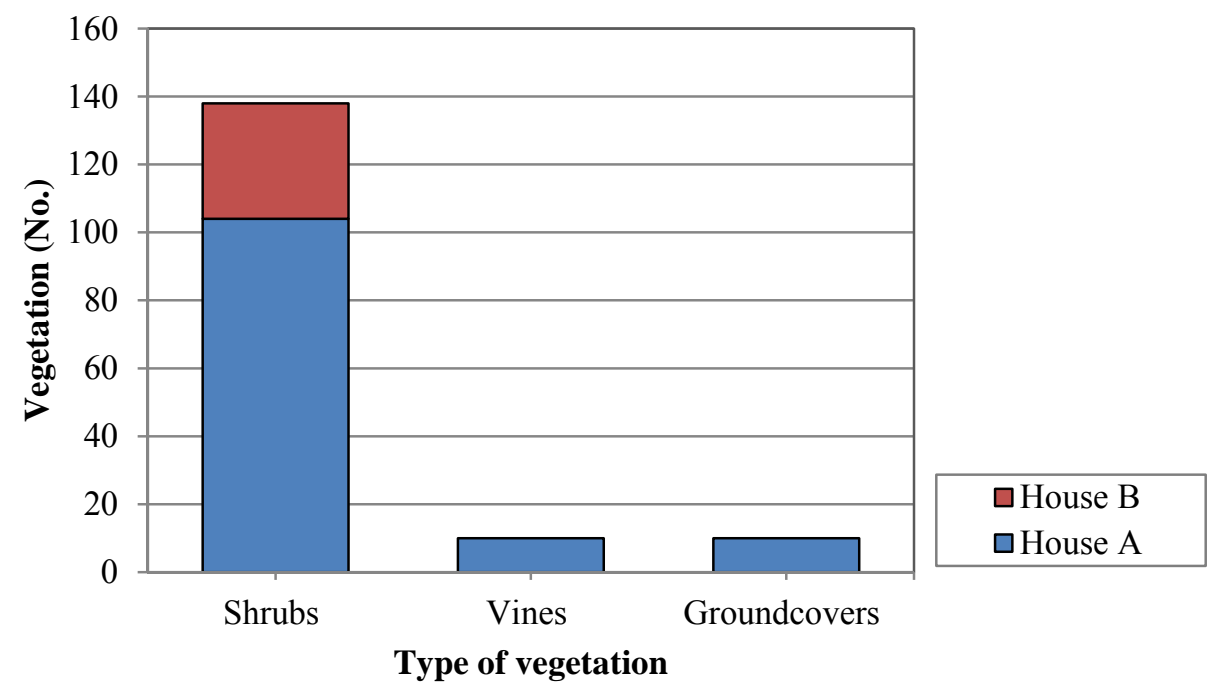

Figure 5.9. Shrubs, vines and groundcover distributions at the heavily landscaped house (A) and the sparsely landscaped house (B)

\section{i) Shrubs}

At the heavily landscaped house there were few categories of shrubs, which included garden, edible and palm shrubs. The majority were from the garden shrub category (75\%), followed by $23 \%$ edible shrubs, and only $1 \%$ palms shrubs. 
Shrubs were scattered along all four sides of the building and garden, with $39 \%$ planted on the east side, $21 \%$ on the north and south sides respectively, and $19 \%$ on the west side. Most of edible shrubs were planted on the north side near the kitchen, which is traditional in Malay culture. Fresh herbs, fruits, and vegetables are required for their everyday cooking activities, and to help provide a fresh and fragrant environment surrounding the house.

Most of the shrubs (54\%) were scattered within $5 \mathrm{~m}$ of the building, with $38 \%$ close to the walls and windows. This was to provide shade during the early morning and late afternoon, especially on the east and west walls that receive direct solar radiation. These shrubs also provided shade for the ground during the peak mid-day sun. The north and south sides of the house, areas where it is also important reduce heat from solar radiation, had only $8 \%$ of the garden's shrubs. These were scattered within 5-9m from the building, underneath trees.

As can be seen in Table 5.5 and Appendix 5.1, approximately half (51\%) of the shrubs were between 0.5 and $0.9 \mathrm{~m}$ tall, $21 \%$ were around $1.0-1.4 \mathrm{~m}$ tall, $17 \%$ were below $0.5 \mathrm{~m}$ tall, and $11 \%$ were between $1.5-1.9 \mathrm{~m}$ tall. The majority of shrubs that are located close to the building's walls were around $1.4 \mathrm{~m}$ tall to provide sufficient shade to the building.

The majority of the shrubs (64\%) were planted in groups of individual species. Twenty-seven percent were in a group of below $5 \mathrm{~m}^{2}, 5 \%$ in a group of $10-20 \mathrm{~m}^{2}, 3 \%$ in a group of $6-10 \mathrm{~m}^{2}$, and $1 \%$ were in a group that was $21-30 \mathrm{~m}^{2}$. The groups of shrubs around this house provided each other with fertilizer, water and shade, which helped to maintain a fertile, lush and green environment. They also protected each other from storm, downpour, and drought.

Most of the shrubs had an upright shape (52\%), 24\% were a spreading shape, and $12 \%$ were rounded and sword leaf respectively. 
The amount of leaves on shrubs influences the shade quality and wind flow into the building. More than half (51\%) of the shrubs had a medium amount of leaves, $32 \%$ had few leaves, and $17 \%$ had a dense amount of leaves. Most of the shrubs had small leaves (73\%), while only $15 \%$ had medium sized leaves and $12 \%$ had large leaves.

On average, the typology of the shrubs around the heavily landscape house were around $0.9 \mathrm{~m}$ high, situated within $5 \mathrm{~m}$ from the building and planted in groups of below $5 \mathrm{~m}^{2}$. Their shape was predominantly upright, and the amount and size of leaves was medium and small respectively, as shown in Table 5.5.

Conversely, the sparsely landscaped house had only 34 individual and groups of shrubs. Sixty-eight percent of these were garden shrubs, $22 \%$ edible shrubs, and $10 \%$ palm shrubs. More than half (56\%) of the shrubs were on the west side of the house garden, $19 \%$ on the east side, $14 \%$ on the south side, and $11 \%$ on the north side. All of the shrubs were located within $5 \mathrm{~m}$ from the building. The majority (62\%) were medium-sized; between $0.5-0.9 \mathrm{~m}$ tall, $19 \%$ were $1-1.4 \mathrm{~m}$ tall, $13 \%$ were $2-2.4 \mathrm{~m}$ tall, and only $6 \%$ were $0.5 \mathrm{~m}$ tall.

Most of the shrubs were planted individually or in pots (56\%), 16\% were in groups of below $5 \mathrm{~m}^{2}, 12 \%$ in groups of $6-10 \mathrm{~m}^{2}, 13 \%$ in groups of $21-30 \mathrm{~m}^{2}$, and only $3 \%$ were in groups of $10-20 \mathrm{~m}^{2}$.

More than half of shrubs (55\%) had an upright shape, 19\% had a spreading shape, $16 \%$ were rounded in shape, and $10 \%$ were sword-leaf shaped. Half of the shrubs had a medium amount of leaves, 31\% a dense amount, and 19\% had few leaves. Fifty-nine percent of the shrubs had leaves that were small in size, $22 \%$ had large size leaves and 19\% medium sized leaves.

On average the typology of the shrubs at the sparsely landscape house was around $0.5-0.9 \mathrm{~m}$ high, situated within $5 \mathrm{~m}$ of the building, planted individually, with an upright shape, a medium number and small size of leaves, as shown in Table 5.6. 
Table 5.5. Shrubs at the heavily landscaped house

\begin{tabular}{|c|c|c|c|c|c|c|c|c|c|}
\hline \multirow[t]{2}{*}{ Azimuth } & \multirow[t]{2}{*}{ No. } & \multirow{2}{*}{$\begin{array}{l}\text { Distance } \\
\text { from } \\
\text { building }{ }^{1}\end{array}$} & \multirow[t]{2}{*}{ No. } & \multirow[t]{2}{*}{ Type $^{2}$} & \multicolumn{5}{|c|}{ Shrub-growth index/typology/biomass structure } \\
\hline & & & & & Height $^{3}$ & Spread $^{4}$ & Shape ${ }^{5}$ & $\begin{array}{l}\text { Amount of } \\
\text { leaves }\end{array}$ & $\begin{array}{l}\text { Size of } \\
\text { leaves }^{7}\end{array}$ \\
\hline \multirow[t]{3}{*}{ North } & \multirow[t]{3}{*}{22} & 1 & 16 & $\mathrm{~F}$ & 1.81 & 1.44 & 2.75 & 1.88 & 1.44 \\
\hline & & 2 & 6 & $\mathrm{~F}$ & 2.83 & 1.67 & 2.33 & 2.33 & 1.67 \\
\hline & & 3 & 0 & - & 0.00 & 0.00 & 0.00 & 0.00 & 0.00 \\
\hline \multirow[t]{5}{*}{ East } & \multirow[t]{5}{*}{42} & \multirow[t]{2}{*}{1} & 8 & $\mathrm{~F}$ & 2.00 & 1.00 & 3.00 & 1.50 & 1.38 \\
\hline & & & 1 & $\mathrm{H}$ & 2.00 & 1.00 & 2.00 & 1.40 & 1.20 \\
\hline & & \multirow[t]{2}{*}{2} & 11 & $\mathrm{~F}$ & 2.30 & 1.60 & 1.70 & 1.40 & 1.20 \\
\hline & & & 22 & $\mathrm{H}$ & 2.44 & 1.33 & 2.06 & 1.72 & 1.44 \\
\hline & & 3 & 0 & - & 0.00 & 0.00 & 0.00 & 0.00 & 0.00 \\
\hline \multirow[t]{3}{*}{ South } & \multirow[t]{3}{*}{22} & 1 & 7 & $\mathrm{~F}$ & 2.00 & 1.57 & 3.29 & 2.29 & 1.29 \\
\hline & & 2 & 7 & $\mathrm{~F}$ & 1.57 & 1.86 & 2.00 & 2.14 & 1.29 \\
\hline & & 3 & 8 & $\mathrm{~F}$ & 3.00 & 1.88 & 2.00 & 1.63 & 2.25 \\
\hline \multirow[t]{5}{*}{ West } & \multirow[t]{5}{*}{18} & \multirow[t]{2}{*}{1} & 7 & $\mathrm{~F}$ & 1.00 & 3.14 & 1.43 & 1.71 & 1.86 \\
\hline & & & 1 & $\mathrm{G}$ & 1.00 & 4.00 & 5.00 & 2.00 & 3.00 \\
\hline & & \multirow[t]{2}{*}{2} & 8 & $\mathrm{~F}$ & 2.75 & 1.63 & 2.00 & 2.00 & 1.25 \\
\hline & & & 2 & $\mathrm{H}$ & 2.00 & 2.50 & 1.00 & 2.00 & 1.00 \\
\hline & & 3 & 0 & - & 0.00 & 0.00 & 0.00 & 0.00 & 0.00 \\
\hline Total & 104 & & 104 & & 234.00 & 157.00 & 232.00 & 193.00 & 144.00 \\
\hline Mean & & & & & 2.25 & 1.51 & 2.23 & 1.86 & 1.38 \\
\hline
\end{tabular}

Table 5.6. Shrubs at the sparsely landscaped house

\begin{tabular}{|c|c|c|c|c|c|c|c|c|c|}
\hline \multirow[t]{2}{*}{ Azimuth } & \multirow[t]{2}{*}{ No. } & \multirow{2}{*}{$\begin{array}{l}\text { Distance } \\
\text { from } \\
\text { building }{ }^{1}\end{array}$} & \multirow[t]{2}{*}{ No. } & \multirow[t]{2}{*}{ Type $^{2}$} & \multicolumn{5}{|c|}{ Shrub-growth index/typology/biomass structure } \\
\hline & & & & & Height $^{3}$ & Spread $^{4}$ & Shape $^{5}$ & $\begin{array}{l}\text { Amount of } \\
\text { leaves }^{6}\end{array}$ & $\begin{array}{l}\text { Size of } \\
\text { leaves }\end{array}$ \\
\hline \multirow[t]{4}{*}{ North } & \multirow[t]{4}{*}{4} & 1 & 0 & - & 0.00 & 0.00 & 0.00 & 0.00 & 0.00 \\
\hline & & \multirow[t]{2}{*}{2} & 2 & $\mathrm{~F}$ & 1.50 & 3.00 & 2.50 & 1.50 & 1.50 \\
\hline & & & 2 & $\mathrm{G}$ & 2.00 & 1.00 & 2.00 & 3.00 & 1.00 \\
\hline & & 3 & 0 & - & 0.00 & 0.00 & 0.00 & 0.00 & 0.00 \\
\hline \multirow[t]{4}{*}{ East } & \multirow[t]{4}{*}{7} & 1 & 0 & - & 0.00 & 0.00 & 0.00 & 0.00 & 0.00 \\
\hline & & \multirow[t]{2}{*}{2} & 5 & $\mathrm{~F}$ & 1.80 & 2.00 & 3.40 & 2.00 & 2.20 \\
\hline & & & 2 & $\mathrm{G}$ & 2.00 & 2.00 & 2.00 & 3.00 & 2.00 \\
\hline & & 3 & 0 & - & 0.00 & 0.00 & 0.00 & 0.00 & 0.00 \\
\hline \multirow[t]{3}{*}{ South } & \multirow[t]{3}{*}{4} & 1 & 0 & - & 0.00 & 0.00 & 0.00 & 0.00 & 0.00 \\
\hline & & 2 & 4 & $\mathrm{~F}$ & 2.25 & 3.00 & 2.50 & 2.25 & 1.25 \\
\hline & & 3 & 0 & - & 0.00 & 0.00 & 0.00 & 0.00 & 0.00 \\
\hline \multirow[t]{4}{*}{ West } & \multirow[t]{4}{*}{19} & 1 & 0 & - & 0.00 & 0.00 & 0.00 & 0.00 & 0.00 \\
\hline & & \multirow[t]{2}{*}{2} & 11 & $F$ & 2.27 & 1.73 & 2.27 & 2.00 & 1.36 \\
\hline & & & 8 & G & 3.38 & 1.75 & 1.88 & 1.75 & 1.75 \\
\hline & & 3 & 0 & - & 0.00 & 0.00 & 0.00 & 0.00 & 0.00 \\
\hline Total & 34 & & 34 & & 82.00 & 67.00 & 80.00 & 70.00 & 54.00 \\
\hline Mean & & & & & 2.41 & 1.97 & 2.35 & 2.06 & 1.59 \\
\hline
\end{tabular}

${ }^{1} 1=$ proximity to walls. $2=$ Below $5 \mathrm{~m}, 3=5-9 \mathrm{~m}$

${ }^{2} \mathrm{~F}=$ garden shrubs, $\mathrm{G}=$ Palm shrubs, $\mathrm{H}=$ edible shrubs

${ }^{3}$ The mean of height scores of all shrubs for the given distance from building

${ }^{4}$ The mean of spread scores of all shrubs for the given distance from building

${ }^{5}$ The mean of shape scores of all shrubs for the given distance from building

${ }^{6}$ The mean of amount of leaves scores of all shrubs for the given distance from building

${ }^{7}$ The mean of size of leaves scores of all shrubs for the given distance from building 


\section{ii) Vines}

The heavily landscaped house had vines climbing close to walls, columns, and or wooden lattices in the balcony areas, pergola and individual garden structures, as listed in Table 5.7. They were located on the north, east and west sides of the house, with $60 \%$ climbing walls on the north and south sides, and $40 \%$ within $5 \mathrm{~m}$ of the building. The main function of these vines was to provide shade for windows and walls.

All the vines have been planted in groups with different group sizes, with approximately $70 \%$ covering an area of below $5 \mathrm{~m}^{2}, 20 \%$ in groups of $10-20 \mathrm{~m}^{2}$, and only $10 \%$ in groups of $6-10 \mathrm{~m}^{2}$.

Fifty percent of the vines had a dense amount of leaves, while $40 \%$ had a medium amount of leaves, and 10\% few leaves. The majority (70\%) had medium sized leaves, which included species such as Bauhinia kockiana and Quisqualis indica. These had grown very well and matured enough to provide shade to the walls of the house. The remaining $30 \%$ of vines had small sized leaves.

\section{iii) Groundcover}

In the heavily landscaped house the purpose of the groundcover was to create a transition between shrubs and turf surfaces. Ten groups of groundcover plants were scattered around the garden, as listed in Table 5.8. Forty percent of the groundcover was on the south side of the house, and $20 \%$ on the north, east, and west sides respectively. Sixty percent was located within $5 \mathrm{~m}$ of the building, $30 \%$ was close to walls, and 10\% was 5-9m from the building.

All groundcover plants were planted in groups underneath the shrubs. Half had been planted in groups of $5-9 \mathrm{~m}^{2}, 30 \%$ were in groups of $10-14 \mathrm{~m}^{2}$, and $20 \%$ were in groups of $15-19 \mathrm{~m}^{2}$. All of them had a medium amount and small size of leaves. 
Table 5.7. List of the vines at the heavily landscaped house

\begin{tabular}{|c|c|c|c|c|c|c|}
\hline & \multirow[t]{2}{*}{ Species } & \multirow{2}{*}{ Azimuth $^{b}$} & \multirow{2}{*}{$\begin{array}{l}\text { Vines } \\
\text { building } \\
\text { distance }^{\mathrm{c}}\end{array}$} & \multicolumn{3}{|c|}{ Vines-growth index/typology/biomass structure } \\
\hline & & & & $\begin{array}{l}\text { Area/ } \\
\text { spread }^{\mathrm{i}}\end{array}$ & $\begin{array}{l}\text { Amount of } \\
\text { leaves }^{j}\end{array}$ & Size of leaves ${ }^{k}$ \\
\hline 1 & Bauhinia kockiana & 5 & 1.00 & 3.00 & 3.00 & 2.00 \\
\hline 2 & Clerodendrum sp. & 5 & 1.00 & 2.00 & 2.00 & 2.00 \\
\hline 3 & Bauhinia kockiana & 1 & 1.00 & 4.00 & 3.00 & 2.00 \\
\hline 4 & Quisqualis indica & 1 & 1.00 & 2.00 & 3.00 & 2.00 \\
\hline 5 & Rosa sp. & 1 & 1.00 & 2.00 & 2.00 & 1.00 \\
\hline 6 & Vallaris glabra & 1 & 1.00 & 2.00 & 3.00 & 1.00 \\
\hline 7 & Bauhinia kockiana & 1 & 2.00 & 4.00 & 3.00 & 2.00 \\
\hline 8 & Rosa sp. & 3 & 2.00 & 2.00 & 1.00 & 1.00 \\
\hline 9 & Psophocarpus tetragonolobus & 3 & 2.00 & 2.00 & 2.00 & 2.00 \\
\hline \multirow[t]{3}{*}{10} & Piper betle & 3 & 2.00 & 2.00 & 2.00 & 2.00 \\
\hline & Total & & 14.00 & 25.00 & 24.00 & 17.00 \\
\hline & Average & & 1.40 & 2.50 & 2.40 & 1.70 \\
\hline
\end{tabular}

Table 5.8. List of the groundcover plants at the heavily landscaped house

\begin{tabular}{|c|c|c|c|c|c|c|}
\hline & \multirow[t]{2}{*}{ Species } & \multirow[t]{2}{*}{ Azimuth $^{b}$} & \multirow[t]{2}{*}{ Distance $^{\mathrm{c}}$} & \multicolumn{3}{|c|}{$\begin{array}{l}\text { Groundcovers-growth index/typology/biomass } \\
\text { structure }\end{array}$} \\
\hline & & & & Area $^{i}$ & $\begin{array}{l}\text { Amount of } \\
\text { leaves }^{j}\end{array}$ & Size of leaves ${ }^{k}$ \\
\hline 1 & Cuphea hyssopifolia & 5 & 3.00 & 3.00 & 2.00 & 1.00 \\
\hline 2 & Selaginella sp. & 5 & 1.00 & 3.00 & 2.00 & 1.00 \\
\hline 3 & Cuphea hyssopifolia & 5 & 2.00 & 3.00 & 2.00 & 1.00 \\
\hline 4 & Selaginella sp. & 5 & 2.00 & 3.00 & 2.00 & 1.00 \\
\hline 5 & Cuphea hyssopifolia & 7 & 1.00 & 5.00 & 2.00 & 1.00 \\
\hline 6 & Cuphea hyssopifolia & 7 & 2.00 & 5.00 & 2.00 & 1.00 \\
\hline 7 & Phyllanthus myrtifolius & 3 & 2.00 & 4.00 & 2.00 & 1.00 \\
\hline 8 & Cuphea hyssopifolia & 3 & 2.00 & 4.00 & 2.00 & 1.00 \\
\hline 9 & Selaginella $s p$. & 1 & 1.00 & 3.00 & 2.00 & 1.00 \\
\hline \multirow[t]{3}{*}{10} & Phyllanthus myrtifolius & 1 & 2.00 & 4.00 & 2.00 & 1.00 \\
\hline & Total & & 18.00 & 37.00 & 20.00 & 10.00 \\
\hline & Average & & 1.80 & 3.70 & 2.00 & 1.00 \\
\hline
\end{tabular}

${ }^{\mathrm{b}} 1$, North, 3, East, 5, South, 7, West.

c $1=$ proximity to walls, $2=$ below $5 \mathrm{~m}, 3=5-9 \mathrm{~m}$.

${ }^{\mathrm{i}} 1=$ below $1 \mathrm{~m}^{2}, 2=1-4 \mathrm{~m}^{2}, 3=5-9 \mathrm{~m}^{2}, 4=10-14 \mathrm{~m}^{2}, 5=15-19 \mathrm{~m}^{2}$

${ }^{\mathrm{j}} 1=$ rare, $2=$ medium, $3=$ dense.

${ }^{\mathrm{k}} 1=$ small, $2=$ medium, $3=$ large

\subsubsection{Other landscape elements}

The heavily landscaped house had a pergola in a backyard garden, which was densely covered by Bauhinia kockiana. The pergola was constructed from hardwood, and was $8 \mathrm{~m}^{2}$ and $2.5 \mathrm{~m}$ high, as shown in Figure 5.6. This garden also had a fish pool for Carassius sp. (Japanese Carp), which was located to the rear of the house alongside the living room walls. This was built of concrete, and was $0.6 \mathrm{~m}$ deep, covering an area of around $10 \mathrm{~m}^{2}$. The fish pool was surrounded and shaded by ferns. 
By contrast, the sparsely landscaped house's only external landscape element was a group of concrete paving blocks with grass inlay as a walkway in the back garden that was $16 \mathrm{~m}^{2}$, as shown in Figures 5.7 and 5.4.

\subsubsection{Energy use}

Both houses operated a split system of 1.5-2 horse power air-conditioning units. Figure 5.10 shows that 10 air-conditioning units were used per house, and were located in private rooms and the common areas. The living rooms had 2 horsepower air-conditioning units, while the other rooms had 1.5 horsepower air-conditioning units. The compressor and evaporator were located in a separate external package, and were connected to the air-conditioning units via two detachable refrigerant pipes. Around $20-45 \%$ of the total electricity used was for cooling. In each house this was influenced by the number of occupants and their living habits. Both households used natural ventilation and ceiling fans for cooling during the day. Air-conditioning systems were only actively used during the night until early morning (from around 20.00 to 7.00 hours).

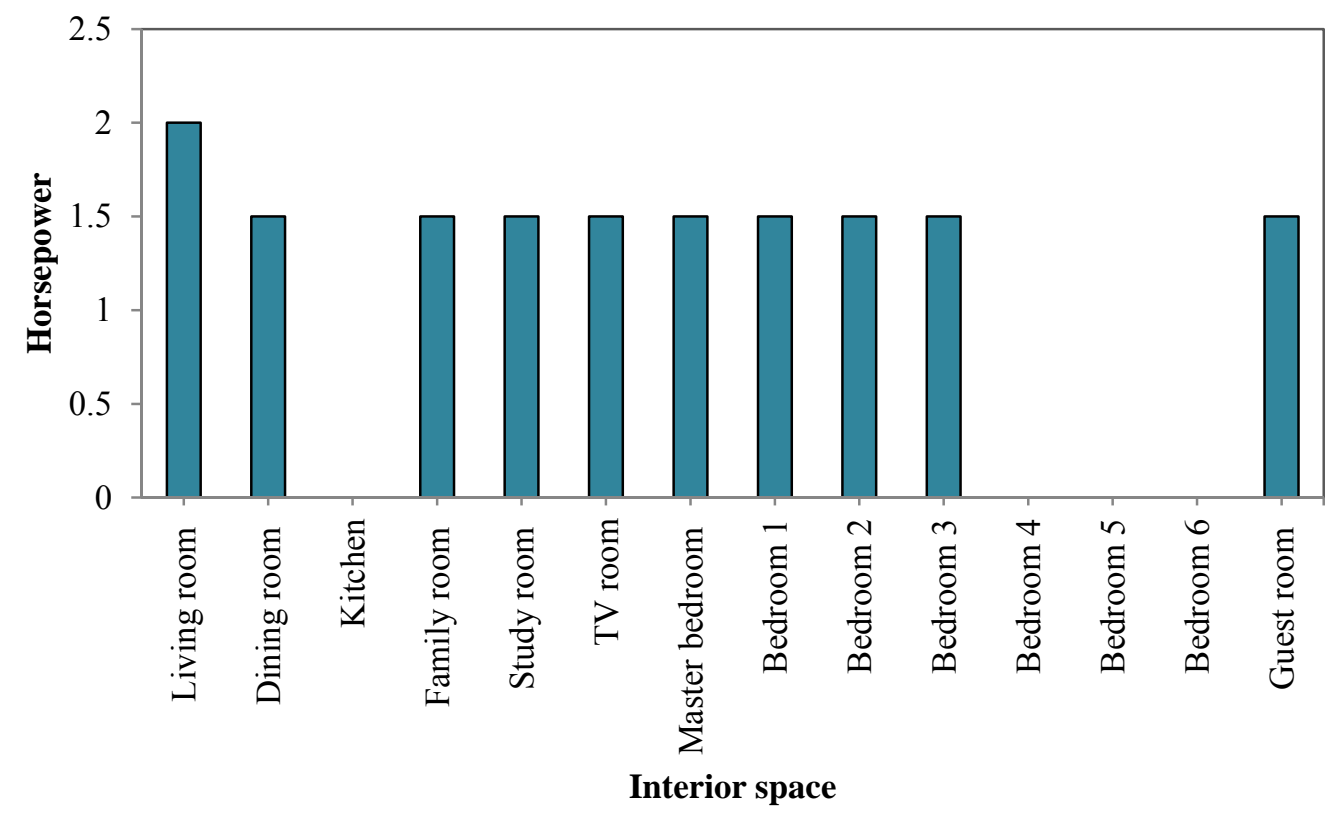

Figure 5.10. Similar capacity of the split system air-conditioning units in both houses 
Table 5.9. Air-conditioning use of the heavily landscaped house (house A) and sparsely landscaped house (house B)

\begin{tabular}{|l|l|l|l|l|l|l|l|l|l|l|l|}
\hline $\begin{array}{l}\text { House } \\
\text { type }\end{array}$ & Occupant & $\begin{array}{l}\text { Cooling } \\
\text { energy } \\
(\mathrm{kWh})\end{array}$ & $\begin{array}{l}\text { House } \\
\text { energy } \\
(\%)\end{array}$ & $\begin{array}{l}\text { HP } \\
\text { per } \\
\text { room }\end{array}$ & $\begin{array}{l}\text { Temp. } \\
\text { setting } \\
\left({ }^{\circ} \mathrm{C}\right)\end{array}$ & $\begin{array}{l}\text { Bed } \\
\text { room } \\
(\mathrm{hrs})\end{array}$ & $\begin{array}{l}\text { Common } \\
\text { room } \\
(\mathrm{hrs})\end{array}$ & $\begin{array}{l}\text { Total } \\
\text { per } \\
\text { month } \\
(\mathrm{hrs})\end{array}$ & $\begin{array}{l}\text { Cooling } \\
\text { Energy } \\
\text { per } \\
\text { person } \\
(\mathrm{kWh})\end{array}$ & $\begin{array}{l}\text { Hours } \\
\text { cooling } \\
\text { per } \\
\mathrm{kWh}\end{array}$ \\
\hline $\mathrm{A}$ & 3 & 0 & 315 & 20 & 1.5 & 26 & 218 & 40 & 258 & 105 & 0.819 \\
\hline $\mathrm{B}$ & 10 & 5 & 746 & 45 & 1.5 & 22 & 600 & 8 & 608 & 50 & 0.815 \\
\hline
\end{tabular}

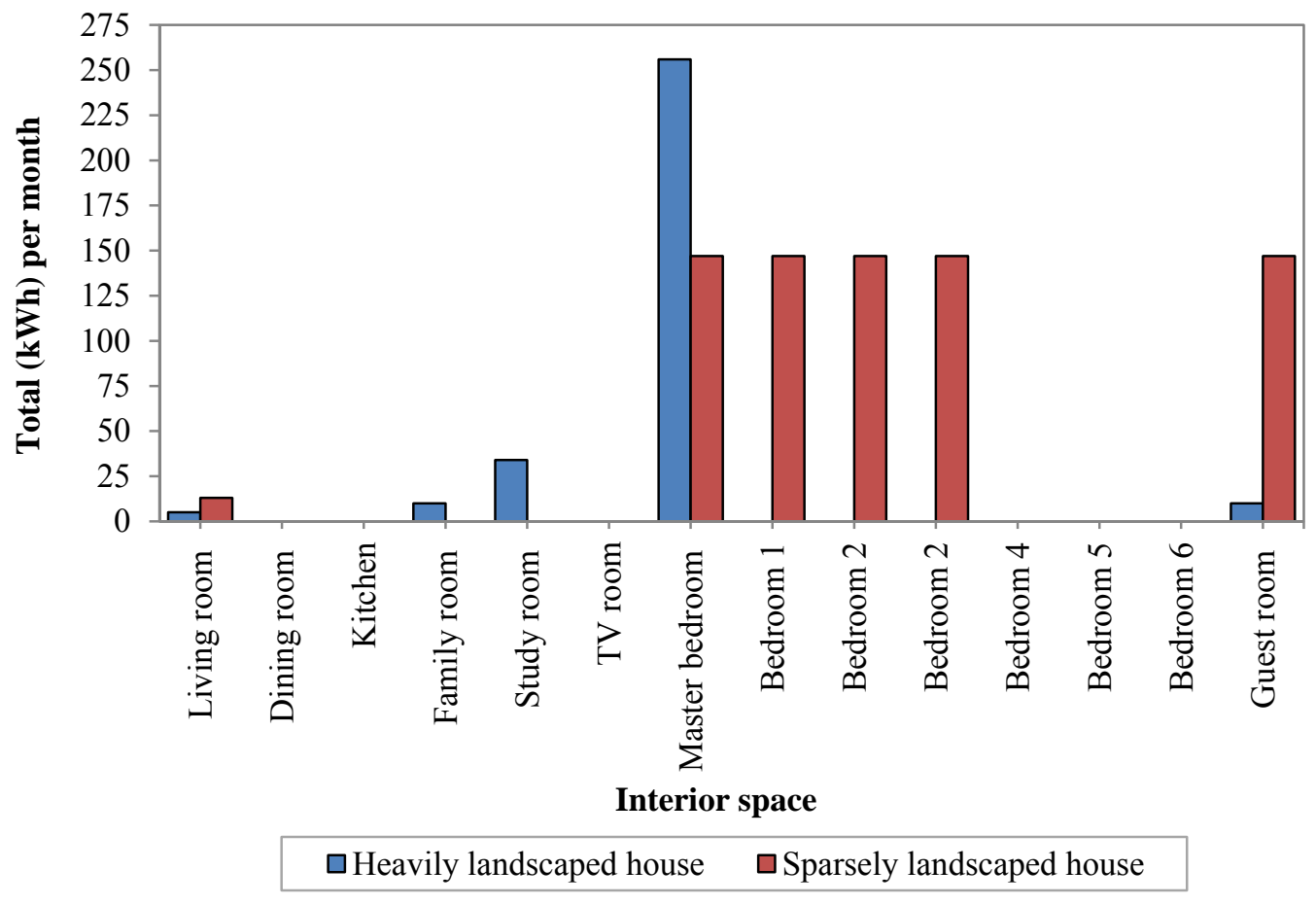

Figure 5.11. Air-conditioning use for the heavily landscaped house (house A) and sparsely landscaped house (house B)

Figure 5.11 shows the total amount of energy use for air-conditioning in $\mathrm{kWh}$ per month for both houses. The heavily landscaped house used around $315 \mathrm{kWh}$ per month for air-conditioning. This house had three adult occupants, who used one airconditioning unit which had its temperature set at approximately $26^{\circ} \mathrm{C}$. This unit was used in the master bedroom during night time. The sparsely landscaped house used $746 \mathrm{kWh}$ for air-conditioning per month. This house had 10 adults and five children in residence, with the air-conditioners used in five bedrooms during the night at a temperature of around $22^{\circ} \mathrm{C}$. Table 5.9 shows the cooling energy per person in the 
sparsely landscape house is lower than in the heavily landscaped house. However the hours of cooling per $\mathrm{kWh}$ were similar at around 0.819 and 0.815 respectively. The temperature setting in the sparsely landscaped house was lower. The calculations for the cooling energy use for both houses are in Appendices 6.3-6.4.

\subsubsection{Weather data}

This investigation was designed to identify the climate parameters around the two houses that have a significant effect on their indoor air temperatures and relative humidity. The climate data measurements were gathered on the ambient air conditions, and include solar radiation and cloud cover, air temperature, wind speed, and relative humidity. This climate data is influenced by the houses' building construction and landscape design.

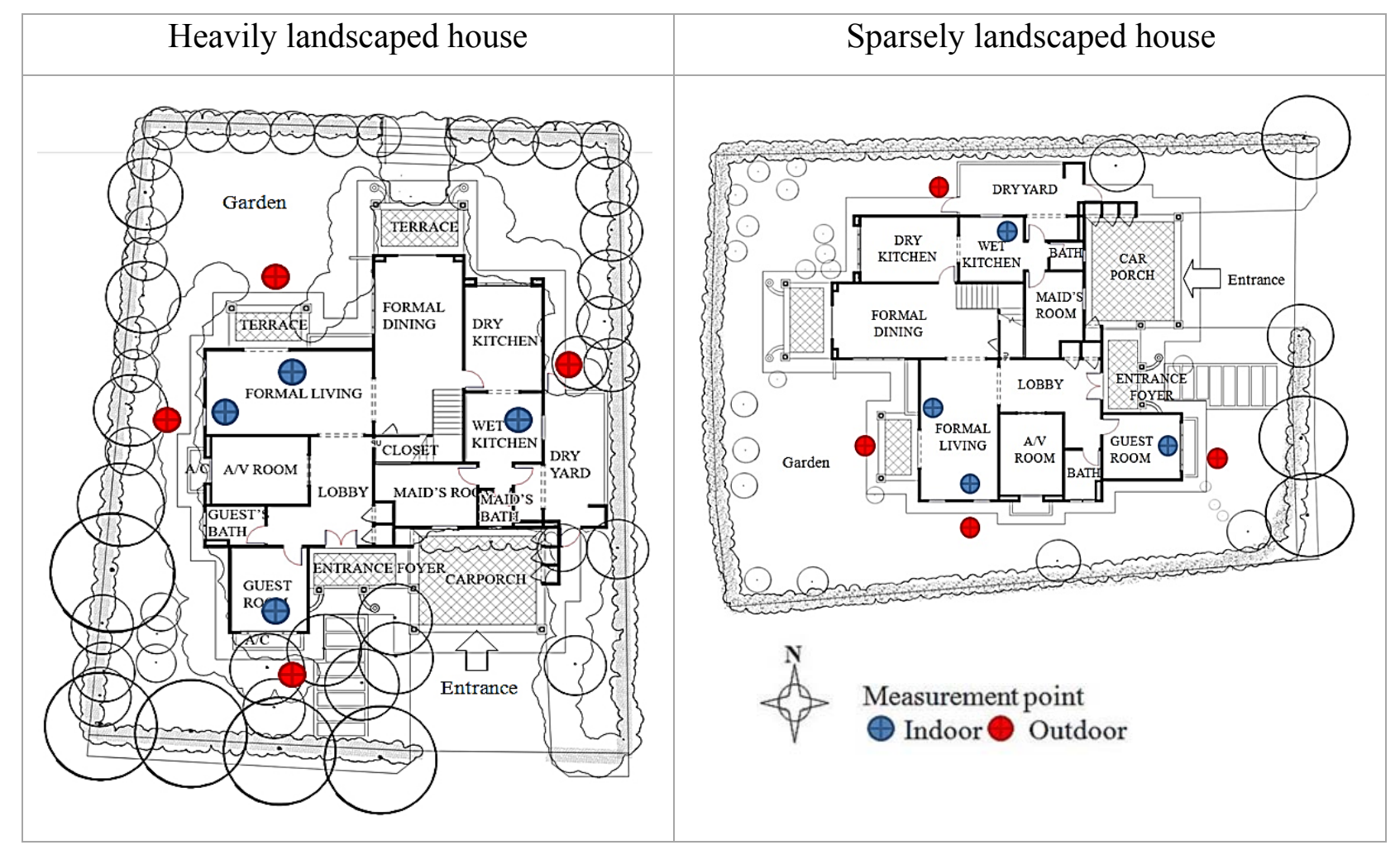

Figure 5.12. Outdoor and indoor measurement points at the heavily landscaped house (house A) and sparsely landscaped house (house B) 


\subsubsection{Solar radiation and cloud cover}

On the day of the case study, the length of the day was around 14 hours, with sunrise at around 7.00 and sunset at around 20.00 hours, as illustrated in Figure 5.13. In the tropics the intensity of solar radiation is considerably higher than other parts of the world all year round. Cloud cover occurs throughout the year, mostly during the rainy season causing this time to be classed as hot-humid.

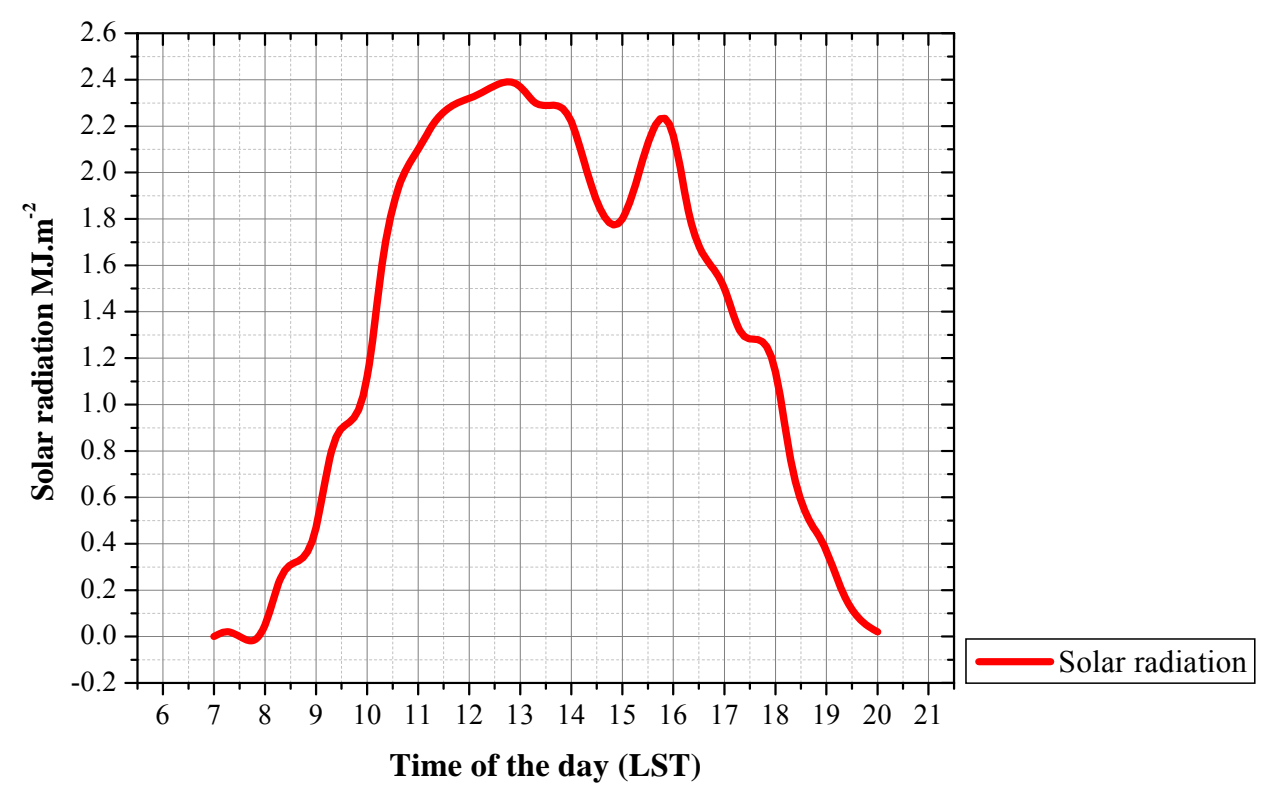

Figure 5.13. The intensity of the surface solar radiation from Sepang

Figure 5.13 shows that solar radiation was recorded as early as 8.00 hours, with the reading as low as $0.05 \mathrm{MJ} . \mathrm{m}^{-2}$. Solar radiation gradually rose over the next four hours to $2.3 \mathrm{MJ} . \mathrm{m}^{-2}$ at 12.00 hours, and continued to rise, reaching the highest level of 2.37MJm-2 at 13.00 hours. Between 13.00-16.00 hours the solar levels fluctuated between 1.8-2.35MJ.m ${ }^{-2}$. According to Mahbar (2011), during this period when cloud cover adds another layer, to become five layers, the temperature drops by around $1^{\circ} \mathrm{C}$. However, solar radiation continued to be normal until 14.00 then dropped by around $0.4 \mathrm{MJ} \cdot \mathrm{m}^{-2}$. Coupled with this, wind flow was at a high of speed of around $2.25 \mathrm{~m} / \mathrm{s}$ and $\mathrm{RH}$ increased to $72 \%$. Solar radiation uniformly decreased throughout the afternoon and evening, to the lowest reading of $0.01 \mathrm{MJ}^{-\mathrm{m}^{-2}}$ at 20.00 hours. Overall, solar radiation in the study area was actively received between 
10.00-18.00 hours; a total of approximately eight hours per day, in the radiation ranges of $1-2.4 \mathrm{MJ} \cdot \mathrm{m}^{-2}$.

Four layers of cloud cover are relatively common in tropical climates, especially during this rainy season. This refers to the fraction of the sky obscured by clouds when observed from the study location. The amount of cloud on the day observed at the study area was 7 Okta.

\subsubsection{Air temperature}

To provide an analytical comparison of air temperature between the heavily landscaped house and the sparsely landscaped house, corresponding average external and internal temperatures were determined at various points and times during the day. Temperatures readings were taken a metre above ground, in four azimuths beside the house wall, between 8.30 and 19.30 hours. These are shown in Figures $5.14-5.17$. 


\section{i) North}

Figure 5.14 shows that the air temperature patterns for the north walls of each house were lower at the heavily landscaped house (House A) than the sparsely landscaped house (House B).

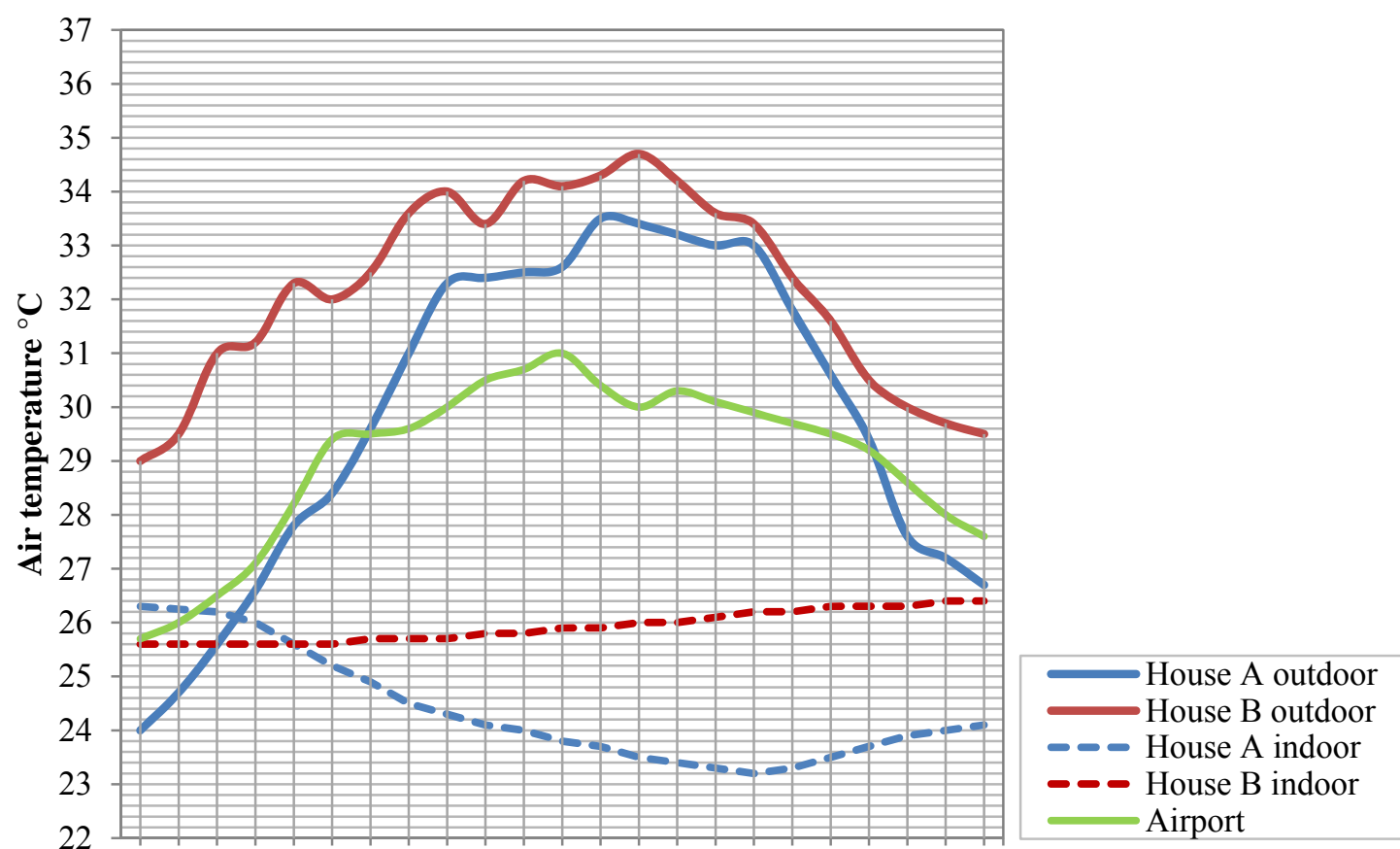

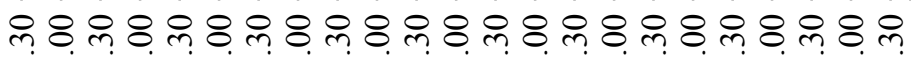

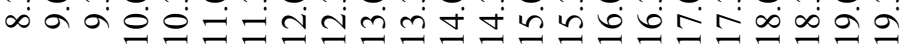

Time of the day (LST)

Figure 5.14. Outdoor and indoor temperature adjacent to the north walls of the heavily landscaped house (house A) and the sparsely landscaped house (house B)

During the morning the exterior air temperatures adjacent to the north walls of the heavily landscaped house ranged from $3.5-5^{\circ} \mathrm{C}$ lower than those at the sparsely landscaped house. The highest temperature was around $33.5^{\circ} \mathrm{C}$ at the heavily landscaped house, and $34.5^{\circ} \mathrm{C}$ at the sparsely landscaped house between 14.00 and 14.30 hours. The temperatures maintained their peak until 16.30 hours, when the ambient temperature for both houses began to gradually fall by approximately $1^{\circ} \mathrm{C}$ every hour until 18.30 hours. The temperature differences for both houses on their north sides varied by between 0.4 and $2.5^{\circ} \mathrm{C}$, with the heavily landscape house having the lower temperatures. The air temperature continued to decline, reaching 
$29.5^{\circ} \mathrm{C}$ at the sparsely landscaped house and $26.5^{\circ} \mathrm{C}$ at the heavily landscaped house at 19.30 hours.

\section{ii) South}

Figure 5.15 shows that the air temperatures readings for the exterior south walls of each house were only slightly different.

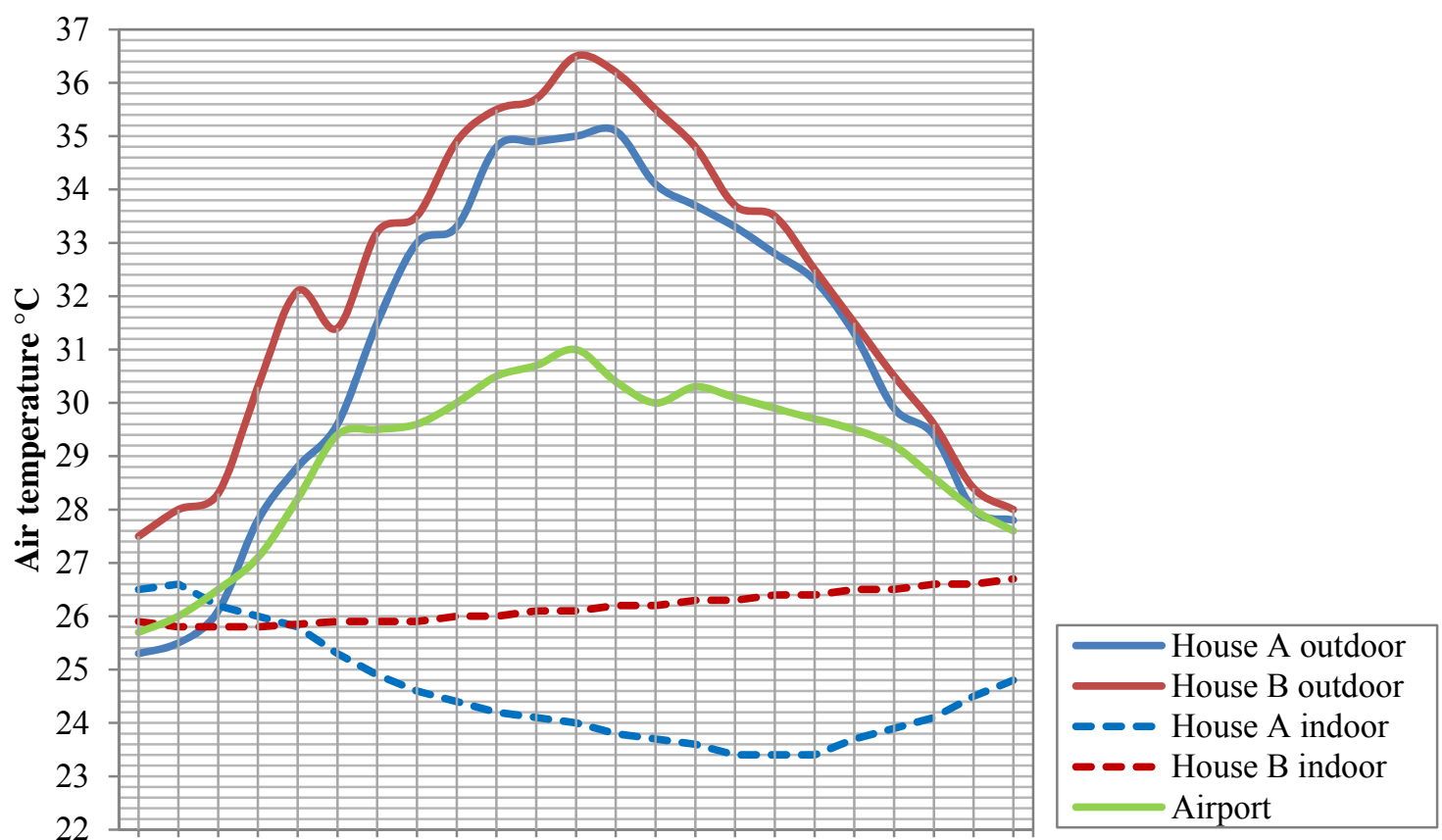

요 요

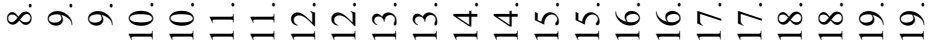

Time of the day (LST)

Figure 5.15. Outdoor and indoor temperature adjacent to the south walls of the heavily landscaped house (house A) and the sparsely landscaped house (house B)

The temperatures adjacent to the south walls of both houses gradually increased through the morning until noon, and were around $2-4^{\circ} \mathrm{C}$ lower at the heavily landscape house. The highest temperature of $36.6^{\circ} \mathrm{C}$ was recorded at the sparsely landscaped house at 14.00 hours. At the same time the temperature at the heavily landscaped house was slightly lower, at approximately $35^{\circ} \mathrm{C}$. The temperatures decreased during the afternoon by around $1-1.6^{\circ} \mathrm{C}$ per hour for both houses until reaching around $28^{\circ} \mathrm{C}$ at both houses at 17.30 hours. On average, the air temperatures 
for both houses on their south sides were different during the peak time of the day, but had a similar reading at late afternoon and toward evening.

The air temperature readings for the exterior south walls of each house were only slightly different because the south wall at the heavily landscape house was near to the main road, an access road and a two-lot car porch. Paving surfaces in these areas influenced the surrounding temperature and increased it compared to the green area.

\section{iii) East}

Figure 5.16 illustrates the air temperature readings for the east walls of the two houses.

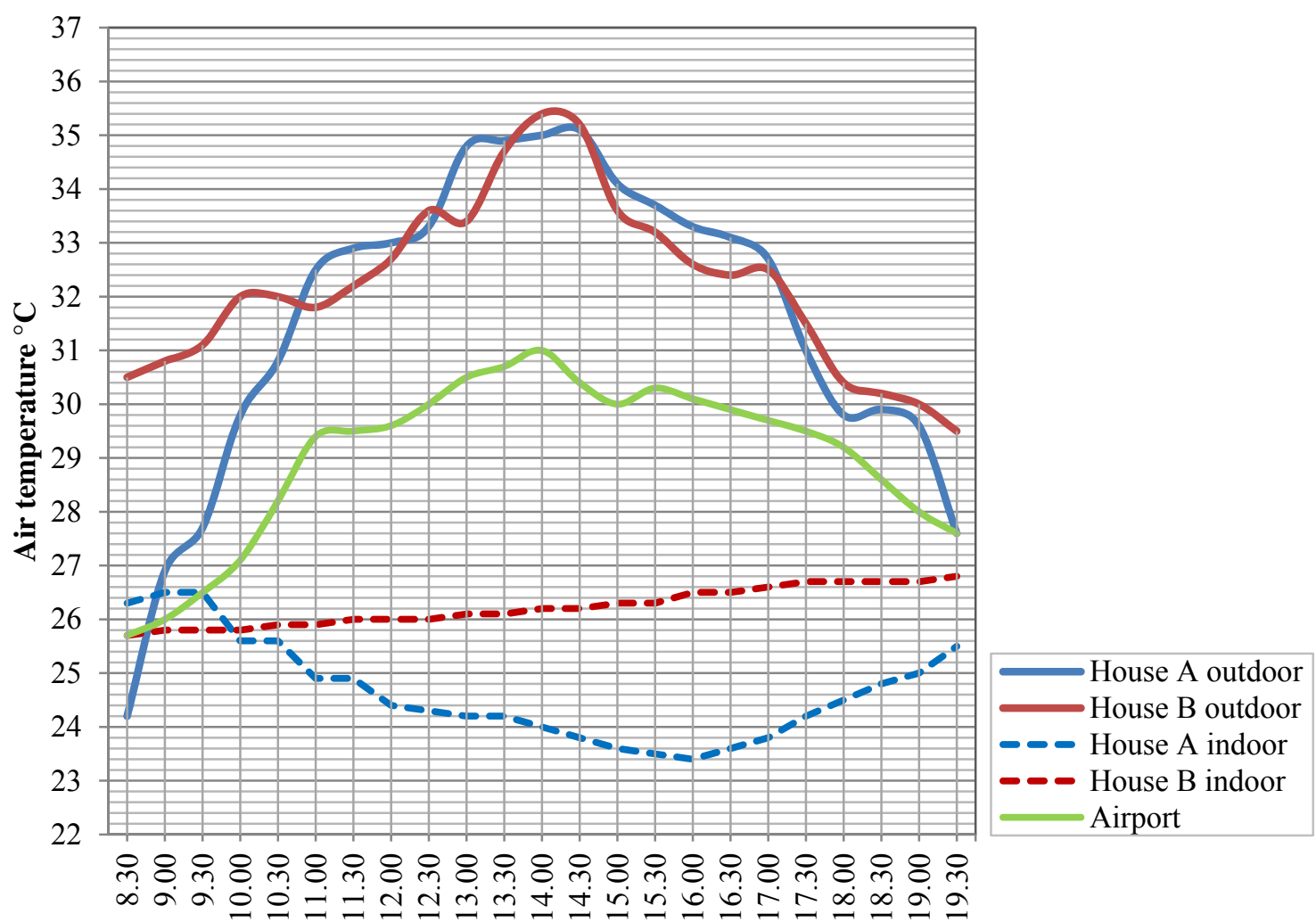

Time of the day (LST)

Figure 5.16. Outdoor and indoor temperature adjacent to the east walls of the heavily landscaped house (house A) and the sparsely landscaped house (house B)

On the east side of the two houses the air temperature patterns fluctuated, but they had similar readings within a range of $0.6-1{ }^{\circ} \mathrm{C}$ between $10.30-17.00$ hours. Air 
temperatures at the heavily landscaped house were lower in the morning, but rose dramatically to $34.8^{\circ} \mathrm{C}$ in the afternoon, while at the sparsely landscaped house the temperature started at a higher point of $30.5^{\circ} \mathrm{C}$, and rose slowly until it reached the highest temperature of $35.4^{\circ} \mathrm{C}$ in the afternoon. The temperatures then gradually decreased reaching $29.4^{\circ} \mathrm{C}$ at the heavily landscaped house and $27.5^{\circ} \mathrm{C}$ at the sparsely landscaped house. On average, the air temperatures for both houses on their east side fluctuated, but were similar from late morning until the late afternoon.

\section{iv) West}

Figure 5.17 shows that the air temperatures on the west side of the heavily landscaped house were lower than those at the sparsely landscaped house.
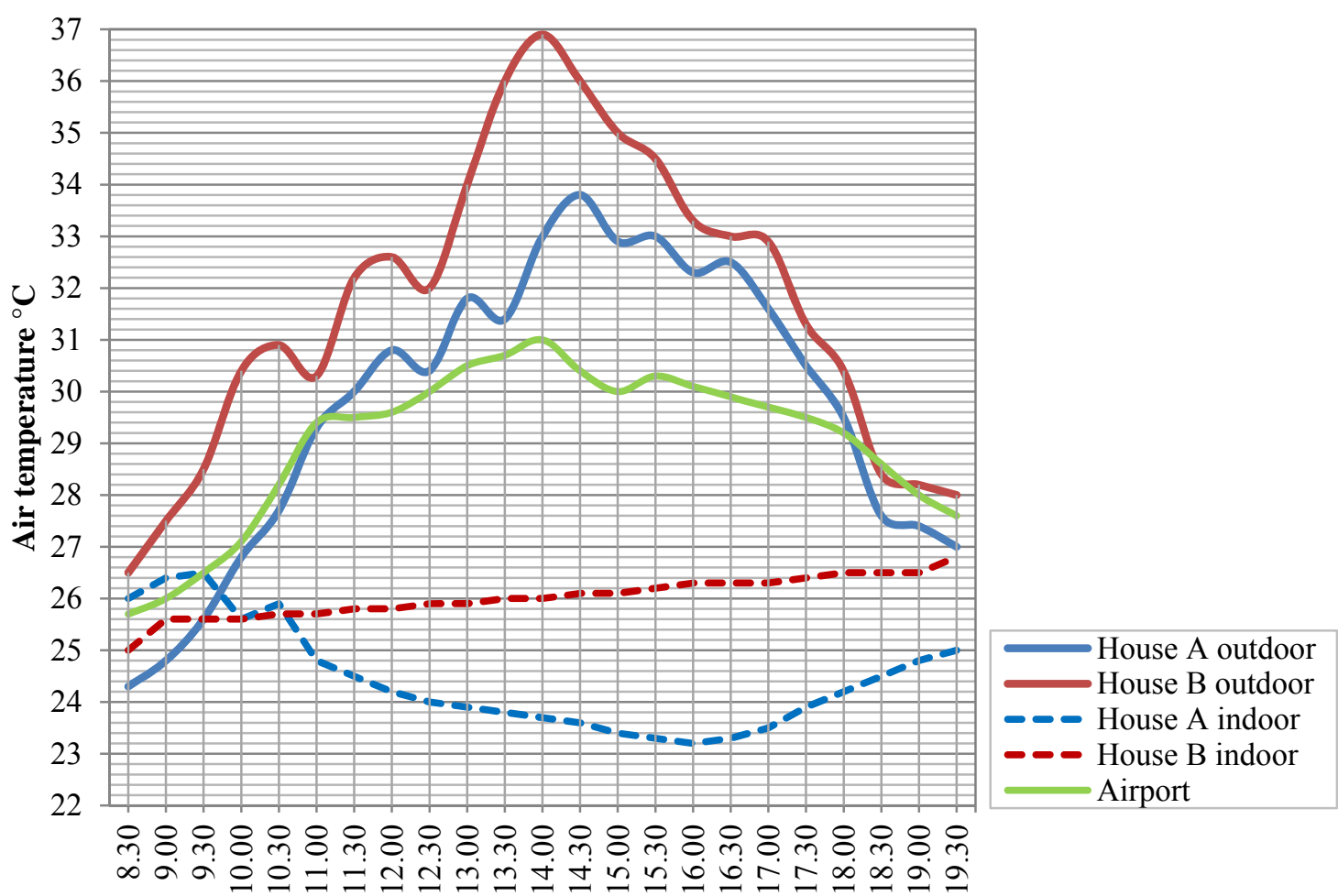

Time of the day (LST)

Figure 5.17. Outdoor and indoor temperature adjacent to the west walls of the heavily landscaped house (house A) and the sparsely landscaped house (house B)

At 8.30 hours the temperature at the heavily landscaped house was $24.3^{\circ} \mathrm{C}$, while at the sparsely landscaped house it was $26.5^{\circ} \mathrm{C}$. The outdoor temperature around the sparsely landscaped house fluctuated until reaching the highest point of $36.9^{\circ} \mathrm{C}$ at 
14.00 hours. The same pattern occurred at the heavily landscaped house, which reached its highest temperature of approximately $33.8^{\circ} \mathrm{C}$ at 14.30 hours. Temperatures at both houses gradually fell during the afternoon until they reached $33^{\circ} \mathrm{C}$ at the sparsely landscaped house and $32.4^{\circ} \mathrm{C}$ at the heavily landscaped house at 16.30 hours. Both houses reached their lowest temperatures at 19.30 hours; $28^{\circ} \mathrm{C}$ at the sparsely landscaped house and $27^{\circ} \mathrm{C}$ at the heavily landscaped house. Overall the exterior temperature on the west side of the heavily landscaped house was as much as $3.9^{\circ} \mathrm{C}$ lower than the sparsely landscaped house during the peak time of the day.

The temperature for all sides of both houses was different to the airport by around 2$6^{\circ} \mathrm{C}$ during the peak time of the day.

In contrast to the exterior temperatures, the interior temperatures on all sides of the heavily landscaped house were relatively different to the sparsely landscaped house. The interior temperature at the heavily landscaped house had a similar pattern and reading for all sides. The temperature gradually declined throughout the day to as low as $23.2^{\circ} \mathrm{C}$ at 16.30 hours, with as much as $3.2^{\circ} \mathrm{C}$ difference when compared to the sparsely landscaped house. After 17.00 hours the temperature gradually rose by $0.4^{\circ} \mathrm{C}$ an hour to reach $23.8^{\circ} \mathrm{C}$ on the north wall at 19.30 hours. At the other walls, the interior temperatures rose to around $24^{\circ} \mathrm{C}$ at 19.30 hours. The clear difference in temperature readings for the interiors of the two houses was influenced by exterior ambient temperatures. Both houses use natural ventilation during the day, combined with electric ceiling fans to circulate air in the interior spaces. Both houses also use natural ventilation during the early morning and late afternoon for rooms facing the main garden area and kitchen spaces. The openings are sliding doors to the living and dining spaces - there were on the north side for the heavily landscaped house and the west side for the sparsely landscaped house. However, the occupants close the openings during the middle of the day to avoid hot air entering the buildings directly. 


\subsubsection{Wind speed}

Wind refers to air in motion, and is expressed in metres per second. Wind flows on the study sites came from various directions during the day. The advantage for both houses was that they faced open spaces and were located at the end site of the housing lot, which was exposed to the prevailing wind. From the early morning through until noon, the wind flow was from the north-east. It then changed in the afternoon to a north-westerly. Towards the evening the wind flows were from the south-west, then returned to the north-east. There were four different patterns of wind speed around the houses, as can be seen at Figures 5.20-5.23.

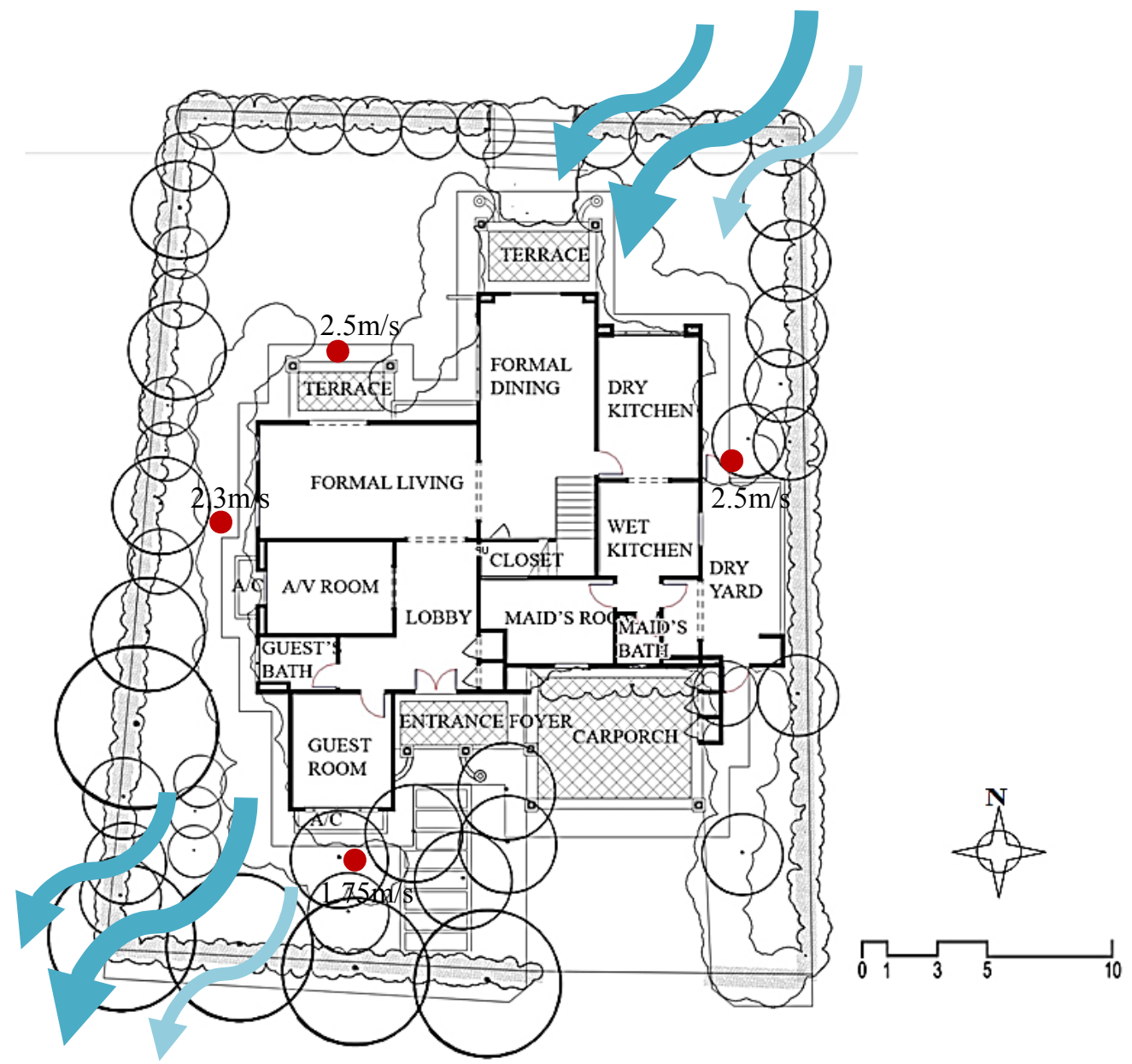

Figure 5.18. Wind flow and average wind speed readings at the heavily landscaped house 


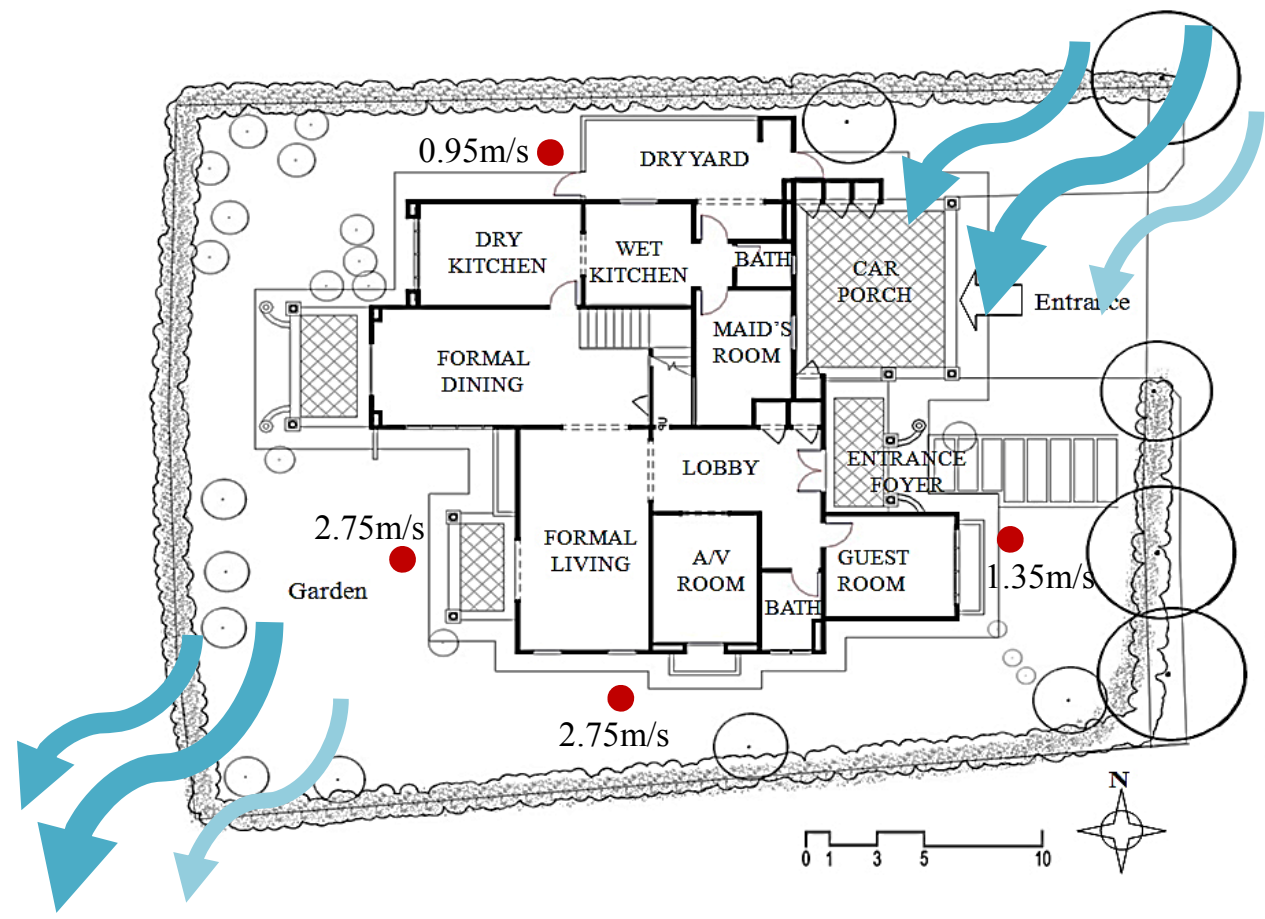

Figure 5.19. Wind flow and average wind speed readings at the sparsely landscaped house 


\section{i) North}

Figure 5.20 shows the wind speed adjacent to the north walls for both houses. Wind passed around the heavily landscaped house in two different patterns.

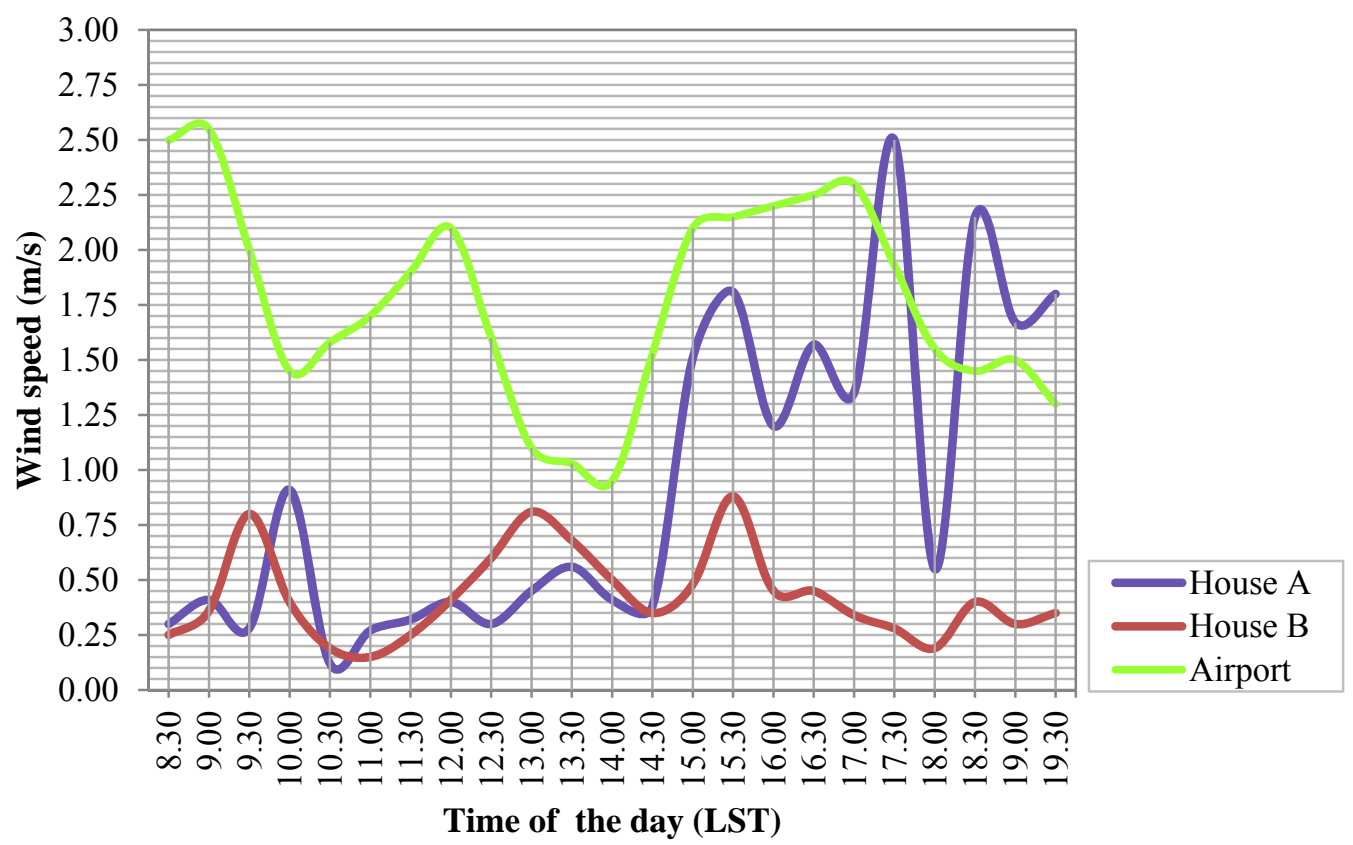

Figure 5.20. Wind speed adjacent to the north walls of the heavily landscaped house (house A) and the sparsely landscaped house (house B)

The wind speed at both houses was slightly different in the morning until noon, in the range of below $1 \mathrm{~m} / \mathrm{s}$, while in the afternoon and evening, the wind speed at the heavily landscaped house was higher than at the sparsely landscaped house, and ranged from 0.55 to $2.5 \mathrm{~m} / \mathrm{s}$. 


\section{ii) South}

The south area of the sparsely landscaped house had higher wind speed during the afternoon and evening than the heavily landscaped house. However, both had a similar pattern of wind speed during the morning. This is shown in Figure 5.21.

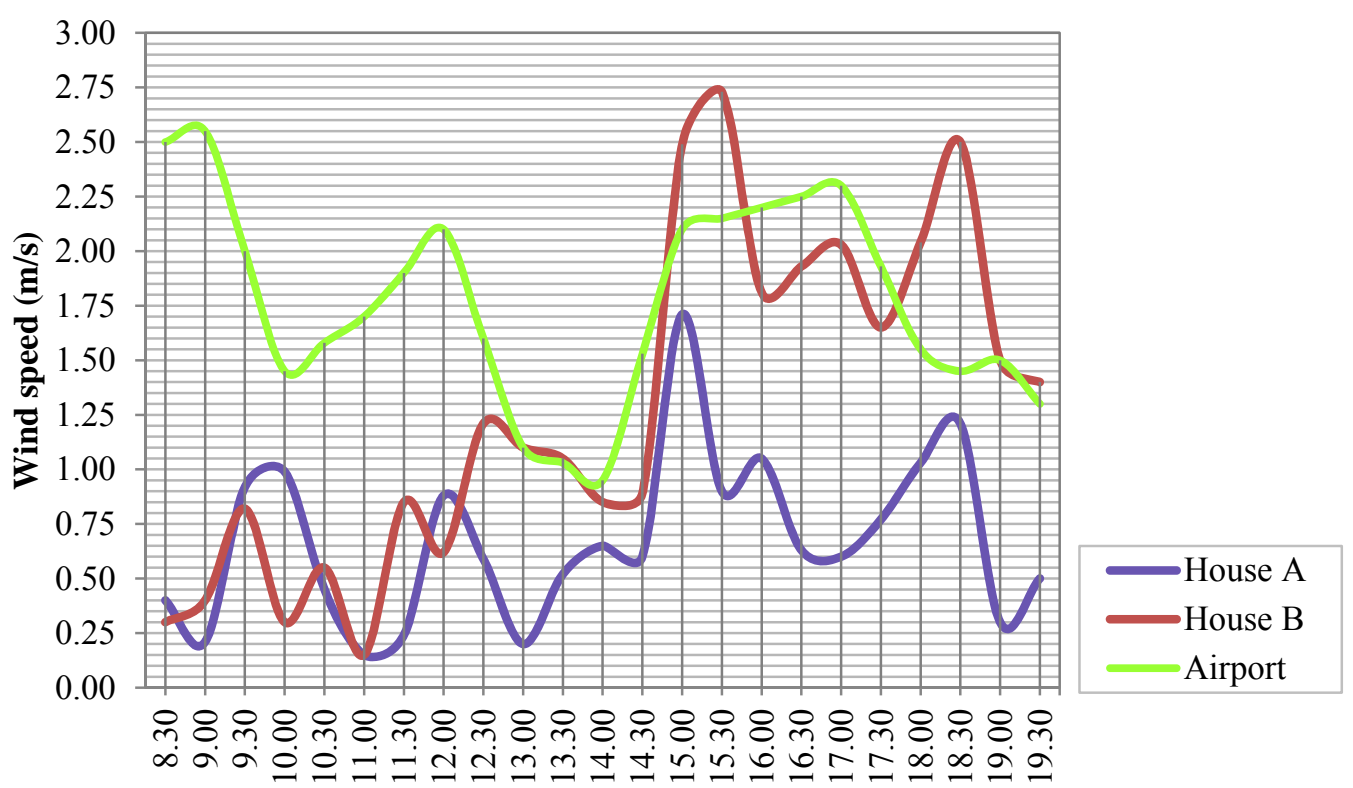

Time of the day (LST)

Figure 5.21. Wind speed adjacent to the south walls of the heavily landscaped house (house A) and the sparsely landscaped house (house B)

During the morning until noon (8.30-11.30 hours) wind speeds were slow and fluctuated between 0.15 and $1 \mathrm{~m} / \mathrm{s}$ adjacent to the south walls of both houses. However, wind speed increased at the sparsely landscaped house by approximately $1.2 \mathrm{~m} / \mathrm{s}$ at 12.30 hours, and dramatically rose until reaching the highest speed of $2.7 \mathrm{~m} / \mathrm{s}$ at 15.30 hours. The wind speed then fluctuated between 1.4 and $2.5 \mathrm{~m} / \mathrm{s}$ between $16.00-19.30$ hours.

The wind speed at the heavily landscaped house fluctuated between $0.2-1.7 \mathrm{~m} / \mathrm{s}$ between 13.00-19.30 hours. The highest wind speed around this house was $1.7 \mathrm{~m} / \mathrm{s}$ at 15.00 hours. Overall, the sparsely landscaped house experienced higher wind speeds on its south side. 


\section{iii) East}

Wind speeds for the exterior east walls of both houses were low compared to their other sides, with the wind only reaching a peak of $1.4 \mathrm{~m} / \mathrm{s}$, as can be seen in Figure 5.22 .

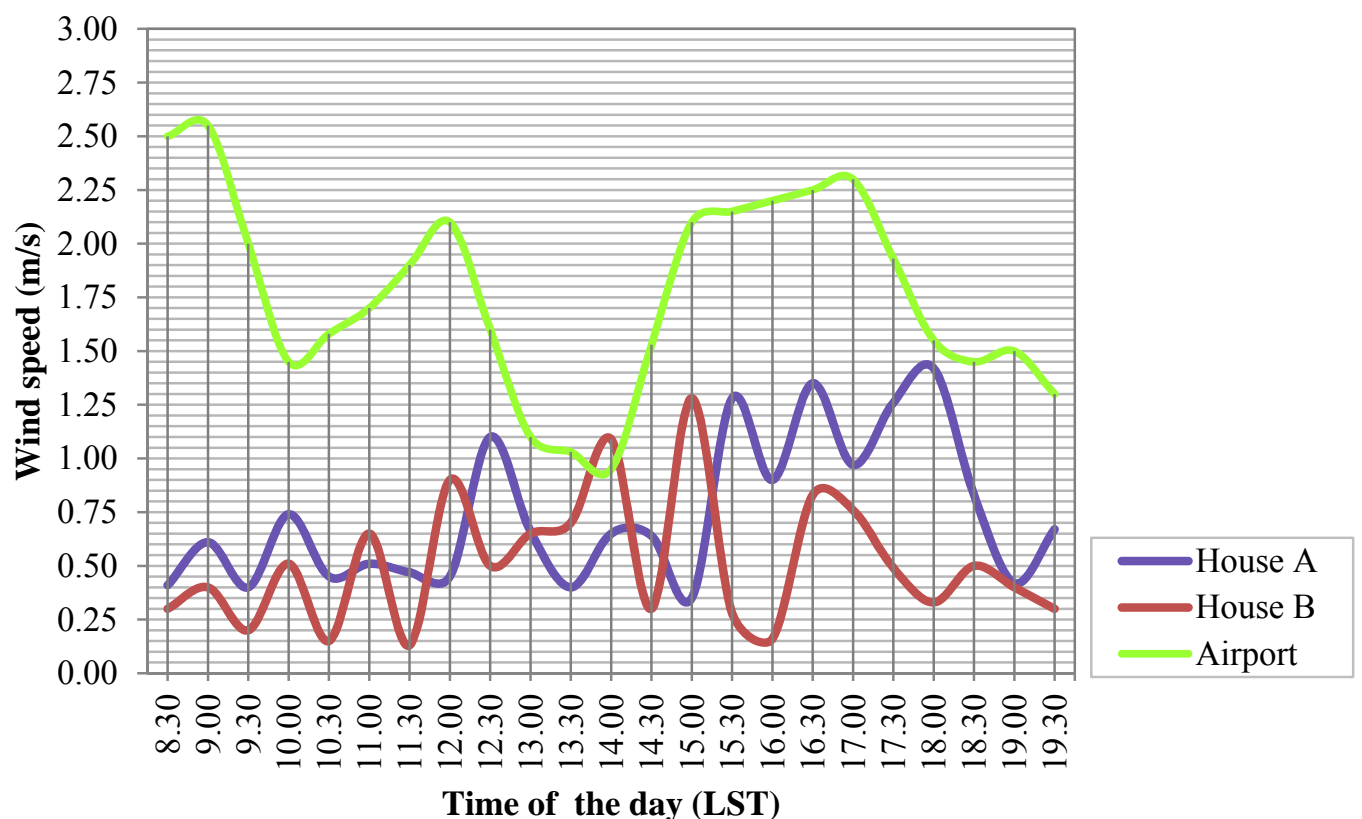

Figure 5.22. Wind speed adjacent to the east walls of the heavily landscaped house (house A) and the sparsely landscaped house (house B)

Wind speeds at both houses fluctuated throughout the day, increasing from 0.2 to $1.4 \mathrm{~m} / \mathrm{s}$ over the day. However, the wind speed at both houses dropped to $0.28 \mathrm{~m} / \mathrm{s}$ at 19.30 hours. Overall, the heavily landscaped house had slightly higher wind speeds on its east side. 


\section{iv) West}

Figure 5.23 shows the wind speeds around the west walls of both houses. The wind speed at the heavily landscaped house was very low, at around $0.5-0.2 \mathrm{~m} / \mathrm{s}$ between 8.30 and 11.30 hours. From 12.00-19.30 hours, wind speeds fluctuated in the range of around $0.25-2.3 \mathrm{~m} / \mathrm{s}$, which was the highest speed recorded (at 17.30 hours).

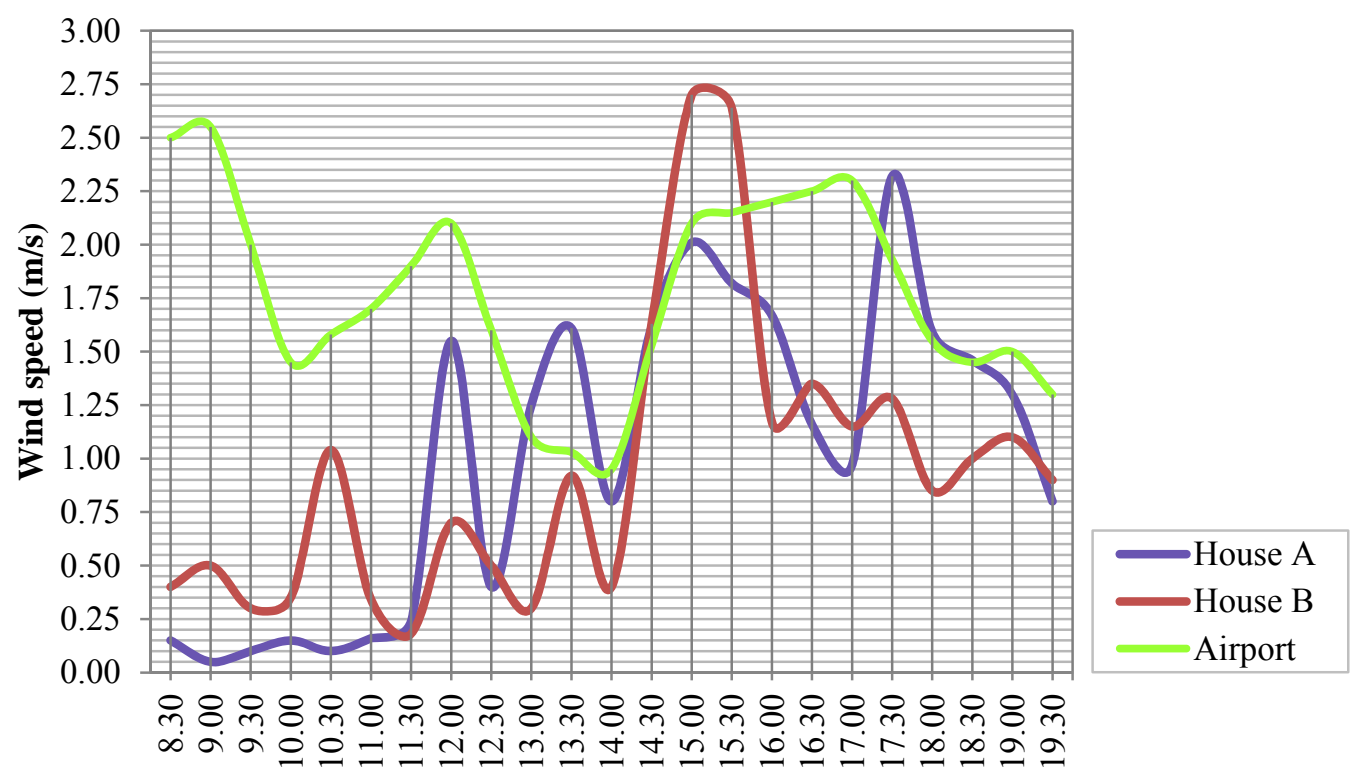

Time of the day (LST)

Figure 5.23. Wind speed adjacent to the west walls of the heavily landscaped house (house A) and the sparsely landscaped house (house B)

On the west side of the houses the sparsely landscaped house received slightly higher wind speeds in the morning, starting at $0.4 \mathrm{~m} / \mathrm{s}$ at 8.30 hours and fluctuating between $0.2-1 \mathrm{~m} / \mathrm{s}$ from 10.00 to 14.00 hours. The wind speed rose dramatically until reaching the highest point at $2.74 \mathrm{~m} / \mathrm{s}$ at 15.00 hours. It then fell to $1.24 \mathrm{~m} / \mathrm{s}$ at 16.00 hours, and fluctuated between $0.9-1.3 \mathrm{~m} / \mathrm{s}$ between $16.30-19.30$ hours.

The wind speed at the airport station was generally more stable than at the two houses. Wind blew in a fluctuating pattern throughout the day, ranging between $0.96-2.55 \mathrm{~m} / \mathrm{s}$. The sparsely landscaped house had a higher wind speed than the airport during the afternoon and evening, with a wind speed of around $0.5-1 \mathrm{~m} / \mathrm{s}$ adjacent to the south walls. The heavily landscaped house had a higher wind speed 
than the airport adjacent to the north walls in the late afternoon, while both houses had higher wind speeds than the airport for the west walls in the afternoon.

\subsubsection{Relative humidity}

Both houses have a different pattern of humidity because of their different landscaping. The heavily landscaped house shows a curved pattern of RH, which started at the highest reading of around $97 \%$ at 8.30 hours, and gradually decreased to reach the lowest reading of around $57 \%$ at 14.30 . It then uniformly rose towards the evening, reaching around $90 \%$ at 19.30 hours.

In contrast, the RH at the sparsely landscaped house started at a low level of around $57 \%$ in the morning, and gradually rose to $64 \%$ at 11.00 hours. The RH level fell to the lowest level of around $54.5 \%$ at $12.30-14.00$ hours, and fluctuated between $70 \%$ and $64 \%$ between $15.30-16.00$ hours. Mahbar (2011) explained that this was probably influenced by the addition of another layer of cloud cover (subtopic 5.5.4.1) at 15.00 hours, with solar radiation dropping by around $0.4 \mathrm{MJ} . \mathrm{m}^{-2}$ as can be seen in Figure 5.13. The RH rose until reaching the highest level of approximately $82 \%$ in the evening at 19.30 hours.

The interior spaces of both houses also had a similar pattern of RH, with stable levels of $\mathrm{RH}$ of between $72-76 \%$ throughout the day. 


\section{i) North}

Figure 5.24 shows the relative humidity adjacent to the north walls for both houses.

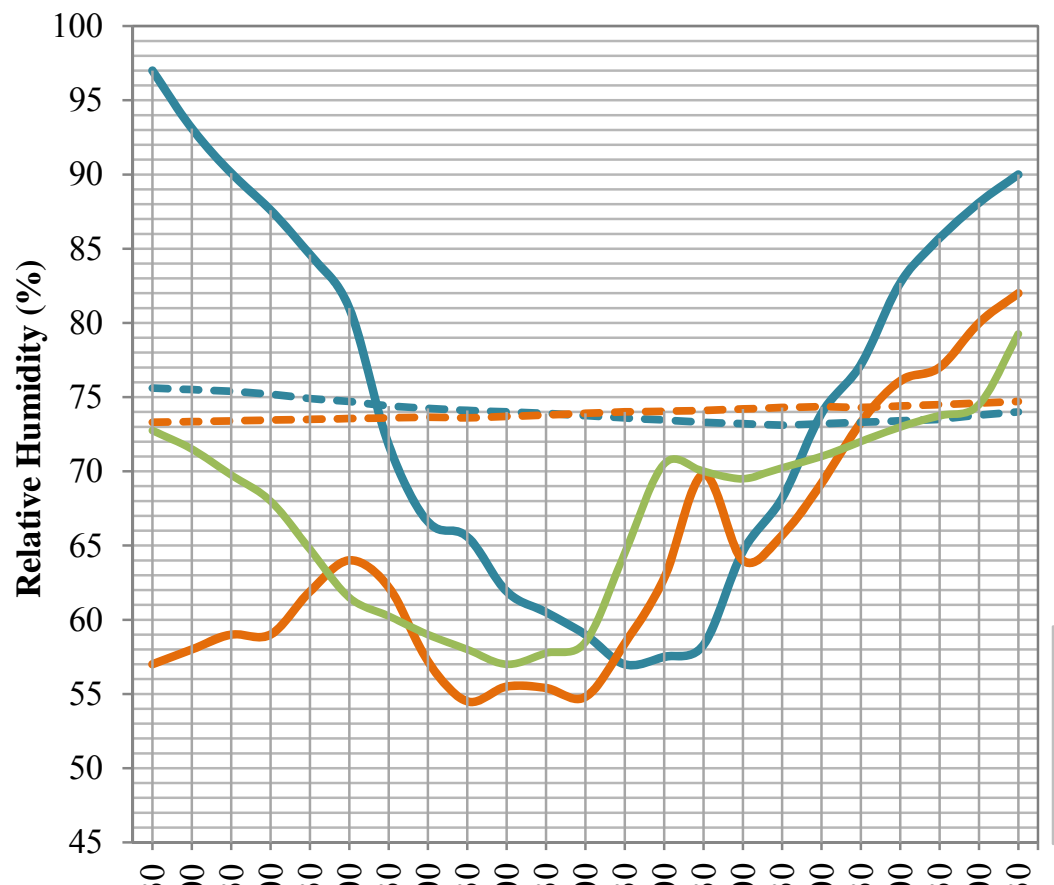

House A outdoor

House B outdoor

- House A indoor

- House B indoor

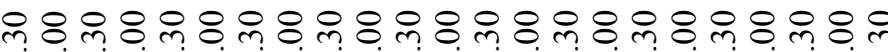

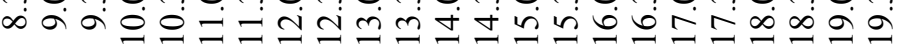

Time of the day (LST)

Figure 5.24. Outdoor and indoor RH levels adjacent to the north walls of the heavily landscaped house (house A) and the sparsely landscaped house (house B) 


\section{ii) South}

Figure 5.25 shows the RH adjacent to south walls of both houses.

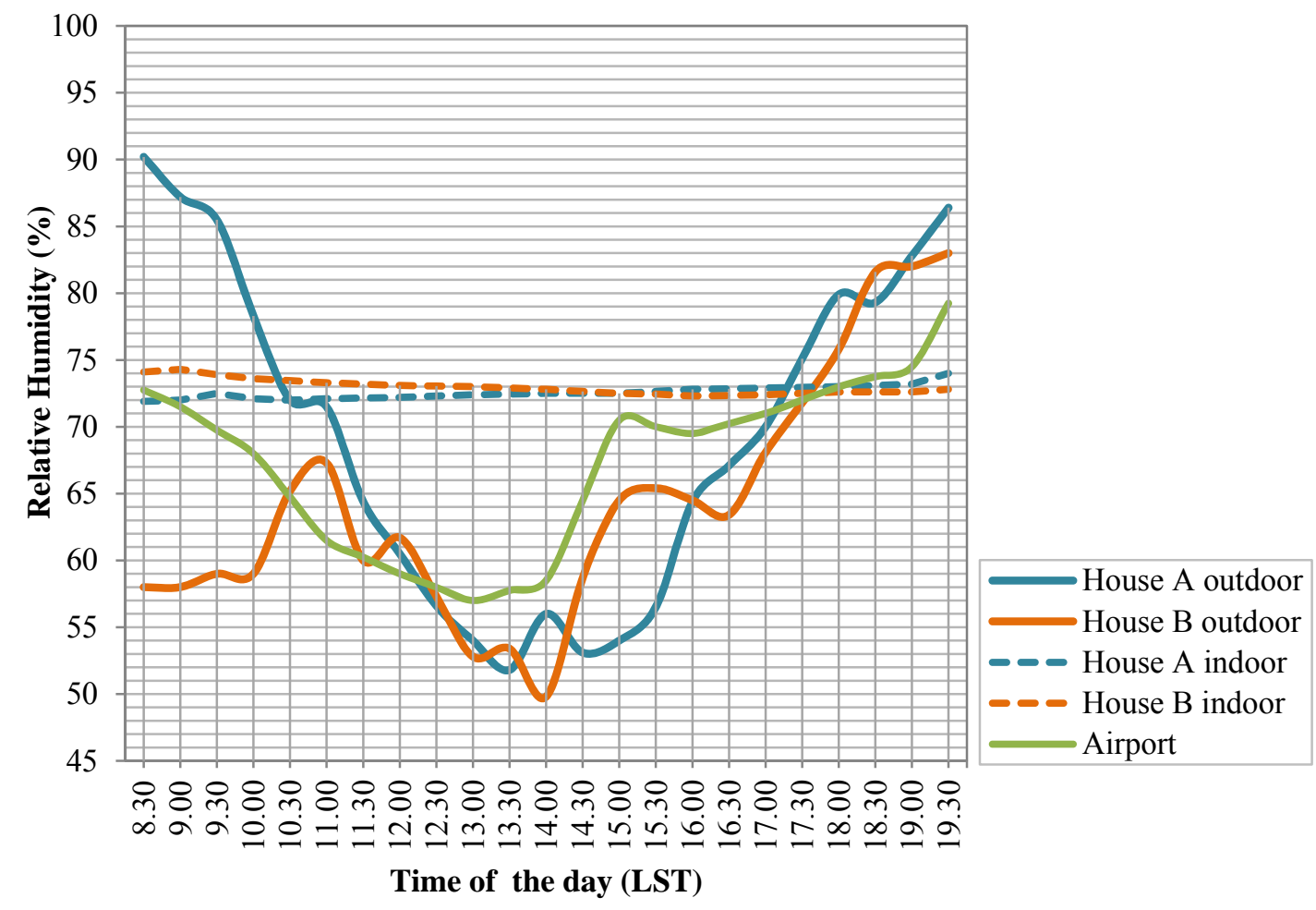

Figure 5.25. Outdoor and indoor RH levels adjacent to the south walls of the heavily landscaped house (house A) and the sparsely landscaped house (house B) 


\section{iii) East}

Figure 5.26 shows RH adjacent to the east walls of both houses.

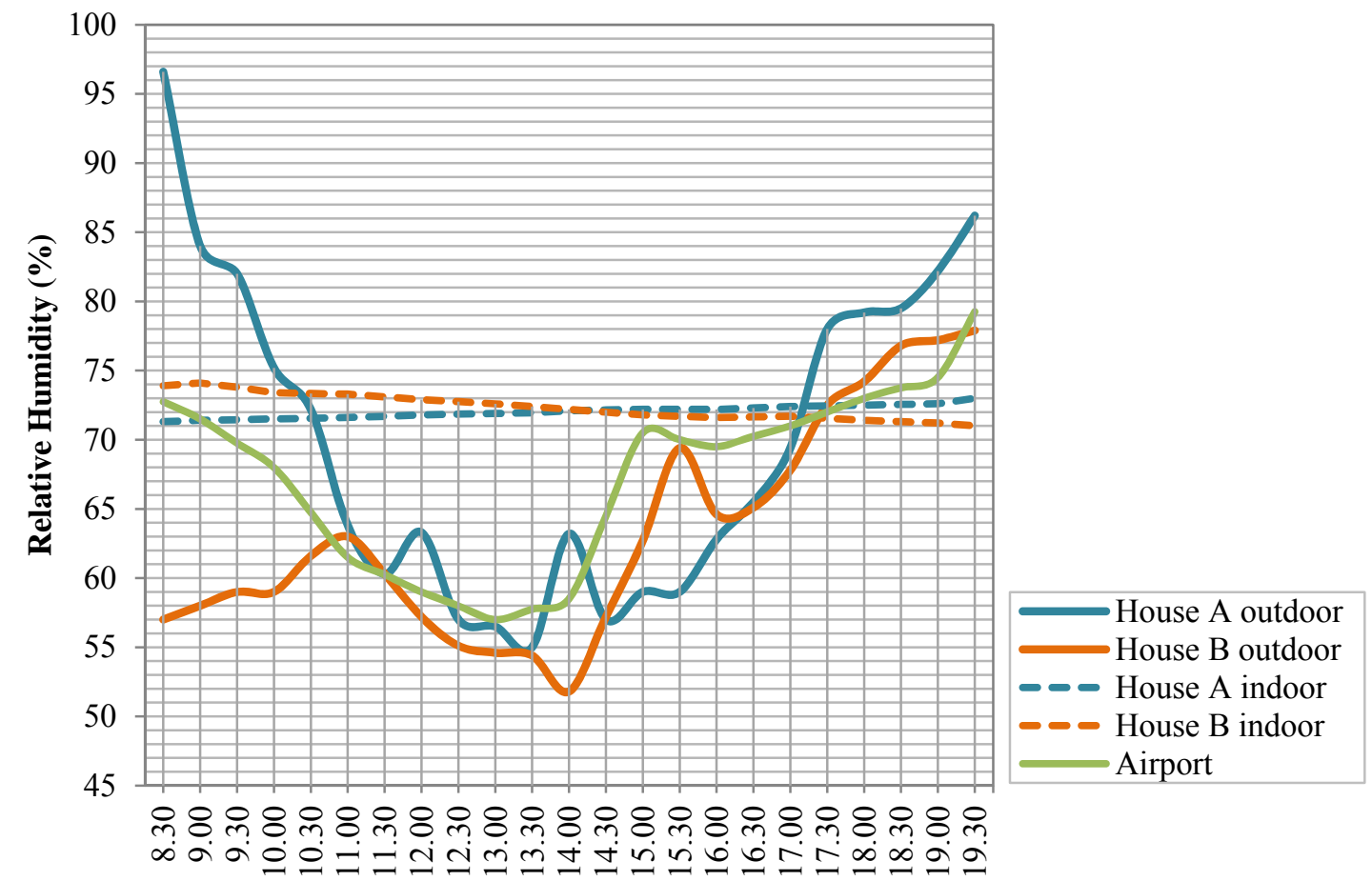

Time of the day (LST)

Figure 5.26. Outdoor and indoor RH levels adjacent to the east walls of the heavily landscaped house (house A) and the sparsely landscaped house (house B) 


\section{iv) West}

Figure 5.27 shows the RH adjacent to the west walls for both houses.
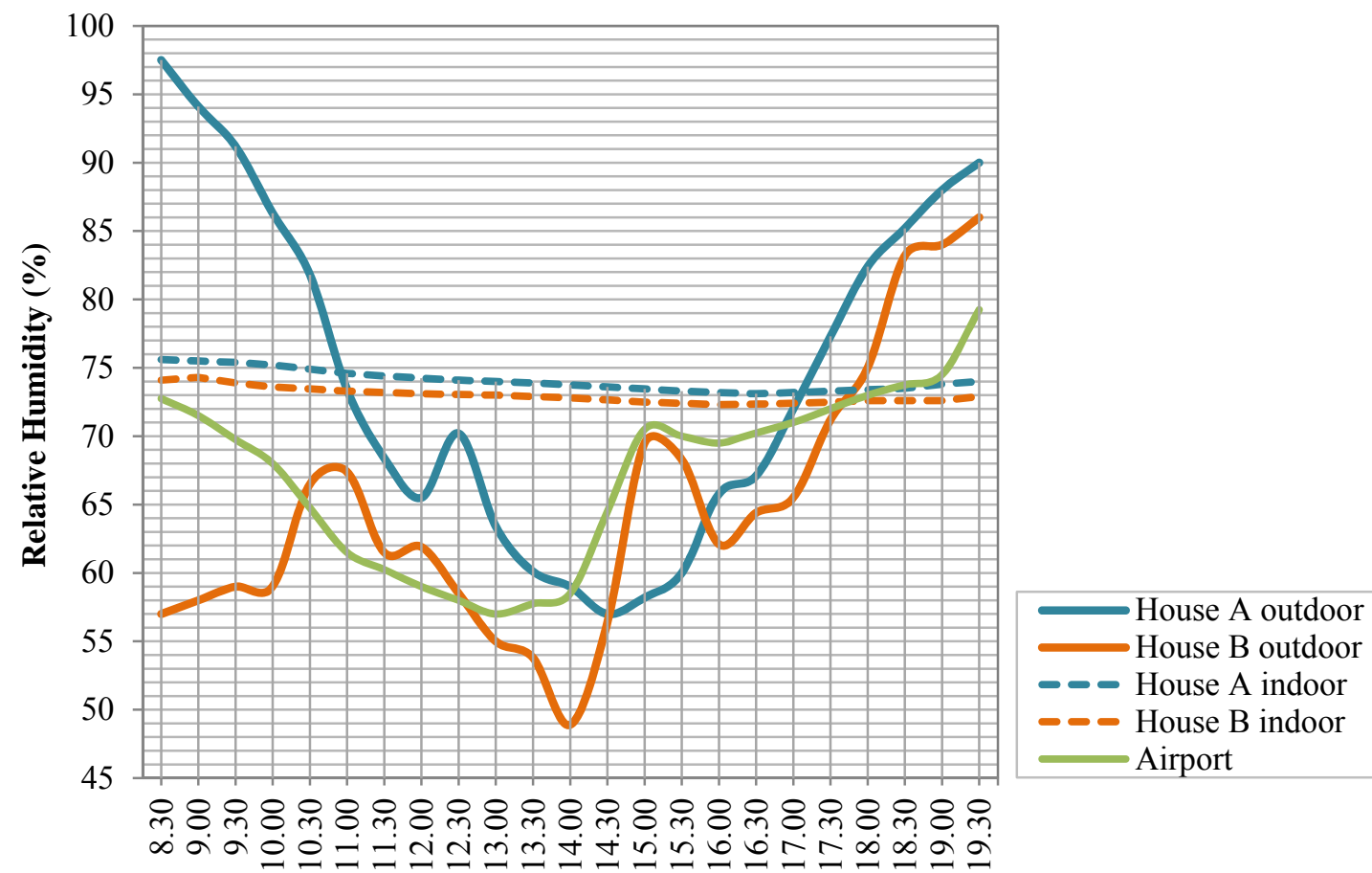

Time of the day (LST)

Figure 5.27. Outdoor and indoor RH levels adjacent to the west walls of the heavily landscaped house (house A) and the sparsely landscaped house (house B)

The RH pattern from the readings taken at the airport, were close to those from the sparsely landscaped house. During the morning the $\mathrm{RH}$ readings from the airport were generally between those from the two houses, with a $10-23 \%$ difference. Towards the evening they were slightly lower by approximately $3 \%$ compared to the sparsely landscaped house. During the middle of the day, the airport RH levels were slightly higher than the sparsely landscaped house, and were around 4-7\% different to the heavily landscaped house. However, all three had a similar reading at 19.30 hours with less than $15 \%$ difference in $\mathrm{RH}$. The dramatic increase in $\mathrm{RH}$ at 15.00 hours was influenced by the drop in global solar radiation of around $0.4 \mathrm{MJ}_{\mathrm{m}} \mathrm{m}^{-2}$, as shown in Figure 5.13. However, the RH at the heavily landscaped house was not affected because its microclimate was controlled by its surrounding landscaping. 


\subsection{Discussion}

The results of this study illustrate that the strategic location and different amount of landscaping in residential areas can modify the ambient air and the thermal performance of the house. Well designed and placed landscaping can also directly influence the microclimate.

On the day the readings at the two houses were taken, the air temperature of the heavily landscaped house was less than the air temperature at the sparsely landscaped house. It also showed the lowest diurnal warming. RH levels had a similar result, with the heavily landscaped having a stable and smooth RH curve that was higher than the sparsely landscaped house.

Wind speed was moderate around the two houses. However, even with the large amount of vegetation around the heavily landscaped house, its good placement allowed prevailing wind flow into the house very well.

\subsubsection{Building construction}

The orientation of the heavily landscaped house is best for a tropical country, as the majority of glass openings should face north-south to minimize the amount of sunlight that enters the internal spaces. This helps to ensure a minimum amount of solar radiation penetrates directly into the interior spaces. The north, or back of the building had major glass openings that covered an area of $29.8 \mathrm{~m}^{2}$, and the south side, which had the house's main entrance, had glass openings that covered an area of $15.2 \mathrm{~m}^{2}$. Conversely at the sparsely landscaped house the majority of the glass openings were located on the west and east sides, which were exposed to the direct sunlight and therefore could account for most of the heat gain to the building. Thus, the building configuration for the heavily landscaped house was properly planned to minimize heat gain. This resulted in the indoor temperature at the heavily landscaped house being relatively lower than the sparsely landscaped house.

Twenty-one percent of each house was glass surfaces, which provided natural ventilation and lighting. Each glazed surface was covered by shading devices, which 
were around $1-3 \mathrm{~m}$ deep. The roof, which projected out from the houses, partially shaded their top storey walls, while a gable or hipped roof was used as a shading device at all windows and doors on all sides on the ground floor. The shading devices on the east and west sides of the houses provided shade from direct solar radiation from late morning until the afternoon, or during the peak time of the day (11-15.00 hours). Shading on the north and south sides also helps to diffuse solar radiation to avoid it entering the house, which helps to reduce heat gain and the resultant load on the air-conditioning systems. However, at the sparsely landscaped house solar radiation could still penetrate during the morning and late afternoon because the walls were not protected by shrubs.

The roof is the biggest surface area exposed to direct sunlight during the day. Both houses in this study have well insulated roofs, which included a layer of aluminium foil underneath the roof coverings. The interior rooms had white ceilings that were $2.9 \mathrm{~m}$ high. Both house also had similar light coloured paint on the exterior walls and concrete roof tiles with an albedo of around 0.25 and 0.33 respectively, which reflects sunlight and reduces the amount of heat absorbed throughout the day.

\subsubsection{Landscape design}

The application of tropical landscape design around the two houses was different. The heavily landscaped house had well designed landscaping, while the sparsely landscaped house had the minimum of landscaping, which was not well organised or ordered. In this study, well designed landscaping is defined as using a sufficient number of plants, that are of a good size and the appropriate species for the area, and that have been situated in a strategic location. Strategic placement is when plants have been placed to maximise their effectiveness in providing shade, channelling wind, and assisting the evapotranspiration process to the surrounding garden area. For both houses, the size, shape and species of each plant, as well as its distance from the building has been listed in Tables 5.3-5.8, Appendices 6.1-6.2, and Figures 5.35.5 . 
The majority of shade trees around the two houses were small, had a rounded shape, and were between $2-4 \mathrm{~m}$ high. These types of trees will not usually provide enough shade for their surroundings, but at the heavily landscaped house the 47 trees had been planted in groups or close to each other in multilayered canopies, as shown in Figure 5.6. This garden had a perfect combination of roadside, garden and fruit trees, and palms that provided a pleasant environment where the trees supported each other, so were able to survive well in all weather conditions. While the average size of leaves on the trees was small, on average most had a medium amount of leaves, which provided shade effectively to the garden areas. Moreover, all of the trees were strategically located at a distance of between 3-9m from the house, for all four azimuths. This created a small microclimate area around the house. Overall, the strategic arrangement of the shade trees in the north and west areas made the air temperature as low as $33.5^{\circ} \mathrm{C}$ and $33.8^{\circ} \mathrm{C}$ respectively, and the difference was as much as $3.9^{\circ} \mathrm{C}$ compared to the sparsely landscaped house.

Conversely the sparsely landscaped house had only 11 trees that were planted individually on the east and south sides of the house. This number was relatively small compared to the size of the garden. In addition, the average amount of leaves was sparse and they were generally small in size. Figure 5.17 shows that the lack of shade provided by trees around the sparsely landscaped house made the air temperature at the west wall up to $3.9^{\circ} \mathrm{C}$ higher than at the heavily landscaped house during the peak time of the day.

The heavily landscaped house had layers of shrubs and groundcover, along with areas of vines underneath shade trees, and next to windows and walls. This included 104 shrubs, 10 plantings of vines and 10 groups of groundcover, and turf that covered every earth surface, which helped to make the whole garden area completely lush and green. These aspects helped to provide a balanced and orderly landscape design

The shrubs had grown well, and were strategically placed to help reduce the effect of solar radiation on walls and windows during the peak time of the day. For example, 
tall shrubs were placed immediately adjacent to a window, which provided shading from direct and indirect radiation throughout the day; and groundcover and turf provided shade along the bottom of the house during the peak time of the day. This arrangement, together with the shade trees, helped to create a relatively cool micro climate via shade and evapotranspiration. The outside temperature differences were as low as 1.5 and $3.8^{\circ} \mathrm{C}$ at the north and west walls respectively during the peak time of the day.

In contrast, the sparsely landscaped house had only 34 shrubs, which were located within $5 \mathrm{~m}$ of the house and scattered around all four azimuths. Some of the shrubs that were planted along the east side of the house were planted underneath shade trees, but most were individually planted around the garden or in groups of shrubs situated on the property's boundary, as shown in Figure 5.7. This landscaping did not prevent solar radiation from reaching the walls and windows. However, on the east side the shrubs worked well with the roadside trees to provide shade and evapotranspiration, and the temperature was as low as $35.4^{\circ} \mathrm{C}$ during the peak time of the day with temperature differences as much as $0.5^{\circ} \mathrm{C}$ compared to the ordinary and new landscape house.

The temperature reductions on the north and west sides of the heavily landscaped house, and the east side of the sparsely landscaped house, show that shrubs positioned in groups, along with shade trees were particularly effective in creating a cooler micro climate.

\subsubsection{Energy saving}

The amount of energy used for cooling was influenced by the building's construction, and the living habits of the occupants of each house and the building construction of the houses, including the number of occupants, their ages and their preferred thermal comfort levels.

The heavily landscaped house had three adult residents, while the sparsely landscaped house was occupied by 10 adults and five children. 
Both houses used active systems during the night for ventilation and cooling, as shown in Figure 5.11. They closed windows at night for security reasons, to prevent noise from outside, and because of climate factors such as rain, storm and strong winds. As a result, they depend entirely on active ventilation systems such as air conditioning and ceiling fans. At night, the heat that has been stored in their building envelop throughout the day is released. This heat contributes to high indoor temperatures, and air-conditioning is the only way to provide a comfortable environment for the occupants. Both houses use air-conditioning at similar times because most of the family members are at home during the night.

Occupants of the heavily landscaped house set the air conditioning at $26^{\circ} \mathrm{C}$ for seven hours per night in one bedroom, while at the sparsely landscaped house the air conditioning was set at the lower temperature of approximately $22^{\circ} \mathrm{C}$ for four to five hours per night in four bedrooms. As a result, the heavily landscaped house used 315 $\mathrm{kWh}$ per month on air conditioning, which was equal to $20 \%$ of the electricity bill, while the sparsely landscaped house used approximately $746 \mathrm{kWh}$ per month, which represented around $45 \%$ of the electricity bill as can be seen in Table 5.9 .

However if the cooling energy was calculated per person, the sparsely landscaped house used less cooling energy, because most rooms were occupied by 3 people ( 2 adults and 1child) while the heavily landscaped house had 1-2 people per room. The 'hours cooling per $\mathrm{kWh}$ ' were similar around 0.819 and 0.815 respectively, for airconditioning as listed in Table 5.9. Moreover, the occupants of the sparsely landscaped house used a lower setting for the air-conditioning system in the evening when higher indoor temperatures were occurring. As a result more cooling energy was used.

These results show that strategic landscaping can provide lower outdoor and indoor temperatures which could reduce the air-conditioning used during day time when natural ventilation was used. In tropical cities, when all openings are closed during 
the night for safety, air conditioning is a basic requirement and need for people to be able to live comfortably.

\subsubsection{Modifying ambient conditions}

The ambient conditions looked at in this case study include air temperature, relative humidity and wind speeds. These were influenced by the surrounding vegetation directly through shading and by channelling wind, and indirectly by evapotranspiration.

\subsubsection{Shading}

The roof is the largest area of the building envelope, and is exposed to solar radiation throughout the day and hard to shade. In the two gardens, the shade trees around their garden were relatively small, so did not currently provide extensive shading to the roofs. The heavily landscaped house had a few Schizolobium parahyba on its south side and Dalbergia oliveri on the west side, which were between 5-9m high and located around $3-5 \mathrm{~m}$ distance from building. These were only able to provide a minimal amount of shade to the roof during late morning until afternoon, as can be seen in Figure 5.7 (left). Because the landscaping does not provide extensive shading of the roof of the houses this may have affected the average external temperatures around the buildings. Air temperatures around the houses rose dramatically in the late morning through until the afternoon, when the sun is high in the sky and more solar radiation reaches the roof surfaces.

To plant for the maximum shading, primary attention should be directed to the walls and windows of houses to reduce heat gain and help maintain low building surface temperatures. If the tree canopies are small or moderately sized the trees should be located fairly close to the building to maximise shading. After five year's growth the canopies of trees planted close to a building will extend to within several metres of the roof, and the walls and windows will be completely shaded during the peak solar radiation periods. 
In the hot-humid tropical city of Putrajaya, the east and west walls of houses are more exposed to the direct sunlight in the morning and afternoon. The north and south walls receive indirect or diffused sunlight. Figure 5.13 shows that even though the cloud cover is 7 Okta, solar radiation is actively radiated for approximately eight hours per day, with maximum radiation $2.4 \mathrm{MJ} . \mathrm{m}^{-2}$ at 13.00 hours.

In areas like these, vegetation could intercept more solar radiation to reduce heat gain to the buildings. According to Hoyano (1988), 95\% of solar radiation at the west side of a building can be intercepted with vegetation

Shrubs, vines and groundcover planted immediately beside a building's exterior walls or windows can also be a barrier to hot outside conditions as they can insulate the building and minimise hot air infiltration into the house

The heavily landscaped house had vines planted along the north, east and south walls that were in good condition and on the north and south sides these had grown up to the first floor. It also had shrubs planted in groups with groundcovers that were green and lush in condition. The average height of shrubs was approximately $1.5 \mathrm{~m}$, which was sufficient to shade walls. A representative example of the insulating properties of shrubs and vines is given in Figure 5.6. This shows that the interior temperature of the heavily landscaped house was lower than that in the sparsely landscaped house throughout the day.

The interior temperature of the heavily landscaped house was as low as $23.2^{\circ} \mathrm{C}$ at 16.30 hours, compared to $26.2^{\circ} \mathrm{C}$ in the sparsely landscaped house $\left(3^{\circ} \mathrm{C}\right.$ lower) during the peak time of the day. Furthermore, its interior temperatures followed a smooth curve daily pattern, achieving the lowest temperature at $23.2^{\circ} \mathrm{C}$. In contrast, the interior temperatures at the sparsely landscaped house had a straight profile, uniformly increasing and $2-3^{\circ} \mathrm{C}$ higher than the heavily landscaped house over the day. This proves that the air beneath the vines, shrubs and groundcover and the air beside the walls of the heavily landscaped house are cooler than that at the sparsely landscaped house. This landscaping delays the heating of the exterior ambient air 
during the day, and helps the interior temperature to remain lower for approximately four hours from midday until 16.30 hours. The temperatures then slowly increased after 17.00 hours. The air beneath the leaves between the shrubs and walls cools down when the heat gain through walls radiates back to the environment during the day. During the night, the temperature increases due to the release of heat stored in the walls during the day. The results of this study show that the cool air was trapped by the leaves of the vines, shrubs and groundcover, and provided insulating properties to the floors, walls, and windows of the house. It also decreased the amount of heat radiated back to the environment from the wall during the night. In general, the exterior air temperature was higher than the interior air temperature, while the fluctuations observed beside the exterior walls were reflected in the interior environment. Furthermore, the air temperature in the interior of the heavily landscaped house was lower than that of walls that were not protected by vegetation in the sparsely landscaped house by up to $3.9^{\circ} \mathrm{C}$.

It can be assumed that air-conditioners were used actively during the night at both houses, as their indoor temperatures increase during this time. This was due to the release of heat stored in the walls during the day, together with the activities of the occupants such as cooking, doing washing, watching television and the use of lighting. Strategic surrounding landscaping at the heavily landscaped house made the heat rise at a slower rate than the sparsely landscaped house. 


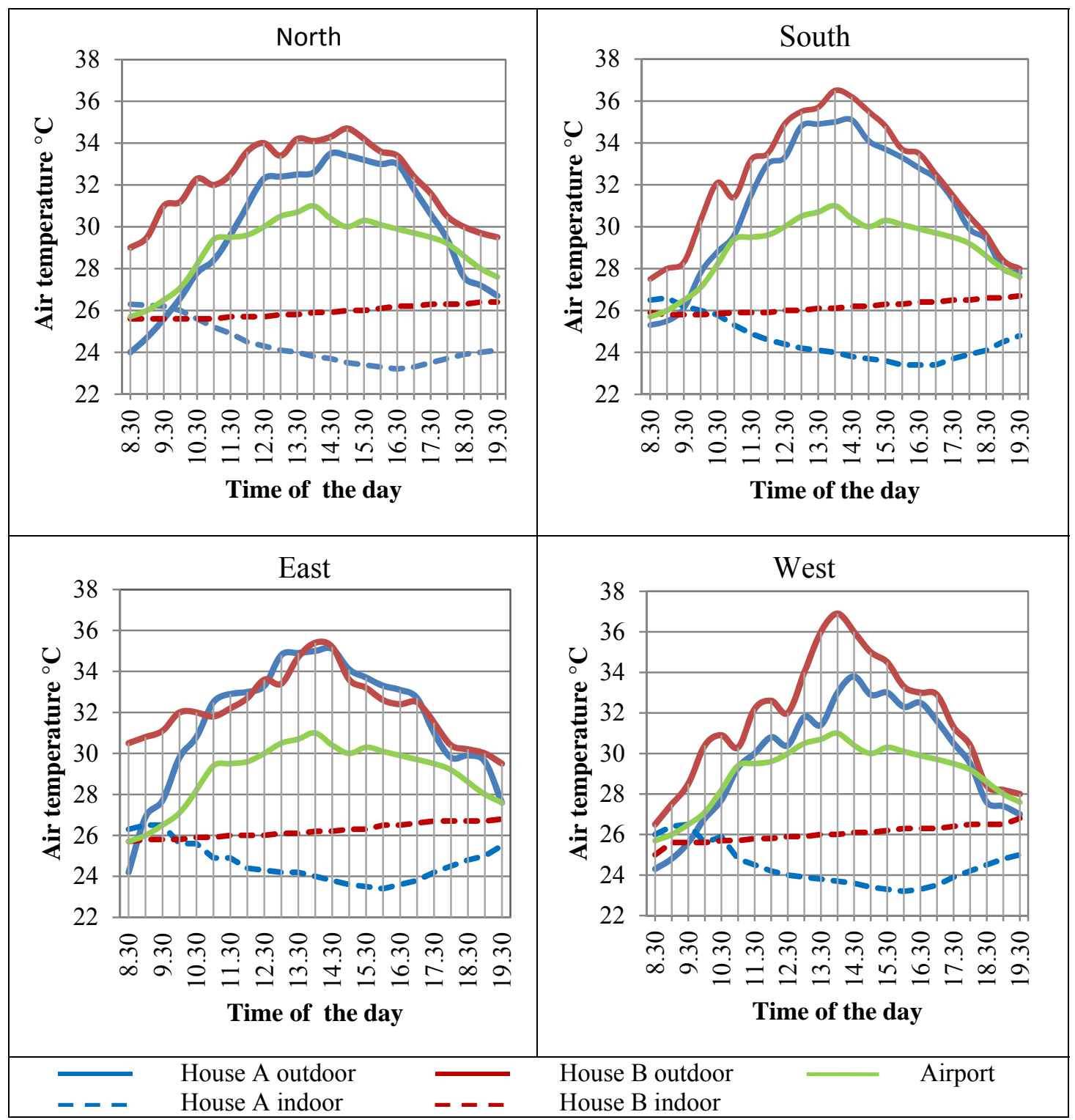

Figure 5.28. A summary of air temperature adjacent to four side walls of the heavily landscaped house (house A) and the sparsely landscaped house (house B)

\subsubsection{Channelling wind}

Appropriate channelling of the prevailing wind is an important part of lowering the surface air temperature of buildings in hot-humid tropical climates. When there is high humidity and temperature conditions, winds are needed to cool the atmosphere. The strategic placement of landscaping and building orientation will allow airflow to cool the building and its surrounding area. 
The predominant wind direction in the study area was from the north and changed three times a day between the north-east and north-west. Wind direction at the study site varied because of the effect of other houses and landscaping elements such as trees and shrubs in the surrounding neighbourhood.

The heavily landscaped house has had its trees and shrubs arranged to channel prevailing winds into its living areas. For example, while the majority of shade trees and palms were still small to moderately sized, most of them had a minimum trunk height of $2 \mathrm{~m}$, which was sufficient to channel wind to the surface of the house and garden areas. On average their leaves were small, and a medium amount, which allowed wind to easily pass through them. The shrubs underneath the trees diverted the prevailing winds from the north-east and north-west side through to the backyard garden, which caused winds to be driven towards the open windows and sliding doors that faced the back yard and windows on the east and west facades. This contributed to cooling the interior of the house and comfortable ambient air conditions.

Figure 5.29 shows that the wind speed in the north area of the heavily landscaped house was higher by $2 \mathrm{~m} / \mathrm{s}$ than in the same section at the sparsely landscaped house during the afternoon and towards the evening. This was because the heavily landscaped house was strategically located to face the wind, and landscaping around the house had channelled wind and allowed it to pass through to the house.

However, when the wind came from the south the sparsely landscaped house was breezier during the afternoon and evening. This was because the winds flowed through the open spaces in north-west and north-east areas of the garden, which had little vegetation around and no other structures to divert the winds from the house. Wind from the east and west were similar in both houses because they were located in end lots, so had similar open space areas to one side and neighbouring houses on the other. 
The result show that the amount of cool breeze that reaches the heavily landscaped house has been influenced by the strategic placement of vegetation and around it. Also, the strategic location of the house facing an open space and towards the prevailing wind was an effective way to allow wind flow to pass through to the house areas. At the sparsely landscaped house some areas of landscaping helped to channel wind effectively to the house, which shows how important strategic landscaping is.

The use of tall shrubs planted strategically around a house next to walls and facing windows can act as a valve to regulate air flow near the openings of the house. For example, at the heavily landscaped house, wind flow from north-west and north-east could pass directly through to the sliding door that was facing the main garden. This was because shrubs blocked or surrounded the other walls. The sliding door opening was strategically located so that cooler winds could be channelled directly to the interior space when it was open. If the sliding door or windows in this area were closed, the cool wind would be channelled away to the garden.

The tall shrubs and vines that were located close to the walls of the house provided insulating pockets of cool air. The gap between these plants and the wall surfaces was around $0.3 \mathrm{~m}$. This ensured that the walls were still exposed to wind, which helped to keep the interior temperature in the heavily landscaped house as low as $23^{\circ} \mathrm{C}$, up to $3^{\circ} \mathrm{C}$ less than the sparsely landscaped house. 


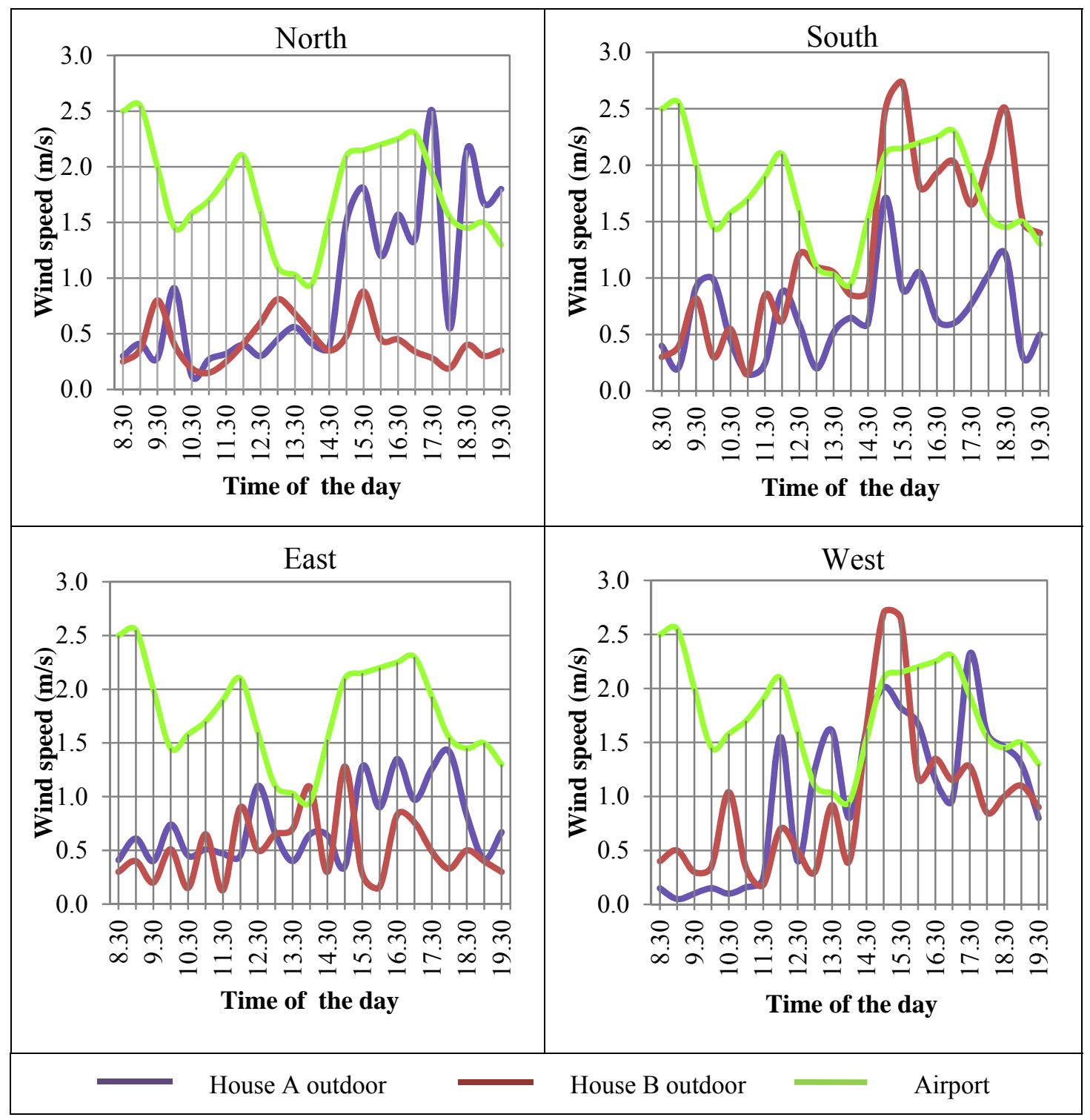

Figure 5.29. A summary of wind speed adjacent to the four side walls of the heavily landscaped house (house A) and the sparsely landscaped house (house B)

\subsubsection{Evapotranspiration}

The RH measurements taken during this study have shown that the increase in RH beside the outdoor walls was due to the surrounding landscaping. The heavily landscaped house had a curved pattern of $\mathrm{RH}$, which shows that the $\mathrm{RH}$ was high during the early morning and late afternoon, and low around noon and through the afternoon. The changes were minimal and consistent around the four sides of the house. The changes were being controlled indirectly by the surrounding heavy 
landscaping with its evaporation and transpiration processes, and directly influenced by solar radiation.

The half hourly RH measurements at the two houses were taken from beside their exterior walls. These measurements are shown in Figure 5.30 and the temperature levels are shown in Figure 5.28. These figures show that evapotranspiration was influenced by the amount, placement and size of plants around the house. The heavily landscaped house had higher RH levels, especially during the morning and afternoon, and these smoothly declined from noon. This was because dense vegetation helps to control the moisture content through the evapotranspiration process.

The different amounts of vegetation around the two houses produced different microclimates. Having a large amount of vegetation can modify net radiation, sensible heat, latent heat and heat storage. In a hot-humid climate, latent heat may exceed net radiation by $10-50 \%$, with sensible heat derived from the air and converted to latent heat (ASCE, 1996).

Evapotranspiration occurs when the net radiation absorbed by the ground surface converts water from the liquid in the soil, standing water, or on plants to gas, as shown by the heavily landscaped house. Subsequently, sensible heat is changed to latent heat and the air temperature falls by lowering dry bulb temperatures through plant evapotranspiration. The more vegetation on the earth's surface, the more the evapotranspiration process will occur, providing a cooler atmosphere. Overall, the vegetation at the heavily landscaped house was in excellent physical condition; fertile, lush and green with medium size and a medium amount of leaves. The arrangement of the vegetation includes trees, shrubs, vines, and groundcover around the house and garden, and this has been done strategically to provide shade to the building envelope and garden area. This contributed to the evapotranspiration cooling of the ambient air surrounding the house, and reduced the ground surface temperature. 


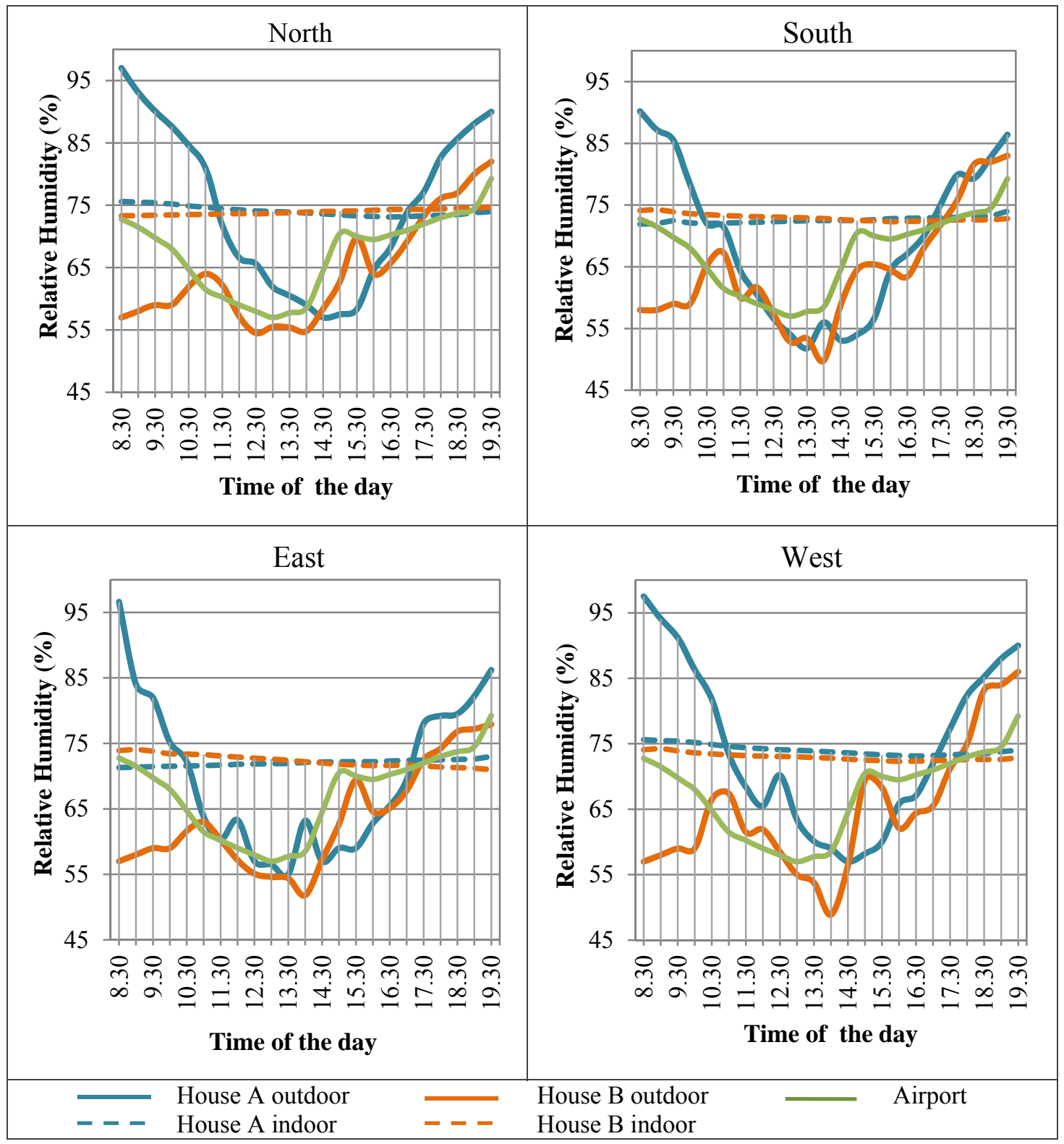

Figure 5.30. A summary of RH adjacent to the four side walls of the heavily landscaped house (house A) and the sparsely landscaped house (house B)

In areas with less vegetation most of the net radiation is returned to the atmosphere as sensible heat, where warm air is carried from the ground or surface, which was evident at the sparsely landscaped house. When latent heat is changed to sensible heat, the air temperature rises. As shown in Figure 5.30, RH at the sparsely landscaped house had low levels of fluctuation from the morning until the afternoon, and was as low as $48.5 \%$ at noon. 
Water elements, such as the fish pool surrounded by shrubs and the pergola structure covered by lush vines in the main garden of the heavily landscaped house, were additional landscape elements that can provide shade and assist the evapotranspiration cooling process. The heavily landscaped house had up to 95 and $85 \% \mathrm{RH}$ levels in the morning and late afternoon respectively, and $58 \%$ at noon. As a result, a greater level of evapotranspiration, combined with shading and a light wind speed could cool the surface temperatures to as low as $33^{\circ} \mathrm{C}$ at the hottest hours of the day (14.00 hours) at the heavily landscaped house. This was compared to temperatures of around $37^{\circ} \mathrm{C}$ at the sparsely landscaped house; a $4^{\circ} \mathrm{C}$ difference.

\subsection{Discussion of the results}

Both houses were of similar design, construction, and age (three years old), but had different façade orientations as can be seen in Table 5.11. The north and south façade orientation of the heavily landscaped house, where the majority of glass openings were located, can minimize the amount of solar radiation penetrating the internal spaces. If east and west façade orientations were experienced, as in the sparsely landscaped house, extensive and strategic shading by trees and shrubs together with wider hipped roof overhangs would be needed to protect the house from sunlight penetration during late morning and afternoon. Otherwise the majority of glass opening design must be changed to north and south orientations and minimal glass openings located on the east and west facades.

Table 5.10. Façade orientation, surrounding vegetation, weather data and human factors at the heavily landscaped house (A) and the sparsely landscaped house (B)

\begin{tabular}{|c|c|c|c|c|c|c|c|c|}
\hline House & $\begin{array}{c}\text { Façade } \\
\text { orientation }\end{array}$ & Surrounding & \multicolumn{2}{|c|}{ Weather data (west) (peak time) } & \multicolumn{2}{|c|}{ Human factors } \\
\cline { 5 - 9 } & & $\begin{array}{c}\text { Vegetation } \\
\text { outdoor } \\
\left({ }^{\circ} \mathrm{C}\right)\end{array}$ & $\begin{array}{c}\text { Temp. } \\
\text { indoor } \\
\left({ }^{\circ} \mathrm{C}\right)\end{array}$ & $\begin{array}{c}\text { Wind } \\
\text { speed } \\
(\mathrm{m} / \mathrm{s})\end{array}$ & $\begin{array}{c}\mathrm{RH} \\
(\%)\end{array}$ & $\begin{array}{c}\text { Cooling } \\
\text { energy } \\
\text { use (\%) }\end{array}$ & $\begin{array}{c}\text { Occupant } \\
\text { number }\end{array}$ \\
\hline $\mathrm{A}$ & $\begin{array}{c}\text { North and } \\
\text { South }\end{array}$ & $\begin{array}{c}\text { Maximum/ } \\
\text { heavy }\end{array}$ & 33 & 23 & 2.4 & 62 & 20 & 3 \\
\hline $\mathrm{B}$ & $\begin{array}{c}\text { East and } \\
\text { West }\end{array}$ & $\begin{array}{c}\text { Minimum/ } \\
\text { sparse }\end{array}$ & 36.9 & 26.3 & 2.7 & 48 & 45 & 15 \\
\hline
\end{tabular}


Huge amounts of vegetation located at strategic positions around the heavily landscaped house create significant effects in providing shade, wind channelling and evapotranspiration cooling. Overall the earth surfaces around this heavily landscaped house were almost $70 \%$ covered by trees, shrubs, vines and groundcover and the rest was covered by turf, while the overall amount of vegetation cover at the sparsely landscaped house was less than one-quarter of the heavily landscaped house. The excellent physical condition of plants which were located strategically to provide shade and wind channelling also contributed to the evapotranspiration cooling of the ambient air surrounding the house and reduced the ground temperature. The heavy amounts of vegetation, positioned at strategic locations, reduced the effect of solar radiation on walls and windows during the peak time of the day, achieving lows of $32.8^{\circ} \mathrm{C}$ outdoor and $23^{\circ} \mathrm{C}$ in indoor spaces.

In this study roof surfaces are the most exposed part of the building envelope to solar radiation, but these are hard to shade by trees. Medium sized trees and a spreading form of canopy can potentially provide shade to roof surfaces, such as by a number of Dalbergia oliveri on the west side of heavily the landscaped house. However, these should be of medium size with heights more than $9 \mathrm{~m}$, and consist of at least two or three trees located on the east and west sides $3-5 \mathrm{~m}$ distant from building. That amount of shade trees will protect at least $50 \%$ of the roof surfaces and furthermore can provide shade and reduce heat gain to the building and decrease the surrounding temperature during the peak time of the day.

Figures 5.6 and 5.31 illustrate the strategic location of shade trees and shrubs for a heavily landscaped house. The results of this study have clearly shown that mature shrubs, vines and ground cover located close to walls provided insulating properties to the floors, walls, and windows of the house (subtopic 5.6.4.1 - shading), as strategically placed surrounding small and small moderate size shade trees, can decrease the temperature up to $3.9^{\circ} \mathrm{C}$ outdoors and $3^{\circ} \mathrm{C}$ indoors. They also keep the interior spaces cool and comfortable after the peak time of the day compared to the house without shrubs along the walls (as around the sparsely landscaped house) by delaying the peak inside temperature by approximately four hours. The cooler 
outdoor environment resulting from heavy landscaping can directly promote cooler indoor spaces as shown in Table 5.11. In both cases the differences in peak temperature between outside and inside was around $10^{\circ} \mathrm{C}$ due mainly to the mass of the building construction, which was similar for both buildings.

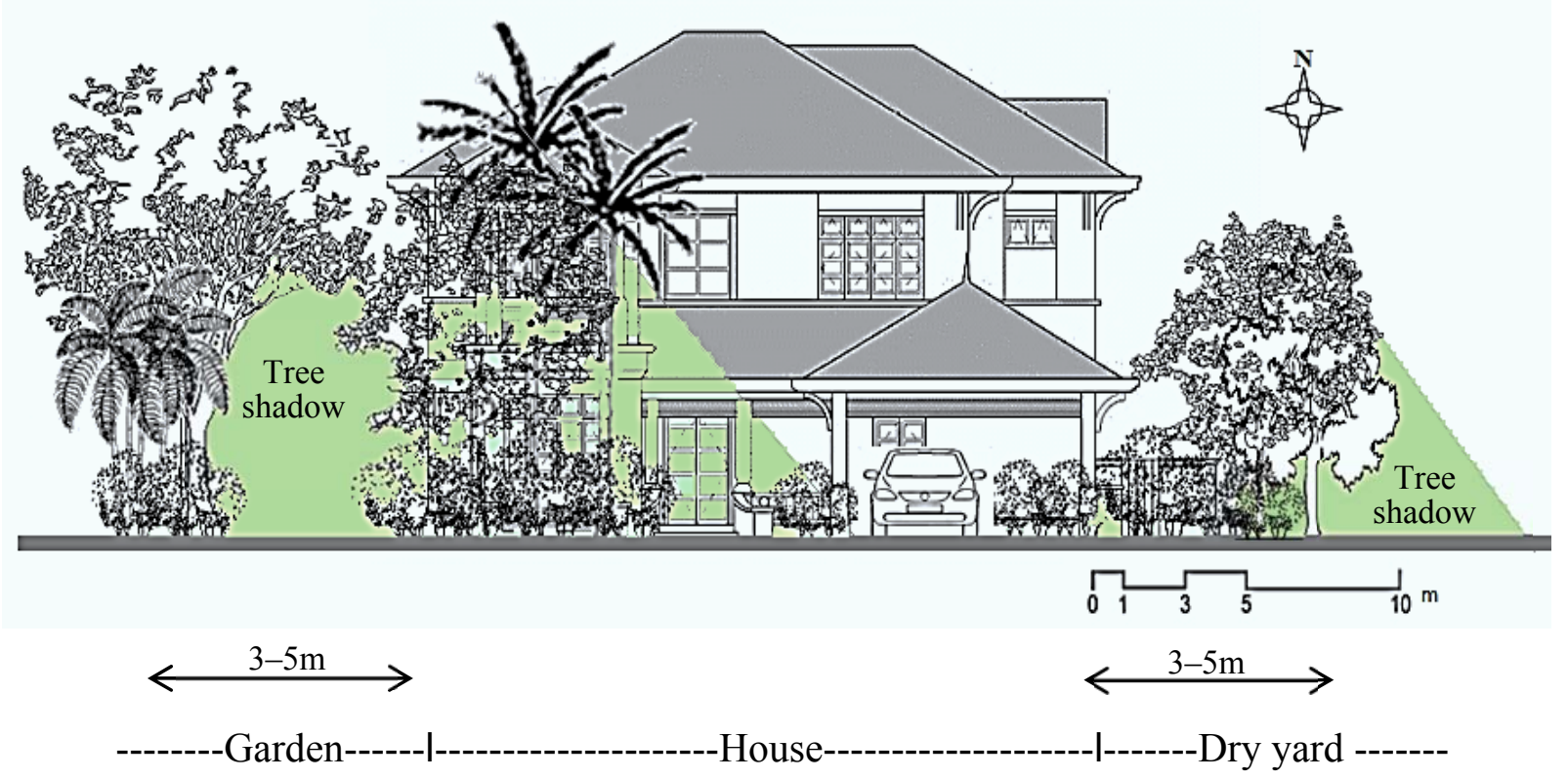

Figure 5.31. Trees and shrubs of heavily landscaped house providing extensive shade to the windows and walls, and ground surfaces close to the house after 15.00 hours (west) in the afternoon and before 11.00 hours (east) in the morning

Both houses faced a large open space at their back façade as well as facing the direction of the prevailing wind which can promote natural ventilation. Table 5.10 shows similar moderate wind speeds during the peak time of the day on the west side of both houses. Both houses use natural ventilation during early morning and late afternoon while they close the sliding doors and windows to avoid hot air entering their house during the peak time of the day. According to Busato (2003), the shape and orientation of the building relative to the direction of the wind can both influence directly the way in which the airflow behaves around buildings. Figure 5.32 shows that the house had the potential to use everyday natural ventilation where the cool air movement can move cross from the living/dining to the lobby area. The position of shrubs can divert the wind flow straight to the interior spaces as shown in Figure 5.32 . 


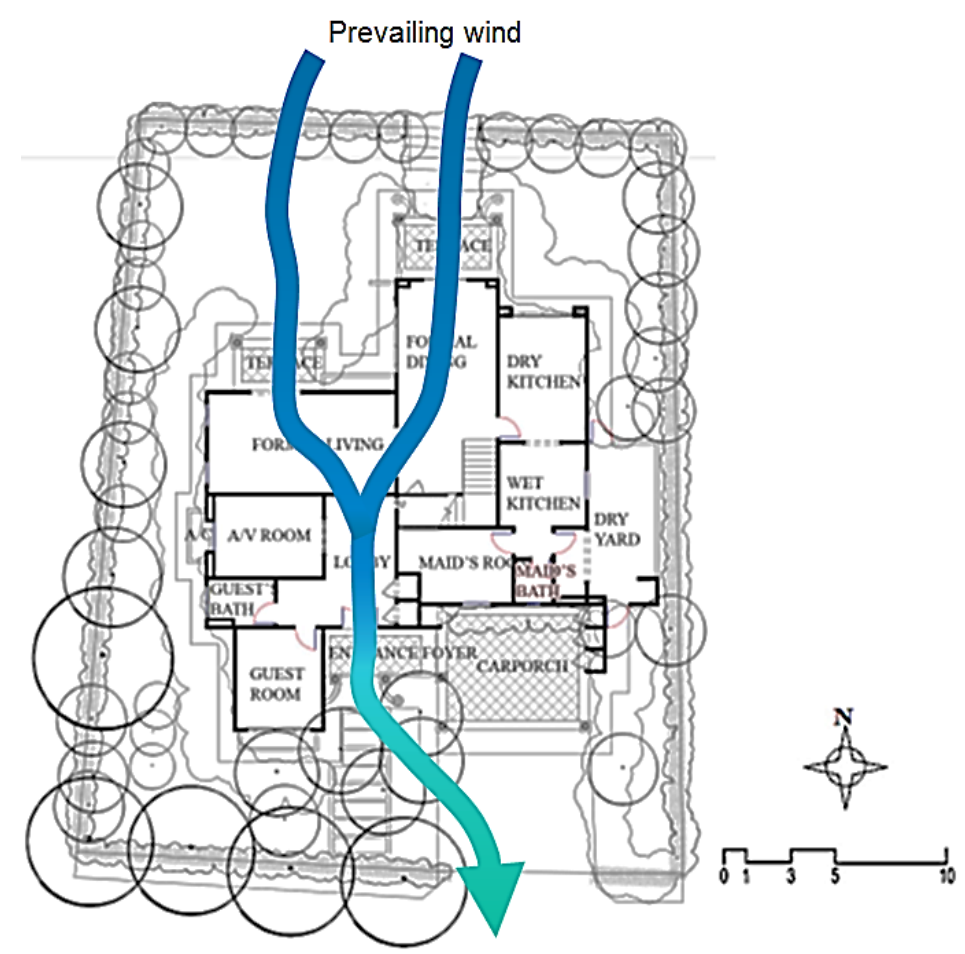

Figure 5.32. The arrangement of shrubs around heavily landscaped house influences the movement of wind from north-east through the building

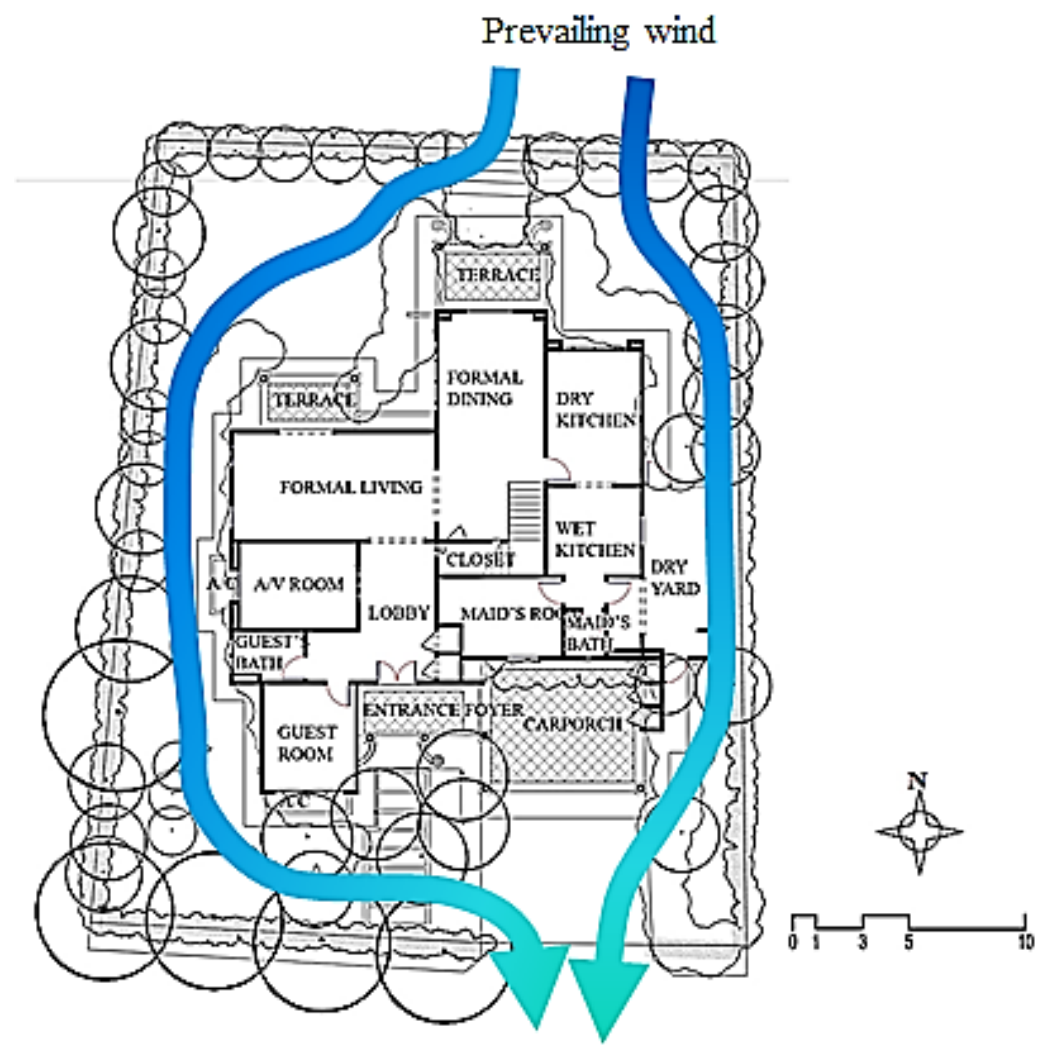

Figure 5.33. The arrangement of trees and shrubs around heavily landscaped house influences the movement of wind from north-east around the building 


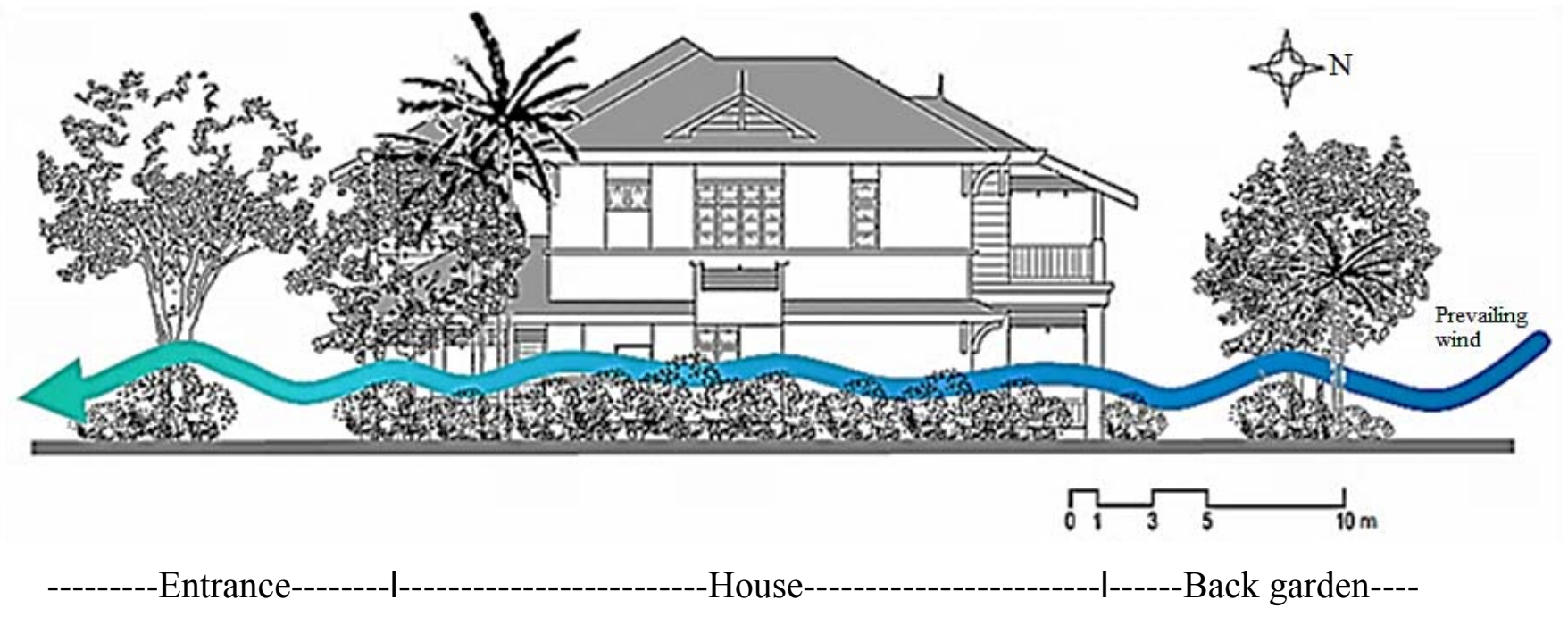

Figure 5.34. The arrangement of trees with sufficient trunk heights and shrubs at heavily landscaped house influences the movement of wind from north-east around and through the building

Figures 5.32-5.34 show, for a heavily landscaped house, that trees with sufficient trunk height and strategically located shrubs, will allow wind to flow to the garden and building surfaces, and through the building (refer to subtopic 5.5.2.1-trees and 5.6.4.2-channelling wind).

The human factors for the two houses were totally different in terms of the number of occupants and total cooling energy use, as can be seen in Table 5.11. The low indoor temperature during day time cannot be maintained until night time. When all the openings were closed during the night, and the indoor temperature was more than $26^{\circ} \mathrm{C}$ the occupants will depend on the air-conditioning system to achieve their comfort level. If the occupants were willing to use natural ventilation during the night time, the use of the air-conditioning system could be decreased.

It is suggested that in the future, architects and landscape architects should work as a team to produce a better overall house design. The house that is surrounded by strategically designed landscaping could potentially use natural ventilation during night time in safety even though the house is located in a city area. 


\section{$5.8 \quad$ Summary}

Both houses in this study were medium sized and built to a typical tropical architecture design. They had similar standards of construction, light coloured walls and roof, and were located at the end of their streets, but they had clear differences in their landscape treatments. The heavily landscaped house was surrounded by various types of plants that were strategically placed around the house and garden, while the sparsely landscaped house had fewer plants, with most located in non-strategic locations.

In a hot-humid tropical climate vegetation is one of the important materials that can be used to alter the microclimate around a house. Quantitative measurements comparing the heavily and sparsely landscaped houses have shown that the landscaping treatments in their private gardens created two different microclimates. The heavily landscaped house had a diverse range of trees, which covered approximately $60 \%$ of the garden area. In comparison, the sparsely landscaped house had a small number of plant species, and the tree canopy only covered around $5 \%$ of the garden's area. Similarly, the heavily landscaped house had 124 individual and groups of shrubs, vines and groundcover, while the sparsely landscaped house had only 34 individual and groups of shrubs planted in all azimuths of the house. Therefore, the heavily landscaped house had a cooler and more comfortable environment than the sparsely landscaped house.

Every piece of vegetation, including trees, shrubs, vines, groundcover and turf, that was used in the landscape design of the heavily landscaped house had their own unique functions to provide high relative humidity, channel wind towards the house and surrounding garden, and lower the air temperature. A greater number of plants, along with a variety of species planted in strategic locations around a garden can clearly reduce air temperature by as much as $3.9^{\circ} \mathrm{C}$ in outdoor areas and $3{ }^{\circ} \mathrm{C}$ for indoor spaces, which helps to provide a cool and comfortable living environment. 
The next chapter will report in detail of the effects of the surrounding landscaping on the cooling load and costs for 50 single-family houses in five neighbourhoods in the two planned cities: Putrajaya and Shah Alam. 


\subsection{THE EFFECTS OF SURROUNDING LANDSCAPING ON THE COOLING LOAD AND COSTS}

\subsection{Overview}

The previous chapter showed how different amounts and placement of the landscaping surrounding two single-family houses (one heavily and the other sparsely landscaped) produced a relative difference in the outdoor and indoor air temperatures. This chapter presents the second stage of fieldwork and site measurements that were conducted at a neighbourhood scale (micro-scale). The objective of this study was to evaluate potential air-conditioning energy savings by conducting interviews and recording data from 50 single-family houses in two well planned cities: Putrajaya and Shah Alam.

In tropical cities, the outdoor environment is extremely warm due to year-round high temperatures. Solar heat passing through windows and absorbed by the walls and roofs of buildings heats the interior of houses, and is the major reason for airconditioner use to create and maintain a comfortable indoor temperature. It has been found that a majority of households in the study areas spend more than $36.7 \%$ of their electricity costs on cooling.

The strategic placement of vegetation around a building has long been recognised as a means of cooling the building. The appropriate amount, type and placement of vegetation can reduce temperature through providing shade, and increasing evapotranspiration and wind channelling to the house. This can reduce residential cooling demand on a hot-humid day. However, the effect of surrounding vegetation on the cooling load and costs for single-family dwellings in a tropical environment has not been widely recognised and quantified.

Monitoring of household electricity use in the study areas has shown that nights are when most of the air conditioning energy expenditure occurs because most households only spend time at home at night. 
Aside from the roof, the critical building envelope surfaces of a house are on the east and west sides. These areas are exposed to the full effect of the sun during the morning and afternoon, and thus provide the highest temperature and heat gain to the building, and retain heat until night time. Proper placement of the right species, size, and form of trees, shrubs, vines, grasses, and turf can greatly reduce the temperature around a building and its heat gain during the day, and therefore the amount of energy used for cooling. This study will determine the potential for energy saving for single-family dwellings in a hot-humid tropical environment, through the strategic planting of vegetation.

\subsection{House study}

Data collection and interviews were carried out on 50 single-family houses in Putrajaya and Shah Alam, Selangor, Malaysia. The houses were randomly chosen, with 70\% from Shah Alam and 30\% from Putrajaya. In general, most of the houses represented a conventional style of tropical architecture that is able to deal with the demanding tropical climate of high temperatures, high humidity and heavy tropical downpours. The main characteristics of these housing estates and houses have hardly changed over the last four decades. They are laid out in a grid-iron manner, and the house construction includes plastered masonry in-fill walls to a reinforced concrete structural frame. The common typology is single-family houses with medium sized fenestration, and hipped-gable timber roofs that are covered with clay or concrete tiles.

Until the advent of air conditioning, single-family houses in Malaysia were designed with local tropical climatic conditions in mind. With greater global awareness of the environment, and a renewed perspective on contemporary Malaysian architecture, this study will show that the use of landscaping around single-family houses is an appropriate solution for reducing the effects of the climate and energy use, alongside good building design and planning. 


\subsection{Weather data}

Two years of weather data was used to obtain an accurate picture of weather conditions in the study areas. Weather data was acquired from the Malaysian Meteorology Department for 2008 and 2009. The data was from two weather stations: Sepang Weather Station for the Putrajaya study area, and Subang Weather Station for Shah Alam. In both study areas, the average annual air temperature and relative humidity $(\mathrm{RH})$ were uniformly high, with heavy rainfall and sunshine for around six to eight hours per day.

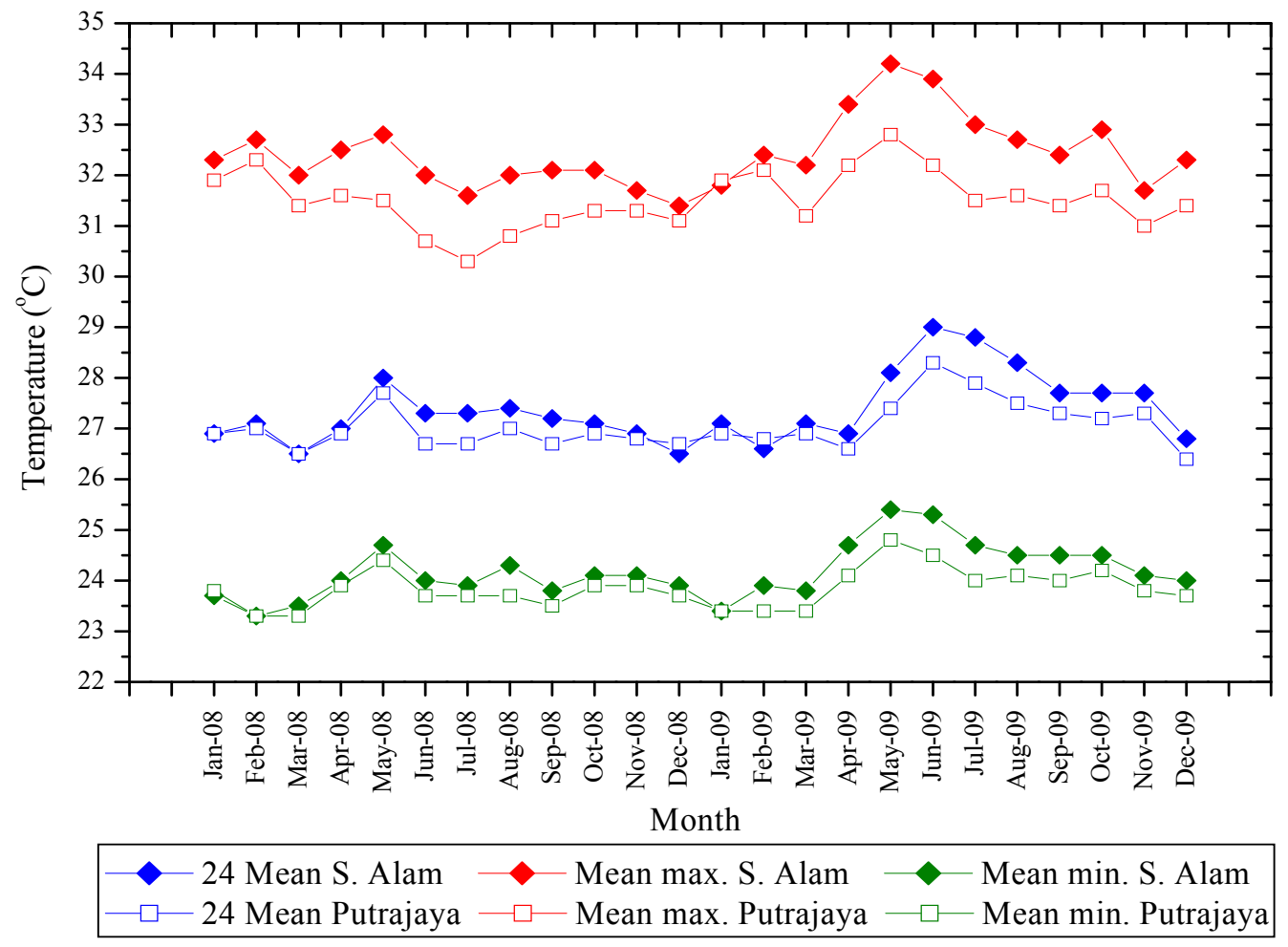

Figure 6.1. Comparison of the monthly average temperature for 2008-2009 at Shah Alam and Putrajaya

Figures 6.1 and 6.2 show the annual weather data for the two cities, which have slightly different air temperatures and relative humidities. The temperatures range from 23.3 to $34.2^{\circ} \mathrm{C}$, with Shah Alam having a slightly higher temperature than Putrajaya. Both cities experienced a relatively high annual RH of between 77 and $81.4 \%$ respectively. Solar radiation was abundant. In Putrajaya it ranged from $16.3-$ $17 \mathrm{MJ} . \mathrm{m}^{-2}$ with 6.9 Okta of cloud cover while in Shah Alam solar radiation was slightly higher, between 18.1 and $18.3 \mathrm{MJ}_{\mathrm{m}} \mathrm{m}^{-2}$, with 7 Okta of cloud cover. 


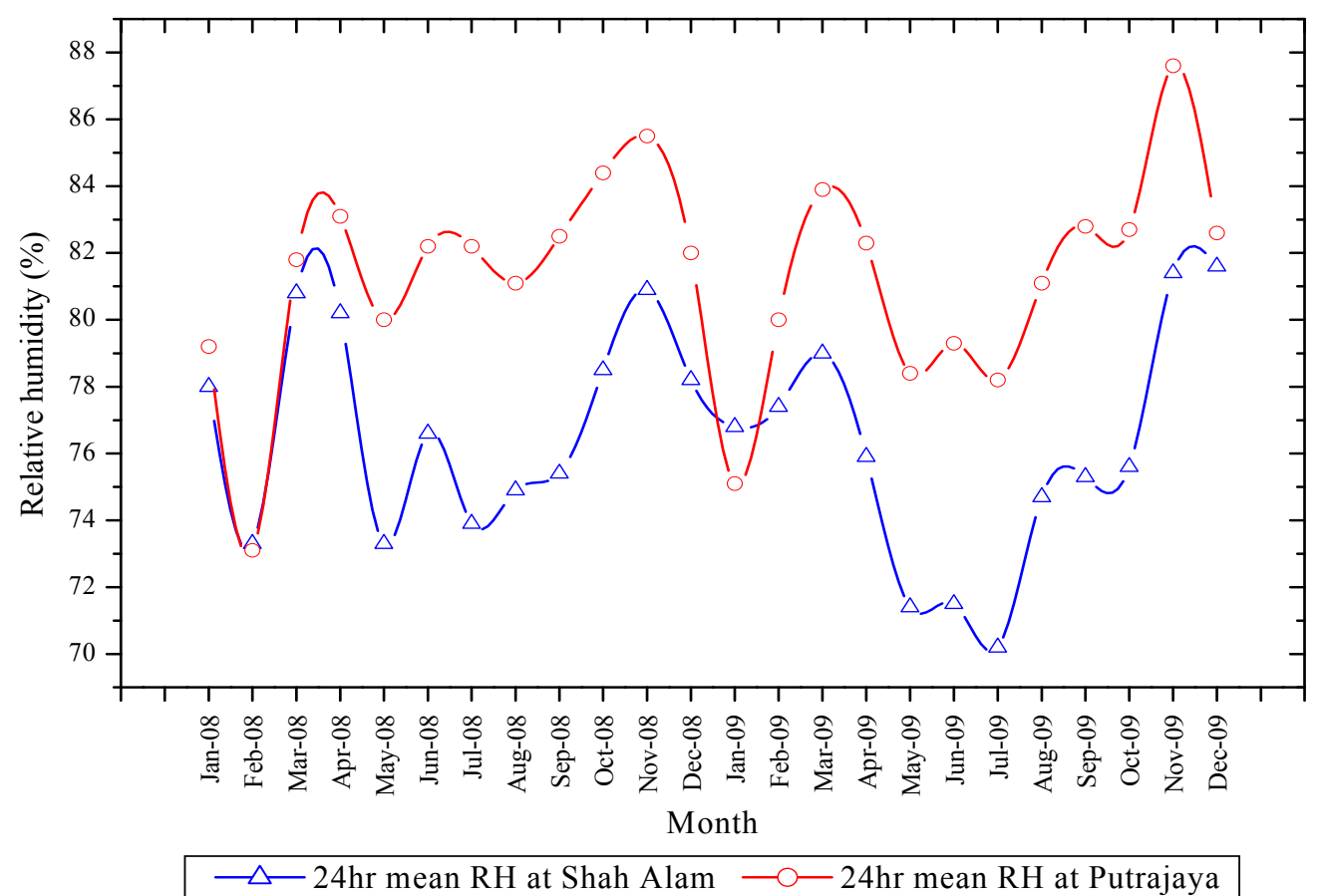

Figure 6.2. Comparison of the monthly average relative humidity for 2008-2009 at Shah Alam and Putrajaya
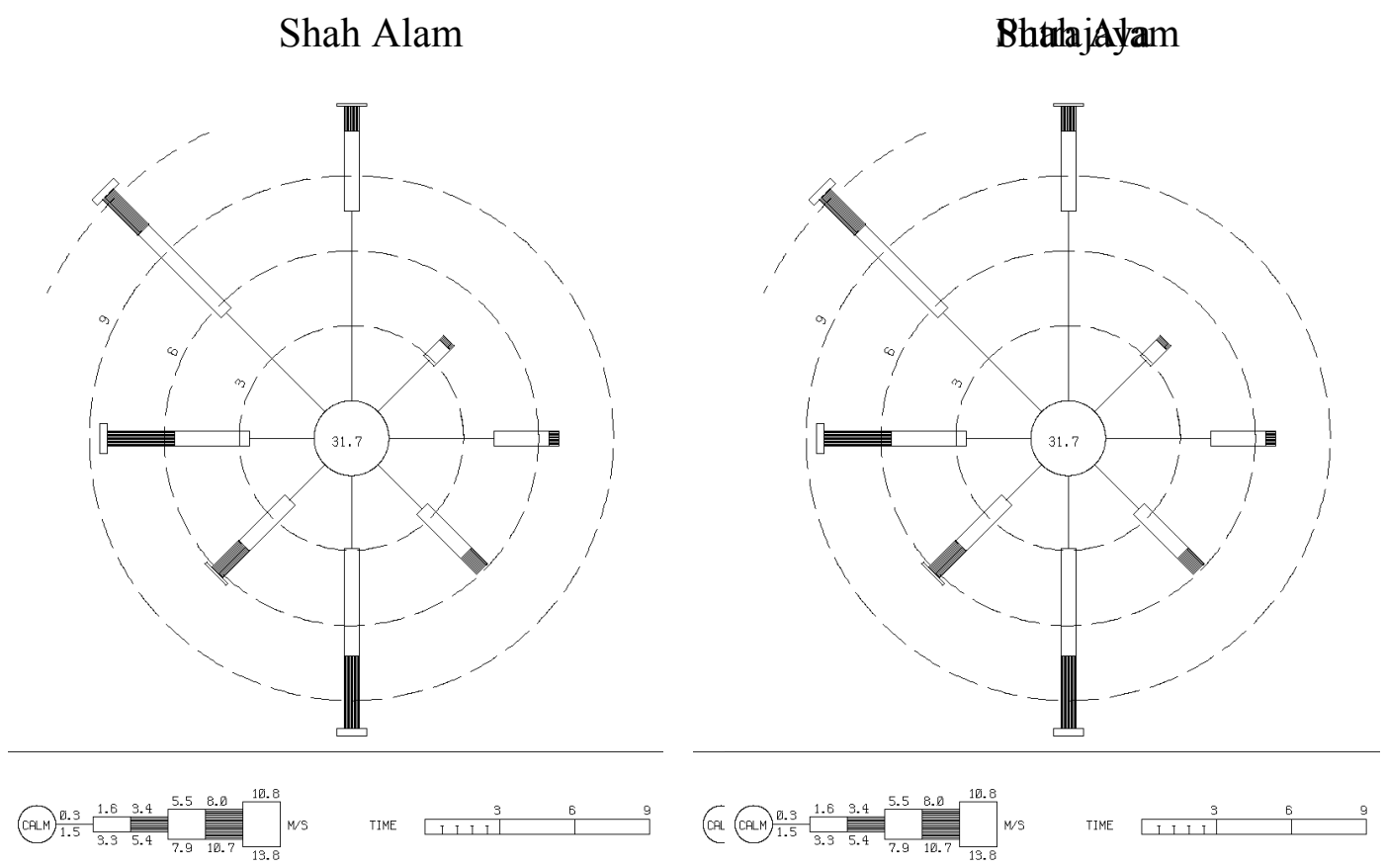

Figure 6.3. Comparison of an average 24 hours of the wind rose summary at Shah Alam (1975-2009) and Putrajaya (1999-2009) 
For the two years from which the weather data was taken, precipitation was high throughout the year, with an annual average of about $2100 \mathrm{~mm}$ in Putrajaya and almost 3300mm in Shah Alam.

In Putrajaya the northeast monsoon rainy season commences in early November and ends in March, with steady north-easterly and north winds prevailing 38\% of the time. The dry season, with the southeast monsoon, is in the latter half of May or early June and ends in September, with a prevailing south-easterly and south wind flow up to $41 \%$ of the time. A calm atmosphere occurs around $7 \%$ of the time.

In Shah Alam, during the rainy season the north and north-westerly monsoon brings a steady wind flow for $27 \%$ of the time, while from May to September the southerly and northerly wind flow was $26 \%$ of the time. The calm scenario is relatively high, at approximately $32 \%$ of the time.

In general, the hot-humid tropical climate for the two cities is slightly different and has fluctuations which are typical of tropical climates.

\subsection{Fieldwork period}

The data collection sheets for building construction and surrounding landscaping, cooling energy use, and interviews of occupants were completed throughout the Shah Alam and Putrajaya areas over 3 months from January through to March 2010 during the rainy season. The data collection for the Puterajaya houses was completed during January 2010, while houses from Shah Alam were completed in February and March 2010. The time for data collection and interviews for the randomly chosen houses averaged 1-2 houses per day.

\subsection{Analysis}

The results of the influences on the amount of cooling energy used have been divided into three parts: building construction, landscaping and human factors. The cooling energy used in the single-family house will be shown based on grouping houses by 
age of construction. These variables will be calculated and evaluated by statistical analyses.

\subsubsection{Building construction data}

Building construction and materials were the main components influencing the cooling energy use of the houses. Fifty medium-sized single-family houses of different ages of construction were analysed to document the physical characteristics of the building and how these might influence the amount of energy used for cooling. These building characteristics included building age and form, construction type, interior and semi open space, ceiling type and building envelope.

\subsubsection{Building form}

The basic building characteristics included the age of construction, how many storeys the building had, floor and roof areas, and porch sizes. A total of 50 buildings were identified, of which $98 \%$ were two storey and $2 \%$ were three storey houses. Table 6.1 shows that around $46 \%$ were aged between 20 and 29 years old, $34 \%$ were between 0 and 9 years, and $14 \%$ and $6 \%$ were 30 to 40 and 10 to 19 year old houses respectively.

Table 6.1. An average floor and roof surfaces

\begin{tabular}{llllllll}
\hline Building & \multicolumn{7}{c}{ Average building area $\left(\mathrm{m}^{2}\right)$} \\
\hline $\begin{array}{l}\text { Age of } \\
\text { construction }\end{array}$ & No. & Percentage & $\begin{array}{l}\text { Ground } \\
\text { floor }\end{array}$ & $\begin{array}{l}\text { Upper } \\
\text { floor }\end{array}$ & $\begin{array}{l}\text { Total } \\
\text { floor }\end{array}$ & Roof & Porch \\
\hline $0-9$ & 17 & 34 & 234.1 & 220.0 & 454.1 & 264.4 & 27.9 \\
$10-19$ & 3 & 6 & 245.0 & 245.0 & 490.0 & 283.3 & 40.0 \\
$20-29$ & 23 & 46 & 206.9 & 201.5 & 411.9 & 254.5 & 30.9 \\
$30-40$ & 7 & 14 & 171.4 & 171.4 & 342.9 & 218.6 & 30.0 \\
\hline All & 50 & 100 & 213.5 & 206.2 & 421.3 & 254.6 & 30.3 \\
\hline
\end{tabular}

On average, the largest total floor area was approximately $490 \mathrm{~m}^{2}$, for houses that were 10 to19 years old. The houses that were 30 to 40 years old were significantly smaller, at around $343 \mathrm{~m}^{2}$, while the 0 to 9 and 20 to 29 year old houses had floor areas of $454 \mathrm{~m}^{2}$ and $412 \mathrm{~m}^{2}$ respectively. This can be seen in Table 6.1 and Figure 6.4. Most of the houses had ground floor areas that were slightly bigger than their 
upper floor areas. Roof areas were in the range of $219 \mathrm{~m}^{2}$ to $283 \mathrm{~m}^{2}$. Almost all of the houses had around $30 \mathrm{~m}^{2}$ of car porch, except for the 10 to 19 year old houses, which generally had car porches that were $40 \mathrm{~m}^{2}$.

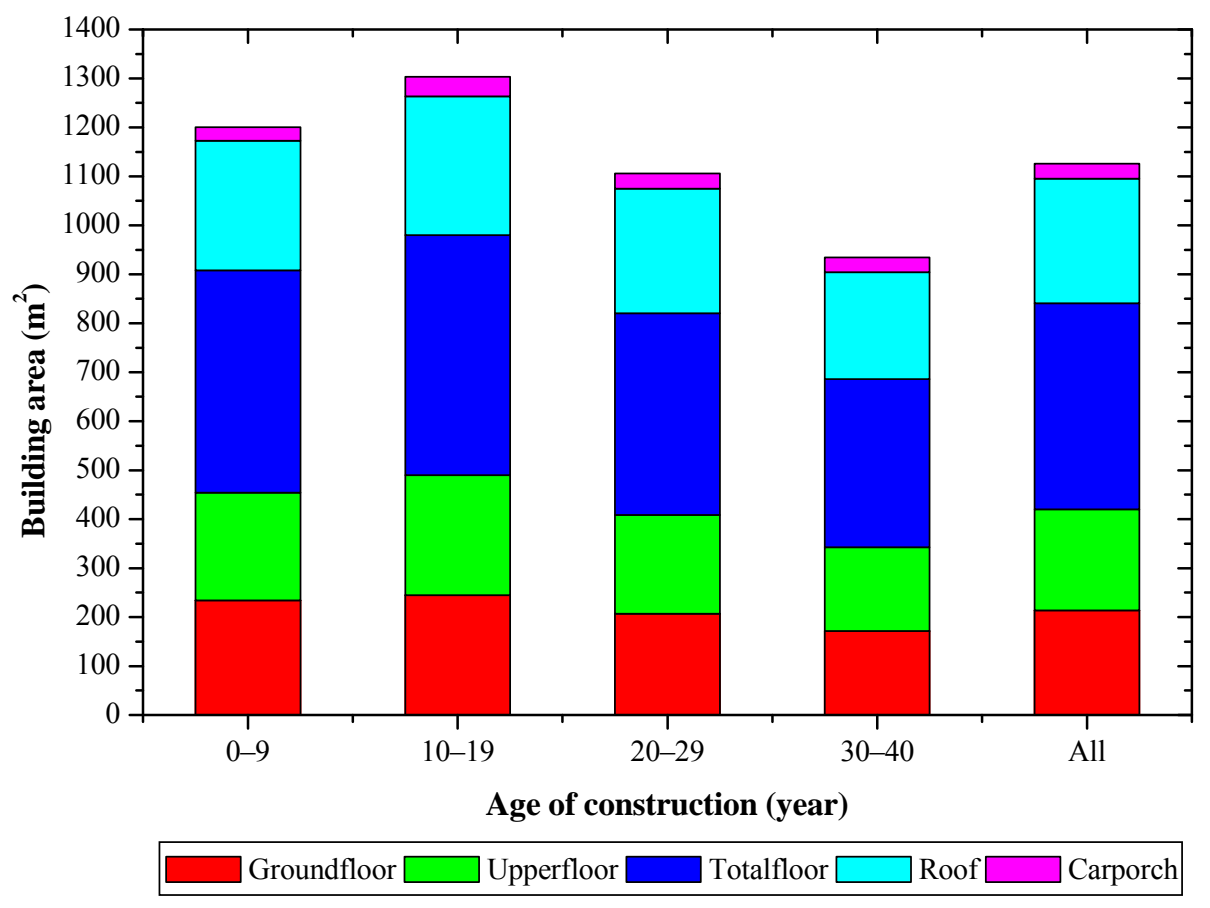

Figure 6.4. The average physical area by age of construction

Almost all (98\%) of the houses were configured to face towards the access road that was located in front of the house. Only $2 \%$ had their sides facing the access road. The access roads were provided by the developers who built the houses in cooperation with the local authority. Underground infrastructure facilities, such as water pipes, electricity cables, telephone lines, and drainage and sewerage systems, were situated beside and parallel to the access roads.

House configuration in the study areas means the direction the main façade faces as shown in Figure 6.6. The directions of house configuration included: north-south, east-west, northeast-southwest, and northwest-southeast. The northwest-southeast configuration was dominant, representing approximately $38 \%$, followed by northsouth $(34 \%)$, northeast-southeast (18\%) and finally east-west $(10 \%)$, as shown in 
Figure 6.5. All houses were rectangular in form, and located in the middle of the land lot. The houses were also surrounded by rectangular garden spaces.

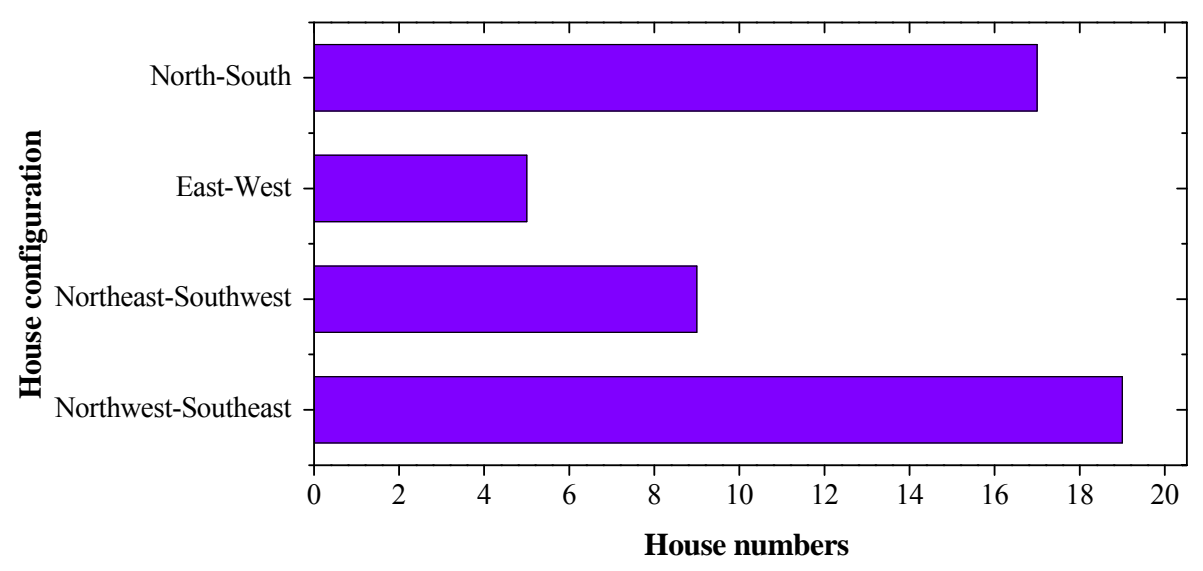

Figure 6.5. House configuration
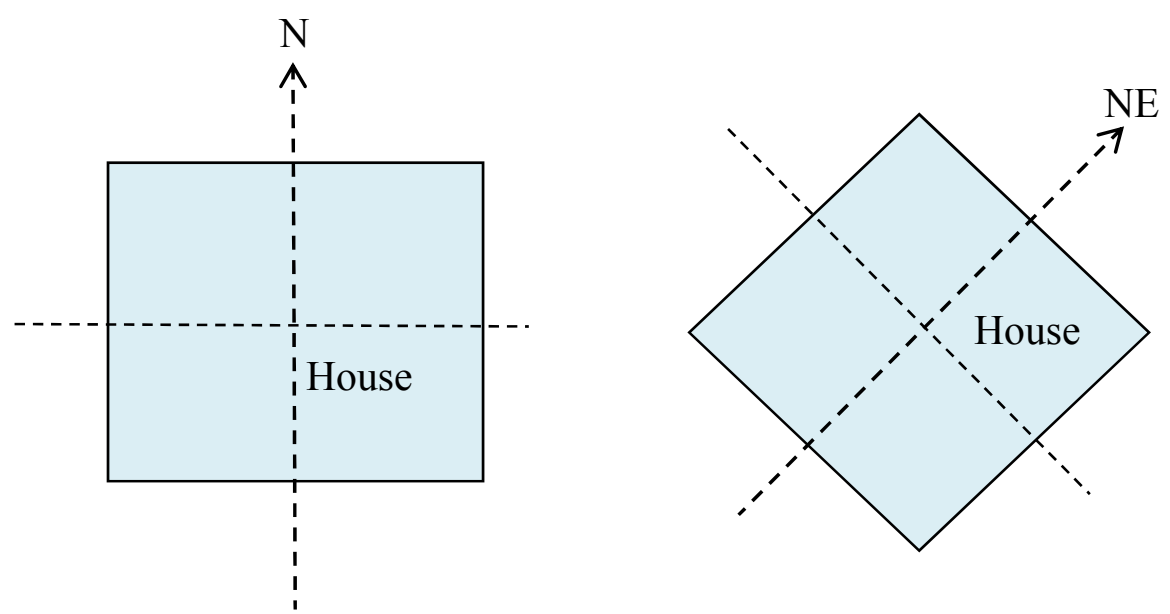

Figure 6.6. Generalized configuration of square/rectangular plan forms of the study houses

\subsubsection{Construction types and materials}

The main materials used for the 50 houses were similar. Table 6.2 shows that the main structures were all reinforced concrete column and beam. The materials used for the building envelopes were flooring built from reinforced concrete, brick walls with cement plaster, and pitched roof structures that were built from preserved hard woods. Approximately $76 \%$ of the houses had concrete tiles on the roof, and $26 \%$ used clay tiles in various colours. 
Table 6.2. Construction types

\begin{tabular}{llccc}
\hline Construction type & Characteristics & Frequency & Percentage & U-value* \\
\hline Column and beam & Reinforced Concrete & 50 & 100 & 3.46 \\
Floor & Reinforced Concrete & 50 & 100 & 4.20 \\
Wall & Bricks & 50 & 100 & 4.37 \\
Roof type & Pitch & 50 & 100 & - \\
Roof structure & Wood & 50 & 100 & - \\
Roof material & Concrete tiles & 37 & 74 & 3.46 \\
(insulation under) & Clay tiles & 13 & 26 & 3.46 \\
\hline${ }^{*}$ U-value unit $=\mathrm{W} / \mathrm{m}^{2 .}{ }^{\circ} \mathrm{C}$ & & &
\end{tabular}

Table 6.2 also shows the U-value of the main material used in the study houses. The $\mathrm{U}$-value is the coefficient of heat transmission used to express the amount of heat that is transferred through materials. The lower the U-value, the greater a product's resistance to heat flow, and the better its insulating value (Darling, 2011). The inverse of the U-value is the R-value. Almost all envelope components in the study houses were constructed without any insulation layer. However, all roof constructions had an insulation layer to help reflect direct heat gain from roof surfaces.

\subsubsection{Interior and semi open spaces}

The interior spaces were divided into two categories: common rooms and private rooms (bedrooms), while semi open spaces consisted of the veranda and covered car porches. These were at the front of the house and designed for two to four cars. However the majority of the study houses have two car porches. Tables 6.3 and 6.4 show the types of common rooms in the study houses. The living, dining and kitchen areas were the basic common spaces for each house located on the ground floor. Most of living or dining rooms were positioned facing the garden, with a veranda to connect them to the garden. The kitchen was usually at the back of the house and consisted of two closed spaces: a wet and a dry kitchen. Most kitchens had a wall facing the side boundary furthest from the access road. Forty percent of the houses had additional common rooms, and usually their function was for specific activities, such as a family room, study, or an audio room. The majority of family and study 
rooms were located on the first floor, while the audio room was generally situated on the ground floor.

Private rooms consisted of six types of bedrooms: master bedroom, bedroom 1, 2, 3, a maid's room, and a guest room. The guest and maid's rooms were located on the ground floor, while the master and bedrooms 1, 2, and 3 were situated on the first floor. This is shown in Table 6.4. Of the houses looked at, 50\% had a maid's room, and $42 \%$ a guest room. The bathrooms in the houses were often connected to the bedrooms.

In all of the houses, the interior walls were built from bricks and plastered with a cement render, and painted with light colours. The interior floors were finished with tiles, terazzo, timber or carpet that ranged from light to medium shades of colour.

\subsubsection{Ceiling type}

In this study the ceiling provides a smooth interior finish, usually to reflect light, and seals the inside from the ventilated roof structure.

In the upper levels of the houses, the ceiling supports the insulation layer and the insulation does the solar radiation reflection. Ceiling heights varied, with $60 \%$ of the houses having a $2.8 \mathrm{~m}$ ceiling height in their common rooms, private rooms, and car porches; while approximately $40 \%$ had $3 \mathrm{~m}$ high ceilings. Porches, which were semi open spaces, had similar heights, with $88 \%$ at $2.8 \mathrm{~m}$. The veranda spaces had a similar height.

There were a variety of ceiling types in the study houses. The most popular ceiling material in the common room was fibrous plaster (50\%), while $24 \%$ of the houses had cement plaster ceilings in the common rooms, and $22 \%$ had asbestos free fibre cement ceiling sheets. In the bedrooms, and semi open porches and verandas, cement plaster and asbestos free fibre cement were the most used ceiling sheets, representing $54 \%$ and $48 \%$ respectively. Almost all ceilings were white. 
Table 6.3. Ceiling characteristics in the common rooms and porch

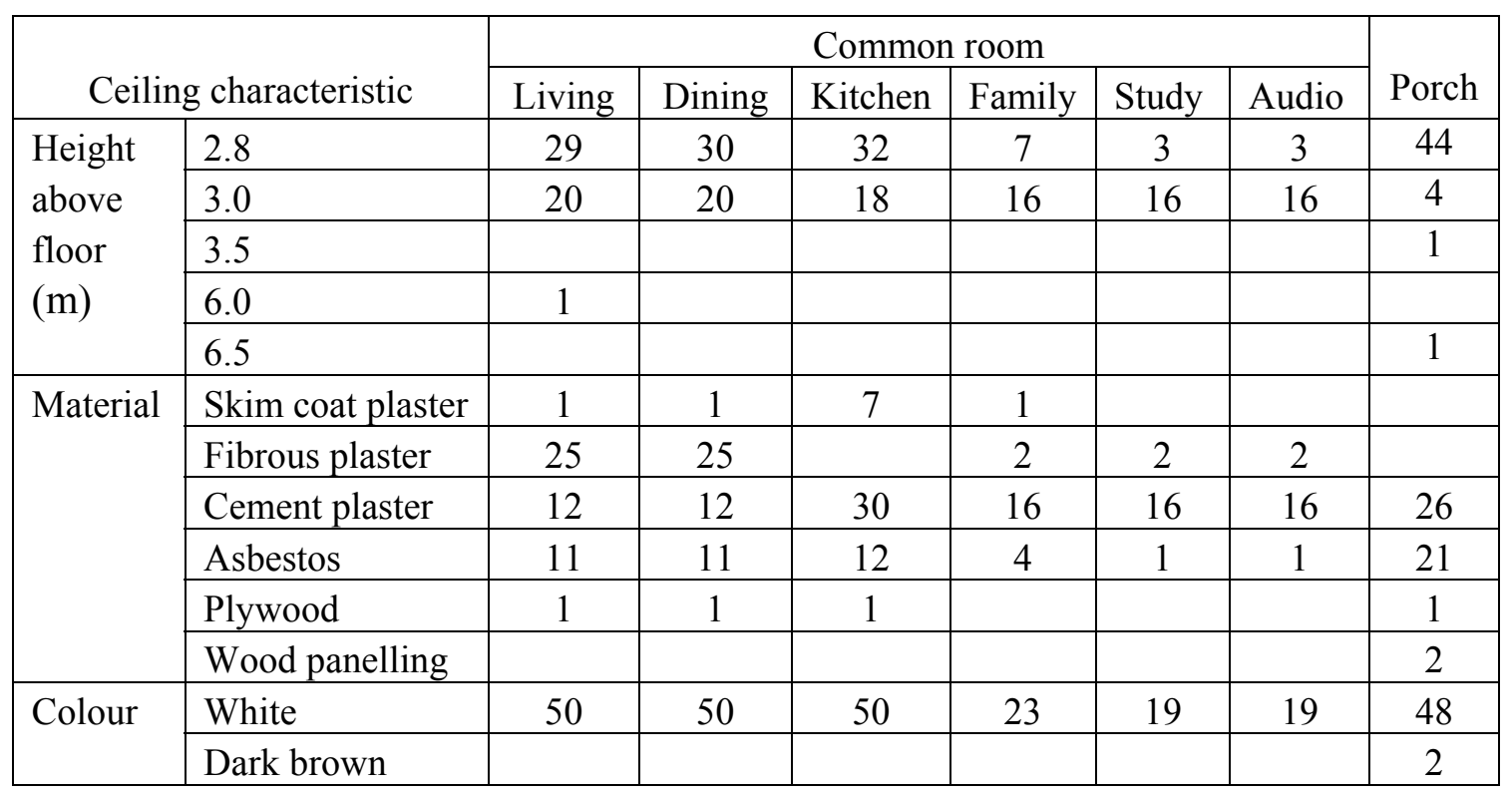

Table 6.4. Ceiling characteristic in the bedrooms

\begin{tabular}{|l|l|c|c|c|c|c|c|}
\hline \multicolumn{2}{|c}{ Ceiling characteristic } & \multicolumn{6}{c|}{ Bedroom } \\
\cline { 2 - 8 } \multicolumn{2}{|c|}{} & Master & 1 & 2 & 3 & Maid & Guest \\
\hline \multirow{2}{*}{$\begin{array}{l}\text { Height above } \\
\text { floor (m) }\end{array}$} & 2.8 & 32 & 32 & 32 & 32 & 9 & 5 \\
\hline \multirow{3}{*}{\begin{tabular}{l} 
Material \\
\cline { 2 - 8 }
\end{tabular}} & 3.0 & 18 & 18 & 18 & 18 & 18 & 16 \\
\cline { 2 - 8 } & Skim coat plaster & 1 & 1 & 1 & 1 & 1 & 1 \\
\cline { 2 - 8 } & Fibrous plaster & 1 & & & & & \\
\cline { 2 - 8 } & Cement plaster & 24 & 27 & 27 & 27 & 21 & 19 \\
\cline { 2 - 8 } & Asbestos & 24 & 22 & 22 & 22 & 5 & 1 \\
\hline Colour & White & 50 & 50 & 50 & 50 & 27 & 21 \\
\hline
\end{tabular}

\subsubsection{Building envelope}

The majority of the building envelopes of the houses in this study were plastered brick walls, with concrete or clay tiled roofs, and reinforced concrete floors.

Table 6.5 and Figure 6.7 show the average exterior wall areas according to the age group of the houses, these areas generally being between 80.3 and $87.5 \mathrm{~m}^{2}$. The highest wall areas among the four ages of house was in the 0 to 9 year old buildings, which were approximately $87.5 \mathrm{~m}^{2}$ per house, while the 30 to 40 year old houses had the lowest wall areas at around $80.3 \mathrm{~m}^{2}$. 


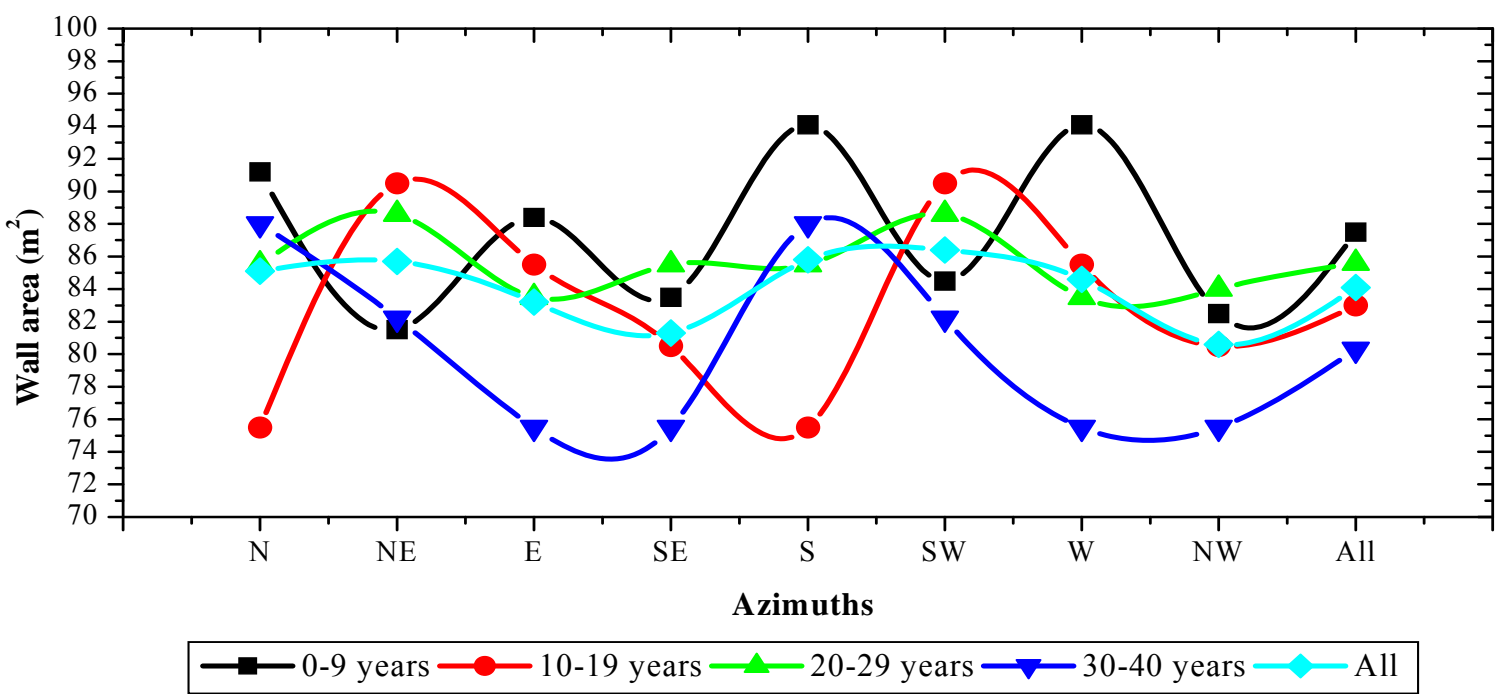

Figure 6.7. The average area for walls for all directions based on ages of construction

All of the walls on each house had similar wall areas. The highest average area among all azimuths was for the south and west walls of the 0 to 9 year old houses at $94.1 \mathrm{~m}^{2}$. The lowest wall area was $75.5 \mathrm{~m}^{2}$ for the east, southeast, west and northwest walls of the 30 to 40 year old houses. The average area for all walls was $84.1 \mathrm{~m}^{2}$.

Table 6.5. Average area for all walls for all house age groups

\begin{tabular}{llllllllll}
\hline \multirow{2}{*}{$\begin{array}{c}\text { Building age } \\
\text { (year) }\end{array}$} & \multicolumn{2}{l}{ Walls area $\left(\mathrm{m}^{2}\right)$} & & & & & & \\
\cline { 2 - 10 } & $\mathrm{N}$ & $\mathrm{NE}$ & $\mathrm{E}$ & $\mathrm{SE}$ & $\mathrm{S}$ & $\mathrm{SW}$ & $\mathrm{W}$ & $\mathrm{NW}$ & All \\
\hline $0-9$ & 91.2 & 81.5 & 88.4 & 83.5 & 94.1 & 84.5 & 94.1 & 82.5 & 87.5 \\
$10-19$ & 75.5 & 90.5 & 85.5 & 80.5 & 75.5 & 90.5 & 85.5 & 80.5 & 83.0 \\
$20-29$ & 85.5 & 88.6 & 83.5 & 85.5 & 85.5 & 88.6 & 83.5 & 84.0 & 85.6 \\
$30-40$ & 88.0 & 82.2 & 75.5 & 75.5 & 88.0 & 82.2 & 75.5 & 75.5 & 80.3 \\
\hline All & 85.1 & 85.7 & 83.2 & 81.3 & 85.8 & 86.4 & 84.6 & 80.6 & 84.1 \\
\hline
\end{tabular}

Glazed windows, sliding doors, and external doors have the main function of letting in daylight and allowing the flow of natural fresh air for ventilation. Table 6.6 and Figure 6.8 show the average area of the aluminium framed, single layer glass windows and sliding doors was in the range of $11.8-15.1 \mathrm{~m}^{2}$ for each side of the house. 
Similar to the average wall areas, the highest average glazed area among all groups of buildings was in the 0 to 9 year old houses, with approximately $16.3 \mathrm{~m}^{2}$ of glazed area, while the 30 to 40 year old buildings had the lowest glazed area at around $10.9 \mathrm{~m}^{2}$. The 0 to 9 year old houses had the highest average glazed area for all azimuths at approximately $16.4 \mathrm{~m}^{2}$, while the lowest glass area was in the 10 to 19 year old houses for the north facing wall at $3.3 \mathrm{~m}^{2}$.

Table 6.6. The average glazed area for all directions

\begin{tabular}{llllllllll}
\hline \multirow{2}{*}{$\begin{array}{c}\text { Building age } \\
\text { (year) }\end{array}$} & \multicolumn{2}{c}{ Glazed area $\left(\mathrm{m}^{2}\right)$} & & & & & & & \\
\cline { 2 - 10 } & $\mathrm{N}$ & $\mathrm{NE}$ & $\mathrm{E}$ & SE & S & SW & W & NW & All \\
\hline $0-9$ & 16.4 & 15.7 & 17.5 & 14.6 & 13.5 & 14.1 & 22.9 & 15.6 & 16.3 \\
$10-19$ & 3.3 & 6.7 & 23.7 & 8.7 & 15.0 & 12.6 & 14.0 & 13.7 & 12.2 \\
$20-29$ & 15.5 & 11.6 & 13.4 & 12.8 & 14.2 & 11.6 & 11.9 & 12.2 & 12.9 \\
$30-40$ & 14.9 & 9.5 & 11.0 & 9.7 & 15.0 & 6.6 & 6.7 & 14.3 & 10.9 \\
\hline All & 15.1 & 12.4 & 14.6 & 12.6 & 13.9 & 11.8 & 14.3 & 13.5 & 13.5 \\
\hline
\end{tabular}

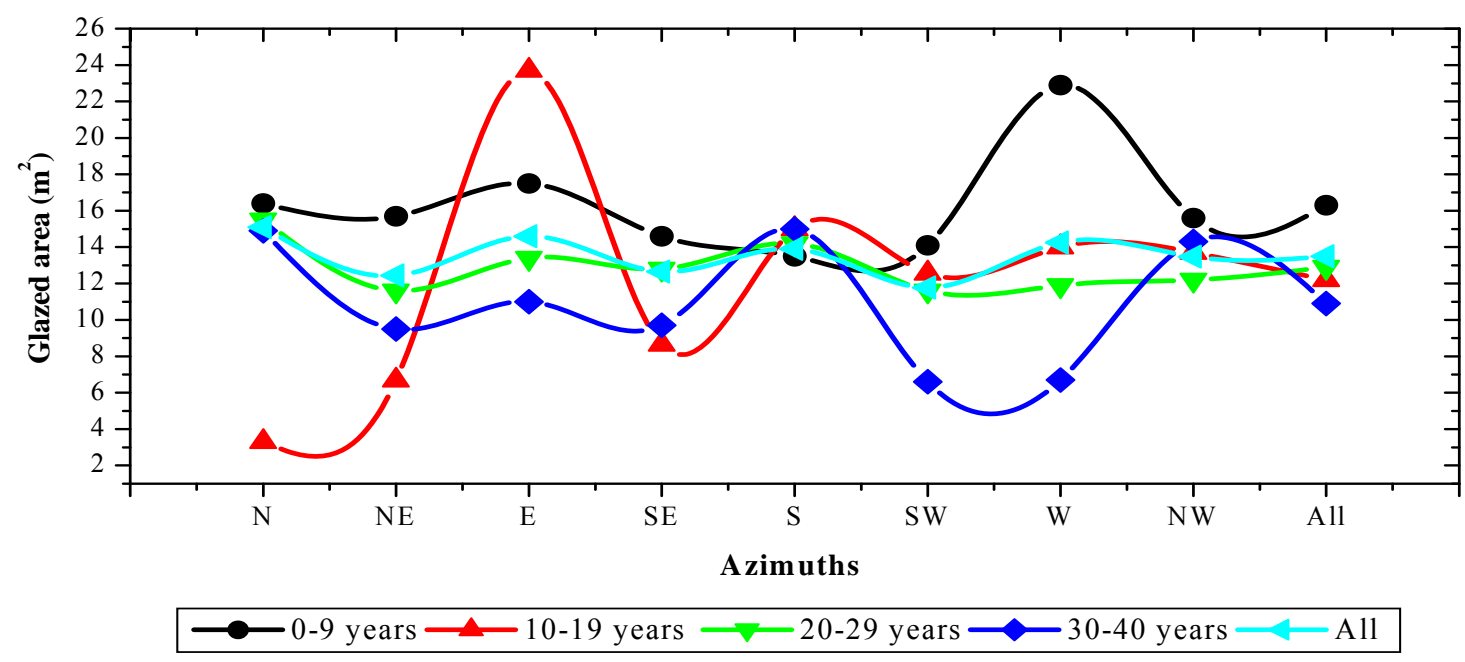

Figure 6.8. The average for glazed areas for all directions based on age of construction

For all houses, the average glazed area over all directions was $13.5 \mathrm{~m}^{2}$. Glazing was located on all sides of the houses in different positions. Glazed areas were equal to $13 \%$ of the floor area, which would allow sufficient natural lighting and ventilation into the building 
The minimum number of external doors was two; a main entrance at the front of the house into the living room and a second door at the back of the house into the kitchen space. However, most houses had more doors that gave access to the garden areas through living or dining rooms. Almost all of the houses had solid hardwood timber doors for the main entrance, and timber flush or hardwood exterior doors in the kitchen. All doors were protected by the roof awning, as the timber doors, like the walls, could gain heat from the ambient weather conditions.

All of the houses used gable or hip roofs, which were built from hardwood trusses and purlins covered by concrete or clay tiles. The roof shape is very suitable for the local climate conditions, where rainfall is heavy throughout the year, with an average pitch of around $30-35^{\circ}$. Rain water down pipes were used to take rainwater from the roof to the drain. The average size of the roof surfaces was approximately $254.6 \mathrm{~m}^{2}$, as shown in Table 6.1. Aluminium foil insulation was placed underneath the roof to act as a thermal resistant or radiant barrier to reflect heat from direct solar radiation throughout the day. Light coloured roof tiles were also used to reflect solar radiation because these cover the uppermost part of the building envelope that is always potentially exposed to solar radiation and could transfer heat to the interior of the building.

At ground level, the study area houses have reinforced concrete floors built on reinforced concrete beam and pad foundations. As with typical Malay houses, their ground floor level was $0.15 \mathrm{~m}$ above ground level to prevent rain water from flowing into the houses. They also had a $0.75-1 \mathrm{~m}$ concrete drainage slope with a small covered concrete drainage channel $(0.15 \mathrm{~m}$ in width) all round the house. The finishes for the indoor floors were tile, wood and carpet. The finish for the veranda floors was tiles. Cement screed, pebbles, or tiles were usually used for the car porch area. Heat gains to the ground floor areas were from the ground and the surrounding earth surfaces.

Albedo is the measurement of a surface's reflectivity, from the fraction of sunshine reflected from the earth for any surface back into space. In this study, the value of the 
albedo ranged from $0.21-0.23$. The average albedo for the building envelope was 0.22, as shown in Table 6.7. The averages of wall and roof colours were based on the colour codes that each house had used. In Malaysia, light colours for walls and roofs have been widely used for 40 years. In this study, the major colours for the building envelope areas were cream walls (2.1) and light red roof tiles (9.4).

Table 6.7. The average wall and roof colour, and albedo values

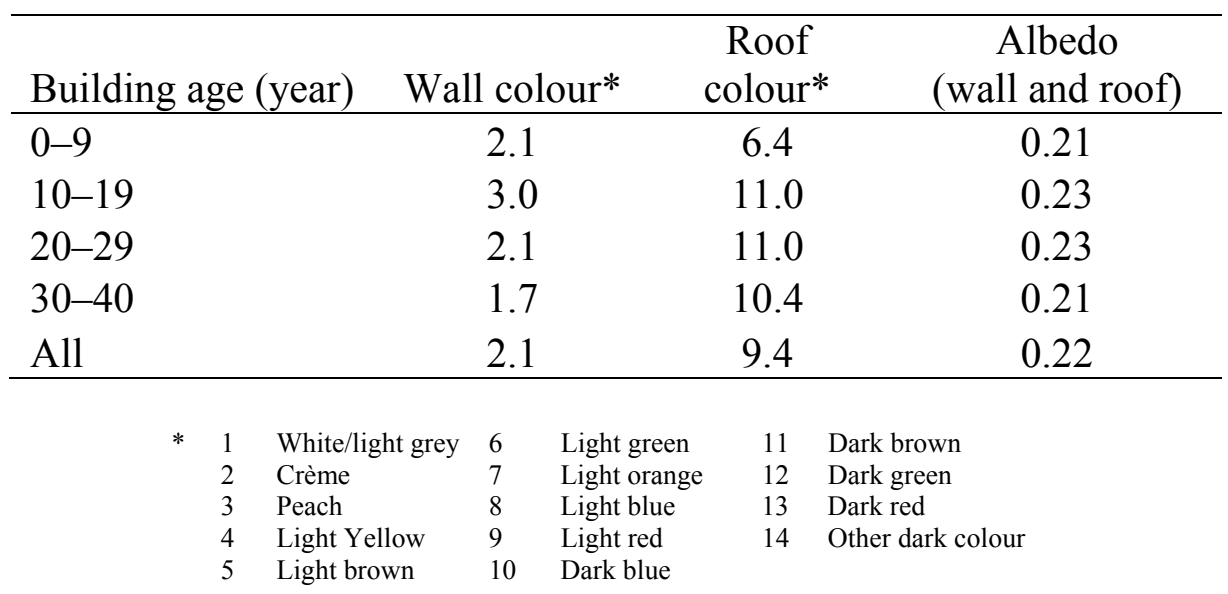

For all the houses, the glazed surfaces on the upper floors were shaded by the roof projection, while those at ground level were shaded by an additional gable, hip, or concrete overhang that were around $0.75-1 \mathrm{~m}$ wide. The shading devices on the east and west sides could provide shade to the glass surfaces between 11.00 and 15.00 hours, which is the peak time of the day, while the shading devices on the north and south sides could provide shade from diffused solar radiation.

Verandas were located adjacent to sliding doors as a transition space between the indoors and outdoors. These had $2-3 \mathrm{~m}$ wide roof covers, which also shaded the sliding doors. Sliding doors on the east and west sides were shaded between 10.00 and 16.00 hours. The covered car porch also provided shading for windows that faced into it. The interior of all windows and sliding doors was also covered by two layers of fabric curtains. One layer was composed of thin cloth and the other of a thick fabric to reduce direct solar radiation. During day time a layer of thick fabric was open, while the thin cloth layer was closed to allow natural daylight and ventilation entry to the buildings. 


\subsubsection{Landscape element (soft and hard) data}

In this study, landscaping refers to the private garden around the single-family houses. All of the houses used a tropical style of landscaping, with a moderate number of plants positioned around the houses. These results include information on the garden size, and five main categories of soft landscaping which include trees, shrubs, vines, groundcover and turf. Data about hard landscape elements was also gathered.

\subsubsection{Garden size}

The private gardens of the study houses were of various sizes that ranged between 180 and $500 \mathrm{~m}^{2}$. Table 4.8 shows the average garden size for the different house age groups.

The 0 to 9 year old houses had the largest average garden size, at around $292.9 \mathrm{~m}^{2}$. The gardens of the 30 to 40 year old houses averaged $218.3 \mathrm{~m}^{2}$, while the 20 to 29 year old houses had an average garden size of $235.5 \mathrm{~m}^{2}$. The 10 to 19 and 30 to 40 year old houses had the lowest average garden size, at $218.3 \mathrm{~m}^{2}$ and $200 \mathrm{~m}^{2}$ respectively. Overall, the average garden size for the houses in the study areas was $258.8 \mathrm{~m}^{2}$.

Table 6.8. Average garden size by age of construction

\begin{tabular}{lccc}
\hline Age of construction & House no. & Percentage & Garden size $\left(\mathrm{m}^{2}\right)$ \\
\hline $0-9$ & 17 & 34 & 292.9 \\
$10-19$ & 3 & 6 & 200.0 \\
$20-29$ & 23 & 46 & 253.5 \\
$30-40$ & 7 & 14 & 218.3 \\
\hline All & 50 & 100 & 258.8 \\
\hline
\end{tabular}

\subsubsection{Trees}

All of the native tropical trees found in the study areas were evergreens, which consisted of a range of plant species, listed in Table 6.9. As the main soft landscape structure in the gardens, the tree canopy sizes were larger, taller and more dominant 
than other soft landscape elements. The tree categories can be divided into six groupings, as shown in Table 6.9.

Table 6.9. Tree category

\begin{tabular}{llcc}
\hline Plant category & Group of plant & Frequency & Percentage \\
\hline Tree & Roadside trees & 68 & 13 \\
& Roadside palm & 29 & 5 \\
& Garden trees & 135 & 25 \\
& Edible Fruit trees & 98 & 18 \\
& Garden palm & 206 & 38 \\
& Bamboo & 6 & 1 \\
\hline Total & & 542 & 100 \\
\hline
\end{tabular}

There are approximately 102 species of tropical trees around the study areas as listed in Appendix 6.2. Palm categories were the most popular, representing approximately $38 \%$ of all trees, followed by garden trees $(25 \%)$ and edible fruit trees $(18 \%)$. Roadside trees were located on access roads, and represent around $13 \%$ of trees in the study area. There were also some roadside palms (5\%) and bamboos (1\%) but less of these were planted at around $5 \%$ and $1 \%$ of total respectively.

The total number of trees across the study area was 542, with between 1 and 48 trees per house. The linear data in Figure 6.9 shows that the tree distribution was in the range of 5.5 and 15.5 per house.

The average number of trees for houses in this study was 11 per house. This consisted of two roadside trees or palms, three garden trees, four garden palms, and two edible fruit trees. 


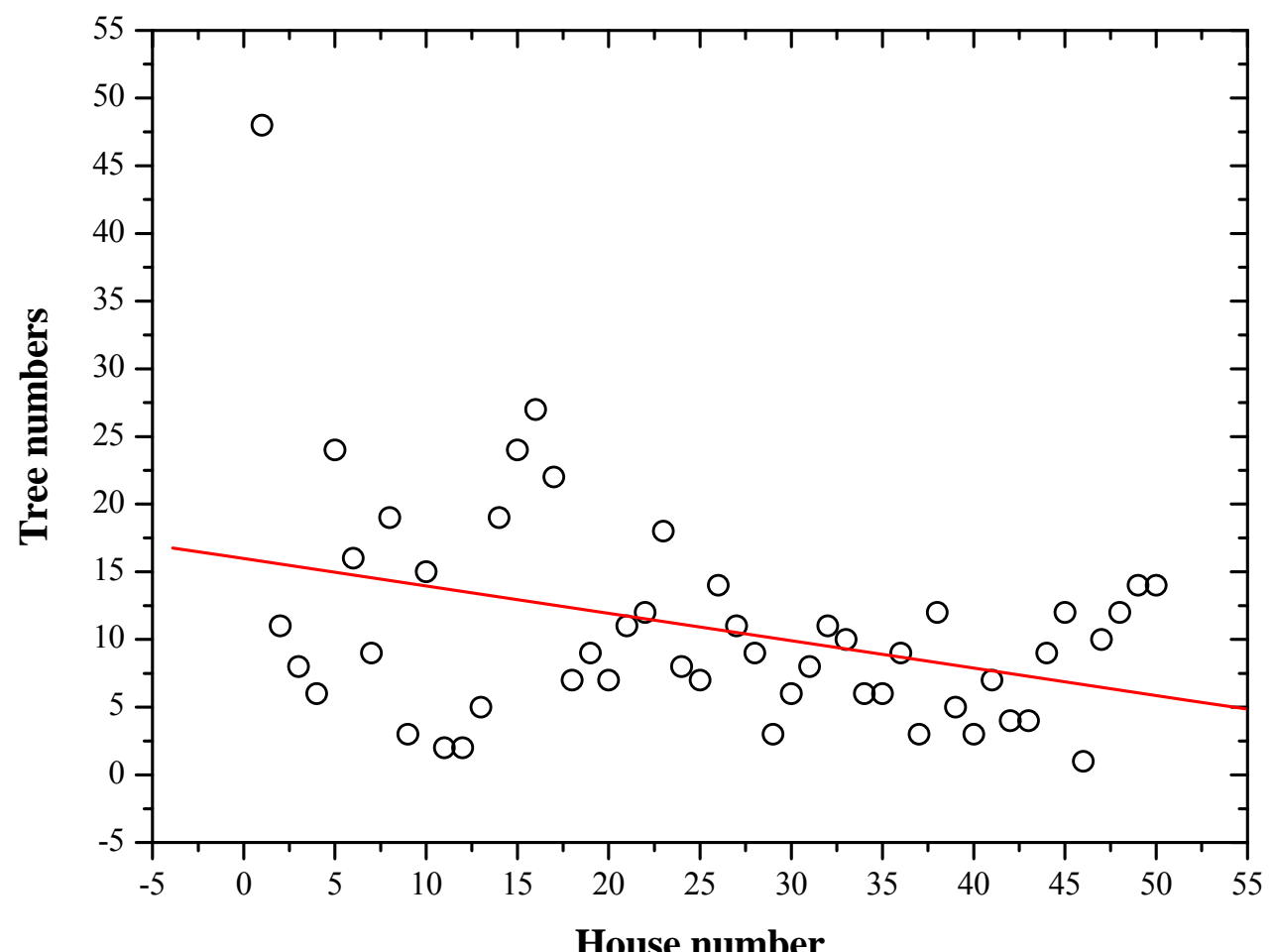

House number

$\circ$ Tree distribution - Linear data (tree distribution)

Figure 6.9. The distribution of trees around the houses

The canopy sizes for the trees in the study area were divided into four groups, where the measurements were of the diameter: small $(2-4 \mathrm{~m}$ diameter, $1-2 \mathrm{~m}$ trunk height and $2-4 \mathrm{~m}$ overall height), small-moderate (4-7m diameter, $2-3 \mathrm{~m}$ trunk height and $5-$ $9 \mathrm{~m}$ overall height), medium ( $8-11 \mathrm{~m}$ diameter, $2.5 \mathrm{~m}$ and above, and $10-14 \mathrm{~m}$ overall height), and large (12-15m diameter, $3 \mathrm{~m}$ and above trunk height, and $15-19 \mathrm{~m}$ overall height).

Tables $6.10-6.11$ and Figure 6.10 show that the majority of trees in the study areas were small, some because this was how they grew, and for others it was because the trees were still young. There were 322 small-sized trees, which represented 59\% of the total number of trees located around the houses; 176 trees were small-moderate sized (33\%); 42 were medium sized (8\%); and only 2 were large $(0.4 \%)$. Figure 6.10 and Table 6.10 shows that the small sized trees were dominant in almost all of the age groupings of houses, followed by small-moderate size. There were two major sizes and amounts of leaves: small and medium. 


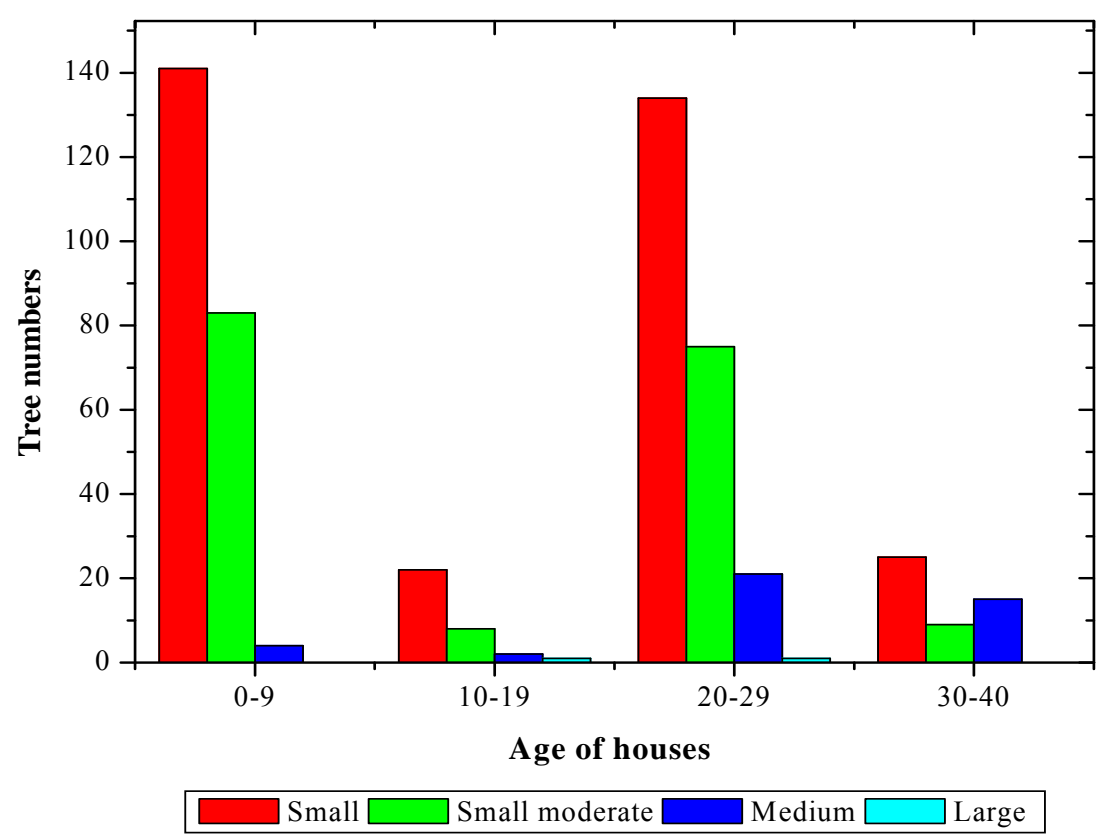

Figure 6.10. Tree sizes for the different age groupings of the houses

Table 6.10. Summary of tree distribution for all age groups of houses

\begin{tabular}{lllllll}
\hline \multirow{2}{*}{ Tree sizes } & \multicolumn{7}{l}{ Age of the houses } & & \\
\cline { 2 - 6 } & $0-9$ & $10-19$ & $20-29$ & $30-40$ & Total & Percentage \\
\hline Small & 141 & 22 & 134 & 25 & 322 & 59 \\
Small moderate & 84 & 8 & 75 & 9 & 176 & 33 \\
Medium & 4 & 2 & 21 & 15 & 42 & 8 \\
Large & 0 & 1 & 1 & 0 & 2 & 0 \\
\hline Total & 229 & 33 & 231 & 49 & 542 & 100 \\
Percentage & 42.3 & 6.1 & 42.6 & 9.0 & 100 & 100 \\
\hline
\end{tabular}

The four groups of houses had a similar number of trees located in each direction around the houses. The majority were situated on the east $(18 \%)$, and west $(15 \%)$ sides. The north and south sides had 13\% and 14\% of trees respectively, while the northeast, southeast, southwest, and northwest areas had between 9 and $12 \%$. This can be seen in Table 6.11. The detailed tree location for each group of house ages is shown in Appendices 6.3.1-6.3.4. 
Table 6.11. Tree sizes and distances from the building for all azimuths for all age groups of houses

\begin{tabular}{|c|c|c|c|c|c|c|c|c|c|c|c|c|}
\hline \multirow[t]{2}{*}{ Tree size } & \multirow[t]{2}{*}{ Distance (m) } & \multicolumn{8}{|c|}{ Tree azimuth } & \multirow[b]{2}{*}{ All } & \multirow[b]{2}{*}{ Total } & \multirow[b]{2}{*}{$\%$} \\
\hline & & $\mathrm{N}$ & $\mathrm{NE}$ & $\mathrm{E}$ & $\mathrm{SE}$ & $\mathrm{S}$ & SW & $\mathrm{W}$ & NW & & & \\
\hline \multirow{4}{*}{ Small } & $3-5$ & 16 & 14 & 35 & 22 & 25 & 13 & 19 & 26 & 170 & 322 & 59 \\
\hline & $6-10$ & 23 & 13 & 24 & 4 & 17 & 13 & 27 & 19 & 140 & & \\
\hline & $11-15$ & 0 & 0 & 1 & 0 & 4 & 1 & 6 & 0 & 12 & & \\
\hline & $16-20$ & 0 & 0 & 0 & 0 & 0 & 0 & 0 & 0 & 0 & & \\
\hline \multirow[t]{4}{*}{$\begin{array}{l}\text { Small } \\
\text { moderate }\end{array}$} & $3-5$ & 4 & 4 & 9 & 10 & 3 & 9 & 5 & 3 & 47 & 176 & 33 \\
\hline & $6-10$ & 15 & 12 & 22 & 7 & 18 & 16 & 18 & 8 & 116 & & \\
\hline & $11-15$ & 0 & 0 & 0 & 1 & 3 & 0 & 2 & 0 & 6 & & \\
\hline & $16-20$ & 0 & 0 & 0 & 0 & 0 & 0 & 7 & 0 & 7 & & \\
\hline \multirow[t]{4}{*}{ Medium } & $3-5$ & 8 & 2 & 3 & 1 & 1 & 4 & 1 & 0 & 20 & 42 & 8 \\
\hline & $6-10$ & 6 & 1 & 1 & 2 & 0 & 6 & 3 & 0 & 19 & & \\
\hline & $11-15$ & 0 & 1 & 1 & 0 & 0 & 0 & 0 & 0 & 2 & & \\
\hline & $16-20$ & 0 & 0 & 0 & 0 & 0 & 1 & 0 & 0 & 1 & & \\
\hline \multirow[t]{4}{*}{ Large } & $3-5$ & 0 & 0 & 0 & 0 & 0 & 0 & 0 & 0 & 0 & 2 & 0 \\
\hline & $6-10$ & 0 & 0 & 1 & 0 & 0 & 1 & 0 & 0 & 2 & & \\
\hline & $11-15$ & 0 & 0 & 0 & 0 & 0 & 0 & 0 & 0 & 0 & & \\
\hline & $16-20$ & 0 & 0 & 0 & 0 & 0 & 0 & 0 & 0 & 0 & & \\
\hline \multicolumn{2}{|c|}{ All tree sizes/distances } & 71 & 47 & 99 & 47 & 78 & 64 & 80 & 56 & 542 & 542 & 100 \\
\hline \multicolumn{2}{|c|}{ Percentage } & 13 & 9 & 18 & 9 & 14 & 12 & 15 & 10 & 100 & 100 & 100 \\
\hline
\end{tabular}

The distance between the trees and buildings was divided into four categories, which ranged between $0-20 \mathrm{~m}$, as can be seen in Table 6.11 and Figure 6.11. The majority of trees in the study area were situated between 6-10m from the houses (51\%), followed by $44 \%$ that were between $0-5 \mathrm{~m}, 3.5 \%$ were within $11-15 \mathrm{~m}$, and $1.5 \%$ between 16-20m, as can be seen in Figure 6.11. 


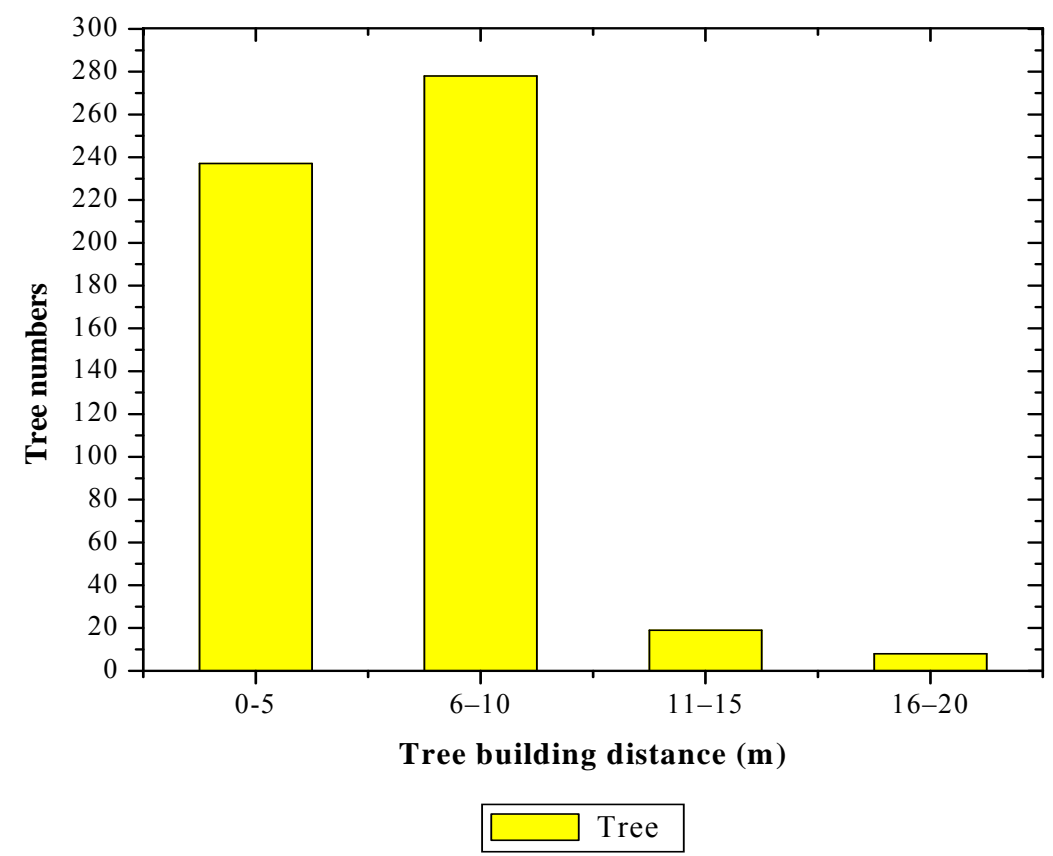

Figure 6.11. Tree to building distance

\subsubsection{Shrubs, vines, groundcover and turf}

Most of the tropical shrubs in the study areas were flowering or edible plants, palms and bamboo. The shrub categories can be divided into four groups, as shown in Table 6.12. Shrubs were dominant on site, with 1715 species (planted individually and in groups), while there were 65 species of vines and 44 groundcover species.

Table 6.12. Categories of shrubs, vines and groundcover

\begin{tabular}{llcc}
\hline Plant category & Group of plant & Plant numbers & Total items \\
\hline Shrubs & Garden shrubs & 1247 & \\
& Edible shrubs & 289 & \\
& Palms & 164 & \\
& Bamboo & 15 & 1715 \\
Vines & Garden vines & 48 & \\
& Edible vines & 17 & 65 \\
Groundcover & Groundcover & 44 & 44 \\
\hline Total & & 1824 & 1824 \\
\hline
\end{tabular}

Appendix 6.5 lists the 316 different species of shrubs, vines and groundcover that were found in the gardens. Of these, there were approximately 200 species of garden 
shrubs that included plants with green and coloured leaves, and flowering shrubs. There were also 59 species of edible shrubs, which included herbs, fruit and vegetable shrubs. The other plant categories, including palms and bamboo, garden and edible vines, and groundcover, amounted to 14 species each.

Figure 6.12 shows the distribution of shrubs, vines and groundcover around the houses. The linear data shows that the average number of shrubs, vines and groundcover ranged between 18 and 53 species per house, with an average of 36 . This included an average of 34 individual and groups of shrubs, and 1 planting of vines and 1 group of groundcover.

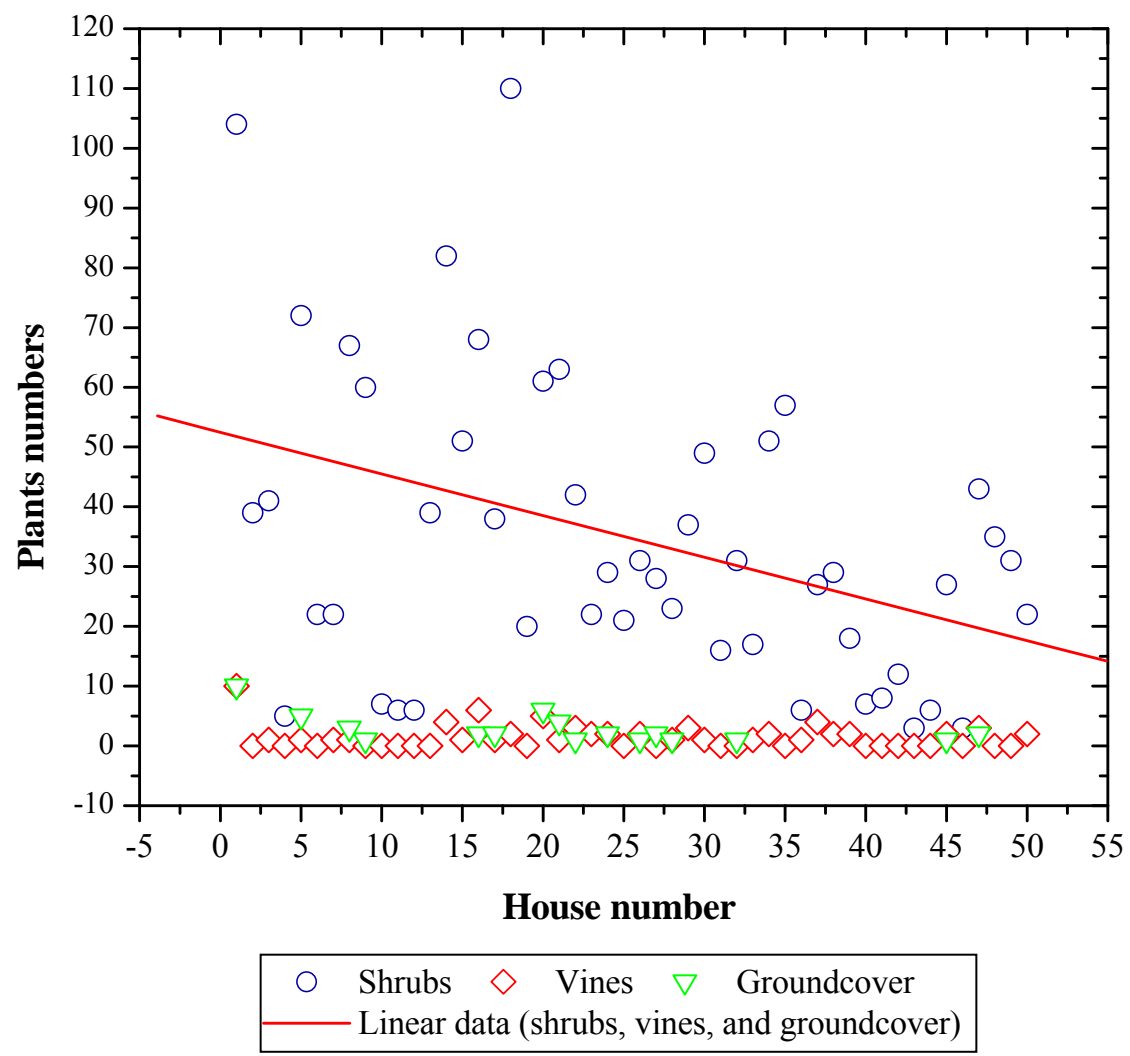

Figure 6.12. Shrubs, vines and groundcover distribution around the houses in the study areas

The shrubs, vines and groundcover were divided into five sizes, including individual plants and groups, where the measurement were taken for the spread of the plant $\left(\mathrm{m}^{2}\right)$ : individual $\left(0.25-1 \mathrm{~m}^{2}\right)$, Group $1\left(0-10 \mathrm{~m}^{2}\right)$, Group $2\left(11-20 \mathrm{~m}^{2}\right)$, Group 3 (21$\left.30 \mathrm{~m}^{2}\right)$, and Group $4\left(31-40 \mathrm{~m}^{2}\right)$. This is shown in Table 6.13 , which along with 
Figure 6.12 shows that the majority of shrubs, vines and groundcover in the study areas were planted individually, but close to other species.

Table 6.13. Shrubs, vines and groundcover by sizes and distances from the building for all azimuths for all age groups of houses

\begin{tabular}{|c|c|c|c|c|c|c|c|c|c|c|c|c|c|}
\hline \multirow{2}{*}{$\begin{array}{l}\text { Plant size } \\
\left(\mathrm{m}^{2}\right)\end{array}$} & \multirow[t]{2}{*}{ Distance (m) } & \multicolumn{8}{|c|}{ Plants azimuth } & \multirow[b]{2}{*}{ All } & \multirow[b]{2}{*}{$\%$} & \multirow[b]{2}{*}{ Total } & \multirow[b]{2}{*}{$\%$} \\
\hline & & $\mathrm{N}$ & $\mathrm{NE}$ & $\mathrm{E}$ & SE & $\mathrm{S}$ & SW & $\mathrm{W}$ & NW & & & & \\
\hline \multirow[t]{5}{*}{ Individual } & Proximity to walls & 22 & 32 & 30 & 24 & 26 & 36 & 24 & 28 & 222 & 12.2 & 1054 & 58 \\
\hline & $2-5$ & 99 & 65 & 140 & 83 & 72 & 69 & 121 & 74 & 723 & 36.6 & & \\
\hline & $5-10$ & 19 & 5 & 24 & 5 & 13 & 6 & 17 & 16 & 105 & 5.8 & & \\
\hline & $11-15$ & 0 & 0 & 0 & 0 & 0 & 0 & 0 & 0 & 0 & 0 & & \\
\hline & $16-20$ & 0 & 0 & 0 & 2 & 0 & 2 & 0 & 0 & 4 & 0.2 & & \\
\hline \multirow{5}{*}{$\begin{array}{l}\text { Group } 1 \\
\left(0-10 \mathrm{~m}^{2}\right)\end{array}$} & Proximity to walls & 16 & 6 & 4 & 3 & 9 & 2 & 1 & 12 & 53 & 2.9 & 534 & 29 \\
\hline & $2-5$ & 52 & 47 & 74 & 37 & 54 & 39 & 59 & 49 & 412 & 22.5 & & \\
\hline & $5-10$ & 11 & 14 & 1 & 5 & 15 & 1 & 12 & 5 & 64 & 3.5 & & \\
\hline & $11-15$ & 0 & 0 & 0 & 0 & 0 & 0 & 4 & 0 & 4 & 0.2 & & \\
\hline & $16-20$ & 0 & 1 & 0 & 0 & 0 & 0 & 0 & 0 & 1 & 0.1 & & \\
\hline \multirow{5}{*}{$\begin{array}{l}\text { Group } 2 \\
\left(11-20 \mathrm{~m}^{2}\right)\end{array}$} & Proximity to walls & 1 & 5 & 0 & 2 & 9 & 2 & 10 & 3 & 33 & 1.8 & 162 & 9 \\
\hline & $2-5$ & 12 & 11 & 16 & 19 & 20 & 10 & 12 & 10 & 110 & 6.0 & & \\
\hline & $5-10$ & 1 & 3 & 1 & 1 & 8 & 0 & 2 & 1 & 17 & 0.9 & & \\
\hline & $11-15$ & 0 & 0 & 0 & 1 & 0 & 0 & 0 & 0 & 1 & 0.1 & & \\
\hline & $16-20$ & 0 & 1 & 0 & 0 & 0 & 0 & 0 & 0 & 1 & 0.1 & & \\
\hline \multirow{5}{*}{$\begin{array}{l}\text { Group } 3 \\
\left(21-30 \mathrm{~m}^{2}\right)\end{array}$} & Proximity to walls & 1 & 0 & 1 & 0 & 0 & 0 & 1 & 0 & 3 & 0.2 & 65 & 4 \\
\hline & $2-5$ & 7 & 7 & 6 & 8 & 7 & 3 & 14 & 4 & 56 & 3.1 & & \\
\hline & $5-10$ & 0 & 2 & 1 & 1 & 0 & 0 & 2 & 0 & 6 & 0.3 & & \\
\hline & $11-15$ & 0 & 0 & 0 & 0 & 0 & 0 & 0 & 0 & 0 & 0 & & \\
\hline & $16-20$ & 0 & 0 & 0 & 0 & 0 & 0 & 0 & 0 & 0 & 0 & & \\
\hline \multirow{5}{*}{$\begin{array}{l}\text { Group } 4 \\
\left(31-40 m^{2}\right)\end{array}$} & Proximity to walls & 0 & 0 & 0 & 0 & 1 & 0 & 2 & 0 & 3 & 0.2 & 9 & 1 \\
\hline & $2-5$ & 1 & 3 & 0 & 0 & 0 & 0 & 2 & 0 & 6 & 0.3 & & \\
\hline & $5-10$ & 0 & 0 & 0 & 0 & 0 & 0 & 0 & 0 & 0 & 0 & & \\
\hline & $11-15$ & 0 & 0 & 0 & 0 & 0 & 0 & 0 & 0 & 0 & 0 & & \\
\hline & $16-20$ & 0 & 0 & 0 & 0 & 0 & 0 & 0 & 0 & 0 & 0 & & \\
\hline \multirow{2}{*}{\multicolumn{2}{|c|}{$\begin{array}{l}\text { All plants si } \\
\text { Percentage }\end{array}$}} & 242 & 204 & 298 & 191 & 234 & 170 & 283 & 202 & 1824 & 100.0 & 1824 & 100 \\
\hline & & 13.3 & 11.2 & 16.3 & 10.5 & 12.8 & 9.3 & 15.5 & 11.1 & 100 & & & \\
\hline
\end{tabular}


Fifty-eight percent, or 1054 of the shrubs, vines and groundcover in the gardens were planted individually, while $29 \%$ were in Group 1 (which included plants sized between $\left.0-10 \mathrm{~m}^{2}\right)$, Group $2\left(11-20 \mathrm{~m}^{2}\right) 9 \%$, Group $3\left(21-30 \mathrm{~m}^{2}\right) 4 \%$ and Group 4 (31$40 \mathrm{~m}^{2}$ ) $1 \%$. The average size and amount of leaves was medium.

The distribution of shrubs, vines and groundcover was spread around all areas around the houses. The majority were situated on the east and west sides, representing around $16 \%$ on each side. The north and south areas had $13 \%$ each, while northeast, southeast, southwest, and northwest had $9 \%$ and $11 \%$ as can be seen in Table 6.13. The detail about the shrubs, vines and groundcover locations for each age group of house is shown in Appendices 6.4.1-6.4.4. The percentage of shrubs, vines and groundcover at the four age groups of house was similar for each.

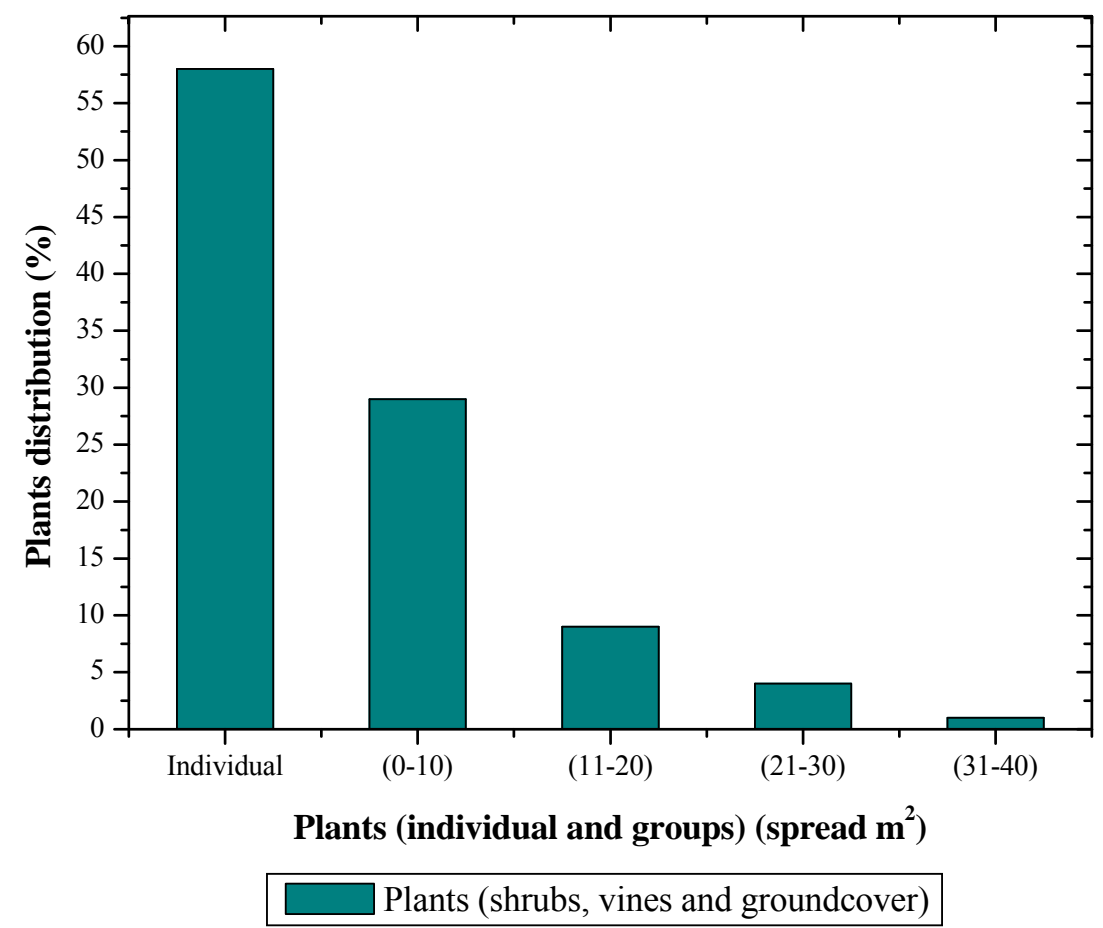

Figure 6.13. Sizes of shrubs, vines and groundcover planted individually and in groups $\left(\operatorname{spread~} \mathrm{m}^{2}\right)$

The distances between the shrubs, vines and groundcover and the buildings have been divided into five categories, as can be seen in Table 6.13 and Figure 6.14. These ranged from close to the walls to $20 \mathrm{~m}$ away. Those that were positioned close 
to the walls had small, shallow roots that could not harm the building's floor or foundations.

The majority of shrubs, vines and groundcover were between $2-5 \mathrm{~m}$ from the buildings $(68.5 \%), 17.3 \%$ were close to walls, $10.5 \%$ were between $5-10 \mathrm{~m}$, and plants between $11-15 \mathrm{~m}$ and $16-20 \mathrm{~m}$ each formed less than $0.3 \%$ of total, as can be seen in Figure 6.14.

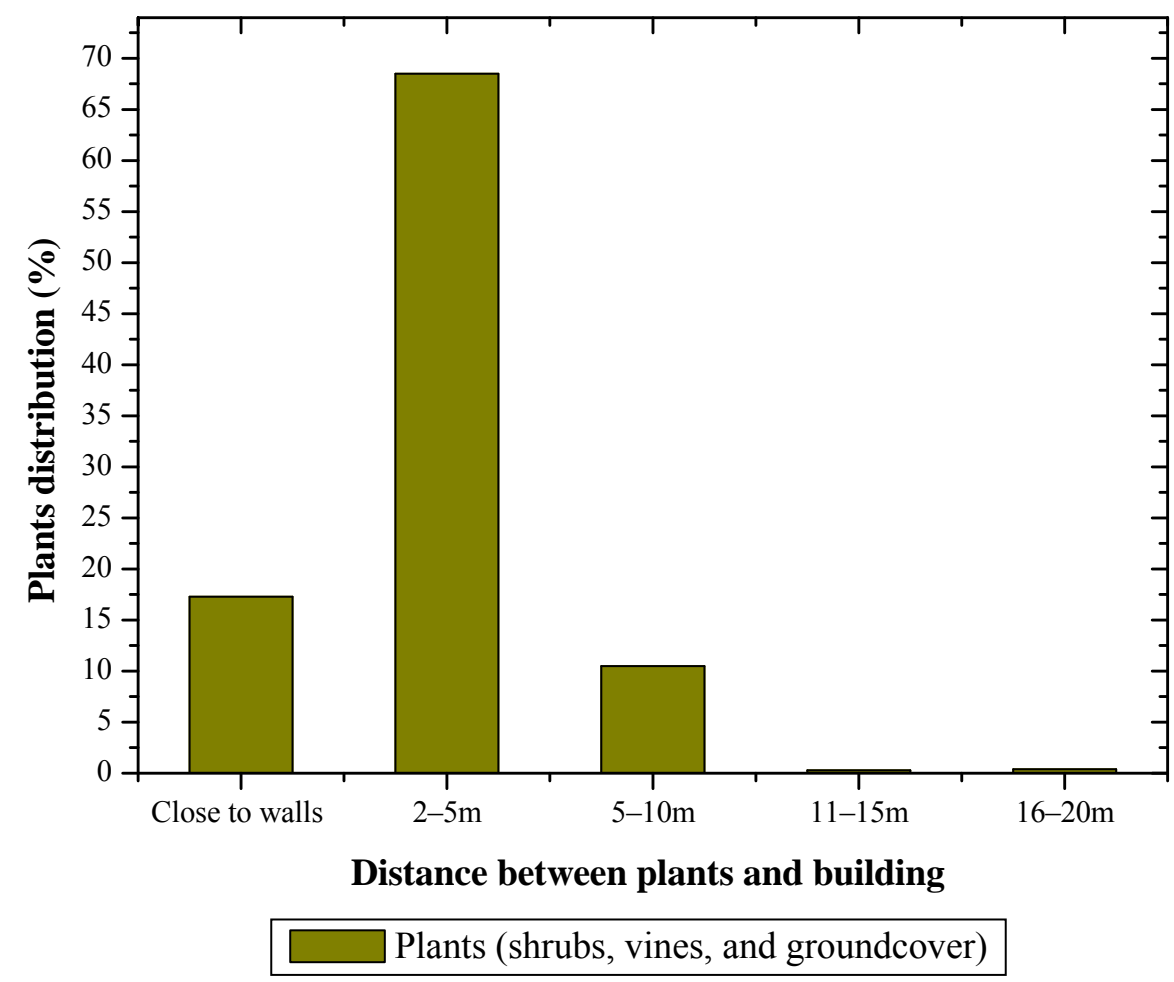

Figure 6.14. Shrubs, vines, and groundcover by distance from the buildings

The average shrub height at the houses in the study area was between 0.7 and $1.15 \mathrm{~m}$, as can be seen in Figure 6.14. The linear data shows that the average height was in the range 0.77 to $0.85 \mathrm{~m}$. 


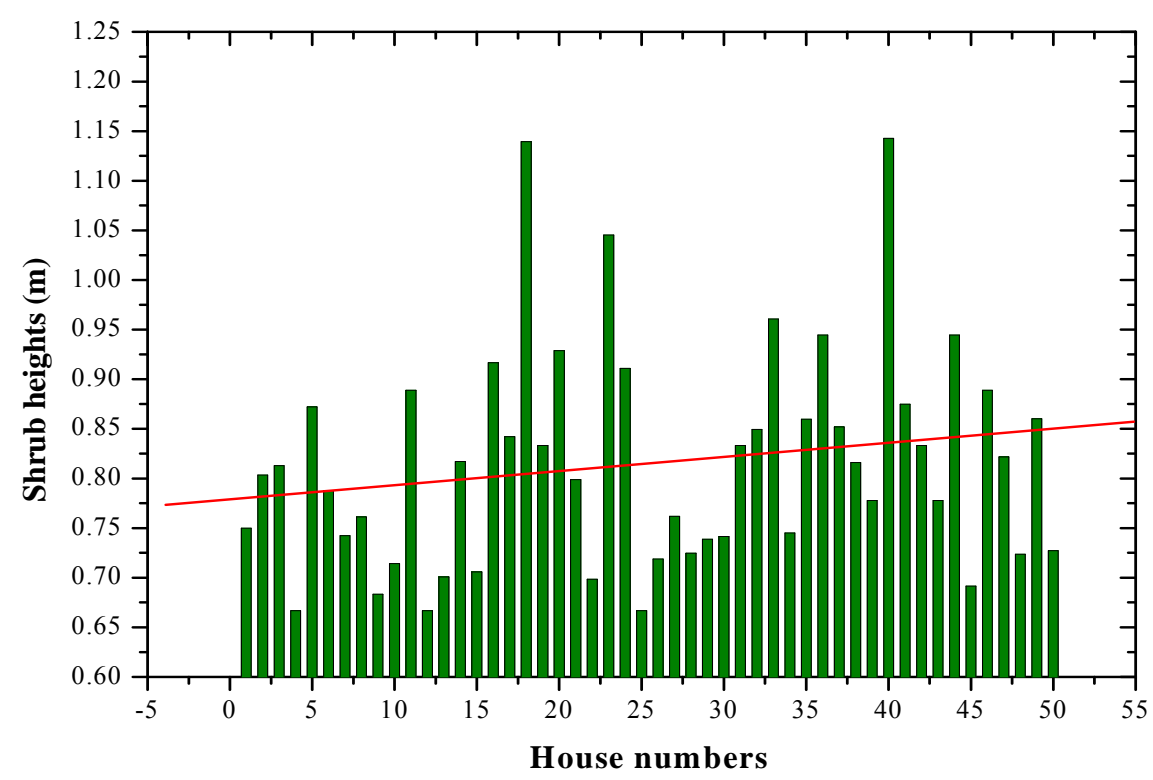

Shrub heights - Linear data (shrub heights)

Figure 6.15. Average shrub heights at each house

Two species of turf were planted in the study areas: $88 \%$ of the houses had Axonopus compressus, while the remaining 12\% had planted Cynadon dactylon. Figure 6.16 shows that up to $75 \%$ of the garden surfaces were covered by turf, while the remaining surfaces were covered with other plants such as trees, shrubs, vines and groundcover, and hard landscape elements.

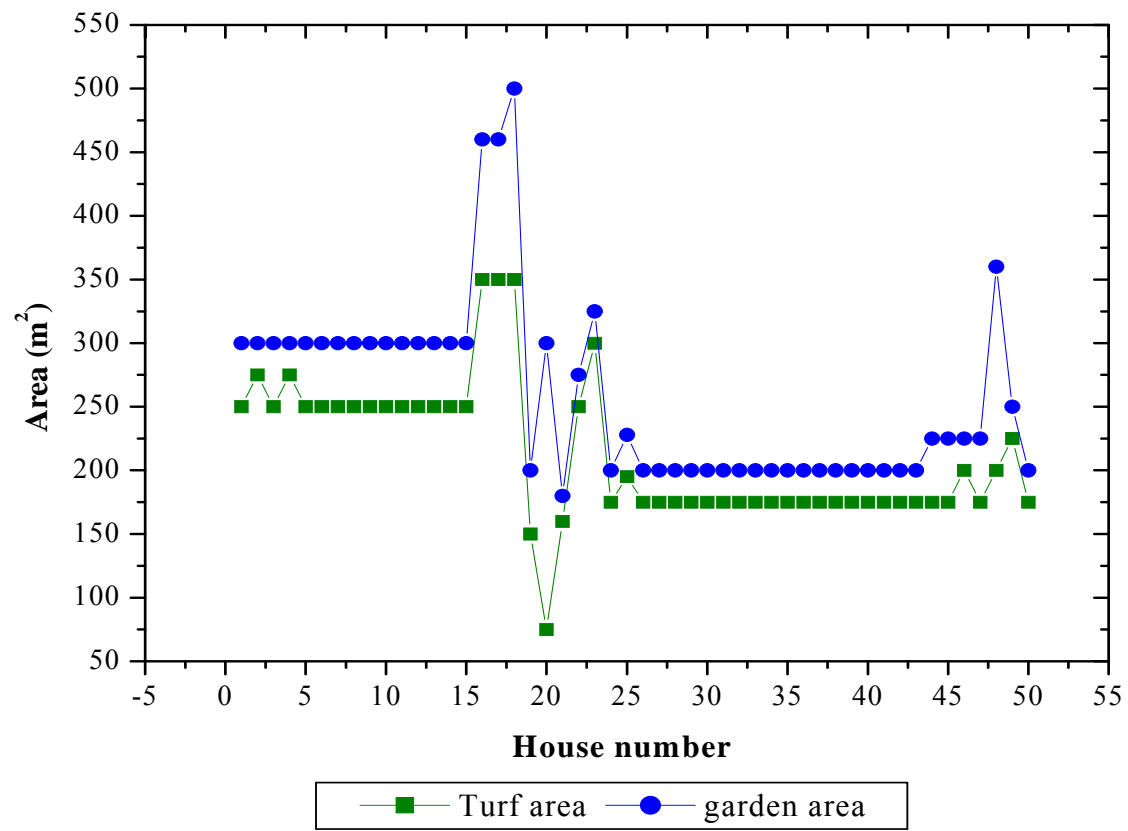

Figure 6.16. Garden and turf cover areas around the houses 


\subsubsection{Hard landscape elements}

Hard landscape elements in the study area included shelters, water elements and footpaths. They have been divided into six types: swimming pool, fountain, fish pool, footpath, gazebo and pergola. The hard landscape elements were located around the gardens, and surrounded by soft landscaping, with $34 \%$ of the houses in the study areas featuring hard landscaping elements in their main garden areas, while the remaining $66 \%$ do not have hard landscaping elements.

Twelve of the gardens included a medium sized pergola $\left(8 \mathrm{~m}^{2}\right.$ by $2.5 \mathrm{~m}$ high), which was used for shelter or to grow vines. Nine gardens had a gazebo, which was 9 to $20 \mathrm{~m}^{2}$ in area and $2.5 \mathrm{~m}$ high, nine had footpaths made from wood, stones, bricks or concrete pieces with grass/turf inlay that were between 10 and $15 \mathrm{~m}^{2}$. Eight gardens had fish pools for Cyprinus carpio (Japanese Carp), which were built from concrete in various shapes and averaged $10 \mathrm{~m}^{2}$ in area and $0.6 \mathrm{~m}$ deep. The least common hard landscape elements found were a fountain (1 example), and swimming pool (1 example) $\left(54 \mathrm{~m}^{2}\right.$ by $1.5 \mathrm{~m}$ deep $)$.

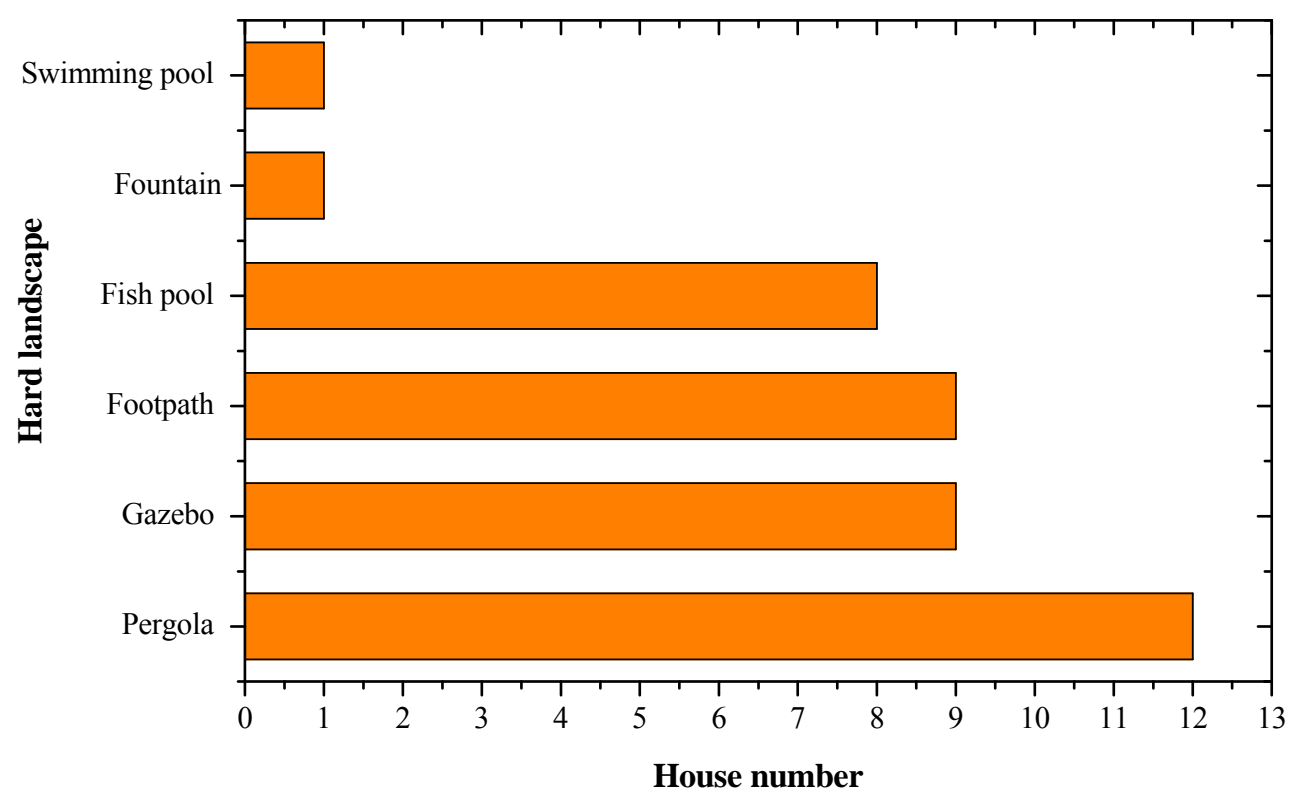

Figure 6.17. Hard landscape elements located around the gardens 


\subsubsection{Energy use data}

Energy use relates to human factors. The focus here is on how the occupants used air-conditioning systems for cooling energy in the 50 single-family houses. The results for cooling energy use have been divided into three parts: number of occupants (users), air-conditioning systems and amount of cooling energy used. The quantitative results of cooling energy used in this study have been compared to information on energy use obtained from National Energy Limited (TNB), Shah Alam for the rainy and dry seasons for 2008-2010.

\subsubsection{Number of occupants}

There were a total of 266 occupants in the 50 single-family houses, which averaged approximately five people per house ( 4 adults and 1 child or adolescent). They have been divided into three groups: children under 12 years of age, adolescents from 13 to 18 years old, and adults aged above 18 years old.

Adults made up the main group of the households (79\%), while $13.0 \%$ were children, and $8.0 \%$ were adolescents, as shown in Table 6.14 . The 20 to 29 year old houses had approximately $45 \%$ of the occupants, followed by $33 \%$ in the 0 to 9 year old houses, $17 \%$ in the 30 to 40 year old houses, and $5 \%$ in the 10 to 19 year old of houses. There is no significant difference in the number of occupants in relation to the different ages of constructions.

Table 6.14. Number of occupants

\begin{tabular}{lllllll}
\hline \multirow{2}{*}{$\begin{array}{l}\text { Age of } \\
\text { construction }\end{array}$} & House no. & \multicolumn{2}{l}{ Household } & & \\
\cline { 3 - 5 } & Adult & Adolescent & Children & Total & Percentage \\
\hline $0-9$ & 17 & 65 & 7 & 15 & 87 & 32.7 \\
$10-19$ & 3 & 11 & 3 & 0 & 14 & 5.3 \\
$20-29$ & 23 & 100 & 6 & 14 & 120 & 45.1 \\
$30-40$ & 7 & 34 & 5 & 6 & 45 & 16.9 \\
\hline Total & 50 & 210 & 21 & 35 & 266 & 100 \\
Percentage & 100 & 78.9 & 7.9 & 13.2 & 100 & 100 \\
\hline
\end{tabular}




\subsubsection{Air conditioning systems}

In hot-humid tropical cities, air conditioning systems are considered a necessity to help moderate temperatures, and are used on a daily basis. In the study areas, $90 \%$ of the houses actively operate an air-conditioning system, while the remaining $10 \%$ do not have air-conditioning systems (these are in 20-40 year old houses). Sixty-seven percent of the houses that had air conditioning systems used window air-conditioning systems, while 33\% used split air-conditioning systems. The cooling effect provided by the two types of systems is similar. Split unit systems were placed in boxes located beside the outdoor walls, while window systems were attached to the wall of the room to be cooled.

In Malaysia the power used by air conditioning systems are stated in horsepower. A horsepower is equal to $0.7457 \mathrm{kWh}$. Table 6.15 shows that a total of 279 air conditioning units were positioned at 45 houses, totalling 378.5 horsepower $(282.25 \mathrm{kWh})$.

Table 6.15. Air conditioning systems

\begin{tabular}{|c|c|c|c|c|c|c|c|c|}
\hline \multirow{2}{*}{$\begin{array}{l}\text { House } \\
\text { Age of } \\
\text { construction }\end{array}$} & \multicolumn{5}{|c|}{ Air-conditioning systems } & \multirow[b]{2}{*}{$\begin{array}{l}\text { Total } \\
\text { unit }\end{array}$} & \multirow[b]{2}{*}{$\begin{array}{l}\text { Total } \\
\text { HP* }\end{array}$} & \multirow[b]{2}{*}{ Percentage } \\
\hline & No. & Split & HP* & Window & $\mathrm{HP}^{*}$ & & & \\
\hline $0-9$ & 17 & 150 & 232.5 & 12 & 18.5 & 162 & 251 & 66.3 \\
\hline $10-19$ & 3 & 0 & 0 & 13 & 17 & 13 & 17 & 4.5 \\
\hline $20-29$ & 19 & 0 & 0 & 80 & 85 & 80 & 85 & 22.5 \\
\hline $30-40$ & 6 & 0 & 0 & 24 & 25.5 & 24 & 25.5 & 6.7 \\
\hline Total & 45 & 150 & 232.5 & 129 & 146 & 279 & 378.5 & 100 \\
\hline
\end{tabular}

Sixty-six percent of the air conditioning units were situated in 17 houses in the 0 to 9 year old house age group, while $23 \%$ were in 19 houses in the 20 to 29 year group. The 30 to 40 and 10 to 19 year old houses had $7 \%$ and $5 \%$ of the air conditioning units respectively. The type and horsepower of air conditioning systems in the 45 houses are illustrated in Tables 6.16-6.17. The majority of air conditioning units (166 or 59\%) were located in bedrooms, and 113 (41\%) were in common rooms. 
The average number of air-conditioning units for each house is approximately six, which includes four for the bedrooms and two for the common rooms, to cater for an average of five occupants per house. The majority of the units in common rooms used 1-2 horsepower $(0.75-1.5 \mathrm{kWh})$, while $1-1.5$ horsepower units $(0.75-1.12 \mathrm{kWh})$ were used for bedrooms.

Table 6.16. Air conditioning systems in the common rooms

\begin{tabular}{|c|c|c|c|c|c|c|c|c|}
\hline \multirow{2}{*}{\multicolumn{2}{|c|}{$\begin{array}{l}\text { Air-conditioning } \\
\text { system }\end{array}$}} & \multicolumn{6}{|c|}{ Common room } & \multirow[b]{2}{*}{ Total } \\
\hline & & \multirow{2}{*}{$\begin{array}{c}\begin{array}{c}\text { Living } \\
\text { room }\end{array} \\
18\end{array}$} & \multirow{2}{*}{$\begin{array}{c}\begin{array}{c}\text { Dining } \\
\text { room }\end{array} \\
8\end{array}$} & \multirow{2}{*}{$\frac{\text { Kitchen }}{1}$} & \multirow{2}{*}{$\begin{array}{c}\text { Family } \\
\text { room }\end{array}$} & \multirow{2}{*}{$\begin{array}{c}\text { Study } \\
\text { room }\end{array}$} & \multirow{2}{*}{$\begin{array}{c}\begin{array}{c}\text { Audio } \\
\text { room }\end{array} \\
2\end{array}$} & \\
\hline Type & Window & & & & & & & 38 \\
\hline & Split & 15 & 15 & 0 & 15 & 15 & 15 & 75 \\
\hline & Total unit & 33 & 23 & 1 & 18 & 21 & 17 & 113 \\
\hline HP* & 1 & 13 & 5 & 1 & 1 & 5 & 0 & 25 \\
\hline & 1.5 & 1 & 15 & 0 & 17 & 16 & 17 & 66 \\
\hline & 2 & 18 & 3 & 0 & 0 & 0 & 0 & 21 \\
\hline & 2.5 & 1 & 0 & 0 & 0 & 0 & 0 & 1 \\
\hline & 3 & 0 & 0 & 0 & 0 & 0 & 0 & 0 \\
\hline & Total HP* & 53 & 33.5 & 1 & 26.5 & 29 & 25.5 & 168.5 \\
\hline
\end{tabular}

Table 6.17. Air conditioning systems in the bedrooms

\begin{tabular}{|c|c|c|c|c|c|c|c|c|}
\hline \multirow{2}{*}{\multicolumn{2}{|c|}{$\begin{array}{l}\text { Air-conditioning } \\
\text { system }\end{array}$}} & \multicolumn{6}{|c|}{ Bedroom } & \multirow{3}{*}{$\begin{array}{c}\text { Total } \\
91\end{array}$} \\
\hline & & \multirow{2}{*}{$\frac{\text { Master }}{30}$} & \multirow{2}{*}{$\frac{1}{26}$} & \multirow{2}{*}{$\frac{2}{17}$} & \multirow{2}{*}{$\frac{3}{13}$} & \multirow{2}{*}{$\frac{4}{2}$} & \multirow{2}{*}{$\begin{array}{c}\begin{array}{c}\text { Guest } \\
\text { room }\end{array} \\
3\end{array}$} & \\
\hline Type & Window & & & & & & & \\
\hline & Split & 15 & 15 & 15 & 15 & 0 & 15 & 75 \\
\hline & Total unit & 45 & 41 & 32 & 28 & 2 & 18 & 166 \\
\hline \multirow[t]{6}{*}{ HP* } & 1 & 24 & 23 & 17 & 12 & 2 & 4 & 82 \\
\hline & 1.5 & 19 & 18 & 15 & 16 & 0 & 14 & 82 \\
\hline & 2 & 1 & 0 & 0 & 0 & 0 & 0 & 1 \\
\hline & 2.5 & 0 & 0 & 0 & 0 & 0 & 0 & 0 \\
\hline & 3 & 1 & 0 & 0 & 0 & 0 & 0 & 1 \\
\hline & Total HP* & 57.5 & 50 & 39.5 & 36 & 2 & 25 & 210 \\
\hline
\end{tabular}

In the common rooms the living room was the most likely place for air-conditioners to be located, with $73 \%$ of the houses operating a cooling system regularly in this 
area. Fifty-one percent were operated in living/dining rooms. For other common rooms, such as family rooms, study rooms and audio rooms, the use of airconditioning was in the range of $38-47 \%$. Figure 6.18 shows the average temperature setting in common rooms, which ranged from 21 to $22^{\circ} \mathrm{C}$ over a four hour period of operation per day.

In the bedrooms, air-conditioning units were operated in all of the master bedrooms, while units in the other three bedrooms were operated between 56 and $82 \%$ of the time. The temperature setting in bedrooms was slightly higher compared to the common rooms, averaging around $23-24^{\circ} \mathrm{C}$. This can be seen in Figure 6.18. Air conditioning was used regularly in the bedrooms of $76 \%$ of the houses, but this use was only during the night when the occupants slept. Use was mainly between 23.00 and 6.00 hours for an average of five hours per night.

On average, three to four bedrooms in each house used air-conditioning systems, to cater for an average of five occupants per house.

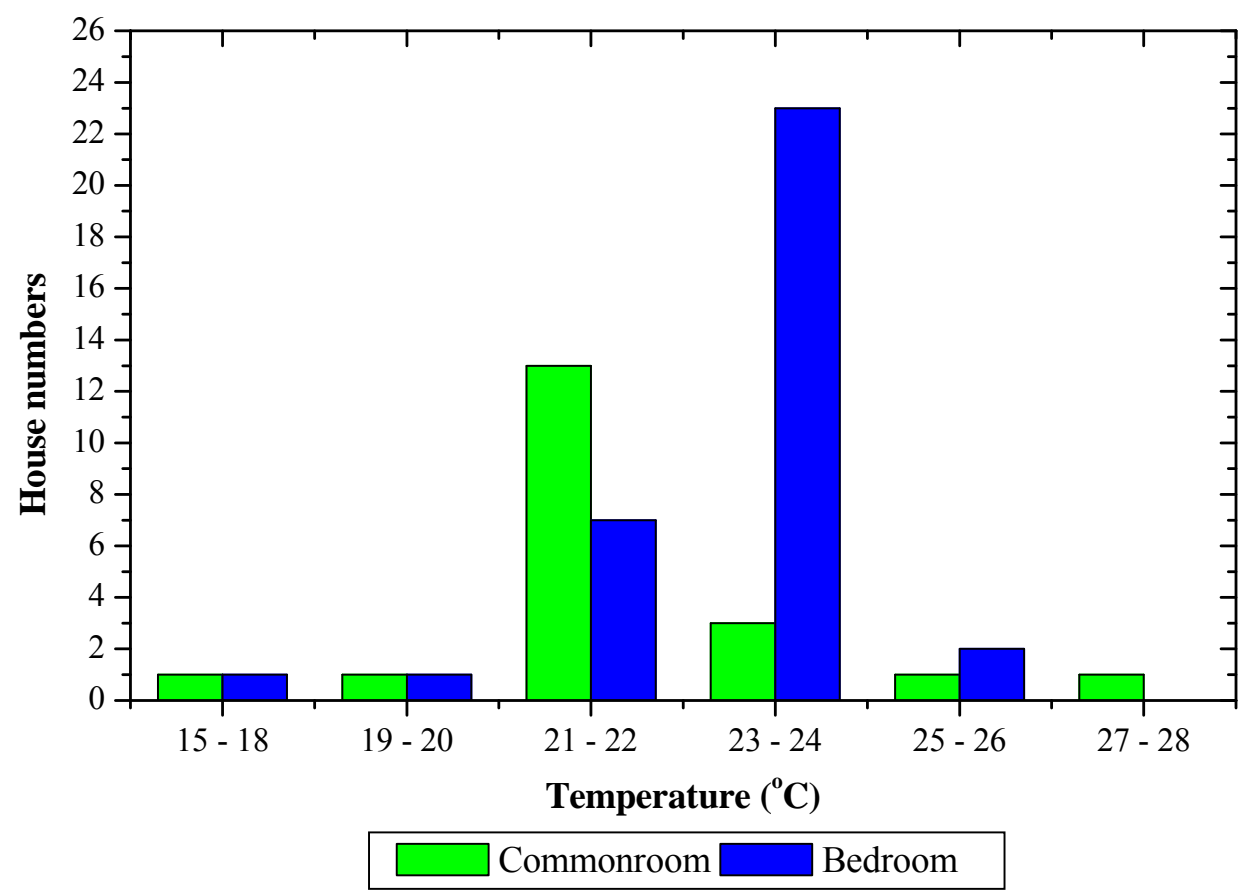

Figure 6.18. Air temperature settings for the common room and bedrooms 


\subsubsection{Cooling energy use}

Table 6.18 illustrates the average electricity bill in Malaysian currency (MYRMalaysian Ringgit), and energy consumption (kWh) for the two seasons: rainy and dry. The electricity bill was calculated based on use of all electrical items in the houses, including the air-conditioning systems. The average percentage of cooling energy use for each house in every month will be determined.

Energy (electricity) use for the single-family houses in the study area was approximately $753,114 \mathrm{kWh}(2,711.21 \mathrm{GJ})$ at a cost of MYR185,040. This was equal to $0.13 \mathrm{GJ} / \mathrm{m}^{2}$ per year or $10.2 \mathrm{GJ} /$ person per year. The energy needed per $\mathrm{m}^{2}$ and per occupant was calculated from the total energy use $(=2,722.21 \mathrm{GJ})$ divided by the total floor area of 50 houses $\left(=21,063 \mathrm{~m}^{2}\right)$, which was equal to $0.13 \mathrm{GJ} / \mathrm{m}^{2}$ per year and $10.2 \mathrm{GJ} /$ person $(2,722 \mathrm{GJ}$ divided by the total of 266 occupants $)$.

Table 6.18. Electricity bills in the rainy and dry seasons

\begin{tabular}{|c|c|c|c|c|c|c|c|}
\hline \multicolumn{2}{|l|}{ House } & \multicolumn{6}{|c|}{ Electricity bill (MYR) } \\
\hline \multirow{2}{*}{$\begin{array}{l}\text { Age of } \\
\text { construction (year) }\end{array}$} & \multirow{2}{*}{ No. } & \multicolumn{2}{|c|}{ Rainy season } & \multicolumn{2}{|c|}{ Dry season } & \multirow{2}{*}{$\begin{array}{l}\text { Increment } \\
\text { (MYR) }\end{array}$} & \multirow{2}{*}{$\begin{array}{l}\text { Increment } \\
(\%)\end{array}$} \\
\hline & & MYR & $\mathrm{kWh}$ & MYR & $\mathrm{kWh}$ & & \\
\hline $0-9$ & 17 & 5400 & 21978.0 & 6865 & 27940.6 & 1465 & 27.1 \\
\hline $10-19$ & 3 & 900 & 3663.0 & 1025 & 4171.8 & 125 & 13.9 \\
\hline $20-29$ & 23 & 5950 & 24216.5 & 7050 & 28693.5 & 1100 & 18.5 \\
\hline $30-40$ & 7 & 1625 & 6613.8 & 2025 & 8241.8 & 400 & 24.6 \\
\hline Total per month & 50 & 13875 & 56471.3 & 16965 & 69047.6 & 3090 & 22.3 \\
\hline Total per year & 50 & 83250 & 338827.8 & 101790 & 414285.7 & 18540 & 22.3 \\
\hline
\end{tabular}

Table 6.19 shows that on average, the total amount of energy for cooling during the rainy season in the study houses was in around 124,376kWh (costing a total of MYR30,559.20). This represented approximately $36.7 \%$ of the total energy consumption for each house per year. Table 6.19 and Figure 6.19 also show the incremental difference in total cooling energy used from the rainy to dry seasons rises by $48 \%$ each month. On average the amount of energy used for cooling for the two seasons was estimated to be $42.5 \%$ of total per house per month. 
Table 6.19. Cooling cost in the rainy season and dry seasons

\begin{tabular}{|c|c|c|c|c|c|c|c|c|}
\hline \multicolumn{2}{|l|}{ House } & \multicolumn{3}{|c|}{ Cooling cost in rainy season } & \multicolumn{3}{|c|}{ Cooling cost in dry season } & \multirow{2}{*}{$\begin{array}{l}\text { Cooling } \\
\text { Difference } \\
(\%)\end{array}$} \\
\hline $\begin{array}{l}\text { Age of } \\
\text { construction (year) }\end{array}$ & No. & MYR & $\mathrm{kWh}$ & $\begin{array}{l}\text { Cooling } \\
(\%)\end{array}$ & MYR & $\mathrm{kWh}$ & $\begin{array}{l}\text { Cooling } \\
(\%)\end{array}$ & \\
\hline $0-9$ & 17 & 1999.0 & 8135.9 & 37.0 & 3464.0 & 14098.5 & 50.5 & 13.5 \\
\hline $10-19$ & 3 & 422.2 & 1718.4 & 46.9 & 547.2 & 2227.1 & 53.4 & 6.5 \\
\hline $20-29$ & 23 & 2045.6 & 8325.6 & 34.4 & 3145.6 & 12802.6 & 44.6 & 10.2 \\
\hline $30-40$ & 7 & 626.4 & 2549.5 & 38.5 & 1026.4 & 4177.5 & 50.7 & 12.2 \\
\hline Total per month & 50 & 5093.2 & 20729.3 & 36.7 & 8183.2 & 33305.7 & 48.2 & 11.5 \\
\hline Total per year & 50 & 30559.2 & 124376.1 & 36.7 & 49099.2 & 199833.9 & 48.2 & 11.5 \\
\hline
\end{tabular}

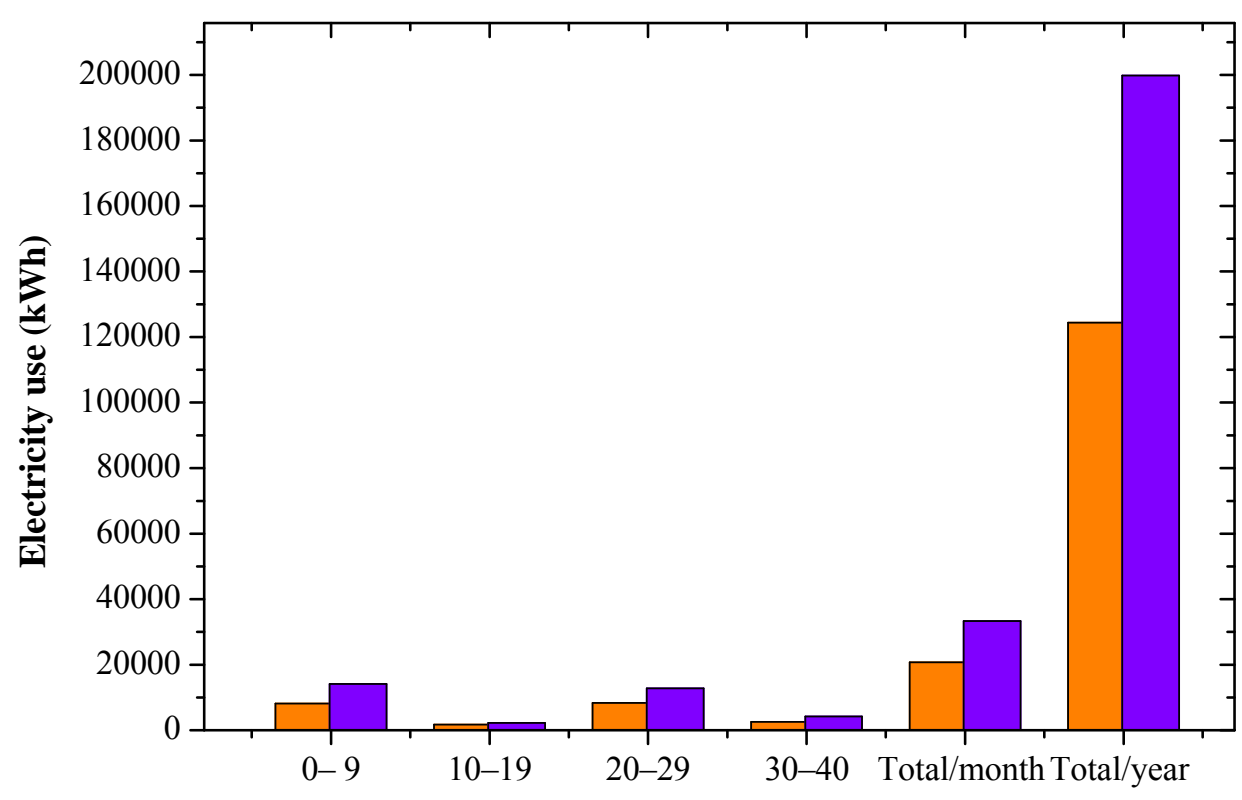

Ages of construction

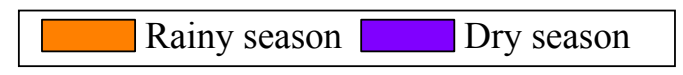

Figure 6.19. Cooling energy use (per month) in the rainy and dry seasons

\subsubsection{General domestic energy use}

The Electric Utility Company, National Energy Limited (TNB) manages the use of electricity in Malaysia. They provide services to commercial, industrial and domestic buildings, and for public areas such as street and park lighting.

Domestic buildings account for around $8-9 \%$ of the total energy used in Shah Alam every month. A monthly summary of energy consumption in kWh from 2008-2010 
in Shah Alam is shown in Figure 6.20. Domestic buildings include all types of residential buildings ranging from low cost to high cost housing. This data is from monthly reports provided by the Sales and Marketing Department of TNB.

In the study area, the rainy season began in October/November 2008 and lasted until March/April 2009, and the dry season went from May to September/October 2009. The average energy consumption per house per month during the rainy season was about 29.2 million kWh. In the dry season energy consumption increased to 33.7 million $\mathrm{kWh}$ per month. The increment of around $4.5 \mathrm{kWh}$ was equal to a $15.4 \%$ change.

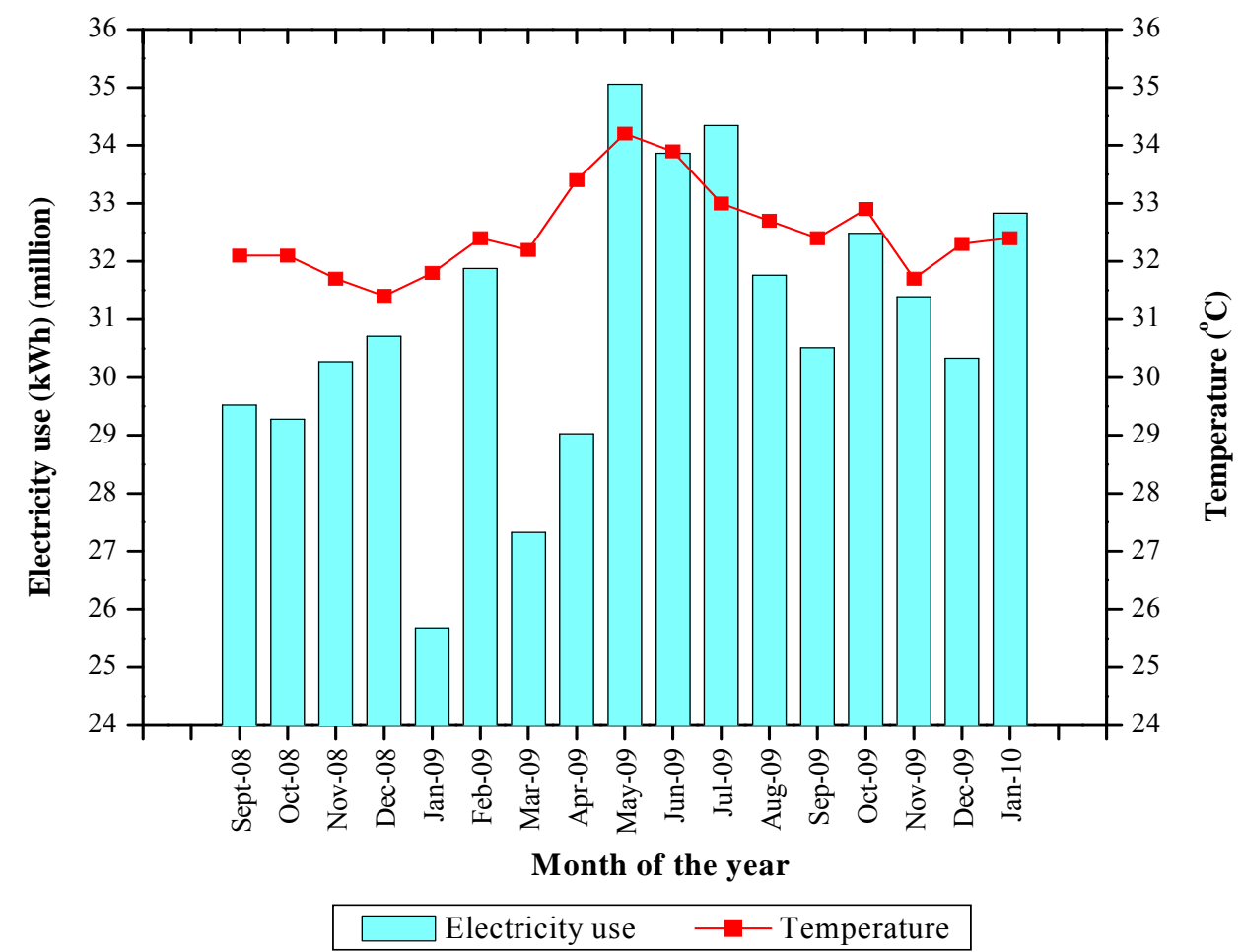

Figure 6.20. Summary of electricity use by tariff $(\mathrm{kWh})$ on a monthly basis for domestic buildings in the Shah Alam records of 24-hour mean temperatures (20082010) (TNB, 2010)

\subsection{Discussion}

The results of studying the building construction, landscaping and human factors that might influence the amount of cooling energy used in single-family houses in the study areas have been shown. All of the houses were medium-sized single-family 
houses, which were typical examples of tropical architecture while having slight differences in size and appearance. Each house had a private garden and different landscaping. Air-conditioning, which needs a substantial amount of energy to run, was needed at almost at all of the houses to achieve thermal comfort.

Strategic surrounding landscaping in sufficient sizes, species and amounts could reduce the air-conditioning energy and therefore energy costs. This section will discuss how climate conditions, building construction, surrounding landscaping and human factors can influence the cooling energy used in a house. Finally, it will focus on how different amounts of surrounding landscaping can save air-conditioning energy and cost.

\subsubsection{Weather}

In the study areas of Shah Alam and Putrajaya temperature was the most noticeable climate characteristic that affected cooling energy use. In these cities air temperature varies according to the two main seasons; the rainy and dry seasons. The weather data showed typical patterns of data, with Shah Alam experiencing slightly higher temperatures than Putrajaya.

During the rainy seasons, from November to March, the temperature in these cities was relatively stable, ranging between a $24^{\circ} \mathrm{C}$ mean minimum temperature and a $33^{\circ} \mathrm{C}$ mean maximum temperature. The 24 hour mean temperature was also stable, at around $26.5-27^{\circ} \mathrm{C}$, as can be seen in Figure 6.1. Temperature was stable because of rain occurring almost every day during this season, which cooled the ambient air.

In contrast, during the dry season from May to September, the temperature was up to $2{ }^{\circ} \mathrm{C}$ higher than usual temperatures during this time, with less rainfall. May was the hottest month, with temperatures ranging from a $25.5^{\circ} \mathrm{C}$ mean minimum temperature to a $34.4^{\circ} \mathrm{C}$ mean maximum temperature, and a mean 24 hour temperature of $29.3^{\circ} \mathrm{C}$. This is shown for each month in Figure 6.1 and annually in Table 6.20. 
According to Olgyay (1963), Givoni (1969) and Montgomery (1987), the best temperature range for human comfort is $20-27^{\circ} \mathrm{C}$. The high temperatures in the study area resulted in most of the single-family houses using air-conditioning to help them achieve thermal comfort.

The comparisons above would show that during the dry season cooling loads would be slightly higher than average. The record showed that while minimum monthly average temperatures remained fairly constant, the maximum temperatures generally increased for most months.

As well as high temperatures high RH levels also cause discomfort. The RH levels for each of the study areas were slightly different, but the humidity patterns were similar. This is shown in Figure 6.2. During the dry season RH was slightly lower than during the rainy season, by up to $10 \%$. RH between $40-70 \%$ does not have a major impact on thermal comfort (Oughton \& Hodkinson, 2002). In interior spaces, humidity is usually kept between this range. However, in places which are not air conditioned or where the climatic conditions outdoors may influence the indoor thermal environment, RH may be higher than $70 \%$ on hot-humid days. On average, the RH in the study areas was between $70-88 \%$, which represents uncomfortable levels.

Olgyay (1963) suggests that wind is particularly important for comfort when temperatures are above $29^{\circ} \mathrm{C}$ and the relative humidity above $50 \%$. In these circumstances, cooling needs are high and landscaping around buildings should help channel cool breezes towards the building to minimise humidity around the building.

In the two study areas, winds were moderate, as is typical of a tropical climate. Moderate winds are very important for cooling during the hot days, and to provide relief from vapour pressure during times of high relative humidity and air temperatures. The direction of windows can help to channel the air flow into buildings when they are arranged towards the prevailing winds, together with strategic surrounding landscaping. 
Table 6.20. Summary of two year's annual weather data for Putrajaya and Shah Alam (2008-2009)

\begin{tabular}{lcccc}
\hline Weather data & $\begin{array}{c}2008 \\
\text { Putrajaya }\end{array}$ & $\begin{array}{c}2009 \\
\text { Shah Alam }\end{array}$ & $\begin{array}{c}\text { Putrajaya } \\
\text { Shah Alam }\end{array}$ \\
\hline Temperature $\left({ }^{\circ} \mathrm{C}\right):$ & & & & \\
1. Maximum & 31.1 & 32.1 & 31.4 & 32.7 \\
2. Minimum & 23.7 & 23.9 & 23.7 & 24.4 \\
Relative humidity (\%) & 82.0 & 78.2 & 82.6 & 81.6 \\
Rainfall (mm): & & & & \\
1. Rainfall amount & 2092.6 & 3278.6 & 2099.2 & 2857.4 \\
2. Number of rain days & 167.0 & 203.0 & 168.0 & 200.0 \\
Global radiation $\left(\right.$ MJ.m $\left.{ }^{-2}\right)$ & 16.3 & 18.1 & 17.0 & 18.3 \\
Cloud cover & 6.9 & 7.1 & 6.9 & 7.0 \\
Wind all directions & & & & \\
(\% of the time): & & & & \\
$\quad$ 1. Total & 98.5 & 98.3 & 98.5 & 98.3 \\
$\quad$ 2. Calm & 6.9 & 31.7 & 6.9 & 31.0 \\
\hline
\end{tabular}

Both areas of the study received wind with similar speeds, with slight differences in a calm atmosphere. As shown in Figure 6.3, average wind speeds are low, but one or two predominant wind directions are common as wind direction varies at different times of the year. This moderate wind speed can increase heat loss through convection, which decreases the air temperature and humidity levels, which with the wind combines to provide gardens and houses with a cool and comfortable environment.

The data that has been looked at for the two year period gives an accurate picture of weather conditions in each of the study areas. They are also probably fairly representative of what could be expected in the future.

\subsubsection{Building construction}

Fifty medium-sized single-family houses were grouped in four ages of construction, covering four decades. The physical characteristic of the houses that influenced cooling energy use included: construction type and materials, building orientation, interior space and semi open spaces, and building envelopes. 


\subsubsection{Construction type and materials}

The results found that in the four ages of construction, similar construction methods and materials were used for each of the houses. The U-value for each material provided moderate thermal resistance for the houses, while an insulation layer of aluminium foil underneath the roof, together with a ceiling, provided thermal resistance for the roof surface, which helped to delay and reduce heat gain to the buildings.

\subsubsection{Building orientation}

For the majority of the houses, the main facades were orientated to face the access roads on northwest-southeast or north-south configurations. Orientation in these directions meant the houses were mainly only exposed to indirect solar radiation, which minimised heat gain. According to Givoni (1994), the main consideration affecting the orientation of the main facades and windows of buildings arises from the relative importance of solar and ventilation issues. Glazed windows and exterior doors were found on all front elevations and back elevations, which were exposed to direct and indirect solar radiation.

However, in the study area the house configuration did not influence the location or size of walls and openings in each house. They had square layouts, which meant different interior spaces could be located in any direction, and provide the required natural lighting, ventilation and views to the garden areas. Overall, the configurations and locations of doors and windows were influenced by their location in relation to the garden area, the type of interior spaces they were part of, and their relation to the front elevation of the building. Most of the houses in the study areas had shading devices for every door and window opening.

\subsubsection{Interior and semi open spaces, and ceiling height}

The common rooms were for living, dining and kitchen. These rooms were usually connected with the surrounding garden by verandas, which provided semi open spaces for the house. The living and dining rooms were connected to the main garden area at the front, side or rear of the houses, while the kitchens were directed to edible 
landscape gardens at the back of the houses. The connectivity between the indoor and outdoor spaces provides good views, can channel wind and provide shade to the interior space. Most of the houses use these semi open spaces as a transition space to get the natural lighting and ventilation to the house during day time and for active activities such as dining in the early morning and late afternoon. Most of the master bedrooms also had a veranda on the upper level of the house, which served similar functions. The houses also had a car porch for two to four cars located at the front of the house, which was connected to the entrance area and provided shade to the entrance doors and windows that faced them.

Similar to the exterior walls, all interior walls were painted light colours, and floors were finished with a combination of light and medium colours, while all ceilings were white. Light colours can reflect light and therefore reduce heat gain in interior spaces.

Ceiling heights of the houses were around $2.8-3.0 \mathrm{~m}$, which are the standard ceiling height for homes in Malaysia as it helps to circulate air. The three types of ceiling, fibrous plaster, cement plaster, and asbestos free fibre cement, also acted to delay the heat gain from the roof space at the upper level of the house.

The light colours of interior surfaces produced a harmonious and a cool interior environment and style of living, which also helped to reflect and reduce interior building heat gain.

\subsubsection{Building envelopes}

In this study area, the main building envelopes were brick walls. Table 6.5 and Figures 6.6 show that the size of the wall areas for all directions was between 80.3 and $87.5 \mathrm{~m}^{2}$, with an average of $84.1 \mathrm{~m}^{2}$. All houses had a square or rectangular layout with similar total areas of walls in all directions. Thus, shading is needed on all sides. 
In the tropics, solar gain through glazed windows is undesirable. According to Olgyay (1963), the highest heat flow comes from glazed windows via direct and diffused solar radiation. Therefore, glazed surfaces should be shaded properly to reduce heat gain to a building. On average, houses in the study area had a medium amount and average size of glazed opening, that covered an area of approximately $13 \%$ of the floor area (UBBL, 2006). These are for natural lighting and ventilation, and to allow views to the outside. The strategic location of windows can minimise solar penetration and allow natural cross-ventilation, which reduces air temperature and relative humidity levels. This natural ventilation can apply in a house equipped with iron grilles for the openings or one using a 'home security system'. The locations of openings for all of the houses were spread on all sides of the house, with an average of $13.5 \mathrm{~m}^{2}$ per side, as can be seen in Table 6.6 and Figure 6.8.

Most of the sliding doors in the houses faced the main areas of the gardens, and the windows faced all directions. In a hot-humid climate excess solar gain might result in high energy use for cooling. Thus, shading glazed areas affects the quantity of incidental radiation and modifies the heat flow to the interior space. All houses in the study areas had gables or hip roof shading devices that were between $0.75-3 \mathrm{~m}$ wide. As well as providing shade, these helped to protect the glass surfaces from direct and diffused solar radiation during the peak time of the day in the late morning until afternoon (11.00-15.00 hours), particularly on the east and west sides.

During the morning and late afternoon, shrubs along the walls also provided shade to the window surfaces, while shrubs, vines and groundcover provided shade to the building ground and garden surfaces.

Despite every house having gable or hipped roofs to shade the windows and doors, solar radiation could still penetrate. The surrounding shrubs were not high enough to prevent this. 


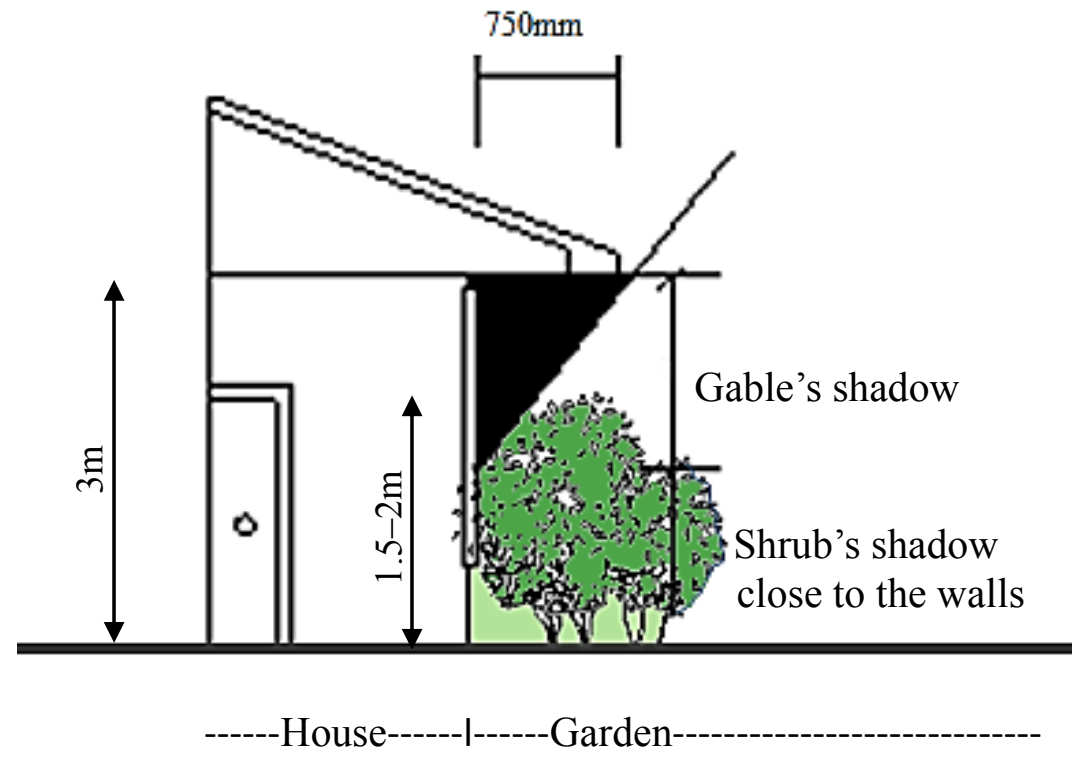

Figure 6.21. Example 1.5-2m shrub heights can provide shade to the windows before 11.00 hours (east) and after 15.00 hours (west) combined with hipped roof

While glazed windows and doors increase the chance of solar radiation entering a house and affecting the temperature, the advantage of having them is that they allow for natural cross ventilation to flow efficiently to the interior spaces, especially when the ambient air is cool. This cool air will increase the comfort levels in interior spaces, and the energy cost for active cooling will be reduced.

Interior shading, such as curtains, were widely use to control glare and as insulation, although they were not as effective as exterior shading. However, light colour curtains can reflect solar radiation through the glass before it heats the building. The combination of exterior and interior shading devices, combined with a sufficient amount and size of shrubs can reflect solar radiation effectively.

The reinforced concrete of ground floors, with their beams and foundations, were the lowest building envelope surfaces that were not directly exposed to solar radiation. However, this surrounding area needs shade to ensure the floor maintains a low temperature, which helps to provide a cool interior environment. 
In a tropical region, the solar reflectance ratio on the surfaces of the walls and roof is very important. This is expressed by the albedo value. The colour of the external envelope surfaces has a tremendous effect on the impact of the sun on the building and on the indoor temperatures. The average albedo values of the building envelopes in the study areas was 0.22 , with the majority of houses having cream walls and light red roof tiles. This value was sufficient to reflect sunlight from the building envelopes.

Each house in this study had a well insulated roof, which included a layer of aluminium foil underneath the roof cover. The $2.9 \mathrm{~m}$ high ceiling construction and materials also acted as an insulation layer. These insulation layers reflected and reduced heat gain from the roof surfaces, which helped to produce a cool and comfortable interior environment.

\subsubsection{Landscaping}

All of the houses in the study area were surrounded by tropical landscaping, that included different combinations and amounts of plants. The analysis conducted for this research included the general landscape design, garden size, data about the five main categories of soft landscaping, including trees, shrubs, vines, groundcover and turf, and information on the hard landscape elements.

\subsubsection{Landscaping in neighbourhood areas}

In Shah Alam, up to $10 \%$ of area in every housing development neighbourhood is for open space, and Putrajaya has more than $38 \%$ of its housing developments as open space. This is to provide balanced environments in areas of housing development, and to maintain the green and natural environment. Both of the study areas were situated on flat or gentle slopes in terms of topography, and had no water elements in their open spaces.

In general, single-family houses in Shah Alam were built by the owner of the house, and their gardens followed a conventional style of tropical landscape architecture. There was a slight gap in construction times among the houses because they were 
built by their owners in their own time frames. The houses in Sections 3 and 6, Shah Alam, were built as early as 40 years ago, with others built 20 to 30 years ago, as can be seen in Appendix 6.1 and Figure 6.1. The houses in Sections 9 and 11 were built between 5 to 20 years ago, as shown in Appendices 4.1-4.2. Overall, the majority of trees around these houses were mature and moderate in size and condition. Trees in the open spaces were also a similar size and condition. However, there were only a few roadside trees along the access roads, some of which were new trees.

Conversely, in Putrajaya the housing development was less than five years old, as shown in Figure 6.1. Houses in this precinct were built by developers with minimum landscaping. Roadside trees were in good condition. In this area the majority of home owners created their own garden around their houses. Trees around the houses and in open spaces were small, and there were only a moderate number that could provide shade to the surrounding garden and house. The shrubs were mature, and also in a moderate condition.

Overall, both groups of houses in Shah Alam and Putrajaya had moderate amounts of trees. The majority of trees in Shah Alam were mature, while in Putrajaya they were immature. Shah Alam was greener than Putrajaya, but in Putrajaya the open spaces were wider. Tropical shrubs were widely planted in both locations and were in good condition.

These small neighbourhood areas can create small microclimates, as the surrounding landscaping and their open spaces can influence the overall surrounding temperatures, $\mathrm{RH}$ and wind speed of the area and its houses. This occurs even though weather analysis has shown there were only slight differences in temperatures, RH and wind speed between the two well-planned cities.

\subsubsection{Landscape design}

In general, the medium-sized single-family houses in the study areas also had medium sized surrounding gardens. Each house was located at the middle of the land lot and was surrounded by tropical landscaping. 
Landscaping has various purposes including aesthetics, leisure, and for cooling the environment. In the study areas, various tropical styles and designs of landscaping were practised. Most of the gardens included tropical and sub-tropical native plants that were planted individually and in groups, in natural looking settings. These native tropical plants are typically lush and exotic, with thick and waxy leaves. The quality of the tropical landscaping in the study areas has been based on the fertility of plants, sizes, and their arrangements and locations around the houses and garden. In this study area fertile tropical plants mean lush, green, fresh, blooming, healthy plants, free from any disease or from insect attack, usually positioned in groups.

Each garden had a wide range of vegetation, which was dependent on the owner's preferences and financial capability. The minimum style of landscaping included only turf or grass to cover the garden surfaces with a few shrubs and a tree. Conversely, the maximum style of landscaping included dense plantings of a combination of many types and species of plants aimed at achieving the maximum environmental impact on their house and garden environments. However, the majority of the houses had a moderate style of landscaping, with a medium amount of shrubs and shade trees, that were medium sized. They were typical of tropical landscape design, with lush, colourful, and constantly growing plants.

Many gardens also had hard landscape structures that suited their tropical design style and concept of landscaping.

The gardens of the houses in the study area generally had different zones around the houses, including an access road area, house entrance, main garden area, an edible garden, and the boundary of the properties. Plants were used naturally to connect each zone. Roadside trees had been planted on the access road facing the house, and the landscaping continued with varieties of shrubs that were positioned at the main entrance of the house to create a welcoming appearance.

Usually the main garden area had a special design that allowed it to become a focal point of the garden, where most of the outdoor activities were focused. The size of 
the area was usually larger than other garden spaces, and was planted with a variety of plant types and species. This area also had hard landscape elements to complete the tropical garden design. The main gardens also faced or were close to the living or dining room, and were connected to these by a veranda and sliding doors.

Table 6.21. Summary of the soft landscape elements in the different age groups of houses

\begin{tabular}{lllllllll}
\hline $\begin{array}{l}\text { Age of } \\
\text { construction }\end{array}$ & $\begin{array}{l}\text { House } \\
\text { no. }\end{array}$ & $\begin{array}{l}\text { Average } \\
\text { garden } \\
\text { size }\left(\mathrm{m}^{2}\right)\end{array}$ & $\begin{array}{l}\text { Tree } \\
\text { no. }\end{array}$ & $\begin{array}{l}\text { Tree no. } \\
\text { per } \\
\text { house }\end{array}$ & $\begin{array}{l}\text { Shrub* } \\
\text { no. }\end{array}$ & $\begin{array}{l}\text { Shrub* } \\
\text { no.per } \\
\text { house }\end{array}$ & $\begin{array}{l}\text { Turf/ } \\
\text { lawn } \\
\left(\mathrm{m}^{2}\right)\end{array}$ & $\begin{array}{l}\text { Turf/lawn } \\
\text { per house } \\
\left(\mathrm{m}^{2}\right)\end{array}$ \\
\hline $3-9$ & 17 & 292.9 & 240 & 13.3 & 800 & 44.4 & 4035 & 237.4 \\
$10-19$ & 3 & 200.0 & 56 & 18.7 & 97 & 32.3 & 525 & 175.0 \\
$20-29$ & 23 & 253.5 & 211 & 8.4 & 763 & 30.5 & 4800 & 208.7 \\
$30-40$ & 7 & 218.3 & 35 & 8.8 & 164 & 41.0 & 1270 & 181.4 \\
\hline All/average & 50 & 258.8 & 542 & 10.8 & 1824 & 36.5 & 10630 & 212.6 \\
\hline \multicolumn{7}{c}{ *Shrubs, vines, and groundcover }
\end{tabular}

The edible garden spaces were located at the back of the houses, close to the kitchens. Edible plants included herbs, vegetable, fruits and medicinal plant species. These types of plants were used for everyday cooking and traditional medicine, as well as providing a naturally fragrant garden area.

Usually a layer of shrubs was planted as a fence along a boundary zone. They were used to soften fence surfaces or act as a fence. Tropical and subtropical plant species were widely planted including trees, shrubs, groundcover, vines and turf.

\subsubsection{Trees}

Trees were the most important components in moderating ground temperature, to produce evapotranspiration cooling, and to provide shade in and under their foliage. Their main function was to provide shade to buildings and the surrounding garden, which helped reduce heat gain to the buildings, especially during the peak time of the day.

All tropical trees in the study areas were evergreen species that were in good conditions. Tropical palms were the most popular species, accounting for almost 
$40 \%$ of trees in the study areas. Palms have become fashionable symbols of luxury because of their aesthetics, unique structure and leaf forms. Although the form and leaf type of palms means leaf cover is low, if palms are planted in groups together with other species of trees, they can produce a similar effect to leafier trees on the houses and surrounding gardens.

Tree distribution ranged between 5.5 and 15.5 per house, with an average of 11 trees per house. With this number of trees, if they are positioned strategically around the garden, this is sufficient to provide shade, channel wind and produce evapotranspiration cooling to the house and surrounding gardens as stated in subtopic 6.5.2.2 - Trees and illustrated in Figure 6.9.

Small sized trees were dominant in almost $60 \%$ of all age groups of houses in the study area. This was followed by small-moderate sized trees, which accounted for around $33 \%$ of trees. The small and small-moderately sized trees were in proportion to the gardens, including the number of trees in each garden, and the size of the houses, which were double storey. Trunk heights also were suitable for promoting wind flow to the surrounding garden and building surfaces. Even though the size of trees were only small to small-moderate, the number of trees in each garden, combined with other soft landscape elements such as shrubs, vines, groundcover and turf, were sufficient to produce shade, channel wind and produce evapotranspiration cooling as suggested by McPherson, Herrington and Heisler (1988) and as shown in Figures 6.9 and 6.12 .

In all of the gardens in the study areas, the trees were scattered around the houses. The majority were situated on the east (18\%) and west $(15 \%)$ sides of the houses, as can be seen in Table 6.11. These areas are the most critical directions around houses and gardens for landscaping, as they receive direct solar radiation. Having the majority of trees in these areas is an example of strategic landscaping, as they provide shade and help minimise direct solar radiation from entering buildings and the surrounding gardens. 
It is also important to shade other areas of the house and garden due to indirect or diffused solar radiation. On average the north sides of the houses had $13 \%$ of the trees and the south side $14 \%$.

The majority of trees (51\%) are located between $6-10 \mathrm{~m}$, and $44 \%$ between $3-5 \mathrm{~m}$, as shown in Figure 6.10. Those located between 3 and $5 \mathrm{~m}$ were close enough to the houses to effectively provide shade, while trees that were between 6 and $10 \mathrm{~m}$ from houses could only provide shade to some part of walls. As the majority of trees were small, most of them could only shade the walls during the early morning and late afternoon. However, the small-moderate and medium sized trees, which were as much as $9 \mathrm{~m}-14 \mathrm{~m}$ high, were able to provide shade to part of the roofs. Figure 6.22 shows that all trees between $3-5 \mathrm{~m}$ and $6-10 \mathrm{~m}$ provided extensive shade to the ground surfaces close to the houses, while the remaining 5\% of trees, which were between $11-20 \mathrm{~m}$ from the houses, provided shade to the surrounding garden. As a result, all trees, regardless of their distance from the houses assisted in keeping the ambient temperature cool and comfortable.

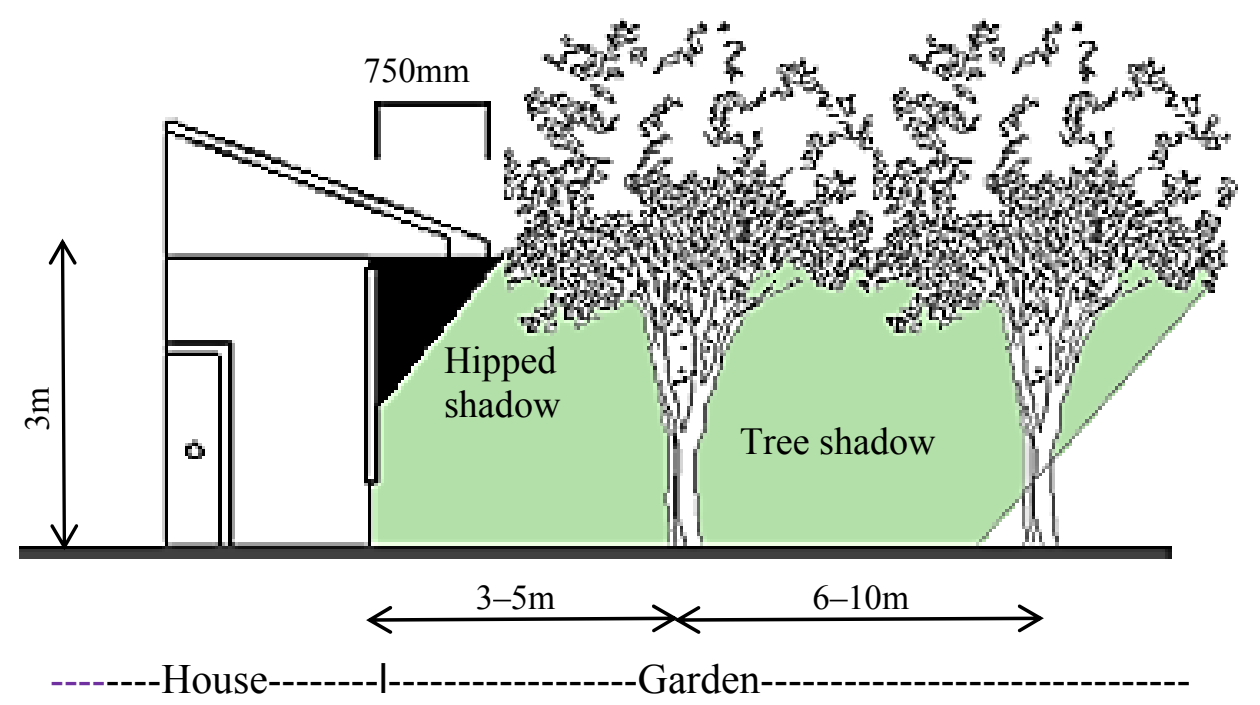

Figure 6.22. Distances of trees providing extensive shade to the ground surfaces close to the house 


\subsubsection{Shrubs, vines, groundcover and turf}

Shrubs, vines, groundcover and turf are important parts of the soft landscape design. Shrubs, which are relatively short with multiple stems, were spread throughout the gardens and around walls, while vines were planted on walls and garden structures such as a pergola to provide shade. Groundcover and turf were planted to cover the earth surfaces. After a minimum of three years, these types of plants should have matured to be big enough to provide shade in and under their foliage to the walls, and garden surfaces. Shrubs, vines, and groundcover help to channel wind flow and moderate ground temperature by producing evapotranspiration cooling to the surrounding environment.

In the study areas shrubs were the dominant types of vegetation, with 1,715 species, while vines were made up of 65 species, and groundcover 44 species. The average was 36 species per house. The diverse range of shrubs, vines and groundcover planted around the houses, together with trees and turf have helped to create pleasant tropical landscape designs.
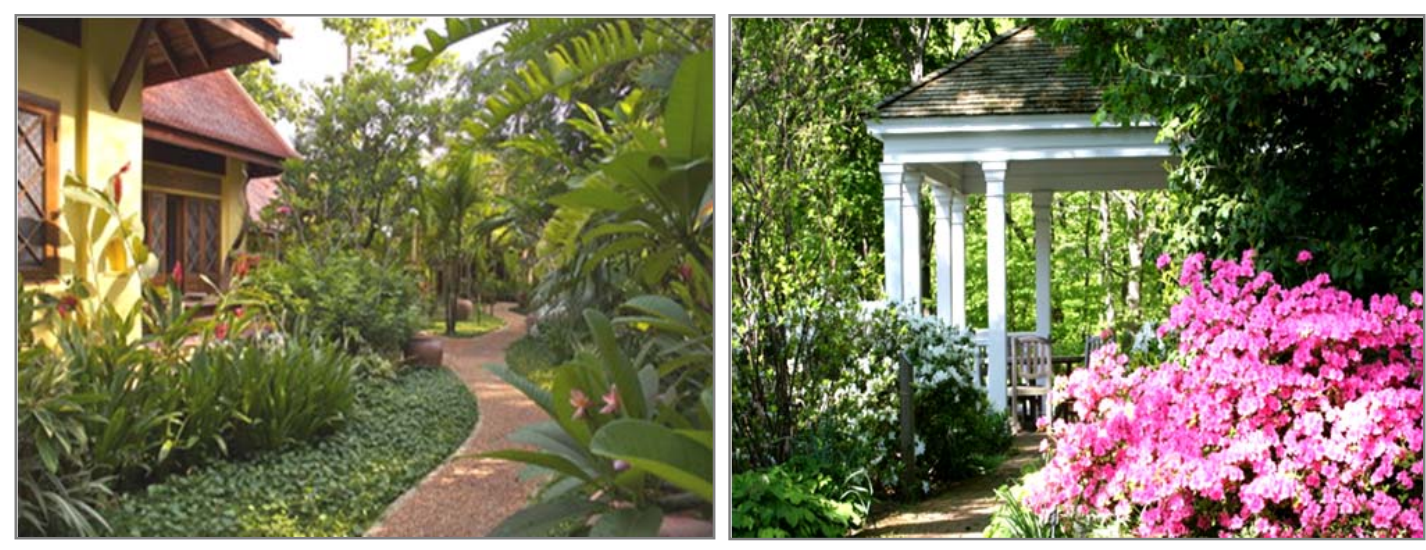

Figure 6.23. Example of pleasant tropical landscape; trees with diverse range of shrubs, groundcover and turf planted around the garden (modified from Kirsch, 2007; Ryan, 2012)

Most of shrubs, vines and groundcover in the study areas were planted in groups and close to other plants. As well as providing a balanced, harmonious and pleasant landscape design, planting shrubs, vines and groundcover in groups helps to make them stronger and more able to survive, especially during dry seasons, as they can 
share water, fertilizer and sunlight. Planting in groups also helps to provide more shade to the surrounding gardens and houses.

The shrubs, vines and groundcover were scattered in all directions around the houses. Similar to the trees, the majority were situated in the east and west areas, with approximately $16 \%$ of all shrubs, vines and groundcover in each area, as can be seen in Table 6.13. The majority of the plants in these areas were in strategic locations to provide shade and minimise the effects of direct solar radiation to houses and their surrounding garden. The north and south sides, where indirect or diffuse solar radiation affects houses, each had around $13 \%$ of shrubs, vines and groundcover.

There was no minimum distance for shrubs, vines and groundcover from the houses, as their shallow and small root systems cannot harm the floor or foundation structures, and their branches could not hurt the wall surfaces. Almost $70 \%$ of shrubs, vines and groundcover in the study areas were located $2-5 \mathrm{~m}$ from the houses, while $17 \%$ were close to the walls, as shown in Figure 6.14 . Between $0-5 \mathrm{~m}$ was close enough to the houses to effectively shade the garden surfaces and the walls during the morning and late afternoon when the sun is low in the sky as shown in Figure 6.21. This is beneficial because during this time external window shading devices cannot shade the windows, as they are designed to provide shade during the peak time of the day.

The average height of the shrubs was between 0.7 and $1.15 \mathrm{~m}$, as can be seen in Figure 6.15 , which was sufficient to provide shade to walls and windows. The shrubs, vines and groundcover also provided shade to the foundation areas of the houses during the peak time of the day. Like trees, they also channelled wind to the houses.

The shrubs, vines and groundcover between 0 and $20 \mathrm{~m}$ from the houses provided extensive shade to the ground garden surfaces close to the houses and gardens. This helped to keep the ambient temperature near the houses cool and comfortable. 
The function of turf surfaces was to cover soil surfaces with cool greenery. Two species, Axonopus compressus and Cynodon dactylon, covered $75 \%$ of all the garden surfaces, while the remainder were covered by groundcovers, shrubs and hard landscape elements. According to Ball (2011), even though turf is small and short, it shades the ground and keeps it cool, as well as reducing glare from reflected solar radiation to the building envelopes. It also plays a role in evapotranspiration cooling of the garden.

Overall, typical tropical landscape design has been widely practised in the study areas. Sufficient amounts and sizes of native tropical evergreen plants species were used to produce lively and harmonious garden designs, which provided a pleasant and comfortable ambient air around the garden. During the rainy season most of the plants were in a fertile and lush condition, which could provide a maximum effect to the surrounding area and the ambient conditions. Due to their size, trees were the most effective at providing useable shade for the houses and garden surfaces, while shrubs, vines, groundcover and turf also provided shade on a small-scale. They all channelled the wind flow towards the houses, and produced evapotranspiration cooling for the houses and surrounding gardens.

\subsubsection{Hard landscape elements}

In the study areas, $34 \%$ of the houses had various hard landscape elements. Their function was to enhance and complement the landscape design, as well as fulfilling the needs of the families for passive and active outdoor activities; the remaining $66 \%$ of the families in the study area did not have these outdoor garden facilities. The hard landscape elements were divided into three categories: shelters, footpaths, and water elements.

Shelters were found in 21 houses in the study areas. The pergola was the most popular type of shelter, serving two functions: to provide shade and a place or structure for vines to grow on. Evapotranspiration cooling from vines, combined with the shade provided by the structure helped to make these shelters and their surrounding garden environment cool, and therefore pleasant and comfortable for the 
occupants. Gazebos were built from wood, and had the main role of providing shade to the garden

Nine houses in the study area had footpaths around their gardens, which were made from materials such as stone or brick, and were designed with grass inlays. The grass inlay helped to minimize heat gain to the surface of the footpath, as well as making the design look green and harmonious with the lawn surfaces.

Water elements in the study areas included concrete fish pools at eight houses, a fountain at one house, and a small swimming pool at another. The fish pools and fountain were surrounded by shrubs, while the swimming pool was open and exposed to solar radiation. These three water elements provided evaporative cooling to the house and surrounding garden.

Overall, the hard landscape elements in the study areas affected the surrounding ambient air around the houses in different ways. Footpaths are often areas that gain heat, but this was minimised by grass inlays; shelters can provided shade; and the water elements provided evaporative cooling to the surrounding gardens and the houses (Penman, 1948).

\subsubsection{Energy use}

The discussions for cooling energy use were closely related to the number of occupants (users) and their life styles related to using air-conditioning systems for each house. The amount of energy use will be validated with energy use from National Energy Limited.

\subsubsection{Number of occupants}

The average number of permanent occupants of the 50 single-family houses in the study areas was five people per house. The occupants regularly used air-conditioning systems for cooling. The quantitative results show that the cooling energy use in the two study areas had similar patterns. 


\subsubsection{Air conditioning systems}

Information gathered from interviews with the occupants of the remaining $10 \%$ of houses that did not have not air-conditioning system revealed many did not have the budget to install an active air conditioning system or pay the monthly costs and do regular maintenance. Others rented the house, and the owner of the house chose not to provide an active cooling system for the tenants. Occupants of these houses used natural ventilation together with electric ceiling fans to circulate air in the interior space during the day and night.

This study focused on the majority of the houses that used active cooling systems. Use of air-conditioning differed at each house, and was according to the occupant's needs for their common rooms and bed rooms. Air-conditioning use during the day and evening was mainly in living and dining rooms, while in the bed rooms the airconditioning was only used during the night. The temperature setting was lower for common rooms because the size of the spaces was bigger than bedrooms. Also these areas were generally used by all the family together and for a relatively short time. Use in the bed rooms was longer and at a higher temperature than in the public areas to help provide a comfortable environment for the occupants sleep in. The results showed that active cooling systems were a basic daily need to help achieve a comfortable environment in the interior spaces of houses in all age groups.

\subsubsection{Cooling energy use}

During the rainy season, the average amount of energy used for cooling was around $36.7 \%$ of total energy use, with minimum to moderate durations of use for the airconditioning systems. Overall, the average spending on energy for cooling was up to one third of the total cost of energy used at each house.

However, throughout the year there are differences in air-conditioning use. For example, during the dry season, air-conditioning is often used for longer periods (moderate to maximum durations), and energy use increases to around $48.2 \%$ of total energy use. The average difference in energy consumption between the rainy and dry seasons was $11.5 \%$ per month. This is because the occupants' living habits and life 
styles change to adapt to high temperatures (approximately $2.5^{\circ} \mathrm{C}$ higher than in the rainy season) and humidity levels (20\% higher), as shown in Figures 6.1 and 6.2.

The difference between energy used from the rainy to dry season was $75,458 \mathrm{kWh}$, at a cost of MYR18,540 per year, which represented a $22.3 \%$ difference, as can be seen in Table 6.18.

\subsubsection{General domestic energy use}

In Shah Alam domestic buildings consumed around $8-9 \%$ of electricity used in the city. The average difference in energy consumption between the rainy and dry seasons was $15.4 \%$ per month.

The single-family houses in the study areas represented around $20 \%$ of all housing types in the city. Figure 6.19 reveals that the patterns of electricity used throughout the year for domestic buildings was parallel with the 24-hour mean temperature in Shah Alam (2008-2010) during the two main seasons: rainy and dry. Air temperature during the dry season is usually higher than in the rainy season by up to $2.5^{\circ} \mathrm{C}$. The hottest month was May, with the 24-hour mean temperature in Shah Alam up to $34^{\circ} \mathrm{C}$. This resulted in the highest level of energy consumption for the study periods of 35 million $\mathrm{kWh}$, which is shown in Figure 6.19.

The energy consumption for air-conditioning is expected to increase every year due to the urban heat island effect, which is increasing as cities grow, and climate change happens. However, sources of energy are reducing and costs are increasing. Strategic landscaping around houses could reduce the use of air-conditioning because it helps to create cool outdoor ambient air, which directly cools the indoor spaces of the houses.

\subsection{Discussion of the results}

The result of the effectiveness of using landscaping to reduce energy used will be discussed by comparing the buildings, surrounding landscape, and cooling energy for each house. 


\subsubsection{The effectiveness of using landscaping to reduce the cooling energy}

The landscaping around each of the houses, including the amount, size, and species of plants, and its distribution were relatively different, as shown in Figures 6.8 and 6.11. To establish the effectiveness of landscaping in reducing the cooling energy used in the single-family houses in a hot-humid tropical climate, a number of comparisons have been made of the energy consumed in the minimum to the moderately landscaped houses. Figure 6.24 shows the fluctuating pattern of cooling energy used compared to the amount of all types of plants around the houses. The linear data for cooling energy used for each house was between 33 and 44\%, compared to the linear data for all types of plants, which was around 20-58 plants per house. In general, the linear data for cooling energy used gradually declined when the amount of plants increased. Trees were dominant in number, and were supported by shrubs, vines, groundcover, and turf.

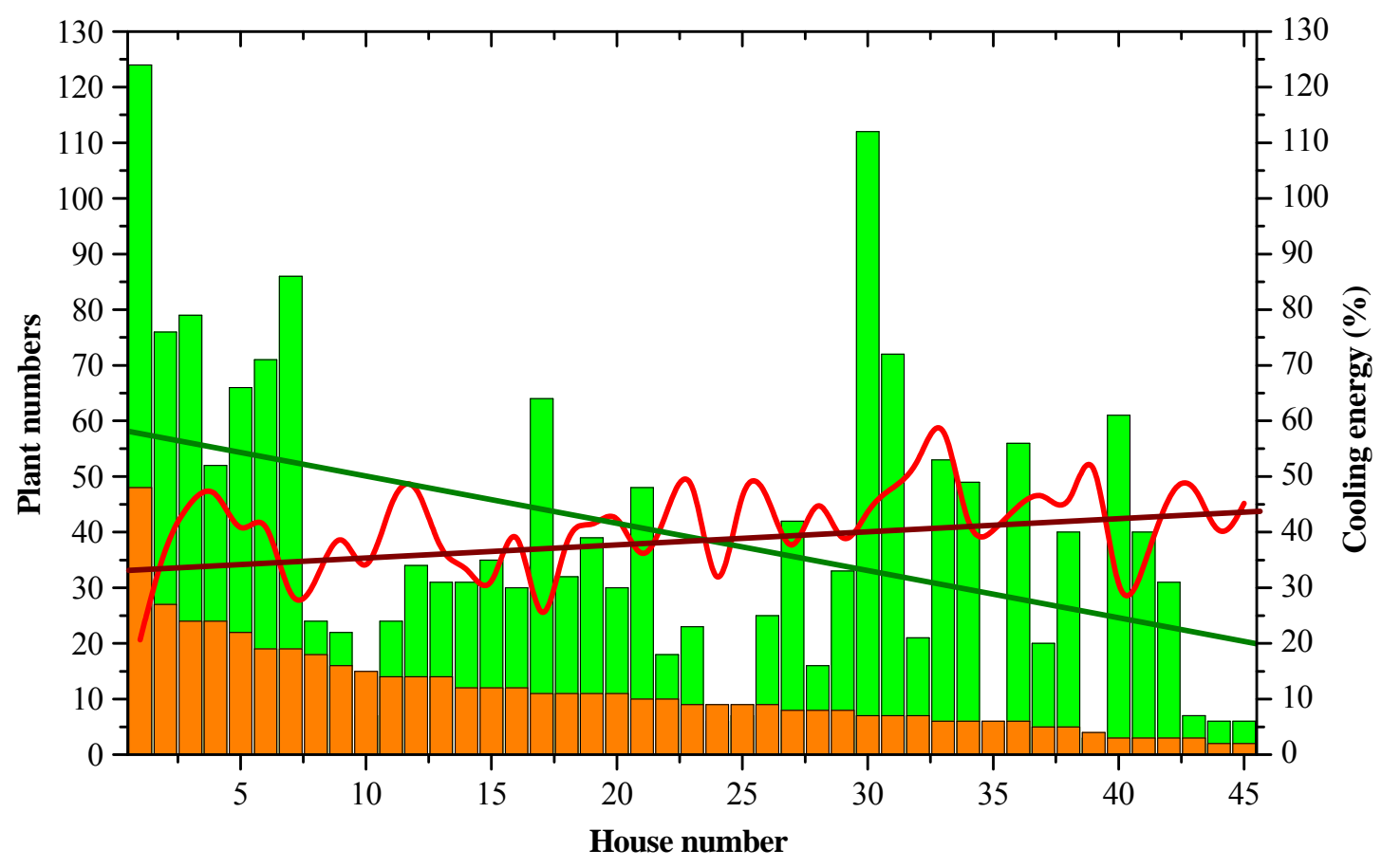

Trees $\square$ Shrubs and others $\longrightarrow$ Cooling energy

Linear data for plants —_ Linear data for cooling energy

Figure 6.24. The distribution of trees, shrubs and other vegetation compared with the cooling energy use 
Trees are considered to be the most important soft landscaping element in a hothumid tropical climate, as they are a primary means of reducing energy consumption for cooling, especially when they are well supported by shrubs, vines, groundcover, and turf. This is because the size of trees means they are able to provide shade to large areas of a house, including the roof, and its garden. They also provide evapotranspiration and channel wind towards the house and around the garden. The function of other plants, such as shrubs, vines and groundcover, is also important in providing shade, evapotranspiration and channelling wind.

The linear data for the amount of cooling energy used by the houses in the study area are shown in Figure 6.24. This shows a gradual increase when the number of trees planted around the houses decreased. Similarly, when the number of trees around the houses increases, a decrease in cooling energy can be gradually generated, which shows that landscaping can significantly influence the amount of energy used for cooling.

In the study areas, the trees were generally a combination of small and smallmoderate sizes. The majority of the trees were strategically located on the east and west sides of houses, along with shrubs, vines and groundcover, as can be seen in Table 6.11 and 6.13. These areas are where the sun has the most impact on the building envelope creating the highest temperature and heat gain to the building in morning and afternoon. These areas retain their heat until night time. As discussed in sub-topics 6.5.2.3 and 6.5.2.4, the majority of trees were in the range of $3-10 \mathrm{~m}$ from the houses, which can provide extensive shade to the garden and the building envelope, including walls, foundation area, and a small amount of shade to the roof.

The majority of shrubs were around $1 \mathrm{~m}$ high, which along with vines and groundcover were located between $2-5 \mathrm{~m}$ from the houses. Eighteen percent of the shrubs, vines and groundcover were located close to the building walls, while $10 \%$ were between $5-10 \mathrm{~m}$ from the houses. These plants were positioned in groups together with trees to promote shade, channel wind and to provide evapotranspiration 
cooling. All earth surfaces were covered by turf to ensure evapotranspiration occurred in every part of the gardens.

Figure 6.25 shows the summary of the four groupings of tree numbers around the houses, compared with the average cooling energy used, The polynominal data for cooling energy used declined when the amount of trees increased. Eighteen percent of houses planted 0-5 trees around their garden, and needed up to $43 \%$ of total energy used for cooling, while $10 \%$ of the houses planted 20 or more trees, and required as little as $36 \%$ of total energy used for cooling. The cooling energy saving for the $0-5$ and 20 and above groups was as much as $7 \%$ of the overall electricity costs, and equal to $20 \%$ of cooling energy. This energy saving was equal to MYR233 (948kWh) per house per year.

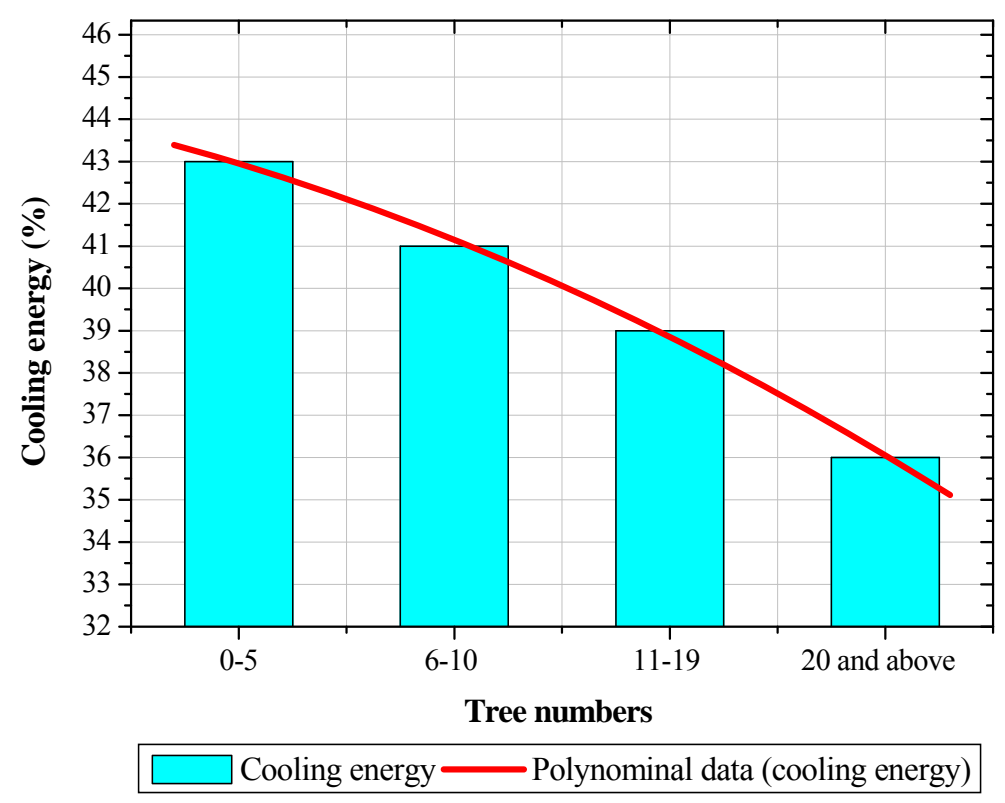

Figure 6.25. Tree numbers by size of group, compared with average cooling energy use

However, the majority of the houses used a moderate style of landscaping, and tended to have groups of between 6-10 trees per house (34\% of houses) or 11-19 trees per house (30\% of houses). These houses could receive a cooling energy saving of approximately $5 \%$ and $11 \%$ respectively, equal to $2-4 \%$ of the overall 
electricity costs, as shown in Figure 6.25. This would be equal to MYR68 (271kWh) and MYR133 (541kWh) per house per year respectively.

\subsubsection{Strategic landscape designed around the house}

Figures 6.26 and 6.27 illustrate a sample of a standard house in the study area. They show a house with a strategic landscape designed around it that will maximise shade and wind flow towards the surrounding garden and produce extensive evapotranspiration cooling.

The square/rectangular plan layout has the building's main façades orientated to face the road access to the building. Figure 6.26 shows the typical conditions where a north-south configuration with respect to the building and landscape design were applied. The amounts of wall and glazing were similar for each direction as listed in Tables 6.5-6.6. North and south facades are mainly only exposed to indirect solar radiation, which can minimise direct sunlight penetration to the openings, while east and west facades need extensive shading.

All of the building envelope elements must be shaded by vegetation as much as possible to reduce heat gain to the building. Most of the vegetation can cover walls and ground building foundations to keep the building surroundings and garden cool, but the tree height is not sufficient to cover roof surfaces. However, roofs in the study houses were properly insulated to reflect solar radiation and prevent it entering the buildings. Light coloured roofs and walls were widely used for the majority of houses in the study area, providing an albedo value $(0.22)$ sufficient to reflect sunlight from the building envelope. 


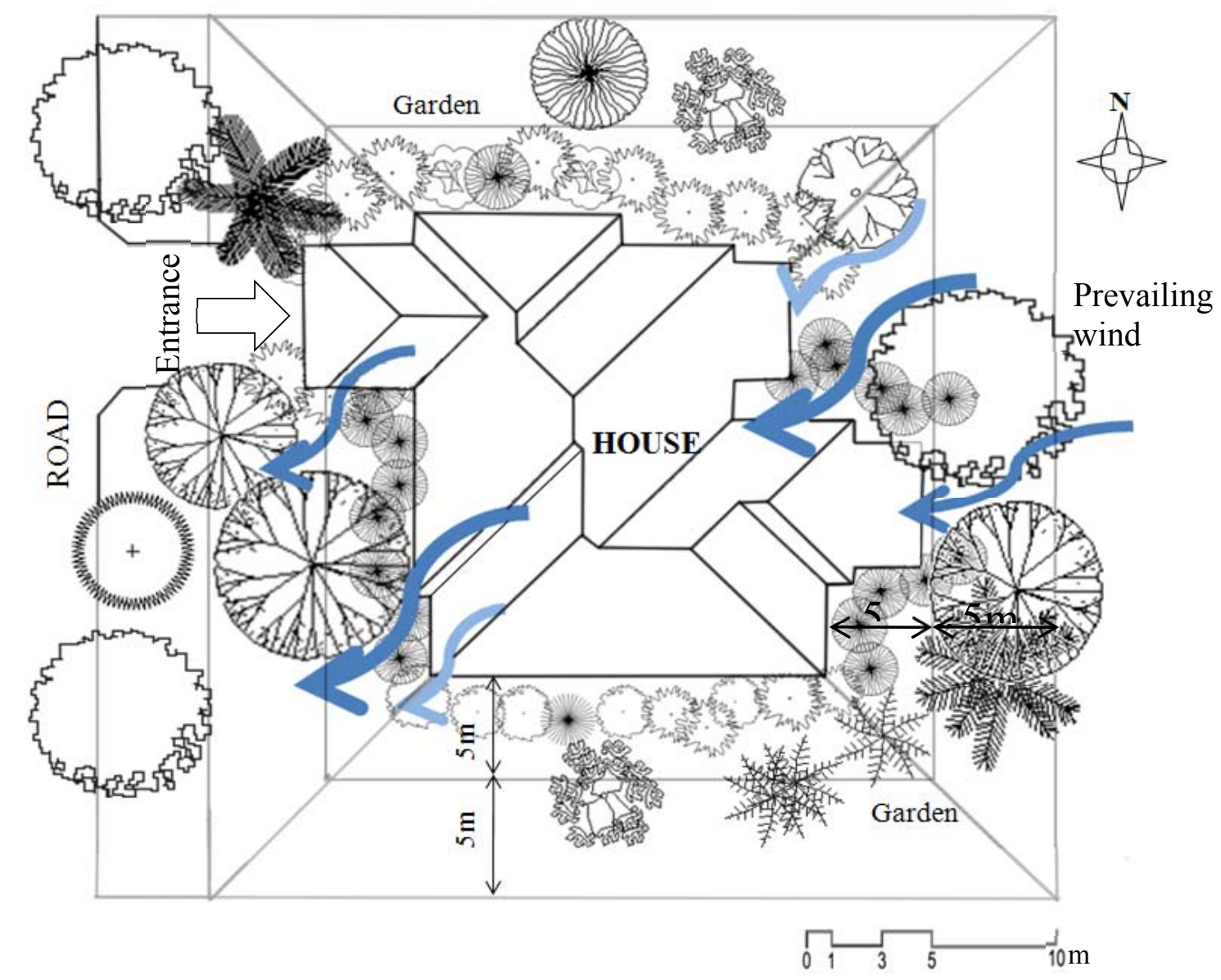

Figure 6.26. Landscape plan for strategically designed landscaping around the house that will maximise shade, wind flow towards the surrounding garden, and produce evapotranspiration cooling

Figure 6.26 shows the average number of trees in this study was 11 per house. Located in strategic locations, these can reduce cooling energy use by up to $11 \%$ equal to $4 \%$ of the overall electricity costs (MYR133/541kWh per house per year) (refer to subtopic 6.7.1 and Figure 6.25). In this study, the majority of shade trees were located on the east and west sides at the distances of 3-5 and 6-10m from the building, which can provide extensive shading to the building and surrounding garden as shown in Figure 6.22. The majority of trunk heights for shade trees were up to $1.5 \mathrm{~m}$ which can encourage and capture wind flow to the surrounding garden. Figure 6.27 shows how the arrangement of trees and shrubs influences the movement of wind for natural or cross ventilation around and through a building. 


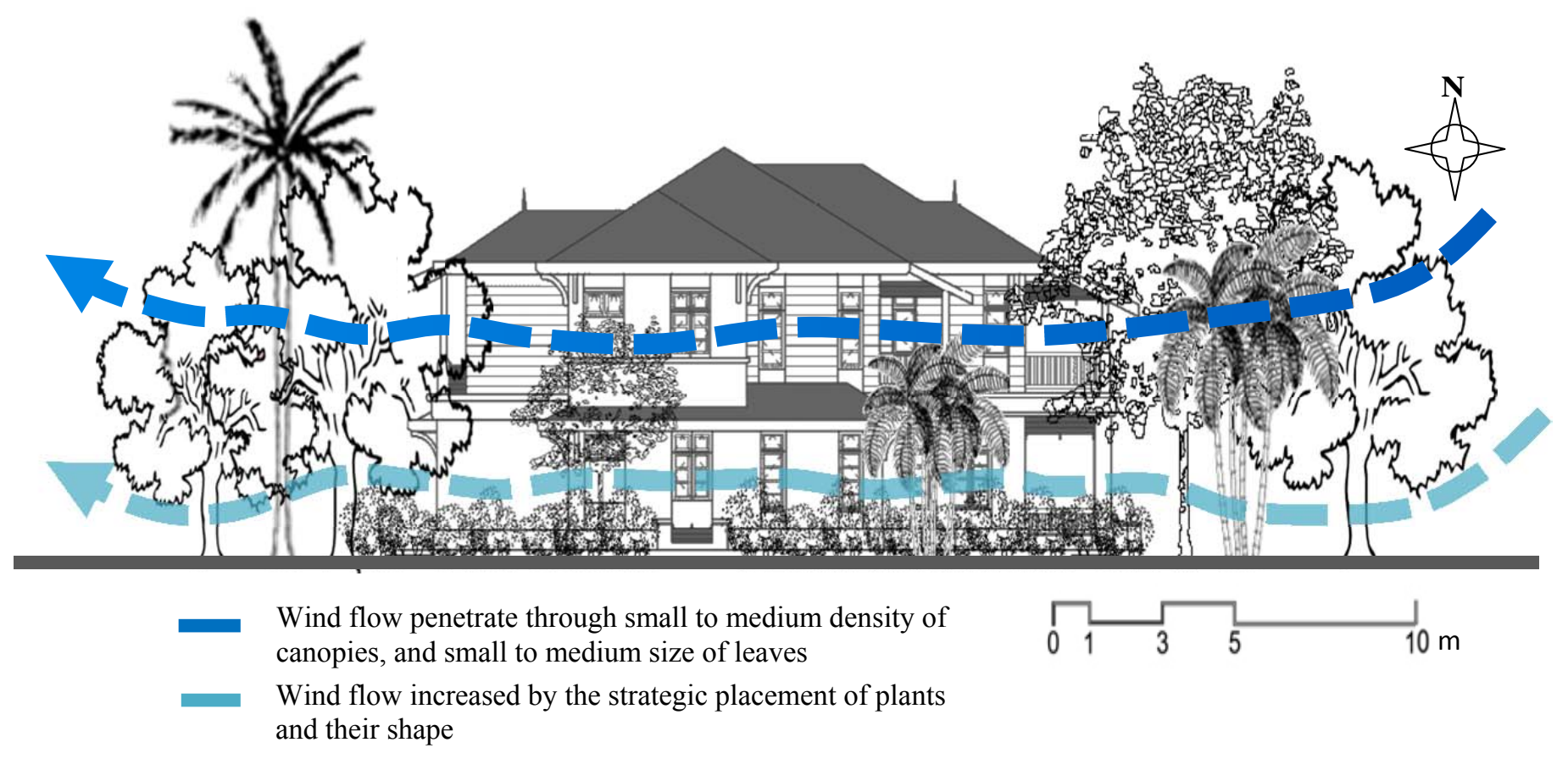

Figure 6.27. Section/elevation of the house with landscaping to enable wind flow to get through to the house and surrounding garden, and to provide shade in the morning and afternoon to the building envelope (roof and walls)

The majority of shrubs, vines, and groundcover were situated on the east and west sides providing shade from the direct sunlight on walls and glass windows. These were supported by fixed shading devices during the peak time of the day as can be seen in Figure $6.21 .1-1.5 \mathrm{~m}$ high shrubs, positioned close to walls and planted in groups can protect from direct and diffuse solar radiation for all directions during morning and afternoon and furthermore keep the interior spaces cool and comfortable. Figure 6.16 shows that turf effectively covered all earth surfaces completely around the garden to help evapotranspiration cooling. Hard landscaping such as a gazebo can help to provide a shaded area, while water elements provide evaporative cooling to the surrounding garden.

This study shows that even though the majority of the trees were in the small and small-moderate size categories, if they were more strategically placed to provide shade and encourage wind flows to the houses, then further cooling energy saving would be achieved. Evapotranspiration cooling from greater numbers of trees, shrubs and other small sized soft landscape elements around the house would also 
help to provide a cool, comfortable, and pleasant environment in the surrounding garden.

\subsection{Summary}

In the study areas, the houses applied similar styles of tropical architecture, and had similar methods of building construction, with the U-values for building materials having moderate levels of thermal resistance. Every house had a medium amount and average size of glazed openings, which covered an area of approximately $13 \%$ of the floor area. They also had well insulated roofs. The overall albedo value of the building envelopes was around 0.22 , which was sufficient to reflect solar radiation.

The majority of trees, shrubs, vines and groundcover surrounding the houses were situated in strategic locations on the east and west sides of the houses, where they received direct solar radiation. They also had a smaller number of plants located on the north and south sides, which could reduce diffuse solar radiation. Sufficient numbers and sizes of trees, which were supported by shrubs, vines and groundcover located in strategic positions, can increase the energy saving potential of a house, particularly during the peak load time of the day. These plants will help to cool the ambient air temperatures, and help decrease air-conditioning use.

During the rainy season, air-conditioning was used for a minimum to moderate period, and accounted for an average of $36.7 \%$ of total energy use. During the dry season, air-conditioning is often used for longer periods, with energy use for cooling estimated to increase to $48.2 \%$. This increment was based on the increased energy use during the dry season. This is because the living habits and life styles of the occupants change to adapt to the high temperatures.

In a tropical environment, landscaping is one of the effective tools for reducing the amount of energy used for air conditioning in a residential space. The data obtained on cooling energy in the study houses was compared with information about the soft landscape elements around the houses that had either a minimum or moderate amount of landscaping. The largest cooling energy saving was as much as $7 \%$ of 
overall electricity costs, equal to $20 \%$ of the average energy used for cooling. This energy saving was equal to MYR233 (948kWh) per house per year. Thus, the savings documented in this study confirm that landscaping is an effective energy saving technique that should be more widely used in the low density residential building developments. 


\subsection{CONCLUSION}

This study has presented several new findings about how to save cooling energy and lower temperatures in residential buildings in Malaysia. The aim of the study was to examine and quantify the relationship between the thermal performance of houses and their surrounding vegetation in a hot-humid tropical environment. The primary objective was to determine, for the tropical residence, the outside temperaturereducing and energy saving potential of vegetation. Two stages of research and data gathering were undertaken: examining planting patterns and structures; and quantifying and validating the results. However, comparison of the results of this study with other similar research in the tropics has not yet proved possible.

This study examined landscaping practices and the resulting energy benefits from shading, channelling wind and evapotranspiration by analysing their effects at several single-family houses. The main fieldwork and analysis was based on the climate's influence on buildings, and the strategies used to reduce the temperature around the exterior and in the interior of buildings to save energy. The results of this study have shown that the planting pattern of tropical landscape designs and styles have different effects on the environment surrounding houses. Those that used strategic landscape design achieved the lowest temperature in the exterior and interior of the house.

Low temperatures, high humidity and moderate wind combine to create a cool and comfortable living environment in hot-humid tropical climate. Trees, shrubs, vines and groundcover play an important role in directly creating this by cooling the environment through shading and channelling wind, while evapotranspiration cooling plays a similar role indirectly in the building-energy balance equation. The open spaces surrounding houses, and neighbourhood landscaping also play a role by contributing to evapotranspiration cooling around the houses and their neighbourhood areas. The physical characteristics of houses, and the specific living habits of their occupants are also important factors for determining the residential energy consumption for cooling. 
This conclusion will show the relationship between the thermal performance of houses and their surrounding vegetation in relation to the outside temperaturereducing and energy saving potential of landscaping. The results have been quantified and validated by examining the landscaping patterns and structures of tropical domestic buildings.

\subsection{Examine and quantify the relationship between surrounding vegetation, and the thermal performance of housing}

This study evaluated how strategic, well designed landscaping around domestic buildings can contribute to reducing the outdoor temperature, which cools the environment and building, and therefore reduces energy use. It also looked at the relationship between the thermal performance of houses and their surrounding vegetation, which can combine to cool the outdoor area and reduce indoor temperatures. Vegetation provides shade to a building and outdoor area, and produces evapotranspiration cooling, which reduces heat build-up. Vegetation also moderates wind flow to the house and provides natural ventilation to the building, which directly helps to produce cool and comfortable interior spaces.

The ambient air temperatures were measured at five houses that had different vegetation configurations in their gardens. Houses with the most strategic landscaping had lower ambient temperatures, high humidity levels and moderate wind flow, which helped to circulate air and produced a cool and comfortable environment for the residents.

The cooling energy saving results were taken from 50 houses that were constructed between 1970 and 2007. These houses had similar types of building construction, but were slightly different in their building envelope, colour and size, with a wide variety of vegetation configurations. The results showed that gardens that had vegetation in strategic locations and higher numbers were the most effective at producing cooling energy savings. 


\subsubsection{Determine the outside temperature reduction}

The results of this study have shown that by using the best combinations of highbranching trees and shrubs, vines, ground cover and turf to ensure shade and moderate wind flow to buildings the temperature could be reduced by as much as $4^{\circ} \mathrm{C}$ in outdoor garden spaces. This directly influenced the temperature in the indoor spaces, which were reduced by up to $3^{\circ} \mathrm{C}$. As well as the direct influence on temperature by shading and channelling wind to the garden and house areas, the temperature is also reduced indirectly by evapotranspiration. This is produced by all types of vegetation around the houses and their surrounding at a microclimate scale.

\subsubsection{Shading, channelling wind and evapotranspiration}

This study presents quantitative findings regarding the effectiveness of the surrounding landscaping for minimising heat gain. It assessed five single-family houses that had used different landscaping strategies in low density residential neighbourhoods in a tropical region. A comparison of the results and analysis of air temperature, relative humidity and wind speed in the outdoor and indoor spaces revealed the passive cooling potential of the surrounding landscaping. The results are grouped into the areas outlined below:

1. The strategic design of landscaping around houses requires the correct selection of the position, number, species and size of vegetation to provide the most reduction in temperature inside and outside of houses. The critical building envelope surfaces of the house to protect are the east and west surfaces, where the sun creates the highest temperature and heat gain to the building.

2. The proper and appropriate combination of trees, shrubs, vines, groundcover and turf to maximise shading, channelling wind and evapotranspiration, reduced the maximum air temperature by as much as $4^{\circ} \mathrm{C}$ during the peak time of the day.

3. Strategic placement of the right amount and size of shrubs, vines and groundcovers close to the walls can shade them and the foundation area of buildings. This also acted as a thermal insulation layer for the walls helping to maintain a cool temperature in this area and channelled wind into the interior 
spaces and other areas in the garden. This could contribute to cooling houses by as much as $3^{\circ} \mathrm{C}$.

4. Trees of a sufficient trunk height that had shrubbery underneath further reduced the ground and garden temperatures, and encouraged wind flow around the garden and through the houses.

5. Winds readily flow through these neighbourhoods. This was dependant on the flow path provided by individual houses to carry humidity and heat out of the house. The strategic placement of vegetation channelled wind to specific areas in and around the house. For garden areas with high relative humidity and low air temperature, moderate breezes are needed to minimise humidity close to the house.

6. Open walls around houses influenced the pattern of the interior temperatures, which tended to gradually increase throughout the day. This phenomenon was predicted because the heat was influenced directly by the surrounding exterior air temperature. It is important that a building's walls and foundation area are shaded to help maintain a cool and comfortable interior temperature during the day.

7. Environmental conditions in nearby neighbourhoods also influence the direction and quantity of wind speed, as well as the evapotranspiration rate in the surrounding garden. Based on the measurements taken at the mature landscape house, it was expected that measurements at the new landscape house would reach higher interior and exterior temperatures during the day than the ordinary landscape house due to its inefficient landscape design. However, the results showed that the air temperature, humidity and wind speed were about the same. This was perhaps because two of the houses were located next to the mature, green, open spaces, which boosted cooling by evapotranspiration.

8. If houses have strategic landscaping this can control interior temperatures better than houses with that have not been strategically landscaped. Landscaping helped to keep interior temperatures within the comfort range, and thus less air conditioning was used. However, during the night air-conditioning is still required to keep house temperatures in the comfort range. 
This study shows that the best way to create a favourable microclimate in a tropical residential landscape is by using appropriately sized and amounts of vegetation, and ensuring they are arranged and positioned in strategic locations and areas around the house. As well as creating a microclimate in the residential areas that is cooler, this also creates an area that is aesthetically pleasing. Strategic and well designed landscaping can also influence the climate at a regional and macroclimate scale.

\subsubsection{Energy saving potential of vegetation}

The quantifying and validation of the result for energy saving were from data of fifty houses that were constructed between 1970 and 2007, with similar building construction, slightly different building envelopes, colours and sizes with a wide variety of vegetation configurations. This combination of characteristics allowed for analysis of vegetation impacts on energy consumption. The factors that influenced the energy saving potential of landscaping were surrounding landscaping, building construction and human factors.

\subsubsection{Surrounding landscaping}

In general, the neighbourhoods surrounding houses used a conventional style of tropical landscape architecture. The houses in this study were surrounded by up to $10 \%$ of open space in Shah Alam and more than $38 \%$ of open space in Putrajaya. This was aimed at providing a balance to residential developments while maintaining the green and natural environment.

The tropical landscaping techniques were widely used in the study areas. Designs were dominated by lush, colourful, and constantly growing plants. Hard landscape structures were included to suit the landscape design style. On average, each singlefamily house in the study areas had a medium-sized garden, and was located in the middle of the land lot and surrounded by landscaping. The majority of the houses made moderate use of landscaping, where trees and shrubs were planted in medium amounts and sizes depending on whether they were there to look at or to cool the environment. 
Numerous tropical and subtropical plant species were planted in various zones around the gardens. Garden zones included the access road area, entrance of the house, main garden area, edible garden, and boundaries of the properties. Plants were used to connect each the different zones. Each type of vegetation and their combination with other plants produced various effects on the ambient air and thermal performance of the house.

In the gardens in the study area, trees were the most important component in the landscape design, while shrubs, vines, groundcover and turf were supporting components. They all worked together to provide a balanced and harmonious design. All of the plants moderated ground temperature, produced evapotranspiration cooling and provided shade to buildings and surrounding garden. Trees were scattered around all directions around the houses, with the majority, along with the shrubs, vines and groundcover, predominantly situated in strategic locations to the east and west. There were also significant amounts of plants in the north and south areas to protect from indirect or diffused solar radiation

Overall, houses in both Shah Alam and Putrajaya had moderate amounts of trees. Shah Alam's trees were generally mature, while those in Putrajaya were immature. Shah Alam was greener than Putrajaya, but Putrajaya had greater areas of open spaces in their neighbourhoods, which can create microclimates as the surrounding landscaping in these areas can influence the overall surrounding temperatures, RH and wind speed to the areas of whole houses.

\subsubsection{Building construction}

Fifty medium-sized single-family houses in the study areas were grouped in four ages of construction, covering four decades. The homes selected had similar construction types and materials. Each home was made of concrete, had plastered brick walls and a wooden roof covered with clay or concrete tiles which provide moderate thermal resistance for the houses. 
In the study areas, tropical architecture and design were used to moderate the hothumid climate. Most of the houses faced an access road on northwest-southeast or north-south sides, which reduced the impact of solar radiation and heat gain to the buildings, as these orientations faced indirect solar radiation, which minimised the heat gain to the buildings. Houses with square layouts were more influenced by their front facade, type of interior spaces, and the connectivity to the main garden areas. The link between indoor and outdoor areas encouraged wind flows, increased natural lighting, and improved ventilation of the house, as well as creating a pleasant view.

The thermal transfer properties of building envelope materials proved to be an important factor in the interaction between vegetation and the thermal performance of the house. The main building envelopes were walls. For the houses in the study area, the average area of brick walls was around $84 \mathrm{~m}^{2}$ at each side, with a moderate number of glazed openings that covered an average of $13.5 \mathrm{~m}^{2}$ per side, which equalled approximately $13 \%$ when compared to the floor area. Windows and doors were shaded by gables or hipped roofs of $0.75-3 \mathrm{~m}$ width, which protected the glass surfaces from direct and diffuse solar radiation at the peak time of the day.

Conductive heat gains through the roofs were an important factor. The average roof size in the study was $254.6 \mathrm{~m}^{2}$, in area and $2.9 \mathrm{~m}$ high, was insulated with aluminium foil, and was light in colour. The insulation layers were intended to reflect and reduce heat gain from the roof to produce cool and comfortable interior spaces.

The average ground floor area was $213.5 \mathrm{~m}^{2}$. The floors were made of reinforced concrete, and the beams and foundations were not directly exposed to solar radiation. The ground around the buildings was shaded, which helped to maintain low floor temperatures and provided a cool interior environment. Landscaping around the gardens provide shade to the walls and ground surrounding the house.

The colour of the external envelope surfaces had a tremendous effect on the impact of the solar radiation on the buildings. To minimise heat gain, the reflectivity value (albedo) for building envelopes should be sufficient to offset the heat gained through 
the roof and walls. In the study areas, light coloured walls and roof surfaces were present in all houses. The average albedo value of the building envelopes of the houses in the study areas was 0.22 . Walls were normally cream coloured, and roof tiles were light red. This value was sufficient to reflect solar radiation on the building envelopes.

The impact of strategically planted vegetation on energy use for household cooling has long been discussed. In tropical environments, good landscaping is a basic requirement for every house for many reasons. The main reason is to reduce the hot ambient temperature around houses and reduce the cooling needs inside the houses. The types of vegetation used, such as trees, shrubs or grasses, make a significant difference on the energy use. Every type of vegetation, if planted in the right place, has different effects on temperature, humidity and wind channelling around houses and in the interior space.

\subsubsection{Human factors}

Air-conditioning systems were used daily need to achieve a comfortable environment inside all of the houses in the study areas regardless of age. On average, the houses had five occupants. Forty percent of air-conditioning units were situated in common rooms, and $60 \%$ in the bedrooms. These units were used between four and five hours respectively, in any 24 hour period. The average temperature setting used in these rooms was different; $22^{\circ} \mathrm{C}$ for common rooms and $24^{\circ} \mathrm{C}$ for bedrooms. The airconditioning used $36.7 \%$ of the household spend on energy.

The study measured cooling energy use in the rainy season. During the dry season people's living habits changed to adapt to the high temperature and humidity. Based on an increase in air temperature and relative humidity of $2.5^{\circ} \mathrm{C}$ and $20 \%$ respectively, and measurement from the study, cooling energy was predicted to increase by $11.5 \%$. In fact in Shah Alam electricity use in domestic buildings (20082009) increased $15.4 \%$ between rainy and dry seasons. The cooling energy consumption in major tropical cities is expected to increase every year due to the 
urban heat island phenomenon. This will cause increased temperature as cities continue to grow.

\subsubsection{The effectiveness of using landscaping to reduce the cooling energy}

The effectiveness of using landscaping to reduce the energy consumed in buildings in a hot and humid tropical climate was calculated by comparing the energy consumed at the moderately landscaped houses. The basic tool for this measurement was looking at the amount of trees, which were also well supported by shrubs, vines and groundcover, in relation to cooling energy use. The cooling energy used gradually increased when there were fewer trees planted around the houses. This shows that landscaping significantly influences the amount of cooling energy used in houses.

The most effective landscaping had the majority of the trees, shrubs, vines and groundcover located on the east and west sides of the buildings, which provided effective shade to the buildings and their surrounding garden. Trees were planted close to building and as far out as $10 \mathrm{~m}$, which provided shade and cooling to the surrounding garden and building. Their average trunk heights were up to $2 \mathrm{~m}$, which would allow breeze to effectively flow to the house and garden. These were supported by shrubs, vines, groundcover and turf cover, which provided evaporative cooling. This resulted in the largest cooling energy saving being was as much as $7 \%$; equal to MYR233 (948kWh) per house per year.

\subsection{Significance of the results}

Energy conservation in Malaysia has become increasingly important. The energy consumption per capita is relatively low, but is expanding at a rapid rate in tandem with the economic development of the country (APEC, 2008). The thrust of Malaysia's energy policy is focused on the aspects of sustainability, using resources efficiently and existing more harmoniously with the environment through the mitigation of negative impacts on the environment. The results of this study were positive in relation to energy saving by creating a cooler and more comfortable environment. The cooler environment may help mitigate the urban heat island effect 
that has occurred in most of major cities around the world, including those in Malaysia.

\subsubsection{Modifying outdoor temperature and reduce urban heat island effect}

The influence of the urban heat island effect in Kuala Lumpur in Malaysia is well documented (Tso, 1996). Shah Alam and Putrajaya are located near to Kuala Lumpur; and share the capital's high air temperatures and year round humidity. Careful design and development of exterior spaces in these cities can reduce the adverse impact of high air temperatures and humidity.

This study found that effective landscaping was very good at reducing the urban heat island effect on homes in a hot-humid climate. The study focused on the impact of shade trees and different types of vegetation on the thermal performance of houses and outdoor air temperatures. Results showed that the outdoor air temperatures of houses with strategic landscaping were lower compared to the minimally-landscaped house. The main findings show that well designed landscaping around single-family houses could reduce temperature in outside houses by up to $4{ }^{\circ} \mathrm{C}$ and by $3{ }^{\circ} \mathrm{C}$ inside at peak times. Shading, evapotranspiration, and wind channelling were the root causes of this reduction. Use of strategic landscaping in all residential zone development will reduce the current urban heat island phenomenon in tropical cities, making living in the cities more pleasant.

While this study was conducted in the wet season, the results should be applicable throughout the year when cooling is required. This study confirms that landscaping should be used more to reduce energy use in low density residential building developments in Malaysia.

\subsubsection{Save energy and costs}

Use of electricity in domestic buildings contributed to almost $10 \%$ of electricity costs in Malaysia, and $30 \%$ of that was spent on cooling energy (Saidur, et al., 2009). The number of medium single-family houses in Malaysia was about 20\%. In this study, 
the average of cooling energy use in a medium-sized single-family house was approximately $36.7 \%$.

This amount of energy could be saved by using strategically designed landscaping around houses. The results revealed that small trees in groups of between five and 20 , planted together with shrubs, vines, groundcover and turf saved as much as $7 \%$ of overall electricity costs, including $20 \%$ of cooling energy. This energy saving was equal to MYR233 $(948 \mathrm{kWh})$ per house per year. An average of 11 trees was planted around the houses in the study area, which resulted in average savings of $4 \%$ of their power consumption per year. Every additional five small trees would reduce energy use by another $2 \%$.

\subsection{Practical application of the results}

If strategically designed landscaping is applied properly around houses it can reduce surrounding air temperatures, and therefore save huge amounts of energy and cost. Appendices 7.1-7.2 show an example of a landscape plan that will promote shade to the building envelope, especially on the east and west sides of the building. This plan will also maximize evapotranspiration cooling and channel wind flow to the house and surrounding garden in a hot and humid tropical climate.

The results from this study to help achieve this has been summarised into the following list of practical applications for the average homeowner:

1. Trees provide extensive shade to gardens and houses, and are therefore the best means of reducing the amount of heating in the building envelope and ambient air. The combination of evergreen tropical native trees, shrubs, vines, groundcover and turf provide a balanced and harmonious residential landscape design.

2. Trees along the access roads of the houses in the study areas numbered on average around two per house. They provided shade to the walkways along the main road as well as the houses. To help look after these trees, which play a role in reducing temperatures in a neighbourhood, local authorities should ensure 
they are well maintained and grow well, and any dead trees should be replaced immediately.

3. The height of shade trees should be proportionate to the height of the houses. They should provide shade to the roof, walls and garden surfaces. Trees should also be within $3-5 \mathrm{~m}$ of the building to provide shade for the roof. Shade trees located 6-10m away will also provide shade to the walls and the upper levels of the house during the morning and late afternoon. The minimum trunk height to channel wind and allow it to flow through the garden to the building surfaces was $1.5 \mathrm{~m}$.

4. Shrubs should be located close to the walls and garden areas of houses. For shrubs close to the walls, the height of shrubs should match the size of the fixedwindow shading devices. These shading devices could provide shade for windows between 11.00-15.00 hours. Outside of these hours, shade to windows should be done by shrubs. Thus the efficient height of shrubs is $1-1.25 \mathrm{~m}$ if planted at the east and west of the house.

5. House orientation and internal configuration is important. The best design is a north-south orientation for openings to receive only indirect solar radiation. Openings on the east and west sides should be minimized to reduce the effect of direct solar radiation. Shade use should be focussed on the east and west of the house. However, plant-made shade reduced solar radiation regardless of position.

6. The comparison of the three different age groups of construction revealed that the houses that were 10 years old had trees that were an ideal age to be of sufficient height and canopy size to shade the roof, while shrubs, vines and groundcover only need to be three years old to reach their optimal size to provide protection to the house.

7. All plant types should be in a fertile and lush condition. Plants with a medium amount of small to medium sized leaves produced maximum shade and evapotranspiration cooling, and channelled the prevailing wind properly. All plants should be planted in groups to survive well in bad weather conditions and make maintenance easier. 
8. Light colours with high albedo values should be used on the walls and roof. Lighter colours reflect more solar radiation, minimising the house's heat gain, which saves on energy used for cooling.

\subsection{Future work}

This study produced models of landscaping design and building configuration of single-family houses with two main functions: to save energy, and reduce hot temperature in a tropical environment. The research collected data on buildings, landscaping and their surrounding environment. However, there were some limitations to the research.

Only a moderate number (50) of participants were interviewed. If more occupants of single-family houses, from more housing estates had participated in the survey, the increased data about different building constructions, landscaping techniques and the living habits of the occupants would improve the results. Future studies will source more data, but still focus on modest sized houses with diverse landscaping and occupants, and will use similar variables for measurement and observation as was done in the case studies. The benefit of this would be that the effect of a larger number and wider variety of landscaping techniques and the user lifestyles on energy saving may be obtained

In this study, the measurements taken from the individual houses were done during the rainy season because this was when the vegetation would be in the best physical condition to provide maximum cooling to buildings and their environments. This decision meant all vegetation was in a lush, fertile condition due to being watered by rainfall almost every day, and the ambient temperature was lower than in the dry season. However, during the dry season the physical condition of vegetation is slightly different as plants are less fertile and sparse because of less rainfall and higher ambient temperatures; there is a $2-5^{\circ} \mathrm{C}$ difference in ambient temperatures between the two seasons. Usually the homeowners spend more on watering their plants during the dry season. To reflect these differences, surveys, interviews and fieldwork should also be made during the dry season. The whole research process 
across the dry and wet seasons will take around a year. From that time commitment, the exact amount of energy use in high temperature and humidity levels during the dry season will be revealed, and a landscaping design that reduces temperature across the two seasons will be recommended.

Data for this study was recorded during the day time; not at night. This was because residents secure their property well at night, and prefer to not have their privacy disturbed during this time. However, most of the nights during the rainy season have light rainfall for few hours, so information about these times should be gathered.

As the majority of occupants use active ventilation systems in indoor spaces regularly the natural air temperature and humidity was set automatically. In future studies, houses without air-conditioning system or semi-active ventilation system will be chosen to record the actual indoor and outdoor temperature, humidity and wind speed over 24 hours. Elto power meters will be used to record and to calculate the energy use for all air-conditioning units in every house in 24 hours.

The equipment to record climate data must be small and able to record it automatically and accurately without disturbing the occupants. The outdoor equipment must be lockable to existing structures within the garden so it does not get stolen. The evapotranspiration measurement will be recoded automatically around the house using Lysimeters. This equipment will be put on the earth surfaces around the garden. It calculates the amount of water in the air produced by evaporation from earth surfaces, including from water, plant and soil transpiration. The unit of evapotranspiration is in litres. $\mathrm{m}^{-2}$. Results will be validated against evaporation readings from Department of Irrigation and Drainage, Ministry of Natural Resource and Environment, Malaysia.

This study recorded the climate data from the four azimuths of the houses and their gardens. The data was calculated based on the averages of the surrounding landscape. In the future research, outdoor climate data must be measured for each vegetation type every 30 minutes. This will allow measurement of the effect of every type of vegetation on air temperature and humidity level. The specific 
locations and types of vegetation will be measured include under shade trees, shrubs along the walls and garden, vines climbing along the walls and under pergolas and other structures, on groundcovers and lawn areas. Different types and groups of vegetation may produce different shade, evapotranspiration cooling and wind movement. The species of vegetation, overall size, and leaf size together with its location will provide different climate data throughout the day. The classification of hard landscape includes areas under gazebos, pergolas, around water elements such as swimming pools, fish pools and artificial waterfalls and fountains, and pavement areas. Likewise, each area will provide different effects on air temperature, humidity and wind flow which will influence the building's thermal performance.

Data about climatic factors around the building envelope and the garden areas will be recorded by infra-red thermograph camera during early morning, mid-noon, afternoon and towards evening. Thermographs represent heat through colour, which will clearly show changes in the temperature of the building envelop and garden. Readings of air temperature, humidity and wind flow will also be produced by computer simulation to show the effect of vegetation on building thermal performance as well as to calculate the cooling energy use.

Housing estates in the city centre contribute to the urban heat island phenomenon. The heat gain in individual houses spreads into whole neighbourhoods. Each neighbourhood joins forces with those adjacent to it to become the city's macroclimate. Climate data for the whole neighbourhood will be produced by Geographic Information System (GIS). Satellite images of the Urban Environmental Climate Map will be obtained from Remote Sensing Data Services. This map will provide some information on urban heat island mitigation. In the specific neighbourhood area, an Urban Climate Analysis Map is needed to manage many layers of data. According to Tanaka \& Moriyama (2011) the Urban Climate Analysis Map represents the existing climate in an area. Map, terrain, klimatope, wind and temperature are overlaid. Terrain is the relief made from the elevation data, while klimatope provides the view of climate on the landscape unit based on land cover. 
From the Urban Climate Analysis Map the urban heat island effect will be identified. Three options can be used to alter the urban heat island effect, namely land cover alteration, anthropogenic heat reduction and wind utilisation. The effect of each of these depends on the geographical features (Tanaka \& Moriyama, 2011). The Urban Climate Analysis Map shows how unwanted heat accumulation, now and in the future, can be prevented or reduced ("Future Cities," 2010). Recommendation maps will also be produced. These maps will show each option for countermeasures to the urban heat island. They will be extracted through the study by the expertise of climatologists, planners and landscape architects. Examples of suggestions include a green area plan for ideal wind flow, green belts along roads parallel to the wind flow, and tree-planting in the recommendation zone map. These maps are an effective aid to decision making on how to mitigate the urban heat island effect for urban planners. Other benefits include improving how the area looks, the increase in property value, and the improved absorption of $\mathrm{CO}_{2}$. 


\section{BIBLIOGRAPHY}

Akbari, H., Davis, S., Dorsano, S., Huang, J., \& Winnet, S. (1992). Cooling our Communities, A Guidebook on Tree Planting and Light-Colored Surfacing Washington: Lawrence Berkeley Laboratory

Akbari, H., Taha, H., Huang, J., \& Rosenfeld, A. (1986). Undoing uncomfortable summer heat islands can save gigawatts of peak power. Paper presented at the The Panel on Small Building Technologies, Washington, DC.

Alread, J., \& Leslie, T. (2007). Design-tech: building science for architects: Architectural Press.

APEC. (2008). Statement on Notable Energy Achievements (Malaysia) Iquitos, Peru

ASCE. (1996). Hydrology Handbook (Second ed.). USA American Society of Civil Engineers

ASHRAE. (2003). Greenguide: The Design, Construction, and Operation of Sustainable Buildings. American Society of Heating, Refrigerating and AirConditioning Engineers. New York ASHRAE Press.

ASHRAE. (2005). Fundamentals (SI ed.). Atlanta American Society of Heating, refrigerating and Air-Conditioning Engineers. Inc. .

Aziz, N. A., \& Hadi, A. S. (2007). Linking urban form to a liveable city. Malaysian Journal of Environmental Management, 8, 87-107.

Bakar, J. A. (2002). A Design Guide for Public Parks in Malaysia Skudai Penerbit Universiti Teknologi Malaysia

Ball, J. (2011). Prevent Sod Webworm Next Year. Retrieved from http://yardener.com/YardenersPlantProblemSolver/DealingWithPestInsects/P estInsectsInTheLawn/SodWebworms/PreventSodWebwormNextYear

Bastide, A., Lauret, p., Garde, F., \& Boyer, H. (2006). Building energy efficiency and thermal comfort in tropical climates Presentation of a numeral approach for predicting the percentage of well-ventilated living spaces in buildings using natural ventilation. Energy and buildings, 38, 1093-1103.

Bernatzky, A. (1982). The contribution of tress and green spaces to a town climate. Energy and Buildings, 5(1), 1-10.

Bobenhausen, W. (1994). Simplified design of HVAC systems. New York WileyIEEE.

Bomberg, M. T., \& Brown, W. C. (1993). Building Envelope and Environmental Control: Part 1-Heat, Air and Moisture Interactions. Construction Canada, 35, 15-18. 
Brandies, M. M. (2004). Landscaping With Tropical Plants. USA: Sunset Publishing Corporation

Brown, L. R. (2008, July). How We Can Decrease the Projected Global Energy Demand for 2020. Mother Earth News. Retrieved from http://www.motherearthnews.com/Renewable-Energy/Decrease-GlobalEnergy-Demand-Lester-Brown.aspx

Brown, R. D., \& Gillespie, T. J. (1995). Microclimatic Landscape Design Creating Thermal Comfort and Energy Efficiency. New York: John Wiley \& Sons, Inc.

Busato, L. (2003). Passive cooling and energy efficient strategies for the design of a hotel on the Sputhern coast of Pernambuco, Brazil London Metropolitan University.

Casper, J. K. (2007). Plants: Life from the Earth. New York Infobase Publishing

CCRR. (2012). Crystal Creek Rainforest Retreat Retrieved from http://www.ccrr.com.au/environment/climate.html

Chan, M. (2008). Thermal Comfort Levels in a Room with Solar Radiation. Indoor and Built Environment, 17(6), 516-524.

Darling, D. (2011). The Encyclopedia of Alternative Energy and Sustainable Living Retrieved 31 March 2011, from http://www.daviddarling.info/encyclopedia/U/AE_U-value.html

Dawn, M. (2011). List of Tropical Plants. Garden guide .com Retrieved 22 May 2011, from http://webcache.googleusercontent.com/

Diekmann, M. (2007). Climate: Fundamentals, History and Projections. Allianz Foundation for Sustainability Retrieved 30 April 2007, from http://knowledge.allianz.com/nopi_downloads/downloads/Allianz_Climate\% 20Change Brochure.pdf

DOE. (1993). Tomorrow's Energy Today for Cities and Counties: Cooling Our Cities U.S. Department of Energy, 1-6.

DOE. (1994). Cooling Your Home Naturally. Energy Efficiency and Renewable Energy Clearinghouse Retrieved 15 September 2009, from http://www.builtonline.com/articles.cfm?P ID $=353$

DOE. (1995). Landscaping for Energy Efficiency. Energy Efficiency and Renewable Energy, 10095(046), 1-8.

DOE. (2008). Building Envelope: U.S. Department of Energy Office of Energy Efficiency and Renewable Energy 
DOE. (2009). The Encyclopedia of Alternative Energy and Sustainable Living Retrieved from http://www.daviddarling.info/encyclopedia/U/AE Uvalue.html

EJCR. (2012). House-Energy Home Overhangs and shade Retrieved from http://www.house-energy.com/Landscape/Overhangs.htm

Evans, D., \& Gruba, P. (2002). How to Write a Better Thesis. Victoria Melbourne University Press

Fanger, P. O. (1970). Thermal Comfort Analysis and Applications in Environmental Engineering. Denmark: McGraw-Hill Book Company.

Fanger, P. O. (1973). Assessment of man's thermal comfort in practice. British Journal of Industrial Medicine, 30, 313-324.

Faulkner, D. (2012). Contemporary Landscaping: The Old Is New. Retrieved from http://EzineArticles.com/?expert=David Faulkner

Foster, R. S. (1994). Landscaping That Saves Energy and Dollars (Revised ed.). USA: First Globe Pequot Edition

Furuta, T. (1978). Properly Placed Plants Can Reduce Energy Use (pp. 3-21). Acadia: Cox Publishing Co.

Future Cities. (2010). Urban climate reccommendation map for the city of Arnhem Retrieved 14 March 2011, from http://www.future-cities.eu/news/newsdetail.html

GAISMA. (2009). Retrieved 5 May, 2009 from http://www.gaisma.com/en/location/kuala-lumpur.html

Givoni, B. (1969). Man, Climate and Architecture England Elsevier Publishing Company Ltd.

Givoni, B. (1976). Man, Climate and Architecture England Elsevier Publishing Company Ltd.

Givoni, B. (1991). Impact of planted areas on urban environmental quality: A review. Atmospheric Environment - Part B Urban Atmosphere, 25(3), 289299.

Givoni, B. (1994). Passive and low energy cooling of buildings New York Van Nostrand Reinhold.

Ha, T. (2009). Landscape Design Sustainable Energy Info i Retrieved 21 August 2009, from http://www.sustainability.vic.gov.au/resources/documents/Landscape design. pdf 
Hackett, B. (1979). Planting Design London E. \& F.N. Spon Limited

Haque, M. T., Tai, L., \& Ham, D. (2000). Landscape Design for energy Efficiency South Carolina: Clemson Extension

Hariri, W. S. W. (2010). Summary of sales by tariff on monthly basis for Shah Alam. : Electric Utility Company (National Energy Limited-TNB)

Hartweg, L. (2007). The Passive Solar Exterior Envelope. Passive Solar Energy Info Retrieved 28 September 2009, from http://www.passivesolarenergy.info/PassiveSolarExteriorEnvelope.html\#S2

Heisler, G. M. (1986). Energy Savings with Trees Journal of Arboriculture, 12(5), 113-125.

Hoyano, A. (1988). Climatological Uses of Plants for Solar Control and the Effects on the Thermal Environment of the Building. Energy and Buildings 11, 181199.

Huang, Y. J., Akbari, H., Taha, H., \& Rosenfeld, A. H. (1987). The potential of vegetation in reducing summer cooling loads in residential building. [Journal article ]. Climate and Applied Meteorology, 26, 1103-1116.

Idris, R. (2008, 14 September). Sentuhan Rasa Bali lebih damai. Berita Harian Retrieved from http://rimbun.weblog.ihsan.com

Kirsch, K. (2007). Kirsch Landscape \& Design. Retrieved from http://www.kirschlandscape.com/images/virtusite/Landscape1.jpg

Koch-Nielsen, H. (2002). Stay Cool a Design Guide for the Built environment in Hot climates. London James \& James (Science Publishers) Ltd

Koenigsberger, O. H., Ingersoll, T. G., Mayhew, A., \& Szokolay, S. V. (1980). Manual of Tropical Housing and Building (Fourth ed.). New York: Longman Inc.

Krigger, J., \& Dorsi, C. (2004). Residential Energy: cost savings and comfort for existing buildings (Fourth ed.). Montana: Saturn Resource Management

Kukreja, C. P. (1978). Tropical Architecture. New Delhi: Tata McGraw-Hill Publising Co. Ltd.

Laumer, J. (2008). Science \& Technology (alternative energy). Retrieved 18 August 2009, from http://www.treehugger.com/files/2008/07/energy-saving-tipshade-air-conditioner_.php

LCC. (2011). Guideline Distances from Development to Trees. Leeds: Leeds City Council 
Lechner, N. (2000). Heating, Cooling, Lighting Design Methods for Architects (Second ed.). New York John Wiley \& Sons, Inc.

Lindinger, K. (2007). What is Global Warming? The Allianz CSR Magazine Retrieved 18 August 2009, from http://knowledge.allianz.com/en/globalissues/climate change/global warming basics/global warming definition.html

Mahbar, S. F. S. (2011). The intensity of surface solar radiation in Putrajaya. Kuala Lumpur: Climatological \& Hydrological Section, Malaysian Meteorological Department.

Mahlia, T. M. I., Masjuki, H. H., Choudhury, I. A., \& Ghazali, N. N. N. (2002). Economical and environmental impact of room air conditioners energy labels in Malaysia. Energy Conversion and Management, 43(18), 2509-2520.

Malaysia. (1993). Gambar Peta Malaysia. Retrieved 5 March, 2011, from http://gambar-peta.blogspot.com/2010/09/gambar-peta-malaysia.html

Malaysia. (2000). Laporan kiraan permulaan banci penduduk dan perumahan 2000. Kuala Lumpur: Department of Statistics Malaysia.

Malaysia. (2007). Malaysian household monthly income distribution. Retrieved 28 September 2009, from http://www.depstc.org/econofc.html

Malek, J. A. (2011, July 14-16). Informative Global Community Development Index of Informative Smart City. Paper presented at the 8th WSEAS International Conference on EDUCATION and EDUCATIONAL TECHNOLOGY, Corfu Island, Greece.

Mallick, F. H. (1996). Thermal comfort and building design in the tropical climates. Energy and Buildings, 23(3), 161-167.

McClenon, C. (1983). Landscape Planning for Energy Conservation New York Van Nostrand Reinhold Company Inc. .

McDowall, R. (2007). Fundamental of HVAC Systems (First ed.). Oxford, UK: Elsevier.

McGinn, C. (1982). Microclimate and energy use in suburban tree canopies. University of California, Davis.

McPherson, E. G., Herrington, L. P., \& Heisler, G. M. (1988). Impacts of vegetation on residential heating and cooling [Journal article ]. Energy and Buildings, $12,41-51$.

McPherson, G., Herrington, L. P., \& Heisler, G. M. (1988). Impacts of vegetation on residential heating and cooling. Energy and Buildings, 12(1), 41-51. 
McPherson, G., \& Simpson, J. R. (1995). Shade Trees as a Demand-Side Resource. Several utilities have embraced trees as a solar-powered demand-side management resource. Home Energy

Meier, A. K. (1990). Strategic landscaping and air-conditioning savings: A literature review. Energy and Buildings, 15(3-4), 479-486.

Micheal, G. (2001). Tropical Rainforest Retrieved 18 August 2009, from http://www.blueplanetbiomes.org/rainforest.htm

Michon, G., Foresta, H. d., Levang, P., \& Verdeaux, F. (2007). Domestic Forests: A New Paradigm for Integrating Local Communities' Forestry into Tropical Forest Science Ecology and Society, 12(2), 1-24.

Mills, J. D. (2003). SPSS Textbooks: A Review for Teachers. Statistics Education Research Journal 2(2), 59-70.

Misni, A., \& Allan, P. (2010). Sustainable Residential Building Issues in Urban Heat Islands - The Potential of Albedo and Vegetation Paper presented at the Sustainable Building Conference (SB10) New Zealand Retrieved from http://www.cmsl.co.nz/assets/sm/5928/61/2.PN003Misni.pdf

MMD. (2010). General climate of Malaysia. Retrieved 13 July 2010, from http://www.met.gov.my/index.php?option=com_content\&task=view\&id=75 $\underline{\text { Itemid }=1089}$

Moffat, A. S., \& Schiler, M. (1981). Landscape Design That Saves Energy. New York: William Morrow and Company, Inc. .

Montgomery, D. A. (1987). Landscaping as a passive solar strategy Passive Solar journal, 4(1), 78-108.

Morgan, R., McLeod, M., Mayfield, J., Richmond-Powers, M., Cohee, B., Gros, L., et al. (2000). Supplement to the Green Building Program. Austin, Texas Green Building Program.

Moss, K. J. (2007). Heat and Mass Transfer in Buildings (Second Edition ed.). New York Taylor \& Francis

Nadel, B. A. (2006). 21st Century Building Envelope Systems: Merging Innovation with Technology, Sustainability, and Function. Architectural Record, Continuing Education Centre, 1-10.

Nasution, H., \& Hassan, M. N. W. (2009). Energy Efficiency at Usage of Air Conditioning Systems In the Effort of Electrics Energy Saving Consumption. Retrieved 27 September 2009, from http://www.he4si.com/Artikel/Abstract_ROVISP05.PDF 
Nervegna, L. (2003). Passive Solar Design Energy Efficiency Through Passive Solar Design. Cement \& Concrete Association of Australia Retrieved 28 September 2009, from www.concrete.net.au

Okba, E. M. (2005). Building envelope design as a passive cooling technique. Paper presented at the International Conference "Passive and Low Energy Cooling for the Built Environment", Santorini, Greece.

Oke, T. R. (1978). Boundary Layer Climates London Methuen \& Co. Ltd.

Oke, T. R. (1988). Boundary Layer Climates. London Routledge Taylor \& Francis Group

Olgyay, V. (1963). Design with Climate Bioclimatic Approach to Architectural Regionalism New Jersey Princeton University Press

Olgyay, V., \& Olgyay, A. (1976). Solar Control and Shading Devices. Connecticut Meriden Gravure Company

Oral, G. K., Yener, A. K., \& Bayazit, N. T. (2003). Building Envelope Design with the Objective to Ensure Thermal, Visual and Acoustic Comfort Conditions. Building and Environment, 39, 281-287.

Oughton, D. R., \& Hodkinson, S. (2002). Faber and Kell's Heating and Airconditioning of Buildings (Ninth ed.). Oxford Butterworth Heinemann

Parker, J. H. (1981). Uses of Landscaping for Energy Conservation Florida: Florida State University System

Paul, G., \& Dieter, A. (1993). Climate Responsive Building Swiss: SKAT.

Penman, H. L. (1948). Natural Evaporation from Open Water, Bare Soil and Grass. Proceeding the royal of society A 193(1032), 120-145.

Pidwirny, M. (2008). Introduction to the Atmosphere, Climate Classification and Climatic Regions of the World. PhysicalGeography, Fundamentals ebook Retrieved 28, September 2009, from http://www.physicalgeography.net/fundamentals/7v.html

Pinet, P. R. (1998). Invitation to Oceanography Massachusetts: Jones and Bartlett Publishers

PJH. (2001). Progress report for residential development at Sub-Precinct 14-9, Federal Territory Putrajaya, Putrajaya.

Prowler, D., \& Prowler, D. (2008). Sun Control and Shading Devices. Whole Building Design Guide Retrieved 28 September 2009, from http://www.wbdg.org/resources/suncontrol.php 
Qiu, K., \& Haghighat, F. (2005, August 15-18, 2005). Assesment of impact of building envelope porosity on energy Paper presented at the Building Simulation 2005, Ninth International IBPSA Conference, Montréal, Canada.

Ross, L. (2011). Homelife. Tropical garden design, (3 August ), 1. Retrieved from http://www.homelife.com.au/garden/outdoor+living/tropical+garden + design, $\underline{4860}$

Ryan, C. (2012). Chiang Mai Lifestyle Retrieved 21 February, from http://www.chiangmailifestyle.com/lodge_picture/HRa_1208608796.jpg

Saaroni, H., Bitan, A., Dor, E. B., \& Feller, N. (2004). The mixed results concerning the [']oasis effect' in a rural settlement in the Negev Desert, Israel. Journal of Arid Environments, 58(2), 235-248.

Saidur, R., Hasanuzzaman, M., Hasan, M. M., \& Masjuki, H. H. (2009). Overall Thermal Transfer Value of residential Building in Malaysia. Journal of Applied Sciences, 1-7.

Salmon, C. (1999). Architectural Design for Tropical Regions New York John Wileys \& Sons, Inc.

Samad, A. M., Zain, M. A. M., Maarof, I., Hashim, K. A., \& Adnan, R. (2011, 4-6 March). Utilization of Artificial Neural Network and GIS for property market valuation. Paper presented at the 2011 IEEE 7th International Colloquium on Signal Processing and its Applications, Penang, Malaysia.

Santamouris, M. (2001a). On the built environment - the urban influence In M. Santamouris (Ed.), Energy and Climate in the Urban Built Environment (pp. 3-18). London James \& James (Sciencs Publisher) Ltd.

Santamouris, M. (2001b). The role of green spaces. In M. Santamouris (Ed.), Energy and Climate in the Urban Built Environment (pp. 145-159). London James \& James (Science Publishers) Ltd.

Santamouris, M., P.Wouters, Loncour, X., Akander, J., GudniJohannesson, Alvarez, S., et al. (2005). Energy Performance of Residential Buildings. London James \& James/Earthscan.

Simpson, J. R. (2002). Improved estimates of tree-shade effects on residential energy use. Energy and Buildings 34, 1067-1076.

Simpson, J. R. (2002). Improved estimates of tree-shade effects on residential energy use. Energy and Buildings, 34(10), 1067-1076.

Simpson, J. R., \& McPherson, G. (1996). Potential of tree shade for reducing residential energy use in California Journal of Arboriculture 22(1), 10-18.

Smith, M., Jarvis, P. G., \& Odongo, J. C. W. (1997). Energy budgets of windbreak canopies in the Sahel. Agricultural and Forest Meteorology, 86(1-2), 33-49. 
Sparrow, J., \& Hanly, G. (2002). Subtropical Plants for New Zealand Gardens. Auckland Random House New Zealand

Starbuck, C. J. (2007). Landscape Plantings for Energy Savings. University of Missouri Extension Retrieved 18 August 2009, from http://extension.missouri.edu/publications/DisplayPub.aspx?P=G6910\#Sun

Szokolay, S. V. (2004). Introduction to architectural science the basis if sustainable design Oxford Architectural Press

Taha, H. (1997). Urban climates and heat islands: albedo, evapotranspiration and anthropogenic heat. Energy and Building, 25, 99-103.

Taha, H., Akbari, H., Rosenfeld, A., \& Huang, J. (1988). Residential Cooling Loads and the Urban Heat Island - the effects of Albedo, Building and Environment (Vol. 23, pp. 271-283).

Taiz, L., \& Zeiger, E. (2006). Plant Physiology (Fourth Edition ed.). Massachusetts: Sinauer Associates, Inc

Talarosha, B. (2002). Menciptakan kenyamanan thermal dalam bangunan Jurnal Sistem Teknik Industri 6(3), 148-158.

Tanaka, T., \& Moriyama, M. (2011). Application of GIS to make "Urban Environmental climate Map" for Urban Planning.

TNB. (2010). Summary of sales by tariff on monthly basis Shah Alam Electric Utility Company (National Energy Limited - TNB)

Tso, C. P. (1994). The Impact of Urban Development on the Thermal Environment of Singapore. Paper presented at the Report of the Technical Conference on Tropical Urban Climates, Dhaka.

Tso, C. P. (1996). A survey of urban heat island studies in two tropical cities. Atmospheric Environment, 30(3), 507-519.

UBBL. (2006). Uniform Building By-Laws, Act 133 (14 ed.). Kuala Lumpur MDC Publishers Sdn Bhd

VanDerZanden, A. M., \& Rodie, S. N. (2008). Landscape Design Theory and Application (Vol. 424). Canada Thomson Delmar Learning

Wijaya, M. (2000). Tropical Garden Design USA: Periplus Editions.

Wong, N. H., \& Yu, C. (2005). Study of green areas and urban heat island in a tropical city Habitat International 29, 547-558.

Yin, R. K. (2003). Applications of Case Study Research (Second ed.). London SAGE Publications 
Zakaria, N. Z., Mokhtar, N., \& Mohamad, R. (2008). Skylight roof for energy savings in residential buildings in the tropical climate of Malaysia. Paper presented at the ANZAScA'08 Innovation, Inspiration and Instruction: New Knowledge in the Architectural Sciences. Retrieved from www.sb08.org 


\section{APPENDICES}

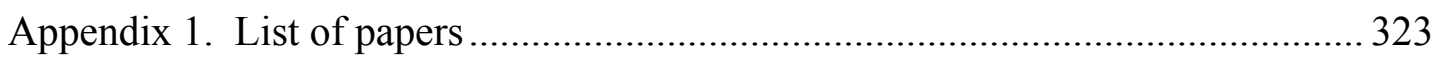

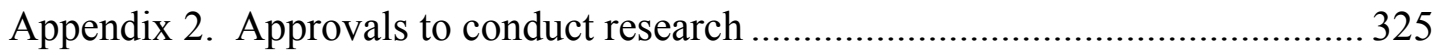

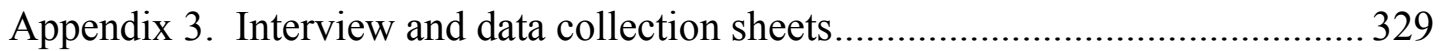

Appendix 4. Landscape elements around mature, ordinary and new

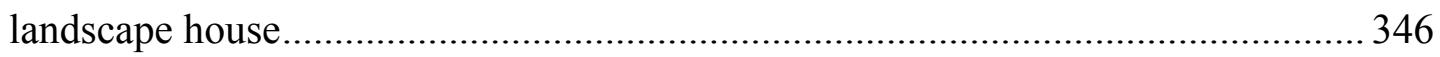

Appendix 5. Landscape elements around heavily and sparsely landscaped

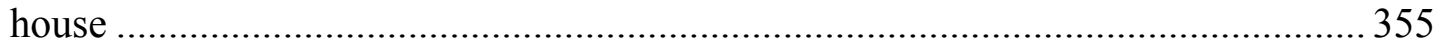

Appendix 6. Soft landscape elements around all houses ...................................... 361 


\section{Appendix 1. List of papers}

1. Misni, A. Baird, G. \& Allan, P. The effect of landscaping on the thermal performance of housing. In International Journal of System Science (IJSSS), Special Issue on Special Issue on: "Urban modelling and social system", IJSSS, revised manuscripts: $31^{\text {st }}$ May 2011, submission of manuscripts: $31^{\text {st }}$ August 2011, Inderscience Publishers, ISSN (Online): 1756-252X - ISSN (Print): 17562511 .

2. Misni, A. Baird, G. \& Allan, P. The effect of landscaping on the thermal performance of housing. In proceedings of International Conference on Spatial Planning and Sustainable Development (SPSD) 2010, Environment Policy and Landscape Design, 29-31 ${ }^{\text {st }}$ July 2011. School of Environmental Design: University of Kanazawa, Japan. ISBN: 978-4-9905800-0-1.

3. Misni, A. \& Allan, P. Sustainable residential building issues in urban heat island - the potential of albedo and vegetation. In proceedings of Sustainable Building New Zealand Conference (SB10) 2010, Innovation and Transformation, organized by International Council for Research and Innovation in Building and Construction (CIB), the International Initiative for a Sustainable Built Environment (iiSBE) and the Division of Technology, Industry and Economics (DTIE) of the United Nations Environment Programme (UNEP), 26-28 ${ }^{\text {th }}$ May 2010, New Zealand: Te Papa Museum, Wellington, http://www.cmsl.co.nz/assets/sm/5928/61/2.pn003misni.pdf.

4. Misni, A. Baird, G. \& Allan, P. A proposed methodology for investigating the effects of the strategic planting of vegetation on the thermal performance of housing in a tropical environment. In proceedings of International Conference, Sustainable Architecture \& Urban Design (ICSAUD) 2010, Issues on Global Energy Crisis and Its Impacts on Design, 3-4 ${ }^{\text {th }}$ March 2010. Hotel Royal Penang, Malaysia: University Science of Malaysia. ISBN: 978-967-5417-67-2.

5. Misni, A. Baird, G. \& Allan, P. Thermal performance of housing in a tropical environment - the effect of the surrounding landscape - a critical review. In proceedings of The International Network for Tropical Architecture (iNTASEGA) 2009, Bridging Innovation, Technology and Tradition, Holistic approaches to (rapid) sustainable architecture and environment, $2-3^{\text {rd }}$ December 2009, Grand Mercure Fortune Bangkok, Thailand: Kasetsart University.

6. Misni, A. The potential of landscaping to save air-conditioning energy. In proceedings of New Zealand Postgraduate Conference (NZPGC) 2009, Let's Get talking: Our Research, Issues \& Opportunities, 20-21 $1^{\text {st }}$ November 2009, Pipitea Campus, New Zealand: Victoria University of Wellington.

7. Misni, A. The effects of vegetation on the thermal performance of housing in a tropical environment. In proceedings of 5th International Conference of the Association of Architecture Schools in Australasia (AASA) 2009 , Conference of Sustainable, theory/theorizing sustainability, $4-5^{\text {th }}$ 
September 2009, School of Architecture, New Zealand: Victoria University of Wellington. 


\section{Appendix 2. Approvals to conduct research}

Appendix 2.1. Ethics Approval: No 17016, from the Human Ethics Committee,

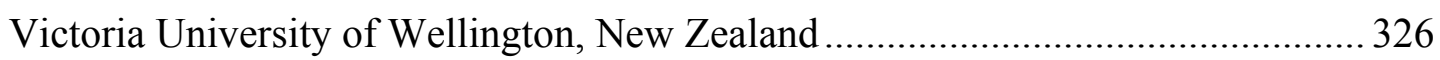

Appendix 2.2. Approval to conduct research in Malaysia from Economic Planning

Unit, Department of Prime Minister, Malaysia.. 
Appendix 2.1. Ethics Approval: No 17016, from the Human Ethics Committee, Victoria University of Wellington, New Zealand
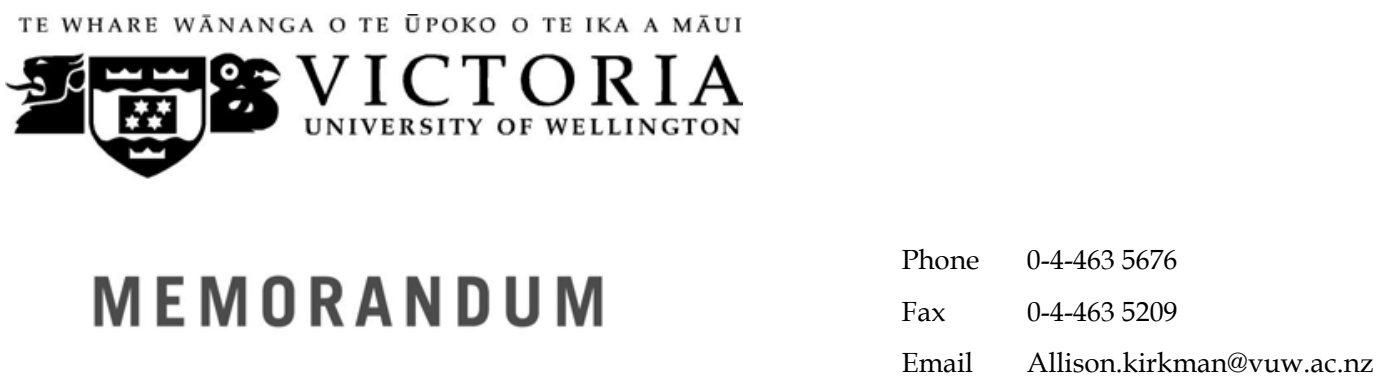

\begin{tabular}{|c|c|}
\hline TO & Alamah Misni \\
\hline COPY TO & George Baird \\
\hline FROM & Dr Allison Kirkman, Convener, Human Ethics Committee \\
\hline DATE & November 172009 \\
\hline PAGES & 1 \\
\hline SUBJECT & $\begin{array}{l}\text { Ethics Approval: No } \mathbf{1 7 0 1 6} \text { - The effects of vegetation, building } \\
\text { construction and human factors on the thermal performance of housing in } \\
\text { a tropical environment. }\end{array}$ \\
\hline
\end{tabular}

Thank you for your application for ethical approval, which has now been considered by the Standing Committee of the Human Ethics Committee.

Your application has been approved from the above date and this approval continues until 28 July 2011. If your data collection is not completed by this date you should apply to the Human Ethics Committee for an extension to this approval.

Best wishes with the research.

Allison Kirkman

Convener 
Appendix 2.2. Approval to conduct research in Malaysia from Economic Planning Unit, Department of Prime Minister, Malaysia

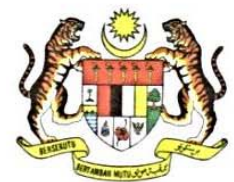

UNIT PERANCANG EKONOMI *

Economic Planning Unit

JABATAN PERDANA MENTERI

Prime Minister's Department

BLOK B5 \& B6

PUSAT PENTADBIRAN KERAJAAN PERSEKUTUAN

62502 PUTRAJAYA

MALAYSIA

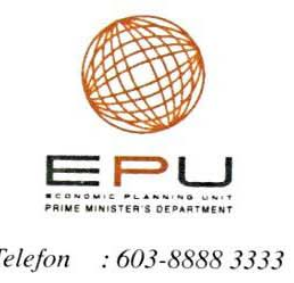

Ruj. Tuan:

Your Ref:

Ruj. Kami:

Our Ref.:

UPE: $40 / 200 / 19 / 2518$

Tarikh:

30 November 2009

Alamah Misni

6/281, The Terrace

Date:

Wellington

New Zealand 6011.

Email: alamahmisni@yahoo.com

\section{APPLICATION TO CONDUCT RESEARCH IN MALAYSIA}

With reference to your application, I am pleased to inform you that your application to conduct research in Malaysia has been approved by the Research Promotion and Co-Ordination Committee, Economic Planning Unit, Prime Minister's Department. The details of the approval are as follows:

Researcher's name : $\quad$ ALAMAH MISNI

Nationality

MALAYSIAN

Title of Research

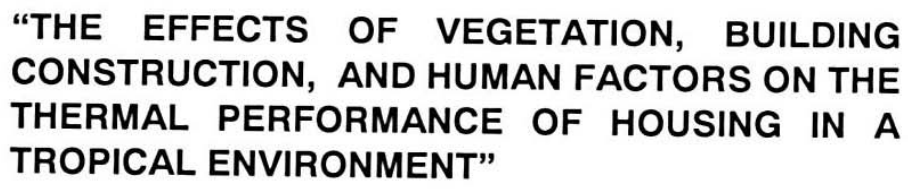

Period of Research Approved: 3 MONTHS

2. Please collect your Research Pass in person from the Economic Planning Unit, Prime Minister's Department, Parcel B, Level 1 Block B5, Federal Government Administrative Centre, 62502 Putrajaya and bring along two (2) passport size photographs. You are also required to comply with the rules and regulations stipulated from time to time by the agencies with which you have dealings in the conduct of your research. 
3. I would like to draw your attention to the undertaking signed by you that you will submit without cost to the Economic Planning Unit the following documents:

a) A brief summary of your research findings on completion of your research and before you leave Malaysia; and

b) Three (3) copies of your final dissertation/publication.

4. Lastly, please submit a copy of your preliminary and final report directly to the State Government where you carried out your research. Thank you.

Yours sincerely,<smiles>C=CCCCCCCCC</smiles>

(MUNIRAH ABD. MANAN)

For Director General,

Economic Planning Unit.

E-mail: munirah@epu.gov.my

Tel: 88725281/88725272

Fax: 88883961

\section{ATTENTION}

This letter is only to inform you the status of your application and cannot be used as a research pass. 
Appendix 3. Interview and data collection sheets

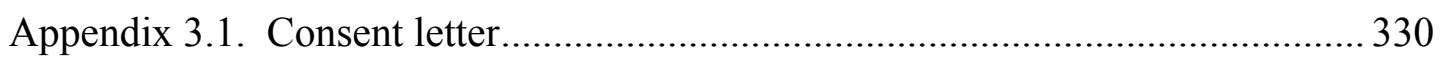

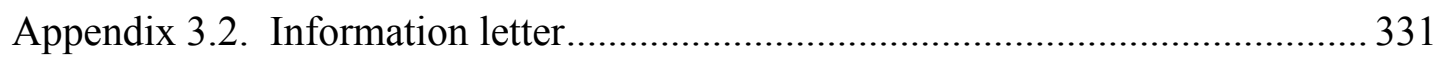

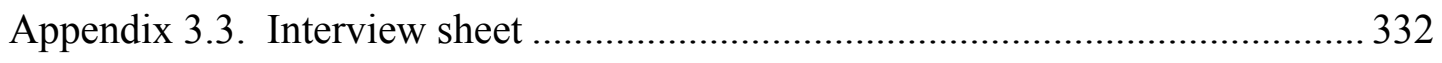

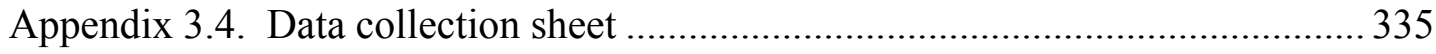




\section{CONSENT FORM}

\section{Informed Consent to Participate in Human Subject Research}

I agree to participate in a study being conducted by Alamah Misni of the School of Architecture under the supervision of Professor Dr. George Baird and Associate Prof. Penny Allan. I have made this decision based on the information I have read in the Information letter. As a participant in this study, I realise that data about the thermal performance, building construction, human factors and landscaping around my house will be collected. I will also be interviewed about my family and general performance of my house.

All information, which I provide, will be held in confidence and I will not be identified in any way in the final report. The data will be stored in a locked file cabinet and will be disposed at the end of the study. I understand that I may withdraw this consent at any time by ceasing to allow data collection of my house. I also understand that this project has been reviewed by and has received clearance through the Human Ethics Committee at the Victoria University of Wellington, New Zealand.

Name

Contact no.

Date

Address

Time: from to

(Signature of subject)

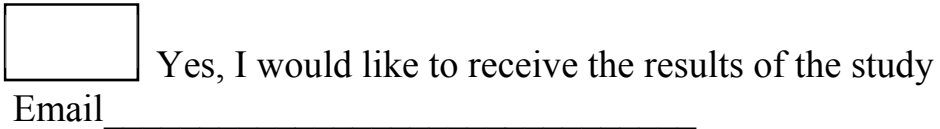

(Please provide your email address, if you would like results to be sent to you)

Alamah Misni

School of Architecture

Faculty of Architecture \& Design

Victoria University of Wellington

PO Box 600

Wellington, NEW ZEALAND

CONTACT:

$+64-4-4636253$ (office phone)

$+64-4-4636204$ (office fax)

+64-21-2531866 (mobile)

misnialam@myvuw.ac.nz (email)

alamahmisni@yahoo.com (email) 


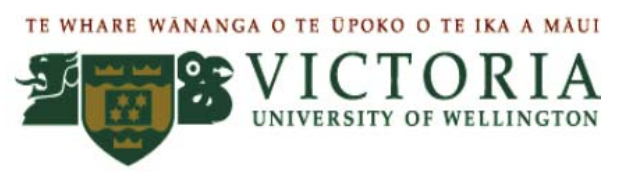

Victoria University of Wellington, New Zealand The effects of vegetation on the thermal performance of housing

\section{INFORMATION LETTER}

\section{The effects of vegetation on the thermal performance of housing in a tropical environment}

The study is being conducted as part of Alamah Misni's $\mathrm{PhD}$ research project through the School of Architecture at the Victoria University of Wellington, New Zealand, and is being supervised by Professor Dr. George Baird and Associate Prof. Penny Allan.

Given the need for air-conditioning in tropical climates, this study is designed to determine the effects of vegetation around domestic buildings in reducing the air-conditioning load. For the purpose of the study, data about thermal performance, building construction, energy use, human factors and landscaping around domestic buildings has to be collected. As a single-family resident of a domestic building in a tropical country, your house has been selected as a case study and you are being asked to participate in the study. It is anticipated that data collection will take approximately forty minutes.

The researcher will use different technical equipment for collection of this data. After the collection of data, you will also be asked a few general questions about your family and general performance of the building which will take approximately five minutes of your time.

The data about the building and your interview answers will be coded so that your name will not appear on any of the forms used for data analysis. No one will be able to identify your building or your answers. Only the researcher and her supervisors will have access to the names associated with the codes and this information will be kept in a locked file cabinet. This information will be use to compile a thesis manuscript which will be submitted to the library and may also be published in conference/journal papers. The entire data will be destroyed at the end of the study which will take approximately two years to complete.

If you want to withdraw from the study, you may do so at any time. Any information collected on your building up to that point will be destroyed. If you have any questions or queries regarding the study, please contact:

Dr George Baird, FIPENZ, FIRHACE, FCIBSE, C Eng

Professor of Building Science, School of Architecture.

MAILING ADDRESS: Victoria University of Wellington,

P O Box 600, Wellington, New Zealand.

LOCATION: 139 Vivian Street, Wellington, New Zealand

CONTACTS:

+6444636231 (office phone)

+6444636204 (office fax)

+64 (0)21394433 (mobile)

George.Baird@vuw.ac.nz (email)

http://www.vuw.ac.nz/architecture/staff/academics/george-baird.aspx 
Appendix 3.3. Interview sheet

\section{INTERVIEW SHEET}

\section{Human factors}

Numbers of household:
a. Children (below 12 years old)
b. Teenager $(12-18$ years old $)$
c. Adult (above 18 years old)

2. Electricity bills (RM):

a. Total average electricity bill per month (MYR) in dry season, (May - September)
1. Below 100

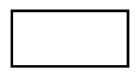
2. $100-150$
3. $150-199$
4. $200-249$

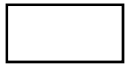
5. 250-299

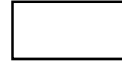
6. $300-349$
7. $350-399$

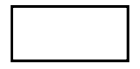
8. $400-449$

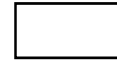
9. $450-499$
10. $500-549$

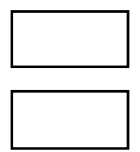
11. $550-599$

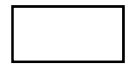
12. $600-649$
14. $700-749$

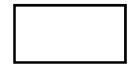
15. 750-799
13. $650-699$

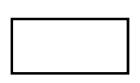
17. $850-899$

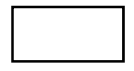
18. $900-949$
19. 950-999
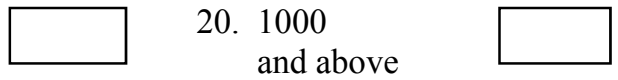
16. $800-849$
20. 1000
and above
$\begin{aligned} & \text { 20. } 1000 \\ & \text { and above }\end{aligned}$
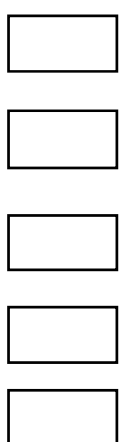

b. Total average electricity bill per month (MYR) during raining season, (November - March)

1. Below 100

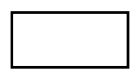

2. $100-150$

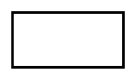

3. $150-199$

4. $200-249$

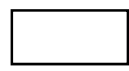

5. $250-299$

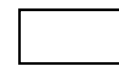

6. $300-349$

8. $400-449$

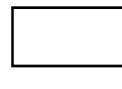

9. $450-499$

7. $350-399$

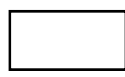

10. $500-549$

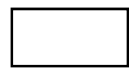

11. $550-599$

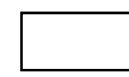

12. $600-649$

14. $700-749$

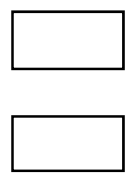

17. $850-899$

20. 1000

15. 750-799

18. $900-949$

17. $800-849$

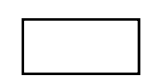

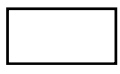
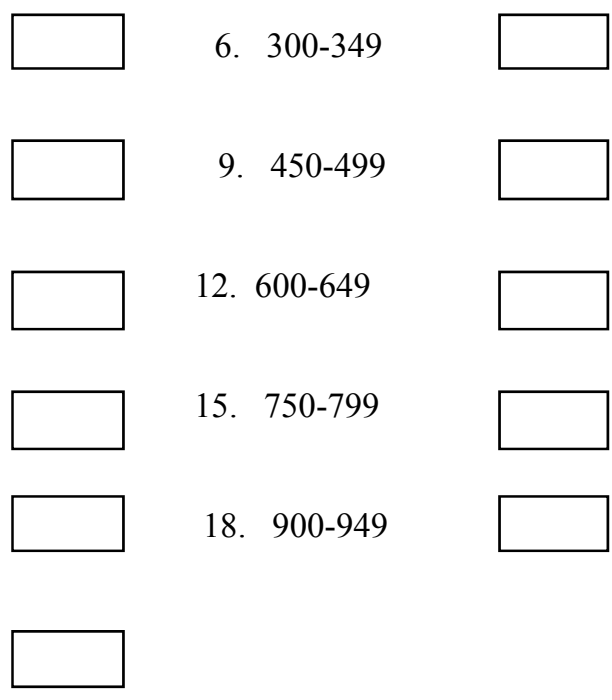


\section{Code for air-conditioning system}

\begin{tabular}{|c|c|c|c|c|c|c|c|c|c|c|c|}
\hline \multicolumn{2}{|c|}{ Type/ system } & \multicolumn{2}{|c|}{ Horsepower } & \multicolumn{2}{|c|}{ No. } & \multicolumn{2}{|c|}{ Setting $\left({ }^{\circ} \mathrm{C}\right)$} & \multicolumn{2}{|c|}{$\begin{array}{l}\text { Total (MYR) per } \\
\text { month* }\end{array}$} & \multicolumn{2}{|c|}{ Total (kW) } \\
\hline 1 & Window & 1 & 1.0 & 1 & 1 & 1 & Below 15 & 1 & Below 50 & 1 & Below 200 \\
\hline 2 & Split & 2 & 1.5 & 2 & 2 & 2 & $15-18$ & 2 & $50-99$ & 2 & $200-399$ \\
\hline & & 3 & 2.0 & 3 & 3 & 3 & $19-20$ & 3 & $100-149$ & 3 & $400-599$ \\
\hline & & 4 & 2.5 & & & 4 & $21-22$ & 4 & $150-199$ & 4 & $600-799$ \\
\hline & & 5 & 3.0 & & & 5 & $23-24$ & 5 & $200-249$ & 5 & $800-999$ \\
\hline & & 6 & 3.5 & & & 6 & $25-26$ & 6 & $250-299$ & 6 & $1000-1199$ \\
\hline & & 7 & 4.0 & & & 7 & $27-28$ & 7 & $300-349$ & 7 & $1200-1399$ \\
\hline & & 8 & 4.5 & & & 8 & $29-30$ & 8 & $350-399$ & 8 & $1400-1599$ \\
\hline & & 9 & 5.0 & & & & & 9 & $400-449$ & 9 & $1600-1799$ \\
\hline & & & & & & & & 10 & $450-499$ & 10 & $1800-1999$ \\
\hline & & & & & & & & 11 & $500-549$ & 11 & $2000-2199$ \\
\hline & & & & & & & & 12 & $550-599$ & 12 & $2200-2399$ \\
\hline & & & & & & & & 13 & $600-649$ & 13 & $2400-2599$ \\
\hline & & & & & & & & 14 & $650-699$ & 14 & $2600-2799$ \\
\hline & & & & & & & & 15 & $700-749$ & 15 & $2800-2999$ \\
\hline & & & & & & & & 16 & $750-799$ & 16 & $3000-3199$ \\
\hline & & & & & & & & 17 & $800-849$ & 17 & $3200-3399$ \\
\hline & & & & & & & & 18 & $850-899$ & 18 & $3400-3599$ \\
\hline & & & & & & & & 19 & $900-949$ & 19 & $3600-3799$ \\
\hline & & & & & & & & 20 & $950-999$ & 20 & $3800-3999$ \\
\hline & & & & & & & & 21 & $\begin{array}{l}1000 \text { and } \\
\text { above }\end{array}$ & 21 & $\begin{array}{l}4000 \text { and } \\
\text { above }\end{array}$ \\
\hline
\end{tabular}

*Note:

a. Every horsepower multiply by MYR 0.20

Example:

1. I horsepower per hour $=$ MYR 0.20

$2 \quad 1.5$ horsepower per hour $=$ MYR 0.30

3. 2 horsepower per hour $=$ MYR 0.40

4. $\quad 2.5$ horsepower per hour $=$ MYR 0.50

5. 3 horsepower per hour $=$ MYR 0.60

6. $\quad 3.5$ horsepower per hour $=$ MYR 0.70

7. 4 horsepower per hour $=$ MYR 0.80

8. $\quad 4.5$ horsepower per hour $=$ MYR 0.90

9. 5 horsepower per hour $=$ MYR 1.00

b. Total $(M Y R)$ per month $=($ Horse power $) \times($ total hour per month $) \times(M Y R$ in different horse power $)$

c. 1 horsepower $=0.7457 \mathrm{~kW}$ 
3. Energy Use: Air conditioning system

\begin{tabular}{|c|c|c|c|c|c|c|c|c|c|c|c|}
\hline \multirow[t]{2}{*}{ No } & \multirow[t]{2}{*}{ Location } & \multirow[t]{2}{*}{ Type } & \multirow{2}{*}{$\begin{array}{l}\text { Horse } \\
\text { power }\end{array}$} & \multirow[t]{2}{*}{ No. } & \multicolumn{3}{|c|}{ The setting range (time) } & \multirow{2}{*}{$\begin{array}{l}\text { Period } \\
\text { time per } \\
\text { week }(\mathrm{hr})\end{array}$} & \multirow{2}{*}{$\begin{array}{l}\text { Setting } \\
\left({ }^{\circ} \mathrm{C}\right)\end{array}$} & \multirow{2}{*}{$\begin{array}{l}\text { Total } \\
\text { (hr) per } \\
\text { month }\end{array}$} & \multirow{2}{*}{$\begin{array}{l}\text { Total } \\
\text { (MYR) } \\
\text { per } \\
\text { month }\end{array}$} \\
\hline & & & & & From & To & $\begin{array}{l}\text { Total } \\
(\mathrm{hr})\end{array}$ & & & & \\
\hline 1 & Living room & & & & & & & & & & \\
\hline 2 & Dining room & & & & & & & & & & \\
\hline 3 & Kitchen & & & & & & & & & & \\
\hline 4 & Family room & & & & & & & & & & \\
\hline 5 & Study room & & & & & & & & & & \\
\hline 6 & TV room & & & & & & & & & & \\
\hline 7 & $\begin{array}{l}\text { Master } \\
\text { bedroom }\end{array}$ & & & & & & & & & & \\
\hline 8 & Bedroom 1 & & & & & & & & & & \\
\hline 9 & Bedroom 2 & & & & & & & & & & \\
\hline 10 & Bedroom 3 & & & & & & & & & & \\
\hline 11 & Bedroom 4 & & & & & & & & & & \\
\hline 12 & Bedroom 5 & & & & & & & & & & \\
\hline 13 & Bedroom 6 & & & & & & & & & & \\
\hline 14 & Guest room & & & & & & & & & & \\
\hline \multicolumn{12}{|c|}{ Total (MYR) per month } \\
\hline To & $\mathbf{k} \mathbf{W})^{*}$ & & & & & & & & & & \\
\hline
\end{tabular}

*Note:

$1 k W=$ MYR 0.2457 
Appendix 3.4. Data collection sheet

Note: This data sheet has been filled out after consent from the subject

BUILDING AND LANDSCAPE DATA COLLECTION SHEET

1. Building construction

a. Year of construction
2. Below 5 years

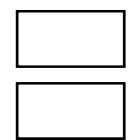
2. 5-9 years

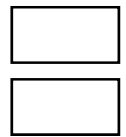
3. 10-14 years
4. 15-19 years
5. 20-24 years
6. 25-29 years
7. 30 years and above

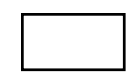

b. Size of the house (floor area in $\mathrm{m}^{2}$ )

1. 250-299

4. $400-449$

7. 550-599

10. $700-749$
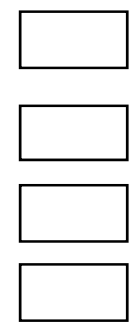

2. $300-349$

5. $450-499$

8. $600-649$

11. $750-799$

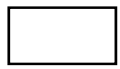

3. $350-400$

6. $500-549$

9. 650-699

12. 800 and above

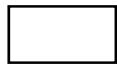

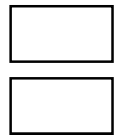

c. How many level/storey

1. Single storey

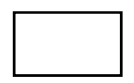

2. Double storey

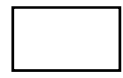

3. Three storey
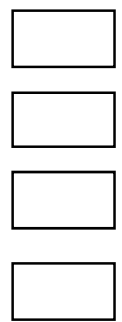

2. Size of the porches $\left(\mathrm{m}^{2}\right)$

1. Below 30

2. $30-59$

3. 60 and above

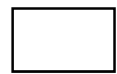

3. Construction type:
a. Column and beam

1. Reinforced Concrete

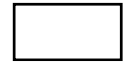

2. Other

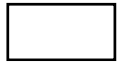

state

b. Wall

1. Bricks

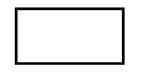

2. Other

state

\section{c. Roof type}

1. Pitch

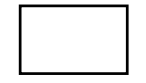

2. Flat

Location 


\section{d. Roof structure}

1. Wood

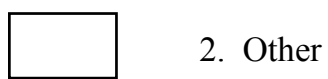

$\square$ state

e. Roof material

1. Concrete tiles

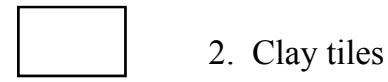

3. Other

Code for window, sliding door and external door, and ceiling

\begin{tabular}{|l|l|l|l|l|l|l|l|}
\hline \multicolumn{2}{|l|}{ Location/azimuth } & \multicolumn{2}{l|}{ Type of window } & $\begin{array}{l}\text { Material } \\
\text { (window \& door) }\end{array}$ & \multicolumn{2}{l|}{ Wall/ceiling colour } \\
\hline 1 & North & 1 & Awning & 1 & Glass & 1 & White/light grey \\
\hline 2 & North East & 2 & Casement & 2 & Wood/timber & 2 & Creme \\
\hline 3 & East & 3 & Double-hung & 3 & Solid timber & 3 & Peach \\
\hline 4 & South East & 4 & Gliding & 4 & Steel/aluminium & 4 & Light Yellow \\
\hline 5 & South & 5 & Picture/Fixed & 5 & PVC/Plastic & 5 & Light brown \\
\hline 6 & South West & 6 & Bay & & & 6 & Light green \\
\hline 7 & West & 7 & Louvers & & & 7 & Light orange \\
\hline 8 & North West & & & & & 8 & Light blue \\
\hline 9 & All sides & & & & & 9 & Light red \\
\hline & & & & & & 10 & Dark blue \\
\hline & & & & & & 11 & Dark brown \\
\hline & & & & & & 12 & Dark green \\
\hline & & & & & & 13 & Dark red \\
\hline & & & & 14 & Dark colour \\
\hline
\end{tabular}

\begin{tabular}{|l|l|l|l|l|l|l|l|}
\hline \multicolumn{2}{|l}{ Ceiling type/material } & \multicolumn{2}{l}{ Space } & \multicolumn{2}{l|}{ Level } & \multicolumn{2}{l|}{ Ceiling height (m) } \\
\hline 1 & Skim coat plaster & 1 & Living room & 1 & Ground floor & 1 & 2.0 \\
\hline 2 & Fibrous plaster & 2 & Dining room & 2 & First floor & 2 & 2.5 \\
\hline 3 & Cement plaster & 3 & Family room & 3 & Second floor & 3 & 2.8 \\
\hline 4 & Asbestos & 4 & Study room & 4 & Basement & 4 & 3.0 \\
\hline 5 & Plywood & 5 & Master bedroom & & & 5 & 3.5 \\
\hline 6 & Wood panelling & 6 & Bedroom & & & 6 & 4.0 \\
\hline 7 & Soft board & 7 & Guest room & & & 7 & 4.5 \\
\hline & & 8 & Kitchen & & & 8 & 5.0 \\
\hline & & 9 & Porch & & & 9 & 5.5 \\
\hline & & 10 & TV/audio room & & & 10 & 6.0 \\
\hline & & & & & 11 & 6.5 \\
\hline & & & & & & 12 & 7.0 and above \\
\hline
\end{tabular}




\begin{tabular}{|c|c|c|c|c|c|}
\hline \multicolumn{2}{|c|}{ Size of window $\left(\mathrm{m}^{2}\right)$} & \multicolumn{2}{|c|}{ Size of sliding door $\left(\mathrm{m}^{2}\right)$} & \multicolumn{2}{|c|}{ Size of door $\left(\mathrm{m}^{2}\right)$} \\
\hline 1 & $\begin{array}{l}1.5 \mathrm{~m} \text { height } \mathrm{x} 0.6 \mathrm{~m} \text { width }(1 \\
\text { leave) }\end{array}$ & 1 & $\begin{array}{l}2 \mathrm{~m} \text { height } \times 1 \mathrm{~m} \text { width }(3 \\
\text { leaves) }\end{array}$ & 1 & $\begin{array}{l}2 \mathrm{~m} \text { height } \mathrm{x} 1 \mathrm{~m} \text { width (1 } \\
\text { leave) }\end{array}$ \\
\hline 2 & $\begin{array}{l}1.5 \mathrm{~m} \text { height } \times 1.2 \mathrm{~m} \text { width }(2 \\
\text { leaves })\end{array}$ & 2 & $\begin{array}{l}2 \mathrm{~m} \text { height } \times 0.8 \text { width ( } 3 \\
\text { leaves) }\end{array}$ & 2 & $\begin{array}{l}2 \mathrm{~m} \text { height } \times 2 \mathrm{~m} \text { width } \\
\text { (2 leaves) }\end{array}$ \\
\hline 3 & $\begin{array}{l}1.5 \mathrm{~m} \text { height } \times 1.8 \mathrm{~m} \text { width ( } 3 \\
\text { leaves) }\end{array}$ & 3 & $\begin{array}{l}2 \mathrm{~m} \text { height } \times 0.8 \text { width ( } 4 \\
\text { leaves) }\end{array}$ & 3 & $\begin{array}{l}2 \mathrm{~m} \text { height } \mathrm{x} 1.5 \mathrm{~m} \text { width } \\
\text { (1.5 leaves) }\end{array}$ \\
\hline 4 & $\begin{array}{l}1.5 \mathrm{~m} \text { height } \times 2.4 \mathrm{~m} \text { width }(4 \\
\text { leaves) }\end{array}$ & 4 & $\begin{array}{l}2 \mathrm{~m} \text { height } \times 1 \mathrm{~m} \text { width (4 } \\
\text { leaves) }\end{array}$ & & \\
\hline 5 & $\begin{array}{l}0.5 \text { height } \times 0.5 \mathrm{~m} \text { width } \\
\text { (1 leave upper window) }\end{array}$ & & & & \\
\hline 6 & $\begin{array}{l}0.5 \mathrm{~m} \text { height } \times 1.0 \mathrm{~m} \text { width }(2 \\
\text { leaves upper window) }\end{array}$ & & & & \\
\hline 7 & $\begin{array}{l}\text { Fix glass } \\
\text { (state size) }\end{array}$ & & & & \\
\hline 8 & $\begin{array}{l}0.5 \mathrm{~m} \text { height } \times 1.5 \mathrm{~m} \text { width ( } 3 \\
\text { leaves upper window ) }\end{array}$ & & & & \\
\hline 9 & $\begin{array}{l}1.5 \mathrm{~m} \text { height } \times 3.0 \mathrm{~m} \text { width }(5 \\
\text { leaves) }\end{array}$ & & & & \\
\hline 10 & $\begin{array}{l}0.5 \mathrm{~m} \text { height } \times 1.0 \mathrm{~m} \text { width }(4 \\
\text { leaves upper window) }\end{array}$ & & & & \\
\hline
\end{tabular}

\section{f. Window}

\begin{tabular}{|l|l|l|l|l|l|}
\hline No. & Type of window & Material & Location/azimuth & level & Size $\left(\mathbf{m}^{2}\right)$ \\
\hline 1 & & & & & \\
\hline 2 & & & & & \\
\hline 3 & & & & & \\
\hline 4 & & & & & \\
\hline
\end{tabular}

\section{g. Sliding door}

\begin{tabular}{|l|l|l|l|l|l|}
\hline No. & Material & Location/azimuth & Space & level & Size $\left(\mathbf{m}^{2}\right)$ \\
\hline 1 & & & & & \\
\hline 2 & & & & & \\
\hline
\end{tabular}

\section{h. External door}

\begin{tabular}{|l|l|l|l|l|l|}
\hline No. & Material & Location/azimuth & Space & Level & Size $\left(\mathbf{m}^{2}\right)$ \\
\hline 1 & & & & & \\
\hline 2 & & & & & \\
\hline
\end{tabular}


Code for wall areas

\begin{tabular}{|l|l|l|l|}
\hline \multicolumn{2}{|l|}{ Location/azimuth } & \multicolumn{2}{l|}{ Wall areas $\left.\mathbf{( m}^{2}\right)$} \\
\hline 1 & North & 1 & Below 20 \\
\hline 2 & North East & 2 & $21-30$ \\
\hline 3 & East & 3 & $31-40$ \\
\hline 4 & South East & 4 & $41-50$ \\
\hline 5 & South & 5 & $51-60$ \\
\hline 6 & South West & 6 & $61-70$ \\
\hline 7 & West & 7 & $71-80$ \\
\hline 8 & North West & 8 & $81-90$ \\
\hline & & 9 & $91-100$ \\
\hline & & 10 & $101-110$ \\
\hline & & 11 & $111-120$ \\
\hline & & 12 & $121-130$ \\
\hline & & 13 & $131-140$ \\
\hline & & 14 & $141-150$ \\
\hline & & 15 & 151 and above \\
\hline
\end{tabular}

\section{i. Wall area}

\begin{tabular}{|l|l|l|}
\hline No. & Location/azimuth & Area $\left.\mathbf{( m}^{\mathbf{2}}\right)$ \\
\hline 1 & & \\
\hline 2 & & \\
\hline 3 & & \\
\hline 4 & & \\
\hline
\end{tabular}

j. $\quad$ Roof area $=$

1. Below 100

4. 151-175

7. $226-250$

8. $301-325$

12. $376-400$

15. $451-475$

$18 \quad 526-550$ $\left(\mathbf{m}^{2}\right)$

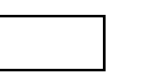

2. $100-125$

3. $126-150$

6. 201-225

5. $176-200$

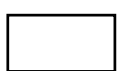

9. $276-300$

8. $251-275$
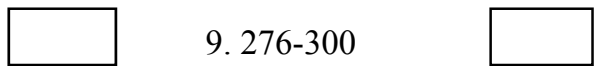

10. $326-350$

11. $351-375$

$13 \quad 401-425$

14. $426-450$

16. $476-500$

$17.501-525$

17. $551-575$

$18.575-600$
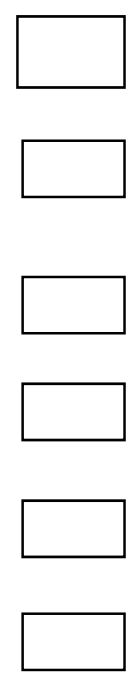


\section{k. The colour of building envelope}

Wall Albedo: $\quad$ Incident sunlight $\operatorname{lux}$

Reflected sunlight $\operatorname{lux}$

Albedo $=$ divide the second measurement (reflected) by the first measurement (incident)
1. $0.00-0.19$
2. $\quad 0.20-0.39$
$0.4-0.59$
4. $0.60-0.79$
5. $0.8-1.0$

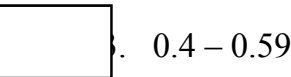

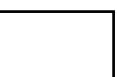

\begin{tabular}{|l|l|l|l|}
\hline No. & Building envelope & Location/azimuth & Colour \\
\hline 1 & Wall & & \\
\hline 2 & Roof & & \\
\hline
\end{tabular}

\section{Ceiling type/material}

\begin{tabular}{|c|c|c|c|c|}
\hline No. & Space & Height (m) & $\begin{array}{l}\text { Ceiling } \\
\text { type/material }\end{array}$ & Colour \\
\hline 1 & Living room & & & \\
\hline 2 & Dining room & & & \\
\hline 3 & Kitchen & & & \\
\hline 4 & Family room & & & \\
\hline 5 & Study room & & & \\
\hline 6 & TV room & & & \\
\hline 7 & Master bedroom & & & \\
\hline 8 & Bedroom 1 & & & \\
\hline 9 & Bedroom 2 & & & \\
\hline 10 & Bedroom 3 & & & \\
\hline 11 & Bedroom 4 & & & \\
\hline 12 & Bedroom 5 & & & \\
\hline 13 & Bedroom 6 & & & \\
\hline 14 & Guest room & & & \\
\hline 15 & Porch & & & \\
\hline
\end{tabular}




\section{Landscaping}

a. Size of the garden $\left(\mathrm{m}^{2}\right)$

1. Below 50

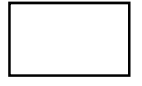

2. 50-99

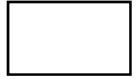

3. 100-149

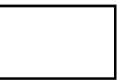

4. 150-199

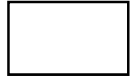

5. 200-249

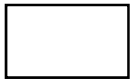

6. 250-299

7. $300-349$

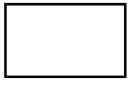

8. $350-399$

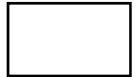

9. $400-449$

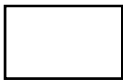

10. $450-499$

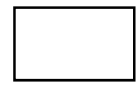

11. $500-549$

12. $550-599$

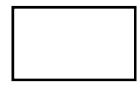

13. $600-649$

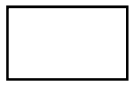

14. $650-649$

15. 650-699

16. 700 and above
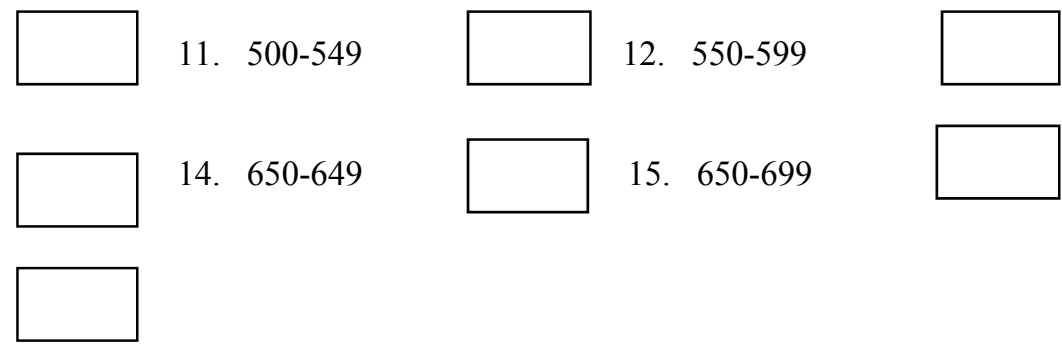

\section{Code for paving surfaces}

\begin{tabular}{|c|c|c|c|c|c|c|c|c|c|}
\hline \multicolumn{2}{|c|}{ Location/ azimuth } & \multicolumn{2}{|c|}{ Space } & \multicolumn{2}{|c|}{ Material } & \multicolumn{2}{|c|}{ Colour } & \multicolumn{2}{|c|}{ Areas $\left(\mathrm{m}^{2}\right)$} \\
\hline 1 & North & 1 & Entrance road & 1 & Tiles & 1 & White/light grey & 1 & Below 10 \\
\hline 2 & North East & 2 & Backyard garden & 2 & Concrete & 2 & Creme & 2 & $11-20$ \\
\hline 3 & East & 3 & Walkway & 3 & Asphalt & 3 & Peach & 3 & $21-40$ \\
\hline 4 & South East & 4 & Garden decoration & 4 & Bricks & 4 & Light Yellow & 4 & $41-60$ \\
\hline 5 & South & & & 5 & Wood & 5 & Light brown/red & 5 & $61-70$ \\
\hline 6 & South West & & & 6 & Pebbles & 6 & Light green & 6 & $71-80$ \\
\hline 7 & West & & & 7 & Stone & 7 & Light orange & 7 & $81-90$ \\
\hline 8 & North West & & & 8 & Bricks with grass inlay & 8 & Light blue & 8 & 91-100 \\
\hline & & & & & & 9 & Dark Grey & 9 & 101 and al \\
\hline
\end{tabular}

Paving areas

\begin{tabular}{|l|l|l|l|l|l|}
\hline No. & Location/azimuth & Space & Material & Colour & Areas ${\left(\mathbf{m}^{2}\right)}^{2}$ \\
\hline 1 & & & & & \\
\hline 2 & & & & & \\
\hline
\end{tabular}


Code for shade trees around building

\begin{tabular}{|l|l|l|l|l|l|l|l|}
\hline \multicolumn{2}{|l|}{ Location/azimuth } & \multicolumn{2}{l|}{$\begin{array}{l}\text { Tree building } \\
\text { distance (m) }\end{array}$} & \multicolumn{2}{l|}{ Trunk height (m) } & \multicolumn{2}{l|}{ Overall height (m) } \\
\hline 1 & North & 1 & Below 5 & 1 & 1.0 & 1 & Below 2 \\
\hline 2 & North East & 2 & $5-9$ & 2 & 1.5 & 2 & $2-4$ \\
\hline 3 & East & 3 & $10-14$ & 3 & 2.0 & 3 & $5-9$ \\
\hline 4 & South East & 4 & $15-19$ & 4 & 2.5 & 4 & $10-14$ \\
\hline 5 & South & 5 & 20 and above & 5 & 3.0 & 5 & $15-19$ \\
\hline 6 & South West & & & 6 & 3.5 & 6 & 20 and above \\
\hline 7 & West & & & 7 & 4.0 & & \\
\hline 8 & North West & & & 8 & 5 and above & & \\
\hline
\end{tabular}

\begin{tabular}{|l|l|l|l|l|l|l|l|l|l|l|}
\hline \multicolumn{2}{|l}{ Canopy size (diametre) } & \multicolumn{2}{l|}{ Shape } & \multicolumn{2}{l|}{ Amount of leaves (density-\%) } & \multicolumn{2}{l|}{ Size of leaves (area - $\left.\mathbf{c m}^{2}\right)$} \\
\hline 1 & Small & Below 3 & 1 & Spreading & 1 & Few & Below 40 & 1 & Small & Below 150 \\
\hline 2 & Small moderate & $3-7$ & 2 & Round & 2 & Medium & $40-70$ & 2 & Medium & $150-1000$ \\
\hline 3 & Medium & $8-11$ & 3 & Columnar & 3 & Dense & Above 70 & 3 & Large & Above 1000 \\
\hline 4 & Large moderate & $12-15$ & 4 & Pyramidal & & & & & & \\
\hline 5 & Large & 16 and above & 5 & Fountain & & & & & & \\
\hline
\end{tabular}

\section{b. Landscaping around the building}

i. Roadside trees:

\begin{tabular}{|l|l|l|l|l|l|l|l|l|l|}
\hline No. & Botanical name & $\begin{array}{l}\text { Common } \\
\text { name }\end{array}$ & Location/azimuth & $\begin{array}{l}\text { Tree } \\
\text { building } \\
\text { Distance } \\
\text { (m) }\end{array}$ & $\begin{array}{l}\text { Trunk } \\
\text { height } \\
\text { (m) }\end{array}$ & $\begin{array}{l}\text { Overall } \\
\text { height } \\
\text { (m) }\end{array}$ & $\begin{array}{l}\text { Canopy } \\
\text { size } \\
\text { (dia.m) }\end{array}$ & $\begin{array}{l}\text { Shape } \\
\text { of leaves } \\
\text { leaves }\end{array}$ & $\begin{array}{l}\text { Amount } \\
\text { leave }\end{array}$ \\
\hline
\end{tabular}

\section{ii. Garden trees}

\begin{tabular}{|c|c|c|c|c|c|c|c|c|c|c|}
\hline No. & $\begin{array}{l}\text { Botanical } \\
\text { name }\end{array}$ & $\begin{array}{l}\text { Common } \\
\text { name }\end{array}$ & Location/azimuth & $\begin{array}{l}\text { Tree } \\
\text { building } \\
\text { Distance } \\
\text { (m) }\end{array}$ & $\begin{array}{l}\text { Trunk } \\
\text { height } \\
\text { (m) }\end{array}$ & $\begin{array}{l}\text { Overall } \\
\text { height } \\
\text { (m) }\end{array}$ & $\begin{array}{l}\text { Canopy } \\
\text { size } \\
\text { (dia.m) }\end{array}$ & Shape & $\begin{array}{l}\text { Amount } \\
\text { of leaves }\end{array}$ & $\begin{array}{l}\text { Size of } \\
\text { leaves }\end{array}$ \\
\hline 1 & & & & & & & & & & \\
\hline
\end{tabular}

iii. Edible fruit trees:

\begin{tabular}{|c|c|c|c|c|c|c|c|c|c|c|}
\hline No. & $\begin{array}{l}\text { Botanical } \\
\text { name }\end{array}$ & $\begin{array}{l}\text { Common } \\
\text { name }\end{array}$ & Location/azimuth & $\begin{array}{l}\text { Tree } \\
\text { building } \\
\text { Distance } \\
\text { (m) }\end{array}$ & $\begin{array}{l}\text { Trunk } \\
\text { height } \\
\text { (m) }\end{array}$ & $\begin{array}{l}\text { Overall } \\
\text { height } \\
\text { (m) }\end{array}$ & $\begin{array}{l}\text { Canopy } \\
\text { size } \\
\text { (dia.m) }\end{array}$ & Shape & $\begin{array}{l}\text { Amount } \\
\text { of leaves }\end{array}$ & $\begin{array}{l}\text { Size of } \\
\text { leaves }\end{array}$ \\
\hline 1 & & & & & & & & & & \\
\hline
\end{tabular}


iv. Roadside palm:

\begin{tabular}{|c|c|c|c|c|c|c|c|c|c|c|}
\hline No. & Botanical name & $\begin{array}{l}\text { Common } \\
\text { name }\end{array}$ & Location/azimuth & $\begin{array}{l}\text { Trees } \\
\text { building } \\
\text { Distance } \\
\text { (m) }\end{array}$ & $\begin{array}{l}\text { Trunk } \\
\text { height } \\
\text { (m) }\end{array}$ & $\begin{array}{l}\begin{array}{l}\text { Overall } \\
\text { height } \\
\text { (m) }\end{array} \\
\end{array}$ & $\begin{array}{l}\text { Canopy } \\
\text { size } \\
\text { (dia.m) }\end{array}$ & Shape & $\begin{array}{l}\text { Amount } \\
\text { of leaves }\end{array}$ & $\begin{array}{l}\text { Size of } \\
\text { leaves }\end{array}$ \\
\hline 1 & & & & & & & & & & \\
\hline
\end{tabular}

v. Garden Palm:

\begin{tabular}{|l|l|l|l|l|l|l|l|l|l|}
\hline No. & Botanical name & $\begin{array}{l}\text { Common } \\
\text { name }\end{array}$ & Location/azimuth & $\begin{array}{l}\text { Palms } \\
\text { building } \\
\text { Distance } \\
\text { (m) }\end{array}$ & $\begin{array}{l}\text { Trunk } \\
\text { height } \\
\text { (m) }\end{array}$ & $\begin{array}{l}\text { Overall } \\
\text { height } \\
\text { (m) }\end{array}$ & $\begin{array}{l}\text { Canopy } \\
\text { size } \\
\text { (dia.m) }\end{array}$ & $\begin{array}{l}\text { Shape } \\
\text { Amount } \\
\text { of leaves } \\
\text { leaves }\end{array}$ & $\begin{array}{l}\text { Size of } \\
\text { liages }\end{array}$ \\
\hline
\end{tabular}

vi. Bamboo:

\begin{tabular}{|c|c|c|c|c|c|c|c|c|c|c|}
\hline No. & Botanical name & $\begin{array}{l}\text { Common } \\
\text { name }\end{array}$ & Location/azimuth & $\begin{array}{l}\text { Bamboos } \\
\text { building } \\
\text { Distance } \\
\text { (m) }\end{array}$ & $\begin{array}{l}\text { Trunk } \\
\text { height } \\
\text { (m) }\end{array}$ & $\begin{array}{l}\text { Overall } \\
\text { height } \\
\text { (m) }\end{array}$ & $\begin{array}{l}\text { Canopy } \\
\text { size } \\
\text { (dia.m) }\end{array}$ & Shape & $\begin{array}{l}\text { Amount } \\
\text { of leaves }\end{array}$ & $\begin{array}{l}\text { Size of } \\
\text { leaves }\end{array}$ \\
\hline 1 & & & & & & & & & & \\
\hline
\end{tabular}

Code for shrub, grass and turf around building

\begin{tabular}{|l|l|l|l|l|l|l|l|}
\hline \multicolumn{2}{|l|}{ Location/azimuth } & \multicolumn{2}{l|}{$\begin{array}{l}\text { Shrubs building } \\
\text { distance (m) }\end{array}$} & \multicolumn{2}{l|}{ Height (m) } & \multicolumn{2}{l|}{} \\
\hline 1 & North & 1 & Proximity to walls & 1 & Below 0.5 & 1 & Individual/potted \\
\hline 2 & North East & 2 & Below 5 & 2 & $0.5-0.9$ & 2 & Group below 5 \\
\hline 3 & East & 3 & $5-9$ & 3 & $1.0-1.4$ & 3 & Group 6-10 \\
\hline 4 & South East & 4 & $10-14$ & 4 & $1.5-1.9$ & 4 & Group 10-20 \\
\hline 5 & South & 5 & $15-19$ & 5 & $2.0-2.4$ & 5 & Group 21-30 \\
\hline 6 & South West & 6 & 20 and above & 6 & $2.5-2.9$ & 6 & Group 31 and above \\
\hline 7 & West & & & 7 & 3 and above & & \\
\hline 8 & North West & & & & & & \\
\hline
\end{tabular}

\begin{tabular}{|c|c|c|c|c|c|c|c|c|c|c|c|}
\hline \multicolumn{2}{|c|}{$\begin{array}{l}\text { Grass/groundcover } \\
\text { area }\left(\mathrm{m}^{2}\right)\end{array}$} & \multicolumn{2}{|c|}{$\begin{array}{l}\text { Turf/lawn area } \\
\left(\mathbf{m}^{2}\right)\end{array}$} & \multicolumn{2}{|c|}{ Shape } & \multicolumn{3}{|c|}{$\begin{array}{l}\text { Amount of leaves (density- } \\
\% \text { ) }\end{array}$} & \multicolumn{3}{|c|}{ Size of leaves (area-cm²) } \\
\hline 1 & Below 1 & 1 & Below 20 & 1 & Spreading & 1 & Few & Below 40 & 1 & Small & Below 150 \\
\hline 2 & $1-4$ & 2 & $20-49$ & 2 & Upright & 2 & Medium & $40-70$ & 2 & Medium & $150-1000$ \\
\hline 3 & $5-9$ & 3 & $50-99$ & 3 & Rounded & 3 & Dense & Above 70 & 3 & Large & Above 1000 \\
\hline 4 & $10-14$ & 4 & $100-149$ & 4 & Vase-shaped & & & & & & \\
\hline 5 & $15-19$ & 5 & $150-199$ & 5 & Sword leaf & & & & & & \\
\hline 6 & $20-24$ & 6 & $200-249$ & 6 & & & & & & & \\
\hline \multirow[t]{2}{*}{7} & 25 and above & 7 & $250-299$ & 7 & & & & & & & \\
\hline & & 8 & $\begin{array}{l}300 \text { and } \\
\text { above }\end{array}$ & & & & & & & & \\
\hline
\end{tabular}


vii. Shrubs:

\begin{tabular}{|c|c|c|c|c|c|c|c|c|c|}
\hline No. & Botanical name & Common name & Location/azimuth & $\begin{array}{l}\text { Shrubs } \\
\text { building } \\
\text { Distance } \\
\text { (m) }\end{array}$ & $\begin{array}{l}\text { Height } \\
\text { (m) }\end{array}$ & $\begin{array}{l}\text { Spread } \\
\left(\mathbf{m}^{2}\right)\end{array}$ & Shape & $\begin{array}{l}\text { Amount } \\
\text { of leaves }\end{array}$ & $\begin{array}{l}\text { Size of } \\
\text { leaves }\end{array}$ \\
\hline 1 & & & & & & & & & \\
\hline
\end{tabular}

viii. Palm (shrubs):

\begin{tabular}{|l|l|l|l|l|l|l|l|l|l|}
\hline No. & Botanical name & Common name & Location/azimuth & $\begin{array}{l}\text { Shrubs } \\
\text { building } \\
\text { Distance } \\
(\mathbf{m})\end{array}$ & $\begin{array}{l}\text { Height } \\
(\mathbf{m})\end{array}$ & $\begin{array}{l}\text { Spread } \\
\left(\mathbf{m}^{2}\right)\end{array}$ & $\begin{array}{l}\text { Shape } \\
\text { omount } \\
\text { of leaves }\end{array}$ & $\begin{array}{l}\text { Size of } \\
\text { leaves }\end{array}$ \\
\hline 1 & & & & & & & & \\
\hline
\end{tabular}

ix. Bamboo (shrubs)

\begin{tabular}{|l|l|l|l|l|l|l|l|l|l|}
\hline No. & Botanical name & Common name & Location/azimuth & $\begin{array}{l}\text { Shrubs } \\
\text { building } \\
\text { Distance } \\
(\mathbf{m})\end{array}$ & $\begin{array}{l}\text { Height } \\
(\mathbf{m})\end{array}$ & $\begin{array}{l}\text { Spread } \\
\left(\mathbf{m}^{2}\right)\end{array}$ & $\begin{array}{l}\text { Shape } \\
\text { Amount } \\
\text { of leaves }\end{array}$ & $\begin{array}{l}\text { Size of } \\
\text { leaves }\end{array}$ \\
\hline 1 & & & & & & & & \\
\hline
\end{tabular}

x. $\quad$ Edible (shrubs):

\begin{tabular}{|l|l|l|l|l|l|l|l|l|l|}
\hline No. & Botanical name & Common name & Location/azimuth & $\begin{array}{l}\text { Shrubs } \\
\text { building } \\
\text { Distance } \\
(\mathbf{m})\end{array}$ & $\begin{array}{l}\text { Height } \\
(\mathbf{m})\end{array}$ & $\begin{array}{l}\text { Spread } \\
\left(\mathbf{m}^{2}\right)\end{array}$ & $\begin{array}{l}\text { Shape } \\
\text { Amount } \\
\text { of leaves }\end{array}$ & $\begin{array}{l}\text { Size of } \\
\text { leaves }\end{array}$ \\
\hline 1 & & & & & & & & \\
\hline
\end{tabular}

xi. Garden Vines:

\begin{tabular}{|l|l|l|l|l|l|l|l|}
\hline No. & Botanical name & $\begin{array}{l}\text { Common } \\
\text { name }\end{array}$ & Location/azimuth & $\begin{array}{l}\text { Vines } \\
\text { building } \\
\text { Distance }(\mathbf{m})\end{array}$ & $\begin{array}{l}\text { Area/ } \\
\text { spread } \\
\left(\mathbf{m}^{2}\right)\end{array}$ & $\begin{array}{l}\text { Amount } \\
\text { of leaves }\end{array}$ & $\begin{array}{l}\text { Size of } \\
\text { leaves }\end{array}$ \\
\hline 1 & & & & & & \\
\hline
\end{tabular}

xii. Edible vines:

\begin{tabular}{|l|l|l|l|l|l|l|l|}
\hline No. & Botanical name & $\begin{array}{l}\text { Common } \\
\text { name }\end{array}$ & Location/azimuth & $\begin{array}{l}\text { Vines } \\
\text { building } \\
\text { Distance }(\mathbf{m})\end{array}$ & $\begin{array}{l}\text { Area/ } \\
\text { spread } \\
\left(\mathbf{m}^{2}\right)\end{array}$ & $\begin{array}{l}\text { Amount } \\
\text { of leaves }\end{array}$ & $\begin{array}{l}\text { Size of } \\
\text { leaves }\end{array}$ \\
\hline 1 & & & & & & \\
\hline
\end{tabular}


xiii. Grass/groundcovers:

\begin{tabular}{|l|l|l|l|l|l|}
\hline No. & Botanical name & Common name & $\begin{array}{l}\text { Location/ } \\
\text { azimuth }\end{array}$ & $\begin{array}{l}\text { Ground covers } \\
\text { building Distance } \\
(\mathbf{m})\end{array}$ & ${\text { Area }\left(\mathbf{m}^{2}\right)}^{2}$ \\
\hline 1 & & & & & \\
\hline
\end{tabular}

xiv. Turff/lawn :

\begin{tabular}{|l|l|l|l|}
\hline No. & Botanical name & Common name & Area $\left(\mathbf{m}^{2}\right)$ \\
\hline 1 & & & \\
\hline
\end{tabular}

Code for swimming pool and other landscape element

\begin{tabular}{|c|c|c|c|c|c|c|c|c|c|}
\hline \multicolumn{2}{|c|}{ Location/azimuth } & \multicolumn{2}{|c|}{ Distance (m) } & \multicolumn{2}{|c|}{$\begin{array}{l}\text { Swimming Pool size } \\
\left(\mathrm{m}^{2}\right)\end{array}$} & \multicolumn{2}{|c|}{$\begin{array}{l}\text { Other landscape } \\
\text { element size } \\
\left(\mathbf{m}^{2}\right)\end{array}$} & \multicolumn{2}{|c|}{$\begin{array}{l}\text { Landscape elements } \\
\text { material }\end{array}$} \\
\hline 1 & North & 1 & Below 5 & 1 & Below 100 & 1 & Below 5 & 1 & Wood/timber \\
\hline 2 & North East & 2 & $5-9$ & 2 & $100-190$ & 2 & $5-9$ & 2 & Steel \\
\hline 3 & East & 3 & $10-14$ & 3 & $200-249$ & 3 & $10-14$ & 3 & Concrete \\
\hline 4 & South East & 4 & $15-19$ & 4 & $250-299$ & 4 & $15-19$ & 4 & Plastic \\
\hline 5 & South & 5 & Above 20 & 5 & $300-349$ & 5 & $20-24$ & 5 & Bricks \\
\hline 6 & South West & & & 6 & $350-399$ & 6 & $25-29$ & 6 & Others \\
\hline 7 & West & & & 7 & $400-449$ & 7 & 30 and above & & \\
\hline 8 & North West & & & 8 & 500 and above & & & & \\
\hline
\end{tabular}

xv. Swimming Pool:

\begin{tabular}{|l|l|l|}
\hline No. & Size $\left(\mathbf{m}^{3}\right)$ & Location/azimuth \\
\hline 1 & & \\
\hline
\end{tabular}

xvi. Other landscape elements:

\begin{tabular}{|l|l|l|l|l|l|}
\hline No. & Landscape elements & Material & Location/azimuth & Distance (m) & Size $\left.\mathbf{( m}^{2}\right)$ \\
\hline 1 & Pergola & & & & \\
\hline 2 & Gazebo & & & & \\
\hline 3 & Fountain & & & & \\
\hline 4 & Fish pool & & & & \\
\hline 5 & 'Wakaf' & & & & \\
\hline
\end{tabular}


xi. Sketches roughly the position of the house and every landscape element and name it.

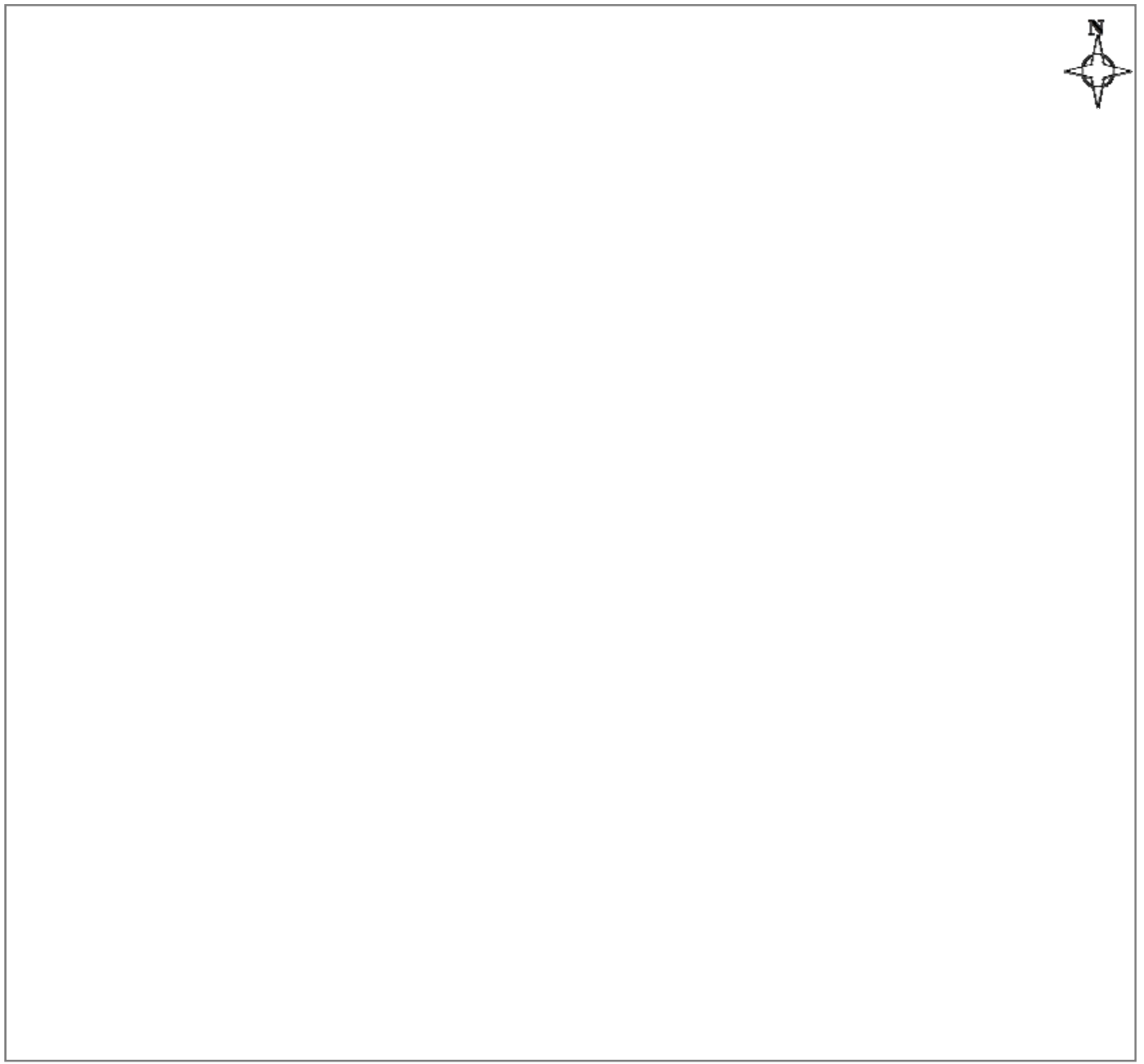

Scale 1:200 


\section{Appendix 4. Landscape elements around mature, ordinary and new landscape house}

Appendix 4.1. Location of ordinary landscape house in Section 11, Shah Alam, Selangor (Google Earth, 2011)

Appendix 4.2. Location of the new landscape house in Section 9 Shah Alam, Selangor (Google Earth, 2011) 348

Appendix 4.3. List of shrubs around the mature landscape house. 349

Appendix 4.4. List of shrubs around the ordinary landscape house 350

Appendix 4.5. List of shrubs around the new landscape house 352 Appendix 4.6. Example calculation of air-conditioning use in the ordinary landscape house 354 
Appendix 4.1. Location of ordinary landscape house in Section 11, Shah Alam,

Selangor (Google Earth, 2011)

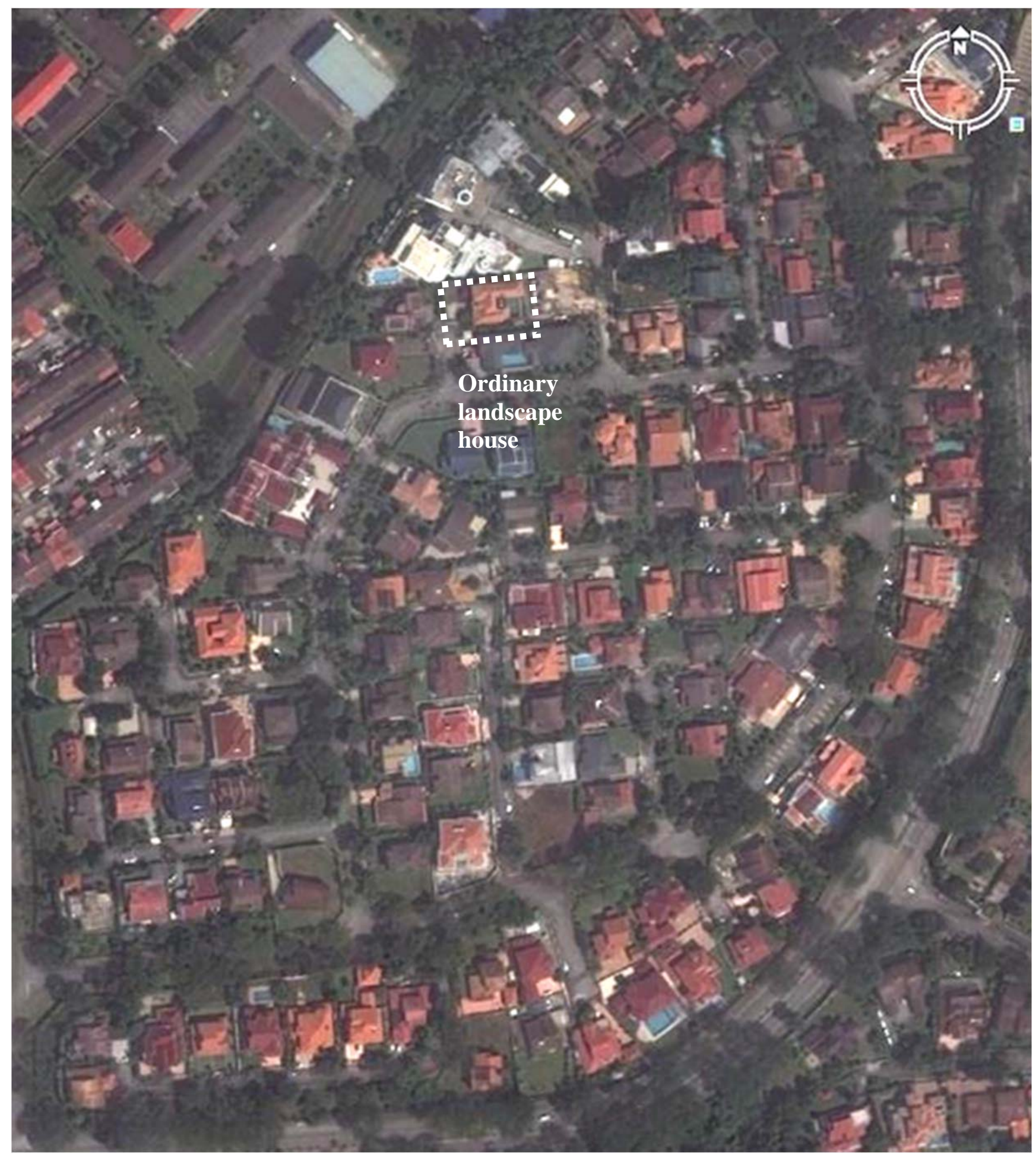


Appendix 4.2. Location of the new landscape house in Section 9 Shah Alam,

Selangor (Google Earth, 2011)

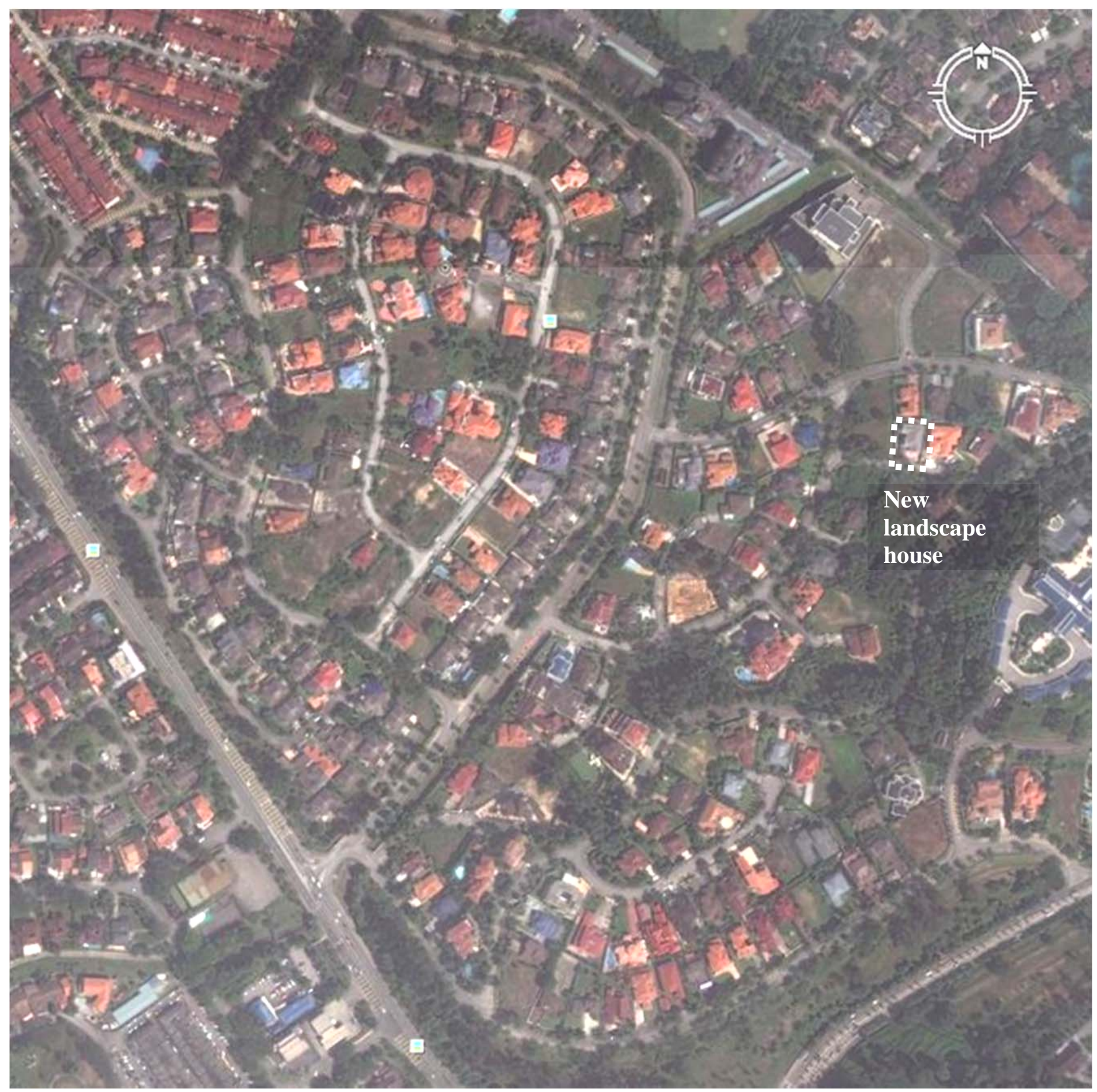


Appendix 4.3. List of shrubs around the mature landscape house

\begin{tabular}{|c|c|c|c|c|c|c|c|c|c|}
\hline \multirow[t]{2}{*}{ No. } & \multirow[t]{2}{*}{ Species } & \multirow[t]{2}{*}{ Type $^{\mathrm{a}}$} & \multirow[t]{2}{*}{ Azimuth $^{b}$} & \multirow{2}{*}{$\begin{array}{l}\text { Shrubs } \\
\text { building } \\
\text { distance }^{\mathrm{c}}\end{array}$} & \multicolumn{5}{|c|}{ Shrub-growth index/typology/biomass structure } \\
\hline & & & & & Height $^{\mathrm{d}}$ & Spread $^{\mathrm{e}}$ & Shape $^{f}$ & $\begin{array}{l}\text { Amount } \\
\text { of leaves }^{\mathrm{g}}\end{array}$ & $\begin{array}{l}\text { Size of } \\
\text { leaves }\end{array}$ \\
\hline 1 & Impatiens namchabarwensis & $\mathrm{F}$ & 7 & 3.00 & 2.00 & 2.00 & 2.00 & 1.00 & 1.00 \\
\hline 2 & Philodendron erubescens & $\mathrm{F}$ & 7 & 2.00 & 2.00 & 1.00 & 2.00 & 2.00 & 3.00 \\
\hline 3 & Jatropha integerrima & $\mathrm{F}$ & 7 & 2.00 & 2.00 & 2.00 & 1.00 & 2.00 & 2.00 \\
\hline 4 & Plumbago auriculata & $\mathrm{F}$ & 7 & 2.00 & 2.00 & 2.00 & 1.00 & 2.00 & 1.00 \\
\hline 5 & Tecomaria capensis & $\mathrm{F}$ & 7 & 2.00 & 2.00 & 2.00 & 1.00 & 2.00 & 1.00 \\
\hline 6 & Mussaenda flava & $\mathrm{F}$ & 7 & 2.00 & 2.00 & 2.00 & 1.00 & 2.00 & 1.00 \\
\hline 7 & Agave neglecta & $\mathrm{F}$ & 7 & 4.00 & 2.00 & 2.00 & 5.00 & 2.00 & 2.00 \\
\hline 8 & Turnera ulmifolia & $\mathrm{F}$ & 7 & 4.00 & 2.00 & 2.00 & 1.00 & 2.00 & 1.00 \\
\hline 9 & Lagerstroemia indica & $\mathrm{F}$ & 5 & 2.00 & 2.00 & 2.00 & 1.00 & 1.00 & 1.00 \\
\hline 10 & Canna sp. & $\mathrm{F}$ & 5 & 2.00 & 2.00 & 2.00 & 2.00 & 1.00 & 2.00 \\
\hline 11 & Cosmos caudatus & $\mathrm{F}$ & 5 & 2.00 & 2.00 & 2.00 & 2.00 & 1.00 & 1.00 \\
\hline 12 & Ixora sp. & $\mathrm{F}$ & 5 & 2.00 & 2.00 & 1.00 & 1.00 & 2.00 & 1.00 \\
\hline 13 & Lagerstroemia indica & $\mathrm{F}$ & 5 & 2.00 & 2.00 & 2.00 & 1.00 & 1.00 & 1.00 \\
\hline 14 & Hymenocallis littoralis & $\mathrm{F}$ & 5 & 3.00 & 2.00 & 2.00 & 5.00 & 3.00 & 2.00 \\
\hline 15 & Spathiphyllum cannifolium & $\mathrm{F}$ & 5 & 3.00 & 2.00 & 2.00 & 2.00 & 3.00 & 3.00 \\
\hline 16 & Duranta gold & $\mathrm{F}$ & 5 & 3.00 & 2.00 & 2.00 & 1.00 & 3.00 & 1.00 \\
\hline 17 & Turnera ulmifolia & $\mathrm{F}$ & 5 & 3.00 & 2.00 & 2.00 & 1.00 & 2.00 & 1.00 \\
\hline 18 & Orchid sp. & $\mathrm{F}$ & 5 & 3.00 & 2.00 & 2.00 & 2.00 & 1.00 & 1.00 \\
\hline 19 & Cordyline terminalis & $\mathrm{F}$ & 3 & 2.00 & 4.00 & 1.00 & 2.00 & 2.00 & 3.00 \\
\hline 20 & Dracaena fragrans & $\mathrm{F}$ & 3 & 2.00 & 4.00 & 1.00 & 2.00 & 2.00 & 2.00 \\
\hline 21 & Bougainvillea sp. & $\mathrm{F}$ & 3 & 2.00 & 3.00 & 1.00 & 1.00 & 1.00 & 1.00 \\
\hline 22 & Philodendron erubescens & $\mathrm{F}$ & 1 & 2.00 & 3.00 & 2.00 & 2.00 & 2.00 & 3.00 \\
\hline 23 & Draceana sp. & $\mathrm{F}$ & 1 & 2.00 & 3.00 & 1.00 & 2.00 & 3.00 & 1.00 \\
\hline 24 & Euphorbia Milli & $\mathrm{F}$ & 1 & 2.00 & 2.00 & 1.00 & 2.00 & 1.00 & 1.00 \\
\hline 25 & Hydrangea macrophylla & $\mathrm{F}$ & 1 & 2.00 & 2.00 & 2.00 & 2.00 & 2.00 & 2.00 \\
\hline 26 & Alocasia macrorrhiza & $\mathrm{F}$ & 1 & 2.00 & 2.00 & 1.00 & 2.00 & 1.00 & 2.00 \\
\hline 27 & Rhapis excelsa & $\mathrm{G}$ & 5 & 2.00 & 2.00 & 1.00 & 2.00 & 3.00 & 1.00 \\
\hline 28 & Rhapis excelsa & $\mathrm{G}$ & 5 & 2.00 & 2.00 & 1.00 & 2.00 & 3.00 & 1.00 \\
\hline 29 & Rhapis excelsa & $\mathrm{G}$ & 5 & 2.00 & 2.00 & 1.00 & 2.00 & 3.00 & 1.00 \\
\hline 30 & Ptychosperma macarthurii & $\mathrm{G}$ & 3 & 2.00 & 2.00 & 1.00 & 1.00 & 1.00 & 1.00 \\
\hline 31 & Dypsis lutescens & $\mathrm{G}$ & 1 & 2.00 & 2.00 & 1.00 & 2.00 & 3.00 & 1.00 \\
\hline 32 & Manilkara zapota & $\mathrm{H}$ & 3 & 2.00 & 6.00 & 1.00 & 1.00 & 1.00 & 1.00 \\
\hline 33 & Pandanus odorus & $\mathrm{H}$ & 3 & 2.00 & 2.00 & 2.00 & 5.00 & 3.00 & 1.00 \\
\hline 34 & Alpinia galanga & $\mathrm{H}$ & 3 & 2.00 & 5.00 & 2.00 & 1.00 & 2.00 & 3.00 \\
\hline 35 & Zingiber officinale & $\mathrm{H}$ & 3 & 2.00 & 5.00 & 2.00 & 2.00 & 2.00 & 2.00 \\
\hline 36 & Citrus hystrix & $\mathrm{H}$ & 3 & 2.00 & 3.00 & 1.00 & 1.00 & 1.00 & 1.00 \\
\hline 37 & Citrofortunella microcarpa & $\mathrm{H}$ & 3 & 2.00 & 3.00 & 1.00 & 1.00 & 1.00 & 1.00 \\
\hline 38 & Musa sp. & $\mathrm{H}$ & 3 & 2.00 & 3.00 & 1.00 & 2.00 & 1.00 & 2.00 \\
\hline & Total & & & 86.00 & 96.00 & 59.00 & 69.00 & 71.00 & 57.00 \\
\hline & Average & & & 2.26 & 2.53 & 1.55 & 1.82 & 1.87 & 1.50 \\
\hline
\end{tabular}

${ }^{\text {a }}$ Type of shrubs; $F=$ garden shrubs, $\mathrm{G}=$ palm shrubs, $\mathrm{H}=$ edible shrubs, $\mathrm{I}=$ =bamboo shrubs

b $1=$ North, $3=$ East, $5=$ South, $7=$ West

c $1=$ Proximity to walls, $2=$ below $5 \mathrm{~m}, 3=5-9 \mathrm{~m}, 4=10-14 \mathrm{~m}, 5=15-19 \mathrm{~m}$

${ }^{\mathrm{d}} 1=$ below $0.5 \mathrm{~m}, 2=0.5-0.9 \mathrm{~m}, 3.0=1.0-1.4 \mathrm{~m}, 4=1.5-1.9 \mathrm{~m}, 5=2.0-2.4 \mathrm{~m}$

e $1=$ individual/potted, $2=$ group below $5 \mathrm{~m}^{2}, 3=$ group $6-10 \mathrm{~m}^{2}, 4=$ group $10-20 \mathrm{~m}^{2}, 5=$ group $21-30 \mathrm{~m}^{2}$

${ }^{\mathrm{f}} 1=$ spreading, $2=$ upright, $3=$ rounded, $5=$ sword leaf.

g $1=$ rare, $2=$ medium, $3=$ dense

${ }^{\text {h }} 1=$ small, $2=$ medium, $3=$ large 
Appendix 4.4. List of shrubs around the ordinary landscape house

\begin{tabular}{|c|c|c|c|c|c|c|c|c|c|}
\hline \multirow[t]{2}{*}{ No. } & \multirow[t]{2}{*}{ Species } & \multirow[t]{2}{*}{ Type $^{\mathrm{a}}$} & \multirow[t]{2}{*}{ Azimuth $^{b}$} & \multirow{2}{*}{$\begin{array}{l}\text { Shrubs } \\
\text { building } \\
\text { distance }^{c}\end{array}$} & \multicolumn{5}{|c|}{ Shrub-growth index/typology/biomass structure } \\
\hline & & & & & Height $^{\mathrm{d}}$ & Spread $^{\mathrm{e}}$ & Shape ${ }^{f}$ & $\begin{array}{l}\text { Amount } \\
\text { of leaves }\end{array}$ & $\begin{array}{l}\text { Size of } \\
\text { leaves }\end{array}$ \\
\hline 1 & Phillodendron selloum & $\mathrm{F}$ & 7 & 2.00 & 2.00 & 2.00 & 1.00 & 2.00 & 3.00 \\
\hline 2 & Asplenium nidus & $\mathrm{F}$ & 7 & 2.00 & 2.00 & 1.00 & 5.00 & 2.00 & 3.00 \\
\hline 3 & Asplenium nidus & $\mathrm{F}$ & 7 & 2.00 & 2.00 & 1.00 & 5.00 & 2.00 & 3.00 \\
\hline 4 & Calathea ornata 'roseo lineata' & $\mathrm{F}$ & 7 & 2.00 & 4.00 & 4.00 & 2.00 & 3.00 & 3.00 \\
\hline 5 & Yucca elephantipes & $\mathrm{F}$ & 7 & 1.00 & 6.00 & 1.00 & 5.00 & 3.00 & 1.00 \\
\hline 6 & Yucca elephantipes & $\mathrm{F}$ & 7 & 1.00 & 6.00 & 1.00 & 5.00 & 3.00 & 1.00 \\
\hline 7 & Thalia geniculata & $\mathrm{F}$ & 7 & 2.00 & 3.00 & 1.00 & 2.00 & 2.00 & 2.00 \\
\hline 8 & Thalia geniculata & $\mathrm{F}$ & 7 & 2.00 & 3.00 & 1.00 & 2.00 & 2.00 & 2.00 \\
\hline 9 & Asparagus densiflorus myersii & $\mathrm{F}$ & 7 & 2.00 & 2.00 & 1.00 & 5.00 & 2.00 & 1.00 \\
\hline 10 & Asparagus densiflorus myersii & $\mathrm{F}$ & 7 & 2.00 & 2.00 & 1.00 & 5.00 & 2.00 & 1.00 \\
\hline 11 & Asparagus densiflorus myersii & F & 7 & 2.00 & 2.00 & 1.00 & 5.00 & 2.00 & 1.00 \\
\hline 12 & Cyperus alternifolius & F & 7 & 2.00 & 2.00 & 1.00 & 2.00 & 2.00 & 3.00 \\
\hline 13 & Bougainvillea sp. & $\mathrm{F}$ & 7 & 2.00 & 3.00 & 1.00 & 1.00 & 1.00 & 1.00 \\
\hline 14 & Neomarica longifolia & $\mathrm{F}$ & 7 & 1.00 & 2.00 & 3.00 & 5.00 & 2.00 & 1.00 \\
\hline 15 & Zingiber zerumbit & $\mathrm{F}$ & 7 & 2.00 & 1.00 & 2.00 & 2.00 & 2.00 & 1.00 \\
\hline 16 & Dracaena godseffiana & F & 7 & 2.00 & 3.00 & 1.00 & 2.00 & 2.00 & 1.00 \\
\hline 17 & Calathea lutea & $\mathrm{F}$ & 5 & 1.00 & 5.00 & 5.00 & 2.00 & 3.00 & 3.00 \\
\hline 18 & Musa ornata & $\mathrm{F}$ & 5 & 1.00 & 5.00 & 2.00 & 2.00 & 2.00 & 3.00 \\
\hline 19 & Pandanus tectorius & $\mathrm{F}$ & 5 & 1.00 & 3.00 & 2.00 & 5.00 & 2.00 & 2.00 \\
\hline 20 & Calathea ornate 'roseo lineata' & $\mathrm{F}$ & 5 & 2.00 & 4.00 & 4.00 & 2.00 & 3.00 & 3.00 \\
\hline 21 & Echinodorus cordifolius & $\mathrm{F}$ & 5 & 1.00 & 1.00 & 1.00 & 1.00 & 1.00 & 2.00 \\
\hline 22 & Pereskia bleo & $\mathrm{F}$ & 5 & 1.00 & 1.00 & 2.00 & 1.00 & 2.00 & 2.00 \\
\hline 23 & Alpinia galangal & $\mathrm{F}$ & 5 & 1.00 & 5.00 & 3.00 & 2.00 & 2.00 & 2.00 \\
\hline 24 & Cyperus alternifolius & $\mathrm{F}$ & 5 & 1.00 & 2.00 & 1.00 & 2.00 & 1.00 & 1.00 \\
\hline 25 & Thalia geniculata & $\mathrm{F}$ & 3 & 2.00 & 2.00 & 1.00 & 2.00 & 1.00 & 2.00 \\
\hline 26 & Alocasia macrorrhiza & $\mathrm{F}$ & 3 & 2.00 & 2.00 & 1.00 & 1.00 & 1.00 & 3.00 \\
\hline 27 & Spathiphyllum cannifolium & $\mathrm{F}$ & 3 & 2.00 & 2.00 & 1.00 & 1.00 & 1.00 & 3.00 \\
\hline 28 & Typha latifolia & F & 3 & 3.00 & 4.00 & 3.00 & 2.00 & 1.00 & 1.00 \\
\hline 29 & Thalia geniculata & $\mathrm{F}$ & 3 & 3.00 & 2.00 & 1.00 & 2.00 & 1.00 & 2.00 \\
\hline 30 & Thalia geniculata & $\mathrm{F}$ & 3 & 3.00 & 2.00 & 1.00 & 2.00 & 1.00 & 2.00 \\
\hline 31 & Thalia geniculata & $\mathrm{F}$ & 3 & 3.00 & 2.00 & 1.00 & 2.00 & 1.00 & 2.00 \\
\hline 32 & Plumeria rubra & $\mathrm{F}$ & 3 & 2.00 & 2.00 & 1.00 & 1.00 & 1.00 & 1.00 \\
\hline 33 & Hymenocallis littoralis & $\mathrm{F}$ & 3 & 2.00 & 1.00 & 2.00 & 5.00 & 1.00 & 1.00 \\
\hline 34 & Calathea ornate & $\mathrm{F}$ & 1 & 2.00 & 2.00 & 1.00 & 2.00 & 1.00 & 2.00 \\
\hline 35 & Dieffenbachia seguine & $\mathrm{F}$ & 1 & 2.00 & 2.00 & 1.00 & 2.00 & 2.00 & 3.00 \\
\hline 36 & Euphorbia milli & $\mathrm{F}$ & 1 & 2.00 & 2.00 & 1.00 & 2.00 & 1.00 & 1.00 \\
\hline 37 & Draceanea reflexa & $\mathrm{F}$ & 1 & 2.00 & 2.00 & 1.00 & 2.00 & 1.00 & 1.00 \\
\hline 38 & Hymenocallis liriosme & $\mathrm{F}$ & 1 & 2.00 & 2.00 & 2.00 & 3.00 & 3.00 & 3.00 \\
\hline 39 & Pandanus sanderi & $\mathrm{F}$ & 1 & 2.00 & 2.00 & 2.00 & 5.00 & 2.00 & 2.00 \\
\hline 40 & Cyperus alternifolius & $\mathrm{F}$ & 1 & 2.00 & 2.00 & 1.00 & 2.00 & 1.00 & 1.00 \\
\hline 41 & Echinodorus cordifolius & $\mathrm{F}$ & 1 & 2.00 & 1.00 & 1.00 & 1.00 & 1.00 & 2.00 \\
\hline 42 & Echinodorus cordifolius & $\mathrm{F}$ & 1 & 2.00 & 1.00 & 1.00 & 1.00 & 1.00 & 2.00 \\
\hline 43 & Rhapis excels & $\mathrm{G}$ & 1 & 2.00 & 4.00 & 4.00 & 2.00 & 2.00 & 1.00 \\
\hline 44 & Rhapis excels & $\mathrm{G}$ & 1 & 2.00 & 4.00 & 1.00 & 2.00 & 2.00 & 1.00 \\
\hline 45 & Musa parasidiaca & $\mathrm{H}$ & 3 & 1.00 & 6.00 & 3.00 & 1.00 & 3.00 & 3.00 \\
\hline 46 & Alpinia galangal & $\mathrm{H}$ & 3 & 1.00 & 5.00 & 3.00 & 1.00 & 3.00 & 3.00 \\
\hline 47 & Carica papaya & $\mathrm{H}$ & 3 & 2.00 & 3.00 & 1.00 & 1.00 & 1.00 & 2.00 \\
\hline 48 & Musa parasidiaca & $\mathrm{H}$ & 1 & 1.00 & 2.00 & 1.00 & 1.00 & 3.00 & 3.00 \\
\hline 49 & Morinda Citrifolia & $\mathrm{H}$ & 1 & 1.00 & 2.00 & 1.00 & 1.00 & 2.00 & 2.00 \\
\hline 50 & Citrofortunella microcarpa & $\mathrm{H}$ & 1 & 2.00 & 2.00 & 2.00 & 1.00 & 2.00 & 1.00 \\
\hline 51 & Curcuma longa & $\mathrm{H}$ & 1 & 2.00 & 2.00 & 1.00 & 2.00 & 2.00 & 3.00 \\
\hline 52 & Capsicum frutescens & $\mathrm{H}$ & 1 & 2.00 & 2.00 & 2.00 & 1.00 & 2.00 & 1.00 \\
\hline 53 & Solanum melongena & $\mathrm{H}$ & 1 & 2.00 & 2.00 & 1.00 & 1.00 & 1.00 & 3.00 \\
\hline 54 & Citrus Hystrix & $\mathrm{H}$ & 1 & 2.00 & 2.00 & 1.00 & 1.00 & 1.00 & 1.00 \\
\hline 55 & Zingiber officinale & $\mathrm{H}$ & 1 & 2.00 & 1.00 & 1.00 & 2.00 & 1.00 & 1.00 \\
\hline
\end{tabular}




\begin{tabular}{|c|l|c|c|c|c|c|c|c|c|}
\hline 56 & Moringa oleifera Lam & $\mathrm{H}$ & 1 & 2.00 & 5.00 & 1.00 & 1.00 & 2.00 & 1.00 \\
\hline 57 & Musa parasidiaca & $\mathrm{H}$ & 1 & 2.00 & 2.00 & 1.00 & 1.00 & 1.00 & 3.00 \\
\hline 58 & Bambusa vulgaris & $\mathrm{I}$ & 7 & 2.00 & 5.00 & 3.00 & 2.00 & 3.00 & 1.00 \\
\hline 59 & Phyllostachys sulphurea & $\mathrm{I}$ & 7 & 2.00 & 5.00 & 2.00 & 2.00 & 2.00 & 1.00 \\
\hline 60 & Phyllostachys sulphurea & $\mathrm{I}$ & 1 & 1.00 & 5.00 & 2.00 & 2.00 & 2.00 & 1.00 \\
\hline 61 & Bambusa vulgaris & $\mathrm{I}$ & 5 & 3.00 & 5.00 & 3.00 & 2.00 & 3.00 & 1.00 \\
\hline & Total & & & 112.00 & 170.00 & 100.00 & 136.00 & 110.00 & 113.00 \\
\hline & Average & & & 1.84 & 2.79 & 1.64 & 2.23 & 1.80 & 1.85 \\
\hline
\end{tabular}

${ }^{\text {a }}$ Type of shrubs; $F=$ garden shrubs, $\mathrm{G}=$ palm shrubs, $\mathrm{H}=$ =edible shrubs, $\mathrm{I}=$ bamboo shrubs

b $1=$ North, $3=$ East, $5=$ South, $7=$ West

c $1=$ Proximity to walls, $2=$ below $5 \mathrm{~m}, 3=5-9 \mathrm{~m}, 4=10-14 \mathrm{~m}, 5=15-19 \mathrm{~m}$

d $1=$ below $0.5 \mathrm{~m}, 2=0.5-0.9 \mathrm{~m}, 3.0=1.0-1.4 \mathrm{~m}, 4=1.5-1.9 \mathrm{~m}, 5=2.0-2.4 \mathrm{~m}$

e $1=$ individual/potted, $2=$ group below $5 \mathrm{~m}^{2}, 3=$ group $6-10 \mathrm{~m}^{2}, 4=$ group $10-20 \mathrm{~m}^{2}, 5=$ group $21-30 \mathrm{~m}^{2}$

${ }^{\mathrm{f}} 1=$ spreading, $2=$ upright, $3=$ rounded, $5=$ sword leaf.

g $1=$ rare, $2=$ medium, $3=$ dense

${ }^{\mathrm{h}} 1=$ small, $2=$ medium, $3=$ large 
Appendix 4.5. List of shrubs around the new landscape house

\begin{tabular}{|c|c|c|c|c|c|c|c|c|c|}
\hline \multirow[t]{2}{*}{ No. } & \multirow[t]{2}{*}{ Species } & \multirow[t]{2}{*}{ Type $^{a}$} & \multirow[t]{2}{*}{ Azimuth $^{b}$} & \multirow{2}{*}{$\begin{array}{l}\text { Shrubs } \\
\text { building } \\
\text { distance }^{\mathrm{c}}\end{array}$} & \multicolumn{5}{|c|}{ Shrub-growth index/typology/biomass structure } \\
\hline & & & & & Height $^{\mathrm{d}}$ & Spread $^{\mathrm{e}}$ & Shape $^{f}$ & $\begin{array}{l}\text { Amount } \\
\text { of leaves }\end{array}$ & $\begin{array}{l}\text { Size of } \\
\text { leaves }\end{array}$ \\
\hline 1 & Plumeria rubra & $\mathrm{F}$ & 5 & 3.00 & 3.00 & 1.00 & 1.00 & 2.00 & 2.00 \\
\hline 2 & Hibiscus sp. & F & 5 & 3.00 & 2.00 & 1.00 & 3.00 & 3.00 & 1.00 \\
\hline 3 & Yucca sp. & $\mathrm{F}$ & 5 & 3.00 & 3.00 & 1.00 & 5.00 & 2.00 & 2.00 \\
\hline 4 & Furcraea gigantea & F & 5 & 3.00 & 2.00 & 1.00 & 5.00 & 2.00 & 3.00 \\
\hline 5 & Yucca elephantipes & $\mathrm{F}$ & 5 & 3.00 & 3.00 & 1.00 & 5.00 & 2.00 & 2.00 \\
\hline 6 & Pandanus sanderi & $\mathrm{F}$ & 5 & 3.00 & 2.00 & 1.00 & 5.00 & 1.00 & 1.00 \\
\hline 7 & Phillodendron selloum & $\mathrm{F}$ & 5 & 1.00 & 3.00 & 1.00 & 1.00 & 3.00 & 3.00 \\
\hline 8 & Bougainvillea sp. & $\mathrm{F}$ & 5 & 2.00 & 2.00 & 1.00 & 2.00 & 1.00 & 1.00 \\
\hline 9 & Bougainvillea sp. & $\mathrm{F}$ & 5 & 2.00 & 2.00 & 1.00 & 2.00 & 1.00 & 1.00 \\
\hline 10 & Licuala grandis & $\mathrm{F}$ & 5 & 2.00 & 4.00 & 1.00 & 3.00 & 3.00 & 3.00 \\
\hline 11 & Heliconia agusta & $\mathrm{F}$ & 5 & 2.00 & 4.00 & 3.00 & 2.00 & 3.00 & 3.00 \\
\hline 12 & Adianthum trapeziforme & $\mathrm{F}$ & 5 & 2.00 & 2.00 & 1.00 & 1.00 & 2.00 & 1.00 \\
\hline 13 & Sannseviera Trifaciata & F & 5 & 2.00 & 1.00 & 2.00 & 3.00 & 2.00 & 2.00 \\
\hline 14 & Dracaena fragrans & $\mathrm{F}$ & 5 & 2.00 & 2.00 & 1.00 & 3.00 & 2.00 & 3.00 \\
\hline 15 & Bougainvillea sp. & $\mathrm{F}$ & 5 & 2.00 & 2.00 & 1.00 & 1.00 & 1.00 & 1.00 \\
\hline 16 & Calathea lutea & $\mathrm{F}$ & 5 & 3.00 & 2.00 & 3.00 & 2.00 & 3.00 & 3.00 \\
\hline 17 & Alpinia purpurata & $\mathrm{F}$ & 5 & 3.00 & 2.00 & 3.00 & 1.00 & 2.00 & 3.00 \\
\hline 18 & Plectranthus scutellarioides & $\mathrm{F}$ & 5 & 2.00 & 1.00 & 2.00 & 1.00 & 2.00 & 2.00 \\
\hline 19 & Bougainvillea sp. & $\mathrm{F}$ & 3 & 2.00 & 2.00 & 4.00 & 1.00 & 1.00 & 1.00 \\
\hline 20 & Hibiscus sp. & $\mathrm{F}$ & 3 & 1.00 & 2.00 & 4.00 & 3.00 & 3.00 & 2.00 \\
\hline 21 & Calathea lutea & $\mathrm{F}$ & 3 & 1.00 & 2.00 & 1.00 & 2.00 & 3.00 & 3.00 \\
\hline 22 & Thalia geniculata & $\mathrm{F}$ & 3 & 2.00 & 1.00 & 1.00 & 3.00 & 2.00 & 2.00 \\
\hline 23 & Jatropha integerrima & $\mathrm{F}$ & 3 & 2.00 & 1.00 & 1.00 & 1.00 & 1.00 & 2.00 \\
\hline 24 & Juniperus chinensis & $\mathrm{F}$ & 3 & 2.00 & 1.00 & 1.00 & 2.00 & 1.00 & 1.00 \\
\hline 25 & Ixora sunkist & $\mathrm{F}$ & 3 & 2.00 & 1.00 & 3.00 & 2.00 & 1.00 & 1.00 \\
\hline 26 & Orthosiphon stamineus & $\mathrm{F}$ & 7 & 1.00 & 2.00 & 4.00 & 1.00 & 1.00 & 1.00 \\
\hline 27 & Ficus benjamina & $\mathrm{F}$ & 7 & 2.00 & 2.00 & 3.00 & 1.00 & 1.00 & 1.00 \\
\hline 28 & Bougainvillea sp. & F & 7 & 2.00 & 1.00 & 3.00 & 1.00 & 1.00 & 1.00 \\
\hline 29 & Murraya Paniculata & $\mathrm{F}$ & 7 & 2.00 & 4.00 & 1.00 & 2.00 & 3.00 & 2.00 \\
\hline 30 & Dracaena fragrans & $\mathrm{F}$ & 7 & 1.00 & 3.00 & 1.00 & 3.00 & 3.00 & 3.00 \\
\hline 31 & Crossandra infundibuliformis lutea & $\mathrm{F}$ & 7 & 1.00 & 2.00 & 3.00 & 3.00 & 2.00 & 2.00 \\
\hline 32 & Calathea lutea & F & 7 & 1.00 & 2.00 & 1.00 & 2.00 & 2.00 & 3.00 \\
\hline 33 & Pandanus sp. & $\mathrm{F}$ & 7 & 1.00 & 2.00 & 1.00 & 5.00 & 2.00 & 3.00 \\
\hline 34 & Phillodendron selloum & $\mathrm{F}$ & 7 & 1.00 & 1.00 & 1.00 & 1.00 & 2.00 & 3.00 \\
\hline 35 & Ixora Sunkist & $\mathrm{F}$ & 7 & 2.00 & 2.00 & 4.00 & 3.00 & 1.00 & 1.00 \\
\hline 36 & Tecoma stans & $\mathrm{F}$ & 7 & 1.00 & 2.00 & 3.00 & 3.00 & 1.00 & 1.00 \\
\hline 37 & Licuala grandis & $\mathrm{G}$ & 5 & 1.00 & 4.00 & 3.00 & 3.00 & 3.00 & 3.00 \\
\hline 38 & Rhapis excelsa & $\mathrm{G}$ & 5 & 1.00 & 4.00 & 3.00 & 2.00 & 3.00 & 2.00 \\
\hline 39 & Rhapis excelsa & $\mathrm{G}$ & 5 & 2.00 & 4.00 & 3.00 & 2.00 & 3.00 & 2.00 \\
\hline 40 & Licuala grandis & $\mathrm{G}$ & 5 & 2.00 & 4.00 & 3.00 & 3.00 & 3.00 & 3.00 \\
\hline 41 & Licuala grandis & $\mathrm{G}$ & 7 & 3.00 & 4.00 & 3.00 & 3.00 & 3.00 & 3.00 \\
\hline 42 & Cyrtostachys renda & $\mathrm{G}$ & 7 & 3.00 & 4.00 & 1.00 & 2.00 & 2.00 & 2.00 \\
\hline 43 & Rhapis excelsa & $\mathrm{G}$ & 7 & 3.00 & 4.00 & 3.00 & 2.00 & 2.00 & 2.00 \\
\hline 44 & Rhapis excelsa & $\mathrm{G}$ & 7 & 1.00 & 2.00 & 3.00 & 2.00 & 2.00 & 2.00 \\
\hline 45 & Cyrtostachys renda & $\mathrm{G}$ & 7 & 3.00 & 4.00 & 1.00 & 2.00 & 2.00 & 2.00 \\
\hline 46 & Licuala grandis & $\mathrm{G}$ & 7 & 1.00 & 4.00 & 3.00 & 3.00 & 3.00 & 3.00 \\
\hline 47 & Rhapis excels & $\mathrm{G}$ & 7 & 1.00 & 2.00 & 3.00 & 2.00 & 2.00 & 2.00 \\
\hline 48 & Carica papaya & $\mathrm{H}$ & 1 & 2.00 & 2.00 & 1.00 & 1.00 & 2.00 & 3.00 \\
\hline 49 & Musa parasidiaca & $\mathrm{H}$ & 1 & 2.00 & 5.00 & 3.00 & 3.00 & 3.00 & 3.00 \\
\hline 50 & Justicia gendarussa & $\mathrm{H}$ & 1 & 2.00 & 1.00 & 1.00 & 3.00 & 1.00 & 2.00 \\
\hline 51 & Pandanus odorus & $\mathrm{H}$ & 1 & 2.00 & 1.00 & 1.00 & 5.00 & 1.00 & 2.00 \\
\hline 52 & Saccharum officinarum & $\mathrm{H}$ & 1 & 2.00 & 2.00 & 1.00 & 2.00 & 1.00 & 1.00 \\
\hline 53 & Orthosiphon stamineus & $\mathrm{H}$ & 1 & 2.00 & 1.00 & 1.00 & 3.00 & 1.00 & 1.00 \\
\hline 54 & Syzygium jambos & $\mathrm{H}$ & 1 & 2.00 & 2.00 & 1.00 & 2.00 & 1.00 & 2.00 \\
\hline 55 & Cymbopogon flexuosus & $\mathrm{H}$ & 1 & 2.00 & 1.00 & 1.00 & 3.00 & 1.00 & 2.00 \\
\hline
\end{tabular}




\begin{tabular}{|c|l|c|c|c|c|c|c|c|c|}
\hline 56 & Ipomoea aquatica & $\mathrm{H}$ & 1 & 2.00 & 1.00 & 1.00 & 2.00 & 1.00 & 1.00 \\
\hline 57 & Mangifera indica & $\mathrm{H}$ & 1 & 2.00 & 4.00 & 1.00 & 2.00 & 3.00 & 2.00 \\
\hline 58 & Morinda citrifolia & $\mathrm{H}$ & 1 & 2.00 & 4.00 & 1.00 & 1.00 & 1.00 & 2.00 \\
\hline 59 & Citrofortunella microcarpa & $\mathrm{H}$ & 7 & 2.00 & 3.00 & 1.00 & 1.00 & 2.00 & 1.00 \\
\hline 60 & Mangifera indica & $\mathrm{H}$ & 7 & 2.00 & 4.00 & 1.00 & 2.00 & 3.00 & 2.00 \\
\hline 61 & Bambusa multiplex & $\mathrm{I}$ & 3 & 2.00 & 1.00 & 1.00 & 2.00 & 2.00 & 1.00 \\
\hline 62 & Bambusa multiplex & $\mathrm{I}$ & 3 & 2.00 & 2.00 & 1.00 & 2.00 & 2.00 & 1.00 \\
\hline 63 & Bambusa multiplex & $\mathrm{I}$ & 3 & 2.00 & 2.00 & 1.00 & 2.00 & 2.00 & 1.00 \\
\hline & Total & & & 123.00 & 151.00 & 113.00 & 149.00 & 123.00 & 123.00 \\
\hline & Average & & & 1.95 & 2.40 & 1.79 & 2.33 & 1.95 & 1.95 \\
\hline
\end{tabular}

${ }^{a}$ Type of shrubs; $\mathrm{F}=$ garden shrubs, $\mathrm{G}=$ palm shrubs, $\mathrm{H}=$ =edible shrubs, $\mathrm{I}=$ bamboo shrubs

${ }^{\mathrm{b}} 1=$ North, $3=$ East, $5=$ South, $7=$ West

c $1=$ Proximity to walls, $2=$ below $5 \mathrm{~m}, 3=5-9 \mathrm{~m}, 4=10-14 \mathrm{~m}, 5=15-19 \mathrm{~m}$

${ }^{\mathrm{d}} 1=$ below $0.5 \mathrm{~m}, 2=0.5-0.9 \mathrm{~m}, 3.0=1.0-1.4 \mathrm{~m}, 4=1.5-1.9 \mathrm{~m}, 5=2.0-2.4 \mathrm{~m}$

${ }^{\mathrm{e}} 1=$ individual/potted, $2=$ group below $5 \mathrm{~m}^{2}, 3=$ group $6-10 \mathrm{~m}^{2}, 4=$ group $10-20 \mathrm{~m}^{2}, 5=$ group $21-30 \mathrm{~m}^{2}$

${ }^{\mathrm{f}} 1=$ =spreading, $2=$ upright, $3=$ rounded, $5=$ sword leaf.

${ }^{\mathrm{g}} 1=$ rare, $2=$ medium, $3=$ dense

${ }^{\mathrm{h}} 1=$ small, $2=$ medium, $3=$ large 
Appendix 4.6. Example calculation of air-conditioning use in the ordinary landscape house

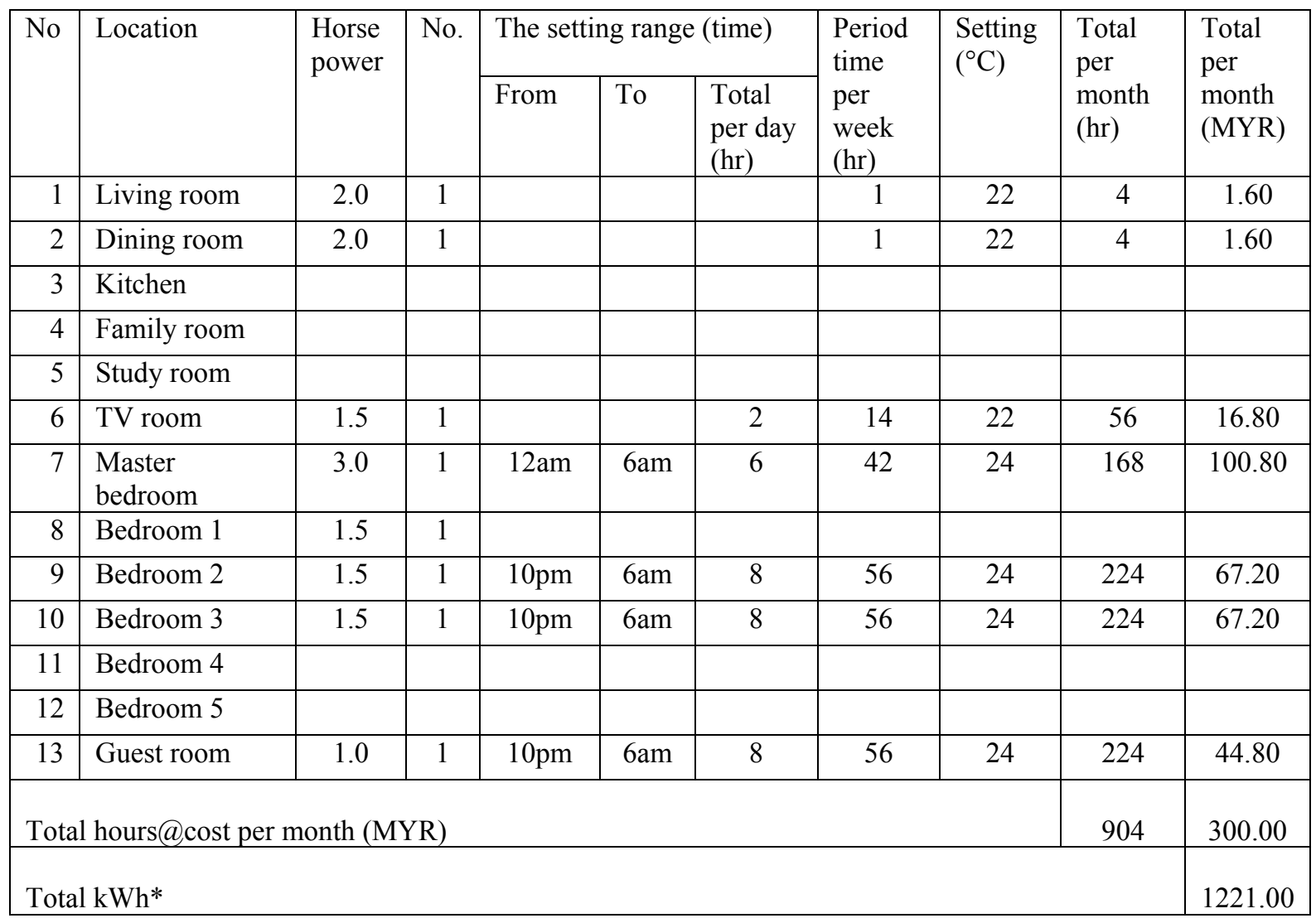

Note (Hariri, 2010):

d. Costs for horsepower per hour (1 horsepower $=$ MYR 0.20)

Example:

1. 1 horsepower per hour $=$ MYR 0.20

$2 \quad 1.5$ horsepower per hour $=$ MYR 0.30

3. 2 horsepower per hour $=$ MYR 0.40

4. 2.5 horsepower per hour $=$ MYR 0.50

5. 3 horsepower per hour $=$ MYR 0.60

e. Total per month $(\mathrm{MYR})=($ Total hour per month) $\mathrm{x}$ (costs per horsepower)

f. 1 horsepower $=0.7457 \mathrm{kWh}$

g. $\quad$ Total $\mathrm{kWh} *=$ Total MYR/0.2457) 


\section{Appendix 5. Landscape elements around heavily and sparsely landscaped house}

Appendix 5.1. List of shrubs in around the heavily landscaped house ................... 356

Appendix 5.2. List of shrubs in around the sparsely landscaped house.................. 358

Appendix 5.3. Calculation of air-conditioning uses in the heavily landscaped house

Appendix 5.4. Calculation of air-conditioning uses in the sparsely landscaped house 360 


\section{Appendix 5.1. List of shrubs in around the heavily landscaped house}

\begin{tabular}{|c|c|c|c|c|c|c|c|c|c|}
\hline \multirow[t]{2}{*}{ No. } & \multirow[t]{2}{*}{ Species } & \multirow[t]{2}{*}{ Type $^{a}$} & \multirow[t]{2}{*}{ Azimuth $^{b}$} & \multirow{2}{*}{$\begin{array}{l}\text { Shrubs } \\
\text { building } \\
\text { distance }^{\mathrm{c}}\end{array}$} & \multicolumn{5}{|c|}{ Shrub-growth index/typology/biomass structure } \\
\hline & & & & & Height $^{\mathrm{d}}$ & Spread $^{\mathrm{e}}$ & Shape $\mathrm{f}^{\mathrm{f}}$ & $\begin{array}{l}\text { Amount } \\
\text { of leaves }\end{array}$ & $\begin{array}{l}\text { Size of } \\
\text { leaves }\end{array}$ \\
\hline 1 & Hymenocallis littoralis & $\mathrm{F}$ & 5 & 3.00 & 1.00 & 4.00 & 5.00 & 3.00 & 1.00 \\
\hline 2 & Melastoma malabathricum & $\mathrm{F}$ & 5 & 3.00 & 3.00 & 2.00 & 1.00 & 2.00 & 1.00 \\
\hline 3 & Bougainvillea sp. & $\mathrm{F}$ & 5 & 3.00 & 2.00 & 1.00 & 1.00 & 2.00 & 1.00 \\
\hline 4 & Aster dumosus & $\mathrm{F}$ & 5 & 3.00 & 2.00 & 2.00 & 2.00 & 3.00 & 1.00 \\
\hline 5 & Bougainvillea sp. & $\mathrm{F}$ & 5 & 3.00 & 2.00 & 2.00 & 1.00 & 2.00 & 1.00 \\
\hline 6 & Rosa sp. & $\mathrm{F}$ & 5 & 3.00 & 2.00 & 1.00 & 1.00 & 2.00 & 3.00 \\
\hline 7 & Rosa sp. & $\mathrm{F}$ & 5 & 3.00 & 2.00 & 1.00 & 1.00 & 2.00 & 3.00 \\
\hline 8 & Thevetia peruviana & $\mathrm{F}$ & 5 & 3.00 & 1.00 & 3.00 & 1.00 & 2.00 & 1.00 \\
\hline 9 & Centella asiatica & $\mathrm{F}$ & 5 & 2.00 & 1.00 & 1.00 & 2.00 & 2.00 & 1.00 \\
\hline 10 & Eugenia oleina & $\mathrm{F}$ & 5 & 2.00 & 2.00 & 4.00 & 3.00 & 3.00 & 1.00 \\
\hline 11 & Eugenia oleina & $\mathrm{F}$ & 5 & 1.00 & 2.00 & 3.00 & 3.00 & 3.00 & 1.00 \\
\hline 12 & Calathea lutea & $\mathrm{F}$ & 5 & 1.00 & 3.00 & 2.00 & 2.00 & 2.00 & 3.00 \\
\hline 13 & Nephrolepis biserrata & $\mathrm{F}$ & 5 & 1.00 & 3.00 & 1.00 & 5.00 & 3.00 & 1.00 \\
\hline 14 & Nephrolepis biserrata & $\mathrm{F}$ & 5 & 1.00 & 3.00 & 1.00 & 5.00 & 2.00 & 1.00 \\
\hline 15 & Bougainvillea sp. & $\mathrm{F}$ & 5 & 2.00 & 2.00 & 2.00 & 1.00 & 2.00 & 1.00 \\
\hline 16 & Nymphaea sp. & $\mathrm{F}$ & 5 & 2.00 & 1.00 & 1.00 & 2.00 & 1.00 & 3.00 \\
\hline 17 & Adianthum raddianum & $\mathrm{F}$ & 5 & 2.00 & 1.00 & 2.00 & 1.00 & 2.00 & 1.00 \\
\hline 18 & Torenia fournieri & $\mathrm{F}$ & 5 & 2.00 & 2.00 & 2.00 & 2.00 & 2.00 & 1.00 \\
\hline 19 & Nephrolepis biserrata & $\mathrm{F}$ & 5 & 1.00 & 1.00 & 1.00 & 5.00 & 2.00 & 1.00 \\
\hline 20 & Thevetia peruviana & $\mathrm{F}$ & 5 & 1.00 & 1.00 & 2.00 & 1.00 & 2.00 & 1.00 \\
\hline 21 & Zamia furfuracea & $\mathrm{F}$ & 5 & 1.00 & 1.00 & 1.00 & 2.00 & 2.00 & 1.00 \\
\hline 22 & Pogonatherum paniceum & $\mathrm{F}$ & 5 & 2.00 & 2.00 & 1.00 & 3.00 & 3.00 & 1.00 \\
\hline 23 & Ixora caesia & $\mathrm{F}$ & 7 & 1.00 & 4.00 & 1.00 & 1.00 & 2.00 & 2.00 \\
\hline 24 & Heliconia rostrata & $\mathrm{F}$ & 7 & 1.00 & 4.00 & 3.00 & 2.00 & 2.00 & 1.00 \\
\hline 25 & Costus speciosus & $\mathrm{F}$ & 7 & 1.00 & 4.00 & 1.00 & 1.00 & 2.00 & 2.00 \\
\hline 26 & Adenium obesum & $\mathrm{F}$ & 7 & 1.00 & 2.00 & 1.00 & 1.00 & 1.00 & 1.00 \\
\hline 27 & Calathea luthea & $\mathrm{F}$ & 7 & 1.00 & 3.00 & 1.00 & 2.00 & 1.00 & 2.00 \\
\hline 28 & Jasminum sp. & $\mathrm{F}$ & 7 & 1.00 & 3.00 & 1.00 & 3.00 & 3.00 & 1.00 \\
\hline 29 & Alocasia macrorrhiza & $\mathrm{F}$ & 7 & 1.00 & 2.00 & 2.00 & 2.00 & 2.00 & 3.00 \\
\hline 30 & Ixora caesia & $\mathrm{F}$ & 7 & 2.00 & 4.00 & 2.00 & 1.00 & 2.00 & 2.00 \\
\hline 31 & Eugenia oleina & $\mathrm{F}$ & 7 & 2.00 & 2.00 & 4.00 & 3.00 & 3.00 & 1.00 \\
\hline 32 & Melastoma malabathricum & $\mathrm{F}$ & 7 & 2.00 & 3.00 & 2.00 & 1.00 & 2.00 & 1.00 \\
\hline 33 & Cyathea latebrosa & $\mathrm{F}$ & 7 & 2.00 & 3.00 & 1.00 & 2.00 & 2.00 & 1.00 \\
\hline 34 & Jasminum sp. & $\mathrm{F}$ & 7 & 2.00 & 2.00 & 1.00 & 2.00 & 2.00 & 1.00 \\
\hline 35 & Streblus asper & $\mathrm{F}$ & 7 & 2.00 & 3.00 & 1.00 & 3.00 & 1.00 & 1.00 \\
\hline 36 & Loropetalum chinense & $\mathrm{F}$ & 7 & 2.00 & 3.00 & 1.00 & 1.00 & 2.00 & 1.00 \\
\hline 37 & Cananga odorata & $\mathrm{F}$ & 7 & 2.00 & 2.00 & 1.00 & 3.00 & 2.00 & 2.00 \\
\hline 38 & Acalypha wilkensiana & $\mathrm{F}$ & 3 & 2.00 & 2.00 & 4.00 & 3.00 & 2.00 & 2.00 \\
\hline 39 & Bougainvillea sp. & $\mathrm{F}$ & 3 & 2.00 & 2.00 & 1.00 & 1.00 & 2.00 & 1.00 \\
\hline 40 & Cordyline terminalis & $\mathrm{F}$ & 3 & 2.00 & 2.00 & 1.00 & 1.00 & 2.00 & 1.00 \\
\hline 41 & Heliconia rostrata & $\mathrm{F}$ & 3 & 2.00 & 3.00 & 1.00 & 2.00 & 1.00 & 1.00 \\
\hline 42 & Sannseviera trifaciata & $\mathrm{F}$ & 3 & 2.00 & 1.00 & 1.00 & 2.00 & 1.00 & 1.00 \\
\hline 43 & Heliconia rostrata & $\mathrm{F}$ & 3 & 2.00 & 3.00 & 2.00 & 2.00 & 1.00 & 1.00 \\
\hline 44 & Gardenia jeminoides & $\mathrm{F}$ & 3 & 2.00 & 2.00 & 1.00 & 1.00 & 1.00 & 1.00 \\
\hline 45 & Musa ornata & $\mathrm{F}$ & 3 & 2.00 & 3.00 & 2.00 & 2.00 & 2.00 & 2.00 \\
\hline 46 & Calliandra emarginata & $\mathrm{F}$ & 3 & 2.00 & 3.00 & 1.00 & 1.00 & 1.00 & 1.00 \\
\hline 47 & Tecoma stans & $\mathrm{F}$ & 3 & 2.00 & 2.00 & 2.00 & 2.00 & 1.00 & 1.00 \\
\hline 48 & Orchid sp. & $\mathrm{F}$ & 3 & 1.00 & 3.00 & 1.00 & 5.00 & 2.00 & 1.00 \\
\hline 49 & Clerodendrum thomsoniae & $\mathrm{F}$ & 3 & 1.00 & 2.00 & 1.00 & 2.00 & 2.00 & 1.00 \\
\hline 50 & Cleome sparkler & $\mathrm{F}$ & 3 & 1.00 & 2.00 & 1.00 & 2.00 & 1.00 & 1.00 \\
\hline 51 & Authorium plowmanii & $\mathrm{F}$ & 3 & 1.00 & 2.00 & 1.00 & 5.00 & 2.00 & 3.00 \\
\hline 52 & Adenium obesum & $\mathrm{F}$ & 3 & 1.00 & 2.00 & 1.00 & 1.00 & 1.00 & 1.00 \\
\hline 53 & Caladium sp. & $\mathrm{F}$ & 3 & 1.00 & 1.00 & 1.00 & 2.00 & 2.00 & 2.00 \\
\hline 54 & Cycas sp. & $\mathrm{F}$ & 3 & 1.00 & 2.00 & 1.00 & 5.00 & 1.00 & 1.00 \\
\hline 55 & Cyperus alternifolius & $\mathrm{F}$ & 3 & 1.00 & 2.00 & 1.00 & 2.00 & 1.00 & 1.00 \\
\hline
\end{tabular}




\begin{tabular}{|c|c|c|c|c|c|c|c|c|c|}
\hline 56 & Costus woodsonii & $\mathrm{F}$ & 3 & 2.00 & 2.00 & 1.00 & 2.00 & 2.00 & 1.00 \\
\hline 57 & Nymphaea sp. & $\mathrm{F}$ & 1 & 2.00 & 2.00 & 1.00 & 2.00 & 2.00 & 3.00 \\
\hline 58 & Nymphaea sp. & $\mathrm{F}$ & 1 & 2.00 & 2.00 & 1.00 & 2.00 & 1.00 & 3.00 \\
\hline 59 & Jasminum sp. & $\mathrm{F}$ & 1 & 2.00 & 4.00 & 1.00 & 3.00 & 3.00 & 1.00 \\
\hline 60 & Jasminum sp. & $\mathrm{F}$ & 1 & 2.00 & 4.00 & 1.00 & 3.00 & 3.00 & 1.00 \\
\hline 61 & Nephrolepis biserrata & $\mathrm{F}$ & 1 & 1.00 & 1.00 & 2.00 & 5.00 & 3.00 & 1.00 \\
\hline 62 & Adianthum raddianum & $\mathrm{F}$ & 1 & 1.00 & 2.00 & 2.00 & 1.00 & 3.00 & 1.00 \\
\hline 63 & Ficus benjamina & $\mathrm{F}$ & 1 & 1.00 & 4.00 & 1.00 & 3.00 & 3.00 & 1.00 \\
\hline 64 & Schefflera arboricola & $\mathrm{F}$ & 1 & 1.00 & 4.00 & 1.00 & 2.00 & 3.00 & 3.00 \\
\hline 65 & Rubia ornamentale & $\mathrm{F}$ & 1 & 1.00 & 1.00 & 1.00 & 1.00 & 1.00 & 1.00 \\
\hline 66 & Calathea luthea & $\mathrm{F}$ & 1 & 1.00 & 2.00 & 2.00 & 2.00 & 2.00 & 3.00 \\
\hline 67 & Blechnum oriental & $\mathrm{F}$ & 1 & 1.00 & 1.00 & 1.00 & 2.00 & 1.00 & 1.00 \\
\hline 68 & Christella parasitica & $\mathrm{F}$ & 1 & 1.00 & 1.00 & 1.00 & 2.00 & 1.00 & 1.00 \\
\hline 69 & Davallia denticulate & $\mathrm{F}$ & 1 & 1.00 & 1.00 & 1.00 & 2.00 & 1.00 & 1.00 \\
\hline 70 & Asparagus densiflorus myersii & $\mathrm{F}$ & 1 & 1.00 & 1.00 & 1.00 & 5.00 & 1.00 & 1.00 \\
\hline 71 & Asplenium nidus & $\mathrm{F}$ & 1 & 1.00 & 1.00 & 1.00 & 3.00 & 1.00 & 2.00 \\
\hline 72 & Orchid sp. & $\mathrm{F}$ & 1 & 1.00 & 2.00 & 2.00 & 5.00 & 1.00 & 1.00 \\
\hline 73 & Draceana sp. & $\mathrm{F}$ & 1 & 1.00 & 2.00 & 2.00 & 2.00 & 3.00 & 1.00 \\
\hline 74 & Draceana sp. & $\mathrm{F}$ & 1 & 1.00 & 2.00 & 2.00 & 2.00 & 3.00 & 1.00 \\
\hline 75 & Authorium plowmanii & $\mathrm{F}$ & 1 & 1.00 & 2.00 & 1.00 & 2.00 & 2.00 & 3.00 \\
\hline 76 & Eugenia oleina & $\mathrm{F}$ & 1 & 2.00 & 2.00 & 4.00 & 3.00 & 3.00 & 1.00 \\
\hline 77 & Orchid ap. & $\mathrm{F}$ & 1 & 1.00 & 2.00 & 2.00 & 5.00 & 1.00 & 1.00 \\
\hline 78 & Malaviscus arboreus & $\mathrm{F}$ & 1 & 2.00 & 3.00 & 2.00 & 1.00 & 2.00 & 1.00 \\
\hline 79 & Rhapis excels & G & 7 & 1.00 & 4.00 & 5.00 & 2.00 & 3.00 & 1.00 \\
\hline 80 & Andrographis paniculata & $\mathrm{H}$ & 3 & 1.00 & 2.00 & 1.00 & 2.00 & 1.00 & 1.00 \\
\hline 81 & Origanum vulgare & $\mathrm{H}$ & 3 & 1.00 & 2.00 & 1.00 & 2.00 & 1.00 & 1.00 \\
\hline 82 & Zingiber officinale & $\mathrm{H}$ & 3 & 1.00 & 2.00 & 1.00 & 2.00 & 2.00 & 1.00 \\
\hline 83 & Curcuma longa & $\mathrm{H}$ & 3 & 1.00 & 2.00 & 1.00 & 2.00 & 2.00 & 2.00 \\
\hline 84 & Citrus hystrix & $\mathrm{H}$ & 3 & 2.00 & 3.00 & 1.00 & 1.00 & 1.00 & 1.00 \\
\hline 85 & Alpinia galangal & $\mathrm{H}$ & 3 & 2.00 & 4.00 & 1.00 & 2.00 & 2.00 & 2.00 \\
\hline 86 & Zingiber sp. & $\mathrm{H}$ & 3 & 2.00 & 2.00 & 1.00 & 2.00 & 2.00 & 2.00 \\
\hline 87 & Tabernaemontana divaricata & $\mathrm{H}$ & 3 & 2.00 & 2.00 & 1.00 & 2.00 & 2.00 & 1.00 \\
\hline 88 & Psophocarpus tetragonolobus & $\mathrm{H}$ & 3 & 2.00 & 3.00 & 2.00 & 2.00 & 2.00 & 1.00 \\
\hline 89 & Telosma cordata & $\mathrm{H}$ & 3 & 2.00 & 2.00 & 1.00 & 2.00 & 1.00 & 1.00 \\
\hline 90 & Piper betle & $\mathrm{H}$ & 3 & 2.00 & 2.00 & 1.00 & 2.00 & 2.00 & 2.00 \\
\hline 91 & Sauropus androgynus & $\mathrm{H}$ & 3 & 2.00 & 3.00 & 1.00 & 2.00 & 2.00 & 1.00 \\
\hline 92 & Curcuma longa & $\mathrm{H}$ & 3 & 2.00 & 2.00 & 1.00 & 2.00 & 2.00 & 2.00 \\
\hline 93 & Cosmos caudatus & $\mathrm{H}$ & 3 & 2.00 & 1.00 & 2.00 & 2.00 & 2.00 & 1.00 \\
\hline 94 & Barringtonia racemosa & $\mathrm{H}$ & 3 & 2.00 & 2.00 & 1.00 & 2.00 & 1.00 & 1.00 \\
\hline 95 & Piper sarmentosum roxb & $\mathrm{H}$ & 3 & 2.00 & 2.00 & 2.00 & 2.00 & 1.00 & 2.00 \\
\hline 96 & Pandanus odorus & $\mathrm{H}$ & 3 & 2.00 & 2.00 & 2.00 & 5.00 & 2.00 & 1.00 \\
\hline 97 & Musa parasidiaca & $\mathrm{H}$ & 3 & 2.00 & 3.00 & 1.00 & 2.00 & 2.00 & 3.00 \\
\hline 98 & Zingiber officinale & $\mathrm{H}$ & 3 & 2.00 & 2.00 & 1.00 & 2.00 & 2.00 & 1.00 \\
\hline 99 & Carica papaya & $\mathrm{H}$ & 3 & 2.00 & 3.00 & 1.00 & 1.00 & 1.00 & 1.00 \\
\hline 100 & Pheaomeria imperialis & $\mathrm{H}$ & 3 & 2.00 & 4.00 & 2.00 & 2.00 & 2.00 & 2.00 \\
\hline 101 & Justicia Gendarussa linn & $\mathrm{H}$ & 3 & 2.00 & 2.00 & 2.00 & 2.00 & 2.00 & 1.00 \\
\hline 102 & Labisia pumila & $\mathrm{H}$ & 3 & 1.00 & 2.00 & 1.00 & 2.00 & 1.00 & 1.00 \\
\hline 103 & Lawsonia inermis & $\mathrm{H}$ & 7 & 2.00 & 3.00 & 1.00 & 2.00 & 1.00 & 1.00 \\
\hline \multirow[t]{3}{*}{104} & Eurycoma longifolia & $\mathrm{H}$ & 7 & 2.00 & 2.00 & 1.00 & 2.00 & 1.00 & 1.00 \\
\hline & Total & & & 172.00 & 234.00 & 157.00 & 232.00 & 193.00 & 144.00 \\
\hline & Average & & & 1.65 & 2.25 & 1.51 & 2.23 & 1.86 & 1.38 \\
\hline
\end{tabular}

a Type of shrubs; $F=$ garden shrubs, $G=$ palm shrubs, $H=$ edible shrubs, $I=$ bamboo shrubs

b $1=$ North, $3=$ East, $5=$ South, $7=$ West

c $1=$ Proximity to walls, $2=$ below $5 \mathrm{~m}, 3=5-9 \mathrm{~m}, 4=10-14 \mathrm{~m}, 5=15-19 \mathrm{~m}$

d $1=$ below $0.5 \mathrm{~m}, 2=0.5-0.9 \mathrm{~m}, 3.0=1.0-1.4 \mathrm{~m}, 4=1.5-1.9 \mathrm{~m}, 5=2.0-2.4 \mathrm{~m}$

${ }^{\mathrm{e}} 1=$ individual/potted, $2=$ group below $5 \mathrm{~m}^{2}, 3=$ group $6-10 \mathrm{~m}^{2}, 4=$ group $10-20 \mathrm{~m}^{2}, 5=$ group $21-30 \mathrm{~m}^{2}$

f $1=$ spreading, $2=$ upright, $3=$ rounded, $5=$ sword leaf.

g $1=$ rare, $2=$ medium, $3=$ dense

${ }^{\mathrm{h}} 1=$ small, $2=$ medium, $3=$ large 


\section{Appendix 5.2. List of shrubs in around the sparsely landscaped house}

\begin{tabular}{|c|c|c|c|c|c|c|c|c|c|}
\hline \multirow[t]{2}{*}{ No. } & \multirow[t]{2}{*}{ Species } & \multirow[t]{2}{*}{ Type $^{\mathrm{a}}$} & \multirow[t]{2}{*}{ Azimuth $^{b}$} & \multirow[t]{2}{*}{ Distance $^{\mathrm{c}}$} & \multicolumn{5}{|c|}{ Shrub-growth index/typology/biomass structure } \\
\hline & & & & & Height $^{\mathrm{d}}$ & Spread $^{\mathrm{e}}$ & Shape ${ }^{f}$ & $\begin{array}{l}\text { Amount } \\
\text { of leaves }\end{array}$ & $\begin{array}{l}\text { Size of } \\
\text { leaves }\end{array}$ \\
\hline 1 & Tabernaemontana divaricata & $\mathrm{F}$ & 3 & 2.00 & 2.00 & 3.00 & 1.00 & 2.00 & 1.00 \\
\hline 2 & Calathea lutea & $\mathrm{F}$ & 3 & 2.00 & 2.00 & 3.00 & 1.00 & 3.00 & 3.00 \\
\hline 3 & Hymenocallis littoralis & $\mathrm{F}$ & 3 & 2.00 & 1.00 & 2.00 & 5.00 & 1.00 & 1.00 \\
\hline 4 & Asplenium nidus & $\mathrm{F}$ & 3 & 2.00 & 2.00 & 1.00 & 5.00 & 2.00 & 3.00 \\
\hline 5 & Asplenium nidus & $\mathrm{F}$ & 3 & 2.00 & 2.00 & 1.00 & 5.00 & 2.00 & 3.00 \\
\hline 6 & Acalypha wilkensiana & $\mathrm{F}$ & 1 & 2.00 & 2.00 & 5.00 & 3.00 & 2.00 & 2.00 \\
\hline 7 & Euphorbia milli & $\mathrm{F}$ & 1 & 2.00 & 1.00 & 1.00 & 2.00 & 1.00 & 1.00 \\
\hline 8 & Platycladus orientalis & $\mathrm{F}$ & 5 & 2.00 & 2.00 & 1.00 & 4.00 & 3.00 & 1.00 \\
\hline 9 & Jasminum sp. & $\mathrm{F}$ & 5 & 2.00 & 3.00 & 1.00 & 2.00 & 1.00 & 1.00 \\
\hline 10 & Thevetia peruviana & $\mathrm{F}$ & 5 & 2.00 & 2.00 & 5.00 & 2.00 & 3.00 & 1.00 \\
\hline 11 & Acalypha wilkensiana & $\mathrm{F}$ & 5 & 2.00 & 2.00 & 5.00 & 2.00 & 2.00 & 2.00 \\
\hline 12 & Eugenia oleina & $\mathrm{F}$ & 7 & 2.00 & 2.00 & 1.00 & 3.00 & 2.00 & 1.00 \\
\hline 13 & Heliconia agusta & $\mathrm{F}$ & 7 & 2.00 & 2.00 & 1.00 & 2.00 & 2.00 & 3.00 \\
\hline 14 & Hibiscus sp. & $\mathrm{F}$ & 7 & 2.00 & 3.00 & 1.00 & 3.00 & 3.00 & 1.00 \\
\hline 15 & Bougainvillea sp. & $\mathrm{F}$ & 7 & 2.00 & 2.00 & 1.00 & 1.00 & 2.00 & 1.00 \\
\hline 16 & Dracaena marginata tricolour & $\mathrm{F}$ & 7 & 2.00 & 3.00 & 1.00 & 2.00 & 1.00 & 1.00 \\
\hline 17 & Baphia nitida & $\mathrm{F}$ & 7 & 2.00 & 2.00 & 4.00 & 2.00 & 2.00 & 2.00 \\
\hline 18 & Thevetia peruviana & $\mathrm{F}$ & 7 & 2.00 & 2.00 & 2.00 & 2.00 & 3.00 & 1.00 \\
\hline 19 & Eugenia oleina & $\mathrm{F}$ & 7 & 2.00 & 2.00 & 1.00 & 3.00 & 2.00 & 1.00 \\
\hline 20 & Dracaena marginata tricolour & $\mathrm{F}$ & 7 & 2.00 & 3.00 & 1.00 & 2.00 & 1.00 & 1.00 \\
\hline 21 & Baphia nitida & $\mathrm{F}$ & 7 & 2.00 & 2.00 & 5.00 & 2.00 & 2.00 & 2.00 \\
\hline 22 & Eugenia oleina & $\mathrm{F}$ & 7 & 2.00 & 2.00 & 1.00 & 3.00 & 2.00 & 1.00 \\
\hline 23 & Dypsis lutescens & $\mathrm{G}$ & 3 & 2.00 & 3.00 & 3.00 & 2.00 & 3.00 & 3.00 \\
\hline 24 & Dypsis lutescens & $\mathrm{G}$ & 3 & 2.00 & 2.00 & 1.00 & 2.00 & 3.00 & 1.00 \\
\hline 25 & Dypsis lutescens & $\mathrm{G}$ & 1 & 2.00 & 2.00 & 1.00 & 2.00 & 3.00 & 1.00 \\
\hline 26 & Dypsis lutescens & $\mathrm{G}$ & 1 & 2.00 & 2.00 & 1.00 & 2.00 & 3.00 & 1.00 \\
\hline 27 & Alpinia galangal & $\mathrm{H}$ & 7 & 2.00 & 5.00 & 2.00 & 1.00 & 2.00 & 3.00 \\
\hline 28 & Zingiber officinale & $\mathrm{H}$ & 7 & 2.00 & 5.00 & 2.00 & 2.00 & 2.00 & 2.00 \\
\hline 29 & Mangifera indica & $\mathrm{H}$ & 7 & 2.00 & 5.00 & 1.00 & 1.00 & 3.00 & 2.00 \\
\hline 30 & Cymbopogon flexuosus & $\mathrm{H}$ & 7 & 2.00 & 2.00 & 2.00 & 1.00 & 2.00 & 1.00 \\
\hline 31 & Saccharum officinarum & $\mathrm{H}$ & 7 & 2.00 & 3.00 & 1.00 & 2.00 & 1.00 & 1.00 \\
\hline 32 & Musa sp. & $\mathrm{H}$ & 7 & 2.00 & 5.00 & 3.00 & 2.00 & 2.00 & 3.00 \\
\hline 33 & Pandanus odorus & $\mathrm{H}$ & 7 & 2.00 & 1.00 & 2.00 & 5.00 & 1.00 & 1.00 \\
\hline 34 & Kaemferia galangal & $\mathrm{H}$ & 7 & 2.00 & 1.00 & 1.00 & 1.00 & 1.00 & 1.00 \\
\hline & Total & & & 68.00 & 82.00 & 67.00 & 80.00 & 70.00 & 54.00 \\
\hline & Average & & & 2.00 & 2.41 & 1.97 & 2.35 & 2.06 & 1.59 \\
\hline
\end{tabular}

${ }^{\text {a }}$ Type of shrubs; $\mathrm{F}=$ garden shrubs, $\mathrm{G}=$ palm shrubs, $\mathrm{H}=$ =dible shrubs, $\mathrm{I}=$ bamboo shrubs

b $1=$ North, $3=$ East, $5=$ South, $7=$ West

c $1=$ Proximity to walls, $2=$ below $5 \mathrm{~m}, 3=5-9 \mathrm{~m}, 4=10-14 \mathrm{~m}, 5=15-19 \mathrm{~m}$

${ }^{\mathrm{d}} 1=$ below $0.5 \mathrm{~m}, 2=0.5-0.9 \mathrm{~m}, 3.0=1.0-1.4 \mathrm{~m}, 4=1.5-1.9 \mathrm{~m}, 5=2.0-2.4 \mathrm{~m}$

e $1=$ individual/potted, $2=$ group below $5 \mathrm{~m}^{2}, 3=$ group $6-10 \mathrm{~m}^{2}, 4=$ group $10-20 \mathrm{~m}^{2}, 5=$ group $21-30 \mathrm{~m}^{2}$

${ }^{\mathrm{f}} 1$ =spreading, $2=$ upright, $3=$ rounded, $5=$ sword leaf.

g $1=$ rare, $2=$ medium, $3=$ dense

h $1=$ small, $2=$ medium, $3=$ large 
Appendix 5.3. Calculation of air-conditioning uses in the heavily landscaped house

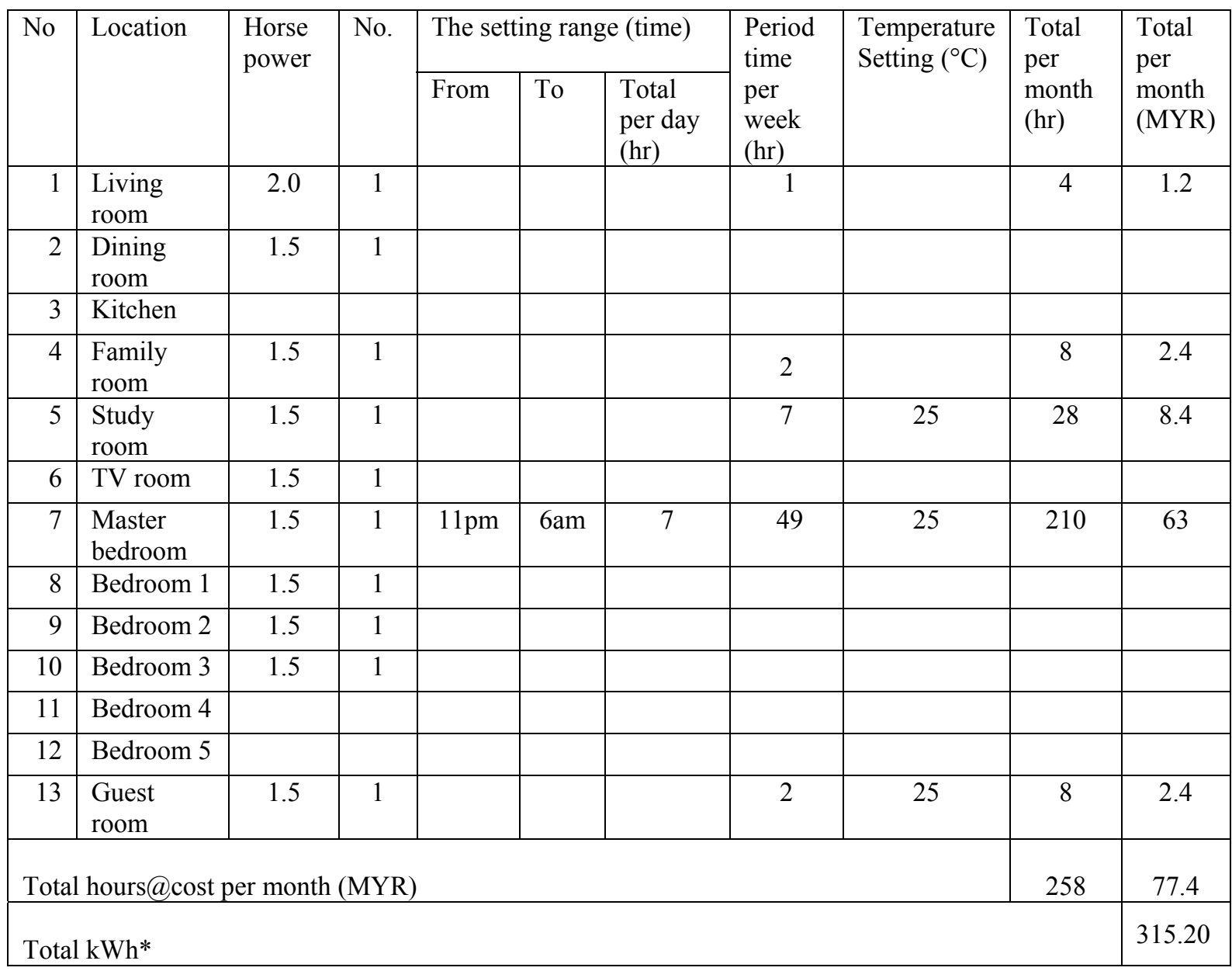

Note (Hariri, 2010):

a. Costs for horsepower per hour (1 horsepower = MYR 0.20) Example:

1. 1 horsepower per hour $=$ MYR 0.20

21.5 horsepower per hour $=$ MYR 0.30

3. 2 horsepower per hour $=$ MYR 0.40

4. 2.5 horsepower per hour $=$ MYR 0.50

5. 3 horsepower per hour $=$ MYR 0.60

b. Total per month $($ MYR $)=($ Total hour per month $) \times($ costs per horsepower $)$

c. 1 horsepower $=0.7457 \mathrm{kWh}$

d. Total $\mathrm{kWh}^{*}=$ Total MYR/0.2457 
Appendix 5.4. Calculation of air-conditioning uses in the sparsely landscaped house

\begin{tabular}{|c|c|c|c|c|c|c|c|c|c|c|}
\hline \multirow[t]{2}{*}{ No } & \multirow[t]{2}{*}{ Location } & \multirow{2}{*}{$\begin{array}{l}\text { Horse } \\
\text { power }\end{array}$} & \multirow[t]{2}{*}{ No. } & \multicolumn{3}{|c|}{ The setting range (time) } & \multirow{2}{*}{$\begin{array}{l}\text { Period } \\
\text { time } \\
\text { per } \\
\text { week } \\
\text { (hr) }\end{array}$} & \multirow{2}{*}{$\begin{array}{l}\text { Temperature } \\
\text { Setting }\left({ }^{\circ} \mathrm{C}\right)\end{array}$} & \multirow{2}{*}{$\begin{array}{l}\text { Total } \\
\text { per } \\
\text { month } \\
\text { (hr) }\end{array}$} & \multirow{2}{*}{$\begin{array}{l}\text { Total } \\
\text { per } \\
\text { month } \\
\text { (MYR) }\end{array}$} \\
\hline & & & & From & To & $\begin{array}{l}\text { Total } \\
\text { per day } \\
\text { (hr) }\end{array}$ & & & & \\
\hline 1 & $\begin{array}{l}\text { Living } \\
\text { room }\end{array}$ & 2.0 & 1 & & & & 2 & 22 & 8 & 3.2 \\
\hline 2 & $\begin{array}{l}\text { Dining } \\
\text { room }\end{array}$ & 1.5 & 1 & & & & & & & \\
\hline 3 & Kitchen & & & & & & & & & \\
\hline 4 & $\begin{array}{l}\text { Family } \\
\text { room }\end{array}$ & 1.5 & 1 & & & & & & & \\
\hline 5 & $\begin{array}{l}\text { Study } \\
\text { room }\end{array}$ & 1.5 & 1 & & & & & & & \\
\hline 6 & TV room & 1.5 & 1 & & & & & & & \\
\hline 7 & $\begin{array}{l}\text { Master } \\
\text { bedroom }\end{array}$ & 1.5 & 1 & $11 \mathrm{pm}$ & $3 \mathrm{pm}$ & 4 & 28 & 22 & 120 & 36 \\
\hline 8 & Bedroom 1 & 1.5 & 1 & $11 \mathrm{pm}$ & $3 \mathrm{pm}$ & 4 & 28 & 22 & 120 & 36 \\
\hline 9 & Bedroom 2 & 1.5 & 1 & $11 \mathrm{pm}$ & $3 \mathrm{pm}$ & 4 & 28 & 22 & 120 & 36 \\
\hline 10 & Bedroom 3 & 1.5 & 1 & $11 \mathrm{pm}$ & $3 \mathrm{pm}$ & 4 & 28 & 22 & 120 & 36 \\
\hline 11 & Bedroom 4 & & & & & & & & & \\
\hline 12 & Bedroom 5 & & & & & & & & & \\
\hline 13 & $\begin{array}{l}\text { Guest } \\
\text { room }\end{array}$ & 1.5 & 1 & $11 \mathrm{pm}$ & $3 \mathrm{pm}$ & 4 & 28 & 22 & 120 & 36 \\
\hline \multicolumn{9}{|c|}{ Total hours@cost per month (MYR) } & 608 & 183.2 \\
\hline \multicolumn{10}{|c|}{ Total kWh* } & 745.62 \\
\hline
\end{tabular}

Note (Hariri, 2010):

a. Costs for horsepower per hour (1 horsepower $=$ MYR 0.20) Example:

1. 1 horsepower per hour $=$ MYR 0.20

21.5 horsepower per hour $=$ MYR 0.30

3. 2 horsepower per hour $=$ MYR 0.40

4. 2.5 horsepower per hour $=$ MYR 0.50

5. 3 horsepower per hour $=$ MYR 0.60

b. Total per month $($ MYR $)=($ Total hour per month) $\mathrm{x}$ (costs per horsepower)

c. 1 horsepower $=0.7457 \mathrm{kWh}$

d. Total kWh* = Total MYR/0.2457 


\section{Appendix 6. Soft landscape elements around all houses}

Appendix 6.1. Location of Section 3 Shah Alam, Selangor (Google Earth, 2011362

Appendix 6.2. Tree species for all categories 363

Appendix 6.3. Tree sizes and distances from the building in all azimuths for 0-9, 10-19, 20-29, and 30-40 years old groups of houses

Appendix 6.3.1. Tree sizes and distances from the building in all azimuths for 0-9

years old group of houses.

Appendix 6.3.2. Tree sizes and distances from the building in all azimuths for 10-19 years old group of houses.

Appendix 6.3.3. Tree sizes and distances from the building in all azimuths for 20-29

years old group of houses.

Appendix 6.3.4. Tree sizes and distances from the building in all azimuths for 30-40 years old group of houses. 370

Appendix 6.4. Shrubs, vines, a Appendix 6.1. nd groundcover, sizes and distances from the building in all azimuths for $0-9,10-19,20-29$, and 30-40 years old groups of houses

Appendix 6.4.1. Shrubs, vines, and groundcover, and distances from the building at all azimuths for 0-9 years old group of houses

Appendix 6.4.2. Shrubs, vines, and groundcover, sizes and distances from the building in all azimuths for 10-19 years old group of houses

Appendix 6.4.3. Shrubs, vines, and groundcover, sizes and distances from the building in all azimuths for 20-29 years old group of houses .....

Appendix 6.4.4. Shrubs, vines, and groundcover, sizes and distances from the building in all azimuths for 30-40 years old group of houses.

Appendix 6.5. Shrubs, vines, and groundcover species for all categories 
Appendix 6.1. Location of Section 3 Shah Alam, Selangor (Google Earth, 2011)

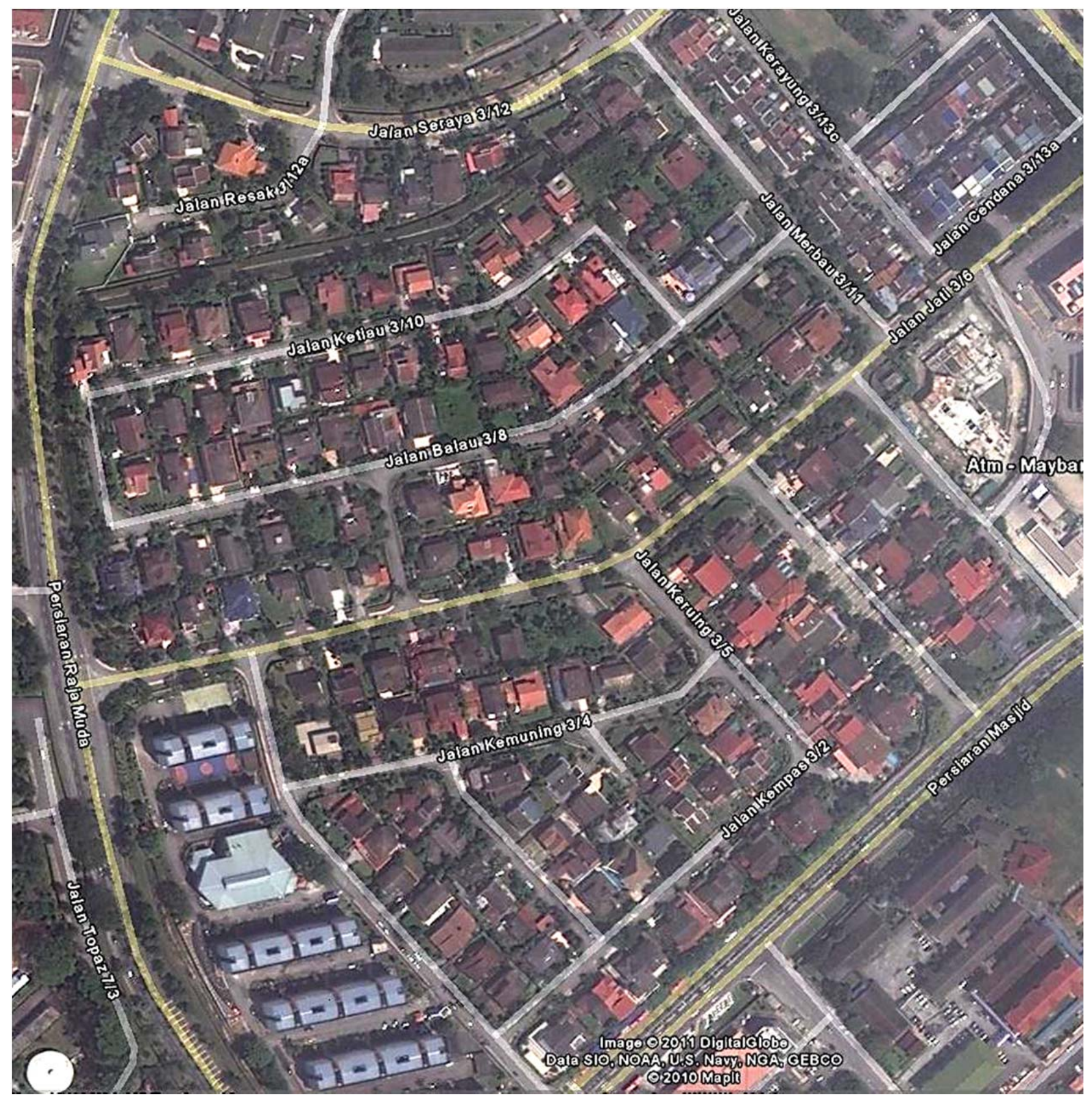


Appendix 6.2. Tree species for all categories

1. Roadside trees

\begin{tabular}{lllc}
\hline No. & Botanical name & Common name & Total \\
\hline 1 & Cinnamomum iners & Cinnamon & 3 \\
2 & Erythrina fusca & Coral bean & 21 \\
3 & Falcataria moluccana & Albizia & 1 \\
4 & Hura crepitans & Possum wood & 2 \\
5 & Mangifera indica & Mango & 1 \\
6 & Mimusops elengi & Spanish cherry & 1 \\
7 & Plumeria rubra & Frangipani & 1 \\
8 & Samanea saman & Rain tree & 27 \\
9 & Spathodea campanulata & African tulip & 3 \\
10 & Tabebuia rosea & Trumpet & 8 \\
\hline & Total & & 68 \\
\hline
\end{tabular}

2. Roadside palms

\begin{tabular}{lllc}
\hline No. & Botanical name & Common name & Total \\
\hline 1 & Bismarkia nobilis & Bismark palm & 1 \\
2 & Golden fruited palm & Golden fruited palm & 4 \\
3 & Cocos nucifera & Coconut & 1 \\
4 & Cyrtostachys renda & Red Palm & 1 \\
5 & Ptychosperma macarthurii & Macarthur palm & 12 \\
6 & Veitchia merillii & Manila palm & 10 \\
\hline & Total & & 29 \\
\hline
\end{tabular}

3. Garden trees

\begin{tabular}{lllc}
\hline No. & Botanical name & Common name & Total \\
\hline 1 & Acacia sp. & Thorn tree & 1 \\
2 & Acasia mangium & Mangium wattle & 1 \\
3 & Alstonia angustiloba & Pulai & 1 \\
4 & Antisdesma ghaesembilla & Black current-tree & 1 \\
5 & Araucaria columnaris & Cook's pine & 2 \\
6 & Bucida buceras & Black olive & 17 \\
7 & Casuarina equisetifolia & Casuarina & 1 \\
8 & Casuarina rumphiana & Casuarina & 5 \\
9 & Casuarina sumatrana & Sumatra casuarina & 13 \\
10 & Cinamomum zeylanicum & Cinnamon & 1 \\
11 & Cinnamomum iners & Cinnamon & 1 \\
12 & Citharexylum spinosum & Fiddlewood & 1 \\
13 & Dalbergia oliveri & Burma tulipwood & 1
\end{tabular}




\begin{tabular}{lllc}
14 & Erioglossum rubiginosum & Rusty sapindus & 1 \\
15 & Erythrina fusca & Coral bean & 4 \\
16 & Fagraea fragrans & Tembusu & 10 \\
17 & Falcataria moluccana & Albizia & 2 \\
18 & Felicium decipiens & Fern-leaf & 1 \\
19 & Ficus benjamina & Benjamin fig & 3 \\
20 & Juniperus chinensis & Chinese juniper & 9 \\
21 & Mancaranga sp. & Mahang & 1 \\
22 & Melaleuca bracteata & Black tea tree & 2 \\
23 & Michelia alba & White champaca & 1 \\
24 & Michelia champaca & Cempaka & 3 \\
25 & Mimusope elengi & Tanjung & 3 \\
26 & Murraya paniculata & Kemuning & 3 \\
27 & Pereskia bleo & Rose cactus & 1 \\
28 & Platycladus orientalis & Oriental arborvitae & 2 \\
29 & Plumeria obtuse & Frangipani & 2 \\
30 & Plumeria rubra & Frangipani & 9 \\
31 & Podocarpus indicus & Podocarpus & 1 \\
32 & Podocarpus rumphii & Podocarpus & 2 \\
33 & Polyalthia longifolia & Indian fir tree & 135 \\
34 & Salix babylonica & Weeping willow & 4 \\
35 & Saraca thaipengensis & Yellow saraca & 2 \\
36 & Schizolobium parahyba & Tower tree & 3 \\
37 & Spathodea campanulata & African tulip & 16 \\
38 & Tabebuia rosea & Trumpet tree & 2 \\
39 & Terminalia catappa & Umbrella tree & 1 \\
\hline & Total & & 1 \\
\hline & & & \\
\hline
\end{tabular}

4. Edible fruit trees

\begin{tabular}{cllc}
\hline No. & Botanical name & Common name & Total \\
\hline 1 & Anacardium occidentale & Cashew tree & 2 \\
2 & Artocarpus altilis & Breadfruit & 2 \\
3 & Artocarpus heterophylus & Jackfruit & 1 \\
4 & Artocarpus integer & Jack tree & 4 \\
5 & Averrhoa bilimbi & Cucumber tree & 8 \\
6 & Averrhoa carambola & Starfruit & 1 \\
7 & Castanea sativa & Sweet chestnut & 1 \\
8 & Citrus aurantifolia & Lime & 1 \\
9 & Diospyros blancoi & Velvet apple & 1 \\
10 & Diospyros discolor & Butter fruit & 1 \\
11 & Durio zibethinus & King of fruits & 2
\end{tabular}




\begin{tabular}{lllc}
12 & Eugenia malaccensis & Malay apple & 3 \\
13 & Eugenia polyantha & Bay-Leaf & 1 \\
14 & Flacourtia Jagomas & Coffee plum & 1 \\
15 & Garcinia mangostana & Mangosteen & 6 \\
16 & Jasminum arabicum & Coffea arabica & 1 \\
17 & Lansium domesticum & Duku & 1 \\
18 & Mangifera indica & Mango & 22 \\
19 & Mangifera odorata & Kuini mango & 1 \\
20 & Manilkara zapota & Brown sugar fruit & 1 \\
21 & Morinda citrifolia & Noni & 3 \\
22 & Moringa oleifera lam & Drumstick-tree & 1 \\
23 & Nephelium lappaceum & Rambutan & 19 \\
24 & Nephelium lithi & Lychee & 1 \\
25 & Persia americana & Avocado & 3 \\
26 & Phyllanthus acidus & Malay gooseberry & 1 \\
27 & Psidium guajava & Guava & 1 \\
28 & Solanum wrightii & Giant potato tree & 2 \\
29 & Syzygium aqueum & Water apple & 3 \\
30 & Syzygium cumini & Black plum & 1 \\
31 & Tamarindus indica & Indian date & 2 \\
\hline & Total & & 98 \\
\hline
\end{tabular}

5. Garden palms

\begin{tabular}{cllc}
\hline No. & Botanical name & Common name & Total \\
\hline 1 & Areca catechu & Betel nut palm & 10 \\
2 & Bismarkia nobilis & Bismark palm & 8 \\
3 & Caryota mitis & Fish tail palm & 5 \\
4 & Chrysalidocarpus lutescens & Golden fruited palm & 15 \\
5 & Cocos nucifera & Coconut & 10 \\
6 & Cyrtostachys renda & Red palm & 28 \\
7 & Elaeis guineensis & Palm oil & 1 \\
8 & Hyophorpbe lagenicaulis & Bottle palm & 4 \\
9 & Licuala Grandis & Ruffled fan palm & 1 \\
10 & Livistona Chinensis & Chinese Fan Palm & 1 \\
11 & Ptychosperma macarthurii & Macarthur palm & 34 \\
12 & Ravenala madagascariensis & Traveller's palm & 13 \\
13 & Veitchia merillii & Manila palm & 32 \\
14 & Wodyetia bifurcata & Fox tail palm & 44 \\
\hline & Total & & 206 \\
\hline
\end{tabular}


6. Bamboo

\begin{tabular}{cllc}
\hline No. & Botanical name & Common name & Total \\
\hline 1 & Bambusa vulgaris & Giant buddha belly & 4 \\
2 & Thyrsostachis siamensis & Fine leaf bamboo & 2 \\
& Total & & 6 \\
\hline
\end{tabular}


Appendix 6.3. Tree sizes and distances from the building in all azimuths for $0-9,10$ $19,20-29$, and 30-40 years old groups of houses

Appendix 6.3.1. Tree sizes and distances from the building in all azimuths for $0-9$ years old group of houses

\begin{tabular}{|c|c|c|c|c|c|c|c|c|c|c|c|c|}
\hline \multirow[t]{2}{*}{ Tree size } & \multirow[t]{2}{*}{ Distance (m) } & \multicolumn{9}{|c|}{ Tree azimuth } & \multirow[b]{2}{*}{ Total } & \multirow[b]{2}{*}{$\%$} \\
\hline & & $\mathrm{N}$ & $\mathrm{NE}$ & $\mathrm{E}$ & $\mathrm{SE}$ & $\mathrm{S}$ & SW & $\mathrm{W}$ & NW & All & & \\
\hline \multirow[t]{4}{*}{ Small } & $3-5$ & 1 & 4 & 16 & 5 & 12 & 2 & 14 & 10 & 64 & 141 & 61.6 \\
\hline & $6-10$ & 14 & 3 & 14 & 0 & 10 & 9 & 22 & 5 & 77 & & \\
\hline & $11-15$ & 0 & 0 & 0 & 0 & 0 & 0 & 0 & 0 & 0 & & \\
\hline & $16-20$ & 0 & 0 & 0 & 0 & 0 & 0 & 0 & 0 & 0 & & \\
\hline \multirow[t]{4}{*}{$\begin{array}{l}\text { Small } \\
\text { moderate }\end{array}$} & $3-5$ & 0 & 1 & 3 & 1 & 0 & 6 & 2 & 2 & 15 & 84 & 36.7 \\
\hline & $6-10$ & 4 & 0 & 16 & 4 & 16 & 15 & 12 & 2 & 69 & & \\
\hline & $11-15$ & 0 & 0 & 0 & 0 & 0 & 0 & 0 & 0 & 0 & & \\
\hline & $16-20$ & 0 & 0 & 0 & 0 & 0 & 0 & 0 & 0 & 0 & & \\
\hline \multirow[t]{4}{*}{ Medium } & $3-5$ & 2 & 0 & 0 & 0 & 1 & 0 & 0 & 0 & 3 & 4 & 1.7 \\
\hline & $6-10$ & 0 & 0 & 0 & 0 & 0 & 0 & 1 & 0 & 1 & & \\
\hline & $11-15$ & 0 & 0 & 0 & 0 & 0 & 0 & 0 & 0 & 0 & & \\
\hline & $16-20$ & 0 & 0 & 0 & 0 & 0 & 0 & 0 & 0 & 0 & & \\
\hline \multirow[t]{4}{*}{ Large } & $3-5$ & 0 & 0 & 0 & 0 & 0 & 0 & 0 & 0 & 0 & 0 & 0.0 \\
\hline & $6-10$ & 0 & 0 & 0 & 0 & 0 & 0 & 0 & 0 & 0 & & \\
\hline & $11-15$ & 0 & 0 & 0 & 0 & 0 & 0 & 0 & 0 & 0 & & \\
\hline & $16-20$ & 0 & 0 & 0 & 0 & 0 & 0 & 0 & 0 & 0 & & \\
\hline \multicolumn{2}{|c|}{ All tree sizes/distances } & 20 & 8 & 51 & 10 & 47 & 32 & 42 & 19 & 229 & 229 & 100 \\
\hline \multicolumn{2}{|c|}{ Percentage } & 8.7 & 3.5 & 22.3 & 4.4 & 20.5 & 14.0 & 18.3 & 8.3 & 100 & 100 & \\
\hline
\end{tabular}


Appendix 6.3.2. Tree sizes and distances from the building in all azimuths for 10-19 years old group of houses

\begin{tabular}{|c|c|c|c|c|c|c|c|c|c|c|c|c|}
\hline \multirow[t]{2}{*}{ Tree size } & \multirow[t]{2}{*}{ Distance (m) } & \multicolumn{9}{|c|}{ Tree azimuth } & \multirow[b]{2}{*}{ Total } & \multirow[b]{2}{*}{$\%$} \\
\hline & & $\mathrm{N}$ & $\mathrm{NE}$ & E & SE & $\mathrm{S}$ & SW & $\mathrm{W}$ & NW & All & & \\
\hline \multirow[t]{4}{*}{ Small } & $3-5$ & 0 & 0 & 1 & 5 & 3 & 1 & 0 & 3 & 13 & 22 & 66.7 \\
\hline & $6-10$ & 0 & 1 & 4 & 3 & 0 & 0 & 0 & 1 & 9 & & \\
\hline & $11-15$ & 0 & 0 & 0 & 0 & 0 & 0 & 0 & 0 & 0 & & \\
\hline & $16-20$ & 0 & 0 & 0 & 0 & 0 & 0 & 0 & 0 & 0 & & \\
\hline \multirow[t]{4}{*}{$\begin{array}{l}\text { Small } \\
\text { moderate }\end{array}$} & $3-5$ & 0 & 0 & 0 & 3 & 0 & 0 & 0 & 1 & 4 & 8 & 24.2 \\
\hline & $6-10$ & 0 & 1 & 0 & 0 & 0 & 0 & 0 & 2 & 3 & & \\
\hline & $11-15$ & 0 & 0 & 0 & 1 & 0 & 0 & 0 & 0 & 1 & & \\
\hline & $16-20$ & 0 & 0 & 0 & 0 & 0 & 0 & 0 & 0 & 0 & & \\
\hline \multirow[t]{4}{*}{ Medium } & $3-5$ & 0 & 0 & 0 & 0 & 0 & 1 & 0 & 0 & 1 & 2 & 6.1 \\
\hline & $6-10$ & 0 & 0 & 0 & 1 & 0 & 0 & 0 & 0 & 1 & & \\
\hline & $11-15$ & 0 & 0 & 0 & 0 & 0 & 0 & 0 & 0 & 0 & & \\
\hline & $16-20$ & 0 & 0 & 0 & 0 & 0 & 0 & 0 & 0 & 0 & & \\
\hline \multirow[t]{4}{*}{ Large } & $3-5$ & 0 & 0 & 0 & 0 & 0 & 0 & 0 & 0 & 0 & 1 & 3 \\
\hline & $6-10$ & 0 & 0 & 0 & 1 & 0 & 0 & 0 & 0 & 1 & & \\
\hline & $11-15$ & 0 & 0 & 0 & 0 & 0 & 0 & 0 & 0 & 0 & & \\
\hline & $16-20$ & 0 & 0 & 0 & 0 & 0 & 0 & 0 & 0 & 0 & & \\
\hline \multicolumn{2}{|c|}{ All tree sizes/distances } & 0 & 2 & 5 & 14 & 3 & 2 & 0 & 7 & 33 & 33 & 100 \\
\hline \multicolumn{2}{|l|}{ Percentage } & 0.0 & 6.1 & 15.2 & 42.4 & 9.1 & 6.1 & 0.0 & 21.2 & 100 & 100 & \\
\hline
\end{tabular}


Appendix 6.3.3. Tree sizes and distances from the building in all azimuths for 20-29 years old group of houses

\begin{tabular}{|c|c|c|c|c|c|c|c|c|c|c|c|c|}
\hline \multirow[t]{2}{*}{ Tree size } & \multirow[t]{2}{*}{ Distance (m) } & \multicolumn{9}{|c|}{ Tree azimuth } & \multirow[b]{2}{*}{ Total } & \multirow[b]{2}{*}{$\%$} \\
\hline & & $\mathrm{N}$ & $\mathrm{NE}$ & E & SE & $\mathrm{S}$ & SW & $\mathrm{W}$ & NW & All & & \\
\hline \multirow[t]{4}{*}{ Small } & $3-5$ & 7 & 10 & 13 & 12 & 9 & 10 & 5 & 9 & 75 & 134 & 58.0 \\
\hline & $6-10$ & 7 & 9 & 4 & 1 & 7 & 4 & 3 & 12 & 47 & & \\
\hline & $11-15$ & 0 & 0 & 1 & 0 & 4 & 1 & 6 & 0 & 12 & & \\
\hline & $16-20$ & 0 & 0 & 0 & 0 & 0 & 0 & 0 & 0 & 0 & & \\
\hline \multirow[t]{4}{*}{$\begin{array}{l}\text { Small } \\
\text { moderate }\end{array}$} & $3-5$ & 3 & 1 & 6 & 3 & 2 & 3 & 2 & 0 & 20 & 75 & 32.5 \\
\hline & $6-10$ & 11 & 11 & 6 & 2 & 2 & 1 & 7 & 4 & 44 & & \\
\hline & $11-15$ & 0 & 0 & 0 & 0 & 2 & 0 & 2 & 0 & 4 & & \\
\hline & $16-20$ & 0 & 0 & 0 & 0 & 0 & 0 & 7 & 0 & 7 & & \\
\hline \multirow[t]{4}{*}{ Medium } & $3-5$ & 2 & 0 & 3 & 1 & 0 & 3 & 0 & 0 & 9 & 21 & 9.1 \\
\hline & $6-10$ & 5 & 1 & 2 & 0 & 0 & 0 & 2 & 0 & 10 & & \\
\hline & $11-15$ & 0 & 1 & 1 & 0 & 0 & 0 & 0 & 0 & 2 & & \\
\hline & $16-20$ & 0 & 0 & 0 & 0 & 0 & 0 & 0 & 0 & 0 & & \\
\hline \multirow[t]{4}{*}{ Large } & $3-5$ & 0 & 0 & 0 & 0 & 0 & 0 & 1 & 0 & 1 & 1 & 0.4 \\
\hline & $6-10$ & 0 & 0 & 0 & 0 & 0 & 0 & 0 & 0 & 0 & & \\
\hline & $11-15$ & 0 & 0 & 0 & 0 & 0 & 0 & 0 & 0 & 0 & & \\
\hline & $16-20$ & 0 & 0 & 0 & 0 & 0 & 0 & 0 & 0 & 0 & & \\
\hline \multicolumn{2}{|c|}{ All tree sizes/distances } & 35 & 33 & 36 & 19 & 26 & 22 & 35 & 25 & 231 & 231 & 100 \\
\hline \multicolumn{2}{|c|}{ Percentage } & 15.2 & 14.3 & 15.6 & 8.2 & 11.3 & 9.5 & 15.2 & 10.8 & 100 & 100 & \\
\hline
\end{tabular}


Appendix 6.3.4. Tree sizes and distances from the building in all azimuths for 30-40 years old group of houses

\begin{tabular}{|c|c|c|c|c|c|c|c|c|c|c|c|c|}
\hline \multirow[t]{2}{*}{ Tree size } & \multirow[t]{2}{*}{ Distance (m) } & \multicolumn{9}{|c|}{ Tree azimuth } & \multirow[b]{2}{*}{ Total } & \multirow[b]{2}{*}{$\%$} \\
\hline & & $\mathrm{N}$ & $\mathrm{NE}$ & E & $\mathrm{SE}$ & $\mathrm{S}$ & SW & W & NW & All & & \\
\hline \multirow[t]{4}{*}{ Small } & $3-5$ & 8 & 0 & 5 & 0 & 1 & 0 & 0 & 4 & 18 & 25 & 51.0 \\
\hline & $6-10$ & 2 & 0 & 2 & 0 & 0 & 0 & 2 & 1 & 7 & & \\
\hline & $11-15$ & 0 & 0 & 0 & 0 & 0 & 0 & 0 & 0 & 0 & & \\
\hline & $16-20$ & 0 & 0 & 0 & 0 & 0 & 0 & 0 & 0 & 0 & & \\
\hline \multirow[t]{4}{*}{$\begin{array}{l}\text { Small } \\
\text { moderate }\end{array}$} & $3-5$ & 1 & 2 & 0 & 3 & 1 & 0 & 1 & 0 & 8 & 9 & 18.4 \\
\hline & $6-10$ & 0 & 0 & 0 & 1 & 0 & 0 & 0 & 0 & 1 & & \\
\hline & $11-15$ & 0 & 0 & 0 & 0 & 0 & 0 & 0 & 0 & 0 & & \\
\hline & $16-20$ & 0 & 0 & 0 & 0 & 0 & 0 & 0 & 0 & 0 & & \\
\hline \multirow[t]{4}{*}{ Medium } & $3-5$ & 4 & 2 & 0 & 0 & 0 & 0 & 0 & 0 & 6 & 15 & 30.6 \\
\hline & $5-10$ & 1 & 0 & 0 & 0 & 0 & 7 & 0 & 0 & 8 & & \\
\hline & $11-15$ & 0 & 0 & 0 & 0 & 0 & 0 & 0 & 0 & 0 & & \\
\hline & $16-20$ & 0 & 0 & 0 & 0 & 0 & 1 & 0 & 0 & 1 & & \\
\hline \multirow[t]{4}{*}{ Large } & $3-5$ & 0 & 0 & 0 & 0 & 0 & 0 & 0 & 0 & 0 & 0 & 0.0 \\
\hline & $6-10$ & 0 & 0 & 0 & 0 & 0 & 0 & 0 & 0 & 0 & & \\
\hline & $11-15$ & 0 & 0 & 0 & 0 & 0 & 0 & 0 & 0 & 0 & & \\
\hline & $16-20$ & 0 & 0 & 0 & 0 & 0 & 0 & 0 & 0 & 0 & & \\
\hline \multicolumn{2}{|c|}{ All tree sizes/distances } & 16 & 4 & 7 & 4 & 2 & 8 & 3 & 5 & 49 & 49 & 100 \\
\hline \multicolumn{2}{|l|}{ Percentage } & 32.7 & 8.2 & 14.3 & 8.2 & 4.1 & 16.3 & 6.1 & 10.2 & 100.0 & 100.0 & \\
\hline
\end{tabular}


Appendix 6.4. Shrubs, vines, and groundcover, sizes and distances from the building in all azimuths for 0-9, 10-19, 20-29, and 30-40 years old groups of houses

Appendix 6.4.1. Shrubs, vines, and groundcover, and distances from the building at all azimuths for 0-9 years old group of houses

\begin{tabular}{|c|c|c|c|c|c|c|c|c|c|c|c|c|c|}
\hline \multirow[t]{2}{*}{ Plant size $\left(\mathrm{m}^{2}\right)$} & \multirow[t]{2}{*}{ Distance (m) } & \multicolumn{8}{|c|}{ Plants azimuth } & \multirow[b]{2}{*}{ All } & \multirow[b]{2}{*}{$\%$} & \multirow[b]{2}{*}{ Total } & \multirow[b]{2}{*}{$\%$} \\
\hline & & $\mathrm{N}$ & $\mathrm{NE}$ & $\mathrm{E}$ & SE & $\mathrm{S}$ & SW & $\mathrm{W}$ & NW & & & & \\
\hline \multirow[t]{5}{*}{ Individual } & Proximity to walls & 17 & 24 & 25 & 4 & 26 & 20 & 19 & 12 & 147 & 18.4 & 483 & 60.4 \\
\hline & $2-5$ & 54 & 24 & 70 & 28 & 27 & 28 & 65 & 19 & 315 & 39.4 & & \\
\hline & $5-10$ & 0 & 0 & 3 & 0 & 11 & 0 & 4 & 3 & 21 & 2.6 & & \\
\hline & $11-15$ & 0 & 0 & 0 & 0 & 0 & 0 & 0 & 0 & 0 & 0.0 & & \\
\hline & $16-20$ & 0 & 0 & 0 & 0 & 0 & 0 & 0 & 0 & 0 & 0.0 & & \\
\hline Group 1 & Proximity to walls & 11 & 1 & 4 & 1 & 7 & 1 & 1 & 9 & 35 & 4.4 & 166 & 20.8 \\
\hline \multirow[t]{4}{*}{$\left(0-10 \mathrm{~m}^{2}\right)$} & $2-5$ & 13 & 9 & 35 & 4 & 11 & 17 & 22 & 13 & 124 & 15.5 & & \\
\hline & $5-10$ & 0 & 0 & 0 & 0 & 6 & 0 & 1 & 0 & 7 & 0.9 & & \\
\hline & $11-15$ & 0 & 0 & 0 & 0 & 0 & 0 & 0 & 0 & 0 & 0.0 & & \\
\hline & $16-20$ & 0 & 0 & 0 & 0 & 0 & 0 & 0 & 0 & 0 & 0.0 & & \\
\hline Group 2 & Proximity to walls & 1 & 1 & 0 & 0 & 9 & 1 & 7 & 1 & 20 & 2.5 & 88 & 11.0 \\
\hline \multirow[t]{4}{*}{$\left(11-20 m^{2}\right)$} & $2-5$ & 5 & 7 & 7 & 9 & 14 & 7 & 6 & 3 & 58 & 7.3 & & \\
\hline & $5-10$ & 0 & 0 & 1 & 0 & 7 & 0 & 2 & 0 & 10 & 1.3 & & \\
\hline & $11-15$ & 0 & 0 & 0 & 0 & 0 & 0 & 0 & 0 & 0 & 0.0 & & \\
\hline & $16-20$ & 0 & 0 & 0 & 0 & 0 & 0 & 0 & 0 & 0 & 0.0 & & \\
\hline Group 3 & Proximity to walls & 1 & 0 & 1 & 0 & 0 & 0 & 1 & 0 & 3 & 0.4 & 57 & 7.1 \\
\hline \multirow[t]{4}{*}{$\left(21-30 \mathrm{~m}^{2}\right)$} & $2-5$ & 7 & 5 & 6 & 5 & 7 & 3 & 12 & 3 & 48 & 6.0 & & \\
\hline & $5-10$ & 0 & 2 & 1 & 1 & 0 & 0 & 2 & 0 & 6 & 0.8 & & \\
\hline & $11-15$ & 0 & 0 & 0 & 0 & 0 & 0 & 0 & 0 & 0 & 0.0 & & \\
\hline & $16-20$ & 0 & 0 & 0 & 0 & 0 & 0 & 0 & 0 & 0 & 0.0 & & \\
\hline Group 4 & Proximity to walls & 0 & 0 & 0 & 0 & 1 & 0 & 2 & 0 & 3 & 0.4 & 6 & 0.8 \\
\hline \multirow[t]{4}{*}{$\left(31-40 m^{2}\right)$} & $2-5$ & 1 & 0 & 0 & 0 & 0 & 0 & 2 & 0 & 3 & 0.4 & & \\
\hline & $5-10$ & 0 & 0 & 0 & 0 & 0 & 0 & 0 & 0 & 0 & 0.0 & & \\
\hline & $11-15$ & 0 & 0 & 0 & 0 & 0 & 0 & 0 & 0 & 0 & 0.0 & & \\
\hline & $16-20$ & 0 & 0 & 0 & 0 & 0 & 0 & 0 & 0 & 0 & 0.0 & & \\
\hline \multicolumn{2}{|c|}{ All plants size/distances } & 110 & 73 & 153 & 52 & 126 & 77 & 146 & 63 & 801 & 100 & 801 & 100 \\
\hline \multicolumn{2}{|l|}{ Percentage } & 13.8 & 9.1 & 19.1 & 6.5 & 15.8 & 9.6 & 18.3 & 7.9 & 100 & & & \\
\hline
\end{tabular}


Appendix 6.4.2. Shrubs, vines, and groundcover, sizes and distances from the building in all azimuths for 10-19 years old group of houses

\begin{tabular}{|c|c|c|c|c|c|c|c|c|c|c|c|c|c|}
\hline \multirow[t]{2}{*}{ Plant size $\left(\mathrm{m}^{2}\right)$} & \multirow[t]{2}{*}{ Distance (m) } & \multicolumn{8}{|c|}{ Plants azimuth } & \multirow[b]{2}{*}{ All } & \multirow[b]{2}{*}{$\%$} & \multirow[b]{2}{*}{ Total } & \multirow[b]{2}{*}{$\%$} \\
\hline & & $\mathrm{N}$ & $\mathrm{NE}$ & E & SE & $\mathrm{S}$ & SW & W & NW & & & & \\
\hline \multirow[t]{5}{*}{ Individual } & Proximity to walls & 0 & 0 & 1 & 7 & 0 & 6 & 0 & 3 & 17 & 17.5 & 62 & 63.9 \\
\hline & $2-5$ & 10 & 1 & 0 & 8 & 4 & 10 & 0 & 2 & 35 & 37.1 & & \\
\hline & $5-10$ & 0 & 2 & 5 & 2 & 0 & 0 & 0 & 0 & 9 & 9.3 & & \\
\hline & $11-15$ & 0 & 0 & 0 & 0 & 0 & 0 & 0 & 0 & 0 & 0.0 & & \\
\hline & $16-20$ & 0 & 0 & 0 & 0 & 0 & 0 & 0 & 0 & 0 & 0.0 & & \\
\hline Group 1 & Proximity to walls & 0 & 2 & 0 & 0 & 0 & 0 & 0 & 1 & 1 & 1.0 & 30 & 30.9 \\
\hline \multirow[t]{4}{*}{$\left(0-10 m^{2}\right)$} & $2-5$ & 3 & 3 & 2 & 6 & 2 & 2 & 4 & 1 & 23 & 23.7 & & \\
\hline & $5-10$ & 0 & 1 & 0 & 3 & 1 & 0 & 0 & 1 & 6 & 6.2 & & \\
\hline & $11-15$ & 0 & 0 & 0 & 0 & 0 & 0 & 0 & 0 & 0 & 0.0 & & \\
\hline & $16-20$ & 0 & 0 & 0 & 0 & 0 & 0 & 0 & 0 & 0 & 0.0 & & \\
\hline Group 2 & Proximity to walls & 0 & 1 & 0 & 1 & 0 & 1 & 0 & 0 & 3 & 3.1 & 5 & 5.2 \\
\hline \multirow[t]{4}{*}{$\left(11-20 m^{2}\right)$} & $2-5$ & 1 & 0 & 0 & 0 & 0 & 1 & 0 & 0 & 2 & 2.1 & & \\
\hline & $5-10$ & 0 & 0 & 0 & 0 & 0 & 0 & 0 & 0 & 0 & 0.0 & & \\
\hline & $11-15$ & 0 & 0 & 0 & 0 & 0 & 0 & 0 & 0 & 0 & 0.0 & & \\
\hline & $16-20$ & 0 & 0 & 0 & 0 & 0 & 0 & 0 & 0 & 0 & 0.0 & & \\
\hline Group 3 & Proximity to walls & 0 & 0 & 0 & 0 & 0 & 0 & 0 & 0 & 0 & 0.0 & 0 & 0.0 \\
\hline \multirow[t]{4}{*}{$\left(21-30 m^{2}\right)$} & $2-5$ & 0 & 0 & 0 & 0 & 0 & 0 & 0 & 0 & 0 & 0.0 & & \\
\hline & $5-10$ & 0 & 0 & 0 & 0 & 0 & 0 & 0 & 0 & 0 & 0.0 & & \\
\hline & $11-15$ & 0 & 0 & 0 & 0 & 0 & 0 & 0 & 0 & 0 & 0.0 & & \\
\hline & $16-20$ & 0 & 0 & 0 & 0 & 0 & 0 & 0 & 0 & 0 & 0.0 & & \\
\hline Group 4 & Proximity to walls & 0 & 0 & 0 & 0 & 0 & 0 & 0 & 0 & 0 & 0.0 & 0 & 0.0 \\
\hline \multirow[t]{4}{*}{$\left(31-40 m^{2}\right)$} & $2-5$ & 0 & 0 & 0 & 0 & 0 & 0 & 0 & 0 & 0 & 0.0 & & \\
\hline & $5-10$ & 0 & 0 & 0 & 0 & 0 & 0 & 0 & 0 & 0 & 0.0 & & \\
\hline & $11-15$ & 0 & 0 & 0 & 0 & 0 & 0 & 0 & 0 & 0 & 0.0 & & \\
\hline & $16-20$ & 0 & 0 & 0 & 0 & 0 & 0 & 0 & 0 & 0 & 0.0 & & \\
\hline \multicolumn{2}{|c|}{ All plants size/distances } & 14 & 9 & 8 & 27 & 7 & 20 & 4 & 8 & 97 & 100 & 97 & 100 \\
\hline \multicolumn{2}{|l|}{ Percentage } & 14.4 & 0.5 & 8.2 & 27.8 & 7.2 & 20.6 & 4.1 & 8.2 & 100 & & & \\
\hline
\end{tabular}


Appendix 6.4.3. Shrubs, vines, and groundcover, sizes and distances from the building in all azimuths for 20-29 years old group of houses

\begin{tabular}{|c|c|c|c|c|c|c|c|c|c|c|c|c|c|}
\hline \multirow[t]{2}{*}{ Plant size $\left(\mathrm{m}^{2}\right)$} & \multirow[t]{2}{*}{ Distance (m) } & \multicolumn{8}{|c|}{ Plants azimuth } & \multirow[b]{2}{*}{ All } & \multirow[b]{2}{*}{$\%$} & \multirow[b]{2}{*}{ Total } & \multirow[b]{2}{*}{$\%$} \\
\hline & & $\mathrm{N}$ & $\mathrm{NE}$ & $\mathrm{E}$ & SE & $\mathrm{S}$ & SW & $\mathrm{W}$ & NW & & & & \\
\hline \multirow[t]{5}{*}{ Individual } & Proximity to walls & 5 & 3 & 1 & 13 & 0 & 3 & 5 & 10 & 40 & 5.2 & 418 & 54.8 \\
\hline & $2-5$ & 25 & 32 & 61 & 46 & 26 & 26 & 41 & 46 & 303 & 39.7 & & \\
\hline & $5-10$ & 17 & 3 & 16 & 3 & 0 & 6 & 13 & 13 & 71 & 9.3 & & \\
\hline & $11-15$ & 0 & 0 & 0 & 0 & 0 & 0 & 0 & 0 & 0 & 0.0 & & \\
\hline & $16-20$ & 0 & 0 & 0 & 2 & 0 & 2 & 0 & 0 & 4 & 0.5 & & \\
\hline Group 1 & Proximity to walls & 5 & 3 & 0 & 2 & 2 & 1 & 0 & 2 & 15 & 2.0 & 282 & 37.0 \\
\hline \multirow[t]{4}{*}{$\left(0-10 \mathrm{~m}^{2}\right)$} & $2-5$ & 30 & 25 & 33 & 26 & 36 & 10 & 26 & 30 & 216 & 28.3 & & \\
\hline & $5-10$ & 9 & 12 & 1 & 1 & 7 & 1 & 11 & 4 & 46 & 6.0 & & \\
\hline & $11-15$ & 0 & 0 & 0 & 0 & 0 & 0 & 4 & 0 & 4 & 0.5 & & \\
\hline & $16-20$ & 0 & 1 & 0 & 0 & 0 & 0 & 0 & 0 & 1 & 0.1 & & \\
\hline Group 2 & Proximity to walls & 0 & 4 & 0 & 1 & 0 & 0 & 2 & 2 & 9 & 1.2 & 53 & 6.9 \\
\hline \multirow[t]{4}{*}{$\left(11-20 m^{2}\right)$} & $2-5$ & 3 & 3 & 6 & 7 & 3 & 2 & 5 & 6 & 35 & 4.6 & & \\
\hline & $5-10$ & 1 & 3 & 0 & 1 & 1 & 0 & 0 & 1 & 7 & 0.9 & & \\
\hline & $11-15$ & 0 & 0 & 0 & 1 & 0 & 0 & 0 & 0 & 1 & 0.1 & & \\
\hline & $16-20$ & 0 & 1 & 0 & 0 & 0 & 0 & 0 & 0 & 1 & 0.1 & & \\
\hline Group 3 & Proximity to walls & 0 & 0 & 0 & 0 & 0 & 0 & 0 & 0 & 0 & 0.0 & 7 & 0.9 \\
\hline \multirow[t]{4}{*}{$\left(21-30 \mathrm{~m}^{2}\right)$} & $2-5$ & 0 & 1 & 0 & 3 & 0 & 0 & 2 & 1 & 7 & 0.9 & & \\
\hline & $5-10$ & 0 & 0 & 0 & 0 & 0 & 0 & 0 & 0 & 0 & 0.0 & & \\
\hline & $11-15$ & 0 & 0 & 0 & 0 & 0 & 0 & 0 & 0 & 0 & 0.0 & & \\
\hline & $16-20$ & 0 & 0 & 0 & 0 & 0 & 0 & 0 & 0 & 0 & 0.0 & & \\
\hline Group 4 & Proximity to walls & 0 & 0 & 0 & 0 & 0 & 0 & 0 & 0 & 0 & 0.0 & 3 & 0.4 \\
\hline \multirow[t]{4}{*}{$\left(31-40 \mathrm{~m}^{2}\right)$} & $2-5$ & 0 & 3 & 0 & 0 & 0 & 0 & 0 & 0 & 3 & 0.4 & & \\
\hline & $5-10$ & 0 & 0 & 0 & 0 & 0 & 0 & 0 & 0 & 0 & 0.0 & & \\
\hline & $11-15$ & 0 & 0 & 0 & 0 & 0 & 0 & 0 & 0 & 0 & 0.0 & & \\
\hline & $16-20$ & 0 & 0 & 0 & 0 & 0 & 0 & 0 & 0 & 0 & 0.0 & & \\
\hline \multicolumn{2}{|c|}{ All plants size/distances } & 95 & 94 & 118 & 106 & 75 & 51 & 109 & 115 & 763 & 100 & 763 & 100 \\
\hline \multicolumn{2}{|l|}{ Percentage } & 12.5 & 12.3 & 15.5 & 13.9 & 9.8 & 6.7 & 14.3 & 15.1 & 100 & & & \\
\hline
\end{tabular}


Appendix 6.4.4. Shrubs, vines, and groundcover, sizes and distances from the building in all azimuths for 30-40 years old group of houses

\begin{tabular}{|c|c|c|c|c|c|c|c|c|c|c|c|c|c|}
\hline \multirow[t]{2}{*}{ Plant size $\left(\mathrm{m}^{2}\right)$} & \multirow[t]{2}{*}{ Distance (m) } & \multicolumn{8}{|c|}{ Plants azimuth } & \multirow[b]{2}{*}{ All } & \multirow[b]{2}{*}{$\%$} & \multirow[b]{2}{*}{ Total } & \multirow[b]{2}{*}{$\%$} \\
\hline & & $\mathrm{N}$ & $\mathrm{NE}$ & E & SE & $\mathrm{S}$ & SW & $\mathrm{W}$ & NW & & & & \\
\hline \multirow[t]{5}{*}{ Individual } & Proximity to walls & 0 & 5 & 3 & 0 & 0 & 7 & 0 & 3 & 18 & 11.0 & 92 & 56.1 \\
\hline & $2-5$ & 10 & 8 & 9 & 1 & 15 & 5 & 15 & 7 & 70 & 42.7 & & \\
\hline & $5-10$ & 2 & 0 & 0 & 0 & 2 & 0 & 0 & 0 & 4 & 2.4 & & \\
\hline & $11-15$ & 0 & 0 & 0 & 0 & 0 & 0 & 0 & 0 & 0 & 0.0 & & \\
\hline & $16-20$ & 0 & 0 & 0 & 0 & 0 & 0 & 0 & 0 & 0 & 0.0 & & \\
\hline Group 1 & Proximity to walls & 0 & 2 & 0 & 0 & 0 & 0 & 0 & 0 & 2 & 1.2 & 55 & 33.5 \\
\hline \multirow[t]{4}{*}{$\left(0-10 \mathrm{~m}^{2}\right)$} & $2-5$ & 6 & 10 & 4 & 1 & 5 & 10 & 7 & 5 & 48 & 29.3 & & \\
\hline & $5-10$ & 2 & 1 & 0 & 1 & 1 & 0 & 0 & 0 & 5 & 3.0 & & \\
\hline & $11-15$ & 0 & 0 & 0 & 0 & 0 & 0 & 0 & 0 & 0 & 0.0 & & \\
\hline & $16-20$ & 0 & 0 & 0 & 0 & 0 & 0 & 0 & 0 & 0 & 0.0 & & \\
\hline Group 2 & Proximity to walls & 0 & 0 & 0 & 0 & 0 & 0 & 1 & 0 & 1 & 0.6 & 16 & 9.8 \\
\hline \multirow[t]{4}{*}{$\left(11-20 m^{2}\right)$} & $2-5$ & 3 & 1 & 3 & 3 & 3 & 0 & 1 & 1 & 15 & 9.1 & & \\
\hline & $5-10$ & 0 & 0 & 0 & 0 & 0 & 0 & 0 & 0 & 0 & 0.0 & & \\
\hline & $11-15$ & 0 & 0 & 0 & 0 & 0 & 0 & 0 & 0 & 0 & 0.0 & & \\
\hline & $16-20$ & 0 & 0 & 0 & 0 & 0 & 0 & 0 & 0 & 0 & 0.0 & & \\
\hline Group 3 & Proximity to walls & 0 & 0 & 0 & 0 & 0 & 0 & 0 & 0 & 0 & 0.0 & 1 & 0.6 \\
\hline \multirow[t]{4}{*}{$\left(21-30 m^{2}\right)$} & $2-5$ & 0 & 1 & 0 & 0 & 0 & 0 & 0 & 0 & 1 & 0.6 & & \\
\hline & $5-10$ & 0 & 0 & 0 & 0 & 0 & 0 & 0 & 0 & 0 & 0.0 & & \\
\hline & $11-15$ & 0 & 0 & 0 & 0 & 0 & 0 & 0 & 0 & 0 & 0.0 & & \\
\hline & $16-20$ & 0 & 0 & 0 & 0 & 0 & 0 & 0 & 0 & 0 & 0.0 & & \\
\hline Group 4 & Proximity to walls & 0 & 0 & 0 & 0 & 0 & 0 & 0 & 0 & 0 & 0.0 & 0 & 0.0 \\
\hline \multirow[t]{4}{*}{$\left(31-40 m^{2}\right)$} & $2-5$ & 0 & 0 & 0 & 0 & 0 & 0 & 0 & 0 & 0 & 0.0 & & \\
\hline & $5-10$ & 0 & 0 & 0 & 0 & 0 & 0 & 0 & 0 & 0 & 0.0 & & \\
\hline & $11-15$ & 0 & 0 & 0 & 0 & 0 & 0 & 0 & 0 & 0 & 0.0 & & \\
\hline & $16-20$ & 0 & 0 & 0 & 0 & 0 & 0 & 0 & 0 & 0 & 0.0 & & \\
\hline \multicolumn{2}{|c|}{ All plants size/distances } & 23 & 28 & 19 & 6 & 26 & 22 & 24 & 16 & 164 & 100 & 164 & 100 \\
\hline \multicolumn{2}{|l|}{ Percentage } & 1.3 & 1.5 & 1.0 & 0.3 & 1.4 & 1.2 & 1.3 & 0.9 & 9.0 & & & \\
\hline
\end{tabular}


Appendix 6.5. Shrubs, vines, and groundcover species for all categories

1. Garden shrubs

\begin{tabular}{|c|c|c|c|}
\hline No. & Botanical name & Common name & Total \\
\hline 1 & Acalypha hispida & Cat's tail & 1 \\
\hline 2 & Acalypha wilkensiana & Cat's tail & 10 \\
\hline 3 & Acalypha wilkensiana 'cultivar' & Cat's tail & 8 \\
\hline 4 & Acalypha wilkensiana 'mosaica' & Cat's tail & 3 \\
\hline 5 & Adenium obesum & Desert-rose & 2 \\
\hline 6 & Adianthum raddianum & Maidenhair & 4 \\
\hline 7 & Adianthum rapeziforme & Diamond maidenhair & 1 \\
\hline 8 & Adianthum tenerum & Black-stick maidenhair & 2 \\
\hline 9 & Aechmea fasciata & Silver-Vase & 5 \\
\hline 10 & Agave angustifolia & Variegated Caribbean Agave & 4 \\
\hline 11 & Agave neglecta & Wild century plant & 5 \\
\hline 12 & Aglaonema costatum & Spotted evergreen & 6 \\
\hline 13 & Aglaonema sp. & Chinese evergreen & 2 \\
\hline 14 & Allmanda cathartica & Golden trumpet & 3 \\
\hline 15 & Alocasia cucullata & Chinese taro & 4 \\
\hline 16 & Alocasia macrorrhiza & Elephant's ear & 18 \\
\hline 17 & Alocasia sp. & Alocasia & 1 \\
\hline 18 & Alocasia xamaazonica & Jewel Alocasia & 2 \\
\hline 19 & Aloe vera & Medicinal aloe & 1 \\
\hline 20 & Alpinia purpurata & Pink ginger & 12 \\
\hline 21 & Alpinia sanderae & Variegated Ginger & 3 \\
\hline 22 & Alpinia sp. & Ginger & 4 \\
\hline 23 & Anthurium andraeanum & Tail Flower & 1 \\
\hline 24 & Araucaria excels & Norfolk island pine & 6 \\
\hline 25 & Artemisia Scoparia & Redstem Wormwood & 2 \\
\hline 26 & Asparagus densiflorus Myersii & Sprengeri fern & 8 \\
\hline 27 & Asparagus plumosus & Emerald Fern & 3 \\
\hline 28 & Asplenium longissimum & Blechnum longicauda & 5 \\
\hline 29 & Asplenium nidus & Bird's nest fern & 52 \\
\hline 30 & Aster dumosus & Pillow aster & 1 \\
\hline 31 & Asystasia sp. & Asystasia & 1 \\
\hline 32 & Authorium plowmanii & Jungle bush & 10 \\
\hline 33 & Baphia nitida & Camwood & 14 \\
\hline 34 & Beaucarnea recurvata & Ponytail palm & 6 \\
\hline 35 & Begonia sp. & Begonia & 2 \\
\hline 36 & Berberis thunbergii & Japanese barberry & 1 \\
\hline 37 & Berringtonia asiatica & Sea putat & 1 \\
\hline 38 & Blechnum oriental & Centipede fern & 1 \\
\hline
\end{tabular}


39 Bougainvillea sp.

40 Breynia nivosa

41 Brunfelsia magnifica

42 Cactus sp.

43 Caladium bicolour

44 Caladium sp.

45 Calathea luthea

46 Calathea ornata

47 Calathea roseo picta

48 Calathea sp.

49 Calliandra emarginata

50 Callistemon citrinus

51 Cananga odorata

52 Canna indica

53 Casuarina sumatrana

54 Catharanthus roseus

55 Centella asiatica

56 Christella parasitica

57 Cleome sparkler

58 Clerodendrum calamitosum

59 Clerodendrum paniculatum

60 Clerodendrum sp.

61 Clerodendrum speciosissimum

62 Clerodendrum thomsoniae

63 Codiaeum variegatum

64 Cordyline fruiticosa

65 Cordyline terminalis

66 Cosmos caudatus

67 Costus speciosus

68 Costus woodsonii

Crossandra infundibuliformis

69 Lutea sp.

70 Ctenanthe setose

71 Cyathea latebrosa

72 Cycas circinalis

73 Cycas revoluta

74 Cycas rumphii

75 Cycas sp.

76 Cyperus alternifolius

77 Davallia denticulate

78 Dieffenbachia seguine

79 Dracaena fragrans
Bougainvillea

Coffee bush

Yesterday today and tomorrow 3

Cactus

Fancy Leaf Caladium

Caladium

Calathea

Calathea

Rose Painted Prayer Plant

Calathea

Powderpuff plant

Bottlebrush Tree

Ylang-ylang

Indian Shot

Casuarina

Rosy Periwinkle

Indian pennywort

Christella

Spider Flower

White Butterfly

Pagoda flower

Clerodendrum

Java Glorybower

Bleeding heart glory-bower

Garden croton

Cordyline

Cordyline 'red ribbons'

King salad

Crape ginger

Red button ginger

Firecracker flower

Ctenanthe

Tree fern

Queen sago palm

King sago palm

Malayan fern palm

Cycas

Umbrella plant

Rabbit's foot fern

Dumbcane

Corn Plant

\section{1}


Gold-dust dracaena

81 Dracaena marginata tricolor

Rainbow Tree

Gold Dust dracaena

83 Draceana reflexa

Draceana

Ribbon dracaena

84 Draceana sanderiana cultivar

85 Draceana sp.

86 Draceanea reflexa

87 Draceanea sanderiana

Draceana

Pleomele

Ribbon dracaena

88 Duabanga grandiflora

Duabanga

Golden dew-drop

89 Duranta gold

90 Duranta repen gold'

Duranta

Creeping burhead

Water Hyacinths

Flame Violet

Eugenia

Crown-of-thorns

Cactus

Pencil plant

Ali's umbrella

Benjamin fig

99 Ficus benjamina

Rubber tree

Indian laurel fig

Ficus

False agave

Cape jasmine

Daisy

Bird of paradise

Parrot's heliconia

Heliconia

Tropical hibiscus

Hibiscus

Homalomena

Hydrangea

Spider lily

Rose balsam

"True blue" impatiens

Needle flower

Needle flower

Needle flower

Needle flower

Needle flower

120 Ixora sunkist

121 Jasminum sambac

Jasmine 


\begin{tabular}{|c|c|c|}
\hline 122 & Jasminum sambac & Arabian jasmine \\
\hline 123 & Jasminum sp. & Jasmine 'bonsai' \\
\hline 124 & Jasminum sp. & Jasmine \\
\hline 125 & Jatropha integerrima & Peregrina \\
\hline 126 & Jatropha multifida & Jatropha tree \\
\hline 127 & Jatropha podagrica & Gout stick \\
\hline 128 & Jatropha sp. & Jatropha \\
\hline 129 & Juniperus chinensis & Chinese juniper \\
\hline 130 & Lagerstroemia indica & Crape myrtle \\
\hline 131 & Lantana camara & Yellow sage \\
\hline 132 & Licuala Grandis & Ruffled fan palm \\
\hline 133 & Loropetalum chinense & Carolina Midnight' loropetalum \\
\hline 134 & Malaviscus arboreus & Turk's cap \\
\hline 135 & Maranta sp. & Prayer Plant \\
\hline 136 & Medinilla myriantha & Malaysian Grapes \\
\hline 137 & Melaleuca bracteata & Black tea tree \\
\hline 138 & Melastoma malabathricum & Straits rhododendron \\
\hline 139 & Michelia alba & White Champaca \\
\hline 140 & Michelia champaca & Champaca tree \\
\hline 141 & Murraya paniculata & Orange jasmine \\
\hline 142 & Musa ornata & Flowering banana \\
\hline 143 & Mussaenda flava & Yellow mussaenda \\
\hline 144 & Mussaenda Philippica & Tropical dogwood \\
\hline 145 & Neomarica longifolia & Yellow walking iris \\
\hline 146 & Nephrolepis biserrata & Giant sword fern \\
\hline 147 & Nerium oleander & Oleander \\
\hline 148 & Nymphaea sp. & Water lily \\
\hline 149 & Opuntia sp. & Prickly pear cacti \\
\hline 150 & Orchid sp. & Orchid \\
\hline 151 & Orthosiphon stamineus & Cat's Whiskers \\
\hline 152 & Pandanus sanderi & Pandanus \\
\hline 153 & Pandanus sp. & Small Screwpine \\
\hline 154 & Pandanus sp. & Pandanus \\
\hline 155 & Pandanus tectorius & Pandanus palm \\
\hline 156 & Pedilanthus tithymaloides & Zigzag Plant \\
\hline 157 & Pereskia bleo & Rose cactus \\
\hline 158 & Phillodendron selloum & Lacy tree philodendron \\
\hline 159 & Philodendron erubescens & Blushing philodendron \\
\hline 160 & Philodendron sp. & Philodendron \\
\hline 161 & Philodendron xanadu & Split leaf philodendron \\
\hline 162 & Phyllanthus myrtifolius & Cyelon myrtle \\
\hline 163 & Pisonia alba & Lettuce tree \\
\hline
\end{tabular}


164 Pithecellobium dulce 'Variegated'

165 Platycerium sp.

166 Platycladus orientalis

167 Plectranthus scutellarioides

168 Plumbago auriculata

169 Plumeria rubra

170 Plumeria rubra cultivars

171 Podocarpus indicus

172 Pogonatherum paniceum

173 Polyscias crispatum

174 Polyscias guilfoylei

175 Polyscias guilfoylei

176 Polyscias scutellaria

177 Pseuderanthemum 'jessica'

178 Pseuderanthemum carruthersii

179 Pseudomussaenda flava

180 Pueraria phaseoloides

181 Rosa sp.

182 Rubia ornamentale

183 Saccharum officinarum

184 Sanchesia nobilis

185 Sanchezia speciosa

186 Sannseviera trifaciata "laurenti"

187 Schefflera arboricola

188 Schefflera arboricola

189 Spathiphyllum cannifolium

190 Spathiphyllum cannifolium

191 Streblus asper

192 Tabernaemontana cumingiana

193 Tabernaemontana divaricata

194 Talinum fruticosum

195 Tecoma stans

196 Tecomaria capensis

197 Thalia geniculata

198 Thalia geniculata

199 Thevetia peruviana

200 Torenia fournieri

201 Turnera ulmifolia

202 Typha latifolia

203 Vanilla planifolia

204 Yucca aloifolia

205 Yucca elephantipes
Madras thorn

Staghorn fern

Oriental arborvitae

Painted nettle

Forget-me-not

Frangipani

Frangipani

Podocarpus

Baby panda bamboo

Geranium-leaf aralia

Shield aralia

Geranium-leaf aralia

Shield aralia

Pseuderanthemum

Yellow-vein eranthemum

Yellow Mussaendada flava

Tropical Kudzu

Rose

Rubia

Sugarcane

Sanchesia

Fire Fingers

Mother-in-law's tongue

Umbrella tree

Dwarf Umbrella Tree

Peace lily

Spathiphyllum

Toothbrush tree

Crape gardenia

Crepe jasmine

Surinum purslane

Yellow trumpet bush

Cape honey suckle

Water canna

Fire-flag

Yellow oleander

Wishbone flower

Yellow alder

Bulrush

Vanilla orchid

Yucca

Adams needle 


\begin{tabular}{lllc}
206 & Yucca gloriosa & Spanish Dagger & 1 \\
207 & Yucca sp. & Adams needle & 2 \\
208 & Zamia furfuracea & Cardboard palm & 6 \\
209 & Zamioculcas zamiifolia & Aroid Palm & 10 \\
210 & Zingiber zerumbit & Shampoo ginger & 1 \\
\hline & Total & & 1247 \\
\hline
\end{tabular}

2. Palm shrubs

\begin{tabular}{lllc}
\hline No. & Botanical name & Common name & Total \\
\hline 1 & Bismarkia nobilis & Bismark palm & 3 \\
2 & Caryota mitis & Fish tail palm & 1 \\
3 & Cocos nucifera & Coconut & 3 \\
4 & Cyrtostachys lakka & Red Palm & 7 \\
5 & Cyrtostachys renda & Red Palm & 15 \\
6 & Dypsis lutescens & Yellow cane palm & 58 \\
7 & Hyophorpbe lagenicaulis & Bottle palm & 2 \\
8 & Johannesteijsmannia altifrons & Umbrella Leaf Palm & 1 \\
9 & Licuala grandis & Ruffled fan palm & 14 \\
10 & Licuala spinosa & Mangrove Fan Palm & 1 \\
11 & Phoenix roebelenii & Pygmy date palm & 1 \\
12 & Ptychosperma macarthurii & Macarthur palm & 21 \\
13 & Ravenala madagascariensis & Traveller's palm & 1 \\
14 & Rhapis excels & Lady palm & 36 \\
\hline & Total & & 164 \\
\hline
\end{tabular}

3. Bambu shrubs

\begin{tabular}{lllc}
\hline No. & Botanical name & Common name & Total \\
\hline 1 & Bambusa multiplex & Hedge bamboo & 6 \\
2 & Bambusa ventroicosa bamboo & Buddha Belly & 2 \\
3 & Bambusa vulgaris & Common banboo & 4 \\
4 & Phyllostachys sulphurea & Budddha's belly & 3 \\
\hline & Total & & 15 \\
\hline
\end{tabular}


4. Edible shrubs

\begin{tabular}{|c|c|c|c|}
\hline No. & Botanical name & Common name & Total \\
\hline 1 & Aloe vera & Medicinal aloe & 2 \\
\hline 2 & Alpinia galanga & Galangale & 14 \\
\hline 3 & Ananas comosus & Pineapple & 4 \\
\hline 4 & Andrographis paniculata & King of bitters & 1 \\
\hline 5 & Annona cherimola & Custard apple & 1 \\
\hline 6 & Annona reticulate & Custard apple & 2 \\
\hline 7 & Averrhoa bilimbi & Cucumber tree & 5 \\
\hline 8 & Barringtonia racemosa & Freshwater mangrove & 1 \\
\hline 9 & Caladium sp. & Caladium & 2 \\
\hline 10 & Capsicum annuum & Chilli & 1 \\
\hline 11 & Capsicum frutescens & Bird chilli & 6 \\
\hline 12 & Carica papaya & Papaya & 20 \\
\hline 13 & Centella asiatica & Indian pennywort & 1 \\
\hline 14 & Citras grandis Osbeck & Grape-fruit pomelo & 1 \\
\hline 15 & Citrofortunella microcarpa & Calamansi limes & 10 \\
\hline 16 & Citrus aurantifolia & Limes & 7 \\
\hline 17 & Citrus hystrix & Kaffir lime & 9 \\
\hline 18 & Cosmos caudatus & King salad & 2 \\
\hline 19 & Curcuma longa & Turmeric & 8 \\
\hline 20 & Cymbopogon flexuosus & Lemongrass & 20 \\
\hline 21 & Eurycoma Longifolia & Ali's umbrella & 1 \\
\hline 22 & Garcinia mangostana & Mangosteen & 3 \\
\hline 23 & Hylocereus undatus & Dragon fruit & 2 \\
\hline 24 & Ipomoea aquatica & Water Morning Glory & 1 \\
\hline 25 & Justicia gendarussa & Gandarusa & 1 \\
\hline 26 & Justicia Gendarussa linn & Black malabar nut & 1 \\
\hline 27 & Kaemferia galanga & Aromatic ginger & 1 \\
\hline 28 & Labisia pumila & Kacip Fatimah & 1 \\
\hline 29 & Lawsonia inermis & Henna & 2 \\
\hline 30 & Mangifera indica & Mango & 18 \\
\hline 31 & Manihot eesculenta & Tapioca & 8 \\
\hline 32 & Manihot eesculenta variegata & Tapioca & 2 \\
\hline 33 & Manilkara zapota & Brown Sugar Fruit & 6 \\
\hline 34 & Momordica charantia & Balsam apple & 1 \\
\hline 35 & Morinda citrifoli & Noni & 8 \\
\hline 36 & Moringa oleifera lam & Horseradish-tree & 3 \\
\hline 37 & Murraya koenigii & Karipulei & 16 \\
\hline 38 & Musa ornate & Flowering Banana & 1 \\
\hline 39 & Musa parasidiaca & Banana & 26 \\
\hline 40 & Musa sp. & Banana & 8 \\
\hline 41 & Nephelium lappaceum & Rambutan & 1 \\
\hline
\end{tabular}


42 Origanum vulgare

43 Orthosiphon stamineus

44 Pandanus odorus

45 Pheaomeria imperialis

46 Piper betle

47 Piper sarmentosum roxb

48 Polygonum minus huds

49 Psidium guajava

50 Psophocarpus tetragonolobus

51 Saccharum officinarum

52 Sauropus androgynus

53 Solanum melongena

54 Syzygium aqueum

55 Syzygium jambos

56 Tabernaemontana divaricata

57 Telosma cordata

58 Zingiber officinale

59 Zingiber sp.
Origano

Cat's whiskers

Fragrant leaves

Ginger flower

Betel leaf

Betel leaf

Kesum

Guava

Winged bean

Sugarcane

Star gooseberry

Tropical eggplant

Water apple

Rose apple

Ceylon jasmine

Tonkinese reeper

Ginger

Indian ginger

289

5. Garden vines

\begin{tabular}{lllc}
\hline No. & Botanical name & Common name & Total \\
\hline 1 & Allamanda cathartica & Golden trumpet & 2 \\
2 & Bauhinia kockiana & Red trailing bauhinia & 6 \\
3 & Clerodendrum sp. & Clerodendrum & 3 \\
4 & Clitoria ternatea & Blue pea vine & 1 \\
5 & Dracaena surculosa & Gold Dust dracaena & 1 \\
6 & Epipremnum aureum & Money plant & 3 \\
7 & Ficus pumila & Creeping Fig & 4 \\
8 & Quisqualis indica & Rangoon creeper & 16 \\
9 & Rosa sp. & Rose & 2 \\
10 & Scindapsus aureus & Money plant & 2 \\
11 & Tristellateia australasiae & Shower of gold climber & 2 \\
12 & Vallaris glabra & Bread Flower & 6 \\
\hline & Total & & 48 \\
\hline
\end{tabular}


6. Edible vines

\begin{tabular}{lllc}
\hline No. & Botanical name & Common name & Total \\
\hline 1 & Clitoria ternatea & Blue pea vine & 2 \\
2 & Piper betle & Betel leaf & 8 \\
3 & Piper sarmentosum roxb & Betel leaf & 1 \\
4 & Psophocarpus tetragonolobus & Winged bean & 4 \\
5 & Vigna sesquipedalis & Long beans & 1 \\
6 & Vitis californica & Grapevines & 1 \\
\hline & Total & & 17 \\
\hline
\end{tabular}

7. Groundcover

\begin{tabular}{lllc}
\hline No. & Botanical name & Common name & Total \\
\hline 1 & Cuphea hyssopifolia & Cuphea & 9 \\
2 & Hemigraphis alternata & Red Flame Ivy & 6 \\
3 & Pandanus pygmaeus & Small Screwpine & 3 \\
4 & Phyllanthus myrtifolius & Cyelon myrtle & 4 \\
5 & Piper sarmentosum roxb & Betel leaf & 1 \\
6 & Pogonatherum paniceum & Baby Panda Bamboo & 1 \\
7 & Portulaca grandiflora & Japanese rose & 2 \\
8 & Rheo discolour & Moses-in-the-cradle & 11 \\
9 & Selaginella sp. & Spikemoss & 3 \\
10 & Wedelia trilobata & Creeping Daisy & 2 \\
11 & Zephyranthes candida & Fairy lily & 2 \\
\hline & Total & & 44 \\
\hline
\end{tabular}

